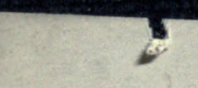


20. 442

BDSCENCES LHBFARY
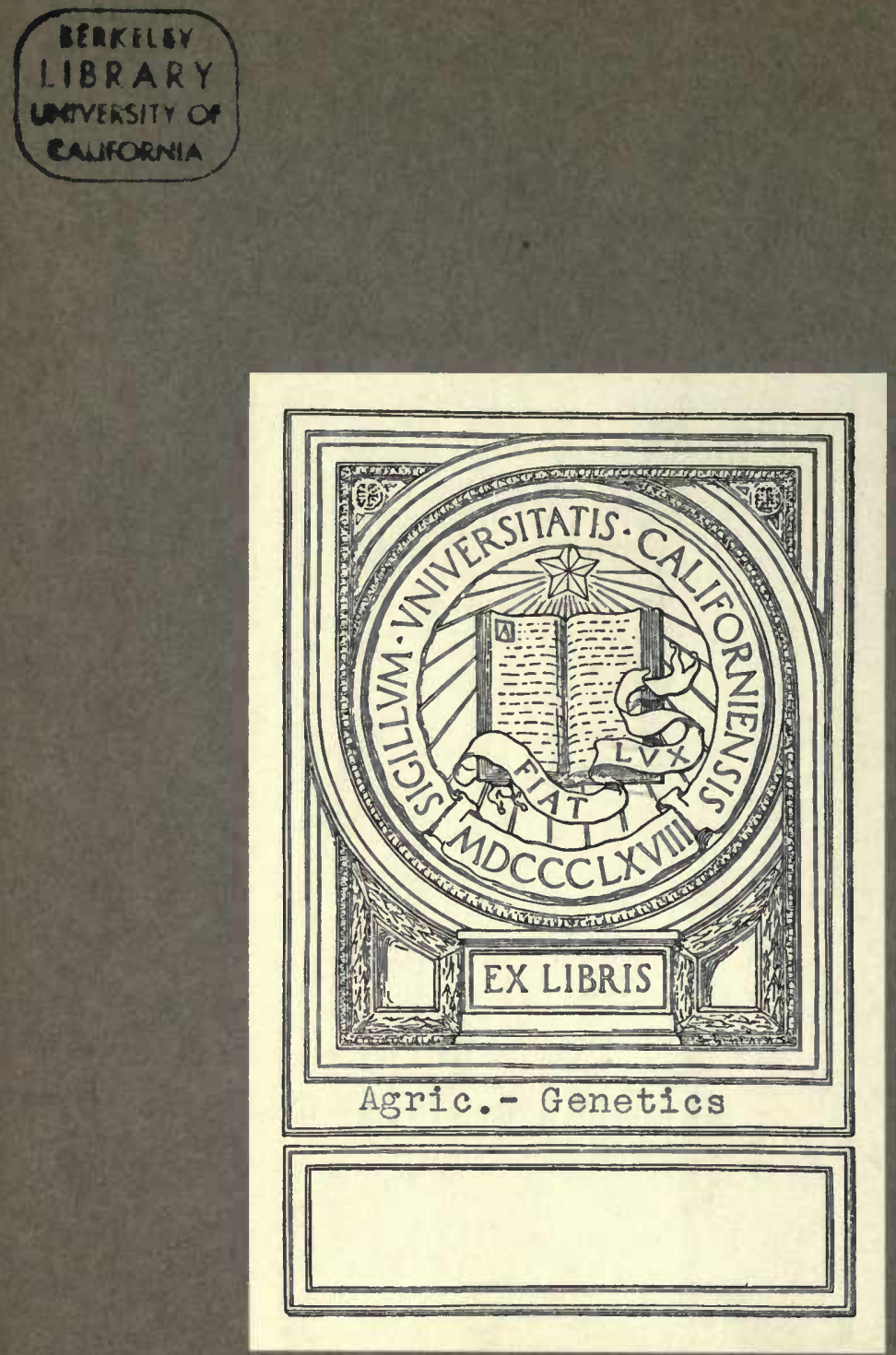
$6 x^{2}$ 



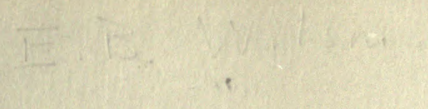




$$
\begin{aligned}
& \text { QH605 } \\
& \text { W } 47 \\
& \text { V.1-8 } \\
& \text { B105 }
\end{aligned}
$$

Agric. - Genetics 
Studies on chromosomes

1. The behavior of the idiochromosomes in Hemiptera.

II. The paired Microchromosomes, idlochromosomes and heterotropic. chromosomes in Hemiptera.

III.The sexual differences of the Chromosomegroups in Hempitera, with some considerations of the determination and inheritance of sex.

IV. The "accessory" chromosome in Syromastes and Pyrrochoris with a comparative review of the types of sexual differences of the chromosome groups.

V. The chromosomes of Metapodius, a contribution to the hypothesis of the genetic continuity of chromosomes.

VI. A new type of chromosome combination in Aepirase. Metapodius.

VII. A review of the chromosomes of Nezara; with some more general considerations.

VIII. Observations on the maturation-phenomena in certain Hempitera and other forms with considerations on synapsis and reduction. 
etrrestrob

Bemosomomilo no aplbutre

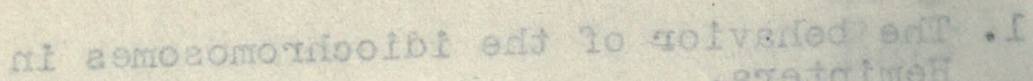
- Brejंcisteg

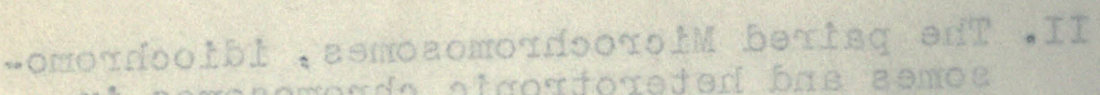

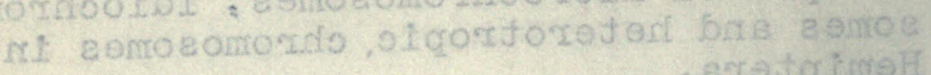
- Bxosqlimel

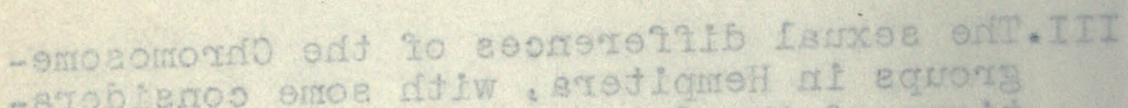

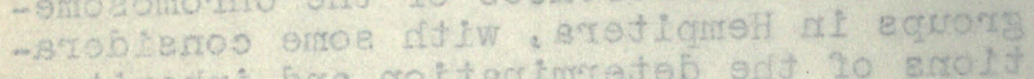

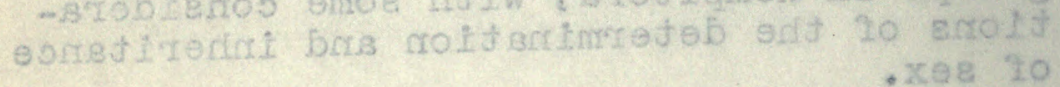

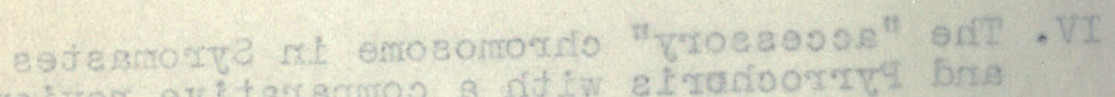

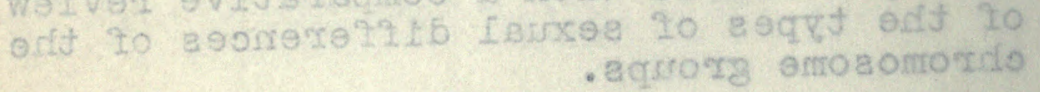

-xdintroo e aulbogstelt io aemoaomorido edT .V - noo oljerreg edt Zo elaediogra edt of nolt - aemoeomondo 20 ktherrith

nl noltsnidmos enrozomords 20 equj wen A .IV

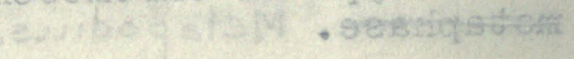

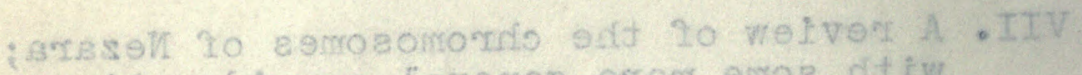

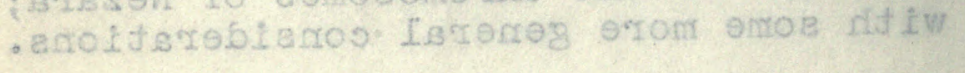

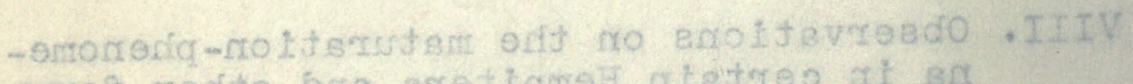
amrol resito brra sqejlqmer aletroo mi sR

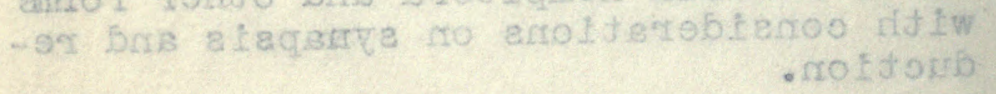




\section{With the complimants of}

EDM. B. WILSON, COLUMBIA UNIVERSITY, NEW YORK.

\section{STUDIES ON CHROMOSOMES}

THE BEHAVIOR OF THE IDIOCHROMOSOMES

IN HEMIPTERA

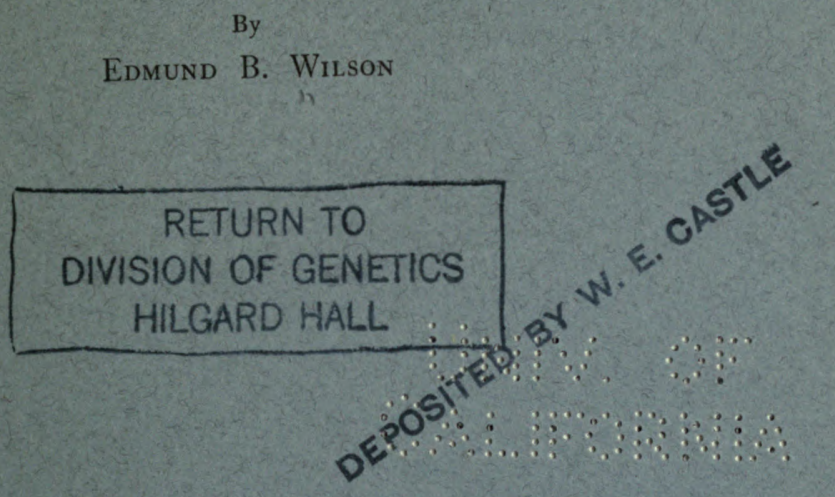

REPRINTED FROM

THE JOURNAL OF EXPERIMENTAL ZOÖLOGY

Volume II

No. 3

BALTIMORE, MD., U. S. A. 
QHileos

W 47

Geuctis

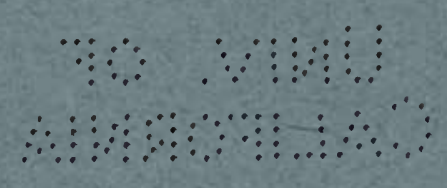




\section{STUDIES ON CHROMOSOMES.}

\section{THE BEHAVIOR OF THE IDIOCHROMOSOMES IN HEMIPTERA. ${ }^{1}$}

BY

EDMUND B. WILSON.

With 7 Figures.

In studying the spermatocyte-divisions in Lygæus turcicus and Cœnus delius, and afterward in several other genera of Hemiptera, my attention was directed to the fact that the number of chromosomes appeared to vary, polar viçws, of the ex̧uatorial plate showing sometimes seven chromosumes; sônetimes eight (cf. Figs. $1 b, \mathrm{i} i, 2 a, 2 i, 3^{d}, 3 f$, etc.). Mont zome tis his extensive comparative paper of I90I, describes and figures a similar variation in a number of cases, including Cœnus delius and Euschistus tristigmus ('OI, I, pp. I6I, I66), and in the latter case considered it as a result of variations in the synapsis of the two "chromatin nucleoli" which he supposed might either conjugate to form a bivalent body before the first division (in which case this division

1This paper is based on a study of some very fine series of sections of the testes of certain Hemiptera, prepared six or eight years ago by Dr. F. C. Paulmier, in connection with his valuable paper on the spermatogenesis of Anasa tristis ('99). Part of the original Anasa sections, with a number of series of the testes of some other insects, were given to the cytological cabinet of the Columbia laboratory at that time; some of the best of the remainder were subsequently loaned to Mr. Sutton, and others to Dr. Dublin, for comparison with their work on the spermatogenesis of other forms. Certain inconsistencies in the literature relating to the accessory chromosome and the microchromosomes or "chromatin-nucleoli" led me to re-examine the preparations of Anasa and some of the other genera, which yielded some new and interesting conclusions in the case of Anasa, and also of Alydus, Lygæus and Cœnus. Dr. Paulmier being preoccupied with other lines of work did not find it practicable again to take up his cytological studies, and he was generous enough to give me, for the laboratory, his entire set of preparations, comprising, in addition to the slides already given or loaned, serial sections of more than a hundred testes representing upward of twenty genera of Hemiptera and other insects. A typical series of the Hemiptera from which these testes were taken had been identified by the eminent specialist, Mr. P. R. Uhler. Much of this material is admirably fixed, sectioned and stained, and the best preparations are a model of technical excellence, showing especially the chromosomes of the spermatogonial and spermatocyte divisions with a clearness and brilliancy comparable with that of the best Ascaris preparations. The 
was described as showing but seven chromosomes) or might remain separate during this division (in which case eight separate chromosomes appear). I soon found, however, that in Lygæus and Cœnus whole cysts differed in this respect, all of the cells of a given cyst constantly showing one number or the other. With this is correlated the fact that in the anaphases of the second division no accessory chromosome in the usual sense of the term is present, all the spermatid-nuclei receiving the same number of chromosomes, namely, seven, which is half the spermatogonial number in both species. Further study conclusively showed that in both of the species the cells with eight chromosomes were primary spermatocytes undergoing the first maturation-division, while those with seven were the secondary spermatocytes undergoing the second division. Of this fact no doubt can exist, since the secondary spermatocytes are much smaller than the primary ones, the spindles are shorter, the chromosomes only half as large, the metaphase-figuresarẹ of ten found in the same cysts with the characteristic late anaphases and telophases, and all the stages of both

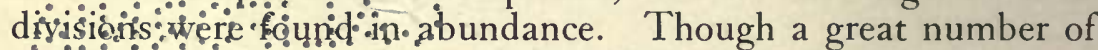
division-figures have been examined, I have never seen seven chromosomes in the first division in any of the forms examined; and though I will not deny that Montgomery may be correct in the statement that such forms occur, I believe he was misled on

most successful preparations are from material fixed with strong Flemming's fluid, and stained with iron hæmatoxylin followed by long extraction, in some cases followed also by counter staining with Congo red or orange G. These show the cytoplasm completely decolorized, the chromosomes intensely black, and with outlines of such regularity and sharpness that the most careful camera drawings give the appearance of being schematized. A few very fine series were stained with Zwaardemaker's saffranin (which gives a splendid transparent stain). Many others were fastened to the slide unstained, and some of these I have stained with saffranin and gentian violet (the method recommended by Montgomery) which have given very valuable control results, especially in regard to the accessory chromosome and plasmosome which in the earlier growth stages are not well differentiated by the hæmatoxylin. I am much indebted to Dr. Paulmier's generosity in placing at my disposal this valuable material, to which I have since added many new preparations of my own.

In a subsequent paper I shall describe the results of a re-examination of some of the maturation phenomena in Anasa and Alydus, in both of which there is demonstrative evidence that the accessory chromosome is not the small central chromosomeor microchromosome ("chromatin-nucleolus" of Montgomery), as Paulmier supposed in the case of Anasa, but the odd or peripheral one, precisely as Gross ('o4) has recently described in Syromastes. While looking over some of the other species for the sake of comparison my attention was directed, first in the spermatogenesis of Lygæus turcicus and Cœnus delius and afterward in that of Euschistus, Podisus and other forms, to the phenomena which form the subject of the present paper. 
this point by failing in some cases to distinguish between the two divisions.

On tracing out the history of the two divisions step by step, decisive proof was obtained that the apparent reduction in number is brought about in the period immediately following the final anaphase of the first division (which coincides with the earliest prophase of the second division) by a conjugation of two unequal chromosomes that occupy the center of the equatorial plate in the first division and evidently correspond to some of the forms designated by Montgomery as "chromatin-nucleoli." This process can be determined with certainty, owing to the fact that in all of the species, with a single exception, one of the two conjugating chromosomes is much smaller than the others, while in Lygæus both are much smaller, and they are very unequal in size. The central dyad of the second division is therefore asymmetrical, one of its constituents being in Lygæus not less than five or six, and in Cœnus not less than two or three, times the bulk of the other. The two unequal constituents of this dyad are then immediately separated again in the ensuing division in such a manner that in both species one half the spermatids receive the smaller, one balf the larger, moiety of the central chromosome (or dyad) of the second division. An essentially similar process was ultimately, found to occur in Euschistus fissilis, in another undetermined species of the same genus, in Brochymena, Nezara, Podisus and Trichopepla. The first four of these show the same chromosomenumbers as in Lygæus and Cœenus. In Podisus the number is in each division one more than in the corresponding divisions of the other genera (i.e., respectively 9 and 8 instead of 8 and 7 , while the spermatogonial number is 16 instead of 14$){ }^{1} \quad$ Nezara differs from the other genera in the fact, which is of importance for a comparison with such forms as Anasa or Alydus, that the two chromosomes which undergo conjugation after the first division are of equal size; so that in this form the two classes of spermatids are indistinguishable by the eye. Since the eight species I have

\footnotetext{
${ }^{1}$ In several of these cases the numbers do not agree with those given by Montgomery ('OI, I). I believe this observer to have been misled by the fact, which he also observed in some cases, that the first division shows one more than half the spermatogonial number of chromosomes; and it is easy to mistake the latter number owing to the fact that the larger spermatogonial chromosomes often show a more or less marked constriction in the middle. Slightly oblique views of the late metaphase, when the chromosomes are double, may also readily give an erroneous result.
} 
examined represent two different families of Hemiptera (Pentatomidx and Lygæidæ) the idiochromosomes will probably be found to be of wide occurrence in the group. ${ }^{1} \quad$ The only other case known to me in any higher plant or animal of the unequal division of a chromosome (or chromatin-body) in karyokinesis occurs in Tingis clavata, regarding which Montgomery states that one of the chromosomes of the first division "very frequently is seen to be characterized in having its two components of very unequal volume" ('OI, 2, p. 262). This author also observed a considerable number of cases in which the "chromatin nucleoli" are unequal in the rest stage of the spermatogonia, and he describes some forms in which a similar condition appears in the growth-period of the spermatocytes (e. g., in Trichopepla, Peribalus and Euschistus tristigmus). In the last-named species he found that a separation of the two unequal "chromatin nucleoli" takes place in the second mitosis ('OI, I, pp. I6I, I62), but expressly states that they are not joined together in the equatorial plate (op. cit., p. I62). It is evident from Montgomery's brief description that this phenomenon is similar to, and probably identical with, the one that forms the subject of this.paper.

\section{TERMINOLOGY.}

Since confusion may readily arise in the terminology, I wish to define clearly the terms that will be employed throughout this paper and its successors. I shall apply the term "chromosome" to each coherent chromatin-mass, whatever be its form, mode of origin or valence, which as such enters the equatorial plate. In the case of compound or plurivalent chromosomes ("tetrads" or "dyads") McClung's term "chronatid" may conveniently be applied to each of their univalent constituents. I may call attention, in connection with this, to the fact that the valence of chromosomes cannot be determined by mere inspection of their form. In many Hemiptera, for example, the chromosomes of the first maturation-division frequently show a dyad-like or dumb-bell shape (typically the case, for example, in Euschistus, Lygxus or Cœnus) even though in earlier stages they are plainly quadripar-

\footnotetext{
'Since writing the above I have found the idiochromosomes in several additional genera. In Mineus they are only slightly unequal, in Murgantia nearly as unequal as in Lygæus. Nezara, Mineus, Brochymena, Euschistus, Murgantia and Lygæus thus show a progressively graded series of stages in the size-differentiation of this peculiar pair of chromosomes.
} 
tite; and such dyad-like forms, agreeing both in mode of origin and in fate with actual tetrads, may occur in the same equatorial plate with obviously quadripartite forms ( $c f$. Fig. 2e). Conversely it will be shown beyond that bivalent and univalent chromosomes occurring in the same equatorial plate may exactly agree in form, though having a wholly different mode of origin.

The purely descriptive term "idiochromosomes" (peculiar or distinctive chromosomes) will be applied to the two chromosomes, usually unequal in size, which, as stated above, undergo a very late conjugation and subsequent asymmetrical distribution to the spermatid-nuclei. These bodies, as already stated, are identical with some of those to which Montgomery ('OI, 'O4) has applied the term "chromatin nucleoli." This use of the latter term is, however, undesirable, since the accessory chromosome also appears in the growth-period (of Orthoptera and some Hemiptera) in the form of a chromatin-nucleolus. I shall, therefore, employ the latter term in a broader sense to designate any compact deeply staining chromatin-mass, present in the resting nucleus, which afterward contributes to the formation of the chromosomes. When, as in case of the accessory chromosome, or the idiochromosomes, such a chromatin-nucleolus represents a single chromosome or pair of chromosomes it may conveniently be called a "chromosome-nucleolus"; but I think this term should be restricted to the resting nuclei and cannot appropriately be applied to the corresponding chromosome of the division-stage. Especially large or small chromosomes may be designated as "macrochromosomes" or "microchromosomes," irrespective of their behavior.

\section{DESCRIPTIVE.}

In the following account Lygæus and Cœnus will be taken as types, a brief comparison of the other forms being added. Some of the latter - especially Brochymena and Nezara-present features of peculiar interest which I hope to make the subject of a special study hereafter.

\section{The Maturation Divisions.}

Lygæus and Cœenus show an extremely close agreement in the general history of the chromosome-group, and especially in the behavior of the idiochromosomes; though the earlier history of 
these bodies shows a more primitive condition in the former genus. I have followed their behavior in the early stages less completely in Euschistus and Podisus, but their behavior during the maturation-divisions in these forms is closely similar to that of the others and leads to an exactly similar result. Lygæus is in some respects the most favorable of all these species owing to the remarkable disparity in size between the idiochromosomes, and to the fact that both are so much smaller than the other chromosomes as to admit of their immediate identification at every period.

In all the species, the chromosomes show distinct and constant size-differences. A largest chromosome or macrochromosome may be distinguished in all, and in most cases a second largest; and in all, the small idiochromosome is the smallest of the group and typically lies near the center of the equatorial plate. (Figs. $\mathrm{I} b, 2 c, 2 d, 3^{a}, d, f$, etc.) It is difficult to be sure of the size-differences in case of the other chromosomes, owing to variations in form and position, which produce various degrees of foreshortening. In all the forms, with the exception of Nezara, the larger chromosomes of the first division are typically arranged in an irregular ring within which lie the two idiochromosomes, side by side, but always quite separate (Figs. I $b, 2 a, 3 a, d$, j, etc.). This grouping is apparently invariable in Lygæus, but in Cœnus and Euschistus the larger idiochromosome frequently lies in the outer ring (Figs. $2 b, 3 e)$. In Lygaus the two idiochromosomes, within the ring, are always much smaller than any of the outer ones, and the smaller is so minute that at first sight I mistook it for a centrosome. ${ }^{1}$ In Cœnus both the idiochromosomes are relatively larger than in Lygæus, and their inequality of size is less striking (Fig. 2, $a, b, g, b$ ). The larger one is about equal in size to the smallest of the peripheral chromosomes and hence cannot. be certainly distinguished when it lies in the outer ring (Fig. 2b). In both species an equatorial plate occasionally occurs in which nine chromosomes clearly appear (Figs. I $d, 2 c)$, but this is exceptional, and I have never found a spindle showing this body in division. The presence of this additional chromosome is probably due to a failure of synapsis between two of the spermatogonial chromosomes which normally conjugate to form a bivalent body, and it is evidently to be regarded as an abnormal condition.

${ }^{1} C f$. Montgomery's Fig. 105, of Corizus, 'or, 1. 

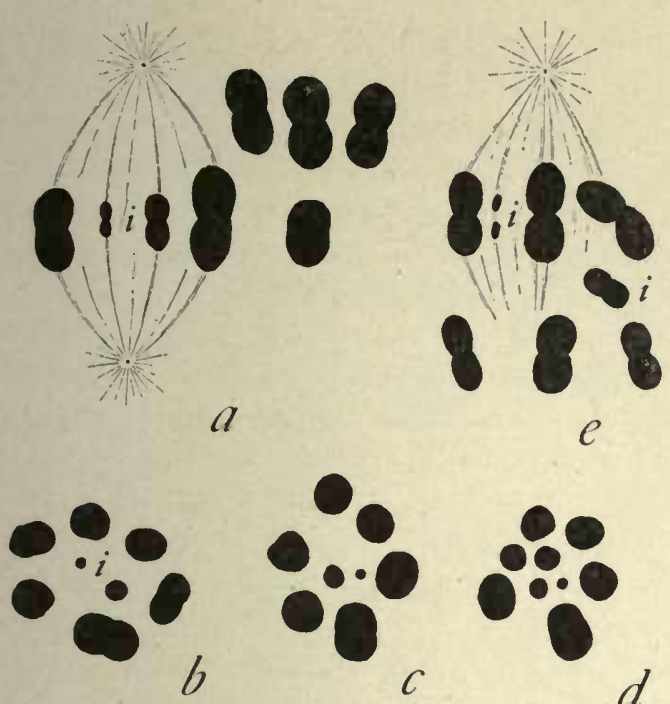

e
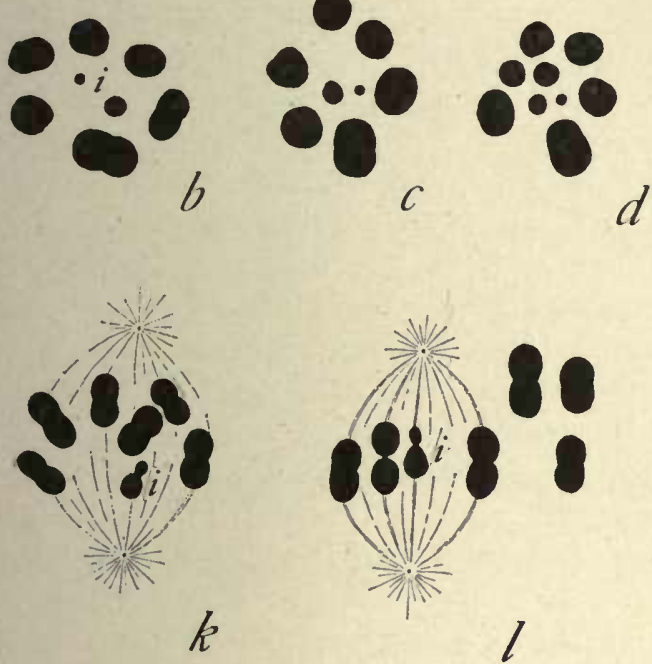

Frg. I. $^{1}$

Lygaus turcicus. $a, e$, metaphase of first division-in the first two figures several of the chromosomes are represented out of their natural positions at one side; $b, c$, normal metaphase-groups in polar view, first division; $d$, abnormal form with nine chromosomes; $f$, the idiochromosomes and two others, in early anaphase, first division; $g, h$, daughter-groups, late anaphase first division, from the same spindle; $i, j$, equatorial plates of second division, at the metaphase, in polar view; $k$, prophase of second division, showing all the chromosomes just before taking up their definitive positions; $l$, metaphase of second division - three of the chromosomes drawn out of position at one side; $m$, separation of the idiochromosomes, second division; $n, o$, daughter-groups, late anaphase of second division, from the same spindle.

${ }^{1}$ All of the figures were drawn as carefully as possible with the camera, a $\frac{1}{12}$ oil immersion, and compensation ocular 12 (Zeiss), enlarged $2 \frac{1}{2}$ diameters with a drawing camera, carefully corrected by renewed comparison with the objects and then reduced in the engraving to one-half. At such an enlargement some error is unavoidable, but great care has been taken to represent the chromosomes 
In side views of the spindles both species usually show the chromosomes of a symmetrical dumb-bell shape (Figs, I $a, e, 2 d$ ), though one or more of them may appear quadripartite, as is especially common in case of the largest one or macrochromosome (Fig. 2b,e). In both forms all of the eight chromosomes are symmetrically divided in the first mitosis (Figs. If, $2 e$, f), giving rise to two exactly similar daughter-groups of eight chromosomes each (Figs. I $g, b, 2 g, b$ ). The rate at which the daughterchromosomes separate varies widely in different cases. Frequently the idiochromosomes lead the way in the march toward the poles and may be widely separated at a time when one or more of the larger chromosomes are only just separating (Fig. If), while the macrochromosome often lags behind the others (Fig. 2e); but now and then a spindle shows the reverse condition, the small idiochromosome being the slowest of the group. In the end the daughter-chromosomes come to lie at the same level, and in the final anaphase are drawn more closely together. At this period the grouping of the chromosomes is exactly the same in the two species, the two idiochromosomes lying close together and closely surrounded by a ring formed by the six larger chromosomes. In spindles that lie vertically or slightly obliquely the two daughtergroups may in both species be seen, with the greatest clearness, to be exact duplicates of each other, proving beyond doubt the equal division of all of the eight chromosomes of the first mitosis (Figs. $\mathrm{I} g, h, 2 g, b)$, and the size-relations of the chromosomes persist without noticeable change. In Lygæus at this period the two idiochromosomes are still as a rule clearly separated; in Cœnus this may be the case, but they sometimes lie in close contact, already forming a dyad almost identical in appearance with the central unequal dyad of the second mitosis (Fig. $2 b$ ).

as accurately as possible, and none of the figures are schematized in this respect, except that in a few cases one or more of the chromosomes have been drawn out of their natural positions in order to avoid confusion in the figures. It should be noted that in the division-stages there is some variation in the actual size of the chromosomes, and this is more or less exaggerated in the figures owing partly to slight differences in position, which cause foreshortening in various degrees, and partly to differences in form (different degrees of elongation of the chromosomes cause corresponding variations in thickness as seen in polar view). No attempt has been made to represent the minuter details of the spindlefibers or asters, though the figures are but slightly schematized in this respect. Some of the stages of the growth-period (especially stages $b, d$ and $f$ ) are difficult to represent adequately in pen drawings: though $I$ have attempted to show them as accurately as possible.

In all the figures the idiochromosomes are marked $i$, the plasmosome $p$. 
In the stage that immediately follows, the chromosomes for a brief period become so crowded that their exact changes cannot be followed. In slightly later prophases of the second division, which follows without a pause, the chromosomes again spread apart,
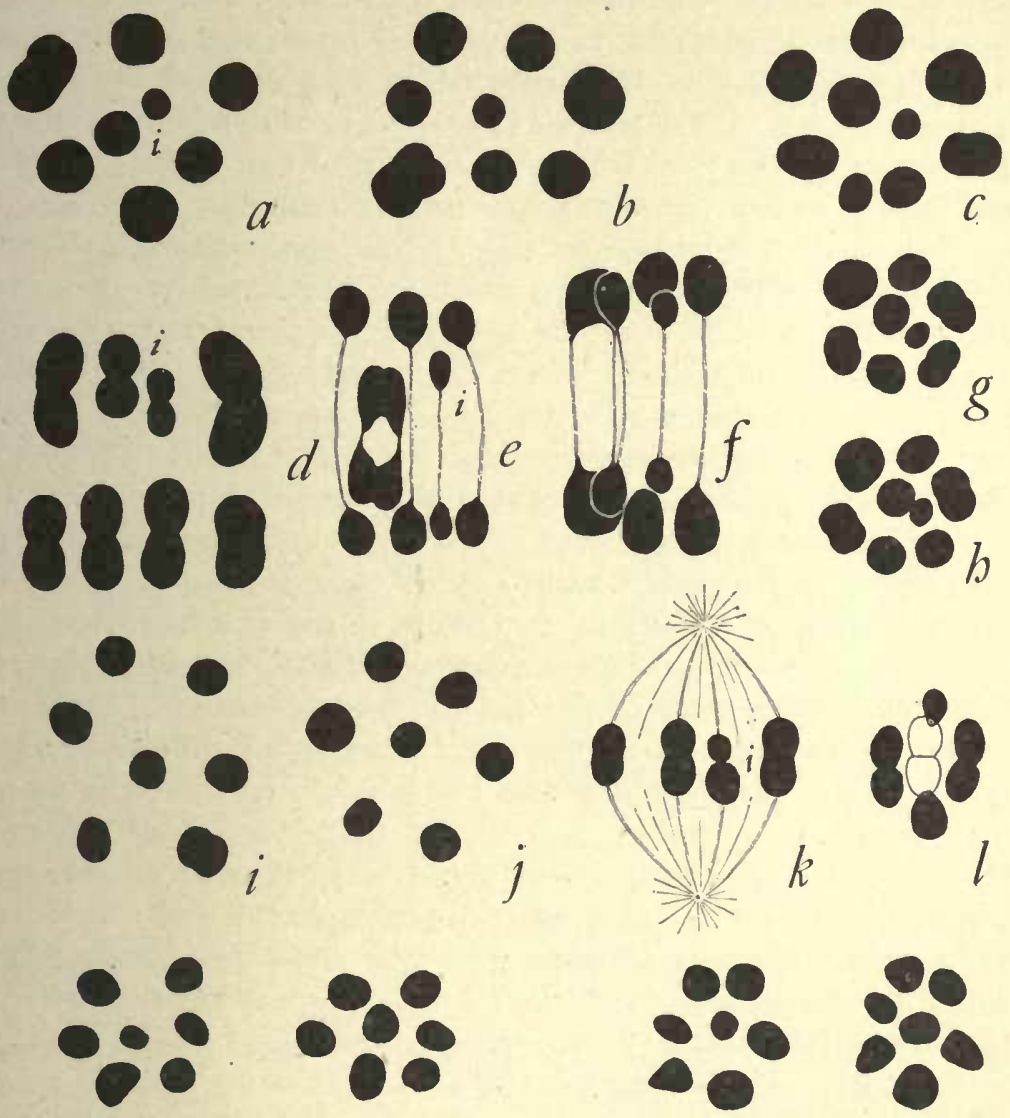

$m$

$n$

0

$p$

Fig. 2.

Cœnus delius. $a, b$, normal equatorial plates, metaphase of first division; $c$, abnormal form with nine chromosomes; $d$, the entire chromosome-group, first division metaphase, in side view, the upper four in their natural position; $e, f$, anaphases in side view showing in each case the idiochromosomes and three others; $g, h$, daughter-groups, late anaphase of first division, from the same spindle; $i, j$, equatorial plates, metaphase of second division, polar view; $k$, metaphase of second division; $l$, later metaphase, separation of the idiochromosomes; $m, n-0, p$, two pairs of daughter-groups, late anaphase, second division, in each case from the same spindle. 
and it may now be seen, even before the equatorial plate is formed, that the two idiochromosomes, retaining their characteristic sizerelations, have conjugated to form an asymmetrical dyad, which shows the most striking contrast to the six other dyads, all of which have a symmetrical dumb-bell shape (Fig. $\mathrm{I} k$ ). The seven dyads are now drawn into the equatorial plate, the asymmetrical one invariably lying at the center of a ring formed by the six symmetrical ones (Figs. I $l, m, 2 k, 3 b$ ). These dyads place themselves with their long axes parallel to that of the spindle, so that when seen in polar view they present a circular or more or less ovoidal outline; if they lie in a slightly oblique position, as is frequently the case, they may give a bipartite appearance. Since in polar view the small idiochromosome lies above or below the large one it is usually invisible, and hence only the larger one appears at the center of the equatorial plate (Figs. $1, j, 2 i, j$ ). In the early metaphase the chromosomes, especially in Conus, are often rather widely separated, so that the equatorial plate may be nearly or quite as wide as in the first division ( $c f$. Figs. $2 a, b, 2 i, j$ ). Such figures might at first sight readily be mistaken for those of the first division, but without exception, in my material, both the chromosomes and the cell-bodies of the 7-chromosome cells are much smaller than those of the 8-chromosome ones; and the completeness of the series and the great number of division-figures that I have had under observation precludes, I think, the possibility of error on this point.

In the ensuing division each of the dyads draws apart into two spheroidal single chromosomes, the peripheral ones dividing equally, while the idiochromosome-dyad separates into its two unequal constituents-invariably, I believe, leading the way in the division (Figs. I $m, 2 l, 3 c, g, k$ ). Owing to this fact the unequal division of the central dyad may be seen with unmistakable clearness. In polar or slightly oblique view of the late anaphases, when both daughter-groups are visible, the asymmetrical result may plainly be seen. In such figures the two daughter-groups show a most striking contrast to those of the first division, being no longer duplicates of each other, and both showing seven instead of eight chromosomes. Each daughter-group shows, as in the metaphase-group, a ring of six larger chromosomes (now single spheroidal bodies) within which lies at one pole the smaller, at the other pole the larger, of the idiochromosomes (Figs. I $n, 0,2 \mathrm{~m}$, 
$n, o, p)$. Each spermatid-nucleus thus receives seven chromosomes, one-half the spermatogonial number, and no accessory chromosome, in the usual sense of the word, is present; but the spermatids nevertheless consist of two groups, equal in number, one of which contains the smaller, the other the larger of the idiochromosomes. In the mature spermatozoa I have not been able to detect any corresponding difference.

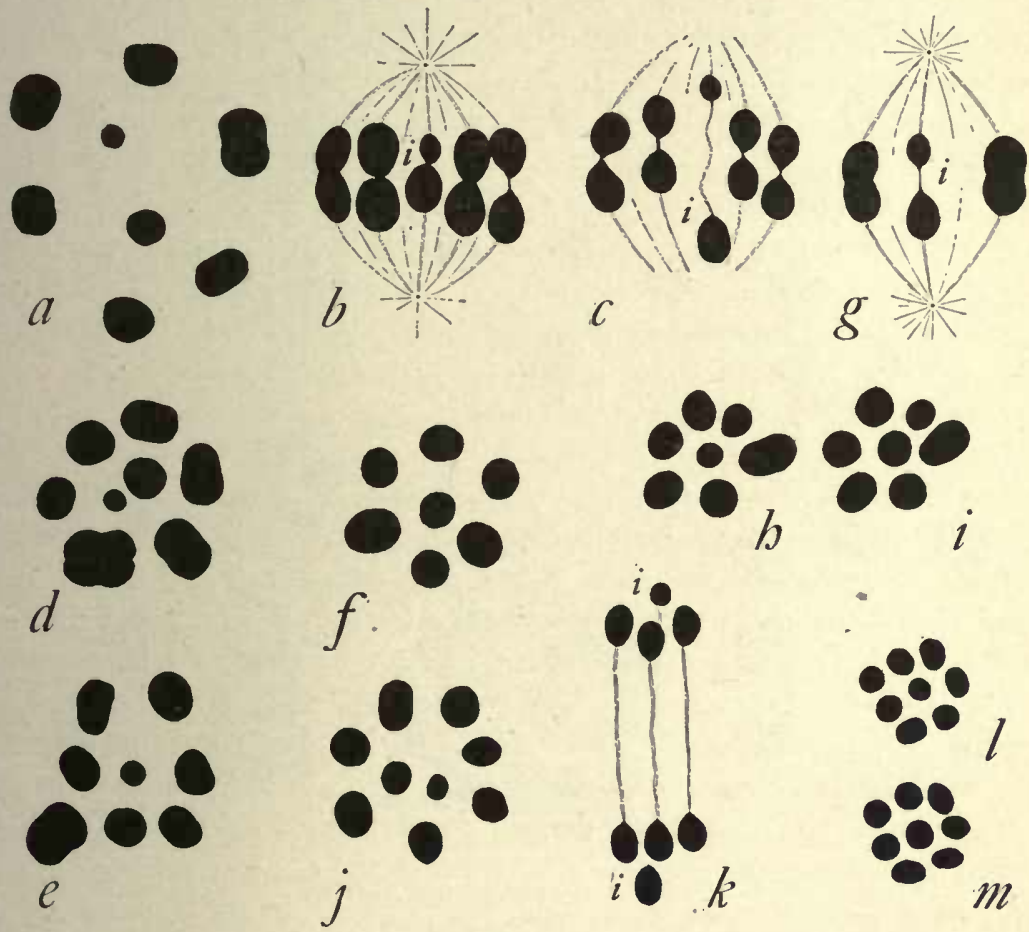

Fig. 3 .

Euschistus fissilis, Euschistus sp., Podisus spinosus. $a-c$, Euschistus fissilis. $a$, metaphasegroup, first division; $b, c$, metaphase-figure, and carly anaphase in side view, second division. $d-i$, Euschistus sp.; $d, e$, metaphase groups, first division; $f$, metaphase-group, second division; $g$, second division, side view; $h, i$, daughter-groups, late anaphase of second division, from the same spindle; $j-m$, Podisus spinosus; $j$, metaphase-group, first division; $k$, late anaphase, second division; $l, m$, daughter-groups from the same spindle, late anaphase, second division.

In Euschistus, Brochymena, Podisus and Trichopepla the facts are, with a few variations of detail, essentially similar. In both species of Euschistus and in Brochymena the number of chromo- 
somes agrees with that of Lygaus, being I 4 in the spermatogonia, eight in the first spermatocyte-division, and seven in the second, and their grouping is similar (Figs. 3, 7), save that in Brochymena, alone among all the forms, the small idiochromosome frequently lies in the outer ring (Fig. $7 k$ ). Podisus differs only in the fact that the numbers are respectively 9 and 8 (Figs. 3j, $l, m$ ), while the spermatogonial number is 16 instead of 14 (Fig. $5^{k}$ ). Trichopepla is a puzzling case which I have not yet fully cleared up. The daughter-groups of the second division show 7 chromo-

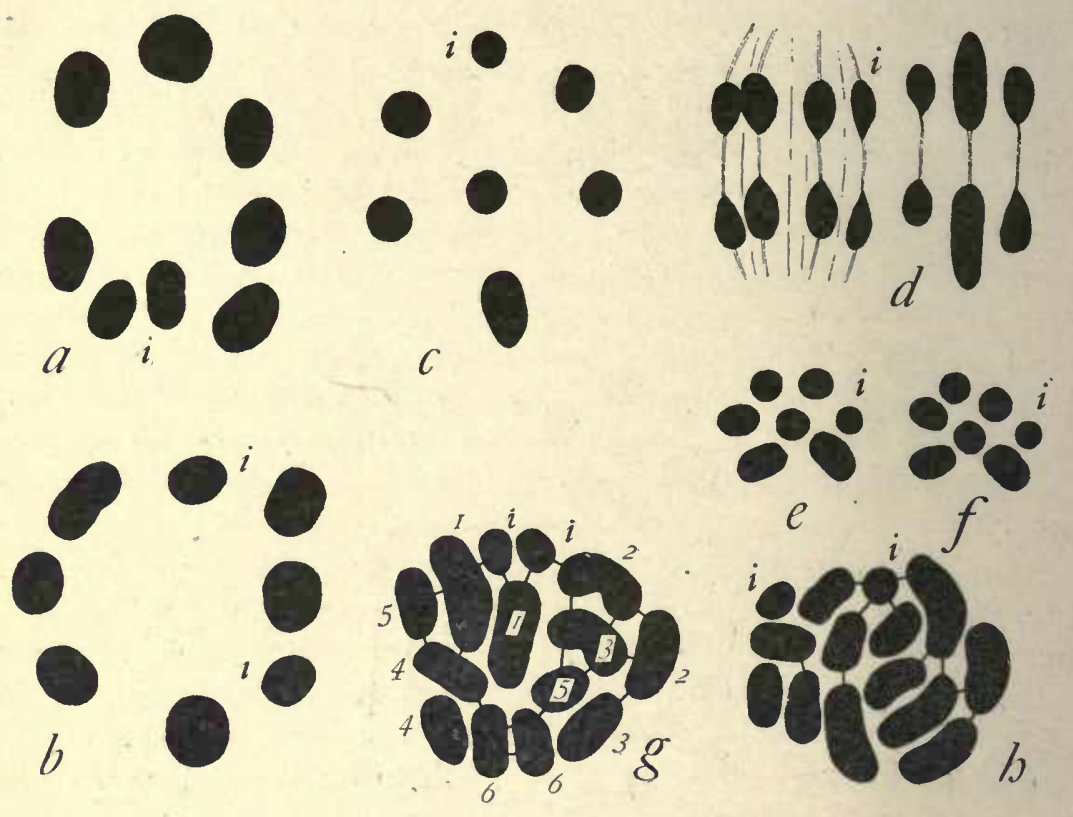

Fig. 4.

Nezara. $a, b$, metaphase-groups, first division; $c$, metaphase-group, second division; $d$, entire chromosome-group, second division, in side view, showing equal division (three chromosome-pairs from a lower level of the spindle shown at the right); $e, f$, duplicate sister chromosome-groups, anaphase of second division, from the same spindle; $g, h$, spermatogonial metaphase-groups.

somes each (Fig. 7q, r), the idiochromosomes being well differentiated. The first division, however, agrees with Montgomery's description in showing either 9 (Fig. 70 ) or 8 , the smallest chromosome being in the latter case wanting.

The conditions in Nezara are of particular interest from a comparative point of view in that the idiochromosomes are of equal 
size. The first spermatocyte-division shows 8 chromosomes, which differ in grouping from that of the other forms in that all usually lie in a ring without a chromosome at its center (Fig. 4 $a, b)$. For this reason no clue to the identification of the idiochromosomes is given by their position. Two of the chromosomes are, however, distinctly smaller than the others; and the relations in the spermatogonia leave little doubt that it is these two that correspond to the idiochromosomes. They may lie side by side (Fig. $4 a$ ) or more or less widely separated $(4 b)$. All these chromosomes are, in side view, seen to have a symmetrical dumb-bell shape, and all are equally halved in the first division. None of the preparations show the stage immediately following; but there can be no doubt that a conjugation of the two small chromosomes takes place at this time, since the second division (of which I have a large number) invariably shows in polar view but 7 chromosomes, which have now assumed the usual arrangement, with one in the center of a ring formed by the 6 others (Fig. 4c). Nezara differs again, however, from all the other forms in the fact that the small chromosome (i.e., the idiochromosome-dyad) lies in the outer ring, in many if not in all cases, while one of the larger chromosomes lies at the center of the ring. Lateral views of the spindle show all of the chromosomes as quite symmetrical dyads; and in the ensuing division all divide equally (Fig. $4 d$ ). In such views the idiochromosome-dyad, which is readily recognizable by its size, may be clearly seen to divide equally; and in this respect Nezara differs from all the others. The anaphase sister-groups of the same spindle, each containing 7 chromosomes (Fig. $4 e, f$ ) are, accordingly, exact duplicates, the idiochromosome retaining its position in the outer ring.

The symmetrical division of the idiochromosome-dyad in Nezara is a fact of importance for the comparison of the idiochromosomes with the microchromosomes of such forms as Anasa or Alydus. Were it not for their failure to unite to form a bivalent body until the end of the first mitosis we should find no ground in this case for designating these chromosomes by a special name.

\section{The Spermatogonial Cibromosomes.}

We have now to examine the relation of the chromosomes of the first maturation to those of the spermatogonia. The material 
for the spermatogonial division is most abundant in the case of Lygæus, which is much the most favorable form for an accurate count, the chromosomes being well separated and showing with almost schematic clearness. In the preparations of this form numerous spermatogonial plates appear, showing, whenever an accurate count can be made, without exception I4 chromosomes (Fig. $5 g, b)$. Nezara, of which numerous spermatogonial divisions are also available, shows the same number and with almost equal clearness, though the chromosomes are in this form more crowded. In the other forms the material is less abundant, but the relations are clearly shown in most of them. Cœnus (Fig. 5i) Euschistus (Fig. 5i), and Brochymena (Fig. 7n) also show I4 spermatogonial chromosomes, while in Podisus (Fig. $5^{k}$ ) the number is $16 .{ }^{1}$ In all these cases, therefore, the spermatogonial number is double that of the chromosomes in the second spermatocyte-division, and two less than double the number in the first division. The most striking fact is that in all these forms, with the exception of Nezara, the spermatogonial groups show but one microchromosome (marked $i$ in Figs. 4, 5, 7); in striking contrast to the fact first determined by Paulmier in Anasa and afterward by Montgomery in many other Hemiptera, that two such bodies ("chromatin-nucleoli" of Montgomery) equal in size, are often present. Did this observation rest only on the examination of a few division-figures (as in the case of Cœnus, Euschistus, Podisus and Brochymena) I should hardly trust in its general applicability to the species; but in Lygæus numerous demonstrative cases remove every doubt regarding this point, and the agreement of the other forms in their later history makes it nearly certain that my observation is not at fault with them. ${ }^{2}$

Distinct, though not very great, size-differences may be observed in the larger spermatogonial chromosomes, as has been indicated by Montgomery in several other genera of Hemiptera. Though these differences are not nearly as marked as those recognized by Sutton in Brachystola, it is nevertheless pretty clearly evident

\footnotetext{
${ }^{1}$ Montgomery ('O1, 1) gives the numbers as follows: Euschistus variolarius 16, E. tristigmus $1_{4}$ Nezara 16, Cœnus 14, Brochymena 16, Podisus 16.

${ }^{2}$ This point is emphasized since Montgomery describes and figures two spermatogonial microchromosomes ("chromatin-nucleoli") in Euschistus variolarius ('or, 1, Figs. 2, 3), E. tristigmus (op. cit., Fig. 20), Cœnus delius (Fig. 55), and Brochymena (Fig. 47). One of the figures of the first-named species shows them equal, the other unequal, in size.
} 
that the larger chromosomes may be grouped in six pairs; and in Figs. $5 h, i, j, 4 g, 7 n^{1}$ I have attempted to indicate these by corresponding numbers; though no pretense to complete accuracy of identification can be made, since the chromosomes vary somewhat in form and their apparent sizes vary somewhat with their position, owing to foreshortening. Allowing for all errors of identification it is obvious that in all but Nezara 12 of the larger chromo-
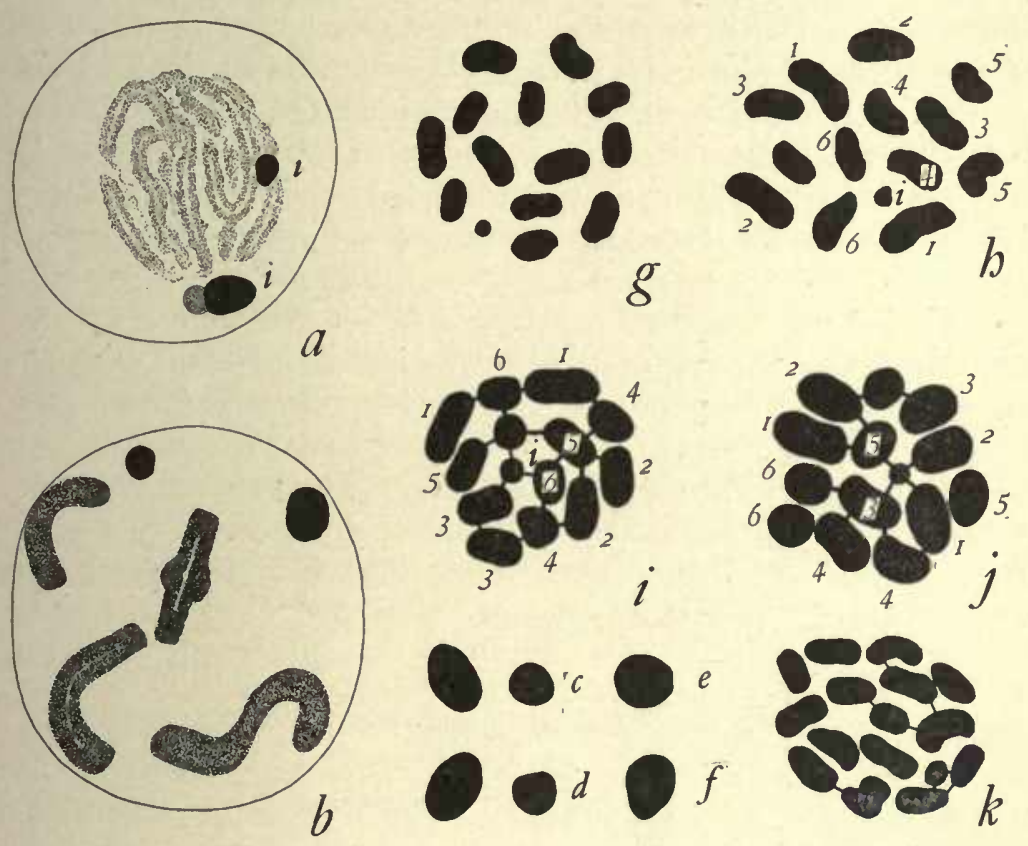

FIG. 5.

Cœnus $(a-f, i)$, Lygæus $(g, h)$, Euschistus $(j)$, Podisus $(k)$. $a$, Cœnus, contraction-phase, showing both idiochromosomes in the form of chromosome-nucleoli; $b$, prophase, corresponding to stage $h$ in Lygaus, the two idiochromosomes and four of the others; $c-f$, chromosome-nucleoli of Cœnus from a stage corresponding to stage $f ; c, d$, both idiochromosomes present, $c, f$, single chromosome-nucleoli; $g$, $h$, spermatogonial metaphase-groups, Lygæus; $i$, spermatogonial group, Cœnus; $j$, spermatogonial group, Euschistus, sp.; $k$, spermatogonial group, Podisus.

somes may be symmetrically paired off, two by two, while a thirteenth (unnumbered in the figures) is left without a fellow of like size. The conclusion is, I think, irresistible that in synapsis

${ }^{1}$ In the last figure the chromosomes have been by inadvertence wrongly lettered. 
twelve of the larger chromosomes unite to form the six peripheral bivalents of the first division, that the thirteenth is the larger idiochromosome and the fourteenth (the microchromosome) the small idiochromosome. These two alone remain separate in the first division as univalent chromosomes, thus giving a group of eight instead of seven. This conclusion is in accordance with Montgomery's interpretation of the facts observed by him in Euchistus tristigmus, as pointed out beyond, except that he believed that in this form the first division might in many cases show only seven chromosomes, the "chromatin nucleoli" having, like the other chromosomes, already conjugated to form a bivalent body. My interpretation is strikingly confirmed by the facts observed in Nezara; for in this case, where the first spermatocytedivision shows two chromosomes of equal size and the smallest of the group, the spermatogonia correspondingly show two equal microchromosomes, as in Anasa, Alydus, or Protenor (Fig. $4 g, b$ ). Since these two are represented in the second division by a single symmetrical dyad, which is again the smallest of the group (Fig. $4 g-f$ ) it is evident that the two equal microchromosomes must conjugate after the first division. They therefore agree in behavior with the unequal idiochromosomes present in the other forms, and differ from those of the Anasa type, in remaining separate during the first maturation-mitosis.

\section{The Growth-Period of the Primary Spermatocytes.}

\section{(a) General History.}

It is not my purpose to describe in detail at this time the general history of the chromatin during the growth-period, but it will be convenient to outline the stages in order to trace the history of the idiochromosomes. In Lygæus ten well marked stages may be distinguished from the early synapsis $(a)$ to the metaphase of the first division $(j)$, inclusive. Though some of these stages are best characterized by the condition of the idiochromosomes, an account of the latter can more readily be given after considering the history of the larger chromosomes. In stage a (early synapsis), which shortly follows the final anaphase of the last spermatogonial division, the chromosomes have the form of rather ragged, longitudinally split loops, the free ends of which converge toward 
one pole of the nucleus. In stage $b$ (contraction-phase, Fig. 6a) they become closely massed in a spheroidal aggregation at the center or toward one side of the nucleus, and surrounded by a large clear space. At this time they stain so deeply that their
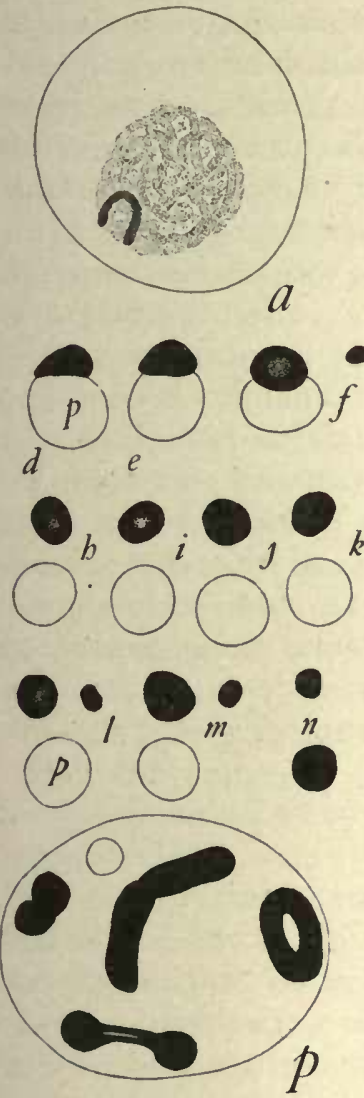

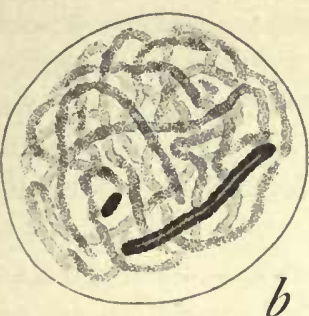

$b$

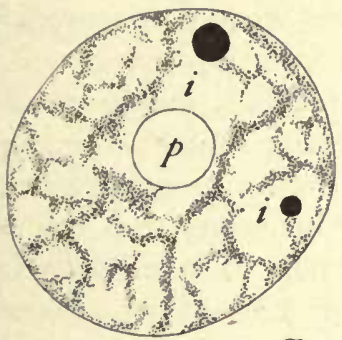

$\xi$

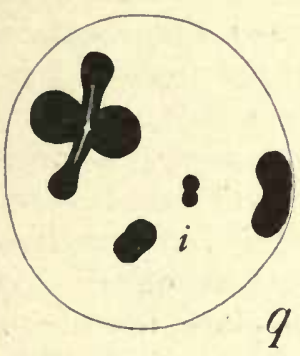

Fig. 6.
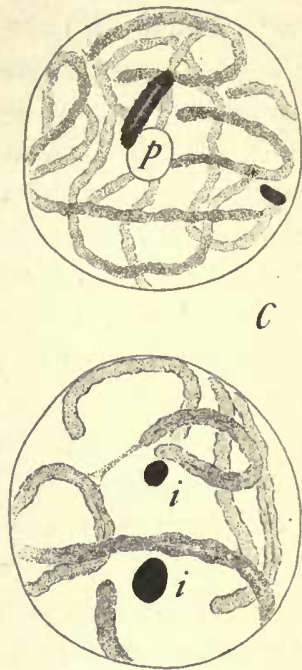

0

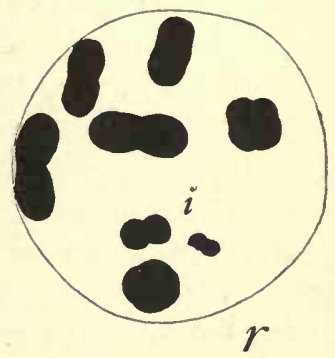

Lygaus turcicus. $a$, contraction-phase of synaptic period (stage $b$ ) showing large idiochromosome; $b$, early post-synapsis (stage $c$ ), botb idiochromosomes shown; $c$, stage $d$, a plasmosome connected with the large idiochromosome; $d-f$, chromosome-nucleoli (with plasmosome) from stage $e ; d$ and $e$ single, $f$, showing the two idiochromosomes; $g$, stage $f$, with plasmosome at its maximum size, and both idiochromosomes; $h-n$, chromosome-nucleoli from stage $f$ (represented side by side near the plasmosome, out of their natural position); $h-k$, four cases in which but one is present, $l-n$ showing both idiochromosomes; 0 , stage $g$, with both idiocbromosomes; $p, q$, stage $h$, two sections of the same nucleus showing all of the eight chromosomes and a small plasmosome; $r$, stage $i$, showing the six larger chromosomes and the two idiochromosomes. 
outlines can only with difficulty be made out, and then only after long extraction. In stage $c$ (early post-synapsis) they again spread through the nuclear cavity, giving the appearance of an interrupted, contorted and more or less confused spireme, composed of delicate and somewhat varicose threads (Fig. 6b). In stage $d$ (Fig. $6 c$ ) the threads become somewhat shorter and thicker and have a more open arrangement, but still show a very marked spireme-like arrangement. I believe the threads to be at this period longitudinally split, though this can only be made out with difficulty. This stage is well characterized by the condition of the large idiochromosome, as described beyond, and by the appearance of a pale plasmosome. In stage $e$ the threads become markedly shorter and thicker, ragged in outlines, often faintly show a longitudinal split, and diminish somewhat in staining-capacity. The chromosomes now undergo a great change, becoming in stage $f$ very loose in texture, showing only vague boundaries, and almost completely losing their staining-capacity, so that it is difficult to represent their appearance in a black and white figure (Fig. $6 g$ ). As may be seen from the figure the chromosomes now give the appearance of a rather vague, pale, finely granular network, in which traces of a spireme-like arrangement can usually be seen, but they cannot be clearly made out as individual bodies. Stage $g$ again shows a great change (Fig. 60) the chromosomes having resumed their staining capacity and definite boundaries, and now appearing as long, coarse, winding threads, often showing rather ragged outlines and a more or less distinct longitudinal split, as in stage $e$; the two stages may, however, at once be distinguished both by the position of the cells in the testis and by the different relation of the plasmosome and the chromosome-nucleoli, as described below. The condensation of the chromosomes now takes place, the succeeding three stages following in rapid succession. In stage $b$ the long split rods shorten and thicken, stain much more deeply, and assume a great variety of forms-curved double rods, dumb-bell shaped figures (sometimes longitudinally split), closed rings, and peculiar cross-forms. (Fig. $6 p, q$, which show all the chromosomes from a single nucleus.) In stage $i$ (early prophase) all these forms condense into quadripartite tetrads or dyad-like bodies, the latter consisting of two symmetrical halves closely joined together and frequently showing no trace of a second division, though in the same nucleus 
may occur one or more distinctly quadripartite forms (Fig. 6r). The history of these dyad-like bodies clearly shows, I think, that with the exception of the idiochromosomes they are derived from bivalent longitudinally split rods, and they have therefore the same valence as the actual tetrads with which they are associated. The nuclear membrane now disappears and the chromosomes are drawn into the equatorial plate of the first mitosis.

The general history of the growth-period in Cœnus is similar to that of Lygxus, but is in some respects abbreviated; and stage $f$ is much less marked, the chromosomes not losing their boundaries or their staining-capacity in so great a degree, and still presenting the appearance of a ragged interrupted spireme.

(b) The Idiochromosomes during the Growth-period.

The foregoing description applies only to the larger or ordinary chromosomes. Throughout the whole of the growth-period in Conus and from stage $e$ onward in Lygæus, at least one of the idiochromosomes can always be distinguished as a compact, spheroidal, intensely staining chromosome-nucleolus, and frequently both idiochromosomes are distinguishable in this form in all of these stages. The early stages of Lygæus ( $b$ to $d$ ) are of especial interest in that the condensation of the idiochromosomes is delayed, and at least the larger one still has the form of an elongate, longitudinally split rod or thread. Even at this time, however, it is immediately distinguishable from the others by its greater thickness and greater staining capacity. It is clear beyond all question, therefore, that at least the large idiochromosome may retain its identity throughout the whole growth-period. With the small idiochromosome the case is not so strong, as will be seen from the following account.

It is convenient to trace the history of the idiochromosomes in the reverse order from stage $i$ backward, again taking Lygæus as the type. In this stage (late prophase), when the six larger chromosomes are in the form of condensed tetrads or dyad-like bodies, both the idiochromosomes have very distinctly the form of dyads. The nucleus now contains therefore eight separate chromosomes, among which the idiochromosomes are at once recognizable by their small size $(6 r)$. In stage $b$ the idiochromosomes have the same general appearance, though their bipartite 
form is often less distinct $(6 q)$. Up to this point both idiochromosomes are apparently always present. In the stages now to be considered both are often plainly distinguishable, but quite as frequently this is not the case, the nucleus showing but a single chromosome-nucleolus the size of which proves it to be either the large idiochromosome or the large and small one united. Between these two possibilities I have not been able to decide in Lygaus and Cœnus; but decisive evidence is given in the case of Brochymena, as described beyond. In stage $g(60)$ the idiochromosomes (or the single chromosome-nucleolus) appear as spheroidal compact bodies, usually not showing a bipartite structure, and in addition one or more pale rounded plasmosomes are often present. In stage $f$, owing to the loss of staining capacity by the larger chromosomes, the idiochromosomes show with brilliant clearness, since they are still stained intensely black, and may very readily be studied with care $(6 g-n)$. When both are present they appear slightly larger than in the later stages, and often the larger one is plainly seen to be hollow (which probably accounts for its larger size) though this is shown still more clearly in Brochymena, as described beyond. A small plasmosome is sometimes attached to it at one side, but in addition to this there is always present a very large pale plasmosome quite free from both idiochromosomes, and free also from the single chromosome-nucleolus when but one of these bodies is present. In stage $e$ the idiochromosomes present the same appearance, but the larger one is now always attached to the large plasmosome, and often more or less flattened against it (as is also the single chromosome-nucleolus when but one appears) while the small one is almost always free from it (Fig. 6d, e, f). The larger body is as before often evidently hollow.

Up to this point Lygæus and Cœnus agree almost exactly. In the earlier stages they differ in that Cœnus still shows the idiochromosomes in the form of compact chromosome-nucleoli, while in Lygæus the larger one certainly, and I believe the smaller one also, assumes the form of a longitudinally split elongate chromosome. In stage $d$ in Lygæus (Fig. $6 c$ ) the large idiochromosome is a rather short, deeply staining rod, longitudinally split, and still attached (usually toward one end) to the plasmosome, which is now considerably smaller. The small idiochromosome is now also more or less elongate, but I cannot be sure whether it 
is longitudinally split. All intermediate stages may readily be observed between this stage and the next earlier one $(c)$ in which the large idiochromosome is an elongate split thread that may extend through more than half the diameter of the nucleus (Fig. 66 ). It is, however, at once distinguishable from the other chromosomes by its straighter course, greater thickness and deeper staining capacity, ${ }^{1}$ which renders it very conspicuous among the thread-like chromosomes. No plasmosome can now be seen. The small idiochromosome can still clearly be distinguished in many of the nuclei as a short rod staining like the larger one. Finally, in the contraction-phase (stage $b$ ) when all the chromosomes are massed together, the large idiochromosome still unmistakably appears as a very distinct deeply staining rod, sometimes nearly straight, more usually curved, and frequently horse-shoe shaped, at one side of the chromosome-mass $(6 a)$. Neither plasmosome nor small idiochromosome can now be made out. In Cœenus at this period (Fig. 5a) both idiochromosomes (or the single chromosome-nucleolus) still appear as compact, deeply staining spheroidal bodies, the larger one typically having a small plasmosome attached to it at one side.

The early history of the large idiochromosome proves most clearly that the chromosome-nucleolus into which it afterward condenses is a modified chromosome (as Montgomery first showed in Euschistus variolarius) and one that forms a connecting link between the ordinary chromosomes and the more usual forms of "chromatin-nucleoli" in Hemiptera, described by Montgomery, or the accessory chromosome of Orthoptera; for in none of these latter is the condensation to a compact body so long delayed. Paulmier ('99) showed, however, that in Anasa the compact chromosome-nucleolus of the synaptic period afterward elongates considerably and appears as a rather short longitudinally split rod, similar to that of Lygæus at stage $d$ (Paulmier, Fig. 22) afterward again condensing into a compact tetrad. In Lygxus there can be little doubt that the central cavity of the spheroidal. chromosome-nucleolus, often visible in stages $e-g$, represents the original longitudinal split. It is therefore hardly open to doubt that the division of the large idiochromosome in the first mitosis is an equation-division, and the same is probably true of the small

'This has been somewhat exaggerated in the engraving. 
idiochromosome. It is, on the other hand, quite certain that the division of the idiochromosome-dyad in the second mitosis is a reduction-division. The order of the divisions in case of the idiochromosomes is thus the reverse of that which occurs in the other chromosomes, according to Paulmier's and Montgomery's accounts; and as pointed out beyond, it is also the reverse of that which takes place in the division of the small central chromosome in Anasa and Alydus.

As already stated, I did not in Lygæus and Cœnus, succeed in finding any certain explanation of the fact that the nuclei of the growth-period may show either one or two chromosome-nucleoli.

In Brochymena, however, there is very clear evidence on this point. Here, too, the nuclei of the middle growth period show either one or two spheroidal chromosome-nucleoli, the former condition being much the more frequent. When both are present they may be widely separated or close together, and both very clearly show a central cavity, which is rendered very conspicuous by the fact that the chromatin is frequently concentrated in a dark zone immediately around it (Fig. $7 c-g$ ). When but one is present it is, as a rule, perfectly spherical, hollow, and shows no evidence of a double nature (Fig. $7 f, g$ ). In the early growthperiod, however, the single chromosome-nucleolus almost always appears bipartite, being composed of two unequal halves, forming an asymmetrical dyad (Fig. $7 a, b)$ very similar to that seen in the second maturation-division (Fig. $7 \mathrm{~m}$ ). At a later period both of the constituents become hollow (and hence appear somewhat larger) and stain less deeply; and all gradations may be observed in the fusion of the two bodies to form a single hollow body (Fig. $7 c, d, f)$ which is plainly as large as the two separate chromosomenucleoli (such as may be seen in cells of the same cyst) taken together. In Brochymena, therefore, there can be no doubt that when only one chromosume-nucleolus is present it is to be considered as a bivalent body arising by the fusion or synapsis of the two idiocbromosomes.

Thus far the facts confirm the interpretation given by Montgomery ('or, I) who observed in Cœnus, Euschistus tristigmus and some other forms that the cells of the growth-period may show either a single "chromatin-nucleolus" or (in Euschistus "apparently more frequently") two such bodies that are unequal in size; and this fact he interpreted to mean that the two corresponding 
spermatogonial "chromatin-nucleoli" may either conjugate at the period of general synapsis to form a bivalent body or may remain separate as univalent bodies. As already pointed out, it was probably this interpretation that led him to conclude that the first division in these forms might show either seven or eight chromosomes. But the later stages observed in Brochymena give conclusive evidence that even though such a primary synapsis

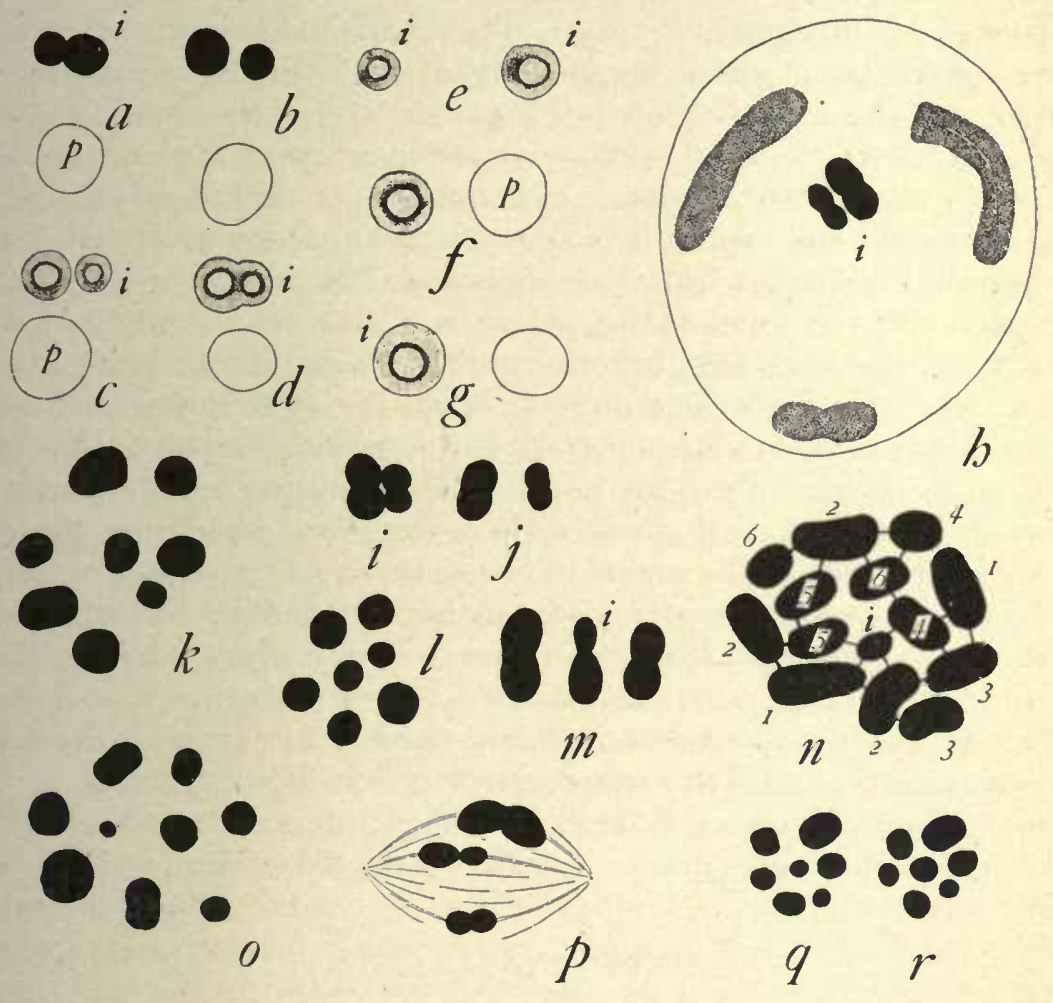

Fic. 7 .

Brochymena, Trichopepla. $a-n$, Brochymena, o-r, Trichopepla; $a, b$, idiochromosomes and plasmosomes, from early growth-period; $c-g$, condition of the idiochromosomes in middle and late growthperiods; $h$, prophase of first division, showing idiochromosome-tetrad; $i, j$, two stages, one following and one preceding the last, in the division of the bivalent chromosome-nucleolus; $k$, metaphase-group, first division; $l$, metaphase-group, second division; $m$, side view, second division, showing idiochromosome-dyad and two other chromosomes; $n$, spermatogonial metaphase-group; 0 , metaphase-group, first division, Trichopepla; $p$, spindle of second division in lateral view; $q, r$, sister-groups, late anaphase of second division. 
of the idiochromosomes takes place the bivalent chromosomenucleolus again separates into its univalent constituents in the early prophases of the first maturation-division. This process, which at first greatly puzzled me, occurs at the time just preceding the concentration of the larger chromosomes into their final condensed form (corresponding to stage $b$ in Lygæus). In cysts of this period every stage may be seen in the transformation of the single chromosome-nucleolus into an asymmetrical tetrad, consisting of two symmetrical dumb-bell shaped bodies of unequal size (Fig. 7i), and the separation of these unequal dyads to form the idiochromosomes of the first division ( $c f$. Figs. $7 h, i, j$ ). It is evident that in these cases the final reunion or conjugation at the end of the first division is not a primary synapsis, but a secondary process. ${ }^{1}$

The facts observed in Brochymena make it very probable that when only a single chromosome-nucleolus is present in Lygxus, Cœnus and the other forms, it is there also a bivalent body as Montgomery assumed; but the uniform separation of the idiochromosomes in the first division of all the eight species I have examined is almost a demonstration that in all the forms a division of the bivalent body must occur. On the other hand it seems equally certain that in many of these forms the idiochromosomes may fail to unite at the period of general synapsis, and may remain separate through the whole growth-period; and in Brochymena the same cysts that show the division of the single idiochromosome-tetrad may also contain nuclei in which the dumb-bell shaped idiochromosomes are widely separated. In such cases it seems probable that the conjugation of the idiochromosomes at the close of the first spermatocyte-division must be regarded as a true or primary synapsis that has been deferred to this late perind.

\section{DISCUSSION OF RESULTS.}

The most essential fact brought out by a study of the idiochromosomes is that in Lygæus, Conus, Podisus, Euschistus, Brochymena and Trichopepla a dimorphism of the spermatozoa exists, there being two groups equal in number, both of which contain

\footnotetext{
${ }^{1}$ The division of the bivalent chromosome-nucleolus is similar to the process described by Gross ('o4) in Syromastes; though it occurs at a much later period. For reasons that will appear in a subsequent paper, I am, however, skeptical in regard to Gross's conclusion which is based on a study not of the idiochromosomes but of paired microchromosomes similar to those of Anasa or Alydus.
} 
the same number of chromosomes, but differ in respect to one of them. In this respect these genera differ from all those that possess an accessory chromosome (Pyrrochoris, Anasa, Alydus, etc.), since in the latter case one-half of the spermatozoa receive one chromosome fewer than the other half. It is remarkable that two types of dimorphism apparently so different should coexist within the limits of a single order of insects. We are thus led to inquire into the relation between the idiochromosomes and the accessory; and this inquiry must also include the "small chromosomes" of Paulmier and the "chromatin-nucleoli" of Montgomery. It will conduce to clearness if the second part of this question be considered first.

It is evident from the figures and descriptions of Montgomery (' 98 , 'or, I, 'or, 2, 'O4) that the bodies I have called idiochromosomes are identical with some of those that this author has described under the name of "chromatin-nucleoli" (which are usually also small chromosomes or microchromosomes); but it is now manifest that the bodies described under the latter name are not all of the same nature. It is evident that two types of these bodies may be distinguished that differ markedly in their behavior during the maturation-mitosis. One of these, typically represented in Anasa, Alydus and Protenor appear in the spermatogonia in the form of two equal microchromosomes ("chromatinnucleoli") which like the other chromosomes sooner or later unite in synapsis to form a bivalent body that lies at the center of the equatorial plate of the first mitosis. Both divisions accordingly show exactly one half the spermatogonial number of chromosomes but it is a very noteworthy fact that the final conjugation of the two microchromosomes is long deferred, taking place in the prophases of the first division (as was first observed by Montgomery in Protenor and some other forms, more recently by Gross in Syromastes and by myself in Anasa and Alydus). In this case there seems to be no doubt that the first division of the bivalent body thus formed is a reducing division. It appears to be further characteristic of this type - at least in all the forms mentioned-that a true accessory chromosome is associated with the microchromosomes, and that only one-half of the spermatozoa receive one-half the somatic number of chromosomes, the other spermatozoa receiving one less than this. The distinction between the accessory and the microchromosomes or "chromatin-nucleoli" first demonstrated 
by Montgomery ('OI) in Protenor, has been more recently shown by Gross ('O4) to exist in Syromastes; and I have also been able to demonstrate the same fact in Alydus and in Anasa (Paulmier having been in error in his identification of the accessory with the small chromosome in the last-named form).

The second type includes the idiochromosomes, which in the forms I have studied differ from the Anasa type in four respects (Nezara being an exception in regard to the first of these), namely, (I) in their unequal size, with which is correlated the fact that only a single microchromosome appears in the spermatogonia; (2) in the fact that the final conjugation or synapsis of these bodies is deferred until the prophases of the second division, a result of which is that the first division shows one more than half the spermatogonial number of chromosomes while the second division shows exactly half the spermatogonial number; (3) in the fact that in case of the idiochromosomes it is manifestly the second division that is the reducing one, while my observations on Lygxus render it practically certain that the first is an equation-division; (4) in the fact that no accessory chromosome in the usual sense is present and all the spermatozoa receive the same number of chromosomes. In view of these differences it seems expedient for the present to place these two types in different categories.

It is evident from Montgomery's figures and descriptions that he observed many of the details of the phenomena described in the present paper; but it is equally clear from the varying interpretations that he adopted that he failed to reach any consistent general result regarding the behavior of the idiochromosomes, or to recognize the dimorphism of the spermatozoa. For example, it is evident, I think, from his descriptions of Euschistus variolarius, E. tristigmus, Cœnus delius, Oncopeltus fasciatus, and Lygus pratensis, that the essential facts in these forms agree with those I have described, the idiochromosomes being in the last named two species of equal size, as in Nezara; but Montgomery offers for each of these cases a different interpretation. In the first named species the idiochromosomes are clearly figured in his first paper ('98, Figs. I7I, I88, I89, etc.), and in Fig. 214 they are shown separating in quite typical fashion in the second division (the smaller one designated as a "chromatin-nucleolus"); but it is evident from the descriptions given in both this and the following paper ('OI, I) that he did not reach a correct interpretation of the 
facts. ${ }^{1}$ In Euschistus tristigmus and Cœnus delius the first division is stated to show either seven or eight chromosomes (the spermatogonial number being I4), but quite different interpretations are given of this in the two species, the conditions in Euschistus being assumed to be due to the frequent failure of the two "chromatin nucleoli" to unite in synapsis, while in the case of Cœnus seven of the chromosomes (including the large "chromatinnucleolus") are assumed to be bivalent, while the eighth is an additional small "chromatin-nucleolus" not distinguishable in the spermatogonia ('OI, I, p. I66). In Euschistus tristigmus the "chromatin-nucleoli" are stated to be of unequal size and to be separated from each other without divison in the second mitosis. This is evidently the same phenomenon that I have described; though Montgomery overlooked the conjugation of the two unequal "chromatin-nucleoli" at the end of the first division, and expressly states that they are not joined together in the second division. In Oncopeltus, likewise, the first division shows one more than half the spermatogonial number, I6 ( $i . e$. , nine instead of eight, precisely as I have described in Podisus), and this is stated to result from the persistence of the two "chromatin-nucleoli" throughout the whole growth period without union; but an interpretation differing from both the foregoing is here sought in the assumption that each of the two "chromatin-nucleoli" is bivalent, even in the spermatogonia ('or, I, p. I86). In Lygus pratensis, finally, the first division shows 18 chromosomes and the second I 7 , the still different explanation being here offered that the two "chromatin-nucleoli" pass undivided one to each pole of the first spindle ('or, I). Of these various interpretations only the one given in the case of Euschistus tristigmus, I believe, con-

'The first mitosis is here clearly shown to have eight chromosomes, grouped in the same way as in my "Euschistus sp." and the anaphase daughter-plate of the second division is shown with seven (Fig. 220), precisely as in the two species I have studied. Montgomery gave the spermatogonial number, correctly I believe, as 14. He nevertheless concluded that all of the eight chromosomes (seven chromosomes +1 "chromatin nucleolus") divide separately in both divisions, apparently overlooking the fact that this would give the spermatozoa one chromosome too many (since he himself demonstrated that the "chromatin-nucleolus" is a modified chromosome). This account of the divisions is not modified in the paper of rgor except in the statement that "in the second maturation-division the chromatin-nucleolus is not always divided" (p. I61), while the spermatogonial number-is now given as 16 . Since the figures of the earlier paper show that the divisions in E. variolarius are evidently the same as in the species I have examined, I think that on both these points the first account was probably more accurate than the later one. 
forms to the true one, and it is probable that all of these cases will be found to agree in the essential phenomena with those I have determined in Lygæus, Cœnus, Nezara and the other forms.

We may now inquire what is the relation of the idiochromosomes to the accessory chromosome. The observations suggest so obvious an answer to this question that I wish to indicate not only the evidence in its favor, but more especially the difficulties it has to encounter. In forms possessing an accessory chromosome the spermatozoa fall into two equal groups that differ only in respect to one chromosome. The same is true of Lygæus and other forms that lack the accessory but possess the idiochromosomes, with the difference that in the former case the distinctive chromosome is present in but one-half the spermatozoa, while in the latter case two such distinctive chromosomes are present, one of which is present in one-half, the other in the other half, of the spermatozoa. It is impossible to overlook the evident analogy between the two cases; and the idiochromosomes may in one sense be considered as two accessory chromosomes that are never allotted to the same spermatozoön since each fails to divide in the second mitosis (precisely as is the case with the single accessory in other Hemiptera). The difference between Lygæus and Conus in the size-ratio of the idiochromosomes obviously suggests the view that the single accessory of other forms may have arisen by the disappearance of one of the idiochromosomes; and in Lygaus the smaller one is already so minute as distinctly to suggest a vestigial structure. We might accordingly assume that in a more primitive type the two idiochromosomes were of equal size (as in Nezara), undergoing synapsis and subsequent reduction in the same way as the other chromosomes; that Cœnus and Lygæus represent successive stages in the reduction of one of these chromosomes; and that by the final disappearance of the smaller one in such forms as Anasa or Pyrrochoris a single accessory chromosome remains.

This hypothesis at first sight seems to give a clear and intelligible view of the origin of the accessory chromosome, and to reconcile the remarkable mode of spermatogenesis occurring in the insects with forms in which no accessory seems to appear. But further reflection shows that it has to encounter a formidable if not insuperable difficulty in the fact that in some of the forms possessing an accessory chromosome the number of spermato- 
gonial chromosomes is an even one (as in Anasa and Syromastes); and there seems to be no escape from the conclusion that the accessory is here a bivalent body arising by the synapsis of two equal spermatogonial chromosomes. Even in cases showing an odd number of spermatogonial chromosomes (as in many Orthoptera and some Hemiptera - for example Alydus or Protenor) it has been assumed, and with good reason, that one of the chromosomes (probably the accessory) is already bivalent, ${ }^{1}$ and Montgomery has shown ('OI, I) that in Protenor the large accessory ("chromosome $x$ ") is sometimes transversely constricted into two equal halves in the spermatogonia. A similar fact was subsequently shown in Harmostes ('OI, 2) which also has normally an odd spermatogonial number. To this should be added the fact that these forms possess the small bivalent central chromosome (which arises by the synapsis of two equal microchromosomes) in addition to the accessory. The difficulty pointed out above cannot be escaped by supposing that the disappearance of one of the idiochromosomes has been effected by its gradual absorption by the other; for this assumption, too, fails to explain the even number of spermatogonial chromosomes. Apparently therefore the hypothesis I have suggested must in the present state of our knowledge be considered untenable. ${ }^{2}$

It appears more probable that the idiochromosomes are comparable to the two equal microchromosomes or "chromatin-

\section{${ }^{1} \mathrm{Cf}$. Montgomery, 'o4.}

${ }^{2}$ Since this paper was sent to press I have determined beyond the possibility of doubt, I think, that the number of spermatogonial chromosomes in Anasa tristis is 21 , not 22 as given by botb Paulmier and Montgomery. This result is based on the study of a large number of preparations, and careful camera drawings of more than twenty perfectly clear metaphase figures have been made. All without exception show 21 chromosomes, and I bave sought in vain for even a single cell that shows 22. (Paulmier's original slides were used.) If corroboratory evidence be needed it is given by the fact that there are always three macrochromosomes, one of which is obviously without a mate of like size, and is probably the accessory. I have, also, positively determined the spermatogonial number to be 21 in a form included in Paulmier's material and labeled "Chariesterus antennator," (since this number disagrees with Montgomery's count of the spermatocytes there may be an error of identification; but the form is certainly different from Anasa) and 15 in Archimerus calcarator (from my own material, identified by Mr. Uhler), both members of the same family, as Anasa. This wholly unexpected result perhaps justifies a certain skepticism in my mind in regard to the accounts of other observers, who give an even spermatogonial number for forms possessing an accessory chromosome; and if this be well founded the objection urged above disappears. I shall return to this subject hereafter. It is needless to say that had I been acquainted with these facts, the discussion that follows would have been different. 
nucleoli" which in such forms as Anasa or Alydus conjugate to form the small central chromosome of the first mitosis. The differences between the two forms have already been pointed out. Their resemblances are, however, no less obvious, namely, their usual central position in the equatorial plate, small size, occasional persistence as chromosome-nucleoli in the growth-period, and their late conjugation. This comparison finds very definite support in the conditions I have described in Nezara, where the idiochromosomes are of equal size and appear as two equal microchromosomes in the spermatogonia. From the analogy of other forms it is very probable that the more primitive and typical form of synapsis is that between chromosomes of like size. It is therefore probable that such a condition as that observed in Nezara is a less modified one than that in which the idiochromosomes are unequal; and that the latter condition has arisen through a secondary morphological differentiation of two chromosomes that were originally of equal size, and perhaps are represented by the two equal microchromosomes that appear in the spermatogonia of such Hemiptera as Anasa, Alydus, Syromastes or Protenor. This comparison involves two assumptions, namely, first that in case of the idiochromosomes the final conjugation of the microchromosomes has been postponed from the prophases of the first division to those of the second; and secondly that a reversal in the order of the reduction- and equation-divisions has taken place in case of these particular chromosomes, the first division being in case of the Anasa-type the reduction- and in case of the idiochromosomes the equation-division. The difficulty apparently involved by the second assumption is less serious than may appear. All the facts at our command indicate that a reduction-division is the necessary, or at least invariable, sequel to a foregoing conjugation; and if, as in the case of the idiochromosomes, the final conjugation is deferred to the second division, the reductiondivision must also be deferred. The univalent idiochromosomesas is shown with certainty in case of the larger one in Lygxusundergo longitudinal division at the same stage of the growthperiod as their bivalent companions and are already double at the time of the first mitosis. There is, therefore, no difficulty in the way of assuming-indeed, the facts seem to admit of no other conclusion - that this is the equation-division.

It must be recognized, however, that the foregoing comparison 
wholly fails to explain the origin or meaning of the accessory chromosome, nor does it account for the surprising fact (of which the phenomena in Brochymena seem to leave no doubt) that two chromosomes may unite in synapsis, subsequently part company so as to divide as univalents in the first mitosis, but again conjugate to form a bivalent in the second mitosis. It seems likely that further comparative study of this phenomenon may throw important light on the general mechanism of karyokinesis and reduction.

The history of the idiochromosomes possesses a more general interest in the strong support that it lends to the general theory of the individuality of chromosomes, to the specific conclusions of Montgomery and Sutton in regard to synapsis, and especially to the correlation of the phenomena of reduction with those of Mendelian inheritance attempted by the last-named author ('02, '०3). It has been assumed by some authors, including some of those who have accepted Montgomery's remarkable conclusion ('OI, I, 'O4) that corresponding paternal and maternal chromosomes unite in synapsis, that in this process the individuality of the conjugating chromosomes is completely lost - "Sie vereinigen sich zu einem einzigen Zygosom, aus dem erst wieder zwei neue Chromosomen hervorgehen." It is undoubtedly true that frequently all visible traces of the duality of the bivalents that emerge from the synapsis stage are for a time lost; and as Sutton suggested ('o3, p. 243), such cases as those of first crosses that breed true-and I may add, perhaps also those in which blended inheritance or weakening of dominance occurs - may be taken to indicate that a permanent fusion, or intermixture of the chromosome-substances, may really take place. But, on the other hand, the history of the idiochromosomes in cases where they remain separate through the whole growth-period leaves not the least doubt that as far as these particular chromosomes are concerned the same two that unite in synapsis persist as distinct individuals to be afterward separated by the reducing division and assigned to different germ-cells. This preliminary conjugation and subsequent separation ensures that the germ-cells shall be "pure" in respect to these particular chromosomes-i. e., that both shall not enter the same spermatozoön-and if this be true of one pair of the conjugating chromo-

'Strasburger, '04, p. 26; cf. also Bonnevie, 'o5. 
somes we have good reason to conclude that it may be true of all, as Montgomery has urged and as Sutton has so cogently argued, from a study of the size-relations. It is a fair working hypothesis that the idiochromosomes represent a pair of corresponding or allelomorphic qualities, or group of qualities, that are respectively maternal and paternal, as Sutton, building on the basis laid by Montgomery and himself, has argued for the chromosome-pairs in general. The argument of Montgomery and Sutton is based, it is true, on the fact that chromosomes of different sizes in the spermatocytes are represented by symmetrical chromosome-pairs of corresponding sizes in the spermatogonia; and to this the idiochromosomes in most of the cases described form an exception in being unequal. If this appears to be a difficulty it is removed by the case of Nezara, where the idiochromosomes are of equal size. Even in the more usual case, where they are unequal, symmetrical synapsis takes place between all the other chromosome-pairs. If the theory of the individuality of chromosomes be granted no other conclusion seems possible, accordingly, than that the remaining two, despite their size-difference, are respectively the paternal and maternal elements of the remaining pair; and if Sutton's general hypothesis be well founded, these elements may be assumed to be physiological correlates or allelomorphs. Their marked difference in size suggests a corresponding qualitative differentiation, and this inevitably suggests a possible connection between them and the sexual differentiation. The visible dimorphism of the spermatid-nuclei in such forms as Lygæus, Cœnus or Podisus shows too obvious a parallel to the sexual dimorphism of the germ-cells, indicated by so much of the recent work on sexdetermination, to be ignored; while in Nezara, where no visible dimorphism exists, the spermatozoa nevertheless fall into two equal groups in respect to the previous behavior of one of the chromosomes. But such a suggestion as to the possible significance of the idiochromosomes immediately encounters the difficulty that both idiochromosomes are present in the male cells (spermatogonia, and spermatocytes), just as McClung's similar hypothesis regarding the accessory chromosome is confronted with the fact, determined by Montgomery and Gross, that in the Hemiptera both sexes show the same number of chromosomes. Whether these difficulties can be met by assumptions of dominance and the like remains to be seen; but the fact should be recognized 
that as far as the Hemiptera are concerned neither the suggestion I have made, nor the hypothesis of McClung has at present any direct support in observed fact. ${ }^{1}$

The practical interest of the idiochromosomes lies in the very definite basis that they give for an examination of the question by the study of fertilization, for their disparity in size gives us the hope of determining their history by direct observation. There is good reason to believe that such a study will yield interesting results.

\section{SUMMARY OF OBSERVATIONS.}

I. In Lygæus turcicus, Cœnus delitis, Euschistus fissilis, Euschistus sp., Brochymena, Nezara, Trichopepla and Podisus spinosus all of the spermatids receive the same number of chromosomes (half the spermatogonial number), and no accessory chromosome is present; but the spermatozoa nevertheless consist of two groups, equal in number, which differ in respect to one of the chromosomes, which may conveniently be called the "idiochromosome."

2. In all of the forms named, excepting Nezara, half the spermatozoa receive a larger, and half a smaller, idiochromosome. In Nezara the idiochromosomes are of equal size, but agree in behavior with the unequal forms.

3. In all of the forms the idiochromosomes remain separate and univalent in the first maturation-division, while the other chromosomes are bivalent; this division accordingly shows one more than half the spermatogonial number of chromosomes. They divide separately in the first mitosis, but at the close of this division their products conjugate to form a dyad, which in all the forms save Nezara is asymmetrical. The number of separate

${ }^{1}$ The discovery, referred to in a preceding foot-note, that the spermatogonial number in Anasa is 21 instead of 22, again goes far to set aside the difficultics here urged. Since this paper was sent to press $I$ have also learned that Dr. N. M. Stevens (by whose kind permission I am able to refer to her results) bas independently discovered in a beetle, Tenebrio, a pair of unequal chromosomes that are somewhat similar to the idiochromosomes in Hemiptera and undergo a corresponding distribution to the spermatozoa. She was able to determine, further, the significant fact that the small chromosome is present in the somatic cells of the male only, while in those of the female it is represented by a larger chromosome. These very interesting discoveries, now in course of publication, afford, I think, a strong support to the suggestion made above; and when considered in connection with the comparison I have drawn between the idiochromosomes and the accessory show that McClung's hypothesis may, in the end, prove to be well founded. 
chromatin elements is thus reduced to one half the spermatogonial number. In the second maturation-division the asymmetrical dyad separates into its two unequal constituents, the larger one passing to one pole and the smaller one to the other pole of the spindle, while the other dyads divide equally.

4. In all the forms excepting Nezara the spermatogonia possess but one microchromosome (the small idiochromosome), while in Nezara two equal microchromosomes are present as in forms like Anasa which possess an accessory chromosome.

5. In the primary synapsis the idiochromosomes may unite to form a bivalent body or may remain separate. In the former case the bivalent body condenses to form a single chromosome-nucleolus that persists throughout the whole growth-period, but again separates into its univalent constituents before the first mitosis (directly proved in Brochymena, inferred in the other forms). If the idiochromosomes fail to unite in the primary synapsis, they remain separate through the growth-period in the form of chromosome-nucleoli. In either case the idiochromosomes divide separately in the first mitosis.

6. In Lygæus the large idiochromosome has in the synaptic and early post-synaptic periods the form of a long longitudinally split thread which afterward condenses into a hollow spheroidal chromosome-nucleolus.

Zoülogical Laboratory, Columbia University, May 5th, 1905.

\section{WORKS CITED.}

Bonnevie, K., '05.-Das Verhalten des Chromatins in den Keimzellen enteroxenos östergreni. Anat. anz., xxvi, 13, 14, 15.

Gross, J., '04.-Die Spermatogenese von Syromastes marginatus; Zool. Jahrb., Anat. u. Ontog., xx, 3.

Montgomery, T. H., '98.-The Spermatogenesis in Pentatoma, etc. Zool. Jahrb., Anat. u. Ontog., xii.

'OI, I.-A Study of the Chromosomes of the Germ-cells of Metazoa. Trans. Amer. Phil. Soc., xx.

'or, 2.-Further Studies on the Chromosomes of the Hemiptera heteroptera. Proc. Acad. Nat. Sci., Phil., March, I9or.

'04.- Some Observations and Considerations upon the Maturation Phenomena of the Germ-cells. Biol. Bull., vi, 3, Feb. 
Paulmier, F. C., '99.-The Spermatogenesis of Anasa tristis. Jour. Morph., $\mathrm{xv}$, supplement.

Strasburger, E., '04.-Ueber Reduktionsteilung. Sitzber. Kön. Preuss. Akad. Wiss., xviii, 24 März, 1904.

Sutton, W. S., 'o2.- On the Morphology of the Chromosome Group in Brachystola magna. Biol. Bull., iv, I.

'o3.-The Chromosomes in Heredity. Biol. Bull., iv, 5 .

Wilson, E. B., '05.-Observations on the Chromosomes in Hemiptera. Rept. N. Y. Academy of Sciences, May 8th, 1905; Science, xxi, 548, June 30 . 

(1)

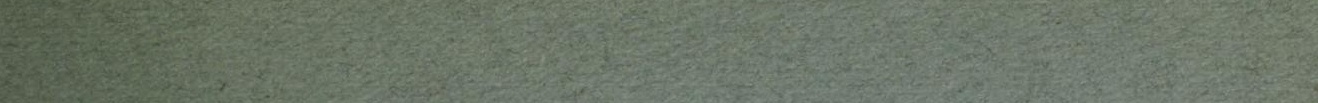

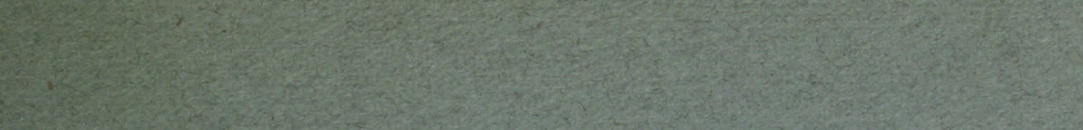

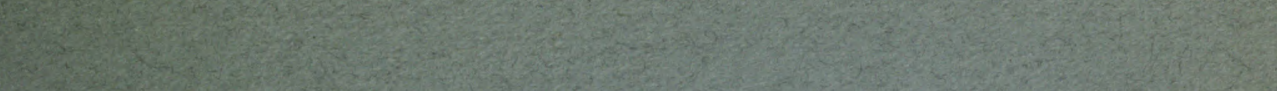

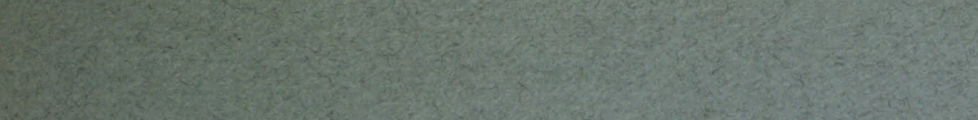
W. 140 .

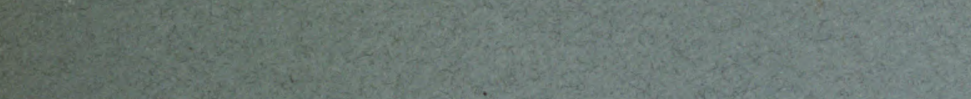
(2)

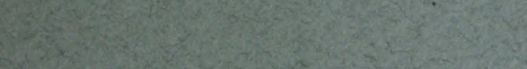

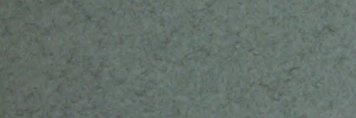
Por.

(3)

ats

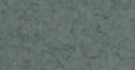

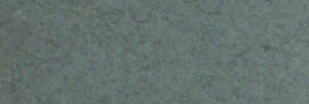

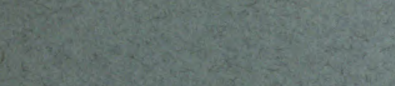

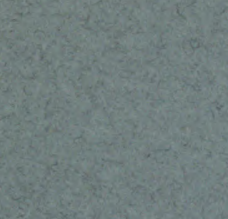

$60 x+4 x^{2}$ 2.

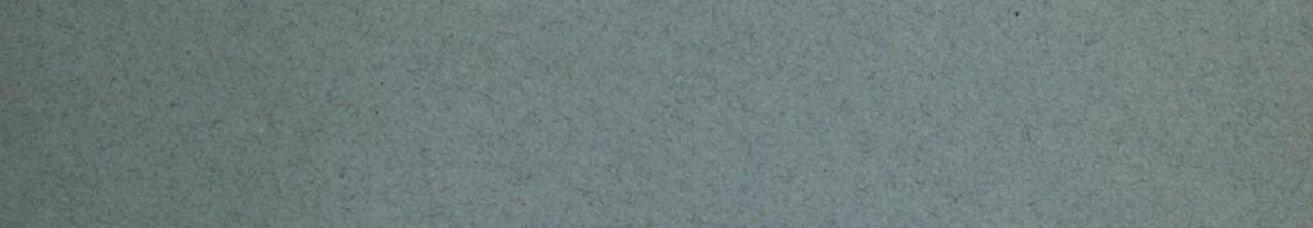

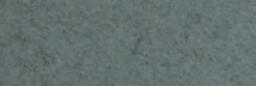

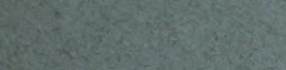

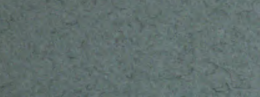

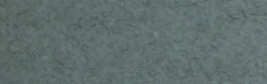

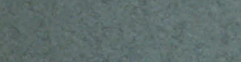

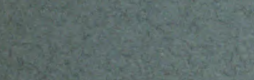

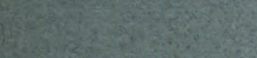

at

$\frac{B}{2}=$

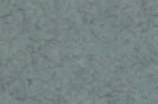

(5)

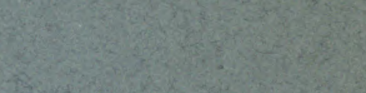

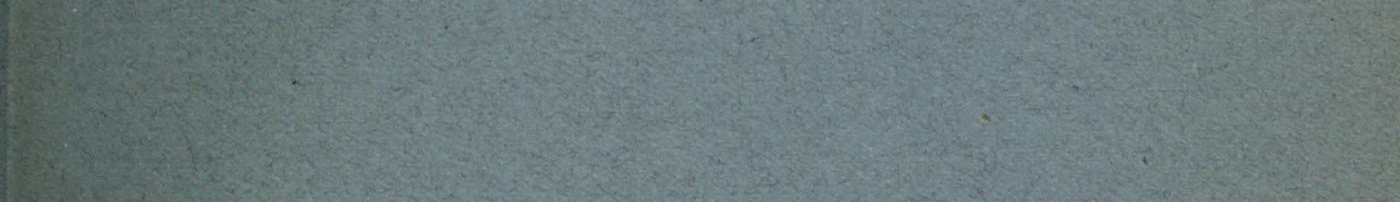

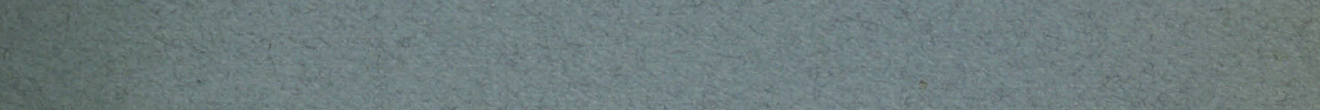

(3) 
THE JOURNAL OF EXPERIMENTAL ZOÖLOGY is issued quarterly. A volume consists of four numbers, containing from 100 to 200 pages each, with numerous illustrations.

\section{PRICE OF SUBSCRIPTION PER VOLUME}

(PAYABLE IN ADVANCE)

To subscribers in the United States, Canada and Mexico, $\$ 5.00$

To subscribers in other countries - $\quad-\quad-\quad-\quad-5.50$

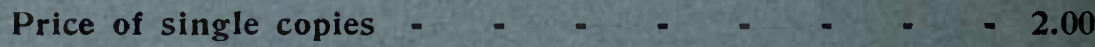

These prices are net and under no condition subject to discount

Remittances should be made by Postal Money Order (Mandat de Poste, Postanweisung) or by draft on New York, payable to THE Journal of Experimental ZoöLogy.

Address all communications to

THE JOURNAL OF EXPERIMENTAL ZOÖLOGY,

N. E. Cor. Wolfe and Monument Streets,

Baltimore, Md., U. S. A. 


\section{STUDIES ON CHROMOSOMES}

II. THE PAIRED MICROCHROMOSOMES, IDIOCHROMOSOMES AND HETEROTROPIC CHRO-

MOSOMES IN HEMIPTERA

By

EDMUND B. WILSON

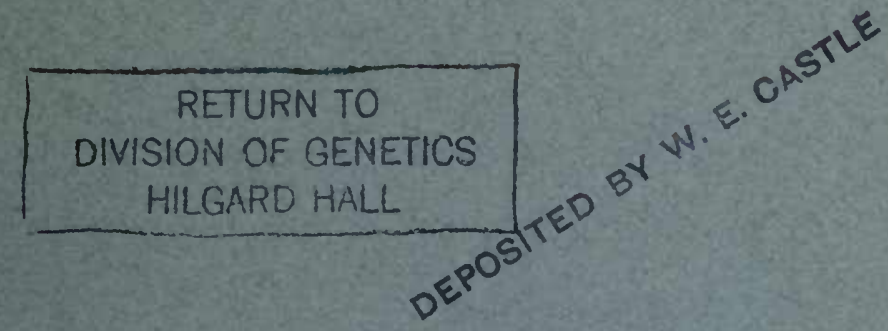

REPRINTED FROM

THE JOURNAL OF EXPERIMENTAL ZOÖLOGY

Volume II

No. 4

BALTIMORE, MD., U. S. A.

November, 1905 . 



\section{STUDIES ON CHROMOSOMES.}

\section{THE PAIRED MICROCHROMOSOMES, IDIOCHRO- MOSOMES AND HETEROTROPIC CHRO- MOSOMES IN HEMIPTERA. ${ }^{3}$ BY \\ EDMUND B. WILSON.}

With 4 Figures.

In investigating the physiological significance of the chromosomes and their individual values in heredity, it is important to determine as accurately as possible how far they are differentiated in respect to individual behavior, and to ascertain by the comparative study of different forms to what extent the chromosomes can be grouped in well-defined classes. The work of Henking, Paulmier, Montgomery, Gross and Stevens on the Hemiptera has shown that this group is peculiarly favorable for such a study; and I believe from my own observation that no group of animals has thus far been examined that offers greater advantages in this direction. ${ }^{2}$ But although the general results obtained by the above-mentioned observers are of great value and interest they nevertheless show many discordances of detail that stand in the way of a consistent general interpretation of the phenomena, while some of Gross's conclusions are a stumbling block in the way of the whole theory of the individuality of the chromosomes. For this reason I propose in this paper to record a series of observations that I hope may serve to clear away some of the confusion that now exists in the accounts of the subject, and that open the way, I believe, to a true interpretation of the "accessory chromosome" and its relation to the determination of sex.

In a series of suggestive papers ('Or, '04, '05) Montgomery

\footnotetext{
${ }^{1}$ Attention is called to the Appendir in which are briefly recorded facts, determined by later observations, that exactly realize the theoretic expectation regarding the sexual differences of the chromosome-groups, stated at p. 539. An abstract of these observations was published in the issue of Science for Oct. 20, 1905 .

${ }^{2} \mathrm{~J}$ am much indebted to Mr. Uhler's kindness in identifying many of the species examined.
} 
has endeavored to bring together under the name of "heterochromosomes" two classes of chromosomes in these insects, namely, the "unpaired heterochromosome" ("accessory chromosome" of McClung) 1 and the "paired heterochromosomes" (or "chromatin nucleoli"), which differ markedly in behavior from the other chromosomes during the maturation process. Montgomery gives as the most essential characteristic of these chromosomes "their difference in behavior from the other chromosomes in the growth period of the spermatocytes and ovocytes, as sometimes during the rest period of the spermatogonia, a difference which appears usually to consist in the maintenance of their compact structure and deep-staining intensity, so that while the other chromosomes become long loops or even compose a reticulum, these do not undergo any such changes or only to slight extent" ('05, p. I9r). "Thanks to this peculiarity they can be followed with extreme certainty from generation to generation, even during rest stages; and so are splendid evidence for the thesis of the individuality of the chromosomes" ('O4, p. I46).

The study of these chromosomes has led Montgomery to some very important conclusions regarding synapsis and reduction with which, as far as their more general features are concerned, I am glad to find my own results in substantial agreement. Considered more in detail, however, there are many points regarding which I think Montgomery's general treatment of the "heterochromosomes" requires emendation.

In a preceding paper (Wilson,'05) the fact was indicated that two types of "paired heterochromosomes" or "chromatin nucleoli" occur in Hemiptera. The first, including what I have called the

"Since there is no reason for considering the "accessory chromosome" as in any sense accessory to the others, it appears to me that McClung's term might well be abandoned in favor of a less compromising one. I suggest that until their physiological significance is positively determined chromosomes of this type may provisionally be called hete otropic chromosomes (in allusion to the fact that they pass to one pole only of the spindle in onc of the maturation-divisions) in contradistinction to amphitropic chromosomes, the products of which pass to both poles in both divisions. There are several objections to this term, one of which is that the "accessory" chromosome behaves as a heterotropic body in only one of the divisions (and probably in one sex only). Another is the fact that the members ("chromatids") of every chromosome-pair are heterotropic in the reducing division, since this only separates univalent chromosomes that were previously in synapsis; but if, as in these studies, the term "chromosome" be consistently applied to each coherent chromatin-element of the equatorial plate, whatever be its valence or mode of origin, this objection is perhaps not serious enough to weigh against the convenience of the term. 
"idiochromosomes" (which occur in such forms as Lygæus, Euschistus, Cœnus, Brochymena, etc.) are typically unequal in size, and differ from all other known forms of chromosomes in the fact that their union in synapsis gives rise to an unequal or asymmetrical bivalent. The spermatogonial groups correspondingly show but one small chromosome, since the larger idiochromosome is not noticeably smaller than the ordinary chromosomes. The second type includes the equal paired "chromatin nucleoli" of such forms as Anasa, Alydus, Syromastes or Archimerus. Since the latter are almost always markedly smaller than the others they may conveniently be called the paired microchromosomes, or better, in order to avoid all ambiguity, simply the $m$-chromosomes; and these are distinguishable in the spermatogonial groups as an equal pair of especially small chromosomes. The most obvious difference of behavior between these two types, so far as is now known, is that the idiochromosomes divide as separate univalents in the first maturation-mitosis, which accordingly always shows one more than half the spermatogonial number of separate chromatin elements, while the $m$-chromosomes, like the other chromosomes, always unite to form a bivalent before the first mitosis-which therefore shows the same number as in the second division. Other no less characteristic differences are described beyond. These two forms are not yet known to coexist in the same species; and, as a rule, forms that possess the idiochromosomes do not have an "accessory" or heterotropic chromosome, while as far as now known such a chromosome is always associated with the $m$-chromosomes.

The confusion that has grown out of the failure to observe these differences arose in the first instance from two conclusions- both of which I shall show to be untenable-reached by Paulmier in his valuable, and, as far as the general history of the maturationprocess is concerned, very accurate, study of the spermatogenesis of Anasa tristis ('99), and was increased by the subsequent efforts of Montgomery ('OI, '04, 'O5) to reduce the behavior of the "chromatin nucleoli" to a uniform scheme. Paulmier, who was the first to reëxamine the history of the "accessory" chromosome since its discovery by Henking, was also the first to describe the $m$-chromosomes (in Anasa) as two very small chromosomes of equal size in the spermatogonial metaphase-groups. These two 
small chromosomes, he believed, united in synapsis to form a single condensed bivalent chromosome-nucleolus which persisted throughout the growth-period of the spermatocytes and later gave rise to the small central "tetrad" of the first maturation-mitosis. $\mathrm{He}$ believed, further, that after an equal division of this small "tetrad" in the first mitosis each of its products passed undivided to one pole of the second spermatocyte-spindle. He therefore compared the "small tetrad" (microchromosome-bivalent) of Anasa to the body, first discovered by Henking in Pyrrochoris, and afterward found in the Orthoptera and some other insects by McClung and others, to which the last-named author gave the name of "accessory chromosome." In identifying the chromosome-nucleolus of the growth-period as the microchromosomebivalent Paulmier has been followed by Montgomery in all of his papers and with some modifications by Gross ('04) in his recent study of Syromastes. Paulmier's conclusion on this point cannot, however, be sustained, as I shall try to show; and the same is true of his identification of the microchromosome-bivalent as the "accessory" or heterotropic chromosome.

I. GENERAL HISTORY OF THE $M$-CHROMOSOMES AND THE HETEROTROPIC CHROMOSOME DURING THE GROWTH-PERIOD AND IN THE MATURATION-DIVISIONS.

The behavior of the $m$-chromosomes in the maturation-divisions may conveniently be considered first.

Paulmier's original preparations, ${ }^{1}$ as well as my own more recent ones, give demonstrative evidence of the equal division of the small central chromosome in both maturation-mitoses, and the same appears no less clearly in Alydus and in Archimerus, precisely as has been shown by Montgomery ('OI) in Protenor and by Gross ('O4) in Syromastes. I was long since led to suspect an error in Paulmier's conclusion in regard to this point from the fact, which clearly appears in his own figures, that the "accessory" is nearly or quite as large as the other chromosomes, and much larger than the products of the first division of the small bivalent.

II have in the previous paper acknowledged my indebtedness to Dr. Paulmier's generosity in placing at my disposal his entire series of preparations of Anasa and other insects. He has since added to this indebtedness by sending me from time to time a large amount of valuable living material. 
(Cf. Paulmier's Figs. 28, 34-36, and my Fig. 2, $k-n$.) Both in Anasa and in Alydus careful search among longitudinal sections of the second division shows in fact in the clearest manner, that the "small dyad" divides into equal halves, so that each of the spermatids received one of its products (Figs. I, $i-m ; 2, m, n$ ). The heterotropic chromosome is a much larger body, as shown by the figures, in Anasa fully equal in size to some of the larger single chromosomes of the anaphases of the second division. Paulmier's failure to observe the second division of the small bivalent is easily explained by the difficulty of observing this body owing to its usually central or subcentral position, and the mistake was a very natural one at the time his paper was written. Had he examined Alydus where there are but seven chromosomes, which show marked and constant size-differences, he could not have failed to observe this division.

We have now to examine a second and more difficult point, namely, the nature of the condensed nucleolus-like body (chromosome-nucleolus) of the growth-period, which so closely simulates the heterotropic chromosome of the Orthoptera at the corresponding period. I have always doubted Paulmier's and Montgomery's conclusion that this body is the microchromosomebivalent, from the fact, clearly shown in the figures of both these authors, that the chromosome-nucleolus of the synaptic and growth-periods is always larger, and in some species very much larger (e. g., in Alydus) ${ }^{\mathrm{i}}$ than the two spermatogonial microchromosomes taken together, or than the small central bivalent to which it was assumed to give rise. ( $C f$. Paulmier's Figs. I6-2 r, with 26,28 .) This fact did not escape Montgomery's attention, but he explained it as due to an increase of volume on the part of the chromatin-nucleolus in the early growth-period and a corresponding decrease in the late growth-period or in the prophases of the first division ('or, p. 203). This explanation was, however, not supported by any sufficient evidence ${ }^{2}$ and the only detailed evidence on this point has been brought forward by Gross ('04) in the case of Syromastes. 'This observer, however, while apparently confirming Paulmier and Montgomery as to

\footnotetext{
${ }^{1}$ Cf. Montgomery, 'or, Figs. 96-98.

${ }^{2}$ Montgomery's study of the facts in Euschistus (' 98 ) is not in point, since he was here undoubtedly dealing with the idiochromosomes and not with the $m$-chromosomes.
} 
the fate of the chromosome-nucleolus, differs entirely from them in regard to its origin, concluding that it is derived from two of the larger spermatogonial chromosomes. In the attempt to reconcile these contradictory results (with both of which my own are in disagreement) he is led to some speculative conclusions that I think must be regarded as highly improbable. ${ }^{1}$

A careful study of all the intermediate stages, not only in Anasa, but also in Alydus, Archimerus, and Chariesterus gives in point of fact, evidence that I believe is quite decisive, that the small central bivalent is not derived from the large chromosome-nucleolus of the growth-period, and that the latter is nothing other than the accessory or beterotropic. chromosome, precisely as in the Orthoptera. To the differences between the idiochromosomes and the $m$-chromosomes already stated may therefore be added the fact that the former, like the heterotropic chromosome, may form a single chromosome-nucleolus during the growth-period, while this is not the case, in the forms I have studied, with the $m$-chromosomes. It may seem strange that Montgomery, after accurately tracing the history of the heterotropic chromosome ("chromosome $x$ ") in Protenor and showing its complete independence of the "chromatin-nucleoli" ( $m$-chromosomes) was not led to suspect a similar relation in the other forms. That he apparently did not do so was doubtless due to his having failed to distinguish between the $m$-chromosomes and the idiochromosomes, which latter bodies he correctly identified (in Euschistus, etc.) as the bivalent chromosome-nucleolus (or two separate univalents) of the growth-period.

The entire independence of the large chromosome-nucleolus and the $m$-chromosomes is most obvious in Alydus and Archimerus, partly because in both these forms the heterotropic chromosome is at every period recognizable by its characteristic size, partly because - in Alydus certainly, and I believe also in Archimerus - the $m$-chromosomes frequently assume a compact condensed form at a much earlier period than in Anasa; they can therefore be recognized in addition to the beterotropic chromosome, throughout the latter part of the growth-period, at a time when the larger chromosomes are still in the pale, vague condition characteristic of so many of the Hemiptera at this period.

In Alydus pilosulus the first mitosis invariably shows seven

${ }^{1}$ Gross ('04) pp. $4^{81}, 4^{82 .}$ 
bivalent chromosomes, which show very marked and characteristic size-differences (Fig. I , $c-g, b)$. There are always (I) a largest chromosome or macrochromosome, which is frequently quadripartite; (2) a second largest; (3) three slightly smaller ones of nearly equal size; (4) a fourth, considerably smaller than the last; and finally (5) the smallest or michrochromosome-bivalent. These show a characteristic grouping, the five larger ones forming an irregular ring with the small bivalent ("chromatin nucleolus") at its center, while the next smallest lies more or less at one side of the ring (Fig. I, $g$ ). In the first division all these chromosomes are equally halved (Fig. I, $f$ ). In the second all are again halved with the exception of the second smallest which passes undivided to one pole of the spindle (Fig. I, $i-0$ ). The size-relations leave not the least doubt that this chromosome is derived from the one of corresponding size in the first division-i. e., the odd or eccentric one-and the latter accordingly is to be identified as the "accessory" or heterotropic chromosome. In the first division this chromosome sometimes shows a quadripartite form (as was described by Paulmier in Anasa) sometimes a dumbbell-shaped or dyad-like form. In the second it is usually unconstricted and often curved (Fig. I, $i, m, n$ ), sometimes into a $\mathrm{U}$-shape so as almost to appear double (Fig. I, o).

A study of the growth-period shows that the heterotropic chromosome may be traced uninterruptedly backward from the metaphase of the first division to the contraction-phase of the synaptic period, being always in the form of a condensed chromosome-nucleolus, which in the early growth-period is attached to a large, pale plasmosome, from which it afterwards separates. It is impossible to mistake this chromosome, owing to the fact that its characteristic size does not noticeably change except that it becomes sligbtly larger as the growth-period advances (probably owing to the presence of a central cavity), again becoming slightly smaller as the general condensation takes place. (Cf. Fig. I, $a-c$.) In the contraction-phase (Fig. I, $a$ ) and in the early postsynaptic spireme the $m$-chromosomes are not visible, but as the larger chromosomes assume the peculiar pale, ragged, clumped condition, characteristic of the middle and late growth-periods, the $m$-chromosomes frequently come into view, in the form of two compact, intensely-staining bodies, that may occupy any relative position (Fig. $\mathrm{I}, b$ ). The period at which these bodies 


\section{Figure 1.}

Alydus pilosulus. $-a$, Contraction-phase of synaptic period, "accessory" ( $h)$ in the form of a condensed chromosome-nucleolus attached to a large plasmosome $(p) ; b$, spermátocyte-nucleus, middle growth-period, showing large diffused chromosomes- "accessory" still attached to the plasmosomeand the two condensed $m$-chromosomes on opposite sides of the nucleus; $c$, early prophase of first division, showing all of the chromosomes, the larger ones condensing; d, late prophase, showing "accessory" $(h)$ and the two $m$-chromosomes still separate; e, slightly later prophase, showing all of the chromosomes; $f$, initial anaphase, first division, the $m$-chromosomes separating; $g$, polar view of metaphase-group, first division; $h$, polar view of metaphase-figure, second division; $i, j$, initial anaphases, second division; $k$, spermatogonial metaphase-group; $l, m, n, 0$, anaphases of second division. 
Studies on Chromosomes.

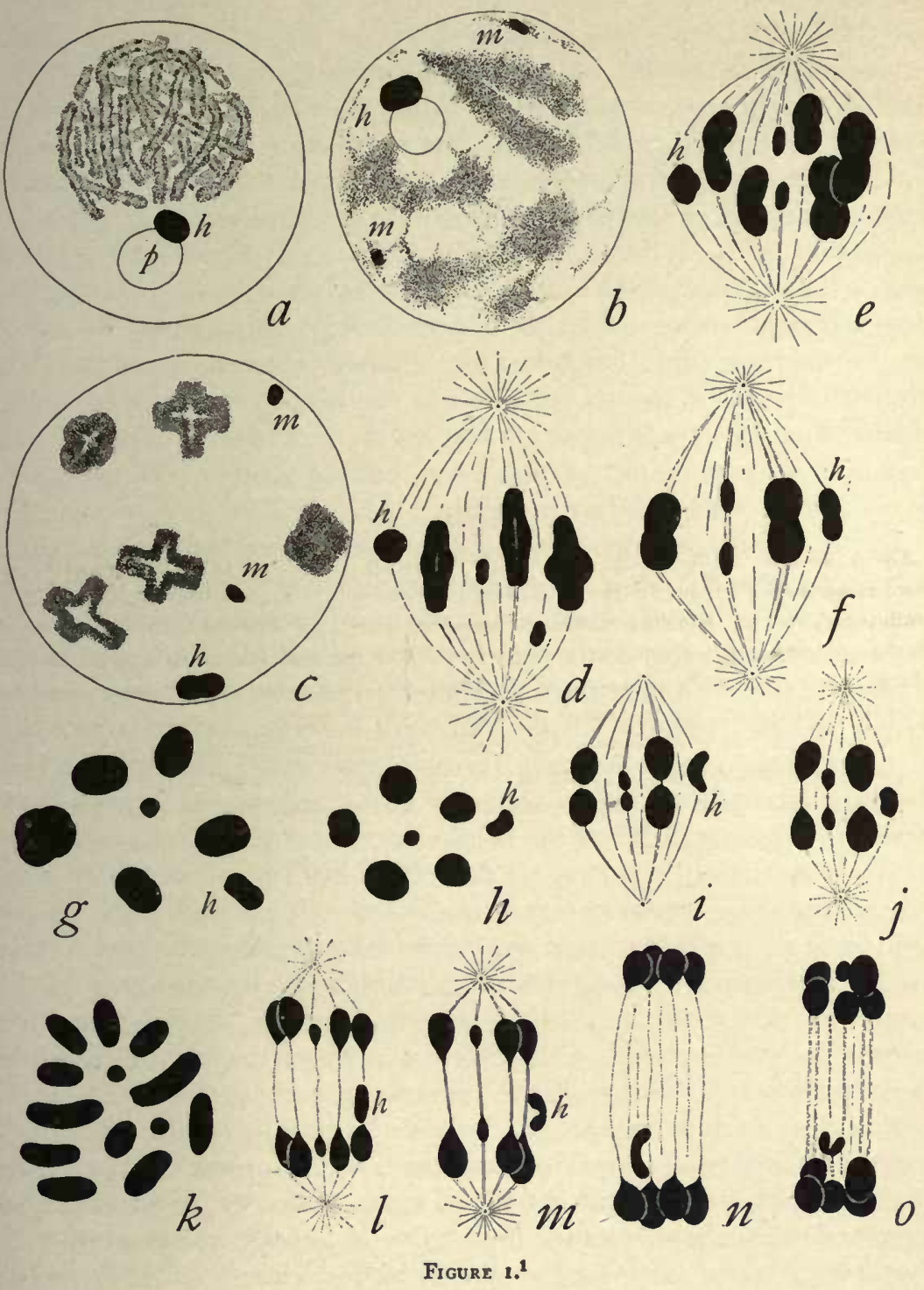

${ }^{1}$ The figures are all drawn to the same scale as those of the preceding study. 
condense into the compact form appears to vary considerably, for they cannot always be distinguished until the later growthperiod, and it should be noted that during the pale period the nuclei often show a variable number of smaller deeply-staining granules. I believe, however, that there can be no doubt as to the nature of the two larger bodies on account of their great constancy, their size, and the completeness of the series that connects the earlier with the later conditions (such as is shown in Fig. I, $c$ ), where no doubt of their nature can exist. The persistence of the larger chromosome-nucleolus ("accessory") throughout all these stages without any considerable change renders it manifestly impossible that it should give rise to the $m$-chromosome bivalent, either directly as assumed by Paulmier and Montgomery, or by division into two univalents that subsequently conjugate, as described by Gross in Syromastes.

In the early prophases the larger chromosomes resume their staining capacity and condense into characteristic cross-forms (Fig. I, $c$ ), and finally into compact quadripartite tetrads or bipartite bodies. At this time the heterotropic chromosome assumes a dumbbell or quadripartite shape, and the $m$-chromosomes, which are still quite separate and may even lie on opposite sides of the nucleus, also frequently become bipartite. The nucleus now contains, accordingly; eight separate chromatin-elements, one more than the number of bivalents in the first 'mitosis; as is also the case in Archimerus and Anasa, as described beyond. As the spindle forms the two microchromosomes lose their bipartite shape, approach each other, and in the stage just preceding the metaphase finally conjugate to form the small bivalent chromosome at the center of the group. Without fusing, the two halves are then immediately separated, the division always taking place more rapidly than in the case of the larger chromosomes (Fig. I, $f$ ).

It is clear to demonstration accordingly, that in Alydus the small central bivalent does not arise from the large chromosome-nucleolus of the growth-period, but is formed by the late conjugation of two separate microchromosomes that have no genetic connection with that body. The same fact is shown no less clearly in Archimerus calcarator (which shows eight chromosomes in the first mitosis), where the $m$-chromosomes, and the corresponding bivalent, are of extraordinary minuteness and are so much smaller than the accessory that they could not possibly be confused with the latter (Fig. 3). 
I believe that in this form, too, the $m$-chromosomes are frequently recognizable as condensed separate bodies in the growthperiod; but owing to their minute size it is difficult to be sure of this. In any case, in the period just before the disappearance of the nuclear membrane they are quite distinct from the "accessory," which is, as in Alydus, immediately recognizable by its size (Fig. 3, g). From this period, as in Alydus, the latter body may be traced continuously backward into the growth-period.

The foregoing facts, observed in Alydus and Archimerus are in close agreement with Montgomery's results on Protenor, differing only in that the condensation of the $m$-chromosomes takes place somewhat later. ${ }^{1}$ In Anasa the condensation of these chromosomes from the diffused condition takes place still later; and this, combined with the fact that the "accessory" cannot be certainly distinguished from the other larger chromosomes by its size, renders the question more difficult of solution than in Alydus, though I believe the result is equally decisive. In Anasa, as in Alydus or Archimerus, the small central bivalent of the first equatorial plate is formed by a very late conjugation of two separate microchromosomes that only come together as the spindle forms, precisely as Gross describes in Syromastes. Of this fact no doubt is left by the study of a large number of preparations that show every stage of the process, step by step. In the late prophases, just before the nuclear membrane disappears, the nuclei invariably show twelve separate, condensed, intensely-staining chromatin-elements (one more than the number of chromosomes in the first mitosis) in addition to one or more pale rounded plasmosomes with which the chromosomes cannot for a moment be confused. Ten of these are larger bivalents which have the form of quadripartite tetrads or dumbbell-shaped bodies. The remaining two are much smaller bodies, irregularly ovoidal or frequently more or less distinctly bipartite $(m$, Fig. $2, e, f)$; they may occupy any relative position. As the spindle forms, the microchromosomes lose their bipartite form, assume an evenly rounded ovoidal shape, and conjugate at the center of the equatorial plate to form a small dyad-shaped bivalent (Fig. 2, $g-i$ ). Without fusion the two halves are then immediately drawn apart

\footnotetext{
In Alydus pilosulus this author believed the $m$-chromosomes, as usual, to be derived from the large chromosome-nucleolus.
} 


\section{Figure 2.}

Anasa tristis. $-a$, Contraction-phase of synaptic period, showing "accessory" $(h)$ and plasmosome ( $p)$; $b$, spermatocyte-nucleus, late gro wth-period, beginning of the condensation, showing "accessory" ( $h)$ and the $m$-chromosomes $(m) ; c$, a slightly later stage than the last; $d$, later stage, immediately before the final condensation, from a long-extracted preparation; $e, f$, two sections of one nucleus, showing all of the twelve chromosomes immediately before the disappearance of the nuclear membrane; - $g$, view of one pole of the late prophase just after disappearance of the nuclear membrane, the $m$-chromosomes still wide apart; $h$, early metaphase-group in side view, showing approach of the $m$-chromosomes; $i$, four chromosomes from the metaphase, conjugation of the $m$-chromosomes to form the small central bivalent; $j$, early anaphase, separation of the $m$-chromosomes, "accessory" at the left; $k$, polar view of metaphase-group, first division; $l$, polar view of metaphase-group, second division; $m, n$, anaphases of second division, showing division of $m$-chromosomes and the undivided heterotropic chromosome; $0, p$, spermatogonial metaphase-groups drawn as carefully as possible to show sizes of the chromosomes. 
Studies on Chromosomes.

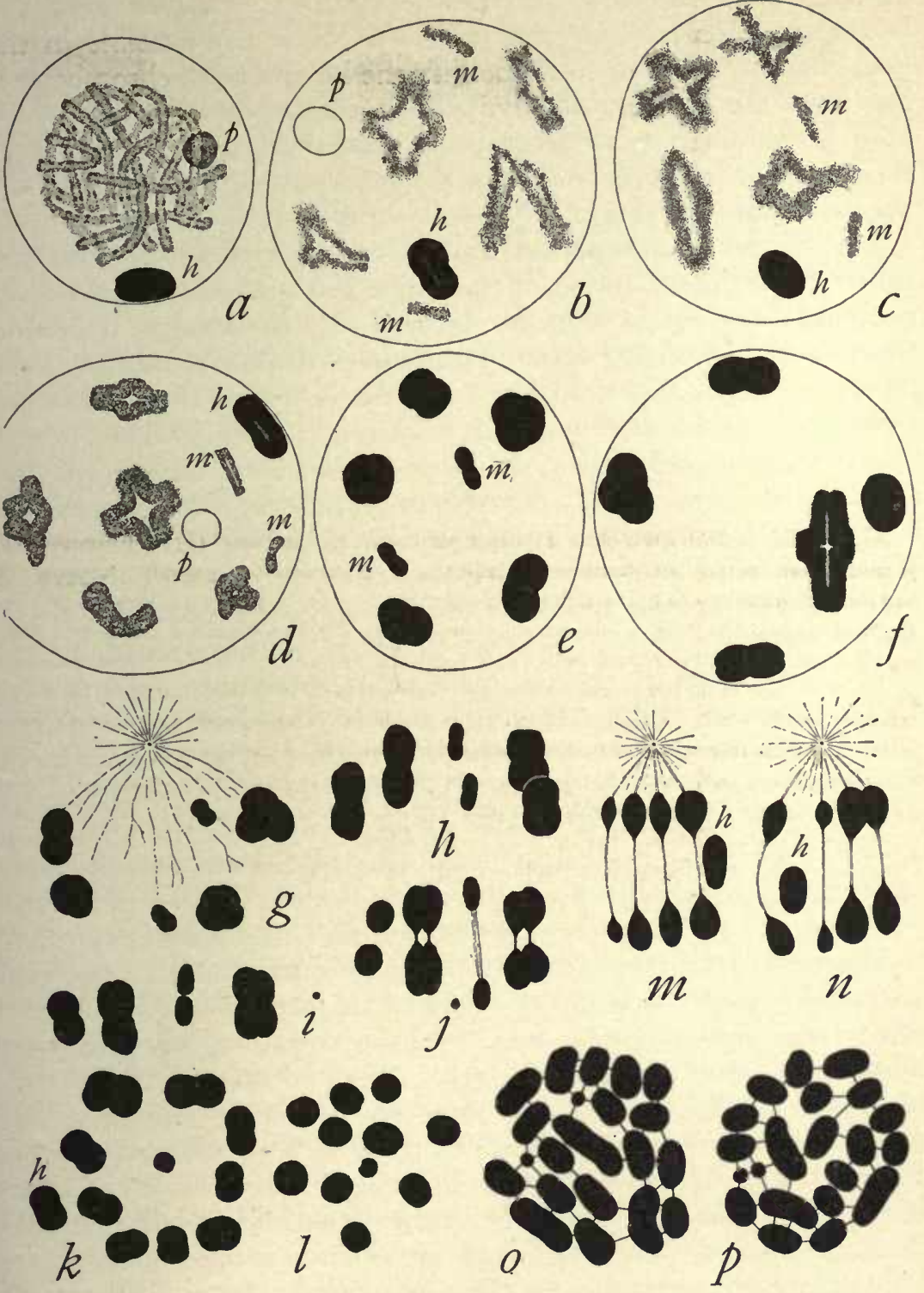

Figure 2. 
in the initial anaphase, always separating in advance of the larger chromosome-halves (Fig. 2, j). It is not possible in the prophases just described to identify the heterotropic chromosome; but from the analogy of Alydus, Syromastes and Archimerus it may be assumed with great probability that it is the "odd or eccentric chromosome which in the metaphase-group lies outside the principal ring (Fig. 2, $k$ ).

During the growth-period, as Paulmier described, the chromosomes, with the exception of the single conspicuous chromosomenucleolus, remain in a loose, diffused, lightly-staining condition from the post-synaptic spireme stage until the condensation of the tetrads begins; and until the end of this period the $m$-chromosomes cannot be distinguished. Throughout this whole period the chromosome-nucleolus is distinctly visible; and it may at every period, even in hematoxylin preparations, if long extracted, be at once distinguished from the true nucleolus or plasmosome (as is shown in Paulmier's figures), since the former stains intensely black, the latter pale blue or in double-stained preparations, pale red or yellow. In the contraction-phase of the synaptic period it is more or less elongated, ovoidal, or sometimes slightly constricted in the middle (Fig. 2, a). In the late post-synaptic period, at a time when the other chromosomes are beginning to shorten and to give rise to the characteristic double cross-figures and V-figures it is usually more or less elongated, the transverse constriction is less obvious or disappears from view, and the body often shows faintly but distinctly a longitudinal split. (Cf. Paulmier, Fig. 22.) Slightly later, as the other chromosomes continue to shorten and thicken, the chromosome-nucleolus also shortens and thickens, of ten assuming a spheroidal form in which a central cavity may sometimes be seen. As the remaining chromosomes condense to form the tetrads it again alters its shape, often becoming bipartite (Fig. 2, $b-d$ ), but sometimes showing a more or less distinctly quadripartite form as described by Paulmier $(e . g$., in his Figs. 23, 24). It now becomes indistinguishable from the other larger chromosomes, since the latter. have also condensed into similar tetrads or dyad-like forms, but the two $m$-chromosomes are immediately recognizable by their small size. It might therefore be supposed that the chromosome-nucleolus has divided to form the two microchromosomes, as Gross believed to be the case in Syromastes. The stage that immediately precedes this gives, 
however, conclusive evidence that such is not the case. In this stage (corresponding to Paulmier's Figs. 22, 23) the chromosomenucleolus is still unmistakably recognizable by its compact and rounded appearance, while the other chromosomes, including the two microchromosomes are still in the form of paler and more diffuse bodies. The $m$-chromosomes at this period (one of them is clearly shown in Paulmier's Fig. 24) appear as short, more or less ragged, paler, irregular rods that give the appearance of being longitudinally split (Fig. 2, b-d). Some of the cysts at this period show every stage in the condensation of these two small diffused chromosomes to form the two small, dyad-like microchromosomes that conjugate to form the small central bivalent. I have studied numerous nuclei in these stages with great care, and believe that they remove every doubt that the two microchromosomes that conjugate to form the small central bivalent in Anasa arise neither from separate small condensed bodies, as in Protenor or often in Alydus, nor from the single large cbromosomenucleolus as assumed by Paulmier, Montgomery and Gross, but from diffused masses similar to the larger ordinary chromosomes during the greater part of the growth-period. The same fact may be seen in Chariesterus, though I have not in this case so complete a series of stages. The chromosome-nucleolus must therefore give rise to one of the larger chromosomes; and the exact agreement of Anasa with Alydus and Archimerus, save in the one point of the later condensation of the microchromosomes in the former form, justifies the confident conclusion that in Anasa the chromosome-nucleolus is the "accessory" or beterotropic chromosome. Anasa, Alydus, Chariesterus, and Archimerus thus fall in line with the facts observed in the Orthoptera, and I believe the same will prove to be the case with other Hemiptera in which an "odd," "accessory" or heterotropic chromosome occurs. ${ }^{1}$ This result, which is wholly at variance with the accounts of previous observers, forms the first step in clearing away the confusion that has hitherto stood in the way of a consistent general interpretation of the heterotropic chromosome.

1 I cannot at present offer a definite explanation of the divergence between this result and that reached by Gross in Syromastes. Without questioning the accuracy of his figures, I feel confident, in view of what I have seen in so many other forms, that further examination of this genus will give a different result, both on this point and on a number of others. 
2. RELATION OF THE CHROMOSOME-NUCLEOLUS TO THE SPERMATOGONIAL CHROMOSOMES.

In view of the foregoing conclusion it will readily be admitted that a derivation of the chromosome-nucleolus from the two spermatogonial microchromosomes is a priori highly improbable; and in point of fact, all the actual observations not only of myself, but also, I believe, of Paulmier and Montgomery, are opposed to such a conclusion.

This question has beén complicated in a most unfortunate way by errors in counting the spermatogonial chromosomes. It was natural that the earlier observers should have expected to find an even number of chromosomes in the spermatogonial divisions; and the number is in point of fact an even one in all the forms that possess the idiochromosomes, as I have shown in the first of these studies. Regarding the forms that possess an accessory or heterotropic chromosome the existing accounts are, however, in conflict in giving sometimes an even number (Anasa, $t$. Paulmier and Montgomery, Syromastes, $t$. Gross, Alydus pilosulus, $t$. Montgomery), and sometimes an odd one (Protenor, Harmostes, Edancala, Alydus eurinus, $t$. Montgomery). A similar difference occurs in the existing accounts of the spermatogonia in Orthoptera, some of which are described as showing an even number and some an odd. This contradiction has enormously increased the complication of the subject; for it has necessarily involved the view that in cases showing an even number the heterotropic chromosome is a bivalent body, formed by the synapsis of two of the spermatogonial chromosomes; and this, in turn, very naturally led Montgomery ('04, '05, etc.) to the further conclusion that in cases showing an odd number one of the chromosomes (presumably the "accessory") is already bivalent in the spermatogonia.

I myself had at first no doubt of the correctness of both these interpretations, and my faith was not shaken even after the discovery that the number is $\mathrm{I} 3$ in Alydus pilosulus (Fig. I, $k$ ), I5 in Archimerus (Fig. 3, i), and $2 \mathrm{I}$ in "Chariesterus." "When, however, demonstrative evidence was obtained that even in Anasa -in opposition to the concordant results of Paulmier and Montgomery on Anasa and those of Gross on the related form Syro-

'The indentification of this form (from Paulmier's material) is doubtful. 
mastes-the number is $2 \mathrm{I}$ instead of 22 ( $\mathrm{Fig} .2,0, p$ ) I confess that surprise at this result was followed by skepticism regarding all of the accounts asserting the occurrence of an even number in other forms. This result, which was totally unexpected to me, rests on the study of a large number of division-figures exactly in the metaphase, many of which are of almost schematic clearness. Of these, twenty-five (selected from six testes from different individuals, including both adults and larval forms) were drawn with the camera, chromosome by chromosome, and subsequently counted. Without one exception these drawings show exactly twenty-one chromosomes; it is therefore out of the question that my result (worked out on Paulmier's original preparations) can be due to an accidental displacement of one of the chromosomes in the process of sectioning, or to other similar sources of error. I believe the error of previous observers on this point is owing to the fact that one or more of the chromosomes sometimes show a more or less obvious constriction near the middle, and the larger ones are not infrequently curved-sometimes almost into a $U$-shape-so that one might readily be mistaken for two.

Quite in harmony with this result is the fact that in Anasa the metaphase groups always show not two but three chromosomes that are distinctly larger than the others, ${ }^{1}$ one of these being obviously without a mate of like size, while all the others may be symmetrically paired, two by two, as a study of Fig. 2, o, p, will show. It is obvious therefore that the heterotropic chromosome, and hence the chromosome-nucleolus of the growth-period, must be compared with one, not two, of the spermatogonial chromosomes.

In Alydus the heterotropic chromosome appears in the contraction phase of the synaptic period as an ovoidal single body, always attached to one side of a large plasmosome and immediately distinguishable from the latter by its different stainingreaction (Fig. I, a). Comparison of this figure with that of the spermatogonial chromosomes (Fig. I, $k$ ), shows that the heterotropic chromosome at this period is much larger than the two spermatogonial microchromosomes united. In the spermatogonial equatorial plates of Alydus or Archimerus it is not possible

II regret to find myself here again in disagreement with Montgomery, who finds only two large spermatogonial chromosomes in Anasa ('04, p. 15 r, Fig. 16). 
positively to identify the heterotropic chromosome by its size; though it is evidently not one of the largest ones, since the latter form a symmetrical pair (Fig. $I, k$ ) which doubtless unite to form the single macrochromosome of the spermatocyte-divisions (in accordance with Montgomery's account of several other forms). In Anasa, however, it may be regarded as highly probable that the heterotropic chromosome is one of the largest three chromosomes, the remaining two of which pair as usual to form the spermatocyte macrochromosome-bivalent (Fig. 2, $0, p$ ). This is confirmed by comparison with the chromosome-nucleolus at the synaptic contraction-period (Fig. 2,a). At this time it varies considerably in form, but is always more or less elongate, often ovoidal, sometimes almost rod-shaped, and sometimes more or less distinctly constricted in the middle; it rarely appears to be composed of two symmetrical halves (described by Gross as the typical condition in Syromastes.) It is rarely attached to a plasmosome, the latter body, when present, being usually separate (as in Fig. 2, a).

The discrepancy in size between the chromosome-nucleolus and the spermatogonial microchromosomes is here still greater than in Alydus. On the other hand, as a comparison of the figures will show, the chromosome-nucleolus of this period is of very nearly the same volume as one of the largest three spermatogonial chromosomes. All the facts therefore point to the conclusion that one of the latter is the heterotropic chromosome, and that it persists throughout the growth-period as the chromosome-nucleolus, precisely as in Alydus or Protenor. Exactly the same result is indicated in Archimerus, where the discrepancy in size between $m$-chromosomes and heterotropic chromosome is even greater than in Anasa (Fig. 3, a, i).

3. BEHAVIOR OF THE HETEROTROPIC CHROMOSOME IN THE MATURATION-DIVISIONS OF ARCHIMERUS CALCARATOR.

In all the Hemiptera thus far described (Pyrrochoris, Anasa, Alydus, Protenor, Syromastes, Harmostes, CEdancala, Chariesterus), the heterotropic chromosome, when present, divides equally in the first spermatocyte-mitosis, but fails to divide in the second, thus showing a marked contrast to the phenomena in the Orthoptera where the reverse order occurs. In the present section I wish 
briefly to record the fact that Archimerus, which agrees so closely with Alydus in most other respects, differs from this and all the above-mentioned forms in that the heterotropic chromosome fails

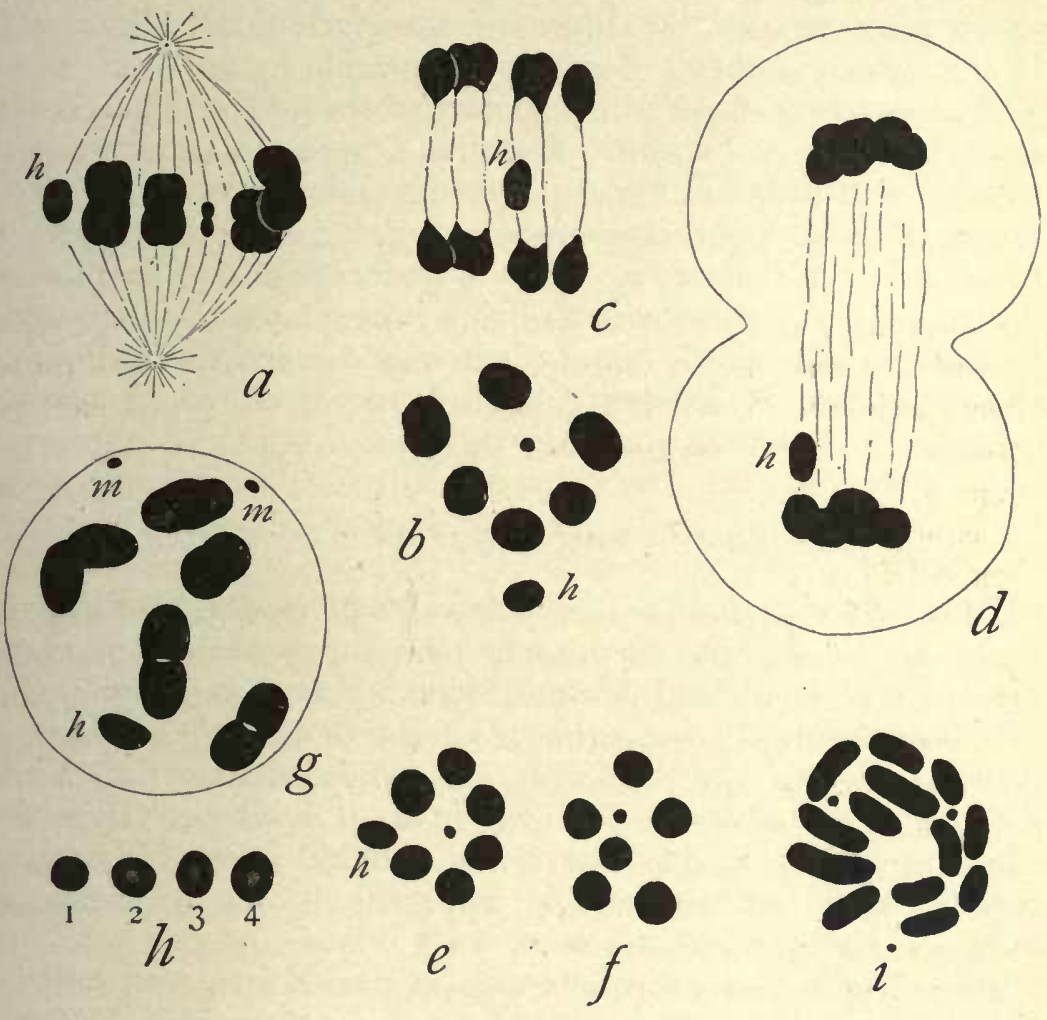

Figure 3.

Archinerus calcarator.- $a$, Side-view of first division metaphase showing heterotropic chromosome and $m$-chromosome bivalent; $b$, polar view of metaphase-group, first division; $c$, anaphase group, first division, side view; $d$, late anaphase, first division; $e, f$, polar views of metaphase-groups, second division, the former including, the latter lacking, the heterotropic chromosome; $g$, spermatocytenucleus, prophase of first division, showing heterotropic chromozome $(h)$, the two separate $m$-chromosomes $(m)$, and five of the sir large bivalents; $h$, views of the chromosome-nucl eolus (heterotropic chromosome) at different periods-I, from the contraction-phase of the synaptic period; 2, middle growth-period; 3, 4, later growth-period (the last three showing central cavity); $i$, spermatogonial metaphase-group.

to divide in the first mitosis, passing over bodily to one pole and dividing equally in the second mitosis, precisely as in the Orthoptera (Fig. 3, c, d). This fact, which at first I myself hardly found 
credible, is placed beyond doubt by numerous preparations showing every stage in the first division, and no less certainly by the occurrence of two forms of the second division, in equal numbers and appearing side by side in the same cyst, one of which shows seven chromosomes, the other eight, the additional chromosome in the latter case being usually recognizable by its size. Fig. 3, $c, d$, shows two stages in the history of the heterotropic chromosome in the first division. Fig. $3, e, f$, gives polar views of the two forms of equatorial plates in the second division, one showing seven, the other eight, chromosomes. A large number of sections from different individuals show no exception to this mode of distribution, the two divisions being immediately distinguishable by the size of the cells and by both the size and the form of the chromosomes. A similar case will be described, in Banasa calva, in the following section.

\section{THE CHROMOSOME-GROUP IN BANASA CALVA.}

In this section I shall briefly describe a remarkable form that is unique among the Hemiptera thus far described in that it possesses both the idiochromosomes and a heterotropic chromosome; and as a consequence of this it is unique among all described animals in possessing not merely two but four visibly different classes of spermatid-nuclei in equal numbers. These four classes are in no visible way distinguishable in the fully formed spermatozoa, but are clearly apparent in the chromosomegroups of the spermatid-nuclei.

No spermatogonial metaphase-groups are shown with sufficient clearness to admit of an accurate count, but there are great numbers of dividing spermatocytes which show every stage of both the maturation-divisions. The first division constantly shows, in polar view of the metaphase, fifteen chromosomes, of which two are markedly smaller than the others (Fig. 4, $a, b$ ). As is demonstrated by their later history, one of these smaller chromosomes is the small idiochromosome (i) and one the heterotropic chromosome $(b)$. One of them frequently, but not invariably, lies at one side of the group, sometimes outside the principal ring of chromosomes (Fig. 4, a); but it may lie inside the ring (Fig. $\mathrm{I}, b$ ). One always lies within the ring; and judging by the analogy of such forms as Lygæus, Euschistus or Conus, a 
much larger chromosome beside which it lies is to be identified as the larger idiochromosome. Besides these fifteen undoubted chromosomes one or more paler rounded bodies are of ten present, lying outside the chromosome-group, sometimes close to it, that are undoubtedly the remains of the plasmosome of the growthperiod.

In side views of the metaphase-figure all of these chromosomes, with one exception, have a symmetrical bipartite (rarely a quadripartite) shape; and in the ensuing division these are equally divided. One of the small chromosomes (heterotropic) never shows a bipartite shape, but is simply elongate and more or less fusiform (Fig. 4, $c, d, e$ ). As the division proceeds, this chromosome at first remains near the equator of the spindle and then passes over bodily toward one pole where it enters the daughter group (Fig. $4, f, g$ ), finally shortening again so as to assume a spheroidal form. One of the secondary spermatocytes therefore receives fifteen chromosomes, the other fourteen.

The failure of this small chromosome to divide in the first mitosis at first seemed to me so anomalous (I had not then observed the similar phenomenon in Archimerus, described in the foregoing section) that for a time I thought that this body must be one of the fragments of the plasmosome; and this suspicion was strengthened by the fact that other plasmosome-fragments are often found lying near or in the spindle (Fig. 4, g). Further study, however, conclusively showed that this suspicion was not well-founded. The plasmosome-fragments are always rounded, paler, wholly inconstant in position and never lie in the equatorial plate. The heterotropic chromosome, on the other hand, is always present (many division-figures in all stages have been studied) and every stage of its asymmetrical distribution has been repeatedly observed. All doubt is, moreover, removed by a study of the metaphase-figures of the second division. Great numbers of these, showing the relations with schematic clearness, are available for study. In polar view these show either fourteen or thirteen chromosomes (Fig. $4, b, i$ ), the two classes existing in approximately equal numbers, and side by side in the same cyst. At first sight neither of the small chromosomes of the first division can be distinguished in polar view of the second. This is owing to two causes: First, the small heterotropic chromosome, having failed to divide while all the others are but half as large as before, 
is sometimes hardly distinguishable from the latter-though, as in Fig. $4, i$, it can often be identified on careful scrutiny. Second, the small idiochromosome, now only half as large as in the first division, has conjugated in typical fashion with the larger one, so as to be visible, as a rule, only in side view (Fig. $4, j$ ), though careful focusing will of ten reveal it also in polar view, especially when the idiochromosome-dyad lies in a slightly oblique position. In this way the idiochromosome-dyad has been positively identified in Fig. 4, $h, i$. In side-view the second division shows with entire clearness the separation of the idiochromosome-dyad into its two unequal constituents, precisely as in Lygæus, Euschistus, etc., while all the other dyads, including the small beterotropic, divide equally (Fig. $4, j-l$ ). From this it follows that four visibly different classes of spermatid chromosome-groups are formed in equal numbers. Two primary classes are formed that possess respectively fourteen and thirteen chromosomes, according to the presence or absence of the heterotropic chromosome; and each of these falls into two secondary classes, one of which contains the large idiochromosome, the other the small. Although this result necessarily follows from the mode of division, it is not a matter merely of inference, but of observed fact; for with a little pains spindles of both classes in the anaphases may readily be found in a vertical position that show both the sister-groups. Such a pair, from the early anaphase of a fourteen-chromosome spermatocyte, are shown in Fig. 4, $m$, the two groups exactly corresponding, chromosome by chromosome, except in case of the idiochromosomes (which are shown by focusing to be more widely separated than the others). A similar pair from a somewhat later anaphase of the thirteen-chromosome class is shown in Fig. 4, o, the relations being as before save that the heterotropic chromosome is lacking. A pair from a later anaphase of the fourteen-chromosome type is shown in Fig. 4, n, showing a principal ring of ten ordinary chromosomes within which lie four others. Two of these (below) are ordinary chromosomes; the other two are, at one pole the heterotropic and the small idiochromosome, at the other pole the heterotropic and the large idiochromosome. 

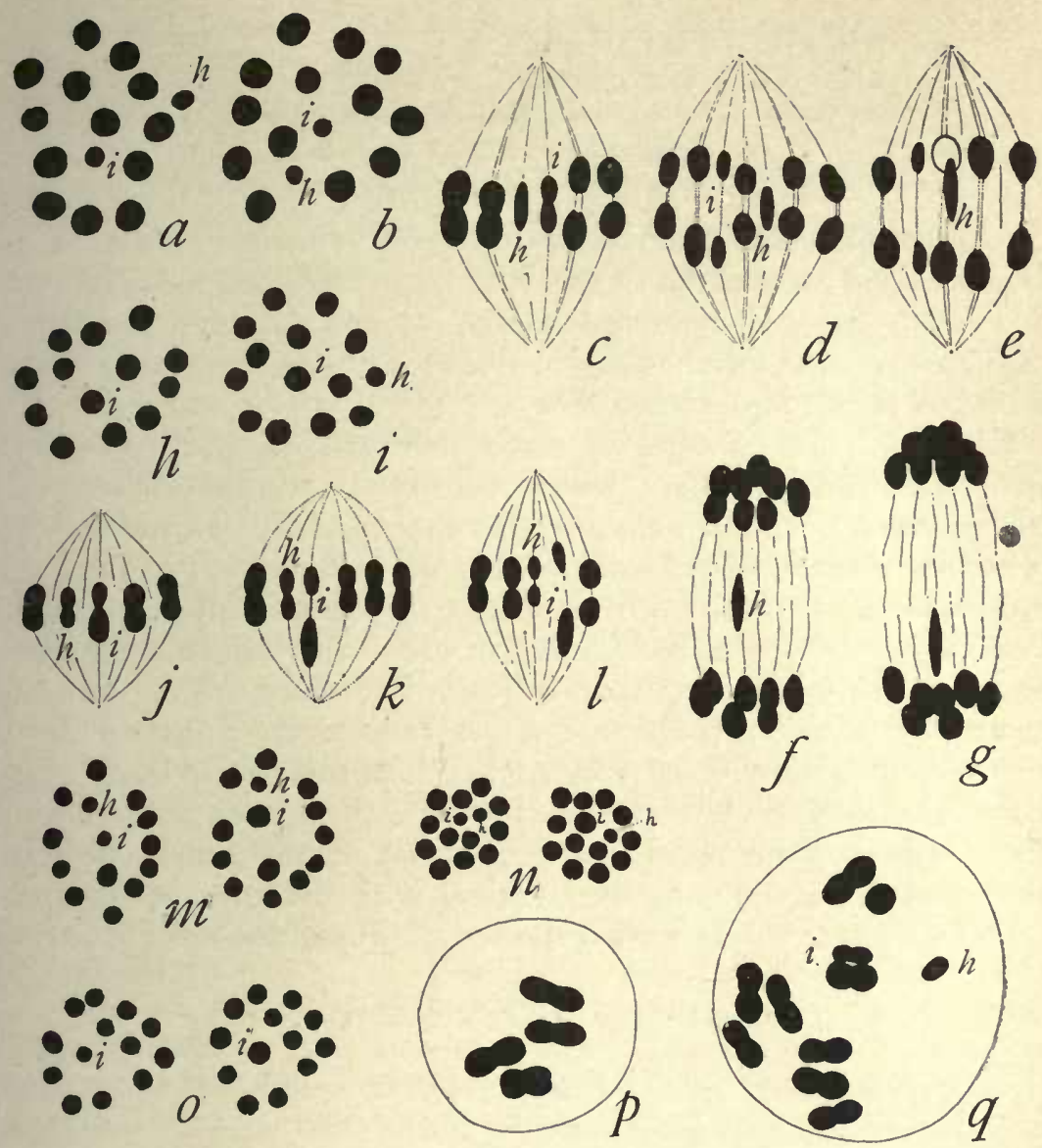

Figure 4.

Banasa calva.- $a, b$, Metaphase-figures, first division, in polar view, showing fifteen chromosomes, including two small ones ( $h$, heterotropic chromosome, $i$, small idiochromosome-the large idiochromosome not distinguishable); $c-g$, successive stages of first division, in side-view, showing division of the small idiochromosome (i), and the unipolar movement of the heterotropic chromosome ( $h$; $h$, metaphase-group of second division, with thirteen chromosomes; $i$, metaphase-group of the same division. with fourteen chromosomes; $j-l$, metaphase and early anaphase of second division, showing separation of the idiochromosomes, and equal division of the heterotropic chromosome; $m$, sister-groups from the same spindle, early anaphase second division, fourteen-chromosome type; $n$, similar pair, late anaphase; 0 , similar pair, middle anaphase, thirteen-chromosome type; $p, q$, entire chromosome-group from a single nucleus at the end of the growth-period, showing idiochromosome-dyad ( $i)$ and heterotropic chromosome $(h)$. 
The four classes thus formed may be tabulated as follows:

Primary Class A $\int(1)$ I2 ordinary chromosomes, 1 heterotropic, 1 large chromosome.

(14 chromosomes) $\{$ (2) 12 ordinary chromosomes, I heterotropic, I small idiochromosome.

Primary Class B $\{(3) 12$ ordinary chromosomes, 1 large idiochromosome.

(13 chromosomes) $\{(4) 12$ ordinary chromosomes, 1 small idiochromosome.

Restating the facts from the point of view of mere size, it appears that class (3) contains no especially small chromosome, class (2) two small chromosomes, and classes (I) and (4) each one small chromosome, the latter being in one case the heterotropic, in the other the small, idiochromosome. ${ }^{1}$

I have not yet studied in sufficient detail the history of this form in the growth-period, which will require additional material; but the main facts are such as might be expected. In the middle growth-period the nuclei show, with great constancy, two unequal chromosome-nucleoli, both of which frequently appear hollow. The larger of these is almost certainly the idiochromosomebivalent; for in the prophases of the first division it may be seen separating into its two unequal constituents, precisely as I described in Brochymena (Fig. $4, p, q$ ). At this period the heterotropic chromosome is unmistakably recognizable by its size and shape, showing no constriction like that of the other chromosomes. I believe this to be identical with the smaller chromosome-nucleolus of the earlier period, but cannot offer decisive proof.

\section{CRITICAL AND COMPARATIVE.}

The three well-defined classes of chromosomes that have been described in this and my preceding paper differ from the others, each in its own way, especially in respect to their behavior in the process of synapsis and during the growth-period. The most characteristic common feature of the first two classes is their long delayed synapsis, which in both cases is deferred to the period

\footnotetext{
It is probable that additional light will be thrown on this form by further study of the related one, Thyanta custator, which I now have under investigation. The general aspect of the chromosome group in this species is closely similar to that of Banasa, and the first mitosis also shows fifteen chromosomes, of which however three, instead of two, are smaller than the others. The second mitosis differs from that of Banasa in showing always but thirteen chromosomes, and I have not thus far found a heterotropic chromosome in either division. Though I cannot yet speak positively, these conditions seem only explicable under the assumption that two pairs of idiochromosomes are present. From such a condition one nearly similar to that observed in Banasa might readily be derived by the disappearance of one of the small idiochromosomes.
} 
immediately preceding the reduction-division-i.e., in case of the $m$-chromosomes to the prophases of the first division, at the very end of the growth-period, and in case of the idiochromosomes to a still later period following the first division (though a temporary or preliminary union frequently occurs at a much earlier period). The "accessory" or heterotropic chromosome, finally, does not undergo synapsis at all, since it is without a mate with which to pair.

As regards their behavior in the growth-period, the idiochromosomes and the heterotropic chromosome agree in being "heterochromosomes" in Montgomery's sense-i.e., are distinguished from the other chromosomes by their compact form and deepstaining capacity. The m-chromosomes, on the other hand, may remain in a diffused condition throughout the early and middle growth-periods, only condensing to the compact form at the same time as the ordinary chromosomes (Anasa, "Chariesterus"); their condensation may, however, take place in the middle growth-period (Alydus), or even earlier (Protenor, according to Montgomery). An analogous difference in the time of condensation exists in case of the idiochromosomes, which in case of Lygæus do not condense as early as in Cœnus or Euschistus.

My observations prove definitely in some cases (Alydus, Anasa, Archimerus, "Chariesterus"), and I think render it probable for all cases, that in those Hemiptera that possess an "accessory" or heterotropic chromosome and two equal spermatogonial microchromosomes ( $m$-chromosomes), the large chromosomenucleolus of the synaptic and growth-periods is not, as other observers have supposed, the microchromosome-bivalent ("chromatin nucleolus" of Montgomery) but the heterotropic chromosome, precisely as in the Orthoptera. This error of identification has led Montgomery to designate three quite distinct kinds of chromosomes by the same name of "chromatinnucleoli." These are (I) the equal paired spermatogonial microchromosomes and the corresponding bivalent of the first spermatocyte division; (2) the idiochromosomes, which are typically unequal and do not form a bivalent in the first division; and (3) the heterotropic chromosome as it appears in the growth-period. It is therefore desirable, despite some repetition, to bring together in brief form the principal distinctions between these three. 
I. The paired microchromosomes-or preferably " $m$-chromosomes," since forms may be found in which they are not smaller than the others-form an equal pair in the spermatogonia, and in most of the forms thus far known are much smaller than the others. These do not, ordinarily conjugate to form a bivalent in the general synaptic period, and may (Alydus, Archimerus) or may not (Anasa, "Chariesterus") condense early in the growthperiod to form two small separate chromosome-nucleoli which can be distinguished in addition to the principal one (heterotropic chromosome). They undergo a very late synapsis (in the prophases of the first maturation division) to form a small symmetrical bivalent, typically central in position, that undergoes a reduction-division in the first mitosis and an equation-division in the second. Each spermatid nucleus therefore receives a single $m$-chromosome. They are always, as far as known, associated with a heterotropic chromosome, and the number of spermatogonial chromosomes is odd (with the more than doubtful exception of Syromastes). The first maturation-division shows a number of chromosomes which when doubled is one more than the spermatogonial number (as in Orthoptera). Known to occur in Anasa, "Chariesterus," Syromastes, Protenor, Alydus, Archimerus, Harmostes, Edancala, and doubtless occur in many others.

2. The idiochromosomes are typically unequal in size (Nezara forms an exception) forming an unequal pair in the spermatogonia (which accordingly show typically but one small chromosome); they may conjugate to form a bivalent at the time of general synapsis, or may remain separate, in either case condensing to form a chromosome-nucleolus (or two separate unequal ones) which persists throughout the greater part or the whole of the growth-period. In either case they are in the Hemiptera always separate univalents at the time of the first maturation-mitosis, and separately undergo an equation-division in that mitosis. This division accordingly shows one more than half the spermatogonial number of separate chromatin-elements, the latter number being in all cases an even one. At the end of the first mitosis their products conjugate to form a bivalent dyad (thus reducing the number of separate chromatin-elements to one-half the spermatogonial number). This dyad, typically unsymmetrical, undergoes a reduction-division in the second mitosis, and all of the spermatozoa receive the same number of chromosomes, 
one-half receiving the larger and one-half the smaller idiochromosome. They are not ordinarily associated with a heterotropic chromosome, the single known exception being Banasa. The idiochromosomes are known to occur in Lygæus, Cœnus, Podisus, Trichopepla, Mineus, Nezara, Murgantia, Brochymena and Banasa and are doubtless of much wider occurrence.

3. The "accessory" or heterotropic chromosome is certainly in most Hemiptera-and I believe will be found to be in allunpaired in the spermatogonia, and its behavior is throughout that of a univalent body. It fails to unite in synapsis with any other chromosome, and persists throughout the spermatocytic growth-period as a chromosome-nucleolus. During the earlier part of this period it resembles the idiochromosome bivalent (or the univalent large idiochromosome) in being attached to a large plasmosome from which it afterward separates. ${ }^{1}$ This chromosome divides in only one of the maturation-divisions, passing undivided to one pole of the spindle in the other. The latter division is usually the second (Pyrrochoris, Anasa, Protenor, Alydus, Chariesterus, Syromastes, Harmostes, Edancala), but in Archimerus and Banasa it is the first. In either case one-half the spermatozoa receive one more chromosome than the other half.

From the foregoing it will be seen that Montgomery correctly identified the chromosome-nucleolus in the growth-period of such forms as Euschistus, Cœenus, Podisus, Brochymena, Trichopepla or Nezara, which possess the idiochromosomes. He was, however, at fault in the conclusion that it gave rise to a small bivalent in the first division, the small chromosome of this division being always a univalent that is not at this time paired with its (usually) larger fellow; and further, owing to a failure to discriminate between these bodies and the paired microchromosomes of the Anasa or Alydus type, he describes and figures the spermatogonial groups in most of these forms as containing a symmetrical pair of "chromatin-nucleoli." Owing to his having overlooked the constant separateness of the idiochromosomes as univalents in the first mitosis he has also, I believe, been misled in several

II is doubtless a similar condition that has led Moore and Robinson ('05) in the case of Periplaneta, to conclude that the "accessory" chromosome is nothing but a "nucleolus." These observers have evidently studied the phenomena in a very superficial manner. 
instances in regard to the spermatogonial number (e. g., in Euschistus variolarius, Nezara and Brochymena). The statement given in the general summing up of his latest paper ('05) "Whenever the heterochromosomes occur in pairs in the spermatogonia they (i.e., the 'chromatin nucleoli') always conjugate to form bivalent ones in the first spermatocytes, and their univalent components become separated in the first maturation mitosis, i.e., divide prereductionally" (p. I95, and elsewhere), is inapplicable to the idiochromosomes; for even though they conjugate to form a bivalent chromosome-nucleolus in the growth-period they again separate to divide as separate univalents in the first mitosis, as I showed in detail in Brochymena, and as must also occur in the other forms (as is proved by the number of the chromosomes and their later history). The statement cited above applies only to the $m$-chromosomes of such forms as Anasa, Chariesterus, Alydus, Archimerus or Protenor; but the name "chromatin nucleoli" is in these cases not very appropriate in view of the fact that in the very form (Anasa) in which they were first discovered they do not appear as chromatin-nucleoli at any time during the growthperiod of the spermatocytes. As to their behavior in the restperiod of the spermatogonia I have at present noopinion to express. It is further probable that the distinction urged by Montgomery between the "odd chromosome" and the accessory ('05, p. I92) is also not valid; for my observations prove that in Alydus and Archimerus the "odd chromosome" ("accessory") is a typical chromosome-nucleolus (i.e., "heterochromosome") in the growthperiod, and it is extremely probable that the same will be found to hold true of the "odd chromosome" of Harmostes and Edancala. I think therefore that Montgomery's general conclusions regarding the "heterochromosomes". require some revision.

We may now briefly consider the nature of the "accessory" or heterotropic chromosome. So long as any of the forms possessing such a chromosome were supposed to have an even number of spermatogonial chromosomes the conclusion drawn by Montgomery ('OI, 'O4, 'O5) that this chromosome is a bivalent seemed an almost necessary one, even in cases where it appears as a single body in the spermatogonia. The observations brought forward in this paper cast grave doubt, I think, on all of the earlier accounts asserting an even spermatogonial number in 
the Hemiptera that possess a heterotropic chromosome. Of. these accounts (in cases positively known to have such a chromosome) there are but four, namely, Henking's original account of Pyrrochoris ('90), Paulmier's ('99), and Montgomery's ('or, '04) accounts of Anasa, Montgomery's of Alydus pilosulus ('OI) and Gross's more recent one of Syromastes ('O4). Henking states that he counted but four cases, one of which seemed to show twenty-three, the other three twenty-four, and it is evident both from the figures and from the frank statement of this able observer, that he adopted the latter number more on account of theoretical considerations than as a result of any adequate study of the facts. I have shown the counts of Paulmier and Montgomery to be erroneous in the case of Anasa, and also that of Montgomery in the case of Alydus pilosulus. There remains therefore the single case of Syromastes; but perhaps, in view of the results I have reached in other forms, I may be allowed the prediction that a reëxamination of this one will lead to a similar conclusion.

If this expectation is verified every ground will be removed for considering the heterotropic chromosome as a bivalent body; and I think that until definite evidence to the contrary is forthcoming we are bound to take this chromosome at its face-value, so to speak, as univalent. This conclusion involves a series of other conclusions and possibilities of which I shall here undertake to indicate only the more important.

I. As was indicated by McClung ('O2, p. 7I), if the "accessory" be univalent, its behavior in the maturation-mitoses at once falls into line with that of the other spermatogonial chromosomes; for each of these, too, undergoes but one division in the course of the two maturation-mitoses. One of these divisions (the reduction division) merely separates the univalent chromosomes that have previously paired in synapsis (as is so convincingly shown in case of the idiochromosomes or the $m$-chromosomes); and only the fact that the "accessory" has no mate with which to pair renders its behavior in one of the divisions apparently different from that of the ones that do pair.

2. The objections that I myself urged to the suggestion made in the first of these studies regarding the origin of the heterotropic chromosome are thus set aside, and my attempt to compare the idiochromosomes with the $m$-chromosomes was made on incorrect 
premises. My suggestion was that a heterotropic chromosome might arise from a symmetrical bivalent by the gradual reduction and final disappearance of one member of the conjugating pair, conditions corresponding to several of the stages of such a reduction being shown to exist in Nezara, Mineus, Cœnus, Euschistus, Murgantia, and Lygæus. All of the facts seem to me to indicate that this interpretation is the true one. Were the small idiochromosome to disappear in such forms as Lygæus or Euschistus, the large idiochromosome would be left as a heterotropic chromosome agreeing, point by point, with that of such forms as Alydus, Protenor or Anasa, namely, in its persistence as a chromosome-nucleolus during the growth-period; its association with the plasmosome in the earlier part of this period and its subsequent separation from it; its equal bipartition by an equationdivision in the first spermatocyte-mitosis, and the failure of the resulting products to divide in the second mitosis; and in correlation with the foregoing the existence of an odd number of spermatogonial chromosomes. The exactness of this correspondence is such, I think, as to lend a high degree of probability to the interpretation.

The only apparent obstacle in its way is the fact that in Banasa a heterotropic chromosome coexists with a typical pair of idiochromosomes; but this difficulty only exists under the assumption that a heterotropic chromosome has arisen but once in the history of the species, and nothing is known to justify such an assumption. I think, on the contrary, that the facts in Banasa may fairly be taken as evidence that a process is here in progress which if continued would lead to the formation of a second heterotropic chromosome. ${ }^{1}$

3. The formation of a heterotropic chromosome in the manner indicated involves a reduction of the total number of chromosomes by one; and it is possible that this may represent one process by which changes from a higher to a lower number or chromosomes have been brought about. But I doubt whether such a process can have gone very far, since, as pointed out beyond, there is reason to believe that it has occurred in only one sex.

\footnotetext{
1Should my surmise (stated in the footnote at $\mathrm{p} .53^{\circ}$ ) be correct that in the related form Thyanta two pairs of idiochromosomes are present without a heterotropic chromosome, I think additional support will be lent to the above interpretation.
} 
It seems, on the other hand, probable that the $m$-chromosomes may be of more general significance in this direction, since the facts distinctly suggest that they are diminishing or disappearing, and perhaps in some cases already vestigial, structures in both sexes. Paulmier was the first, as far as I am aware, to suggest that a reduction in the size of particular chromosomes might foreshadow their total disappearance; that chromosomes might in this way assume a vestigial character; and further, that such chromosomes might represent "somatic characters which belonged to the species in former times, but which characters are disappearing" ('99, p. 26I). This conception was applied by him to the small $m$-chromosomes (which he believed to represent the "accessory"), but was further supported by his observation of a very small chromatin-body that may divide like a chromosome (Paulmier, Fig. $28, a$ ) but is only rarely visible. ${ }^{i}$ Paulmier's suggestion, which I suspect may prove to embody one of the most important results of his paper, has been further developed by Montgomery. This author first suggested that an uneven number of chromosomes "represents a transition stage between a higher number and a lower" ('OI, p. 215); and he has more recently assumed that the "unpaired heterochromosomes" ("accessory" or heterotropic chromosomes) have arisen from paired heterochromosomes ("chromatin nucleoli") or ordinary chromosomes by fusion of the members of a pair to form a bivalent body ('05, p. 197). Both the paired and the unpaired heterochromosomes are considered to be chromosomes on the way to disappearance. Though my conclusion regarding the origin of the unpaired or heterotropic chromosome is an entirely different one, it agrees with that of Montgomery in assuming a reduction in the original number of chromosomes; and it is possible that by a subsequent disappearance of the heterotropic chromosome a further reduction may take place, though as indicated above there are difficulties in the way of this assumption. My conclusion is, however, distinctly opposed to the view that heterotropic chromosomes have arisen from "paired heterochromosomes" ( $m$-chromosomes), and although they have some features in common the evidence is opposed to

${ }^{1}$ It seems quite possible that this body may be the last remnant of a small idiochromosome, of which the corresponding larger one has remained as the heterotropic chromosome; but definite evidence of this is lacking. 
any direct relationship between these two classes of chromosomes. Montgomery has called attention to the fact that the $m$-chromosomes vary greatly in size in different species, graduating down to excessively minute forms (such as those occurring in Archimerus.) It is evident that these chromosomes have undergone a symmetrical reduction which, if continued, might lead to the disappearance of both; and such a process, if repeated, would lead in the history of a species to a progressive and parallel reduction of the number in both sexes. When these facts are compared with those presented by the idiochromosomes the thought can hardly be avoided that the reduction of the $m$-chromosomes may be correlated with a corresponding change that is taking place equally in both sexes; while the reduction of the small idiochromosome may represent a change that is taking place more rapidly in one sex than in the other, or affects one sex only.

4. How the foregoing conclusions and suggestions regarding the idiochromosomes and heterotropic chromosomes will square with McClung's hypothesis ('02, 2) and my own similar suggestion ('05) that these bodies may be in some way concerned with sex-determination, does not yet clearly appear from the known data; but there are some considerations that are too interesting in this connection to be ignored. If the heterotropic chromosome be a univalent body the conclusion is unavoidable (since the spermatogonial number is odd) that in the production of males, the number of chromosomes contributed by the two germ-cells cannot be the same. To this extent the facts harmonize with the view of McClung; but further consideration gives reason to doubt some of the more specific features of his hypothesis. The presence of the heterotropic chromosome in the male by no means proves that it is of paternal origin in fertilization, still less that it is specifically the male sex-determinantindeed, I believe the facts point in the opposite direction. In Anasa, for example, where the spermatozoa possess either ten or eleven chromosomes, offspring (males) having twenty-one would be produced by the fertilization of an egg having ten chromosomes by a spermatozoön having eleven (as McClung would assume); but the same result would follow from the fertilization of an egg having eleven by a spermatozoön having ten. I believe the second of these alternatives to be the more probable one for the following reasons: According to my view, the heterotropic 
chromosome has assumed its unpaired character by the reduction and final disappearance of its parental mate or homologue (i.e., a small idiochromosome); and it is highly probable that this process has occurred in one sex only, namely, the male. ${ }^{1}$ If this be the fact, it is evident that the heterotropic chromosome that remains in the male is the maternal mate or homologue of that which has vanished. I think therefore that we may expect to find that the heterotropic chromosome present in the male is derived in fertilization from the maternal group of chromosomes; and also that the female will be found to possess one more chromosome than the male (exactly the opposite of McClung's assumption), the additional chromosome being the homologue of that which has vanished in the male. ${ }^{2}$ If this be the fact, it follows with great probability that in the egg-synapsis this chromosome pairs with its paternal homologue (originally the heterotropic chromosome) to form a symmetrical bivalent, and that all the eggs receive eleven chromosomes; while in the male the heterotropic chromosome fails to pair (having no mate) and hence remains univalent. The expectation may therefore be stated as follows:

Egg $11+$ spermatozoön $10=21$ (male).
Egg $11+$ spermatozoön $I 1=22$ (female)..$^{3}$

Important direct evidence in favor of this expectation is given by the discovery by Stevens, briefly referred to in my preceding paper, that in the beetle Tenebrio a small chromosome, evidently analogous to the small idiochromosome of Hemiptera, is present in the somatic cells of the male only, while in the female

II will here not go into the somewhat intricate difficulties encountered under the supposition that it has occurred in both sexes, except to point out that if an unpaired heterotropic chromosome be present in the female and is allotted to only half the eggs (as in the male) it is necessary to assume a fertilization of each form of egg by the opposite form of spermatozoön, since otherwise three forms of offspring would result. Such a mode of fertilization is a priori very improbable. Still greater difficulties stand in the way of assuming that an unpaired heterotropic chromosome, present in the female, is retained in all of the eggs.

${ }^{2}$ Montgomery ('O4) has in fact found in the oögonia and follicle-cells of the female Anasa twentytwo chromosomes, and Gross ('O4) reports the same number in those of the female Syromastes. But since the first-named observer is certainly, and I believe the second-named is probably, in error as to the number in the male, both these cases require reëxamination. On the other hand Sutton has found twenty-two in the oögonia and follicle-cells of the Orthoptera (Brachystola) while the spermatogonial groups show twenty-three; but here again I think a result so important should be supported by more adequate evidence than he has brought forward. I now have this subject under investigation.

${ }^{3}$ For the confirmation of this, see Appendix. 
it is represented by a corresponding larger one (both sexes having the same number of chromosomes). Were the small chromosome to disappear, the female would show one more chromosome than the male in accordance with my general assumption.

We have now therefore good reason to hope that observation will directly determine whether sex is predetermined in the chromosome-group; and further, whether the sex-determining function can be localized in a particular chromosome or pair of chromosomes, as McClung suggested.

5. The foregoing offers no specific suggestion as to the meaning of the four classes of spermatozoa observed in Banasa. But it may be remarked that the existence of two or four (or more) classes of germ-cells in the same sex is in itself nothing anomalous; for as Sutton has pointed out, under the conception of himself and Montgomery there may be as many classes of spermatozoa as there are combinations of paternal and maternal chromosomes (in accordance with the Mendelian ratios). Forms which possess idiochromosomes or heterotropic chromosomes differ from the more usual ones only in that two or four of these classes are made visible by a greater or less differentiation of the members of one or two of the chromosome-pairs. It seems admissible to suppose that such a visible differentiation of the members of particular chromosome-pairs may stand for a corresponding differentiation of corresponding or allelomorphic qualities in the adult. I would therefore suggest the possibility that such a visible polymorphism of the male germ-nuclei as exists in Banasa may be accompanied by a visible polymorphism in the adults; and, while I am not aware that such a polymorphism has been observed in the Hemiptera, I believe this subject should be carefully examined.

It is hardly necessary to point out, finally, how strong a support the foregoing observations lend to, the general hypothesis of the individuality of chromosomes, and to the conception of synapsis and reduction first brought forward by Montgomery and developed in so fruitful a way by Sutton and Boveri. I must frankly confess that until I had followed step by step the behavior of the idiochromosomes and the $m$-chromosomes in the Hemiptera I did not appreciate how cogent is the argument brought forward in Montgomery's paper of 'or in support of his conclusion that synapsis involves an actual conjugation of chromosomes two by two, and that the 
chromosomes thus uniting are the paternal and maternal homologues. In the case of the $m$-chromosomes, no less clearly than in that of the idiochromosomes, the conjugation is not in any way an inference but an easily observed fact; and in both cases it is equally clear that the subsequent reducing division separates, with their individuality unimpaired, the same chromosomes that have previously united in synapsis.

I believe that any observer who will take the trouble to study in detail the history of the chromosomes in these insects must sooner or later in his task acquire the firm conviction that he is dealing with definite, well characterized, entities which show the most marked individual characteristics of behavior, which in some manner persist from one cell-generation to another without loss of their specific character, and which unite in synapsis and are distributed in the ensuing maturation-divisions in a perfectly definite manner. All the facts indicate that these phenomena are the visible expression of a preliminary association, and subsequent distribution to the germ-cells, of corresponding hereditary characters. It is evident, therefore, that the time has come when cytologists must seriously set themselves to the task of working out a comparative morphology and physiology of the chromosomes, with the ultimate aim of attempting their specific correlation with the phenomena of heredity and development.

\section{SUMMARY.}

I. The chromosomes that have been called "heterochromosomes" in Hemiptera (Montgomery) include three distinct forms that may provisionally be called $(a)$ the paired microchromosomes or $m$-chromosomes; $(b)$ the idiochromosomes; $(c)$ the "accessory" or heterotropic chromosomes.

2. The $m$-chromosomes are usually very small, form a symmetrical pair in the spermatogonia, and do not unite (in the forms I have studied) to form a bivalent chromosome-nucleolus in the growth-period. At an earlier or later period they condense to form two separate chromosomes that finally pair to form the small bivalent central of the first division, but are immediately separated without fusion. Each divides equally in the second division.

3. The idiochromosomes are typically unequal, and hence do not form a symmetrical pair in the spermatogonia. They may 
or may not pair at the time of general synapsis to form a bivalent; in the former case they appear in the growth-period as a single bivalent chromosome-nucleolus, in the latter case as two separate univalent chromosome-nucleoli. In either case they undergo equal division as separate univalents in the first maturationmitosis, their products conjugating at the close of this division to form an asymmetrical dyad the two constituents of which are, without fusion, immediately separated in the second division.

4. The heterotropic chromosome is without a mate in the spermatogonia (which accordingly show an odd number of chromosomes) and hence fails to undergo synapsis. Its behavior is throughout that of a univalent body. It divides only once in the course of the two maturation mitoses, this division taking place usually in the first, but in some species in the second, mitosis. It has probably arisen by the reduction and final disappearance of one member of a symmetrical chromosome-pair, this process having taken place in the male only.

5. The $m$-chromosomes are always associated with a heterotropic chromosome, while the idiochromosomes and heterotropic chromosomes are known to coexist in only a single case (Banasa). This case indicates that the formation of heterotropic chromosomes may have taken place more than once in the history of the species and possibly represents one mode of change from a higher to a lower number of chromosomes.

6. In forms possessing the idiochromosomes two classes of spermatozoa exist in equal numbers, which receive the same number of chromosomes but differ in respect to the idiochromosome. In forms possessing a heterotropic chromosome two classes of spermatozoa likewise exist, one of which possesses one more chromosome than the other. When both idiochromosomes and heterotropic chromosomes are present (Banasa) four classes of spermatozoa are formed, two having one more chromosome than the other two, each of these groups again differing in respect to the idiochromosome.

7. The facts support the general theory of the individuality of chromosomes, the theory of Montgomery in regard to synapsis, and that of Sutton and Boveri regarding its application to Mendelian inheritance; and they point toward a definite connection between the chromosome-group and the determination of sex. 
APPENDIX.

During the summer, and since the foregoing paper was entirely completed in its present form, I have obtained new material which shows decisively that the theoretic expectation in regard to the relations of the nuclei in the two sexes, stated at p. 539, is realized in the facts. In Anasa, precisely in accordance with the expectation, the oögonial divisions show with great clearness one more chromosome than the spermatogonial, namely, twenty-two instead of twenty-one; and the same number occurs in the divisions of the ovarian follicle-cells. Again in accordance with the expectation, the oögonial groups show four large chromosomes instead of the three that are present in the spermatogonial groups. In other respects the male and female groups are closely similar. In like manner, the oögonial divisions in Alydus and Protenor show fourteen chromosomes, the spermatogonial but thirteen; and in Protenor the spermatogonial chromosome-groups have but one large chromosome (unquestionably the heterotropic) while the oögonial groups have two such chromosomes of equal size.

The interpretation is unmistakable. Taking Protenor as a type, all of the matured eggs must contain seven chromosomes, of which one, much larger than the others, corresponds to the heterotropic chromosome present in one-half of the spermatozoa. These spermatozoa (seven-chromosome forms) contain a chromosome-group exactly similar to that of the egg; and fertilization by a spermatozoön of this class produces a female having fourteen chromosomes. The other half of the spermatozoa (six-chromosome forms) lack the heterotropic chromosome; and fertilization of an egg by a spermatozoön of this class produces a male having but thirteen chromosomes, the unpaired one being derived from the egg and appearing in the maturation of this male as the heterotropic chromosome since it is without a mate. There can, therefore, be no doubt that a definite connection exists between the chromosomes and the sexual characters, and I believe that the conclusion can hardly be escaped that the chromosomecombination, established at the time of fertilization, is, in these insects, the determining cause of sex.

The result reached in Anasa is confirmed by a comparison of the male and female chromosome-groups in Lygæus, Cœnus and Euschistus, all of which possess in the male a pair of unequal 
idiochromosomes in place of an unpaired heterotropic chromosome. In all of these forms, as I showed in my first paper, the spermatogonial groups show fourteen chromosomes that may be equally paired with the exception of a small and a large idiochromosome. The oögonial groups in these forms also show fourteen chromosomes, but all may be equally paired, the small idiochromosome being represented by a larger one that has a mate of equal size. In these forms, accordingly, males are produced as a result of fertilization by spermatozoa containing the small idiochromosome, females by fertilization by spermatozoa containing the large idiochromosome (which accords with Stevens' result in Tenebrio). This proves the correctness of my conclusion that the size-reduction and final disappearance of the small idiochromosome has taken place in the male sex only, and that the large idiochromosome corresponds to the heterotropic chromosome. Complete disappearance of the small idiochromosome in the male has led to each a condition as exists in Anasa and other forms possessing a heterotropic chromosome. These facts will be described and discussed in the third of these studies.

October 4, 1905 . 


\section{LITERATURE. ${ }^{1}$}

Baumgartner, W. J., '04.-Some new Evidences for the Individuality of the Chromosomes. Biol. Bull., viii, I.

Boveri, Тн., '04.-Ergebnisse über die Konstitution der Chromatischen Substanz des Zellkerns. Jena, I904.

Gross, J., '04.-Die Spermatogenese von Syromastes marginatus. Zoöl. Jahrb., Anat. Ontog., xx, 3 .

Henking, H., '9o.-Ueber Spermatogenese und deren Beziehung zur Entwickelung bei Pyrrochoris apterus. Z. wiss. Zoöl., li.

McClung, C. E., 'oo.-The Spermatocyte Divisions of the Acrididx. Bull. Univ. Kansas, ix, I.

'02, I. - The Spermatocyte Divisions of the Locustidx. Ibid., xi, 8.

'O2, 2.-The Accessory Chromosome. Sex Determinant? Biol. Bull., iii, I, 2.

Montgomery, T. H., '98.-The Spermatogenesis in Pentatoma, etc. Zoöl. Jahrb., Anat. Ontog., xii.

'or.-A Study of the Chromosomes of the Germ-cells of Metazoa. Trans. Amer. Phil. Soc., xx.

'04.- Some Observations and Considerations upon the Maturation Phenomena of the Germ-cells. Biol. Bull., vi, 3.

'05.-The Spermatogenesis of Syrbula and Lycosa, etc. Proc. Acad. Nat. Sci. Phil., Feb., 1905. Issued May 18, I905.

Moore and Robinson, '05.-On the Behavior of the Nucleolus in the Spermatogenesis of Periplaneta Americana. Q. J. M. S., xlviii, 4 .

Paulmier, F. C., '99.-The Spermatogenesis of Anasa tristis. Jour. Morph., $\mathrm{xv}$, supplement.

Stevens., N. M., '05.-A Study of the Germ-cells of Aphis rosæ and Aphis œnotherx. Journ. Exp. Zoöl, ii, 3

Sutron, W. S., 'oo.-The Spermatogonial Divisions in Brachystola magna. Bull. Univ. Kansas, ix, I.

'02.-On the Morphology of the Chromosome Group in Brachystola magna. Biol. Bull., iv, I.

'03.-The Chromosomes in Heredity. Biol. Bull., iv, 5 .

Wilson, E. B., '05.-The Behavior of the Idiochromosomes in Hemiptera. Journ. Exp. Zoöl., ii, 3 .

\footnotetext{
Including only works directly cited in the text. A full literature-list is given in the works of McClung ('02, 2) and Montgomery ('05).
} 

The

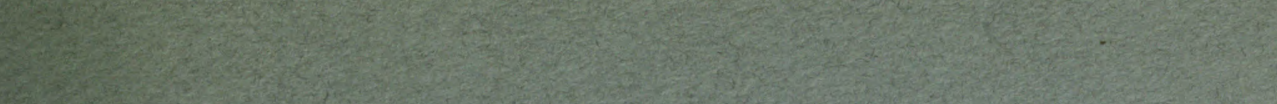
2.

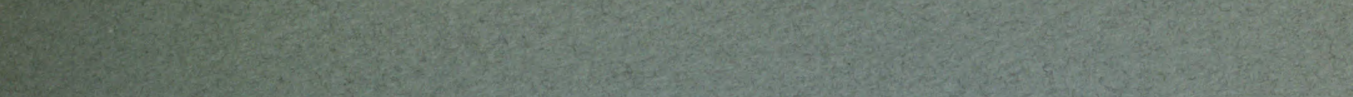
H. 2.

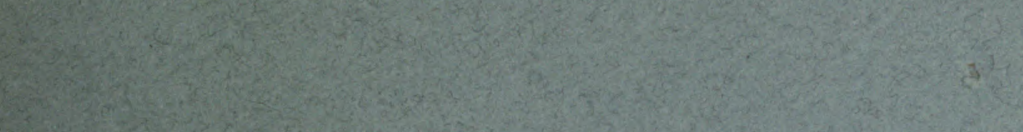

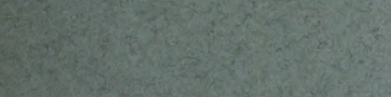

Titer.

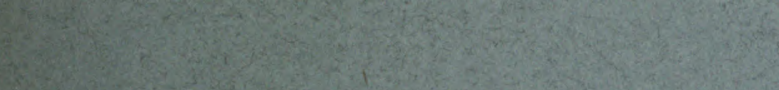

W.

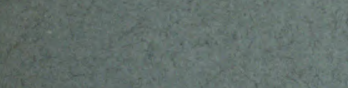

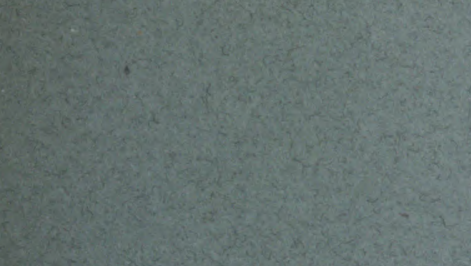

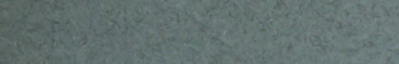

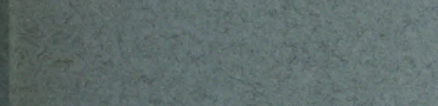

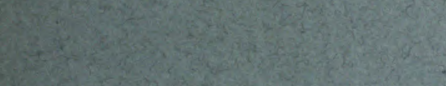

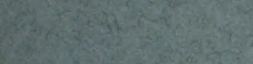

We

W.

1.5.

W.

II:

(7)

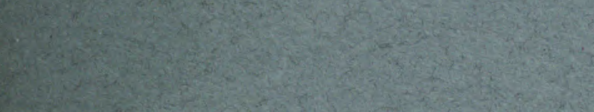

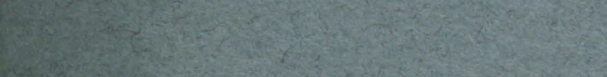

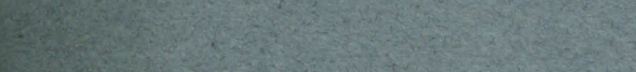

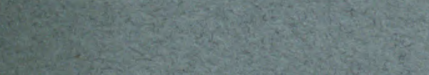

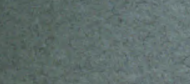

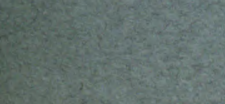

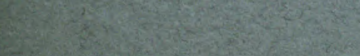


THE JOURNAL OF EXPERIMENTAL ZOÖLOGY is issued quarterly. A volume consists of four numbers, containing from 100 to 200 pages each, with numerous illustrations.

\section{PRICE OF SUBSCRIPTION PER VOLUME}

(PAYABLE IN ADVANCE)

To subscribers in the United States, Canada and Mexico, $\$ 5.00$ To subscribers in other countries - $\quad-\quad+\quad-\quad-5.50$ Price of single copies - - - - - - - $\quad-\quad-2.00$

These prices are net and under no condition subject to discount

Remittances should be made by Postal Money Order (Mandat de Poste, Postanweisung) or by draft on New York, payable to THB JoURNAL OF EXPERIMENTAL ZOÖLOGY.

Address all communications to THE JOURNAL OF EXPERIMENTAL ZOÖLOGY, N. E. Cor. Wolfe and Monument Strect; Baltimore, Md., U. S. A. 


\section{STUDIES ON CHROMOSOMES}

11. THE SEXUAL DIFFERENCES OF THE CHROMOSOME-GROUPS IN HEMIPTERA, WITH SOME CONSIDERATIONS ON THE DETERMINATION AND INHERITANCE OF SEX

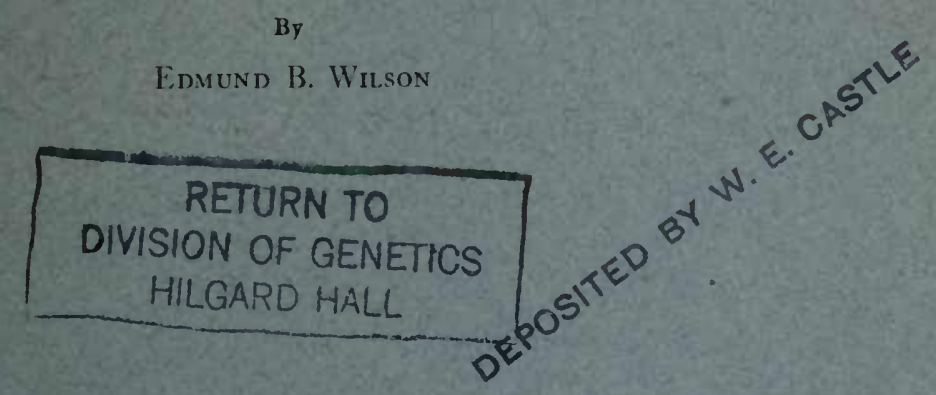

REPRINTED FROM

THE JOURNAL OF EXPERIMENTAL ZOÖLOGY

$$
\text { Volume III }
$$

No. 1

BALTIMORE, MD., U, S. A.

February, 1906 



\section{STUDIES ON CHROMOSOMES}

III. THE SEXUAL DIFFERENCES OF THE CHROMOSOMEGROUPS IN HEMIPTERA, WITH SOME CONSIDERATIONS ON THE DETERMINATION AND INHERITANCE OF SEX

BX

\section{EDMUND B. WILSON}

With Six Figures

Since the time of Henking's able paper on the spermatogenesis of Pyrrochoris ('9I), it has been known that in certain Hemiptera, and in some other insects, a dimorphism exists in the nuclear constitution of the spermatozoa, one-half of them containing the socalled "accessory" or "heterotropic" chromosome, while in the other half this chromosome is lacking. The meaning of this fact has hitherto remained undetermined. McClung in 1902 developed an hypothesis of sex-production based on the conjecture that the heterotropic chromosome is a sex-determinant, and more specifically that spermatozoa containing this chromosome produce males, for the very obvious, yet fallacious, reason that it is present in the male. This hypothesis was based simply on the fact that the spermatozoa are of two numerically equal classes, like the sexes of the adults; and it was apparently overthrown by subsequent observation. The hypothesis implied that the cells of the female must contain one chromosome less than those of the male; and although McClung did not specifically place his assumption in this form, he considered it extremely improbable that the accessory chromosome, or "any such-element," is present in the egg. Sutton ('O2) believed that he had found a confirmation of this in the grasshopper Brachystola, where he showed that the number in the male (spermatogonia) is twentythree, and stated that in the female (oögonia and follicle-

Journal of Experimental Zoölogy, Vol. II, No. 1. 
cells) the number is twenty-two, supporting this statement by a single figure (op. cit., Fig. I I). Sutton was, however, able to examine only a very few of the female groups, and the object is an unfavorable one as compared with the Hemiptera, owing to the less compact form of the chromosomes. McClung's hypothesis seemed to be rendered completely untenable by the later observations of Montgomery on Anasa ('O4), and of Gross on Syromastes ('04), both these authors describing and clearly figuring the same number of chromosomes (twenty-two) in the male and the female cells. Gross and Wallace ('05) were thus independently led to the conclusion that only one of the two classes of spermatozoa was functional, namely, that in which the heterotropic chromosome is present. Those of the other class were assumed to degenerate after the fashion of polar bodies.

I am now able to bring forward decisive proof that the apparently adverse evidence brought forward by Montgomery and Gross was based on errors of observation, and that the sexes in Hemiptera of this type do in fact show a constant difference in the number of chromosomes. As far as these animals are concerned, however, McClung's conjecture as to the mode of fertilization proves to have been the reverse of the truth; for it is the female, not the male, that possesses the additional chromosome, as I have determined beyond all doubt in four genera, namely, Anasa, Alydus, Harmostes and Protenor. The facts leave no doubt that both forms of spermatozoa are functional; that all of the eggs possess the same number of chromosomes; that all contain the homologue, or maternal mate, of the accessory or heterotropic chromosome of the male; and that fertilization by spermatozoa that possess this chromosome produces females, while males are produced upon fertilization by spermatozoa that do not possess it.

A second type of dimorphism of the nuclei of the spermatozoa was made known in the first of these studies. In this type all of the spermatozoa contain the same number of chromosomes, but half of them contain a large "idiochromosome" and the other half a corresponding small one. I was led in that paper to suggest the possibility that the idiochromosomes might play a definite 
rôle in sex-production, but could at that time produce no evidence in support of the suggestion. I have now the evidence to show that this suggestion was in accordance with the facts; for in at least four genera, Lygæus, Euschistus, Coenus and Podisus, both sexes show the same number of chromosomes, but the small idiochromosome is present only in the male. Somewhat earlier, and independently, Stevens ('05) determined a precisely similar fact in the case of a beetle, Tenebrio, which indicates that the phenomenon is of wide occurrence in the insects. These results confirm the correctness of my conclusion that the heterotropic or "accessory" chromosome has become unpaired in the male sex through the disappearance in that sex of its mate, and give a complete explanation of the fact that in forms possessing the heterotropic chromosome the male number is odd and one less than the female number. I believe that these facts may give the basis for a general theory of sex-production.

\section{DESCRIPTIVE}

\section{A. General Character of the Chromosome-groups}

In two preceding papers (Wilson, '05, I; 05, 3,) (where due acknowledgment is made to previous observers in this field) I have described in some detail the general nature of the chromosomes in these insects. For such an investigation as the present one, the Hemiptera present peculiar advantages, owing above all to the short and regular form of the chromosomes, and the relative lack of crowding in the equatorial plate. I have employed almost exclusively Flemming's strong fluid as a fixative, staining the sections with iron-hæmatoxylin and extracting until the cytoplasm is nearly or quite colorless. The best preparations thus obtained leave nothing to be desired in point of brilliancy and clearness, and show the chromosomes with a distinctness that is hardly exaggerated by the black and white figures here reproduced. The very large number of sections now at my disposal (including all those of Paulmier and a still greater number of new preparations of my own) has enabled me in the case of nearly every species to examine numerous division-figures (of which only the 
best have been selected for illustration) and to satisfy myself thoroughly of the constancy of the relations as described. Everyone familiar with such objects will, however, realize that in regard to such matters as the arrangement and size-differences of the chromosomes certain apparent variations appear that are due to slight differences in the form and position of the chromosomes, and to the various degrees of foreshortening thus caused. This introduces a slight error, into both the observations and the drawings, that can hardly be avoided. A second source of error lies in the degree of extraction, which produces surprising variations in the apparent size of the chromosomes-I have found, for instance, that by successive extraction the chromosomes may be reduced almost to one-half their original apparent size, and the smaller chromosomes may thus be caused almost to disappear from view. Camera drawings at successive stages of the extraction show, however, that the relative sizes of the chromosomes remain substantially unchanged, and the comparison of the same object after a shorter and a longer extraction has thus, in a number of cases, given a more certain result than could otherwise have been obtained. I have, whenever it was possible, figured different stages of the same species from the same slide, so as to avoid the error due to different degrees of extraction; but this is not always possible, since as a rule longer extraction is required to give a perfectly clear view of the spermatogonial groups than is desirable for the spermatocyte-divisions. For the comparison of the two sexes, different slides must of course be used, and to this is due, I am sure, some of the size-differences between the ooggonial and spermatogonial groups that appear in the figures.

Making all due allowance for the sources of error mentioned, it remains perfectly clear that the chromosomes in each species show among themselves constant and characteristic size-differences; and further, that with the special exceptions in the male described beyond, the chromosomes of the unreduced groups (i. e., those of the oögonia and spermatogonia) may be paired off, two by two, to form equal or symmetrical pairs. The pairing of the chromosomes is most evident in the case of especially small chromosomes (such as the $m$-chromosomes of Anasa, Alydus, 
Harmostes, etc., or the small pair of ordinary chromosomes of Cœnus and Euschistus, described beyond) or especially large ones such as the largest pair in Alydus, and in some of the species of Euschistus. Those of intermediate size are also obviously paired in some of the forms (e. g., in Protenor, Fig. I); but in many of the species the several pairs are not sufficiently marked in size to admit of certain recognition. Nevertheless, a comparative study of many species has convinced me of the correctness of the conclusion, first indicated by Montgomery ('OI) and afterward more fully worked out by Sutton ('O2), that all the chromosomes (again with the special exceptions referred to above) may be thus paired, and that the chromosome-group as a whole includes two parallel series of chromosomes that undoubtedly represent respectively the descendants of those that originally are brought together in the union of the gametes. This is very clearly brought out by making camera drawings of the chromosomes, and arranging them as nearly as practicable in pairs of equal size. This arrangement conspicuously shows the sexual differences, as may be seen by a comparison of Figs. 2, $f$ and $b$ (Anasa) and 5, $c$ and $g$ (Lygæus). There is, of course, a large error to be allowed for in the series as thus arranged, and no pretense to complete accuracy in the selection of the members of most of the pairs can be made. Nevertheless, when all due allowance for differences of form, foreshortening and the like is made, the fact that such a double series exists is unmistakable. When it is borne in mind that the spermatid-nuclei in each case contain a single series of chromosomes showing the same size-relations ( $c f$. for instance, Figs. I, $b$, $c, d ; 2, a, d, e ; 3, a, e, f ; 4, b, f, d, b)$, it becomes in a high degree probable that the corresponding pairs of the somatic groups consist each of a paternal and a maternal member, in accordance with Montgomery's original and fundamental assumption ('or). As may be seen by a comparison of the figures, the members of each pair when in their natural position, do not as a rule lie in juxtaposition but may occupy any relative position. Only at the period of synapsis do they actually couple, two by two, to form the bivalents whose members are subsequently separated by the reducing division. 
In order to give a wider basis of comparison I have given new figures of the chromosome-groups of nearly all the species, even in the case of forms already figured in my preceding papers. Since the idiochromosomes or the heterotropic chromosome form the distinctive differential between the nuclei of the two sexes, I shall in the descriptive part of this paper call them the "differential chromosomes."

\section{B. First Type. Forms Possessing an "Accessory" or Hetero- tropic Chromosome}

As stated above, I have compared the males and females in respect to the chromosome-groups in four genera, selecting for this purpose the most available cells, which are the dividing oögonia and ovarian follicle-cells in the female, the spermatogonia and investing cells of the testis-cysts in the male. The general result is the same in all, but owing to the conspicuous size-difference of the chromosomes in Protenor, this form gives the most obvious and striking evidence. ${ }^{1}$

\section{a. Protenor belfragei}

Montgomery ('oI) first made known the general character of the chromosome-groups in this interesting species, showing that the spermatogonial groups show an odd number, thirteen, that the heterotropic chromosome (Montgomery's "chromosome $x$ ") is immediately recognizable by its enormous size-it is fully twice the size of the largest of the other chromosomes-and that it is unpaired (though he considered it a bivalent). My own observation confirms his description in every point, except that I have never seen this chromosome transversely constricted into two halves. The first glance at a good preparation of the spermatogonial metaphase, as seen in polar view, shows this huge chro-

'There can be no doubt of the identification of the follicle-cells; but there is some uncertainty regarding the cells here called oögonia, since they are from the undifferentiated region of the ovary in which the distinction between oögonia and follicle-cells cannot be made out. It is therefore quite possible that some of the groups here described as oögonia may be from very young follicle-cells or nutritive cells; but this does not affect the main result. 
mosomed a sa long worm-shaped body obviously without a mate, (Fig. I, $d-f$ ). The remaining twelve chromosomes may be grouped in symmetrical pairs (indicated by numbers in Fig. I,
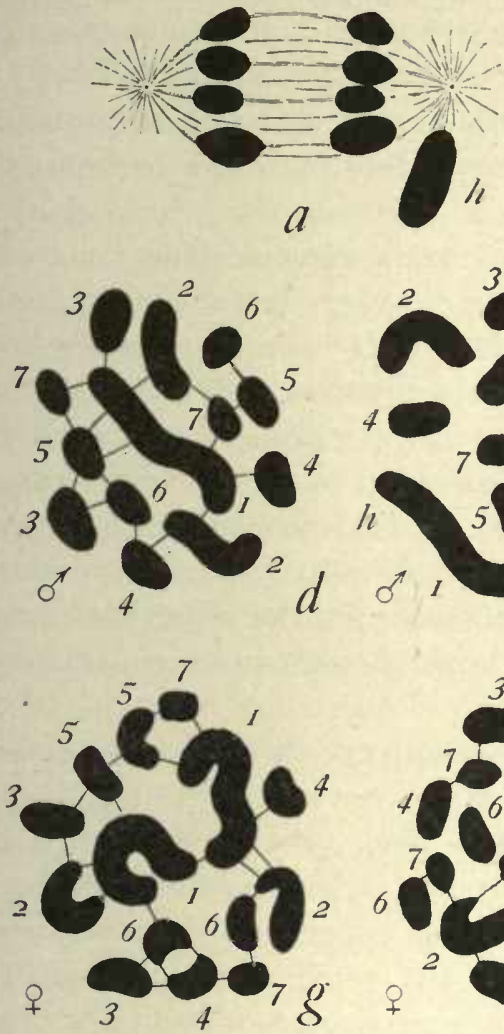
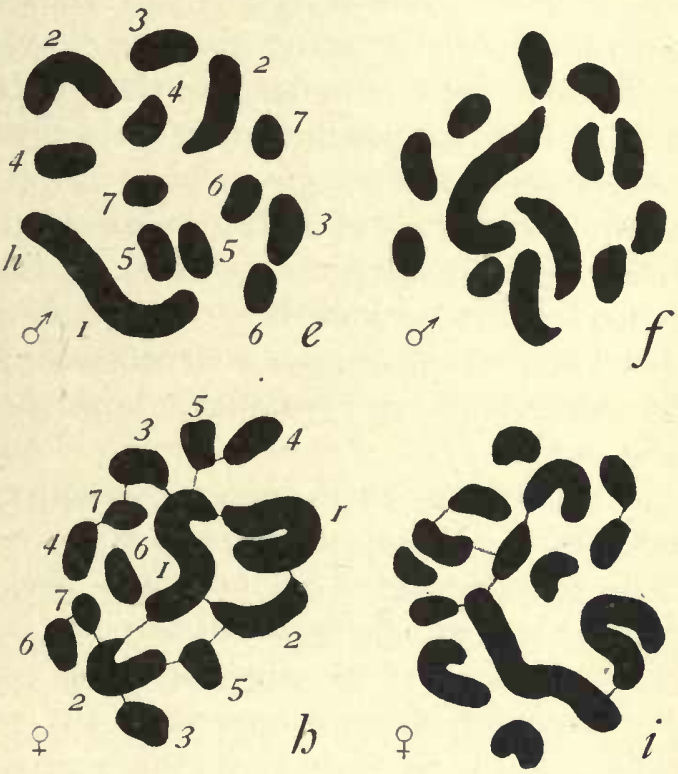

Figure ${ }^{1}$

Protenor belfragei-- $a$, Anaphase of second spermatocyte-division; $b, c$, sister groups, from the same spindle, polar view, second spermatocyte-division; $d, e, f$, spermatogonial groups; $g, h$, groups from immature ovaries, probably oögonia; $i$, group from dividing follicle-cell.

$d, e)$, though the members of each pair may occupy any relative position. Of these six pairs, one $(2,2)$ is always much larger than the others, its members being approximately half the size of the

'All the figures are drawn to the same scale. In all, $h$ denotes the heterotropic chromosome, $i$ the idiochromosomes (large and small in some cases lettered $I$ and $i$ respectively), $m$ the paired microchromosomes, and $s$ the smallest pair of ordinary chromosomes. 
heterotropic. A second pair $(3,3)$ may usually be distinguished as the next largest, and a third pair $(7,7)$ as the smallest, though this is not always obvious. This pair probably correspond to the " $m$-chromosomes" of my preceding paper. The remaining three pairs are of nearly equal size, though sometimes they clearly show a progressively graded series as in Fig. I, $d, e$. In synapsis the six paired chromosomes become coupled, as usual, to form six corresponding bivalents, while the large chromosome remains as an unpaired univalent. During the whole growthperiod of the spermatocytes this chromosome remains in a condensed spheroidal state, forming a very large chromosomenucleolus. In the prophases of the first division it again elongates and divides longitudinally in this division. Each secondary spermatocyte accordingly receives seven chromosomes. In the second division six of these (the products of the bivalents) again divide equally, while the seventh (the large chromosome) passes undivided to one pole (Fig. I, a). One-half of the spermatid nuclei accordingly receive six chromosomes, the other half seven, the additional one being the large heterotropic chromosome (Fig. I, $b, c$ ).

In the female the chromosome-groups of the dividing oögonia and follicle-cells appear with a clearness not inferior to that shown in the spermatogonial groups (Fig. I, $g-i$ ). It is at once apparent that in these groups there are two very large chromosomes, equal in size, in place of the single one that appears in the male, while the remaining chromosomes show the same relations as in the male. There are accordingly fourteen chromosomes in all, which may be equally paired off, two by two, and no chromosome is without a mate of corresponding size. Since the largest two are of the same relative size as the single heterotropic chromosome of the male, it is quite clear that one of them must have been derived from a spermatozoön containing this chromosome, while the other is its maternal mate or homologue.

I have not been able to follow by actual observation the phenomena of reduction, maturation and fertilization in the egg; but the data are sufficient to show, with a degree of probability only short of certainty, what must be the history of the chromo- 
somes in these processes. Since the oögonia contain fourteen equally paired chromosomes, synapsis in the oöcyte must result in the formation of seven symmetrical bivalents- $i$. $e$., seven couples of equal chromosomes - and each egg after maturation contains seven univalent chromosomes, one of which is the maternal representative or mate of the heterotropic chromosome of the male. This group contains one chromosome of each of the original pairs, and is precisely similar to the group present in those spermatozoa that contain the heterotropic chromosome (Fig. I, c). Fertilization by such a spermatozoa doubles this group, giving the condition observed in the female-i.e., fourteen chromosomes equally paired, the largest pair consisting of the heterotropic chromosome and its maternal mate $(I, I, \mathrm{Fig}$. I, $g, b)$. Fertilization by a spermatozoön that lacks the heterotropic chromosome will give the condition observed in the male, namely, thirteen chromosomes, of which twelve are equally paired, while the thirteenth is the large unpaired one which is obviously derived from the egg. There is therefore no escape from the conclusion that both forms of spermatozoa are functional, that females are produced upon fertilization by spermatozoa that contain, and males upon fertilization by spermatozoa that lack, the heterotropic chromosome. Since the two classes of spermatozoa are equal in number, fertilization will in the long run produce males and females in approximately equal numbers.

\section{b. Anasa tristis}

A comparison of the nuclei of the two sexes in this species gives a precisely concordant result, though the size-differences do not allow of so exact an identification of the differential chromosomes. In the preceding study I showed that the number of chromosomes in the male (spermatogonia) is twenty-one, not twenty-two as stated by previous observers. Study of the spermatogonial metaphase groups shows that twenty of the chromosomes may be equally paired, two by two, while the remaining one is, of course, without a mate (Fig. 2,e, f). The unpaired heterotropic chromosome is one of three largest chromosomes, 
but which particular one cannot be determined by simple inspection, since the three are of nearly equal size. In synapsis two of these large chromosomes unite to form the largest of the ten bivalents ( $I$, Fig. 2, a) that appear in the first spermatocyte division. The third, which retains its compact form as a chromosome-nucleus during the growth-period, remains as the univalent heterotropic chromosome ( $b$, Fig. 2, $a$ ). The first spermatocyte division accordingly shows eleven chromosomes, ten of which are bivalent, and one (heterotropic) is univalent. The distribution of these chromosomes in the maturation-division takes the usual course, the heterotropic chromosome dividing equally with the ten bivalents in the first mitosis while its products pass undivided to one pole of the spindle in the second (Fig. 2,b). Half the spermatozoa accordingly receive ten chromosomes, one of which ( $I$, Fig. 2, $C$ ) is larger than the others, and half an exactly similar group plus the large heterotropic chromosome, or eleven in all (Fig. 2, d).

The oögonial groups show invariably twenty-two chromosomes, which may be arranged in eleven equal pairs (Fig. 2, g, b). In place of the three large chromosomes of the spermatogonial groups appear four similar chromosomes, forming two equal pairs. Two of these four are obviously the large chromosome, common to all the spermatozoa, and its maternal mate, while the other two must be the heterotropic chromosome (derived in fertilization from the spermatozoön) with its maternal mate. It is, therefore, clear that all of the matured eggs must contain eleven chromosomes, that females are produced upon fertilization by those spermatozoa that contain a similar group-i. e., by those containing the heterotropic-males upon fertilization by spermatozoa that lack the heterotropic.

The ovarian follicle-cells often show chromosome-groups identical with those of the oögonia (Fig. 2, j). Not infrequently, however, the number of chromosomes is much greater, and the same is true of the nuclei of the investing cells of the ovary, of the oviduct and of the fat-body. In the male similar multiple groups are not uncommon in the interstitial and investing cells of the testis. Only in a single case have I succeeded in gaining 

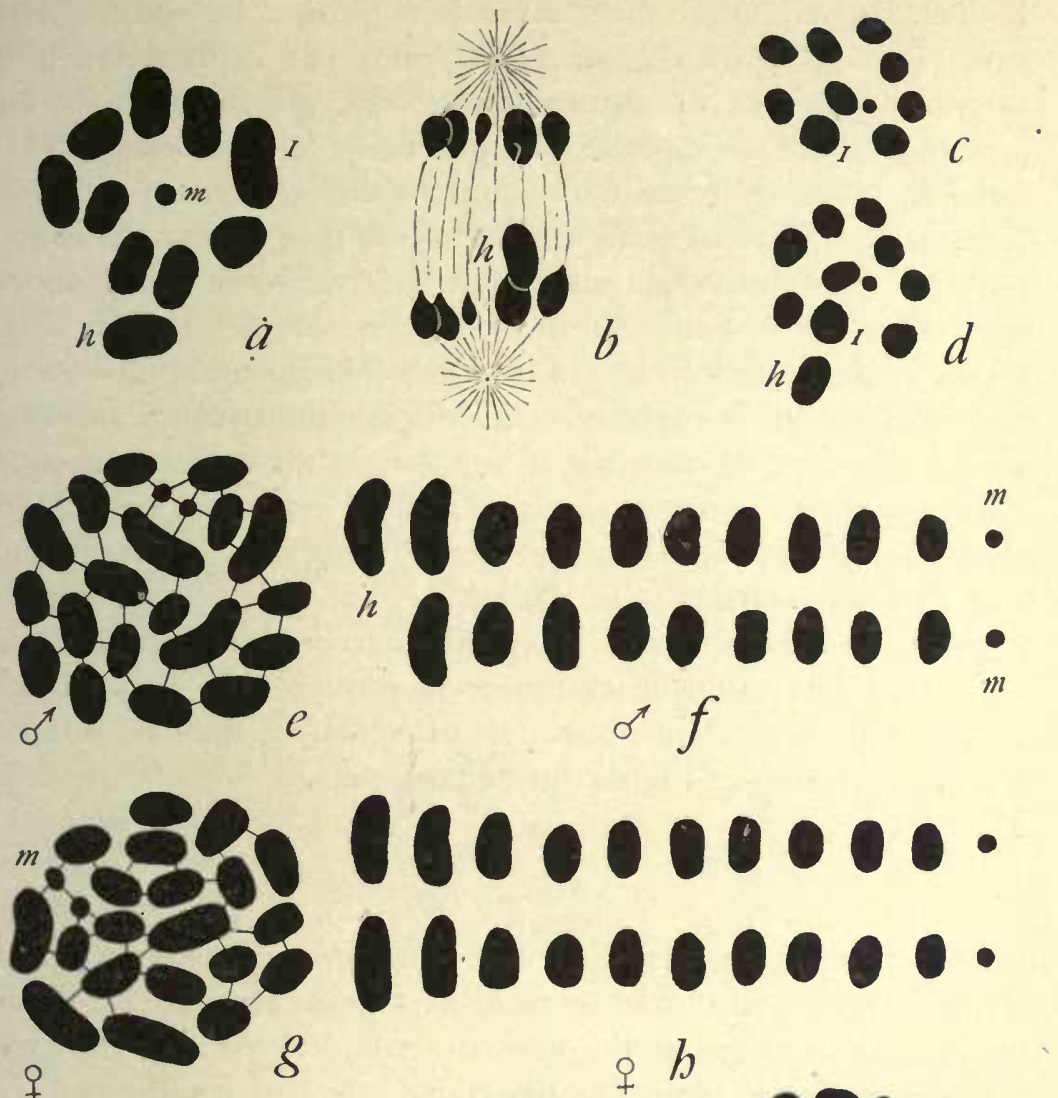

$+h$
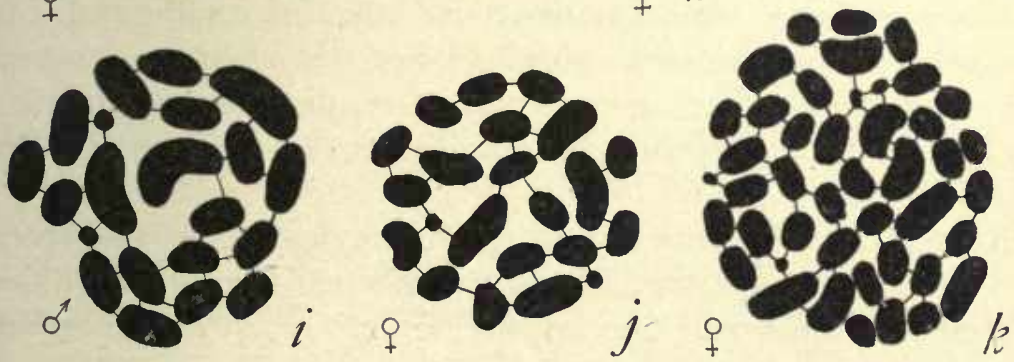

FIGURE 2

Anasa tristis. $-a$, Metaphase of first spermatocyte-division, in polar view, showing the nine large bivalents in a ring, the univalent heterotropic chromosome below it, and the $m$-chromosome bivalent in the center; $b$, anaphase of second division; $c, d$, sister-groups from the same spindle, polar view, second division ( 1 the macrochromosome); e, spermatogonial group; $f$, the same chromosomes arranged in pairs; $g$, oögonial group from a larva; $h$, the same group arranged in pairs; $i$, spermatogonial group; $j$, group from a dividing follicle-cell; $k$, double group, from a cell toward the periphery of a larval ovary. 
a clear and complete view of such a group; but this one case suffices to give, with great probability, the explanation of the increased number of chromosomes. In this case every chromosome of the metaphase group may be clearly seen, and the number is exactly twice the oögonial number, namely, forty-four (Fig. 2, k). Careful study clearly shows that this group contains four microchromosomes and eight macrochromosomes, in each case twice the number of those present in the ooggonia. This leaves no doubt that in this case all the chromosomes have divided once without the occurrence of a cytoplasmic division, and makes it probable that the increase in number in the cells in question is always due to a process of this kind. I have not been able to obtain faultless preparations of the dividing cells of other tissues, and can only state that in the ectodermal cells of the larva the number of chromosomes is approximately the same as in the oögonia. The multiple chromosome-groups were only observed in the cells mentioned above, all of which, it may be observed, are degenerating or highly specialized cells.

\section{c. Alydus pilosulus}

Despite the small number of chromosomes ( $9 \mathrm{I}_{4}, \mathrm{o}^{\mathrm{T}} \mathrm{I} 3$, as in Protenor) this genus is in some respects less favorable for detailed analysis than either of the ones described above, for the size of the heterotropic chromosome does not distinguish it sufficiently from the other chromosomes to allow of its certain identification in the spermatogonia. The main fact appears, however, as clearly as in Protenor or Anasa that the female has one more chromosome than the male.

In polar views of the second spermatocyte-division this species shows the sister spermatid-groups with great beauty, one having six chromosomes and one seven (Fig. 3,e, f). These chromosomes show at least five distinguishable sizes that are constant, namely, (I) a largest; (2) an extremely small one ( $m$-chromosome); (3) a second smallest (the heterotropic); (4) a second largest, and (5) three others intermediate in size between (3) and (4), one of which is frequently a little larger than the other two. 
The sister groups are practically exact duplicates save for the heterotropic which varies considerably in appearance as seen from the pole owing to foreshortening ( $c f$. the side-views given in my preceding paper). The spermatogonia correspondingly show always thirteen chromosomes (Fig. 3,a), of which the largest and the smallest pair are at once distinguishable. Next follow four chromosomes nearly equal in size, two of them often appreciably smaller than the other two. Of the remaining five, one must be the unpaired heterotropic; but, as already stated, it cannot be positively identified by inspection. Closely similar groups may
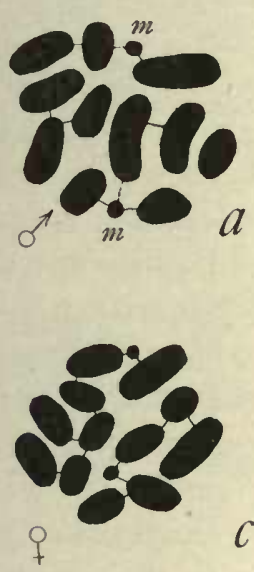
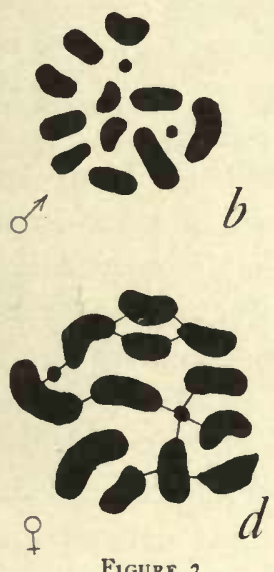

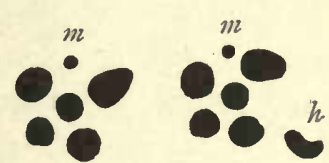

$e$

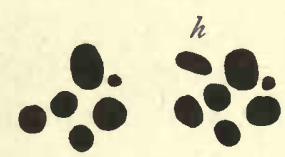

$$
\text { f }
$$

Alydus pilosulus. $-a$, Spermatogonial group; $b$, group from a dividing investing cell of the testis; $c$, oögonial group; $d$, from a dividing cell of an egg-follicle; $e, f$, two pairs of sister-groups, each from a single spindle, anaphase of second spermatocyte-division, in polar view.

occasionally be found in dividing cells of the enveloping cells of the testis (Fig. 3, b). Whether multiple groups occur like those described in Anasa, I cannot say.

The dividing oögonia and follicle-cells, of which a large number have been observed, always show fourteen chromosomes that may be arranged in seven equal pairs (Fig. $3, c, d$ ). As in the spermatogonia, the largest and the smallest pair are usually at once recognizable, and also the four second largest. The remaining six, of nearly equal size, must of course include the heterotropic chromosome and its maternal mate. 


\section{d. Harmostes reflexulus}

My material of this species is much less abundant than that of the three preceding, and the preparations are not of the same excellence. They nevertheless show beyond doubt that the numbers are here the same as in Protenor and Alydus, viz., thirteen in the male and fourteen in the female. In my sections of both sexes the chromosomes appear less regular in contour than in the other species examined (probably owing to somewhat defective fixation). They show clearly, however, in both sexes a largest pair and a smallest ( $m$-chromosomes), as in the other forms.

\section{Second Type. Forms Possessing Unequal Idiochromosomes}

The sexual differences of these forms have been worked out in Lygæus turcicus, five species of Euschistus (variolarius, ictericus, tristigmus, fissilis and servus), Cœnus delius and Podisus spinosus. In the last named species the unreduced number is sixteen, in the others fourteen. In all, the number of chromosomes is the same in both sexes, but while the males show a large and a small idiochromosome, the females show two large idiochromosomes that are equally paired. This difference clearly appears in all the species examined but is most conspicuous in Euschistus variolarius, E. ictericus and Lygæus turcicus, where the inequality of the idiochromosomes is most marked. The relative size of the idiochromosomes varies somewhat (perhaps owing to differences in the degree of extraction of the dye) but on the whole is characteristic of the different species, as described below.

In all of the species of Euschistus examined, and in Conus delius, a largest and a smallest pair of ordinary chromosomes (the latter marked $s$ in some of the figures) are readily distinguishable. These give rise to corresponding large and small bivalents in the first mitosis, and are recognizable as single chromosomes in the spermatid-groups (Figs. 4, 5). The small chromosomes are in every case smaller than the large idiochromosome, and in Mineus bioculatus (Fig. 4, p, q) are actually smaller than the small idiochromosome. It is possible that this pair of chromosomes 

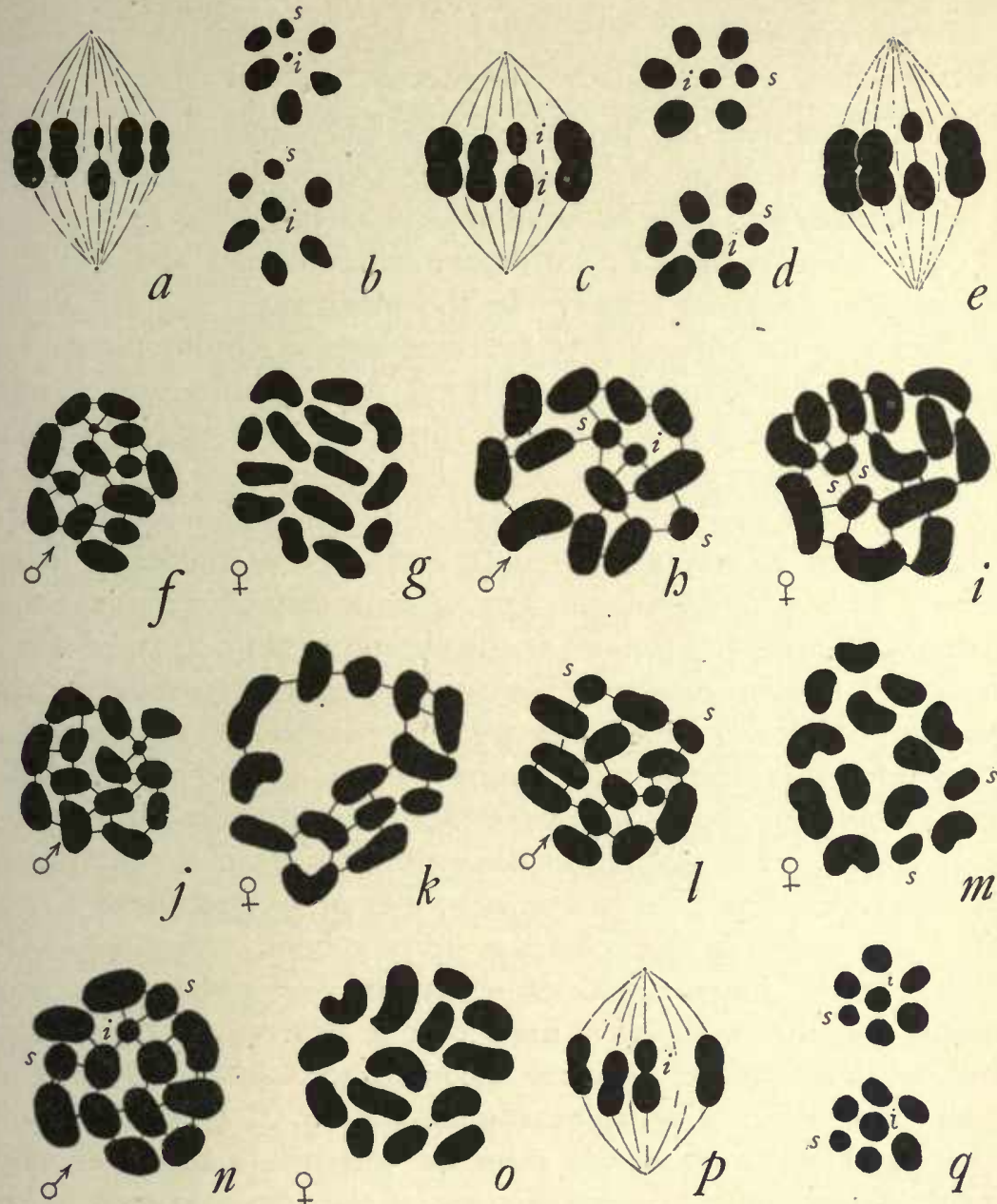

Figure 4

Euschistus, Mineus. $-a$, E. variolarius, second spermatocyte-division; $b$, sister-groups, second division; $c, d$, corresponding views of E. servus; e, second spermatocyte-division, E. tristigmus; $f, g$, E. variolarius, spermatogonial and oögonial groups respectively; $h, i$, corresponding views of E. servus; $j, k$, the same, E. ictericus; $l, m$, the same, E. tristigmus; $n, o$, the same, E. fissilis; $p$, Mineus bioculatus, second spermatocyte-division; $q$, sister-groups, from the same spindle, second division. 
may correspond to the microchromosomes, or $m$-chromosomes, that are so characteristic of the first type ( $m$, in Figs. 2,3$)$.

\section{e. Euschistus}

In $\mathrm{E}$. variolarius the inequality of the idiochromosomes (Fig. 4, a) is greater than in any other of the observed forms excepting Lygæus turcicus. The sister spermatid-groups (Fig. 4, $b$ ) consist in each case of a ring of six ordinary chromosomes with the idiochromosome near its center. In the outer ring may be distinguished as a rule four or five different sizes of chromosomes, the largest and smallest $(s)$ being always recognizable, and usually also a second largest and second smallest. The large idiochromosome is always distinctly larger than the smallest chromosome $(s)$ of the outer ring, while the small idiochromosome is very much smaller than either, and in long extracted preparations looks exactly like a centrosome. The spermatogonial groups correspondingly show seven pairs of chromosomes (Fig. 4, f), of which the small idiochromosome, the smallest pair of ordinary chromosomes, and two large pairs are recognizable. The remaining seven include three equal pairs, while the seventh is the large idiochromosome, but it is impossible to identify this chromosome more nearly. The oögonial groups show fourteen equally paired chromosomes, as shown in Fig. 4, $g$; but my preparations do not show this so well in this species as in the others.

E. ictericus shows a similar spermatogonial group (Fig. $4, j$ ) except that the small idiochromosome is relatively a little larger and the small pair of ordinary chromosomes but slightly smaller than the others. The oögonial groups (Fig. 4, $k$, an unusually open specimen) very clearly show the absence of the small idiochromosome, but the equal pairing of the chromosomes is less obvious than in the following species.

In E. tristigmus (Fig. 4, $e, l, m$ ) the small idiochromosome is relatively much larger than in the foregoing species, while in E. servus, it is usually a little larger still (Fig. 4, $c, d, b$ ). In both these forms the smallest pair of ordinary chromosomes are at once recognizable in the spermatogonia $(s$, Fig. $4, h, l)$ and the equal pairing of the others is evident. In E. servus the oögonial groups 
show the equal pairing of all the chromosomes with equal clearness, the absence of the small idiochromosome being evident (Fig. $4, i)$. The small pair $(s)$ evidently correspond to the small pair in the male $(4, b)$ and the large idiochromosome-pair must therefore be represented by one of the larger pairs. Fig. 4, n, o, show the spermatogonial and oögonial groups of E. fissilis, showing the same relation's as in E. servus, save that the small pair are relatively larger.

The above-described species of Euschistus, while agreeing precisely in the general relations, present individual differences so marked as to show that even the species of a single genus may be distinguishable by the chromosome-groups. In this case the most interesting feature is the series shown in the inequality of the idiochromosomes, which becomes progressively greater in the series (I) E. servus, (2) tristigmus, fissilis, (3) ictericus, (4) variolarius, the inequality in the last case being fully as great as in Lygæus. I may again mention the fact that in the opposite direction the genus Brochymena often shows the idiochromosomes less unequal than in E. servus; in Mineus they are sometimes of nearly equal size (Fig. 4, p, q), while in Nezara no inequality exists. Practically all intermediate conditions are therefore shown within the limits of a single family between the extreme inequality shown in E. variolarius and no inequality at all. . It is quite clear from the observations here brought forward that this progressive differentiation has occurred only in the male sex, as I conjectured in my first paper.

\section{f. Cœnus delius}

The relations in this form are so closely similar to those seen in Euschistus servus or fissilis, as described above, as hardly to require separate description. Fig. $5, b$, shows the spermatogonial metaphase-group; $5, i$, the corresponding oögonial group. Both these preparations show very clearly the small pair $(s)$ of ordinary chromosomes (not so well shown in the figure of the spermatogonial group in my first paper). Here, as in Euschistus, it is evident that the large idiochromosome is much larger than the members of the small pair. 


\section{g. Lygæus turcicus}

In this species the inequality of the idiochromosomes is nearly or quite as great as in Euschistus variolarius, but the differentiation of the chromosome-pairs is less marked than in that species, and the small pair cannot be distinguished with certainty in any of the stages. In the spermatogonial groups, accordingly, only the small idiochromosome is markedly smaller than the others (Fig. 5, c, d); and hence its lack of an equal mate is rendered very conspicuous. In the female the small idiochromosome is absent as usual and all the chromosomes are equally paired (Fig. 5, $f, g$ ). The idiochromosomes cannot be distinguished from the ordinary chromosomes.

\section{b. Podisus spinosus}

In this species both sexes show sixteen chromosomes. In the spermatogonial groups (of which I am now able to give a better figure than the one in my first paper) the small idiochromosome appears relatively larger than in any of the foregoing species, though still not more than half the size of any of the others (Fig. $5, j$ ). In the female (follicle-cells, Fig. 5, $k$ ) all the chromosomes are equally paired and the small idiochromosome is absent, but owing to the relatively large size of the latter in the male the chromosome-groups of the two sexes do not show so obvious a contrast as in the foregoing cases.

\section{Resumé and Conclusions Regarding the Second Type}

In all the forms described under this type the two sexes show the same number of chromosomes but differ in that the male groups include a large and a small idiochromosome while the female groups have two large idiochromosomes of equal size. This result agrees with that already reached by Stevens ('05) in the case of the beetle Tenebrio, and involves the same conclusions that she has indicated. Since all the chromosomes of the ooggonial groups are equally paired, it is evident that all the matured eggs must contain half such a group, one of the chromosomes being the maternal representative, or mate, of the large idiochromosome 

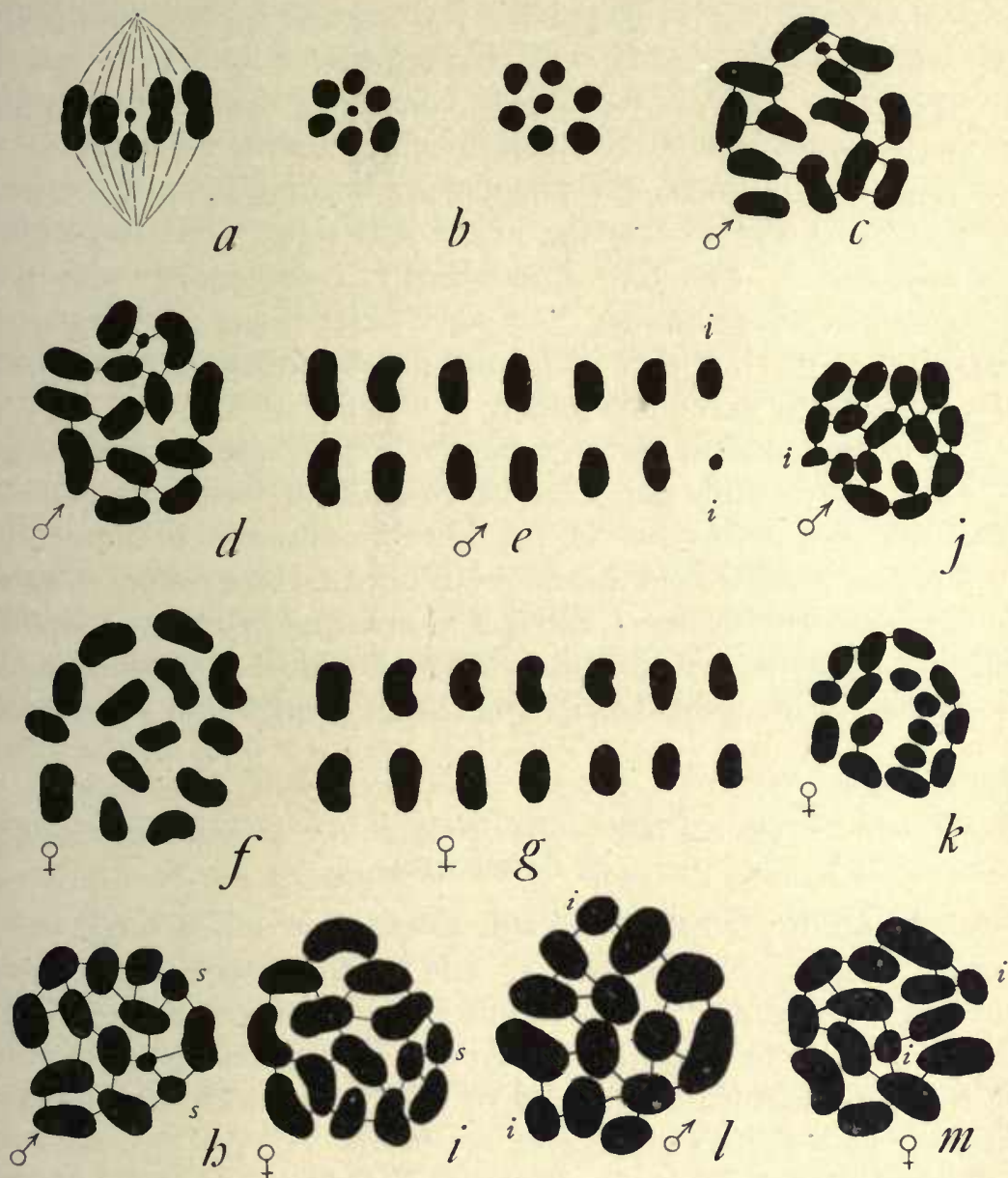

Figure 5

Lygaus, Conus, Podisus, Nezara.- $a$, Lygæus turcicus, second spermatocyte-division; $b$, sistergroups, second division; $c, d$, spermatogonial groups; $e$, the chromosomes of $d$ arranged in pairs; $f$, oögonial group; $g$, the same in pairs; $h, i$, Conus delius, spermatogonial and follicle-cell groups; $l, m$, Nezara hilaris, spermatogonial and oögonial groups respectively. 
of the male. Fertilization of such an egg by a spermatozoön containing the small idiochromosome will produce a group identical with that occurring in the male; fertilization by one containing the large idiochromosome will produce the characteristic female group. This result is thoroughly consistent with that obtained in the first type; for if the small idiochromosome be supposed to disappear in the male, the phenomena become in every respect identical with those occurring in the first type. The large idiochromosome is therefore undoubtedly homologous with the heterotropic chromosome, and the latter owes its unpaired character to the fact that its former paternal mate has vanished, as I conjectured in my first paper.

It is further evident that in synapsis, in both sexes, the members of each chromosome-pair become coupled to form symmetrical bivalents, except in case of the idiochromosomes of the male. In this case alone do chromosomes of unequal size couple to form an asymmetrical bivalent; and it is a consequence of this coupling that the subsequent distribution allots the small idiochromosome to one-half of the spermatozoa and the large one to the other half.

\section{Third Type. Forms in which the Idiochromosomes are of Equal Size}

Of these forms I have been able to examine only a single case, namely, that of Nezara hilaris; and in the course of a whole summer's collecting I obtained but a single female in the proper stage to show the oögonial divisions. Fortunately both ovaries show a considerable number of division-figures which demonstrate the facts with perfect clearness.

A particular interest attaches to this form on account of the fact, described in my first paper, that the idiochromosomes are of equal size and hence give no visible differential between the two classes of spermatozoa. This form gives therefore a test case concerning my general conclusion that the differentiation of the idiochromosomes has occurred only in the male; for since these chromosomes are here alike in all the spermatozoa, it might with some plausibility be assumed that the differentiation had in this 
species taken place in the female. The facts conclusively show that such is not the case.

The spermatogonial groups (Fig. $5, l$ ) show fourteen chromosomes, all of which may be symmetrically paired. The smallest pair, $i, i$, (as I showed in my first paper) are the idiochromosomes as is shown by their characteristic behavior during the growthperiod and in the maturation-divisions. In synapsis the twelve larger chromosomes couple to form six bivalents, while the idiochromosomes divide as separate univalents in the first spermatocyte-division. Their products then conjugate as usual to form the idiochromosome-dyad, which differs from all the forms hitherto observed in being composed of two equal members. All the spermatid-nuclei are accordingly exactly similar in appearance and no visible dimorphism exists ( $c f$. Fig. 4 of my first paper, Wilson, '05, I). We should accordingly expect to find the oögonial groups exactly similar to the spermatogonial; and such is clearly shown to be the fact by the preparations, the ooggonial groups showing fourteen equally paired chromosomes among which the idiochromosomes are readily recognizable by their small size (Fig. $5, m$ ).

In this case, therefore, alone among all those examined, no visible differences are shown by the nuclei of the two sexes. One pair of the chromosomes are, however, different in nature from the others, as is shown by their different behavior in the male in the growth-period and in synapsis; and it is quite clear that the two members of this pair are always assigned to different spermatozoa. In respect to this chromosome, therefore, the spermatozoa fall into two classes as truly as the other forms, though they cannot be distinguished by the eye. It is hardly necessary to point out how important this case is in giving a firm basis of comparison with the more usual forms in which, if we can trust the existing accounts, all of the functional spermatozoa are exactly alike in appearance, and no sexual differences of the chromosome-groups are apparent. 
E. The Differential Cbromosomes in the Synaptic and Growthperiods

I will now briefly consider a very marked difference between the sexes in respect to the behavior of the differential chromosomes during the contraction-phase of synapsis and the succeeding early growth-period. ${ }^{1}$ In the male, as was fully described in my last paper, both the heterotropic chromosome and the idiochromosomes condense early in the growth-period (usually as early as the contraction-phase of synapsis) to form rounded, condensed, intensely-staining chromosome-nuclei. In this condition they persist throughout the whole growth-period of the spermatocyte, without ever assuming the looser texture and more elongate form of the other chromosomes. In the earlier part of this period they are as a rule closely associated with a large pale plasmosome, but later become separated from it.

In the female no trace of such a chromosome-nucleus can be found in the contraction-figure of the synaptic period. My best preparations of this stage are from the ovaries of the larval Anasa, which show a distinct synaptic zone of oöcytes intervening between the zone of multiplication and the growth-zone; but I have observed the same condition in the ovaries of recently emerged adults of Harmostes, Alydus, Euschistus, Cœnus and Podisus. In all these forms the contraction-figure is very similar to that of the spermatocytes, the chromosomes being in the form of deeply staining, ragged, and apparently longitudinally split loops that are crowded into a spheroidal mass toward the center or one side of the nucleus and surrounded by a large clear space. The nuclei at this time occasionally show one or two small deeply-staining nucleolus-like bodies (probably plasmosomes); but these are much smaller than the chromosome-nuclei of the spermatocytes at this period, and in many of the nuclei are absent. The contrast between these nuclei and those of the male at the corresponding period is so striking as to be at once apparent. In later stages the chromosomes spread through the nuclear cavity, become looser in texture and finally give rise to a fine reticular structure. In 
these stages a variable number of deeply-staining nucleoli make their appearance; but their true nature can only be determined positively when the whole ovarian life of the egg has been followed and the process of maturation observed. I can, therefore, only state that no chromosome-nucleolus is present in the contraction period of synapsis, or in the early growth-period; and even though it be present in later stages, which I think is very doubtful, a wide difference between the sexes would still exist in respect to the earlier period.

\section{F. General Resumé}

The foregoing results may be given a general formulation as follows: If $n$ be the unreduced number of chromosomes in the female, the matured eggs in all cases contain half this number $\left(\frac{n}{2}\right)$. The males are of three types. In the first, one of the chromosomes (the heterotropic or "accessory") is without a mate, and the unreduced number is accordingly one less than that of the female. Half the spermatozoa possess, and half lack, the heterotropic chromosome, the first class having the same number as the matured eggs $\left(\begin{array}{l}n \\ 2\end{array}\right)$, the second class one less $\left(\frac{n}{2}-I\right)$. In the second type the male has the same number of chromosomes as the female, but possesses one large and one small idiochromosome while the female possesses two large ones. In maturation half the spermatozoa receive the small and half the large idiochromosome. The third type differs from the second in that the idiochromosomes are of equal size in both sexes, and no visible differences exist between the two classes of spermatozoa or the somatic groups of the two sexes. Designating the large and small idiochromosomes as $I$ and $i$ respectively, the relations in fertilization and sex-production are as follows:

TYPE I

(Protenor, Anasa, Alydus, Harmostes)

Egg $\frac{n}{2}+$ spermatozoön $\frac{n}{2}$ (including heterotropic) $=n$ (female).

Egg $\frac{n}{2}+$ spermatozoön $\frac{n}{2}-\mathrm{I}$ (heterotropic lacking) $=n-\mathrm{I}$ (male).

TYPE II

(Lygaeus, Euschistus, Conjus, Ponisus)

Egg $\frac{n}{2}$ (including $I$ ) + spermatozoön $\frac{n}{2}$ (including $\left.I\right)=n$ (including $I I$ ) (female).

Egg $\frac{n}{2}$ (including $I$ ) + spermatozoön $\frac{n}{2}$ (including $i$ ) $=n$ (including $I i$ ) (male). 


\section{TYPE III}

(NEZARA)

Egg $\frac{n}{2}+$ spermatozoön $\frac{n}{2}=n$ (male or female, including in each case two equal idiochromosomes).

These relations are graphically shown in the following diagram (Fig. 6) in which the differential chromosomes are black and the ordinary ones unshaded (only two pairs of the latter shown). For the sake of simplicity only the final result of synapsis (second column) and the ensuing process of reduction (third column) are shown, without regard to variations of detail. The matured eggs (ov) are represented with a single polar body (the result of the reduction-division) which is greatly exaggerated in size. The female-producing and male-producing spermatozoa $(s p)$ are lettered $a$ and $b$ respectively. It will be evident from an inspection of this diagram that the second type may readily be derived from the third, and the first from the second by the reduction (second type) and final disappearance (first type) of one of the differential chromosomes. This I believe to represent the actual relations of the three types.

\section{GENERAL.}

In recent years evidence has steadily accumulated to strengthen the view that the general basis of sex-production is given by a predetermination existing at least as early as the fertilized egg, but there is a wide divergence of opinion in regard to the conditions preëxisting in the gametes prior to their union. ${ }^{1}$

The fact that in some organisms (such as Dinophilus, Hydatina or Phylloxera) the unfertilized eggs, sometimes even in the ovary, are visibly distinguishable as male-producing and femaleproducing forms, has led a number of recent writers to deny that the spermatozoön can play any part in sex-determination. Beard, for example, asserts that "The male gamete, the spermatozoön, has and can have absolutely no influence in determining the sex

'The general question of sex-determination, with its literature, has within the past five years been so ably and thoroughly reviewed by Cuénot, Strasburger, Beard, von Lenhossék, O. Schultze and others, that $I$ shall here limit myself in the main to an analysis of the new observations brought forward. 
Studies on Chromosomes .

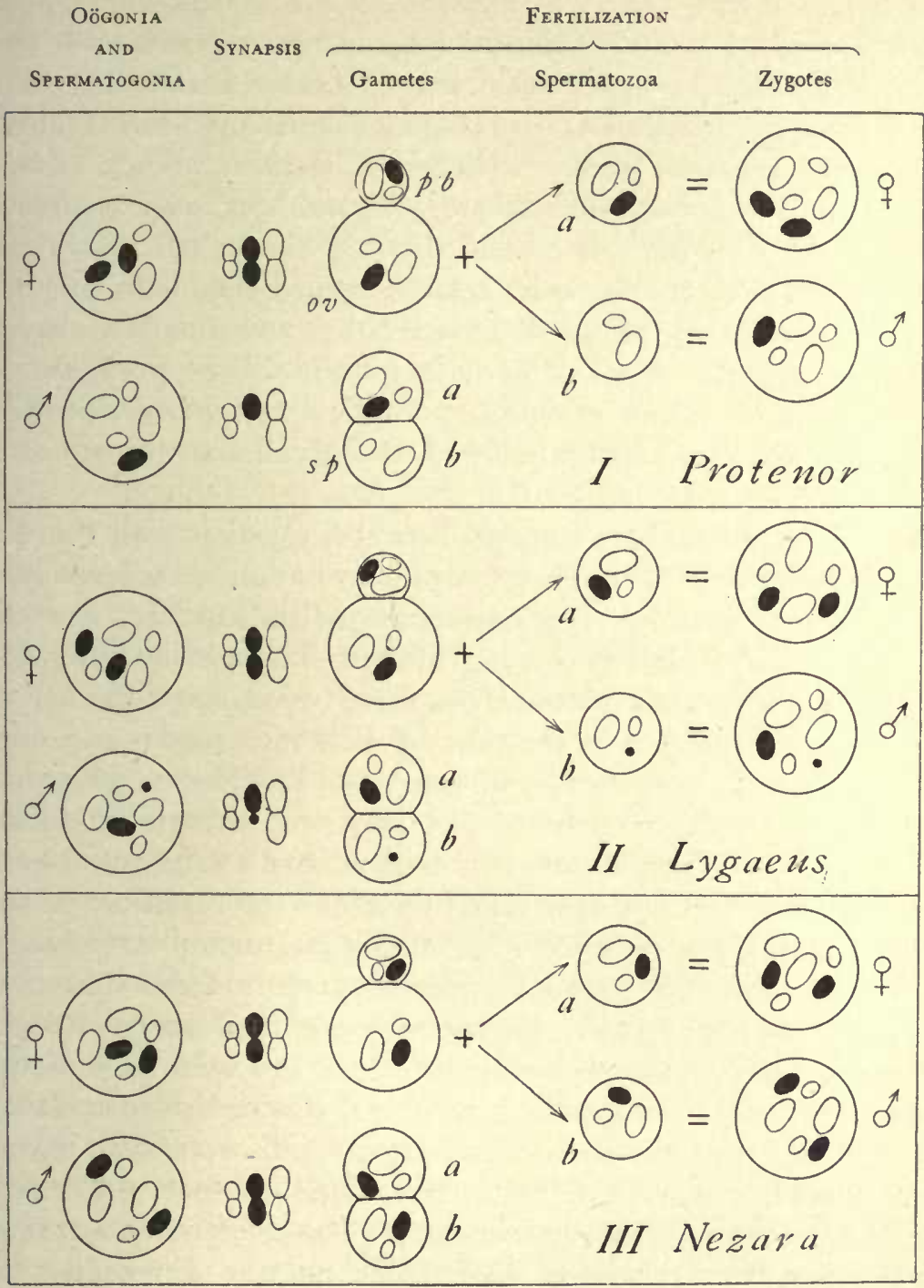

Fra. 6. 
of the offspring" ('02, p. 7I2); and a similar conclusion, though less dogmatically stated, is reached in the general reviews of Lenhossék ('o3) and O. Schultze ('०3). The opposite view that the spermatozoön alone is concerned in sex-determination (which like the preceding one, is of very ancient origin) has, however, been maintained by some recent writers, for instance, Block (whose work I know only from Cuénot's review) and McClung, as already mentioned.' On the other hand, both Cuénot ('99) and Strasburger ('o) in their able reviews, have argued that both gametes may be concerned in sex-determination; and the last named author urged the view, afterward recognized as probable by Bateson and developed in detail by Castle ('03), that sex-production takes place in accordance with the Mendelian principles of inheritance.

The observations here brought forward, together with those of Stevens on Tenebrio, establish the predestination (in a descriptive sense) of two classes of spermatozoa, equal in number, as maleproducing and female-producing forms. Though indistinguishable to the eye in their mature state, these two classes differ visibly in nuclear constitution at the time of their formation; and since this occurs in the same order of insects as Phylloxera, where the eggs are visibly distinguishable (by their size) as male-producing and female-producing forms, it is evident that a substantial basis now exists for the views expressed by Cuénot and Strasburger, and for the Mendelian interpretation of sex-production worked out by Castle. Whether in the Hemiptera that form the subject of this paper the eggs are, like the spermatozoa, predestined as maleproducing and female-producing forms can at present be a matter of inference only. I have not been able to distinguish such classes by their size, and the data show, almost with certainty, that if they exist they do not exhibit any visible nuclear differences like those present in the spermatozoa. But this gives no ground for denying their existence. No visible nuclear dimorphism of

\footnotetext{
1"By exclusion then, it would seem that the determination of this difference (the sexual one) is reposed in the male element" (McClung, 'o2, p. 78). McClung nevertheless maintained the existence of a selective power on the part of the egg such that "the condition of the ovum determines which sort of spermatozoön shall be allowed entrance into the egg substance" (op. cit., p. 76).
} 
the spermatozoa exists in Nezara, yet this condition is connected, by an almost continuous series of intermediate forms, with one in which a conspicuous difference of nuclear constitution is to be seen. It seems hardly open to doubt that sex-production conforms to the same essential type throughout this series. At least a possibility is thus established that in organisms generally both eggs and spermatozoa may be predestined as male-producing and female-producing forms, whether they are visibly different or not. In any case, it is evident that in the Hemiptera the chromosome-combination characteristic of each sex is established by union of the gametes and is a result of fertilization by one or the other of the two forms of spermatozoa. Sex must therefore already be predetermined in the fertilized egg, and it is difficult to conceive how it could subsequently be altered in these animals by conditions external to the egg or embryo. Since the idiochromosomes or heterotropic chromosomes form the distinctive differential between the nuclei of the two sexes, it is obvious that these chromosomes are definitely coördinated with the sexual characters. We must therefore critically inquire into the causal relation between sex-production and the chromosomes, of which this coördination is an expression.

That sex-production may be interpreted as the result of a Mendelian segregation, transmission and dominance of the sexual characters has been shown by Castle ('O3). The history of the differential chromosomes in synapsis and reduction evidently affords a concrete basis for such an interpretation in the terms of the Sutton-Boveri chromosome-theory. Analysis of the facts now known will, however, show even more clearly than the more general considerations adduced by Castle, that this interpretation is only admissible under the assumption that a selective fertilization occurs, such that eggs containing the female-determinant are fertilized only by spermatozoa containing the male-determinant and vice versâ. Until I had read Cuénot's recent interesting paper ('05) on the breeds of mice and their combinations, the necessity for making this assumption seemed to me an almost fatal difficulty in the way of the interpretation, but if Cuénot's conclusions be well founded the $\grave{a}$ priori objections to such a 
selective fertilization are in large measure set aside. I therefore think that the possibility of a Mendelian interpretation of sexproduction should be carefully examined, though as will be shown, an alternative interpretation is possible.

I. In such an examination the distinction between sex-determination and sex-inheritance should be clearly drawn; for it is well known that each sex may contain factors capable of producing the characters of the opposite sex, and it may well be that the patency or latency of the sexual characters is determined by factors quite distinct from those concerned with their transmission from parent to offspring. For the purpose of analysis it will, however, be convenient to speak of the idiochromosomes or their homologues as "sex-determinants," this term being understood to mean that these chromosomes are the bearers of the male and female qualities (or the factors essential to the production of these qualities) respectively. They may also be designated (whenever it is desirable to avoid circumlocution) as sex-chromosomes or "gonochromosomes." As a basis of discussion the Mendelian interpretation may be taken to postulate, further, that the two sex-chromosomes, which couple in synapsis and are subsequently disjoined by the reducing division, are respectively male-determinants and female-determinants in the sense just indicated. The most convenient approach to the question is offered by the heterotropic chromosome, since its unpaired condition in one sex renders its mode of transmission more clearly obvious than that of the idiochromosomes. The facts (especially as observed in Protenor) clearly prove that this chromosome alternates between the sexes in successive generations, passing from the male to the female in the production of females, and from the female to the male in the production of males (Fig. 6). The important bearing of this on both sex-inheritance and sex-determination will appear beyond.

Since the heterotropic chromosome is without a fellow in the male it must, if it be a sex-determinant at all, be the male-determinant, which exerts its effect uninfluenced by association with a female-determinant. But since the spermatozoa that contain 
this chromosome produce only females, it must be assumed that the maternal mate or fellow, with which it becomes associated on entering the egg, is a dominant female-determinant. Further, since males result from fertilization by spermatozoa that do not contain the heterotropic chromosome, the latter must in maleproducing eggs be derived from the egg-nucleus ( $c f$. the diagram, Fig. 6). The general interpretation, therefore, must include the assumption that there are two kinds of eggs (presumably in approximately equal numbers) that contain respectively the maleand the female-determinant, ${ }^{1}$ and that the former are fertilized only by spermatozoa that lack the heterotropic chromosome (i.e., the male determinant) and vice vers $\hat{a},{ }^{2}$ giving the combinations $(m) f$ (female) and $m$-(male). Such a selective fertilization is therefore a sine qua non of the assumption that the heterotropic chromosome is a specific sex-determinant.

A nearly similar, though somewhat more complex, result follows in the case of the idiochromosomes. In respect to sex-production the large idiochromosome is identical with the heterotropic chromosome, and the morphological evidence is nearly or quite decisive that the heterotropic chromosome is actually a large idiochromosome, the smaller mate of which has disappeared. The small idiochromosome may therefore be regarded as a disappearing, or even vestigial, female-determinant that is recessive to its larger fellow (the male-determinant); and its reduction in size may plausibly be regarded as an atrophy resulting from its invariably recessive nature (this chromosome being strictly confined to the male). Precisely as in case of the heterotropic chromosome, the large idiochromosome of the male (maledeterminant) must be derived in fertilization from the egg-nucleus (Fig. 6); and, as before, it must be assumed that eggs that contain this chromosome are fertilized only by spermatozoa that contain the small idiochromosome, those that contain the female-determi-

'This would follow from the coupling of the two sex-chromosomes in synapsis to form the bivalent $(m) f$, and its division in such a way as to leave in the egg either the male- or the female-determinant indifferently.

${ }^{2}$ Otherwise the combinations $m m$ or $f-$ might result, which is contrary to observation, since the sexchromosomes are in this type never paired in the male or unpaired in the female. 
nant only by spermatozoa containing the large idiochromosome. In this type, accordingly, it is clear that the large idiochromosome (like the heterotropic chromosome to which it corresponds) passes alternately from one sex to the other, while the small one never enters the female; and this would remain true even did selective fertilization not occur (Fig. 6). The same interpretation may finally be extended to Nezara, where the idiochromosomes are of equal size in both sexes, the relations of dominance being the same as before.

The two vital points in this result are first, the assumption of selective fertilization, and second the relations of dominance and recession in the two sexes. As regards the first point, until the appearance of Cuénot's paper, referred to above, almost no definite evidence had been produced of an infertility between particular classes of gametes in the same species; though it has long been known that many plants are in a greater or less degree infertile to their own pollen, and an analogous fact has been more recently demonstrated in Ciona by Castle ('96) and Morgan ('04). Correns ('02), in his study of hybrid maize, was led to suggest that in this case there might be a somewhat diminished fertility between the gametes bearing the recessive character (thus accounting for a relative deficiency of extracted recessives in the second generation of crosses, $F_{2}$ ). In studying the breeds of mice Cuénot has found it impossible to obtain pure or homozygous yellow forms. Yellow mice are invariably heterozygotes (the yellow being dominant over gray, black or brown) and when crossed with a pure race of a different color ( $e . g$., gray) give the typical Mendelian result, yellow and gray offspring appearing in equal numbers. This proves that a complete Mendelian disjunction of the yellow and gray determinants takes place in maturation. When yellow mice of known constitution $(e \cdot g ., \mathrm{Y}(\mathrm{G}))$ are paired with like forms, the first offspring include pure gray forms (extracted recessives) slightly in excess of the normal ratio of 25 per cent., and yellow forms; but contrary to the Mendelian expectation the latter, when paired with one another, never give pure dominants (YY), but again produce pure grays (GG) and heterozygous yellows $(\mathrm{Y}(\mathrm{G}))$. Cuénot therefore concludes that although complete segregation of both the gray and yellow 
characters takes place in the gamete-formation, and the resulting yellow-bearing gametes unite freely with those bearing the recessive color, they do not unite with each other: "Ceux-ci (the yellow heterozygotes) forment bien des gamètes de valeur $\mathrm{CJ}$ ou $\mathrm{AJ}$, mais ces gamètes ne peuvent pas s'unir les uns aux autres pour donner des zygotes ayant les formules CJCJ, AJAJ ou CJAJ; par autre, ils s'unissent facilement a tous les autres gamètes que j'ai essayés pour former avec eux des hétérozygotes mono- ou dihybrides" (op. cit., p. cxxx). This conclusion is sustained by the fact that the combination $Y(G) \times Y(G)$ (CYCG $x$ CYCG in Cuénot's terminology) produces a relative deficiency of yellows in the offspring, as is to be expected. ${ }^{1}$ In pairing $Y(G)$ with $\mathrm{Y}(\mathrm{G})$, accordingly, the Y-bearing spermatozoa unite only with the G-bearing eggs, and vice versâ, which is exactly analogous to the selective fertilization assumed in case of the sex-bearing gametes. Perhaps it may be possible to find a different explanation of the facts; but if Cuénot's interpretation be well-founded the case goes far to remove the scepticism which I think one must otherwise feel in regard to a selective fertilization of the gametes in sex-production.

An examination of the question of dominance involved in the Mendelian interpretation leads to some interesting conclusions. In forms possessing unequal idiochromosomes the sexual formulas would be for the female $(m) f$ and for the male $m(f)$ ( $f$ being the small idiochromosome). Applying the same interpretation to Nezara, where the idiochromosomes are of equal size, the corresponding formulas are $(m) f$ and $m(f)$, giving the gametes $(m)$, $f, m$ and $(f)$. Assuming likewise a selective fertilization the facts would be:

$$
\begin{array}{cl}
\text { EgGs SpermatozoA } & \\
(m)+(f) & =(m)(f), \text { producing a male, } m(f) \\
f+m & =m f, \text { producing a female }(m) f .
\end{array}
$$

'The deficiency, though constant, is very slight. Cuénot himself seems to consider this a difficulty, but I believe a very simple explanation may be given. With equal numbers of the gametes of both sexes the ratio of yellows to grays should be two to one, instead of three to one as in the typical Mendelian case (since the class YY is missing). If, however, the spermatozoa be in large excess, as they undoubtedly are, all or nearly all the Y-bearing eggs will be fertilized by G-bearing spermatozoa, and vice versâ, thus bringing the ratio of yellows (Y(G)) to grays (GG) more or less nearly up to three to one. 
Now it is clear that if the relations of the chromosomes to sexproduction be the same here as in the second type, the chromosome $m$ must alternate in successive generations between the male and the female (like the large idiochromosome or the heterotropic chromosome to which it corresponds), and hence also shows an alternation of dominance, being dominant in the former sex and recessive in the latter. If, therefore, dominance and recession be inherent in the chromosomes, there must be such a relation between them that $m$ is always dominant to the chromosome $(f)$ of the male, and always recessive to the chromosome $f$ of the female, and that the latter two chromosomes $(f$ and $(f))$ are never interchanged between the sexes. This last assumption is not so improbable as it may at first sight appear; for in the second type it is certain, as already pointed out, that the small idiochromosome $((f)$ under the general assumption) never enters the female, while the large idiochromosome, $m$, like the heterotropic, alternates between the two sexes in successive generations.

A strict Mendelian interpretation of sex-production may unquestionably, I think, be constructed upon the foregoing assumptions. But an interesting suggestion for a somewhat modified Mendelian interpretation is given by the possibility that the dominance of the sex-chromosomes is determined by extrinsic factors, namely, by conditions in the protoplasm of the zygote. If this were the case it is evident that the idiochromosomes could not be considered as sex-determinants in the strict sense of the word. The determination of sex would in this case be due to factors preëxisting in one or both of the gametes, irrespective of the sex-chromosomes, and the latter could only be considered as a means by which the sex-characters are transmitted or inherited. The possibility is here clearly offered that either or both forms of gametes may be predetermined as males or females (or at least male-producing and female-producing) prior to fertilization and irrespective of the chromosomes; and thus an interpretation of the ordinary forms of gametes would be reached in harmony with such cases as Dinophilus and other forms in which male-producing and femaleproducing eggs are distinguishable in size prior to fertilization. Such an interpretation would further be perfectly consistent with 
the modification of sex-production in some cases by external conditions, and with the production of both males and females in parthenogenesis (though this may be otherwise explicable); and it might also give the explanation of selective fertilization.

II. It has not been my intention to advocate the foregoing interpretation, but only to set forth as clearly as possible, the assumptions that it involves. It is nevertheless my opinion that the analysis places no insuperable obstacles in its way, and that, however dominance be determined, the Mendelian interpretation may in fact give the true solution of the problem. I have, however, endeavored to seek for a different interpretation that may escape the necessity for assuming a selective fertilization; and although I have to offer nothing more than suggestions, some of which undoubtedly encounter serious difficulties, I shall make them in the hope that they may afford some clue to further inquiry. Some of these suggestions are equally applicable to the Mendelian interpretation considered above, but for the purpose of discussion this interpretation may for the time be laid aside.

It seems possible that the differential chromosomes may perform a definite and special function in sex-production without being in themselves specifically male-determining and female-determining or even qualitatively different save in the degree of their special activity (whatever be its nature). This suggestion is given by the fact that the presence of one heterotropic chromosome or large idiochromosome is associated with the production of a male, while if two such chromosomes are present a female is produced. This very obviously suggests that the same kind of activity that produces a male will if reinforced or intensified produce a female; and with this would accord the production of males from unfertilized eggs, and females from fertilized ones, in the case of the bee. In these cases the decisive factor may be a merely quantitative difference of chromatin between the two sexes. But it is obvious that such a difference cannot give the basis for a general explanation, since in Nezara, and presumably in many other organisms, both the number of chromosomes and the quantity of chromatin is the same in both sexes. And yet 
the existence of a quantitative difference in some cases raises the question whether it is not the result or expression of some more deeply lying nuclear difference which may still be present in those cases where no quantitative difference exists. I find it altogether incredible that two animals as nearly related as Nezara and Euschistus should differ fundamentally in the relation of the chromosomes to sex-production; and if there is any reason to conclude that sex-determination is effected by the idiochromosomes (or by the combination of which they form a part) in the case where they are visibly different, I cannot avoid the belief that this conclusion applies with equal reason to the case in which they appear to the eye alike in all the spermatozoa. It therefore seems to me an admissible hypothesis that a physiological or functional factor may be present that differentiates the spermatozoa into male-producing and female-producing forms irrespective of the size of the differential chromosomes; and further, that the morphological difference that has arisen in some forms may have been a consequence of such an antecedent functional difference. If we could assume for instance that the differential chromosome-pair in the male includes a more active and a less active member (the latter having in many cases become reduced in size or even having entirely disappeared) the suggestion might be greatly extended in application. Under this assumption the facts might receive a general formulation in the statement that the association of two more active chromosomes of this class produces a female, while the association of a more active and a less active one (or the absence of the latter, as in case of the heterotropic chromosome) produces a male. Reduction of the less active member to form a small idiochromosome would introduce a quantitative difference of chromatin as well as a qualitative one. Its complete disappearance in the male, leaving only the active member as the heterotropic chromosome, would reduce the difference to a merely quantitative one. The assumption of such a physiological difference is admittedly a purely speculative construction, and may seem à priori very improbable. But from the $a$ priori point of view it would seem equally improbable that a morphological dimorphism of the spermatozoa, affecting 
only one pair of the chromosomes, should have arisen; yet this is an observed fact. I therefore think the suggestion is worthy of serious consideration. If it could be adopted the necessity of selective fertilization would be avoided, for the observed results would follow from the fertilization of any egg by any spermatozoön.

But even if in accordance with fact the suggestion is still obviously incapable of direct application to cases in which sex is determined independently of fertilization-for instance, sexproduction in parthenogenetic development or in hermaphrodites, and in forms (such as Dinophilus) where male-producing and female-producing eggs are distinguishable in size before fertilization. It is possible that these cases may be explicable (under either general interpretation) as a result of some forms of differential distribution of the chromosomes occurring at the time of the formation of the polar bodies (parthenogenesis) or at some earlier period in the cell-lineage of the germ-cells; and this possibility should of course be tested by a close cytological study of the facts. On the other hand, there is nothing in the facts to negative the assumption that in some cases the chromosome-combination, established at fertilization, may be in something like a balanced state that is capable of modification by conditions external to the nucleus (as already suggested in the case of dominance).

Boveri's interesting observations on the dispermic eggs of Ascaris ('04) have given direct evidence that the chromosomes react to their cytoplasmic surroundings; and the same fact is even more clearly shown by the difference of behavior of the differential chromosomes in the two sexes of Hemiptera during the synaptic and growth-periods. Hence, even though a preëstablished basis of sex-determination be given in such a physiological dimorphism of the spermatozoa as I have suggested, the sex of the fertilized eggs may in many cases be only a matter of greater or less predisposition and not an immutable predetermination. The nuclei, and hence the primordial germ-cells, may in such cases be in a state of approximate equilibrium, and still retain the power of response to varying conditions in the cellular environment. The production of eggs or spermatozoa in hermaphrodites may thus be explicable as a result of greater or less nuclear 
activity in the two cases, incited by intra-cellular conditions that are external to the chromosome-groups; and a similar explanation may apply to the related case of the formation of visibly different female-producing and male-producing eggs in the same organism.

It would not, I think, be profitable to speculate further in regard to these special cases, but I have wished to indicate that a hypothesis of sex-production which recognizes in some cases a fixed predetermination in the chromosome-groups of the fertilized egg is not inconsistent with the control of sex-production in other cases by conditions external to the nucleus. The constant chromosomal differences of the sexes existing in many Hemiptera, therefore, by no means preclude experiments on the modification or control of sex-production.

I have intentionally excluded from the foregoing suggestions any discussion of the specific nature of the activities of the differential chromosomes, since we are almost wholly ignorant of the functions of chromosomes in general. But although we here enter upon still more debatable ground, I think we should not hesitate to consider such possibilities in this direction as the facts may suggest.

One of the principal, or at least most obvious, differences between the germ-cells of the two sexes is their great contrast in constructive activity, evinced by the enormous growth of the primary oöcyte as compared with the primary spermatocyte. This growth of the oöcyte involves the production of a mass of protoplasm (including under this term the yolk or metaplasm as well as the active protoplasm) thousands of times the bulk of the spermatocyte; and although the latter also increases noticeably in size during the growth-period, the accumulation of protoplasm is almost insignificant as compared with that which takes place in the female. Now, as described above, the idiochromosomes and heterotropic chromosome remain during this period in the male in a relatively passive condition as compared with the other chromosomes, while this is not the case in the female. The thought cannot be avoided that there is a definite causal connection between the greater activity of these chromosomes in the 
oöcytes and the great preponderance of constructive activity in these cells; and it is especially this coincidence that leads me to the general surmise that one of the important physiological differences (I do not say the only one), between the chromosomegroups of the two sexes, may be one of constructive activity. I have elsewhere (The Cell, Chapter VII) reviewed at some length the evidence pointing toward the conclusion that the nucleus (more specifically, the chromatin) is especially concerned with the constructive processes of cell metabolism; and while I no longer hold the view that the nucleus can be considered as the actual formative center of the cell, it still seems to me very probable that the formative processes are directly or indirectly under its control, as has been advocated by many students of cell-physiology. If this view be well-founded, the facts observed in Hemiptera give a very definite and concrete basis for assuming a greater constructive activity in the cells of the female generally, which reaches a climax in the growth-period of the oöcyte. ${ }^{1}$ It seems possible that some of the specific differentiations that take place in the later history of the germ-cells may be directly traceable to the primary difference in the growth-process. It is well known that the young oöcytes and spermatocytes show a very close similarity, not only in size but also in many details of structure. The enormous accumulation of cytoplasm in the oöcyte as compared with the spermatocyte leaves the latter with a great relative excess of the kinoplasmic or archoplasmic material in which the most characteristic differentiations of the spermatozoa-such as the acrosome, middle-piece, axial filament and tail-envelopestake their origin. Perhaps a direct causal relation here exists.

'This suggestion recalls the theory developed by Geddes and Thomson, in their well known work on the "Evolution of Sex," that "the female is the outcome and expression of relatively preponderant anabolism, and the male of relatively preponderant katabolism" (op. cit., revised ed., I901, p. 140). As dedeloped by these authors, this theory has always seemed to me to have too vague and general a character to have much practical value, though it expresses a certain physiological contrast between the sexes that undoubtedly exists. My suggestion is only remotely connected with that theory, since it refers the differentiation of the sexes to a functional difference that preërists in the cells of the male, and involves no contrasted processes of anabolism and katabolism. Nevertheless, the observations here brought forward may harmonize with that side of the theory which lays stress on the preponderant constructive activity of the female cells. 
III. Though I have found it convenient to consider the two foregoing interpretations separately, they evidently have many points of agreement, and perhaps may be reduced to a common basis. Both assign to the differential chromosomes a specific function in sex-production, both recognize the possibility of a determination of sex (as opposed to its transmission), by conditions external to the chromosome-groups, and both assume, in one sex, a specific difference in the sex-chromosomes, followed by a Mendelian disjunction in the formation of the gametes. The essential point in which the second interpretation diverges from the first is that the sex-chromosomes are not conceived as bearing the male or female qualities respectively but as differing only in the degree of their activity, and this difference is assumed to exist in the male only (owing to the relation of fertilization to sex-production). It must be admitted that each interpretation involves a considerable element of pure conjecture, and that each includes assumptions which without additional data must be considered as serious difficulties. The principal one involved in the first interpretation is the assumption of selective fertilization; but if this assumption be granted I believe that it may give an adequate solution of the problem of sex-production in the sexual reproduction of dinecious organisms. The second interpretation avoids this difficulty; it may explain the primary difference between the gametes of the two sexes, the latency of female characters in the male, and the development of such secondary female characters as may be regarded as an exaggeration or intensification of corresponding characters in the male. It seems conspicuously to fail to explain the reverse case of characters that are more highly developed in the male; and to many this will doubtless appear a fatal difficulty. But we are still ignorant of the action and reaction of the chromosomes on the cytoplasm and on one another, and have but a vague speculative notion of the relations that determine patency and latency in development. Additional data will therefore be required, I think, to show whether the difficulty in question is a fatal one, and in what measure either of the two general interpretations that have been considered may approach the truth. The positive result of the 
observations of Stevens and myself is to demonstrate the existence of a constant and definite correlation between the chromosomes and the sexual characters, which is visibly expressed in the relations of a single pair of chromosomes. These relations unquestionably afford a concrete basis for an interpretation of sex-production that assumes a Mendelian segregation and transmission of the sex-characters and to this extent they accord with the general assumption of Castle. The validity of both this and the alternative interpretation suggested must be tested by further inquiry.

Zoölogical Laboratory of Columbia University,

December 8, 1905 .

\section{WORKS CITED}

BeArd, John, '02.-The Determination of Sex in Animal Development. Fischer, Jena.

Boveri, T. H., '04.-Protoplasmadifferenzierung als auslösender Faktor für Kernverschiedenheit. Sitzungsber. der Physikal.-med. Ges. Würzburg, I904.

Castle, W. E., '96.-The Early Embryology of Ciona intestinalis. Bull. Mus. Comp. Zool., xxvii.

'03.-The Heredity of Sex. Ibid., xl, 4 .

Correns, C., '02.-Scheinbare Ausnahme von der Mendel'schen Spaltungsregel für Bastarde. Ber. d. deutschen Bot. Ges., xx.

Cúnot, L., '99.-Sur la détermination du sexe chez les animaux. Bull. Sci. de la France et de la Belgique, xxxii, v, I.

'05.-Les races pures et leurs combinaisons chez les souris. Arch. Zool. Exp. et Gén. (4), iii, Notes et Revue, No. 7.

Gross, J., 'O4.-Die Spermatogenese von Syromastes marginatus. Zool. Jahrb., Anat. und Ontog., xx, 3 .

Henking, H., '91.-Ueber Spermatogenese und deren Beziehung zur Eientwicklung bei Pyrrochoris apterus. Zeitschr. Wiss. Zool., li.

Lenhosś́k, M. v., 'o3.-Das Problem der geschlechtsbestimmenden Ursachen. Fischer, Jena.

McClung, C. E., '02.-The Accessory Chromosome. Sex-determinant? Biol. Bull., iii, I, 2.

Montgomery, T. H., 'or.-A Study of the Germ Cells of Metazoa. Trans. Am. Phil. Soc., xx.

'04.- - Some Observations and Considerations on the Maturation Phenomena of the Germ-cells. Biol. Bull., vi, 3 . 
Morgan, T. H., '04.-Self-fertilization Induced by Artificial Means. Jour. Exp. Zoöl., i, r.

Schultze, O., '03.-Zur Frage von den geschlechtsbildenden Ursachen. Arch. mik. Anat., lxiii.

Stevens, N. M., '05.-Studies in Spermatogenesis with especial Reference to the "Accessory Chromosome." Publication No. 36, Carnegie Institution of Washington, Sept., I905.

Strasburger, E., I900.--Versuche mit diöcischen Pflanzen in Rücksicht auf Geschlechtsverteilung. Biol. Centralbl., xx, 20-24.

Sutron, W. S., '02.- On the Morphology of the Chromosome-group in Brachystola magna. Biol. Bull., iv, I.

Wallace, L. B., '05.-The Spermatogenesis of the Spider. Biol. Bull., viii, 3.

Watase, S., '92.-On the Phenomena of Sex-differentiation. Journ. of Morphology, vi, 3 .

Wilson, E. B., '05, I.-Studies on Chromosomes. I. The Behavior of the Idiochromosomes in Hemiptera. Journ. Exp. Zoöl., ii, 3.

'05, 2.- The Chromosomes in Relation to the Determination of Sex in Insects. Science, xxii, 564 , Oct. 20, 1905 .

'05, 2. Studies on Chromosomes. II. The Paired Microchromosomes, Idiochromosomes and Heterotropic Chromosomes in Hemiptera. Journ. Exp. Zoöl., ii, 4. 
THE JOURNAL OF EXPERIMENTAL ZOÖLOGY is issued quarterly. A volume consists of four numbers, containing from 100 to 200 pages each, with numerous illustrations.

\section{PRICE OF SUBSCRIPTION PER VOLUME}

(PAYABLE IN ADVANCE)

To subscribers in the United States, Canada and Mexico, $\$ 5.00$ To subscribers in other countries $\quad-\quad-\quad-\quad-5.50$ Price of single copies - - - - - - - - -2.00

These prices are net and under no condition subject to discount

Remittances should be made by Postal Money Order (Mandat de Poste, Postanweisung) or by draft on New York, payable to THE JoURNAL OF EXPERIMENTAL ZOÖLOGY.

Address all communications to

THE JOURNAL OF EXPERIMENTAL ZOÖLOGY,

N. E. Cor. Wolfe and Monument Streets, Baltimore, Md., U. S. A. 


\section{STUDIES ON CHROMOSOMES}

IV THE "ACCESSORY" CHROMOSOME IN SYROMASTES AND PYRROCHORIS WITH A COMPARATIVE REVIEW OF

THE TYPES OF SEXUAL DIFFERENCES OF THE CHROMOSOME GROUPS

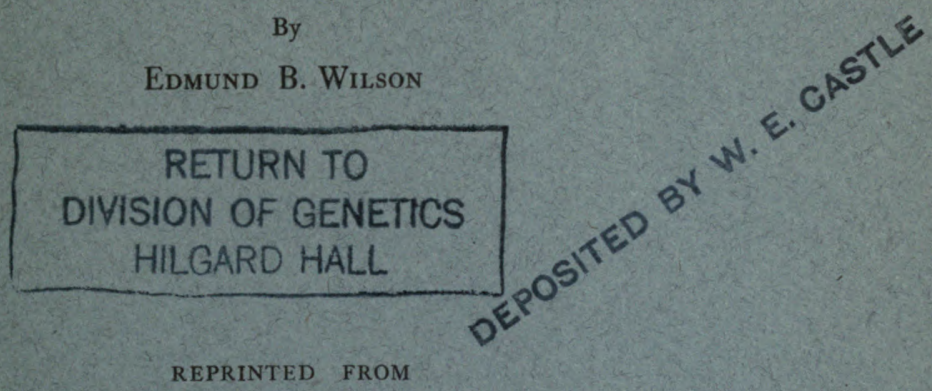

THE JOURNAL OF EXPERIMENTAL ZOÖLOGY

Volume VI No. I :

JANUARY, 1909

BALTIMORE, MD., U. S. A. WILLIAMS \& WILKINS COMPANY 



\title{
STUDIES ON CHROMOSOMES
}

\section{THE "ACCESSORY" CHROMOSOME IN SYROMASTES AND PYRROCHORIS WITH A COMPARATIVE REVIEW OF THE TYPES OF SEXUAL DIFFERENCES OF THE CHROMOSOME GROUPS}

\section{EDMUND B. WILSON}

\author{
With Two Plates and Two Figures in the Text
}

Since the unpaired idiochromosome ("accessory chromosome") was first discovered by Henking (' $9 r$ ) in Pyrrochoris apterus L. this species has been reëxamined by only one observer, Dr. J. Gross ('07), with results that are in substantial agreement with those that pe had reached in an earlier investigation ('O4) on the coreid species Syromastes marginatus L. In both cases his conclusions hre in conflict with the view advanced by McClung ('O2), and first

1 Terminology. With the advance of our knowledge of the chromosomes that form the distinctive differential between the chromosome groups of the two sexes, and between the male producing and the female producing spermatozoa, it becomes increasingly difficult to find a common name that will apply equally to their various modifications. Terms such as the "accessory," "odd" or "heterotropic" chromosome, or "monosome," that are based on the condition of these chromosomes in the male only, are misleading or inappropriate; and some of them are in certain cases inapplicable, even in the malee. g., in Syromastes, where the "accessory" chromosome is not univalent but bivalent. Such terms as "heterochromosome" or "allosome" (Montgomery) seem to me unsatisfactory, since they designate the $m$-chromosomes as well as the differential chromosomes, though these are obviously of quite different nature. Since it has now become evident that a univalent "accessory" chromosome in the male is exactly equivalent to what I have called the "large idiochromosome" in other forms, I think these chromosomes should be designated by the same name, and one that will apply equally to both sexes. While there are some objections to the word "idiochromosome" as a general term for this purpose I am not able to suggest a better one; and since it has already been thus employed by some writers, I shall use it hereafter in a broader sense than that in which I first proposed it, to designate the differential chromosomes in general, whether they are paired or unpaired in the male, and whether one or more pairs are present. A univalent or odd idiochromosome in the male will be called the unpaired idiochromosome (or simply the idiochromosome), while the word "heterotropic" may perhaps conveniently be used as descriptive of its passage without division to one pole in one of the maturation divisions. In Syromastes, as will appear, the "accessory" or heterotropic chromosome represents a pair of idiochromosomes; while in Galgulus there are several pairs of these chromosomes.

The Journal of Experimental Zoölogy, vol. vi, No. I. 
shown to be correct in principle by the work of Stevens and myself, that half the spermatozoa are male producing and half female producing. This view rests on the following facts. When the male somatic chromosome groups contain an odd number, including an odd or unpaired idiochromosome (as in Anasa, Alydus, or Protenor) the female groups have one more chromosome, being duplicates of the male groups with the addition of another chromosome like the unpaired one of the male. When the male groups contain an even number, including a large and a small idiochromosome (as in Lygæus, Cœnus or Tenebrio) the female groups contain the same number, but include two large idiochromosomes in place of a large and a small one. In the first type half the spermatozoa receive the odd idiochromosome while half do not, the former accordingly containing one chromosome more than the latter. In the second type all the spermatozoa receive the same number of chromosomes, but half receive the large idiochromosome and half the small. It follows from these relations that eggs fertilized by spermatozoa containing the odd chromosome, or its homologue the large idiochromosome, must produce females, those fertilized by the other spermatozoa males. These cytological results, first reached by Stevens ('05) in Tenebrio (which has a pair of unequal idiochromosomes in the male) and myself ('o5b, 'o5c, 'o6) in Anasa, Protenor, Alydus and Harmostes (which have an unpaired idiochromosome in the male) and in Lygæus, Cœnus, Podisus and Euschistus (which agree essentially with Tenebrio), have since been confirmed in a considerable number of species and extended to several other orders of insects. ${ }^{2}$ They have recently received indirectly a striking experimental confirmation in the important work of Correns ('o7), which proves that in the diœcious flowering plant, Bryonia dioica, the pollen grains are likewise male determining and female determining in equal numbers.

Gross's conclusion in the case of Syromastes and Pyrrochoris is opposed to all these results in that only one of the two forms of spermatozoa is supposed to be functional (those containing the

${ }^{2}$ See the tabular review in the sequel. 
"accessory" chromosome) the others being regarded as in a certain sense comparable to polar bodies (as was also supposed by Wallace ('05). ${ }^{3}$ This result was based mainly on the numerical relations, and especially on the belief that in both these forms the number of chromosomes is an even one and the same in both sexes-twentytwo in Syromastes, twenty-four in Pyrrochoris. Since the complete reduced number (eleven and twelve in the two respective cases) is present only in those spermatozoa that contain the "accessory" chromosome, Gross argues that this class alone can be concerned in fertilization, as follows:

$$
\begin{aligned}
& \text { Syromastes....Egg II + spermatozoön } 11=22\left(\sigma^{7} \text { or } \&\right) \\
& \text { Pyrrochoris ... Egg } 12+\text { spermatozoön } 12=24\left(J^{7} \text { or } q\right)
\end{aligned}
$$

whereas in Anasa or Protenor the relations are:

$$
\begin{aligned}
& \text { Anasa...... Egg } 11 \text { + spermatozoön IO }=21\left(\sigma^{7}\right) \\
& \text { Egg II + spermatozoön II }=22(\%) \\
& \text { Protenor.... Egg } 7+\text { spermatozoön } 6=1_{3}\left(0^{7}\right) \\
& \text { Egg } 7+\text { spermatozoön } 7=14(\%)
\end{aligned}
$$

In the hope of clearing up this perplexing contradiction I endeavored to procure material for a reëxamination of the two forms in question, and through the great kindness of Professor Boveri, to whom my best thanks are due, was fortunate enough to obtain an abundant supply of both, though unluckily it includes no female material." As far as the relations can be worked out on the male alone they give, I believe, the solution of the puzzle and bring the two species in question into line with the general principle that has been established for other forms. This is evidently true of Pyrrochoris. Syromastes, however, constitutes a new

3 At first thought this seems to be in harmony with the remarkable discovery of Meves ('o3, '०7) that in the male honey bee actual polar bodies are formed which produce abortive spermatids. Butobviously the two cases are not parallel, for in the bee the fertilized eggs produce only females; and this finds a natural explanation, in accordance with the general conclusions of McClung, Stevens and myself, in the assumption that it is the male producing class that degenerate as polar bodies.

1 The material, fixed in Flemming's fluid and in Bouin's picro-acetic-formol mixture, is of excellent quality and gave preparations of perfect clearness. The Flemming material is on the whole the best. For single stains Zwaardemaker's safranin and iron hæmatoxylin were employed (the latter especially for photographs). Various double stains were also used. One of the best, which I can strongly recommend to other workers in this field, is the combination of safranin and lichtgrü, which gives preparations of admirable clearness and is also easy to use and certain in its results. 
type that is not yet known to be exactly paralleled in other forms; though, as will appear, the genus Galgulus presents a somewhat analogous case. It does not seem to have occurred to Dr. Gross (as it did not to me until I had carefully studied both forms) that Syromastes and Pyrrochoris might be of different type, but such is evidently the case. I shall endeavor to show that Pyrrochoris is of quite orthodox type, having an odd somatic number in the male and a typical unpaired idiochromosome. Since I am compelled to differ with Dr. Gross in regard to this species, I am glad to admit that the doubts I formerly expressed as to his account of the spermatogonial number in Syromastes, were unfounded. In regard to the female number, on the other hand, I believe he was misled by a wrong theoretic expectation (as he evidently was in case of the male Pyrrochoris), though it is possible that his determination of the apparent number was also correct, as indicated beyond.

\section{SYROMASTES MARGINATUS L.}

Gross's account of this form was as follows: The somatic groups in both sexes are stated to show twenty-two chromosomes. The "accessory" chromosome arises by the synapsis of two spermatogonial chromosomes, and is therefore a bivalent. It divides equationally in the first spermatocyte division but fails to divide in the second, passing bodily to one pole in advance of the other chromosomes without even entering the equatorial plate. All of the spermatid-nuclei thus receive ten chromosomes and half of them in addition the "accessory." These are the essential conclusions; but they are complicated by the following singular view of the relations between the "accessory" and the microchromosomes or " $m$-chromosomes." The chromosome nucleolus of the growth period is supposed not to give rise (as it does in Pyrrochoris and other forms) to the heterotropic or "accessory" chromosome of the spermatocyte divisions, but to the $m$-chromosome bivalent-the same view as the earlier one of Paulmier ('99) which has since been shown to be erroneous (Wilson '05c). But, on the other hand it is believed to arise, not from the 
$m$-chromosomes of the spermatogonia, but from two larger chromosomes, while the spermatogonial $m$-chromosomes are supposed to be converted into the "accessory" (!). I will not enter upon the very ingenious, if somewhat fantastic, conclusions that are based on these results, for, as I shall attempt to show, the results themselves cannot be sustained in some important particulars. But apart from this I am glad to be able to give the most positive confirmation of Gross's interesting discovery in regard to the numerical relations in the male. Syromastes is indeed a case in which the spermatogonial number is an even one (twenty-two), while there is a heterotropic chromosome in the second division. Half the spermatozoa seem to receive ten chromosomes and half eleven, as in so many otherspecies of Coreidx. But as Gross also correctly described, the heterotropic chromosome is here a bivalent which represents two chromosomes united together. The true numbers characteristic of the two classes of spermatozoa are therefore ten and twelve, respectively. For the sake of clearness I will here point out that this becomes at once intelligible under the assumption that the female number is not twenty-two, as Gross believed, but twenty-four; and such I believe will be found to be the fact.

That Gross was mistaken-doubtless misled by the earlier conclusion of Paulmier ('99), in which he was at first followed by Montgomery ('or) - in supposing that the chromosome nucleolus of the growth period divides to form the $m$-chromosomes, is I think thoroughly demonstrated by my preparations. In the case of Anasa and Alydus I showed ('O5c) that the $m$-chromosomes are not formed in the way Paulmier believed, but arise from two small separate rod-like chromosomes that are in a diffused condition during the growth period and only condense to form compact bodies at the same time that the condensation of the larger chromosomes takes place. I have since found this to be true of many other species. It is confirmed in the case of Anasa by the smear preparations of Foot and Strobell ('o7), and I have also since fully established the same conclusion by this method, by means of which every chromosome in the nucleus may be demonstrated. ${ }^{5}$

8 This is opposed to the conclusion of Montgomery ('o6). 
Although I have no smear preparations of Syromastes it is perfectly clear from the sections that the facts are the same here as in Anasa Alydus, and other forms. In the early prophases of the first division (at a period corresponding to Gross's Figs. 3 I to 37) when the plasmosome has disappeared or is greatly reduced in size, the nuclei contain both the chromosome-nucleolus and the $m$-chromosomes. This is shown in great numbers of cells with unmistakable clearness and after various methods of staining, particularly after safranin alone or combined with lichtgrün. In the early part of this period the chromosome nucleolus is at once recognizable by its intense color and sharp contour and is not for a moment to be confused with a plasmosome. The ordinary bivalents are still in the form of ragged pale bodies, having the form of longitudinally split rods or double crosses. The $m$-chromosomes have the same texture and staining reaction, but are much smaller and never show the cross form. While it is difficult to show the facts to demonstration in photographs of sections they may be fairly well seen in the following. Photo I 8 shows the chromosome nucleolus (not quite in focus,) one of the large bivalents (two others barely appear) and both $m$-chromosomes. Photo 19 is a similar view (the $m$-chromosomes more condensed), while Photo 20 shows the $m$-chromosomes and three of the ordinary bivalents. The succeeding changes must be rapidly passed through, since the successive steps are often seen in the same cyst, passing from one side to the other. In these stages the large bivalents rapidly condense and regain their staining capacity, finally assuming a bipartite or quadripartite form. The $m$-chromosomes undergo a similar condensation, being finally reduced to ovoidal or spheroidal bodies. The chromosome nucleolus, on the other hand, becomes somewhat looser in texture and assumes an asymmetrical quadripartite shape, in which form it enters the equatorial plate to form the eccentric "accessory" chromosome. The period at which the $m$-chromosomes condense varies considerably, and the same is true of their relative position; sometimes they are in contact, sometimes more or less widely separated, even lying on opposite sides of the nucleus. Photo 2I shows two nuclei, one above the other, in each of which appear both $m$-chromosomes, 
the chromosome nucleolus and a number of the other bivalents. Photo 22 shows the same condition. Photo 23, from the same cyst, is slightly later, showing the two spheroidal $m$-chromosomes wide apart, the chromosome nucleolus, and several of the other chromosomes. (The chromosomes nucleolus, perfectly recognizable in the preparation, is in the photograph hardly distinguishable from the other bivalents seen endwise.) Up to this point, which shortly precedes the dissolution of the nuclear membrane, the chromosome nucleolus is still immediately recognizable by its deeper color (especially after safranin). There follows a brief period in which this distinction disappears, but the chromosome nucleolus is still recognizable by its asymmetrical form. That it gives rise to the eccentric "accessory" is, I think, beyond doubt. The evidence is demonstrative that it does not divide to form the $m$-chromosomes, and that the latter arise from separate rods as described. Gross appears to have seen these rods at a much earlier period (cf. his Fig. Io) and correctly identifies them with the spermatogonial $m$-chromosomes; but he believed them to give rise to the "accessory."

The relation of the chromosome nucleolus to the spermatogonial chromosomes cannot be determined in Syromastes with the same degree of certainty as in Pyrrochoris (as described beyond), but the size relations leave hardly a doubt that Gross was right in asserting its origin from two of the larger of these chromosomes. The study of these relations is of importance because I believe they justify the conclusion that the chromosome nucleolus, and hence the "accessory," is nothing other than a pair of slightly unequal idiochromosomes, which can readily be recognized in the spermatogonial groups.

Study of the spermatogonial groups in detail shows that twenty of the chromosomes may be equally paired, while the remaining two are slightly but distinctly unequal in size. These can always be recognized as the smallest of the chromosomes except the $m$-chromosome. Photos $\mathbf{I}$ and 2 show two groups in which this clearly appears. These photographs are reproduced in Text Figs. $\mathbf{I} a, \mathrm{I} b$, with two others, $c$ and $d$, the chromosomes in question being designated as I and $i$. 
It is evidently this pair that give rise to the bivalent "accessory" (eccentric) chromosome of the first division and hence to the chromosome nucleolus of the growth period. Gross correctly describes this bivalent as a quadripartite body or tetrad, but overlooked the fact that it is composed of two slightly unequal halves, and these correspond in relative size to the unequal pair in the spermatogonia. This appears unmistakably in a great number of polar views of the first division metaphase (though it is not always apparent) and is clearly shown in Photos 3,4 and 5. It is evident that the bivalent is so placed in the equatorial
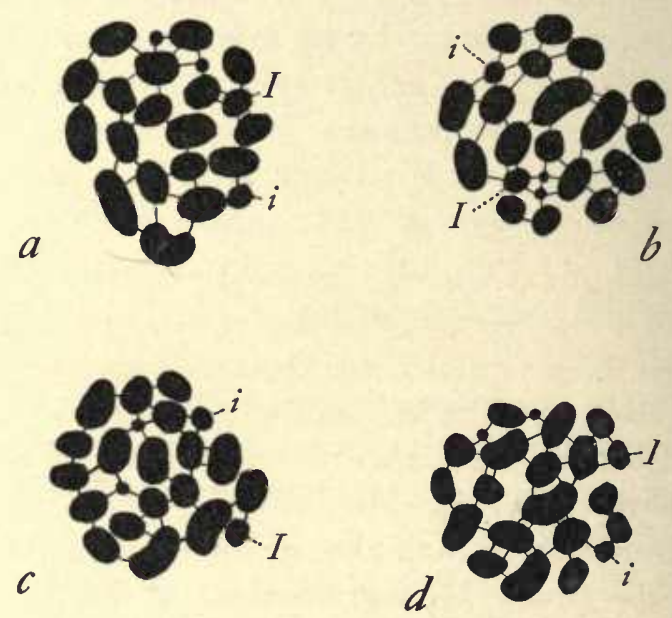

Fig. I. Four spermatogonial chromosome-groups of Syromastes marginatus; $a$ and $b$ are reproductions of Photos $I$ and 2.*

* The drawings are not made from the microscope with the camera lucida but directly upon enlarged photographs of the objects. Since I believe this method to be superior in accuracy for the representation of such small objects I will briefly describe it in the hope that others may find it useful. The original negatives are taken directly from the sections at an enlargement of 1500 diameters ( $2 \mathrm{~mm}$. oil immersion, compensation ocular 6). From these negatives enlarged bromide prints are made (with a photographic camera) three times the size of the original negatives (i. e., 4500 diameters) upon double weight paper, which gives a good surface for pen drawings. The drawing is then made directly on the print with waterproof ink, and when thoroughly dry the remains of the photograph are bleached out in a mixture of sodium hyposulphite and potassium ferricyanide. The enlarged prints of course show the chromosomes with more or less blurred outlines (though they are clearer than might be supposed); but by working with an ordinary print and the object before one for comparison the drawings may nevertheless be made with great accuracy. They may be tested and if necessary corrected, by the use of a reducing glass. 
plate as to undergo an equation division, like the idiochromosomes of other Hemiptera heteroptera. In uniting to form a bivalent before the first division these chromosomes differ from those of most other Hemiptera, but in all other respects up to the end of the first division they correspond exactly with them. But even this difference is bridged by a condition occasionally seen in other forms, for instance in Lygæus and Metapodius. ${ }^{B}$ In the last named form the typical and usual condition is that the idiochromosomes are in the first division quite separate, lying eccentrically outside the principal ring of chromosomes like the unpaired idiochromosomes of other coreids (Photos 6 and 7), and in this position they separately divide. Exceptionally, however, they lie in close contact (Photos 8 and 9), forming an asymmetrical bivalent precisely like that of Syromastes. In both cases this bivalent divides equationally, giving two asymmetrical daughter-dyads, thus $\frac{I i}{I i}$.

The exactness of the correspondence up to this point seems to leave no doubt of the homology of this pair of chromosomes in the two forms. In the second division, however, the two species show a remarkable contrast. In Metapodius, as in Lygæus or Euschistus, the two idiochromosomes are always united to form an unsymmetrical bivalent which enters the equatorial plate and is separated into its two components, half the spermatids receiving the large one and half the small. In Syromastes, on the other hand, the idiochromosomes remain united and do not enter the equatorial plate at all, but pass directly to one pole where they are included in the daughter-nucleus, as Gross has described (Photos II to 17). Owing to this behavior of the idiochromosome bivalent, polar views of the second division always show but ten chromosomes instead of eleven (Photo IO). In this case therefore half the spermatid nuclei receive two more chromosomes than the others, the two classes having respectively ten and twelve chromosomes. As the idiochromosome bivalent passes to the pole its two components are usually closely united, and often cannot be

\footnotetext{
- The latter remarkable genus, which presents the phenomenon of the "supernumerary chromosomes" (Wilson 'orc), will form the subject of a forthcoming fifth "Study."
} 
distinguished; but in some cases they may still be seen, as in Photo 12. As the nuclear vacuole forms the ordinary chromosomes rapidly diminish in staining capacity, while the idiochromosome bivalent retains its compact form and dark color, like a nucleolus, and thus comes conspicuously into view, particularly after safranin. Its double nature is at this time often more clearly apparent than in the preceding stages. It disappears from view some time after the reconstruction, at a much earlier period than in Pyrrochoris.

Only exceptionally in my preparations do the chromosomes of the second division show a quadripartite form as Gross figures them. Their usual form is dumb-bell shaped or dyad-like; though as the two halves separate they are often connected by double fibers, as is the case with many other species of Hemiptera.

\section{PYRROCHORIS APTERUS L.}

As already stated, Pyrrochoris is of different type from Syromastes and agrees precisely with other forms having an unpaired idiochromosome, such as Anasa or Protenor. Aside from the interest that this species possess as the one in which Henking first discovered the idiochromosome, it is in other respects a peculiarly interesting form for the study of the general spermatogenesis, particularly in respect to the presynaptic and synaptic periods. I shall here, however, confine myself mainly to the numerical relations and the history of the idiochromosome. Henking himself somewhat doubtfully concluded that the spermatogonial number was twenty-four: "Ich habe in drei Fällen die Zahl 24 erhalten, in einem Falle die Zahl 23. Da die Bilder überall die gleichen sind, so habe ich das Zahlgeschäft nicht an einer grösseren Zahl vorgenommen und glaube die theoretisch zu erwartende Zahl 24 als das Normal ansehen dürfen" (op. cit., p. 688, italics mine). It is clear enough from this that Henking, too, was misled by a false theoretic expectation; and a study of his figures (op. cit., Figs. 6, $a, b . c, 7$ ) will show that they are very far from decisive. In the case of the female, Henking speaks much more positively ('92) and there is hardly a doubt that his count of twenty-four chromosomes was correct, since he found this number "unverkenn- 
bar" in the dividing oögonia, and in the connective tissue cells of the ovary, and also figured (Fig. 39) a double group (exactly such as I described in Anasa, Wilson '06, Fig. 2, $k$ ), showing forty-eight chromosomes.

Gross accepts Henking's account without question, treating the numerical relations in rather summary fashion as follows: "Die Aequatorialplatte der sich teilenden Spermatogonien enthält 24 Chromosomen. Dieselbe Zahl hat Henking ausser in den Spermatogonien auch in den Oogonien gefunden. Ebenso konnte ich in den Follikelzellen der Eiröhren, also in somatischen Zellen, konstatieren. 24 ist also die Normalzahl der Species" ('o7, p. 277). In support of this are given two polar views of spermatogonial metaphases (the female groups are not figured) each showing eight small and sixteen large chromosomes (Figs. 9 and 10). His account continues as follows: The idiochromosome appears aready in the syanaptic period (synizesis) as a double nucleolus-like body, assumed to be a bivalent body that arises by the synapsis of two of the spermatogonial chromosomes, though none of the earlier stages were followed out. At a later period it appears as a single spheroidal body owing to the close apposition of its two halves. This chromosome divides in the first spermatocyte division, but in the second lags behind the others and passes undivided to one pole, as Henking described. All of the spermatid-nuclei thus receive eleven chromosomes, while half of them receive in addition the idiochromosome. Since both sexes were supposed to contain twenty-four chromosomes, Gross drew the same conclusion as the one previously reached in the case of Syromastes, namely, that only the twelve-chromosome spermatozoa are functional.

In regard to the spermatocyte divisions my own results are perfectly in accord with Henking's and Gross's. As to the spermatogonial number, I must say that after having immediately confirmed Gross's account of Syromastes-(which I examined first) I was fully prepared to find a similar relation in Pyrrochoris. It was therefore with, astonishment that I found everywhere twentythree instead of twenty-four spermatogonial chromosomes. This number appears with diagrammatic clearness in a great number of spermatogonia from different individuals (testes from 35 different 
individuals have been sectioned) and is shown both in camera drawings and in photographs. Eight of the latter are shown (Photos 24 to $3 \mathrm{r}$ ), and these same groups are also represented in the drawings, Text Figs. 2, $a, b, c, d, e, j, k, l$, together with four others $(f, g, h, i)$, also from photographs. Inspection of these photographs and drawings will show that the unpaired idiochromosome is at once recognizable by its large size, which renders
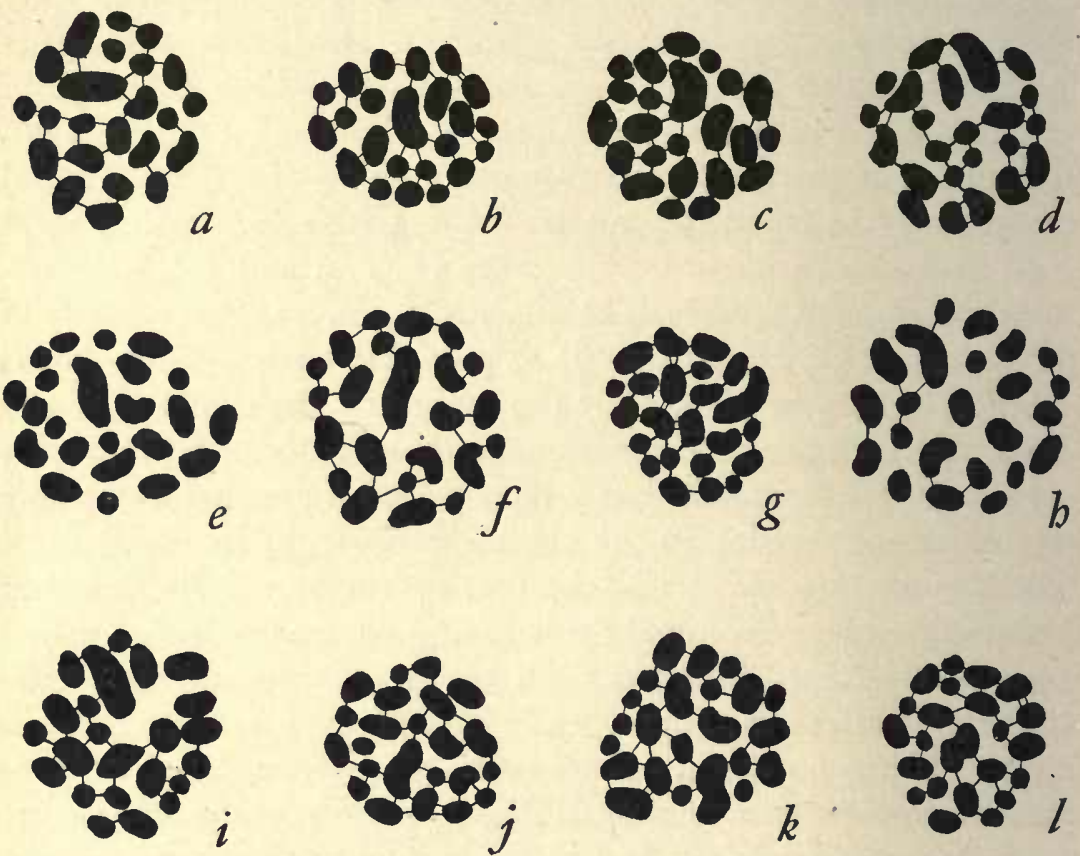

Fig. I. Spermatogonial groups of Pyrrochoris apterus (drawn on photographic enlargements, as explained under Fig. 1); $a, b, c, d, e, j, k$ and $l$ are reproductions of Photos 24, 25, 26, 27, 28, 29, 30 and $3 \mathrm{x}$, respectively.

it almost as conspicuous as in Protenor (heretofore described by Montgomery and myself). I find the size relations not quite the same as Gross describes them. There are, as he states, eight chromosomes that are considerably smaller than the others; but two of the others are but slightly larger. The remaining twelve paired chromosomes are much larger, though the contrast is in my material not so great as Gross figures it. The idiochromo- 
some is nearly twice as large as any of the others, and is obviously unpaired. I have examined a large number of spermatogonial groups with great care with a view to the possibility that this chromosome might in reality be double, but am thoroughly convinced that such is not the case. This is unmistakably evident when this chromosome has the form of a straight or only slightly curved rod (Photos 24 to 28, Text Fig. 2, $a$ to $i$ ), and these constitute the great majority of observed cases. I have, however, found a few cases where it has a very marked sigmoid curvature; two or three of these give at first sight the appearance of two chromosomes in contact (Photos 29, 30, 3 I ; Text Fig. 2, j, $k, l$ ). Even here close study shows that it is a single body; but such forms might readily mislead an observer having a preconceived idea of the number to be expected.

That this is a single chromosome that is identical with the idiochromosome of the growth period and the maturation divisions is placed beyond doubt by a study of the presynaptic stages, which were not examined by either Henking or Gross. This period is of such interest in Pyrrochoris as to merit a special study. With only a single exception I know of no other form in which the history of the idiochromosome and the succession of the stages can be so completely and readily followed at this time. Throughout this whole period, beginning with the telophases of the last spermatogonial division, the idiochromosome can be traced step by step as a single body, and it is evidently identical with the large unpaired spermatogonial chromosome.

In the stages that immediately follow the last spermatogonial telophase (Photos 32 and 33) the chromosomes still retain their boundaries, though they show a looser texture, vaguer outlines and diminished staining capacity (by which characters the postphases are readily distinguishable from the prophases). The large chromosome (idiochromosome) is clearly distinguishable at this time, both by its size and by its deeper color. In the stages that immediately follow a remarkable contrast appears between this chromosome and the others. The latter rapidly lose their visible boundaries and their staining capacity, breaking up into a fine netlike structure in which traces of a spireme-like arrangement may 
sometimes be seen. The idiochromosome, on the other hand, retains its identity and deep color and now appears as a conspicuous elongated body ("caterpillar stage"). Though its outlines are still somewhat ragged and its color less intense than in the succeeding stages, it already appears in sharp contrast to the pale reticulum (Photos 34 and 35). It sometimes extends straight across the whole diameter of the nucleus; but beside such forms, in the same cysts, are often curved and shorter forms. At this time it is usually surrounded by a distinct clear space or vacuole, as I hope the photographs may show; and there are also in the nucleus from one to three much smaller nucleolus-like bodies which (on account of the staining reactions) I believe to be plasmosomes, but these soon disappear. Splendid pictures of these and the following stages are given by the safranin-lichtgrün combination, which shows the idiochromosome at every stage bright red, while in properly differentiated preparations the reticulum is pure green. ${ }^{7}$ The idiochromosome now takes up a peripheral position and the clear space surrounding it disappears. It acquires a more definite contour, stains still more intensely, and rapidly shortens until it is converted into a condensed ovoidal or spheroidal chromosome nucleolus that may be traced without a break through every stage up to the prophases of the first spermatocyte division. As it shortens it may undergo a variety of form changes. In what I regard as the typical process it shows no indication of duality at any period up to the full contraction phase (synizesis) being progressively reduced to a short rod and finally to an ovoidal or spheroidal body (Photos 36 to 42 ). In the meantime the nuclear reticulum contracts more and more, usually towards one side of the nucleus, becomes coarser in texture, and increases in staining capacity, until at the climax of the process

\footnotetext{
7 The effect of this stain depends in some measure, of course, on the relative degree of extraction of the two dyes. My method is to stain in safranin for two to four hours and then to place the slide at once in strong alcoholic solution of lichtgrün for ten to twenty seconds. This is at once followed by rapid washing in 95 per cent and absolute alcohols. The alcohol is then replaced by clove oil and the latter by zylol. In all cases the chromosomes of dividing cells and the chromosome nucleolus of all stages appear brilliant red, the achromatic fibers and general cytoplasm pure green. The relative intensity of red and green depend on the length of immersion in the green solution. The description here given applies to sections rather strongly stained in the green.
} 
it forms a close knot, or rounded mass, staining almost black in hæmatoxylin, at one side of which is the idiochromosome (now a condensed chromosome nucleolus). These structures lie in a large clear nuclear vacuole, as shown in Photos 39 to 42 . The stage thus attained is the characteristic contraction phase or synizesis, which in this species is extremely marked. ${ }^{8}$

In the safranin-lichtgrün preparation at this period the chromosome nucleolus is, as always, intensely red. The synaptic knot varies with the relative intensities of the red and green, being in some preparations distinctly red, in others pure green, in still others of mixed appearance. In the succeeding stage the chromatin emerges from the synaptic knot in the form of separate spireme threads which lose their staining capacity for hæmatoxylin and in the double stain are again pure green (Photos 43 and 44). In the middle and late growth period they are still more or less green but contain red granules. In the prophases of the first division they at last lose their affinity for the green and finally appear pure red; but this does not occur until just before the dissolution of the nuclear membrane. Since the idiochromosome always retains its intense red color it may thus be followed from stage to stage with great certainty.

The study of the whole cycle of changes from the last spermatogonial division onward gives certain very definite results in regard to synapsis in general, and especially in regard to the idio-

8 Many recent writers have expressed the opinion that the synizesis stage is an artifact produced as a shrinkage product, though Miss Sargant ('96) stated very explicitly that she had seen it in the living cells, and this has recently been confirmed by Overton ('05). I can fully substantiate this in the case of Anasa tristis. The perfectly fresh testis, gently teased apart in a Ringer's fluid in which the spermatozoa continue their active movements, very clearly shows nearly all the features of the spermatogenesis, including the number, shape and size relations of the chromosomes, their characteristic grouping and behavior in the spermatocyte divisions, the double rods, crosses and other prophase figures, the spindle fibers and asters, and even, I believe, the centrosomes. In this fresh material the synizesis stage appears in essentially the same form as in the sections, the nuclear knot lying in a large clear vacuole. These nuclei only appear in the same region of the testis as in sections, and they show a conspicuous contrast to those of earlier and later stages that lie near by them. In the post synaptic stages the chromosomes, in the form of spireme threads can be seen again spreading through the nuclear cavity. These observations leave no doubt in my mind that the synizesis is a normal phase of the spermatogenesis in these animals, though it is not improbable that the contraction may be somewhat exaggerated by the reagents. It is evident, however, from such studies as those of the Schreiners ('06) and others that the synizesis does not occur in some forms. 
chromosome. Concerning the first point I will here only indicate one principal conclusion. It is quite clear that in Pyrrochoris (and I think the same holds true in other Hemiptera) synapsis, or the conjugation of chromosomes two by two, does not occur in the closing anaphases of the last spermatogonial division as was described by Montgomery ('o) in Peripatus and Euschistus ("Pentatoma"), by Sutton ('O2) in Brachystola, by Stevens ('03) in Sagitta, and by Dublin ('o5) in Pedicellina. Although the number of chromosomes in the postphases immediately following this division (Photos 32 and 33 ) cannot be exactly made out, it is perfectly evident that it is not the reduced number but approximates to the somatic number (twenty-three). The chromosomes, therefore, have not paired two by two in the spermatogonial anaphases. It is equally certain that this stage does not pass directly into the synizesis but is separated from it by a long "resting period" (Photos 34 to 38 ) - as is demonstated by the topographical relations as well as by the progressive stages of the idiochromosome-in which the ordinary chromosomes lose their sharp boundaries and their affinity for nuclear stains. In this respect Pyrrochoris shows a close similarity to Tomopteris, as described by the Schreiners ('o6), whose original preparations, by the kindness of Dr. Schreiner, I have had opportunity to examine. This comparison has convinced me that synapsis occurs at the same period in both - whether by parasynapsis (side to side union) or telosynapsis (end to end union ${ }^{9}$ ) or in some other way I am not prepared to say. There can be no manner of doubt that the first division of the bivalents is a transverse one, as described by Paulmier and Montgomery; but it has been rendered evident enough by recent studies on reduction that this in itself gives no trustworthy evidence regarding the mode of synapsis. The direct investigation of the process in the Hemiptera presents great difficulties.

The foregoing general conclusion regarding the time of synapsis is of importance for the more specific one in regard to the idiochromosome. During the entire earlier presynaptic period the

-I have for some years made use of these terms in my lectures on cytology. 
elongated idiochromosome is manifestly a single body. As it shortens and condenses to form the chromosome nucleolus, it shows a considerable variety of forms; and the rate of condensation also varies, cells that are already enteringthe synizesis stage being sometimes seen in which the idiochromosome is still distinctly a rod (Photos 35 and 36 ). In most cases it is at this time a single ovoidal or spheroidal body; but not infrequently it appears more or less distinctly double (Photos 37 to 39). This condition is however not produced by a previous synapsis of two chromosomes, as Gross believed, but arises, I think, from a tendency of the chromatin to accumulate towards the ends of the rod; and when this is very marked it may assume an appearance of duality, even in the earlier stages (Photo 37, below), though this is relatively rare. In the later stages a double appearance is not infrequent, dumb-bell forms being thus produced, which sometimes give in the synizesis stage apparently double bodies. The earlier stages conclusively show that this is a secondary appearance. In the later (postsynaptic) stages (Photos 34 and 35), and throughout the growth period, it always appears as a single spheroidal body. In view of these facts I think the conclusion inevitable that the chromosome nucleolus is a univalent chromosome that arises by the condensation of the unpaired large chromosome of the spermatogonia.

I have little to add to Henking's and Gross's acounts of the maturation divisions. As will be seen from Photos 45, 46, 50, $5^{\mathrm{I}}$, the size relations are correlated with those of the spermatogonial chromosomes. In polar views of the first division appear with great constancy four smallest chromosomes, one slightly larger one, and seven still larger ones, or twelve in all. The idiochromosome is one of the largest, but cannot be distinguished from the others (as is also the case in Protenor). This is obviously due to the fact that the idiochromosome is still a univalent or single chromosome, while each of the others represents two of the spermatogonial chromosomes united. Since all have nearly the. same dumb-bell shape as seen in side view, the idiochromosome appears from the pole approximately but half as large, relative to the others, as in the spermatogonia. The same size-relations, appear in the second division, but all the chromosomes are much smaller, as the photographs clearly show. 
I have not succeeded with Pyrrochoris (as I have with several other genera) in obtaining photographs of both anaphase daughter groups showing all the chromosomes; but it is perfectly evident that all divide equally in the first division, and all but the idiochromosome in the second. This chromosome lags behind the others and then passes undivided to one pole where it is included in the daughter nucleus (Photos 47 to 49 ) as Henking described. This pole thus receives twelve chromosomes, the other but eleven. As in a number of other species the idiochromosome retains its compact form and deep-staining capacity long after the reconstruction of the nuclei and the breaking up of the other chromosomes. It may thus be distinguished (especially well in safranin preparations) up to a rather late period stage of the spermatids, even after the tails have grown out. It finally disappears from view, and the mature spermatozoa show no visible indication of their dimorphism.

\section{GENERAL}

If my conclusions are correct, Pyrrochoris agrees exactly with other forms in which an unpaired idiochromosome is present. Syromastes however presents a new type in which the "accessory" chromosome is not univalent but bivalent, and in which accordingly half the spermatozoa receive two more chromosomes than the other half. If we may apply the same rule to Syromastes as that which holds for other Hemiptera we may expect the spermatozoa that receive the "accessory" to be female-producing, the others male-producing. The fertilization formulas for the two species considered in this paper should therefore be as follows:

PYRROCHOR1S

Egg $12+$ spermatozoön $11=$ zyote $23\left(\sigma^{7}\right)$

Egg $12+$ spermatozoön $12=$ zygote 24 (\%)

SYROMASTES

Egg 12 (including $I$ and $i$ )* spermatozoön $10=$ zygote 22 (including $I$ and $i)\left(D^{7}\right)$

Egg 12 (including $I$ and $i$ ) + spermatozoön 12 (including $I$ and $i$ ) = zygote 24 (including $I, I, i, i),($ ㅇ)

* The formation of a reduced female group of this composition may readily be explained if it be supposed that in synapsis the two small idiochromosomes couple with each other to form the bivalent $\dddot{i i}$, the two large ones to form the bivalent $I I$. 
The correctness of my deduction may readily be tested by a reëxamination of the female groups. Gross, it is true, states that he has found but twenty-two chromosomes in the female (follicle cells); but I think no one is likely to consider as in any way conclusive the single figure that he gives in support of this (op. cit., Fig. II I). Not less than five of the twenty-two chromosomes figured are deeply constricted; and any one of these might in reality be two chromosomes in contact. I hope that Dr. Gross himself may be willing to reëxamine this point, in view of the possibility here suggested. It is however also possible that the two members of each of the idiochromosome pairs in the female may be united to form a bivalent, in which case the female would apparently show but twenty-two chromosomes; but even if this be so the two members must separate again when transferred to the male.

In regard to Pyrrochoris, there is little doubt that the determination of the female number by Henking and Gross as twenty-four was correct; and since the idiochromosome in the male is the largest of the chromosomes we may expect the female groups to show two such chromosomes. ${ }^{10}$

I should state the expectation less confidently in the case of Syromastes if it stood entirely alone; but another case has now been made known in which the male and female groups differ by more than one chromosome. This occurs in the genus Galgulus, which has been worked out in my laboratory by Mr. F. Payne (whose results are now in press) ${ }^{11}$ on material collected by myself. The following facts are very clearly shown in this form. The spermatogonial number is thirty-five, the female number thirty-eight. In the second division five of the chromosomes are always asso-

\footnotetext{
${ }^{10}$ Henking's figures ('92) give considerable evidence that such is really the case. His Fig. 83 of the first polar metaphase shows one of the twelve bivalents fully twice the size of the others; and the same is true of Fig. 68, which shows a side view of the second polar spindle, though not all the chromosomes are shown. With this accords his Fig. 39 of a double group from a connective tissue cell of the female showing forty-eight chromosomes, of which four, of nearly equal size, are nearly twice the size of the others. This agrees precisely with the relation shown in a double group of Anasa figured by me in a former paper ('o6, Fig. 2, $k$ ) which shows twice the normal number of both the largest and the smallest chromosomes.
}

${ }^{11}$ Since published in Biol. Bull., riv, 5 . 
ciated to form a definite pentad element of which four pass to one pole, one to the other, while the remaining fifteen chromosomes divide equally. Half the spermatozoa thus receive sixteen chromosomes and half nineteen. From these facts it is clear that the sixteen-chromosome class must be male producing, the nineteenchromosome class female producing, according to the formula:

GALGULUS

Egg $\frac{n}{2}+$ spermatozoön $\frac{n}{2}-3=$ zygote $n-3\left(0^{7}\right)$
Egg $\frac{n}{2}+\operatorname{spermatozoön} \frac{n}{2} \quad=$ zygote $n(\%)$

This case, together with that of Syromastes (if my inference regarding this form be correct) shows that we must considerably enlarge our previous conceptions as to the relations between sex production and the chromosomes; for we can no longer hold that only a single pair are involved. In Syromastes there are two such pairs, in Galgulus several pairs.

A COMPARATIVE REVIEW OF THE TYPES OF SEXUAL DIFFERENCES OF THE CHROMOSOMES

It is evident that a greater variety of types exists in regard to the sex differences than was indicated in the brief general review given in the third of my "Studies" (Wilson '06.) In that paper I distinguished three types, examples of which are given by Protenor, Lygæus and Nezara; but the number must now be increased to at least five, and possibly to seven, of which I will now give a brief synopsis. With the exception of Syromastes and Diabrotica this synopsis includes only species of which both sexes have been accurately determined. Forms like the aphids, in which idiochromosomes have not yet been positively identified, have been omitted. Seventeen of the species are here reported for the first time (one or both sexes) from my own results hitherto unpublished. I am indebted to Dr. Stevens for permission to include her results on the Diptera and on Diabrotica, which are now in press ('o8a, 'o8b). 


\section{I}

Both sexes with the same number of chromosomes; a pair of equal idiochrosomes present in both. No visible difference between the two classes of spermatozoa or between the male and female somatic groups.

\section{FERTILIZATION FORMULA}

Egg ${ }_{2}^{n}+$ spermatozoon ${ }_{2}^{n}=$ zygote $n\left(\sigma^{\top}\right.$ or $\left.\uparrow\right)$

Described Case

\begin{tabular}{|c|c|c|c|c|c|}
\hline Specie & Order & Family & 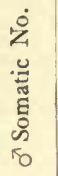 & 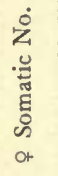 & Authority \\
\hline Nezara hilaris Say & Hemiptera heteroptera & Pentatomidæ & 14 & 14 & Wilson ('o6) \\
\hline
\end{tabular}

To this type belongs also Oncopeltus fasciatus Dall, one of the Lygæidx. It is further probable that here belong many forms in which no visible sexual differences are to be seen, and in which idiochromosomes have not been identified. If a particular pair of chromosomes, corresponding to idiochromosomes, are of general occurrence, though not visibly distinguishable from the others, it is probable that this type represents the most frequent condition in animals generally.

II

Both sexes, and both classes of spermatozoa, with the same number of chromosomes. The male with a pair of unequal idiochromosomes, half the spermatozoa receiving the large one and half the small. In the female a pair of equal idiochromosomes like the large one of the male.

\section{FERTILIZATION FORMULA}

Egg $\frac{n}{2}$ (including $I$ ) + spermatozoön $\frac{n}{2}$ (including $\left.i\right)=$ zygote $n$ (including $\left.I i\right) 0^{*}$ Egg $\frac{n}{2}$ (including $\left.I\right)+\operatorname{spermatozoön} \frac{n}{2}$ (including $\left.I\right)=\operatorname{zygote} n($ including $I I)$ ๆ 
Described Cases

\begin{tabular}{|c|c|c|c|c|c|}
\hline Species & Order & Family & 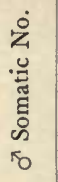 & 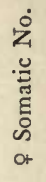 & Authority \\
\hline Oebalus pugnax $\mathrm{Fab}$. & Hemiptera heteroptera & Pentatomidæ & 10 & 10 & Wilson \\
\hline fissilis Uhl. & Hemiptera heteroptera & Pentatomidæ & 14 & 14 & Wilson '05b, 'o5c, '06 \\
\hline ictericus L. & Hemiptera heteroptera & Pentatomidæ & 14 & 14 & Wilson'o6 \\
\hline servus Say & Hemiptera heteroptera & Pentatomidx & 14 & 14 & Wilson \\
\hline tristigmus Say & Hemiptera heteroptera & Pentatomidæ & 14 & 14 & $\left\{\begin{array}{l}\delta^{\top} \text { Montgomery 'or } \\
\text { o Wilson 'o6 }\end{array}\right.$ \\
\hline variolarius P. B. & Hemiptera heteroptera & Pentatomidæ & 14 & 14 & Wilson'o6 \\
\hline Cœnus delius Say & Hemiptera heteroptera & Pentatomidæ & 14 & 14 & Wilson 'o5b, 'o5c, 'o6 \\
\hline Stiretrus anchorago $\mathrm{Fab}$ & Hemiptera heteroptera & Pentatomidæ & 14 & 14 & Wilson \\
\hline $\left.\begin{array}{l}\text { Podisus } \\
\quad \text { maculiventris Say } \\
\quad \text { (spinosus) }\end{array}\right\}$ & Hemiptera heteroptera & Pentatomidæ & 16 & 16 & $\left\{\begin{array}{l}\delta^{7} \text { Montgomery 'or } \\
\text { o Wilson 'o5b, o5c, 'o6 }\end{array}\right.$ \\
\hline $\begin{array}{l}\text { Banasa } \\
\text { dimidiata Say } \\
\text { calva Say }\end{array}$ & $\begin{array}{l}\text { Hemiptera heteroptera } \\
\text { Hemiptera heteroptera }\end{array}$ & $\begin{array}{l}\text { Pentatomidæ } \\
\text { Pentatomidæ }\end{array}$ & $\begin{array}{l}16 \\
26 *\end{array}$ & $\begin{array}{l}16 \\
26\end{array}$ & $\begin{array}{l}\text { Wilson'o7b } \\
\text { Wilson'o7b }\end{array}$ \\
\hline $\begin{array}{l}\text { Lygaus } \\
\text { turcicus Fab. } \\
\text { bicrucis Say }\end{array}$ & $\begin{array}{l}\text { Hemiptera heteroptera } \\
\text { Hemiptera heteroptera }\end{array}$ & $\begin{array}{l}\text { Pentatomidæ } \\
\text { Pentatomidæ }\end{array}$ & $\begin{array}{l}14 \\
14\end{array}$ & $\begin{array}{l}14 \\
14\end{array}$ & $\begin{array}{l}\text { Wilson' } 05 \text { b, 'o5c, 'o6 } \\
\text { Wilson }\end{array}$ \\
\hline Tenebrio molitor & Coleoptera & Tenebrionidæ & 20 & 20 & Stevens'05 \\
\hline Chelymorpha argus & Coleoptera & Chrysomelidx & 22 & 22 & Stevens 'o6 \\
\hline $\begin{array}{l}\text { Trirhabda virgata } \\
\text { canadense }\end{array}$ & $\begin{array}{l}\text { Coleoptera } \\
\text { Coleoptera }\end{array}$ & $\begin{array}{l}\text { Chrysomelidæx } \\
\text { Chrysomelidæ }\end{array}$ & $\begin{array}{l}28 \\
30\end{array}$ & $\begin{array}{l}28 \\
30\end{array}$ & $\begin{array}{l}\text { Stevens 'o6 } \\
\text { Stevens' } 06\end{array}$ \\
\hline Drosophila ampelophila & Diptera & & 8 & 8 & Stevens'o8a \\
\hline Musca domestica & Diptera & & 12 & 12 & Stevens'o8a \\
\hline Calliphora vomitoria & Diptera & & 12 & 12 & Stevens'08a \\
\hline Sarcophaga sarraciniæ & Diptera & & 12 & 12 & Stevens'o8a \\
\hline Scatophaga pallida & Diptera & & 12 & 12 & Stevens'o8a \\
\hline Tetanocera sparsa & Diptera & & 12 & 12 & Stevens'o8 a \\
\hline Eristalis tenax & Diptera & & 12 & 12 & Stevens'o8a \\
\hline
\end{tabular}

* See Type IIa.

\section{III}

The female chromosome groups with one more chromosome than the male. Male with an unpaired idiochromosome and an odd spermatogonial number, half the spermatozoa receiving the idiochromosome and half being without it. Female with an equal pair of idiochromosomes like the unpaired one of the male.

FERTILIZATION FORMULA

Egg $\frac{n}{2}$ (including $\left.I\right)+$ spermatozoön $\frac{n}{2}-1=$ zygote $n-1$ (including $\left.I\right) \sigma^{n}$

Egg $\frac{n}{2}($ including $I)+\operatorname{spermatozoön} \frac{n}{2}$ (including $I$ ) $=$ zygote $n$ (including $\left.I I\right)$ ? 
Described Cases

\begin{tabular}{|c|c|c|c|c|c|}
\hline Species & Order & Family & 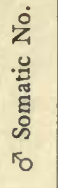 & 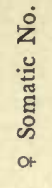 & Authority \\
\hline Largus cinctus H. S. & Hemiptera heteroptera & Pyrrochoridæ & II & 12 & Wilson \\
\hline succinctus $\mathrm{L}$. & Hemiptera heteroptera & Pyrrochoridæ & 13 & 14 & Wilson \\
\hline Pyrrochoris apterus L. & Hemiptera heteroptera & Pyrrochoridæ & 23 & 24 & $\left\{\begin{array}{l}\text { o Henking' } 9 \mathbf{r} \\
\sigma^{\top} \text { Wilson }\end{array}\right.$ \\
\hline Alydus pilosulus H.S. & Hemiptera heteroptera & Coreidx & 13 & 14 & Wilson 'o5b, '05c, 'o6 \\
\hline $\left.\begin{array}{l}\text { Harmostes } \\
\text { reflexulus Std. }\end{array}\right\}$ & Hemiptera heteroptera & Coreidæ & 13 & 14 & $\left\{\begin{array}{l}\sigma^{7} \text { Montgomery'or } \\
\text { of Wilson'o6 }\end{array}\right.$ \\
\hline Protenor belfragei Hag. & Hemiptera heteroptera & Coreidæ & 13 & 14 & $\left\{\begin{array}{l}\sigma^{\top} \text { Montgomery 'or } \\
\text { o Wilson 'o5b, 'o5c, 'o6 }\end{array}\right.$ \\
\hline $\begin{array}{l}\text { Leptocoris trivittatus } \\
\text { Say }\end{array}$ & Hemiptera heteroptera & Coreid $\boldsymbol{x}$ & 13 & 14 & Wilson \\
\hline Archimerus & & & & & \\
\hline calcarator Fab. & Hemiptera beteroptera & Coreidæ & 15 & 16 & Wilson \\
\hline Pachylis gigas Burm. & Hemiptera heteroptera & Coreidæ & 15 & 16 & Wilson \\
\hline $\begin{array}{l}\text { Anasa tristis DeG. } \\
\text { armigera Say }\end{array}$ & $\begin{array}{l}\text { Hemiptera heteroptera } \\
\text { Hemiptera heteroptera }\end{array}$ & $\begin{array}{l}\text { Coreidæ } \\
\text { Coreidæ }\end{array}$ & $21 *$ & 22 & $\begin{array}{l}\text { Wilson 'o5b, 'o5c, 'o6, 'o7a } \\
\left\{\sigma^{\top} \text { Montgomery 'o6 }\right.\end{array}$ \\
\hline armigera Say & $\begin{array}{l}\text { Hemiptera heteroptera } \\
\text { Hemiptera beteroptera }\end{array}$ & $\begin{array}{l}\text { Coreidæ } \\
\text { Coreidæ }\end{array}$ & 21 & $\begin{array}{l}22 \\
22\end{array}$ & $\begin{array}{l}\text { of Wilson } \\
\text { Montgomery 'o6 }\end{array}$ \\
\hline $\begin{array}{l}\text { Euthoctha galeator } \\
\text { Fab. }\end{array}$ & Hemiptera heteroptera & Coreidæ & 21 & 22 & Wilson \\
\hline $\begin{array}{l}\text { Leptoglossus phyllopus } \\
\text { L. } \\
\text { Margus }\end{array}$ & Hemiptera heteroptera & Coreidæ & $2 I$ & 22 & Wilson \\
\hline $\begin{array}{l}\text { inconspicuus H.S. } \\
\text { Chariesterus }\end{array}$ & Hemiptera heteroptera & Coreidæ & 23 & 24 & Wilson \\
\hline $\begin{array}{l}\text { antennator Fab. } \\
\text { Corynocoris }\end{array}$ & Hemiptera heteroptera & Coreidæ & 25 & 26 & Wilson \\
\hline $\begin{array}{l}\text { distinctus Dall. } \\
\text { Aprophora quadrang- }\end{array}$ & Hemiptera heteroptera & Coreidæ & 25 & 26 & Wilson \\
\hline $\begin{array}{l}\text { ularis } \\
\text { Pociloptera }\end{array}$ & Hemipter a bomoptera & Jassidæ & 23 & 24 & Stevens'o6 \\
\hline septentrionalis & Hemiptera homoptera & Fulgoridæ & 27 & 28 & Boring'07 \\
\hline pruinosa & Hemiptera homoptera & Fulgoridæ & 27 & 28 & Borging'o7 \\
\hline Elater, sp. & Coleoptera & Elateridæ & 19 & 20 & $\begin{array}{l}\text { Stevens'o6 } \\
\int^{\top} \text { Stevens' } 06\end{array}$ \\
\hline Blatta germanica & Orthoptera & Blattidæ & 23 & 24 & $\left\{\begin{array}{l}\text { \& Wassilieff'o7 } \\
\text { f }\end{array}\right.$ \\
\hline Anax junius & Odonata & Aeschindæ & 27 & 28 & LeFevre and McGill'o8 \\
\hline
\end{tabular}

*This number, disputed by Foot and Strobell ('o7a, b), has since been confirmed by my own reëxamination ('o7 a, 'o8) and by that of Lefevre and McGill ('o8) and others. 
Female groups (by inference only) with two more chromosomes than the male. In the male a pair of unequal idiochromosomes, half the spermatozoa receiving both these chromosomes, and hence two more than the other half. In the female (by inference only) two such pairs.

FERTILIZATION FORMULA

Egg $\frac{n}{2}$ (including $\left.I, i\right)+$ spermatozoö $\frac{n}{2}-2=2$ ygote $n-2$ (including $\left.I, i\right) \sigma^{7}$

Egg $\frac{n}{2}$ (including $I, i$ ) + spermatozoön $\frac{n}{2}$ including $I, i$ ) $=$ zygote $n$ (including $I, I, i, i$, ) 우 (by inference only)

Described Case

\begin{tabular}{|c|c|c|c|c|c|}
\hline Specie & Order & Family & 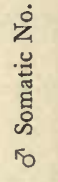 & 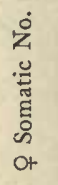 & Authority \\
\hline $\begin{array}{l}\text { Syromastes } \\
\text { marginatus L }\end{array}$ & Hemiptera heteroptera & Coreidæ & 22 & 24 & $\left\{\begin{array}{l}\sigma^{\top} \text { Gross'o4 } \\
\text { o Wilson (inferred) }\end{array}\right.$ \\
\hline
\end{tabular}

V

Female groups with three more chromosomes than the male. Half the spermatozoa receiving three more chromosomes than the other half.

$$
\begin{aligned}
& \text { Egg } \frac{n}{2}+\text { spermatozoön } \frac{n}{2}-3=\text { zygote } n-3\left(0^{7}\right) \\
& \operatorname{Egg} \frac{n}{2}+\text { spermatozoön } \frac{n}{2}=\text { zygote } n(f)
\end{aligned}
$$

\begin{tabular}{|c|c|c|c|c|c|}
\hline Specie & Order & Family & 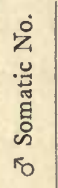 & $\begin{array}{l}\dot{0} \\
\text { ż } \\
\tilde{J} \\
\tilde{J} \\
\tilde{\Xi} \\
\tilde{D} \\
\text { o. }\end{array}$ & Authority \\
\hline Galgulus oculatus Fab. & Hemiptera heteroptera & Galgulidæ & 35 & $3^{8}$ & Payne'o8 \\
\hline
\end{tabular}

Described Case

At least two of the foregoing types may be complicated by the presence of certain additional chromosomes, present in some individuals but not in others of the same species, to which I have applied the name of "supernumerary chromosomes." 12 The number of these varies from one to six in different individuals but is constant in the same individual. In some forms (Metapodius, Banasa) these supernumer-

${ }^{12}$ Wilson 'o7b, 'o7c. A detailed description is now in preparation. 


\section{Studies on Chromosomes}

aries accompany a typical pair of unequal idiochromosomes (as in Type II). In other forms (Diabrotica), the supernumeraries accompany an unpaired idiochromosome (as in Type III). In these cases definite numerical formulas cannot be given, since the distribution of the supernumeraries is variable and both sexes show a variable number of chromosomes in consequence (directly known only in Metapodius.) For the present these cases may most conveniently be treated as sub-types as follows:

\section{IIa}

Forms that agree with Type II except that certain individuals may possess, in addition to a pair of idiochromosomes, one or several supernumerary chromosomes. The cases described, with the numbers of chromosomes observed, are as follows:

\begin{tabular}{|c|c|c|c|c|c|}
\hline Species & Order & Family & $\begin{array}{c}\sigma^{7} \\
\text { Somatic } \\
\text { No. }\end{array}$ & $\begin{array}{c}\stackrel{+}{\text { Somatic }} \\
\text { No. }\end{array}$ & Authority \\
\hline Banasa calva & $\begin{array}{l}\text { Hemiptera } \\
\text { heteroptera }\end{array}$ & Pentatomidæ & $\begin{array}{c}26 \\
{[26+1]}\end{array}$ & 26 & Wilson'o7b \\
\hline Metapodius terminalis & $\begin{array}{l}\text { Hemiptera } \\
\text { heteroptera }\end{array}$ & Coreida & $\begin{array}{l}21 * \\
22 \\
22+1 \\
22+2 \\
22+3 \\
22+4\end{array}$ & $\begin{array}{l}22 \\
22+1 \\
2+2 \\
22+3\end{array}$ & Wilson'opb, '08 \\
\hline femoratus & $\begin{array}{l}\text { Hemiptera } \\
\text { beteroptera }\end{array}$ & Coreidx & $\begin{array}{l}22 \\
22+2 \\
22+3 \\
22+4\end{array}$ & $\begin{array}{l}22+1 \\
22+2 \\
22+4 \\
22+6\end{array}$ & Wilson 'o pb, 'o8 \\
\hline granulosus & $\begin{array}{l}\text { Hemiptera } \\
\text { beteroptera }\end{array}$ & Coreidæ & $\begin{array}{l}22 \\
22+1 \\
22+2 \\
22+3 \\
22+4 \\
22+5\end{array}$ & $\begin{array}{l}22+3 \\
22+4\end{array}$ & Wilson 'o7b, '08 \\
\hline
\end{tabular}

* This number occurs only in Montgomery's ('06) material of this species, identification of which though probably correct, is not absolutely certain. This case will be considered in a later publication.

\section{IIIa}

Forms that agree with Type III except that certain individuals may possess, in addition to an unpaired idiochromosome, one or several supernumerary chromosomes. Described cases as follows: 


\begin{tabular}{c|c|c|c|c|c}
\hline \multicolumn{1}{c|}{ Specie } & Order & Family & $\begin{array}{c}\sigma^{*} \\
\text { Somatic } \\
\text { No. }\end{array}$ & $\begin{array}{c}+ \\
\text { Somatic } \\
\text { No. }\end{array}$ & Authority \\
\hline $\begin{array}{c}\text { Diabrotica 12-punctata } \\
\text { soror }\end{array}$ & Coleoptera & Chrysomelidæ & $\begin{array}{l}19 \\
19+1 \\
19+2 \\
19+3 \\
19+4\end{array}$ & & Stevens'o7, '08 \\
& & & \\
\hline
\end{tabular}

Despite the apparent diversity of the types that have been enumerated all conform to the common principle that the spermatozoa are of two classes, equal in number, that are respectively male producing and female producing. In the case of Type I this is no more than an inference, since the two classes cannot be distinguished by the eye; but its great probability will be admitted in the fact that the forms with equal idiochromosomes are connected by forms (such as Mineus) in which only a slight inequality exists, with those in which the inequality is very marked (Wilson '05a). The facts now show that the difference between the two classes of spermatozoa is not always confined to a single pair of chromosomes, but may affect two pairs (Syromastes) or even a larger number (Galgulus). It is noteworthy that in every case where a quantitative difference of chromatin exists between the sexes it is always in favor of the female, whether it appear in a larger number of chromosomes or in the greater size of one of them. But I must again emphasize the fact that this quantitative difference cannot be considered as the primary factor that differentiates the two classes, for in the first class such a difference does not exist, ${ }^{13}$ while in Metapodius, even in the same species, it is some-

${ }^{13} \mathrm{I}$ based this type on the facts observed in Nezara, where the idiochromosomes are equal in size in both sexes. This is not in accordance with the later observations of Montgomery ('o6) who believes that in the Hemiptera generally the two components (paternal and maternal) of every chromosome pair are at least slightly unequal - though he finds the idiochromosomes of Oncopeltus equal as I have also since observed. A reëxamination of Nezara confirms my original account of this form, though in some individuals the idiochromosomes often appear very slightly unequal. A careful examination of the other chromosomes, particularly the small $m$-chromosomes (which are most favorable for the purpose) in Alydus, Anasa, Archimerus, Pachylis, and other genera, leads me to a very skeptical view of Montgomery's general conclusion on this point. It is true that the two members of each pair vary slightly in relative size, and are not always exactly equal; but, in my material at least, it is clear that 
times the female, sometimes the male, that has the larger number and quantity. I therefore adhere to the view that if the primary and essential difference between the two classes of spermatozoa inhere in the chromosomes (there is of course room for difference of opinion on this point) it must be, or originally have been, qualitative in nature.

Since the appearance of my third "Study," in which some general discussion of the sex chromosomes was offered, there has appeared an important paper by Correns ('o 7 ) on the higher plants, the results of which, as he points out, harmonize remarkably with those based on the cytological evidence. The most important of his results is the experimental proof obtained by hybridizing experiments on Bryonia, that in the diœcious species the pollen grains are male producing and female producing in equal numbers, quite in accordance with the view put forward by McClung ('02) in regard to the spermatozoa of insects and proved to be correct in principle by the work of Stevens and myself. That the same result should appear from investigations carried out on such different material and by such different methods certainly gives good ground for the belief that as far as the male is concerned the phenomenon is at least a very general one. Professor Correns points out in some detail the extraordinarily close parallel between his experimental results and the cytological ones of Stevens and myself; but the interpretation that he offers differs materially from both those that I suggested in an analysis of my observations (Wilson 'o6). According to my first interpretation (Castle's) both sexes are assumed to be sex hybrids or heterozygotes. The conclusion of Correns is that, in respect to the active sexual tendencies of the gametes that produce them, only the male is a sex hybrid or heterozygote $\left(\sigma^{7}(q)\right)$, while the female is a homozygote (우) ${ }^{14}$ This interpretation explains the numerical equality

this is merely a casual fluctuation, the general rule being equality. This variation appears in different cells of the same cyst (as may be seen with especial clearness in the $m$-chromosomes in side views of the second division where errors due to foreshortening may be eliminated). It would be indeed strange if these relations were subject to no variation whatever.

${ }^{14}$ It is necessary to an understanding of Correns's view to bear in mind that the gametes are not considered to be "pure" in the original Mendelian sense, but to bear both sexual possibilities, one of which is "active," the other "latent." 
of the sexes in accordance with the Mendelian principle without the necessity for assuming selective fertilization. It is so simple, and seems to be so clearly demonstrated in the case of Bryonia, that its application to the interpretation of sex production in general is very tempting. Correns himself believes it "very probable" that his conclusion will apply to all the diœcious flowering plants, and possible that it may also hold true of animals (o $\dot{p}$. cit., pp. 65,66 ). It is evident that in their superficial aspects the cytological results seem to bear this out. Wherever the sexes show visible differences in the somatic chromosome groups the female groups consist of two series in duplicate, while the male groups show two series that are not duplicates, only one of them being identical with one of the female series. As far as the chromosomes are concerned, and from a purely morphological point of view, the female is therefore in fact a homozygote, the male a heterozygote, in these animals. But when more closely scrutinized from this standpoint the interpretation seems by no means so clear As I showed in my third "Study" the odd chromosome of the male must be derived from the egg; and if this chromosome bears the sexual tendency, it must under Correns's hypothesis carry the female tendency-which is a reductio ad absurdum, since it is not accompanied by a male-bearing mate or partner in the male. I think this brings clearly into view the following alternative. Either the females of these insects must be physiologically heterozygotes (as I assumed), or the so-called "sex chromosomes" (idiochromosomes) do not bear the sexual tendencies but only accompany them in a definite way. Which of these possibilities is the true one may be left to further research to decide. I will only point out that Professor Correns carefully considers the difficulties that his interpretation encounters in some other directions, and admits that it must be modified in certain cases-for example in the honey bee and in Dinophilus, in which latter case he too is compelled to admit the possibility of selective fertilization. The parthenogenetic females of such forms as the aphids and phylloxerans, which produce both males and females without fertilization, are still considered by Correns as homozygotes, the production of males being assumed to be determined, if I under- 
stand his conception, by the activation of the "latent" (not to be confused with the "recessive") male possibility in the male producing eggs. This is doubtless an admissible assumption, though it seems to me to put a considerable strain upon the general hypothesis. The more natural view would seem to be the one directly suggested by the facts, i.e., that the parthenogenetic stemmother aphid is a heterozygote, the male tendency being in the condition of a Mendelian recessive. But I will not enter upon a discussion of this question, which is now in a condition where a little observation and experiment will outweigh a large amount of hypothesis. I think, however, that the first of the interpretations that I suggested (following Castle) should not be rejected without further data, and especially not until the question of selective fertilization has been put to the test of direct experiment.

Zoölogical Laboratory

Columbia University

February 13, 1908

\section{WORKS REFERRED TO}

Boring, Alice M. '07-A Study of the Spermatogenesis of twenty-two Species of the Membracidæ, Jassidæ, Cercopidæ and Fulgoridæ. Journ. Exp. Zoöl., iv, 4.

Correns, C.'o7-Die Bestimmung und Vererbung des Geschlechtes. Berlin, 1907. Also (in abbreviated form) in Arch. f. Rassen- u. Ges.Biologie, iv, 6.

Dublin, L. I. '05-The History of the Germ-cells in Pedicellina Americana. Ann. N. Y. Acad. Sci., xvi, I.

Foor, K., and Strobell, E. C. 'o7a-The "Accessory Chromosome" of Anasa tristis. Biol. Bull., xii.

'o7b-A Study of Chromosomes in the Spermatogenesis of Anasa tristis. Am. Journ. Anat., vii, 2.

Gross, J. '04-Die Spermatogenese von Syromastes marginatus. Zool. Jahrb., Anat. u. Ontog., xx.

'o7-Die Spermatogenese von Pyrrochoris apterus. Ibid., xxiii.

Henking, H. 'gI-Ueber Spermatogenese und deren Beziehung zur Eientwicklung bei Pyrrorhoris apterus. Zeitschr. f. Wiss. Zool., li.

'92-Untersuchungen über die ersten Entwicklungs-vorgänge in den Eiern der Insekten, Ill. Ibid, liv, I. 
Lefevre, G., and McGill, C. '08-The Chromosomes of Anasa tristis and Anax. junius. Am. Journ. Anat., viii, 4 .

Meves, F. '03-Ueber “Richtungskörperbildung” im Hoden von Hymenopteren. Anat. Anz., xxiv.

'o7-Die Spermatocytenteilungen bei der Honigbiene, etc. Arch. mik. Anat., $\mathrm{lxx}, 3$.

Montgomery, T. H. 'oo-The Spermatogenesis of Peripatus, etc. Zool. Jahrb., Anat. u. Ontog., xiv.

'OI-A Study of the Chromosomes of the Germ-cells of Metazoa. Trans. Am. Phil. Soc., xx.

'o4-Some Observations and Considerations upon the Maturation Phenomena of the Germ-cells. Biol. Bull., vi, 3 .

'o6-Chromosomes in the Spermatogenesis of the Hemiptera Heteroptera. Trans. Am. Phil. Soc., xx.

McClung, C. E. 'O2-The Acessory Chromosome-Sex Determinant? Biol. Bull. iii.

Overton, J. B. '05-Ueber Reduktionsteilung in den Pollenmutterzellen einiger Dikotylen. Jahrb. wiss. Bot., xlii, I.

Paulmier, F. C. '99-The Spermatogenesis of Anasa tristis. Journ. Morph., Supplement.

Payne, F. '08-On the Sexual Differences of the Chromosome groups in Galgulus oculatus. Biol. Bull., xiv, 5 .

Sargant, Ethel ' 96 - The Formation of the Sexual Nuclei in Lilium martagon. I, Ö̈genesis. Ann. Bot., x.

Schreiner, K. E. and A. 'o6-Neue Studien über die Chromatinreifung der Geschlechtszellen (Tomopteris). Arch. Biol., xx.

Stevens, N. M. '03-On the Ovogenesis and Spermatogenesis of Sagitta bipunctata. Zool. Jahrb., Anat. u. Ontog., xviii.

'05-Studies in Spermatogenesis with Especial Reference to the "Accessory Chromosome." Carnegie Institution, Washington, Pub. no. 36 .

'o6-Studies in Spermatogenesis. II. A Comparative Study of the Heterochromosomes in certain Species of Coleoptera, Hemiptera and Lepidoptera, with Especial Reference to Sex Determination. Ibid., Pub. 36, II.

'o8a-A Study of the Germ-cells of Certain Diptera, etc., Journ. Exp. Zoöl., v, 3 .

'o8b-The Chromosomes in Diabrotica, etc. Ibid., v, 4.

Sutron, W. S. 'O2-On the Morphology of the Chromosome group in Brachystola magna. Biol. Bull., iv, $x$. 
Wallace, L. B. '05-The Spermatogenesis of the Spider. Biol. Bull., viii.

Wassiliefr, A. 'o7-Die Spermatogenese von Blatta germanica. Arch. mik. Anat., lxx.

Wilson, E. B. 'o5a-Studies on Chromosomes. I. The Behavior of the Idiochromosomes in Hemiptera. Journ. Exp. Zoöl., ii.

'o5b-The Chromosomes in Relation to the Determination of Sex in Insects. Science, xx, p. 500 .

'05c-Studies on Chromosomes. II. The paired Microchromosomes, Idiochromosomes and Heterotropic Chromosomes in the Hemiptera. Ibid., ii.

'o6-Studies on Chromosomes. III. The Sexual Differences of the Chromosome Groups in Hemiptera, with some Considerations on the Determination and Inheritance of Sex. Ibid., iii.

'o7a-The Case of Anasa tristis. Science, xxv, p. Igr.

'o $\mathrm{b}$-Note on the Chromosome groups of Metapodius and Banasa. Biol. Bull.

'o7c-The Supernumerary Chromosomes of Metapodius. Read before the May Meeting of the N. Y. Acad. of Sci. Science, xxvi, 677 .

'08-The Accessory Chromosome of Anasa tristis. Read before the Am. Soc. of Zoölogists, December, '07. Science, xxvii, 690. 


\section{EXPLANATION OF PLATES}

All of the figures are reproduced directly from photographs by the author, without retouching. The originals were taken with a Spencer $\frac{1}{x 2}$ oil-immersion, Zeiss ocular 6, which gives an enlargement of 1500 diameters. The admirable method of focusing devised by Foot and Strohell was employed. They are reproduced at the same magnification.

\section{Plate I}

(Photos I to 5, 10 to 23 , Syromastes marginatus; 6 to 10, Metapodius terminalis; 24 and 25, Pyrrochoris apterus).

I and 2. Spermatogonial groups of Syromastes; copied in Text-fig. I, $a, b$.

3 to 5 . Polar views, first maturation metaphase; $m$-chromosome at the center, idiochromosomebivalent ("accessory" chromosome) outside the ring at the left.

6 and 7. Corresponding views of Metapodius, typical condition with the two separate idiochromosomes outside the ring at the left.

8 and 9. The same; exceptional condition, with the idiochromosomes (at the left) in contact.

ro. Polar metaphase, second division, Syromastes.

II to 17 . Side views of the same division. The duality of the idiochromosome appears in 12,16 and 17 .

18 to 23. Early prophases of first maturation division, Syromastes. Each of these shows the separate $m$-chromosomes, and in all but No. 20 the chromosome nucleolus (idiochromosome bivalent) also appears.

24 and 25. Spermatogonial metaphases of Pyrrochoris (copied in Text-figs. 2, a,b). 
Edmond B. Wilson.
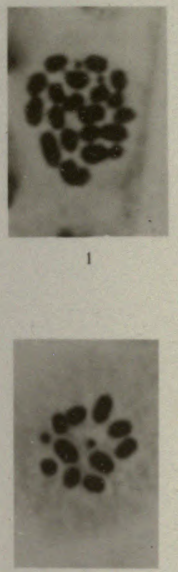

6

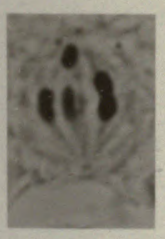

11

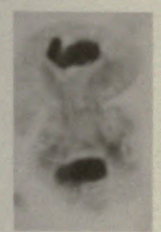

16

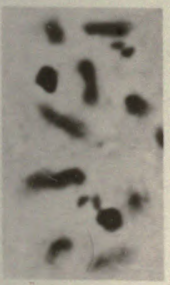

21

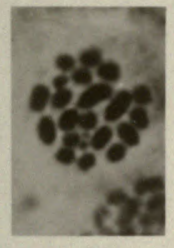

2
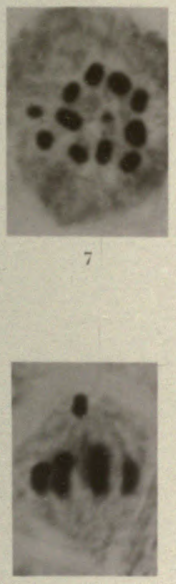

12

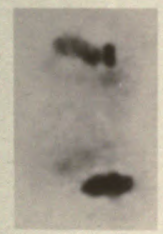

17

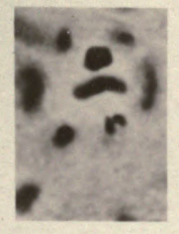

22
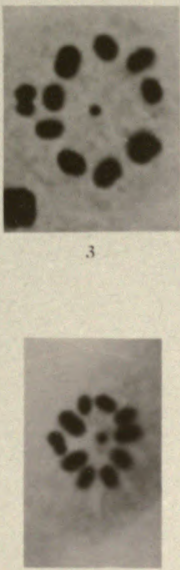

8

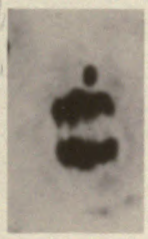

13

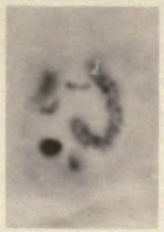

18

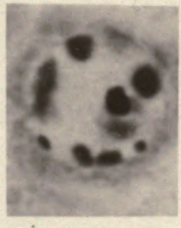

23
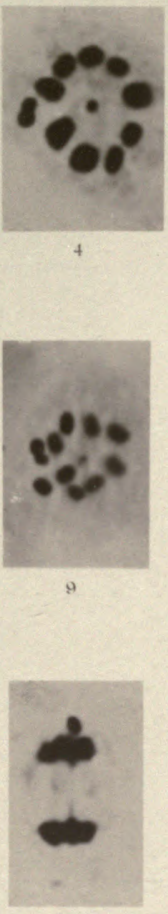

14

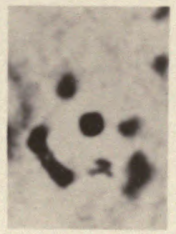

19

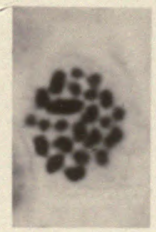

24
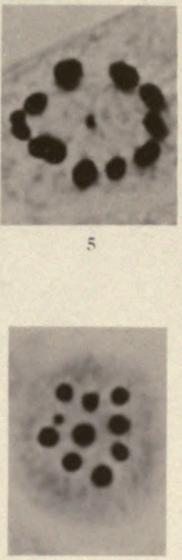

10

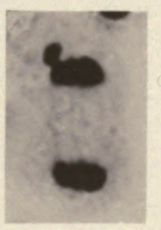

15

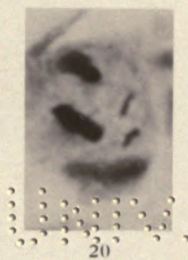

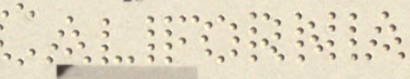

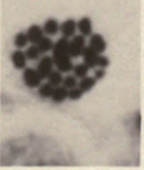

25

The Journal of Experimental Zoology, Vol. VI, No 1. 




\section{Ni.B. A defoct in the plate (not prosen ono chromosome at the right side of thi shors it corroctiy.}

\section{Plate II}

\section{Pyrrochoris apterus}

26 to 31 . Spermatogonial groups, each showing twenty-three chromosomes, including the large unpaired idiochromosome; $30,3^{1}$ illustrate the rare case in which the latter appears double, owing to marked sigmoid curvature. These photos are copied in Text-figs. $2, c, d, e, j, k$ and $l$, respectively. 32 and 33. Post-phases shortly following last spermatogonial division; the chromosomes still distinct, idiochromosome recognizable by its large size and deeper color.

34 and 35. Presynaptic stages following the last, showing "caterpillar" stage of idiochromosome and small nucleoli. In the last two the shortening has begun.

$3^{6}$ to $3^{8}$. Further condensation of the idiochromosome; initial stages of synizesis; apparent duality of the idiochromosome in two of the cells.

39 to 42 . Synizesis, showing various forms of the chromosome nucleolus.

43 and 44. Early post-synaptic stages.

45 and 46 . Polar metaphases, first spermatocyte division.

47 to 49 . Side views of second division.

50 and 51 . Polar metaphases, second division. 


\section{the original negativo) causes}

29 to appear double. Fig.1j

\section{Edmond B. Wilson.}

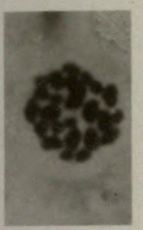

26

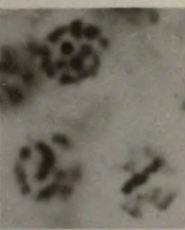

32
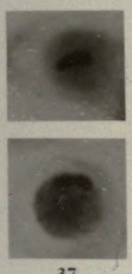

37

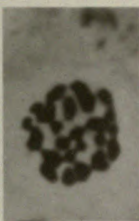

27

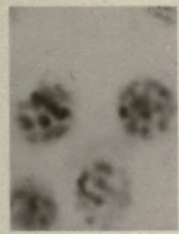

33
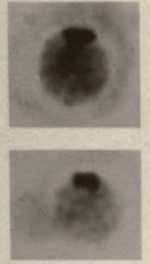

38

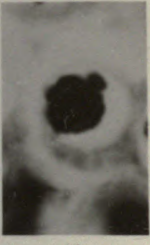

42

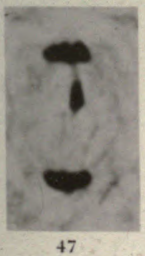

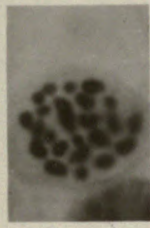

28

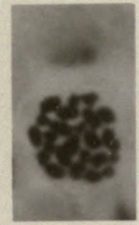

29

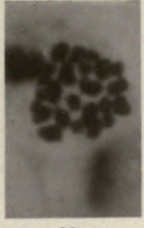

30

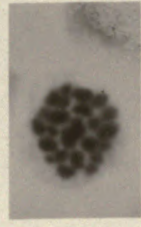

31

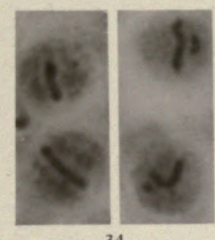

34
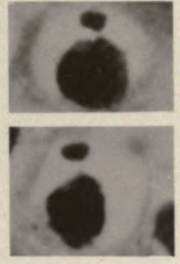

39

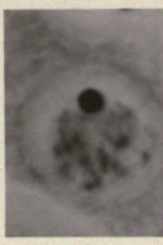

44

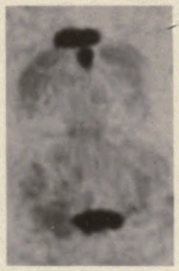

49)
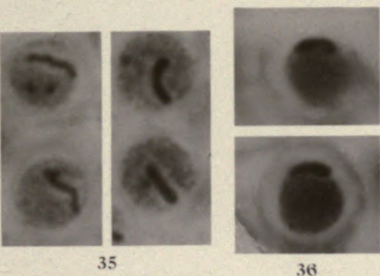

36
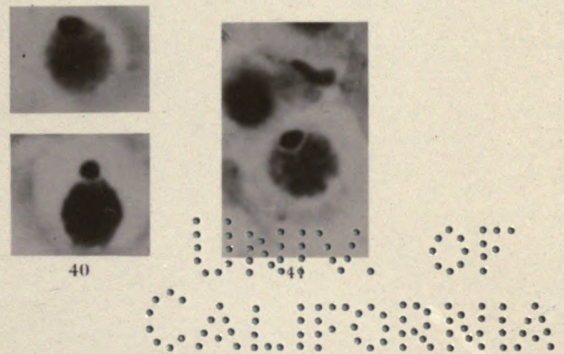

$\therefore \therefore \therefore: \because \because \because \because \because \vdots \vdots \vdots \vdots \vdots$

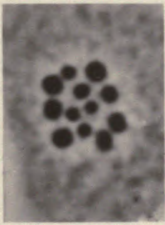

45
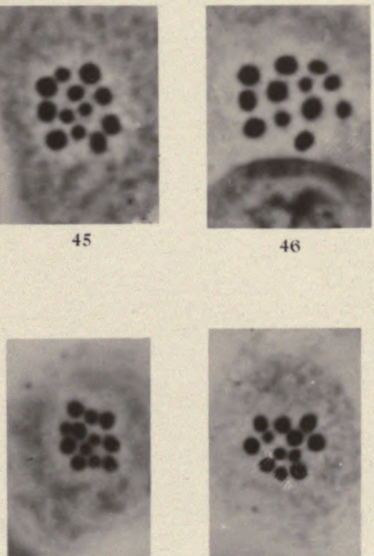

50

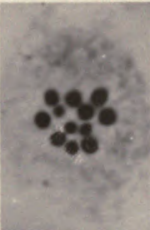

51

The Journal of Experimental Zoology, Vol. VI, No 1. 
$\rightarrow$

$\because$\begin{tabular}{l}
$\cdots$ \\
$\because$ \\
\hdashline
\end{tabular}

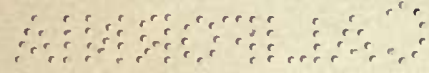

a 
(1)

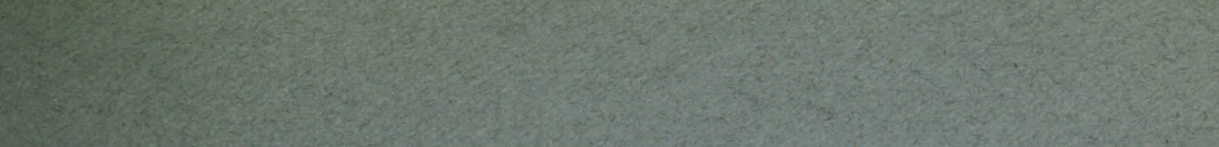

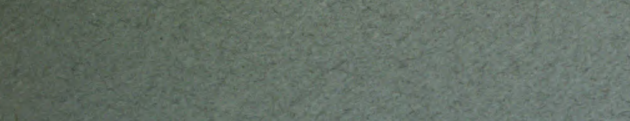

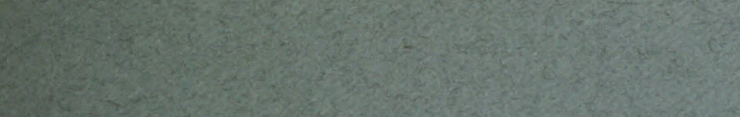

W.

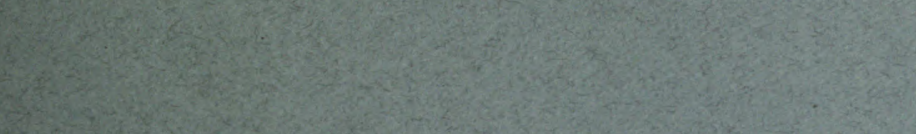

Q.

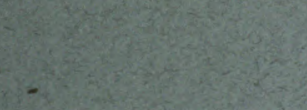

$\sin 2 x+2$

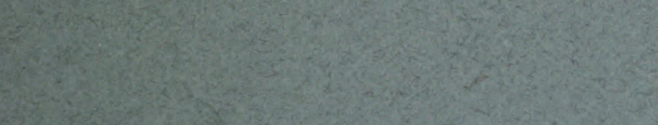

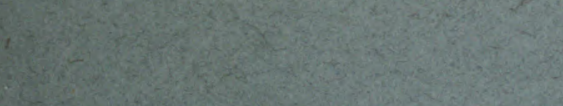

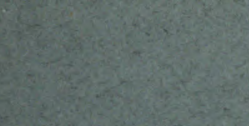

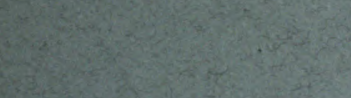

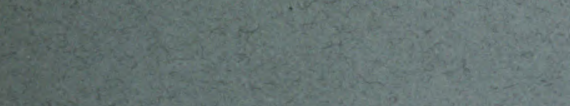

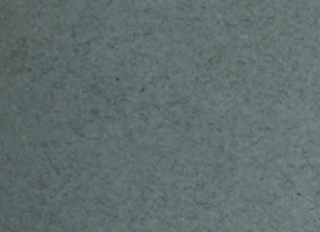

S.

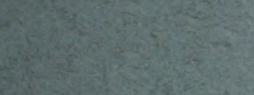

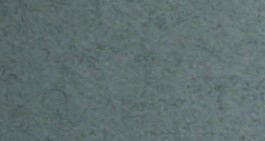

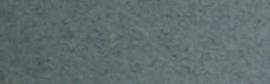

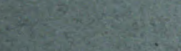

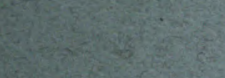

(1)

1.

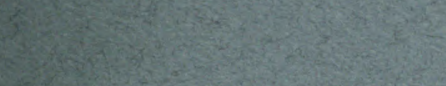

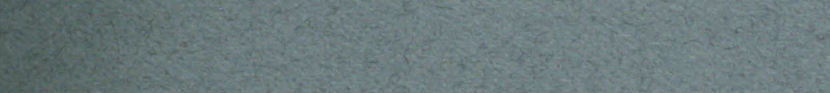

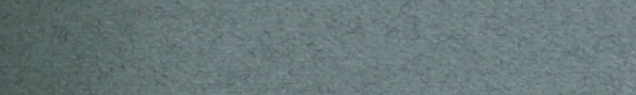

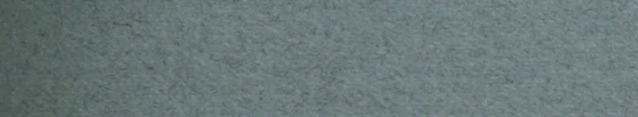

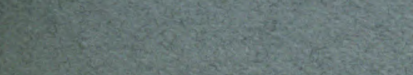

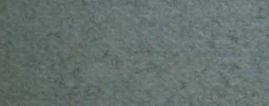





\section{STUDIES ON CHROMOSOMES}

V THE CHROMOSOMES OF METAPODIUS, A CONTRIBUTION TO THE HYPOTHESIS OF THE GENETIC CONTINUITY OF CHROMOSOMES

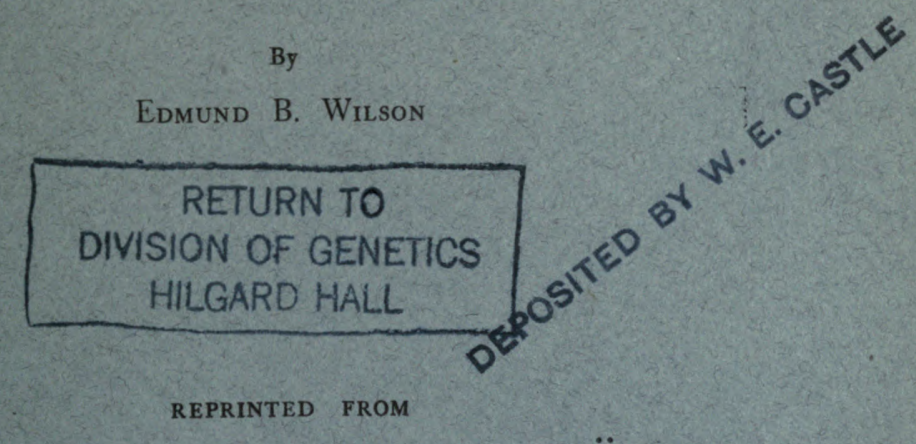

THE JOURNAL OF EXPERIMENTAL ZOÖLOGY

Volume VI No. 2

FEBRUARY, $19 \circ 9$

BALTIMORE, MD., U. S. A. WILLIAMS \& WILKINS COMPANY 



\section{STUDIES ON CHROMOSOMES}

\section{THE CHROMOSOMES OF METAPODIUS. A CONTRI- BUTION TO THE HYPOTHESIS OF THE GENETIC CONTINUITY OF CHROMOSOMES ${ }^{1}$}

\section{BY}

EDMUND B. WILSON

\section{With One Plate and Thirteen Figures in the Text}

The genus Metapodius (Acanthocephala), one of the coreid Hemiptera, shows a very exceptional and at first sight puzzling relation of the chromosome-groups which has seemed to me worthy of attentive study by reason of its significance for the hypothesis of the "individuality" or genetic continuity of the chromosomes. The most conspicuous departure from the relations to which we have become accustomed lies in the fact that different individuals of the same species often possess different numbers of chromosomes, though the number in each individual is constant. An even more surprising fact is that in all of my own material every male individual possesses at least 22 spermatogonial chromosomes, including a pair of unequal idiochromosomes like those of the Pentatomidx, while in Montgomery's material of M. terminalis every male has but 2 I spermatogonial chromosomes, one of which is a typical odd or "accessory" chromosome (unpaired idiochromosome). ${ }^{2}$

The present paper presents the results of an investigation of these relations that has now extended over nearly four years, in the course of which serial sections of more than sixty individuals

${ }^{1}$ Part of the cost of collecting and preparing the material for this research was defrayed from a grant of $\$ 500$ from the Carnegie Institution of Washington, made in I906. I am indebted to Rev. A. H. Manee, of Southern Pines, N. C., for valuable coöperation in the collection of material, and to Dr. Uhler, Mr. Heidemann, Mr. Van Duzee, and Mr. Barber for aid in its identification.

${ }^{2}$ By Professor Montgomery's courtesy I have been enabled to study thoroughly his original preparations and to satisfy myself of the correctness of his account (Montgomery 'o6). I also owe to him a number of unsectioned testes of the same type.

The Journal of Experimental Zoölogy, vol. vi, No. 2. 
have been carefully studied. These individuals belong to three well marked species-M. terminalis Dall. and M. femoratus Fab. from the Eastern and Southern States, M. granulosus Dall. from the Western-all of which show a similar numerical variation. ${ }^{3}$ My first material, including sections of two testes of $M$. terminalis (Nos. I, 2) from the Paulmier collection, long remained a complete puzzle and led me to the suspicion that the material was pathological. This possibility was eliminated by the study of additional material of the same type; but the contradiction with Montgomery's results on the same species suggested that his specimens were not correctly identified (Wilson 'o $7 a$ ). Continued study at length convinced me that this supposition too was probably unfounded. If the identification was correct, as I now believe it was, M. terminalis is a species that varies not only in respect to the individual chromosome number but also in respect to the sexchromosomes, certain individuals having an unpaired "accessory" chromosome, while others have an unequal pair of idiochromosomes. The latter condition alone has thus far been found in M. femoratus and M. granulosus. The essential facts, and the general history of the spermatogenesis, are otherwise closely similar in the three species.

The range of variation in the number of chromosomes is in M. terminalis from $2 \mathrm{I}$ to 26 , in M. femoratus from 22 to 27 or 28 , and in M. granulosus from 22 to 27 , the particular number (or its equivalent in the reduced groups) being a characteristic feature of the individual in which it occurs. I do not mean to assert that there is absolutely no fluctuation in the individual. In this genus, as in others, apparent deviations from the typical number frequently are seen, and real fluctuations now and then appear; but the latter are so rare that they may practically be disregarded. That the number may be regarded as an individual constant (subject to such deviations as are hereafter explained (p. 185) is abundantly demonstrated, not only by the agreement of large numbers of cells from the same individual but perhaps even more

\footnotetext{
${ }^{3} \mathrm{~A}$ complete list of the individuals examined, arranged by localities, is given in the Appendix at p.-202. Each individual is there designated by a number by which it is referred to in the tert and description of figures.
} 
convincingly by the definite correlation of the spermatocytegroups with those of the spermatogonia of the same individual. This is shown in the following table, which summarizes the facts thus far observed. ${ }^{4}$

SUMMARY

\begin{tabular}{|c|c|c|c|c|c|c|c|}
\hline \multirow{2}{*}{$\begin{array}{l}\text { Somatic number } \\
\text { (spermatogonia or } \\
\text { ovarian cells) }\end{array}$} & \multirow{2}{*}{$\begin{array}{c}\text { First spermatocyte } \\
\text { division }\end{array}$} & \multicolumn{2}{|c|}{ terminalis } & \multicolumn{2}{|c|}{ femoratus } & \multicolumn{2}{|c|}{ granulosus } \\
\hline & & $0^{\pi}$ & 우 & व & 우 & ठ & ㅇ \\
\hline $21 \ldots$ & I I & 9 & 0 & 0 & 0 & 0 & 0 \\
\hline $22 \ldots$ & 12 & 3 & 4 & 3 & 0 & I & 0 \\
\hline $23 \cdots$ & 13 & 5 & 2 & 0 & 2 & 2 & 0 \\
\hline $24 \ldots \ldots \ldots$ & 14 & 3 & 3 & 2 & $\mathbf{I}$ & 4 & 0 \\
\hline $25 \ldots$ & 15 & 2 & 2 & 0 & 0 & I & I \\
\hline 26. & 16 & I & 0 & 2 & I & 4 & 2 \\
\hline$(27)$ & 17 & 0 & 0 & 0 & 0 & $\mathbf{I}$ & 0 \\
\hline $28(27$ ? $) \ldots \ldots$ & & 0 & 0 & o & I & 0 & 0 \\
\hline
\end{tabular}

Distribution in the whole group

\begin{tabular}{|c|c|c|c|}
\hline Total somatic number & Number of males & Number of females & Totals \\
\hline ............ & 9 & 0 & 9 \\
\hline $22 \ldots \ldots \ldots \ldots \ldots \ldots \ldots \ldots \ldots \ldots$ & 7 & 4 & II \\
\hline $23 \ldots \ldots \ldots \ldots \ldots \ldots \ldots \ldots \ldots \ldots \ldots \ldots$ & 7 & 4 & II \\
\hline $24 \ldots \ldots \ldots \ldots$ & 9 & 4 & 13 \\
\hline 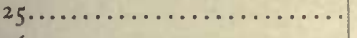 & 3 & 3 & 6 \\
\hline $26 \ldots \ldots \ldots \ldots \ldots$ & 7 & 3 & 10 \\
\hline$(27) \ldots \ldots \ldots \ldots \ldots \ldots \ldots \ldots \ldots$ & I & ○ & I \\
\hline $28(27 ?) \ldots \ldots \ldots \ldots \ldots \ldots \ldots$ & $\circ$ & $\mathbf{I}$ & $\mathbf{I}$ \\
\hline Total & 43 & 19 & 62 \\
\hline
\end{tabular}

${ }^{4}$ The somatic numbers of the males are in each case determined from the dividing spermatogonia. Those of the female are from dividing cells in various parts of the ovary-mainly from the region just above or below the end-chamber-some of them undoubtedly folicle-cells, others probably young nutritive cells or oögonia. The chromosome-groups from different regions differ considerably in size, but otherwis show the same general characters. With a very few exceptions the number of chromosomes has been determined by the count of several groups from the same gonad, in many cases by the count of a very large number. In many individuals hundreds of perfectly clear equatorial plates may be seen and the evidence is entirely demonstrative. In seven of the males (owing to lack of mitoses, or to defective fixation) the somatic number has been inferred from that shown in the spermatocyte divisions, or vice versa; but with a single exception both numbers have been directly observed in other individuals of the same type. I am therefore confident that the numbers are substantially correct as given. In case of the female, only the somatic numbers can be given, since the maturation-divisions are not available for study. 
The material of terminalis is from New Jersey, Pennsylvania, Ohio, North Carolina, South Carolina and Georgia; that of femoratus from the three states last named; that of granulosus from Arizona. The variation of number is independent of locality, and individuals of the same species showing different numbers were often taken side by side on the same food plants. It is equally independent of sex, as the table at once shows. I am unable to find any constant correlation between the number of chromosomes and any other visible structural characters of the adult animals.

Such an astonishing range of variation in the chromosome number in the same species seems at first sight to present a condition of chaotic confusion. But, as I shall endeavor to show, the first impression thus created disappears upon more critical examination. Detailed study of the facts proves that the variation is not indiscriminate but affects only a particular class of small chromosomes that are distinguishable from the ordinary ones both by size and by certain very definite peculiarities of behavior. These chromosomes are absent in all of Montgomery's material; in my own they are sometimes present, sometimes absent, the total number varying accordingly. The chromosomes in question are the ones which in earlier papers I have called the "supernumeraries." In behavior they show an unmistakable similarity to the idiochromosomes; and for reasons given beyond I believe them to be nothing other than additional small idiochromosomes, the presence of which has resulted from irregularities of distribution of the idiochromosomes in preceding generations. The relations seen in Montgomery's material form the converse case, the small idiochromosome having disappeared or dropped out. I shall try to show that both cases are probably due to the same initial cause.

\footnotetext{
${ }^{5}$ Wilson 'o7a, 'o7b. I first discovered this phenomenon in the pentatomid species Banasa calva ('o5b) describing the single supernumerary as a "heterotropic chromosome." Later ('o7a) a single supernumerary was found in certain individuals of Metapodius terminalis, and other numerical variations in this species and in femoratus and granulosus were briefly recorded; but at that time I did not yet fully understand the facts. Banasa calva is the only form oustide the genus Metapodius, in a totalof more than seventy species of Hemiptera I have examined, in which supernumerary chromosomes have been found. Miss Stevens ('o 8 b) has recently found in the coleopteran genus Diabrotica a condition that is in some respects analogous to that seen in Metapodius.
} 


\section{A GENERAL DESCRIPTION}

Since the phenomena as a whole are somewhat complicated, I have thought it desirable to bring the most essential facts together for ready comparison in a preliminary general account illustrated by a limited number of selected figures (Figs. I, 2). The fundamental type of the genus is, I believe, represented by individuals that possess 22 chromosomes in the somatic groups of both sexes, and in which no supernumeraries are present (Fig. I, $d-f$ ). Two of the chromosomes are a pair of very small $m$-chromosomes, like those of other coreids; two are a pair of idiochromosomes consisting in the male of a large and a small member, in the female of two large ones; while the remaining 18 are ordinary chromosomes or "autosomes." These chromosomes have in the spermatogenesis the same general history as in other Hemiptera heteroptera. In the first division the idiochromosomes are separate univalents, their position being typically (but not invariably) outside a ring formed by the nine larger bivalents within which lies the small $m$-chromosome bivalent (Fig. 1, $d$, Photo 2). This division accordingly shows 12 separate chromosomes (one more than the reduced or haploid number.) In the second division, as described beyond, they are always united to form a dyad or bivalent, composed of two unequal halves, and the number of separate chromosomes is II. The spermatogonial groups possess 22 chromosomes (Fig. I, $e$ ) of which the small idiochromosome may often be recognized as the smallest of the chromosomes next to the $m$-chromosomes; but it does not differ sufficiently in size from the other chromosomes to be always certainly distinguishable. ${ }^{6}$ In the growth period the idiochromosomes, as usual, have the form of condensed deeply-staining chromosome-nucleoli, while the other chromosomes are in a vague, faintly staining condition. They are usually in contact but not fused (Fig. 1, f, Photo 25), thus form-

- In considering the relative size-relations it is important to bear in mind that the apparent size, as seen in polar view, varies considerably with the degree of polar elongation. Still more important is the fact (which I have emphasized in a preceding paper) that in the first division univalent chromosomes always appear relatively much smaller than they do in the spermatogonia. This is the case with the idiochromosomes and the supernumeraries, which are always readily recognizable in the spermatocytedivisions, but are often difficult to distinguish in the spermatogonia. 


\section{EXPLANATION OF FIGURES}

Fig. I

About one-fourth of the figures were drawn upon enlarged photographs by the method described in a preceding paper (Wilson 'og). The others are from camera lucida drawings. In all cases the form, size, and grouping of the chromosomes are represented as accurately as possible. The form, size, and general appearance of the spindles are shown, but no attempt bas been made to represent the exact details of the fibrillæ. Figs. I and 2 are enlarged about 3300 diameters, the others a little less than 3000 diameters.

\section{Lettering, in all the Figures}

$I$, large idiochromosome or odd chromosome; $i$, small idiochromosome; $m, m$-chromosome; $p$, plasmosome; $s$, supernumerary chromosome. In cases where $s$ and $i$ are both present and of equal size it is impossible to distinguish between them. In such cases $I$ have as a rule designated as $i$ the one lying nearest to $I$; but this is quite arbitrary. It should be noted also that $I$ cannot always be distinguished from the smaller of the ordinary bivalents. 

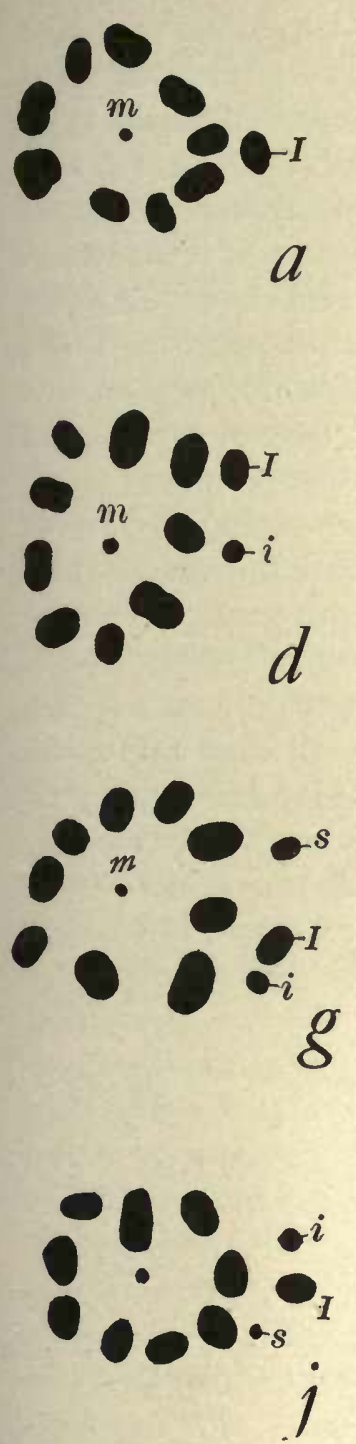

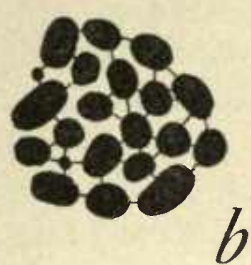

b
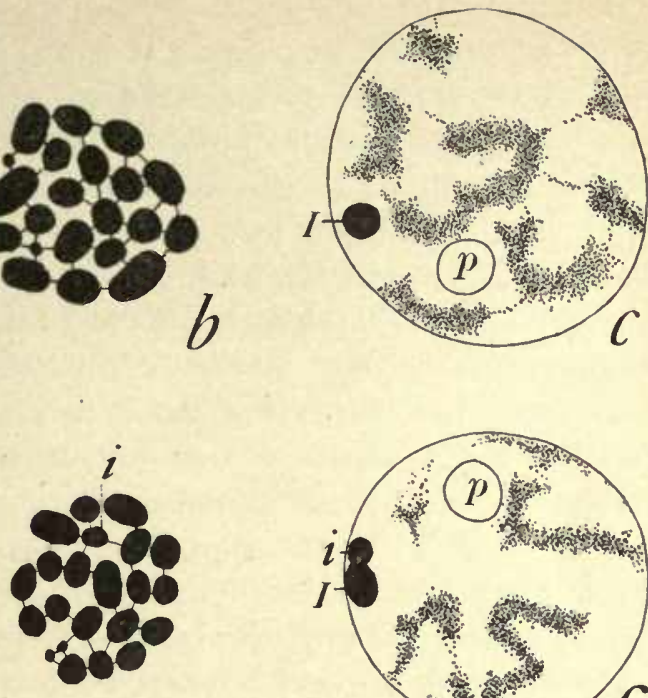

e
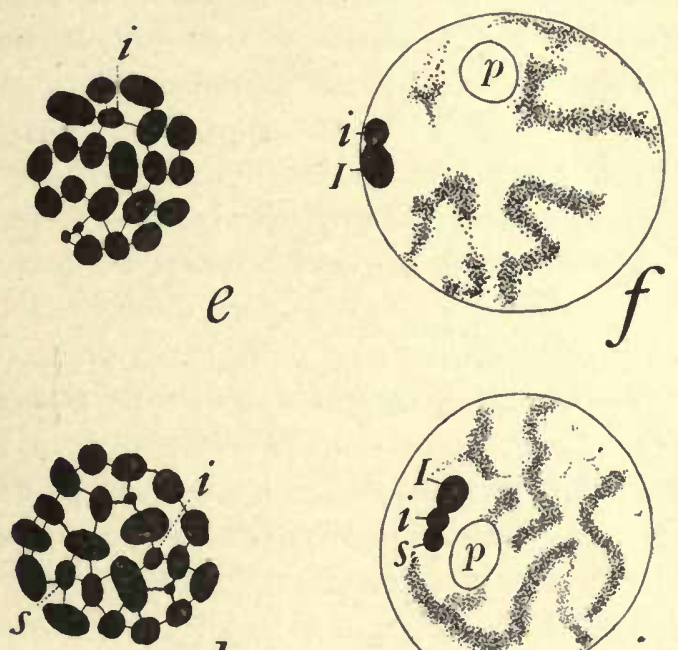

b
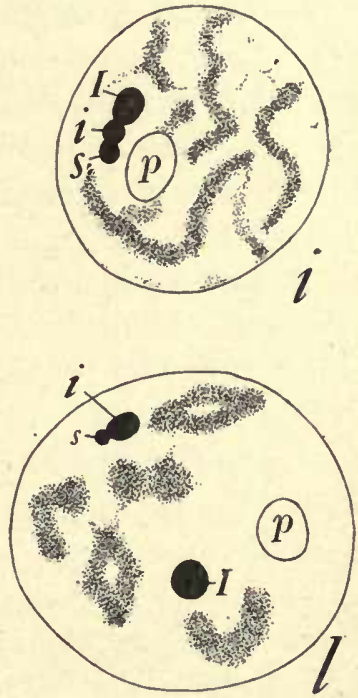

Fig. I

M. terminalis

$a-c$ (No. 3), 2I-chromosome form; $a$, first spermatocyte metaphase; $b$, spermatogonial metaphase; $c$, nucleus from the growth period.

$d-f$ (No. 19), 22-chromosome form, stages corresponding to above.

$g-i$ (No. 20, Photo 4), 23-chromosome form, one large supernumerary.

$j-l$ (No. 43), 23-chromosome form, one small supernumerary. 
ing a very characteristic bipartite body; but in a good many cases they are separate (Fig. 6, $c, d$, Photo 26). A large and very distinct plasmosome is also present.

Such a group of 22 chromosomes may be regarded as the type of which all the other forms may be regarded as variants, and probably as derivatives. In forms having more than 22 chromosomes the increase in number is due to the presence of from one to six supernumeraries. These vary in number and size in different individuals, but both are constant in a given individual. Their maximal size is equal to that of the small idiochromosome (in which case they are indistinguishable from the latter); such forms will be called "large supernumaries." Their minimal size, ("small supernumeraries") is about the same as that of the $m$ chromosomes; but from the latter they are always distinguishable, in the male, by a quite different behavior in the maturation process. When a single supernumerary is present it may be either large or small, its size being (with slight variation) constant in the individual. When more than one is present all may be of the same size (the most usual condition) or they may be of different sizes, the relation being again an individual constant. Whatever their number or size their behavior is essentially the same as that of the idiochromosomes. In the growth-period they have a condensed form and are typically united with the idiochromosomes to form a compound chromosome-nucleolus, the components of which are often distinctly recognizable and vary in number with the number of the supernumeraries. In the first division they divide as separate univalents, and this division accordingly shows as many chromosomes above $\mathrm{I} 2$ as there are supernumerariesi.e., if the spermatogonial number be $22+n$, the number in the first division is typically $12+n$. Their typical position in this division is, like that of the idiochromosomes, outside the ring of larger bivalents, though there are many exceptions. In the second division they are, as a rule, again associated with the idiochromosomes to form a compound element, though not infrequently one or more of them may be free from the others.

A definite correlation thus appears in each individual between the number and relative sizes of the chromosomes seen in the 
maturation-divisions and in those of the spermatogonia; and it also appears in the number and size of the components of the chromosome-nucleoli when these can be distinctly recognized. Figs. I and 2 illustrate this correlation and epitomize the most essential facts. These figures have been selected from a much larger number to show the clearest and most typical conditions. Some of them are enlarged from the photographs reproduced in Plate I. Many others, with an account of secondary variations, are given beyond. Each horizontal row of figures represents three stages of the same type which, with two exceptions, are all from the same individual. The left hand figure in each row shows the typical arrangement of the chromosomes in the metaphase of the first spermatocyte-division, the middle figure a spermatogonial group, and the right hand one a nucleus from the growth period, to show the chromosome-nucleolus together with some of the diffused ordinary chromosomes.

Fig. I, $a-c$ (terminalis, No. 3), represent these three stages in an individual of the 2I-chromosome type (Montgomery's material) showing II chromosomes in the first division, 2 I in the spermatogonia, and a single chromosome-nucleolus in the growth period. (Additional figures of this individual in Fig. 3.) Fig. I, $d-f$ (terminalis, No. r9), show the 22-chromosome type, with a small idiochromosome present in addition to the large one. The small idiochromosome $(i)$ is distinguishable in Fig. $1, e$. (Additional figures in Figs. 4-6.)

Fig. I, $g-i$ (terminalis, No. 20), show the 23-chromosome type, with one large supernumerary. In the spermatogonial group $(h)$ this chromosome and the small idiochromosome are probably represented by the two designated as $i$ and $s$. The nucleus from the growth-period (i), shows the plasmosome $(p)$ and a tripartite chromosome-nucleolus formed by the idiochromosomes and the supernumerary attached in a row ( $c f$. Photo 27; additional figures in Figs. 7-8). Fig. I, $j-l$ (terminalis, No. 43), show a 23-chromosome group with one small supernumerary. This clearly appears in the spermatogonial group $(s)$; and the small idiochromosome $(i)$ is also distinguishable. In the nucleus from the growth-period $(l)$, the supernumerary and small idiochromosome 
are united $(i, s)$ the large idiochromosome $(I)$ being separate. (Additional figures in Figs. 7,8 .)

Fig. 2, $a-c$ (terminalis, No. 2I), show the corresponding stages in an individual of the 24-chromosome type, with two large supernumeraries. Their identification in the spermatogonial group is somewhat doubtful. (Additional figures in Fig. IO.)

Fig. 2, $d, e$ (terminalis No. 34), show a 25-chromosome type with three large supernumeraries. The growth-period $(f)$ is from an individual of granulosus (No. 54) that is possibly of the 26chromosome type. (Additional figures in Fig. 12.)

Fig. 2, $g, h$ (femoratus No. 42), and $i$ (granulosus, No. 60) show the 26-chromosome type with four large supernumeraries. (See Photo. 28, additional figures in Figs. 9, 10.)

Fig. $2, j-l$ (femoratus, No. 40), are from a very interesting individual of the 26-chromosome type, with two large and two small supernumeraries (additional figures in Figs. 9, 10). The spermatogonia of this individual $(k)$ uniformly show 26 chromosomes, including four very small ones (two $m$-chromosomes, two small supernumeraries), but the large supernumeraries and the small idiochromosomes are doubtful. No case was found in which all of the six components of the chromosome-nucleolus could be seen; $l$ shows five of them, including the two small ones.

\section{B ADDITIONAL DESCRIPTIVE DETAILS}

I will now give a somewhat more detailed and critical account of the facts. Taken as a whole, the series (including nearly 300 slides of serial sections) presents a profusion of evidence on many cytological questions that could not be adequately described save in a large monograph; but I will here limit the account mainly to the numerical and topographical relations of the chromosomes. The clearness of the preparations is such that nearly all the principal phenomena might have been illustrated by photographs (of which upwards of 200 have been prepared). Thirty of these are reproduced in Plate I, less for the purpose of giving the evidence in detail than of illustrating its character to those not directly familiar with this material. 


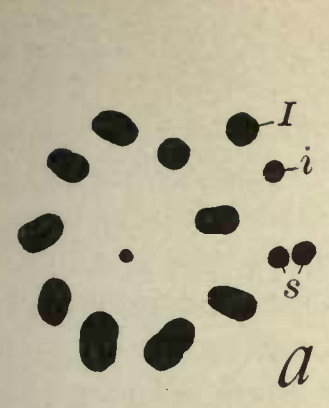

Studies on Chromosomes
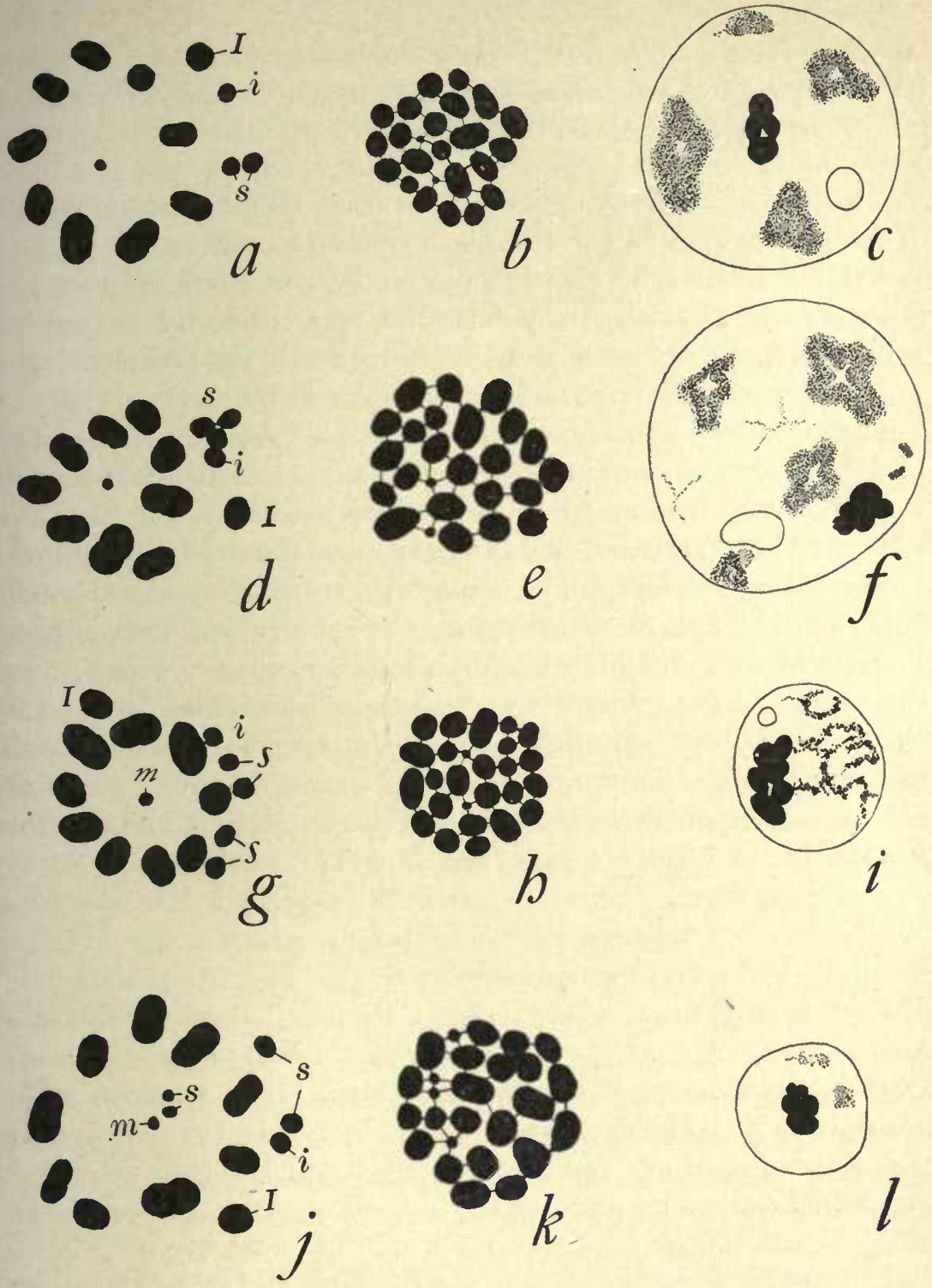

Frg. 2

$a-e$, M. terminalis; $f, i$, granulosus; $g-h, j-l$, femoratus.

$a-c$ (No. 21), 24-chromosome form, two large supernumeraries. $d-c$ (No. 34), 25 -chromosome form, three large supernumeraries.

$f$ (No. 54), growth-period, 25 - or 26-chromosome form.

$g-h$ (No. 42 Photo 8), 26-chromosome form, four large supernumeraries.

i (No. 60), 26-chromosome form, growth-period.

$j-l$ (No. 40), 26-chromosome form, two large and two small supernumeraries. 
I Individuals having twenty-one spermatogonial Chromosomes, including an unpaired Idiochromosome. Small Idiochromosome and Supernumeraries absent

To this group belong only the specimens, all males, collected by Montgomery at West Chester, $\mathrm{Pa}$., of which I have examined nine individuals, all of which have essentially the same characters. ${ }^{7}$ Montgomery ('OI) originally described these forms as having 22 spermatogonial chromosomes but subsequently ('o6) corrected this to $2 \mathrm{I}$, describing the phenomena as agreeing in all essential respects with those seen in Anasa and other coreids. A study of the original preparations has enabled me to confirm this later account in every essential point. After the synizesis or contraction phase of synapsis (as in all individuals of the genus) the ordinary chromosomes appear in the form of rather delicate spireme-like threads, longitudinally split. In later stages of the growth-period they shorten, become irregular, lose their staining capacity, and assume the vague, pale condition characteristic of so many other forms. In the early prophases of the first division they become more definite, stain more deeply, and appear as coarse longitudinally split rods that often show an indication of a transverse division at the middle point, or in the form of the double crosses as described by Paulmier in Anasa ('99). In the later prophases they condense still further to form nine compact bivalents which finally arrange themselves in a more or less regular ring. The equatorial plate of the first division always shows in polar view II chromosomes (Fig. 3, $a, b$, Photo $\mathbf{I}$ ). In the most typical case the univalent idiochromosome lies outside this ring, but it sometimes lies in or inside it. The small $m$-chromosome bivalent is always near the center of the ring. In side view the larger bivalents are either dumb-bell shaped or more or less distinctly quadripartite, in the

\footnotetext{
7 These were taken from magnolia trees. In the summer of 1907 I collected in the same locality two males and three females, all from blackberry bushes. To my disappointment, these differ from Montgomery's specimens, one male having 22 spermatogonial chromosomes, the other 23; while the ovarian cells have in one female 23 and in the other two 24 chromosomes. It is possible that a different species fell into Montgomery's hands, perhaps an introduced form; but both the structure of the testis and the character of the chromosome-groups agree so exactly with my own material that I now believe that Montgomery's identification was probably correct.
} 
latter case appearing dumb-bell shaped as seen in polar view. The eccentric idiochromosome is of nearly the same size as the smallest of the large bivalents and is often indistinguishable from the latter except by its position. All these chromosomes divide equally in this division, the $m$-chromosomes usually leading the way in the march towards the poles, while the idiochromosomes often lag slightly behind the others.

The second division likewise shows II chromosomes in polar view $(3, c, d)$; but the regular grouping characteristic of the first division is now usually lost, the ring formation being often no longer apparent, while either the $m$-chromosome or the idiochromosome may now occupy any position. ${ }^{8}$ In this mitosis all the chromosomes divide except the idiochromosome which lags behind the others and finally passes undivided to one pole (Fig. $3, e-h$, Photos 14, 15) as Montgomery described. The nucleus formed at this pole thus receives II chromosomes, the sister nucleus but ro, precisely as in Anasa, Narnia, Chelinidea or Leptoglossus. This is proved beyond all doubt by polar views of the anaphases, showing the sister groups lying one above the other in the same section (Fig. 3, h). In the particular example figured the idiochromosome lies eccentrically, but this is quite inconstant.

The spermatogonia (Fig. 3, $i, j$ ) always show 2 I chromosomes, a largest and a smallest pair being always distinguishable. The unpaired idiochromosome cannot be distinguished from the others. The m-chromosomes are usually equal, but sometimes appear slightly unequal.

In the growth-period the $m$-chromosomes and the idiochromosome have the same history as in other coreids. The former are

8 The regrouping of the chromosomes in the second division, first described by Paulmier ('99) in Anasa tristis, is characteristic of the Coreida generally, an eccentric position of the idiochromosome being a nearly constant feature of the first division but not of the second. Failure to recognize this fact in the case of Anasa tristis seems to have been one of the main sources of error in the entirely mistaken conclusions of Foot and Strobell ('O7a,'o7b) regarding this species. (Cf. Lefevre and McGill, 'o8.) Demonstrative evidence on this point is given by polar views of rather late anaphases in which every chromosome of each daughter plate may be seen in the same section. Such views, of which I have studied many, both in Anasa and in other genera, show that oneof the chromosomes may indeed occupy an eccentric position, and may there divide; but in such cases the odd chromosome is always found elsewhere in the group, lying either in or near one of the daughter-groups and not in the other. When the odd chromosome is eccentric it is found in one of the daughter groups but not in the other. 
typically separate, and at first diffuse (as in Anasa or Alydus). Later they condense to from two spheroidal bodies that conjugate in the late prophase to form the central small bivalent and are almost immediately separated again by the division. The idio-
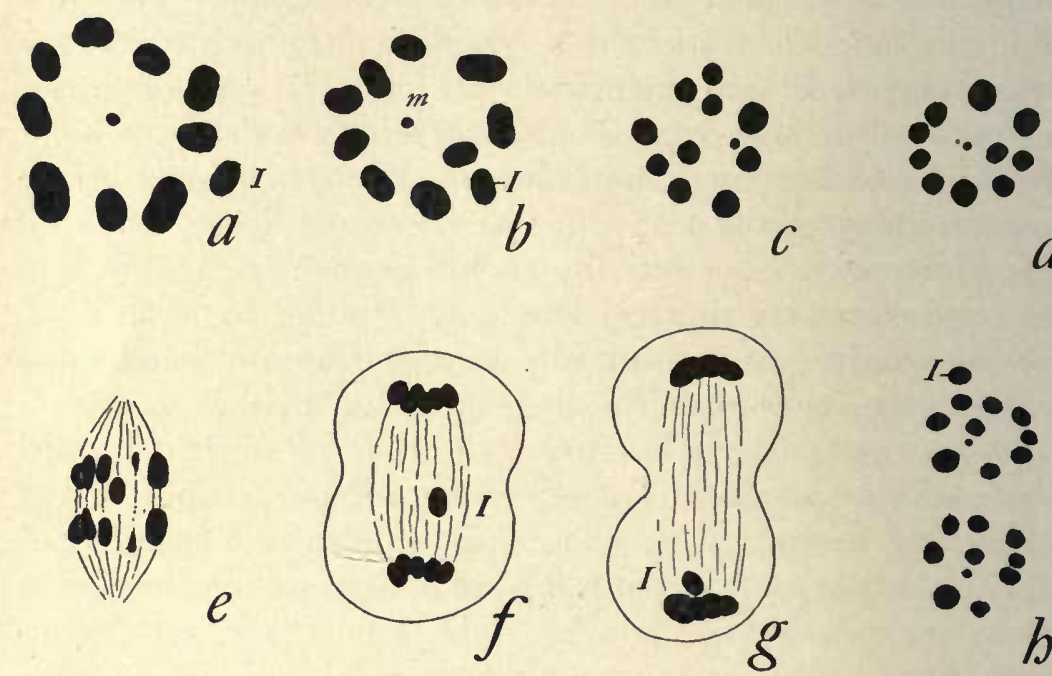

\section{$e$}
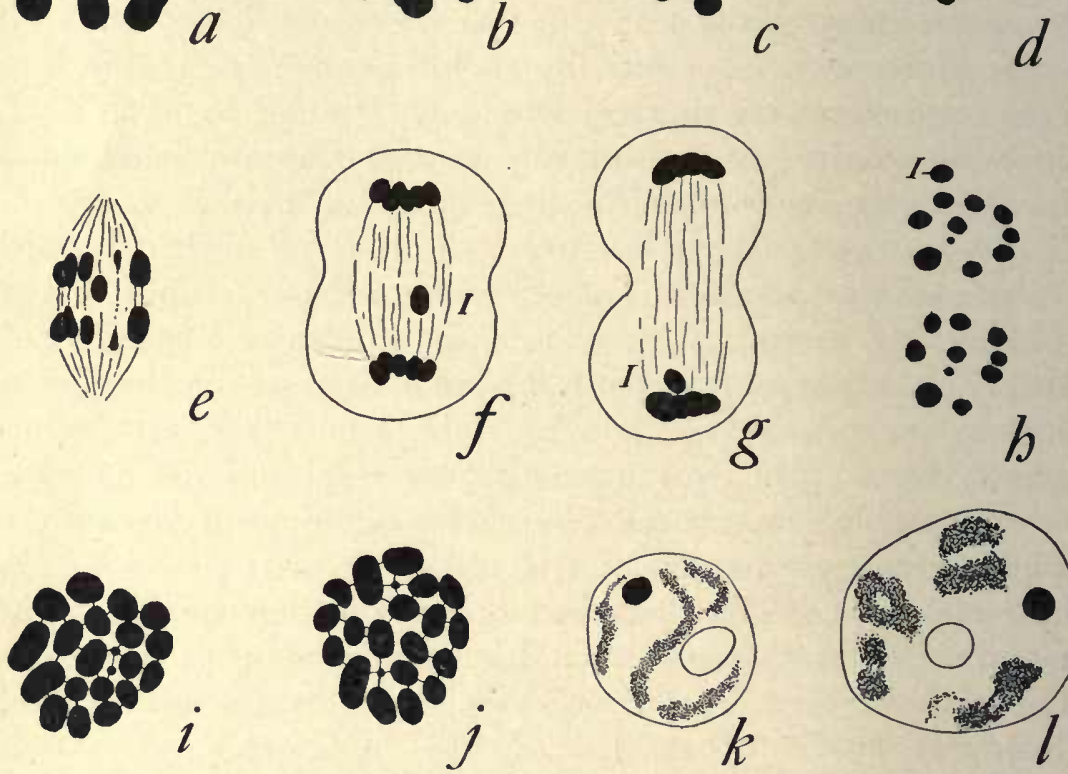

$b$

Fig. 3

M. terminalis (Montgomery's material (Nos. 3-II), 2 I-chromosome form)

$a, b$, first division, polar view (Photo $\mathrm{I}$ ); $c-d$, second division; $e, f, g$, side views of second division (Photos 14, I 5); $h$, sister-groups from the same spindle, in one section, anaphase second division, one showing ro chromosomes the other II.

$i-j$, spermatogonial groups, 2 I chromosomes; $k-l$, early and late growth-period.

chromosome has throughout the early and middle growth-period the form of a single spheroidal or ovoidal intensely staining chromosome-nucleolus, which shows in brilliant contrast to the other chromosomes (Fig. 3, $k, l$, Photo 24). This body is sometimes slightly constricted in the earlier period. Later it is always con- 
stricted, assuming the bipartite form in which it enters the equatorial plate to form the eccentric chromosome. Throughout the growth-period a large plasmosome is also present, usually separate from the chromosome-nucleolus. In properly stained sections these two bodies differ so markedly in staining reactions that they cannot for a moment be confused. In hæmatoxylin preparations the chromosome-nucleus is intensely black, the plasmosome pale yellowish, bluish or gray. In Montgomery's safranin-gentian preparations (though now somewhat faded) the former is bright red, the latter bluish or nearly colorless.

There are no females in Montgomery's material; but inview of the relations known in many other related forms it may safely be concluded that the II-chromosome spermatozoa are female-producing, and that the female somatic number in this race is 22.

2 Individuals with twenty-two Chromosomes in the somatic Groups of both Sexes including a pair of unequal Idiochromosomes in the Male, and a Pair of equal large ones in the Female

This condition has been found in seven males and four females, all three species being represented. The three species closely agree in all the phenomena.

To the males of this type precisely the same description applies as to the foregoing case except that a small idiochromosome is present in addition to the "odd" or "accessory" chromosome. The latter is now indistinguishable from a "large idiochromosome," and the identity of these two forms of chromosomes, on which I have laid stress in former papers, is thus fully demonstrated. This appears most clearly in the maturation divisions. In the first division the chromosomes show the same grouping as in the $2 \mathrm{I}-$ chromosome forms, but a small idiochromosome accompanies the "accessory," frequently lying beside it outside the principal ring, though sometimes being in or inside the latter (Fig. 4, $a-j$, Photos 2, 3). This chromosome is always recognizable as the smallest of all the chromosomes except the $m$-chromosomes, and it is in general about half the size of the large idiochromosome or slightly less. All the chromosomes now divide equally (Fig. 4, $l$, 
Photo II), I2 chromosomes passing to each pole. The second division immediately follows without the intervention of a "resting stage," and the chromosomes undergo the same regrouping as that described for the 2I-chromosome forms. As this takes place; the two idiochromosomes conjugate to form an unequal bivalent (precisely as in Lygaeus or Euschistus); so that when the equatorial plate reforms but II (instead of 12) chromosomes appear in polar view (Fig. 5, $a-c$, Photo I2). The idiochromosome-bivalent now usually lies near the center of the group (contrasting with the first division), and the $m$-chromosome is usually not far from it. Such views are almost indistinguishable from those of the $2 \mathrm{I}$-chromosome individuals, since the small idiochromosome is covered by the large one and only appears in side view. In the course of the division the idiochromosome bivalent separates into its two components, which pass to opposite poles, while all the other chromosomes divide equally. The idiochromosomes at first separate more rapidly than the other daughter-chromosomes(Fig. $5, f, h)$, as in other genera, but as the division proceeds the reverse condition prevails, so that the two idiochromosomes are seen lagging on the spindle between the diverging daughter groups (Fig. 5 $i-l)$. In the later stages one passes to each pole. There is much variation in this process. Often the two move at the same rate so that in the late anaphases one may be seen entering each pole (Fig. $k, l$, Photo I 7 ). Not uncommonly, however, one or the other lags behind upon the spindle (usually the large one, though Fig. 5, j, shows the reverse case) giving a condition that exactly resembles that seen in the $2 \mathrm{I}$-chromosome forms (Fig 5, $m, n)$, but earlier anaphases in the same cysts at once show the difference. It is no less conclusively shown by polar views of the late anaphases, in which each daughter-group is seen to consist of I I chromosomes, ten of which are duplicated in the two while the the eleventh is in one case the large, in the other the small idiochromosome (Fig. 5, q, r, s, t).

The difference between the two types is shown with almost equal clearness by the chromosome-nucleoli of the growth-period. In the $2 \mathrm{I}$-chromosome type, as already stated, this body is single. A similar appearance is sometimes given in the 22-chromosome indi- 

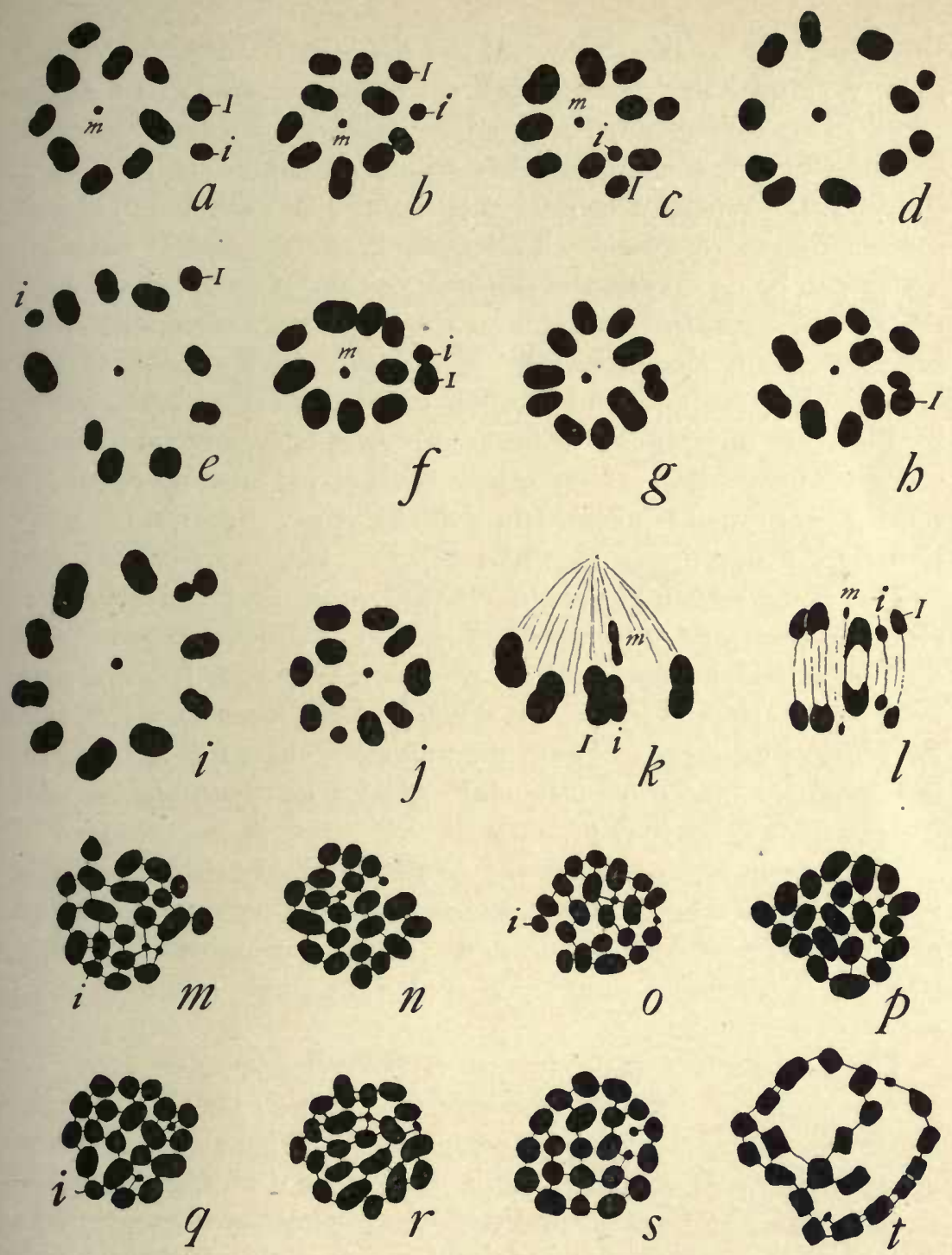

FIG. 4

22-chromosome forms

$a-l$, first division; $a, b$, term. No. 19, typical (Photo 2); c, term. No. 12 (Photo 3); $d, e$, fem. No. 29 $f$. term. No. $12 ; g, h$, term. No. 19 , idiochromosomes united; $i$, fem. No. 29 , same condition; $j$, gran. No. 47 ; $k$, fem. No. 29, first division, side view, idiochromosomes united; $l$, fem. No. 46 (Photo I1), first division, anaphase, division of both idiochromosomes.

$m-q$, spermatogonial groups; $m$, term. No. $19 ; n, o$, fem. No. $46 ; p, q$, fem. No. 29 .

$r-t$, ovarian groups; $r$ term. No. $24: s$, term. No. $44 ; t$, term. No. 23 , exceptional form and grouping. 
viduals, owing to close union of the two idiochromosomes. But in very many cells of this period the chromosome-nucleolus consists of two very distinct unequal moieties, in contact (Fig. 6, a, $b$, Photo 25), or not infrequently widely separated (Fig. 6, $c, d$. Photo 26). When in contact they form a double body closely similar to the idiochromosome-bivalent of the second division. There can be no question of confusing either of these bodies with the plasmosome, since the latter, showing its characteristic staining reactions, is also present.

In the late prophases of the first division the idiochromosomes, if previously united, almost invariably part company to divide as separate univalents, as in other Hemiptera; but they usually remain near together outside the principal ring. Only very exceptionally do they divide together.

The spermatogonial groups (Fig. 4, $m-q$ ) uniformly show 22 chromosomes, and in some cases the small idiochromosome may be recognized by its small size $(m, q)$. This is, however, not nearly so marked as in the first division, since it now appears relatively twice as large, owing to the univalent character of the other chromosomes, and often it cannot certainly be distinguished from the smaller of these $(n, p)$.

These facts make it clear that if the small idiochromosome be supposed to disappear, the entire series of phenomena would become identical with those shown in the 2 I-chromosome individuals, the large idiochromosome now appearing as the odd or "accessory" chromosome.

The unreduced female groups of this type (ovarian cells) are closely similar to those of the male (Fig. $4, r-t$ ) but a small idiochromosome can never be distinguished. The absence of this chromosome cannot be so convincingly shown in Metapodius as in such forms as Lygaeus or Euschistus, owing to its greater relative size. Nevertheless, after the detailed study of many female groups I am convinced that this chromosome is not present, and that all the chromosomes may be equally paired. Apart from analogy, therefore, I think the conclusion reasonably safe that in Metapodius, as in other forms, the unequal idiochromosomepair of the male is represented in the female by a large equal pair, 

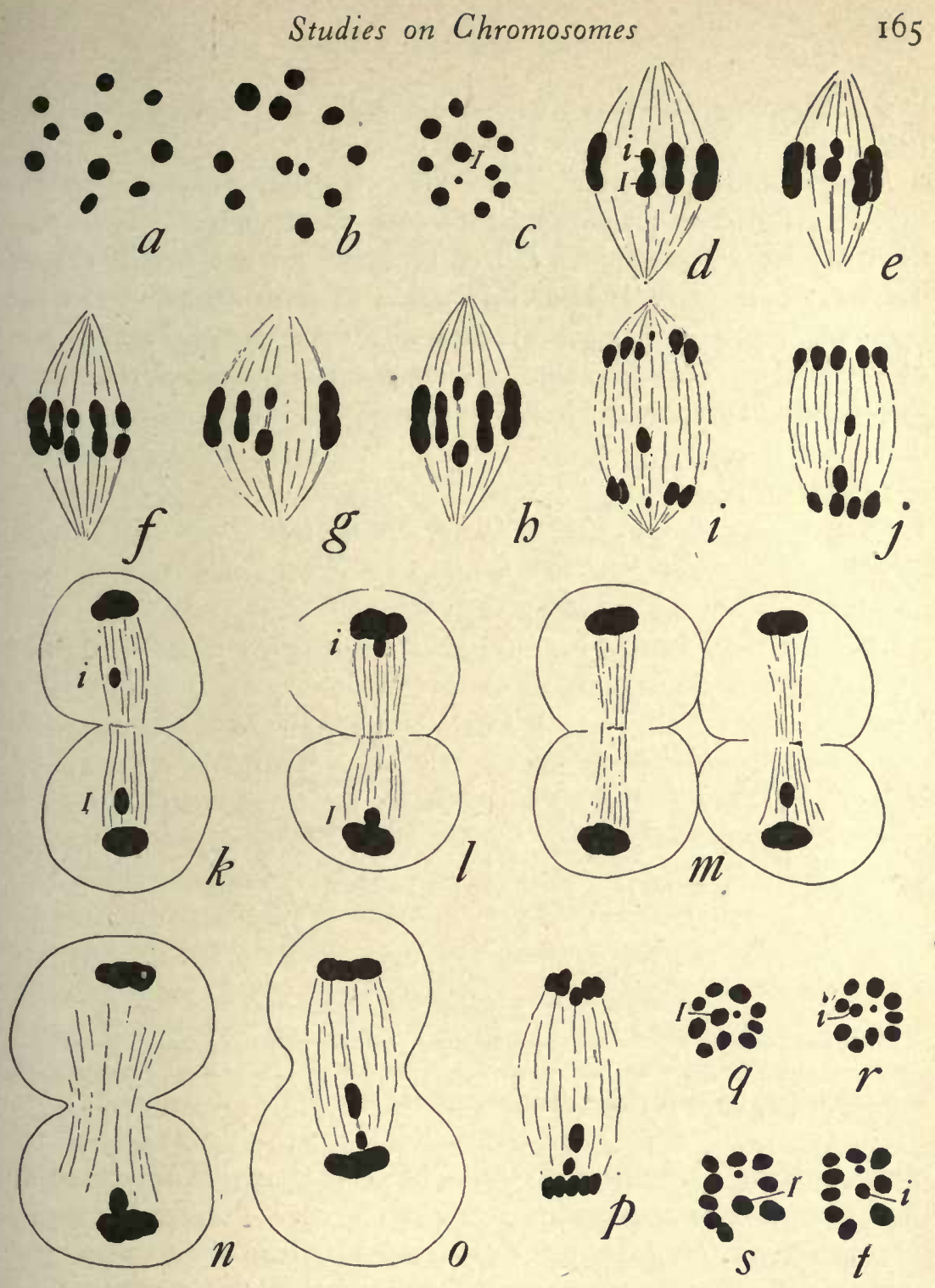

Fig. 5

22-chromosome forms

$a-c$, second division, polar view; $a$, fem. No. 19; $b$, fem. No. 28; c, gran., 47 (Photo 12).

$d-p$, second division, side view; $d-h$, fem. No. 29 , metaphases, separation of idiochromosomes; $i j$, term. No. 19, anaphases, lagging of one idiochromosome; $k-m$, gran., No. 47 , late anaphases (Photo 17 ); $n$, term., No. I9, late anaphase, lagging large idiochromosome; 0 , fem., No. 46, exceptional condition, both idiochromosomes passing to one pole (Photo I8); $p$, term. No. I 9 , similar form; $q, r$, term., No. I9, sister anaphase groups, from the same spindle; $s, t$, fem., No. 29 , the same. 
and that, accordingly, the usual rule holds in regard to fertilization.

Exceptional conditions. There are two conditions, rarely seen, that are of interest for comparisons with other species. Now and then the idiochromosomes fail to separate for the first division, but remain in more or less close union to form an asymmetrical bivalent, which in side view is seen to form a tetrad (Figs. $4, f-i$, $k$, Photo 3). This bivalent undergoes an equation division, in this respect agreeing with the conditions uniformly seen in Syro-
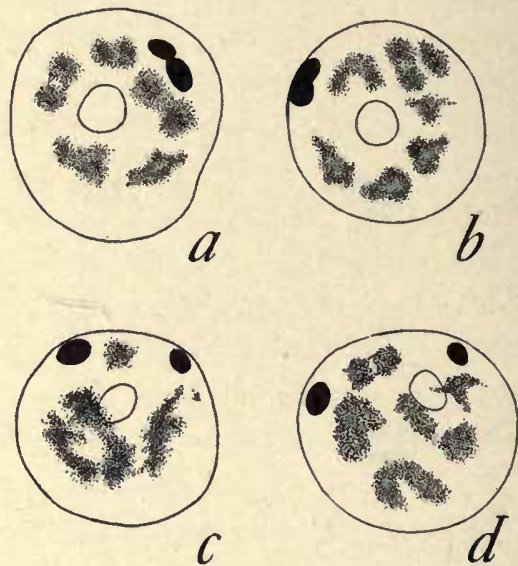

Fig. 6

M. femoratus (No. 29) 22-chromosome form

Four nuclei from growth-period showing diffused ordinary chromosomes, condensed chromosomenucleoli and plasmosome; in $a$ and $b$ the two idiochromosomes are united to form double chromosomenucleoli (Photo 25); in $c$ and $d$ they are separate (Photo 26).

mastes (Gross '04, Wilson 'o9), and differing from that occurring in the Coleoptera or Diptera (Stevens 'o6, '08a). A rarer but more interesting deviation from the type is the failure of the idiochromosomes to separate in the second division, both passing together to the same pole (Fig. 5, o, p, Photo I8). Since the other chromosomes divide equally it may be inferred that in this case one pole receives 12 chromosomes and the other but IO. This has been seen in only three cells and is doubtless an abnormality. It may however, possess a high significance as forming a possible point 
of departure for the origin of the whole series of relations observed in the genus.

\section{Individuals possessing twenty-three Chromosomes; one Supernumerary}

This condition exists in all three species and has been found in seven males and four females. In four of these males the supernumerary is large (of approximately the same size as the small idiochromosome, as in Fig. $\mathrm{I}, g-i$ ); in three it is no larger than the $n t$-chromosomes (as in Fig. $\mathrm{I}, j-l$ ), and is indistinguishable from the latter save in behavior. In each case, as already described, the spermatogonia show 23 chromsomes and the first division I3; and in those showing a small supernumerary in the first division the spermatogonia always show three very small chromosomes.

The grouping in the first division, though conforming to the same general type, shows many variations of detail, as may be seen from Fig 7, $a-l$, Photos $4-6$. It is a curious fact that the form of grouping is to some extent characteristic of the individual. For example, the typical arrangement, with both idiochromosomes and supernumerary outside the ring, is very common in Nos. 43 (Fig. I, $j-l$ ) and $20(7, a-c)$, very rare in Nos. I, 2 (Fig. 7, $i$ ) and 49 (Fig. $7, f-h$ ). In No. 49 , very many of the first division metaphases show both supernumerary and small idiochromosome lying inside the ring (Fig. $7, g-h$ ). I am unable to suggest an explanation of this.

In this division all the chromosomes divide equally (Fig. $7, m-p$ ), so that each secondary spermatocyte receives 13 chromosomes. The usual regrouping now takes place, and the idiochromosomes couple as usual to form. an asymmetrical bivalent. The supernumerary sometimes remains free (i. e., not attached to any other), in which case 12 chromosomes appear in polar view (Fig. 8, $b, d$ ). Much more frequently the supernumerary attaches itself to the idiochromosome bivalent to form a triad element, polar views now showing but II chromosomes $(8, a, c)$, one of which is compound. The three components of such triads usually lie in a straight line, the supernumerary being attached sometimes to the small idio- 
F1G. 7

23-chromosome forms, one supernumerary

$a-h$, first division, polar views, one large supernumerary; $a-c$, term., No. 20, typical grouping; $d-e$, gran., No. $48 ; f, g, h$, gran., No. 49 (Photo 5 ).

$i-l$, first division, polar views, one small supernumerary; $i$, term. No. I. (Photo 6 ); $j-l$, term. No. 43 typical grouping in $k$.

$m-p$, first division, side-views; $m$ and $n$ (term. No. 43 ) show division of $I, i, m$, and small s; 0 , term., No. 20, division of $I, i$, and large $s ; p$, term., No. 43 , division of $m, i$, and small s.

$q-s$, spermatogonial groups from individuals with one large supernumerary; $q, r$, term., No. 20; $s$, gran., No. 49.

$t-y$, spermatogonial groups from individuals with one small supernumerary; $t, u$, term., No. 43 ; v-y, term., No. 2 (Photo 29). 


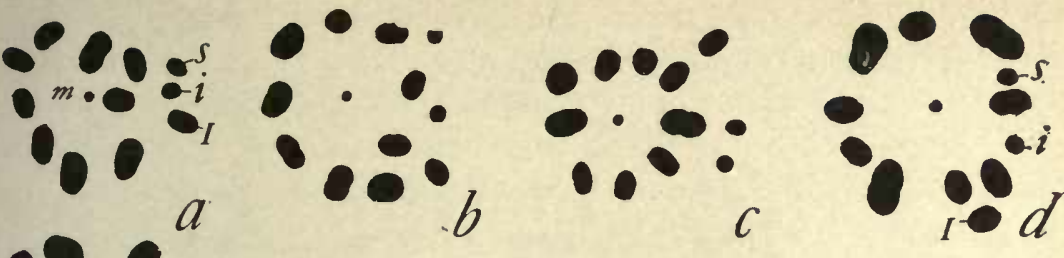

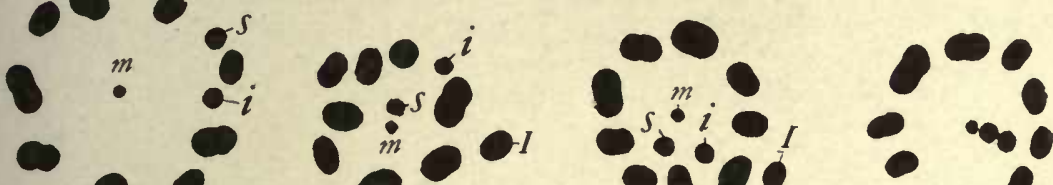

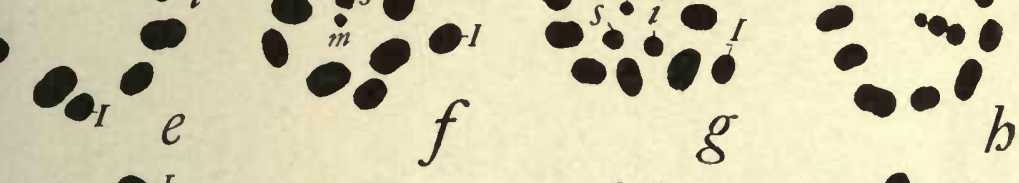

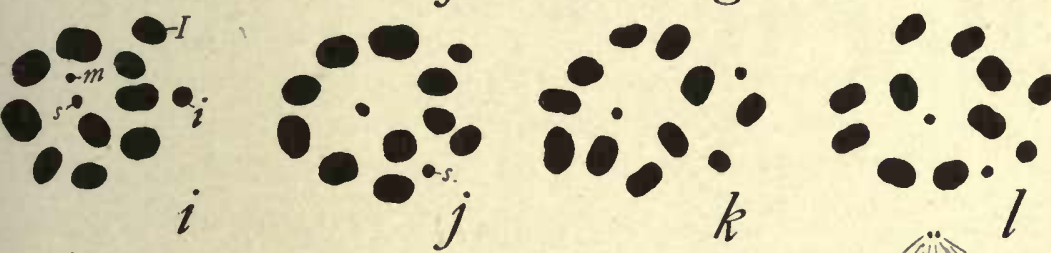

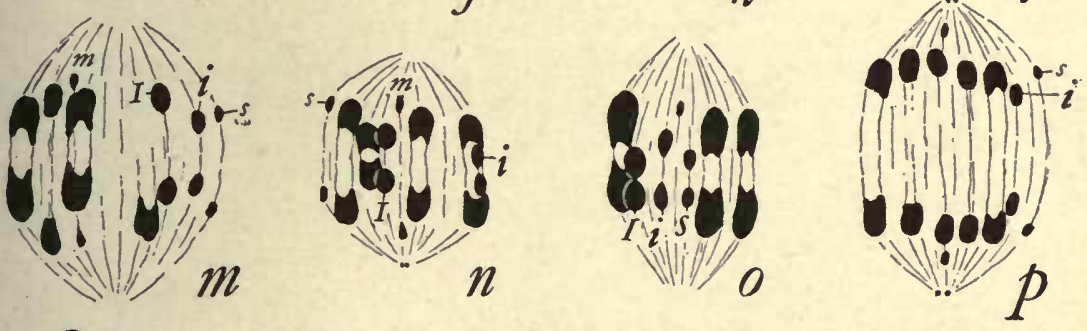

अ8.
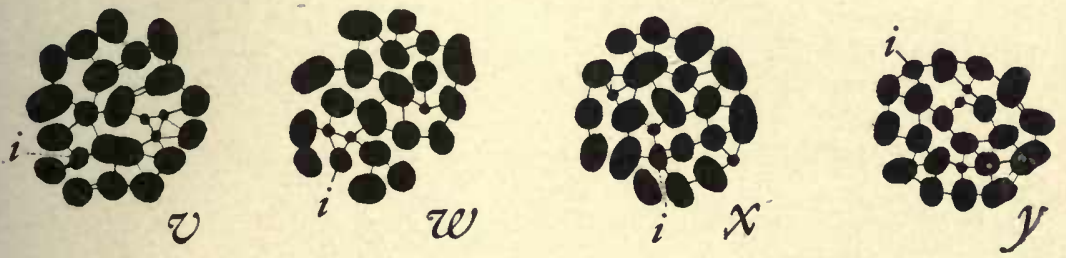

Frg. 7 
chromosome, sometimes to the large, or not infrequently lying between the two (Fig. 8, $g, h, o-q$ ).

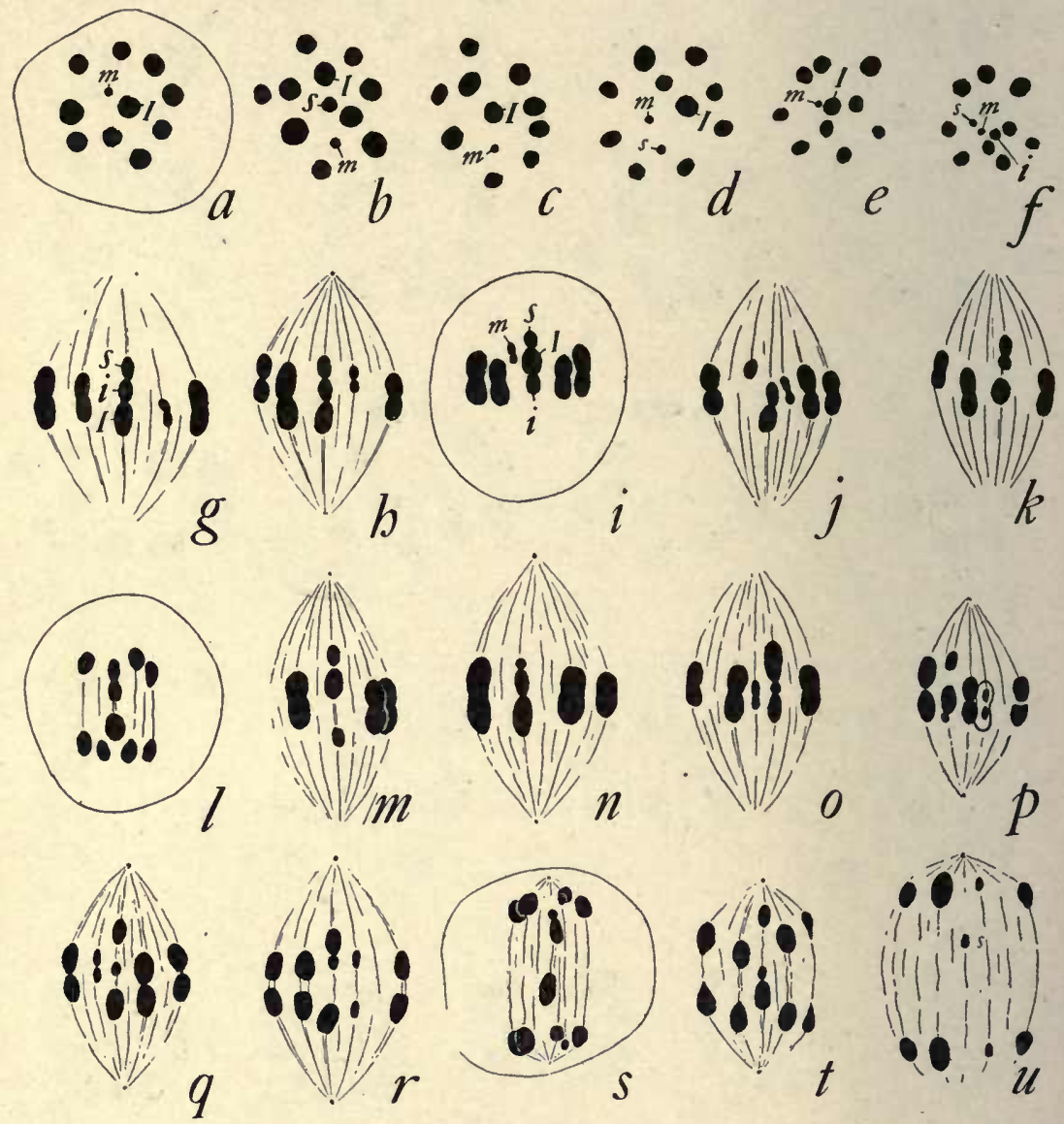

Fig. 8

23-chromosome forms, one supernumerary

$a-f$, polar views, second division; $a$, gran., No. 49, large supernumerary attached; $b$ (same cyst)supernumerary free; $c-d$, similar views of terminalis, No. 43 , with small supernumerary; $e-f$ (No. 43$)$, sister groups from same spindle, pclar views.

$g-m$, side-views, second division, from gran., No. 49 , with large supernumerary, free in $j$, attached in the others.

$n-u$, similar views from individual (term., No. 43) with small supernumerary; in $u$ the supernumerary is free. 
In the ensuing division, if the supernumerary lies free it passes without division as a heterotropic chromosome to one pole $(8, u)$. When connected with the idiochromosome bivalent it passes to one pole attached to one or the other of the idiochromosomes (Fig. $8, k-m, p-t)$. In either case one pole receives I I chromosomes and one 12 (Fig. $8, e, f$ ); but since the supernumerary may accompany either idiochromosome four classes of spermatid nuclei are formed, namely:
(1) $10=I=1 \mathrm{I}$
(2) $10+i+s=12$
(3) $10+i=11$
(4) $10+I+s=12$

As described in an earlier paper ('o7a), there is a tendency for the supernumerary to be associated more often with the small idiochromosome than with the large, and classes I and 2 are accordingly more numerous than 3 and 4 . I was formerly inclined to attribute importance to this as pointing to the more frequent occurrence of the supernumerary in the male than in the female. The larger series of data now available leads me to doubt whether it has much significance; for if (leaving the 2I-chromosome forms out of account) the whole series of forms be taken together, one or more supernumeraries are found in 27 out of 34 males, and in I 5 out of 19 females - about 80 per cent in each case. It appears therefore that in the long run the supernumeraries are distributed between the two sexes with approximate equality.

Figs. $7, q-s$ show spermatogonial groups from individuals with one large supernumerary, but in none of them can this chromosome or the small idiochromosome be certainly distinguished. Fig. $7, t-y$ are from individuals with one small supernumerary, each showing three very small chromosomes. In $t$ and $u$ the small idiochromosome is doubtful. Fig. $7, v-y$, on the other hand, are from an individual (terminalis, No. 2), showing great numbers of very fine spermatogonial groups, in almost all of which the small idiochromosome is at once recognizable. The same is true of a second individual from the same locality. These two individuals, from the Paulmier collection, were the first material I examined and found so puzzling until the examination of another similar individual, No. 43 , cleared up the nature of the second division. 


\section{Individuals with twenty-six Chromosomes; four Supernumeraries}

It will be convenient to consider this type before the 24- and 25chromosome forms, since the material is more favorable for an account of the remarkable phenomena occurring in the second division. Of these individuals there are seven males and three females, all three species being represented. Unfortunately very few perfectly clear spermatogonial groups are shown; but the spermatocyte-divisions and cells of the growth-period are particularly well shown and in large numbers of cells. In all but one of these individuals the four supernumeraries are large and of nearly equal size. In one (femoratus No. 40) two are large and two small. The latter case, already shown in Fig. $2, j-l$, is further illustrated by Fig. $9, h, i, j, n, o$. Two of these ( $h$ and $i$ ) show but three supernumeraries in the first division, a common appearance in this individual (see p. 186). Fig. 9, $a-l$, show varying arrangements of the 16 chromosomes that appear in the first division, the most typical ones being $k$ and $l$. In $9, a-c, k, l$, both idiochromosomes and the four supernumeraries lie outside the ring. In $9, g$, all but the large idiochromosome are inside the ring.

In some of these slides the compound chromosome-nucleoli are shown with great distinctness in many cells of the growth-period. This body usually has the form of a flat plate that lies next the nuclear wall (Fig. Io, $q, r$ ) so that a clear view of all the components can only be had in tangential sections. Thus viewed (Fig. ro, $s-u$, Photo 28 ) it may often be seen to consist of six components one of which (the large idiochromosome) is about twice the size of the others and is usually at one side or end of the group. The other five evidently represent the small idiochromosome and the four supernumeraries. In side view (Fig. 10, $q, r$ ) not more than three or four of the components, can as a rule be recognized. In a considerable number of cases these six chromosomes are not aggregated to form a single body but form two or more simpler bodies.

The second division in these forms presents an extraordinary 


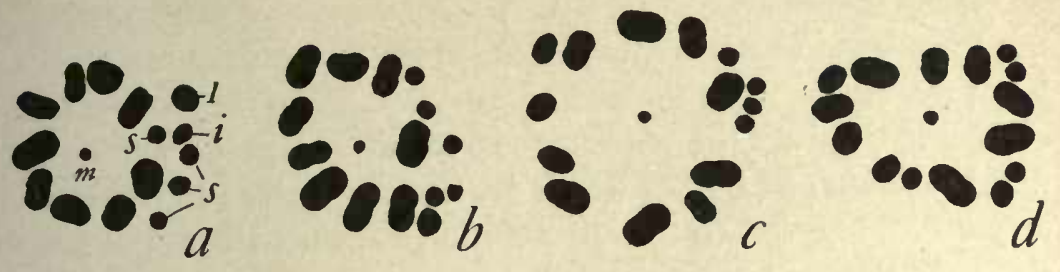

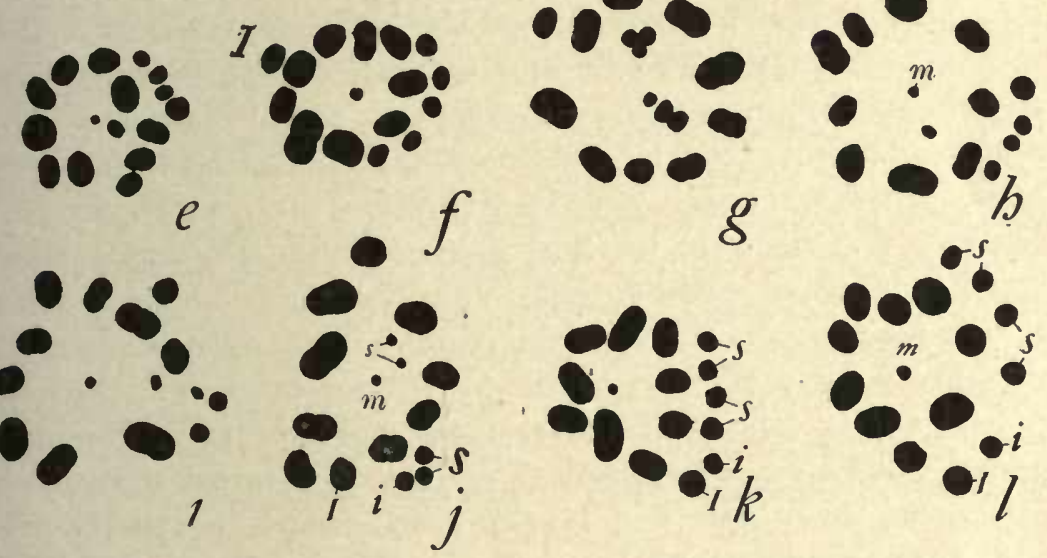

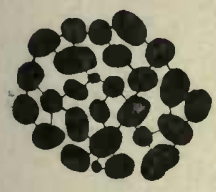

m

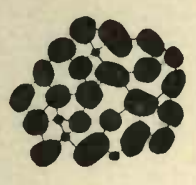

n

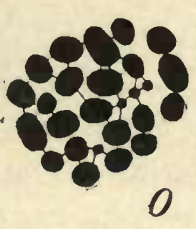

0

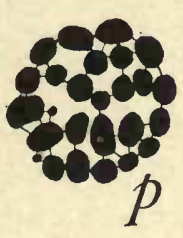

$p$

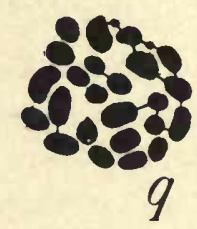

Fig. 9

26-chromosome forms, four supernumeraries

$a-g$, first polar, supernumeraries large and equal; $a-d$, fem., No. $+2 ; e$, gran., No. $55 ; f$, gran., No. 59 ; g, gran., No. 60.

$h-j$ first polar, from (fem., No. 40, with two large supernumeraries and two small; all of these are shown in $j$, ( $f f$. Fig. 2, j), while in $h$ and $i$ one is missing (see p. 186).

$k$, first polar, term., No. $36 ; l$ from same individual (Photo 9).

$m-0$, spermatogonia groups; $m$, fem., No. 42, abnormal group with 27 chromosomes; $n, 0$, fem., No. 40. showing two small supernumeraries.

$p-q$, ovarian groups, gran. No. 61 . 
appearance which I at first thought must be due to an artificial clumping together of the chromosomes through defective fixation; but the study of very many of these figures convinced me that such is not the case. As in the preceding types, ten of the chromosomes, including the $m$-chromosomes, have the form of symmetrical dumb-bell shaped bodies which are equally halved in the ensuing division. The remaining chromosomes are usually aggregated to form a compound element (Fig. I0, $h-l$, Photos 22, 23) in which may be very clearly distinguished the same components as those that appear in the chromosome-nucleoli of the growth-period; and the size-relations make it evident that one of them is the large idiochromosome, one the small, while four are the supernumeraries. In other words, these six chromosomes, which divide as separate univalents in the first division, have now again conjugated to form a hexad group. This compound element almost always lies near the center of the group. Polar views of this division accordingly show typically I I chromosomes, of which the central one is compound (Figs. Io, $a-g$, Photo I3). Not infrequently, however, one or more of the supernumeraries may be separate from the others (Fig. Io, $f, g$ ), the apparent number in polar view varying accordingly.

In side views the grouping of the components of the hexad element is seen to vary considerably though the large idiochromosome is more frequently at one end of the group. In the ensuing division the other ten chromosomes divide equally, while the hexad element breaks apart into two groups that pass to opposite poles (Fig. Io, $l-p$ ). The distribution of the various elements is difficult to determine exactly, since they always lag behind the others in the anaphases and are scattered along the spindle in such a way as often to give confusing pictures. The study of many such anaphases leads me to conclude, however, that at least one of the smaller components always passes to the opposite pole from the larger one, while the other four undergo a variable distribution. In Fig. Io, $l$, the group is just separating into three toward each pole; in $10 \mathrm{~m}$, it is quite clear that three of the small ones are passing to one pole, while the large one and two small ones are passing to the other, and Fig. Io, $n$, is probably a similar case. In these 
cases it seems clear that each pole receives 13 chromosomes, as follows:

$$
\text { a } 10+I+2 s=13 \quad b \quad \text { 10 }+i+2 s=13
$$

Fig. I0, 0 , on the other hand, shows a perfectly clear case in which the hexad element has separated into a 2-group and 4-group: Fig. Io, $p$, shows what is probably a later stage of the same type. In both these cases one pole appears to receive 12 and one 14 as follows:

$$
\text { a } 10+I+3^{s+14} \quad b \quad \text { } 10+i+s=12
$$

one pole receiving but one supernumerary, and the other three. The cases in which all of the components may be clearly recognized in the anaphases are comparatively rare, and in the greater number of them the distribution of the supernumeraries appears to be symmetrical. Of their unsymmetrical distribution in some cases there can be no doubt (and the same is true of the I4-chromo, some form, as described beyond). The few undoubted cases of this all show one to one pole and three to the other (as in Fig. Io, o$p$ ), and I have never found a case in which all four pass to the same pole.

It seems, therefore, probable that in the 26-chromosome type there are at least six classes of spermatozoa, as follows:
(1) $10+I+2 s=13$
(3) $10+I+s=12$
(2) $10+i+2 s=13$
(5) $10+I+3 s=14$
(4) $10+i+3 s=14$
(6) $10+i+s=12$

It is possible that the following four additional classes may be produced:
(7) $10+I+4 s=15$
(9) $10+I=11$
(8) $10+i=11$
(10) $10+i+4 s=15$

Perfectly clear spermatogonial figures of this type were rarely found, though many of them show approximately 26 . The normal group of fem., No. 42, is shown in Fig. 2, $h$. Two groups from fem. No. 40 (with two small and two large supernumeraries) are shown in Fig. 9, $n$, o, each having 26 chromosomes including four small ones (cf. Fig. 2, $k$ ). Two ovarian groups from gran., No. 6I, 
Fig. I0

26-chromosome forms

$a-g$, second division, polar, $d$ from fem. No. 40 , the others from fem. No. $42 ; a$, (Photo 13 ) $b, c$, show a single central hexad; in $e$ and $g$ the components are more loosely united; in $d$ and $f$ one supernumerary is free.

$h-p$, side-views, second division, from fem. No. 42 (Photos 22, 23) explanation in text.

$q-u$, growth-period, gran.. No. $60 ; q$ and $r$ show the compound chromosome-nucleolus in oblique and side-view, $s, t, u$, en face. 


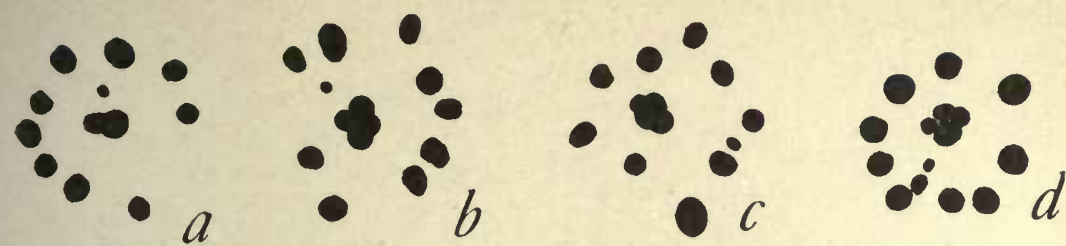

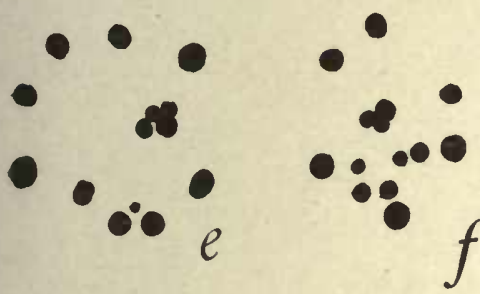

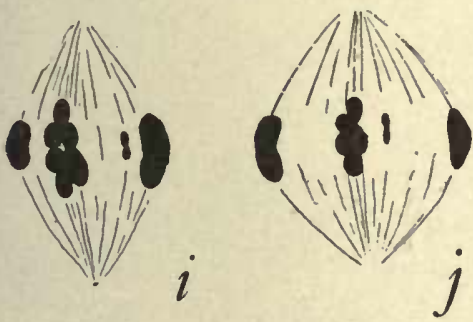
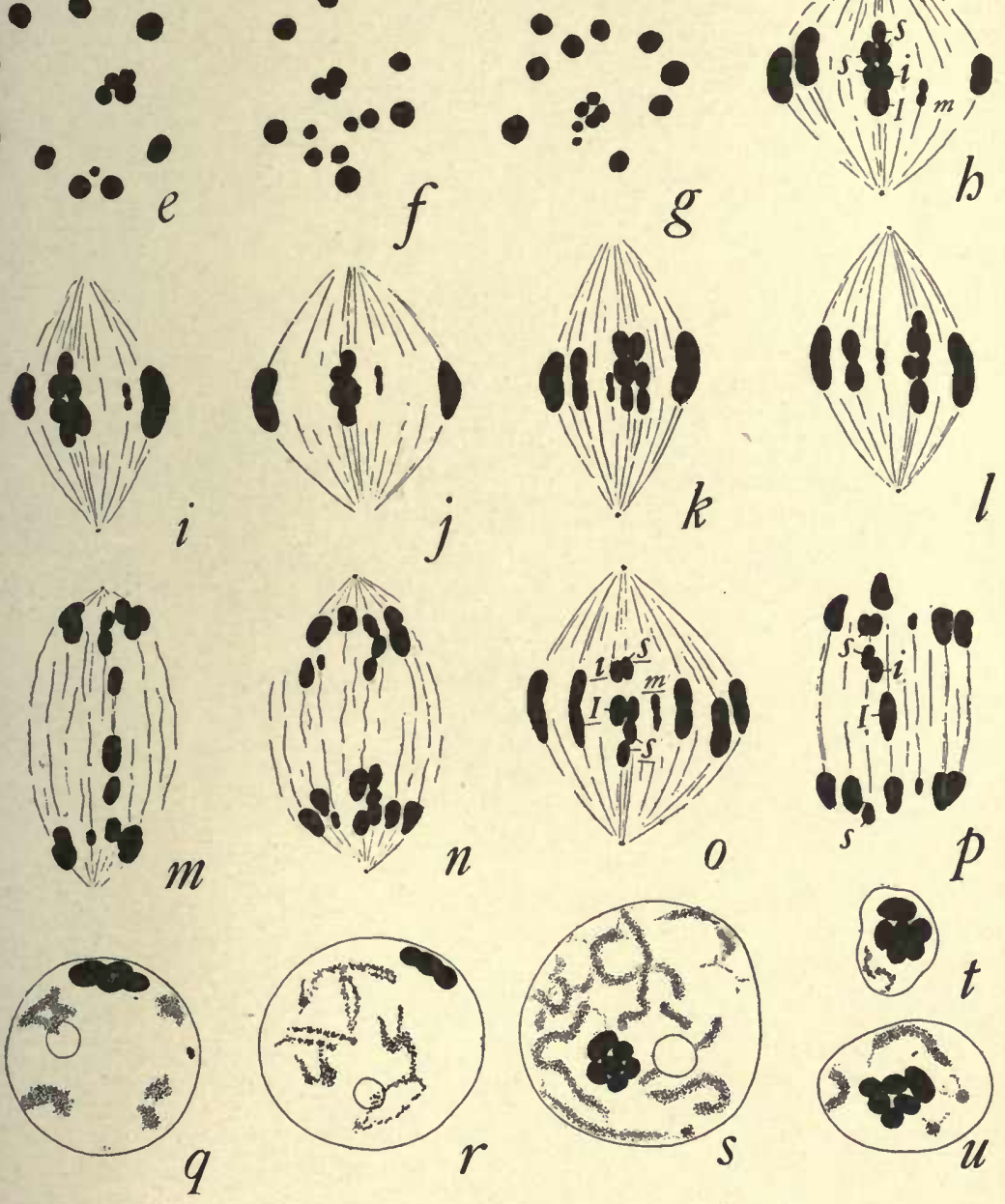
b
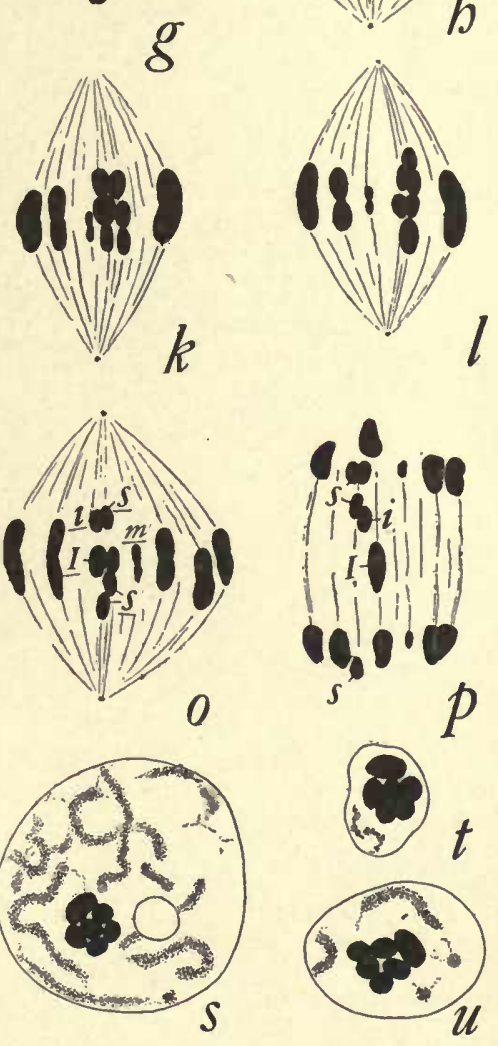

FIG. 10 
FIG. II

24-chromosome forms, two supernumeraries.

$a-e$, term., No. 21, first polar, showing various groupings; $g$, the same, gran., No. 52 (Photo 7 ). $h$, term., No. 21, second polar, tetrad element near center.

$i-0$, somatic groups from individuals with two large supernumeraries; $i-l$, spermatogonial groups from term. No. $21 ; m, n$, ovarian groups from fem. No. 31 ; o, ovarian group, fem., No. 45 .

$p-r$, spermatogonial groups from fem., No. 22, with one large supernumerary and one small; Photo 30).

$s-w$, second division, side-view; $s$, term., No. 21; $t-w$, gran., No. $5^{2}$ (Photo 21 ). 


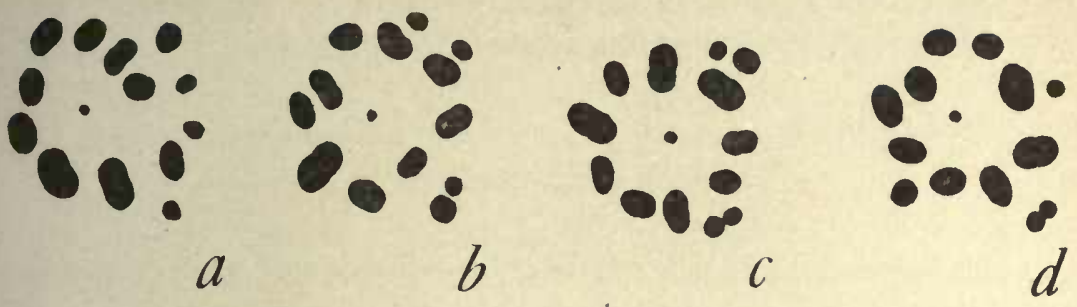

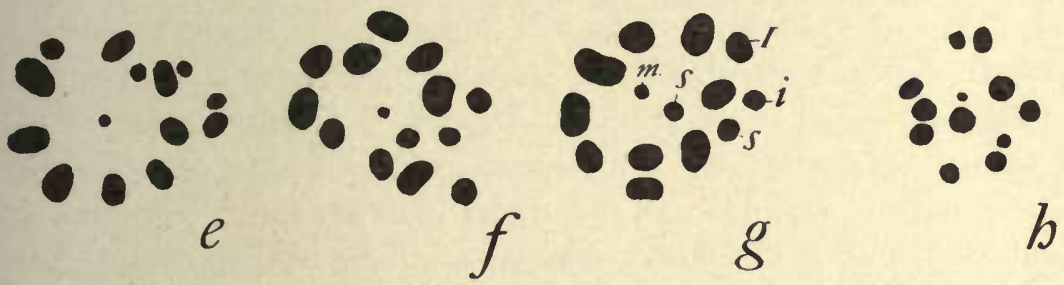

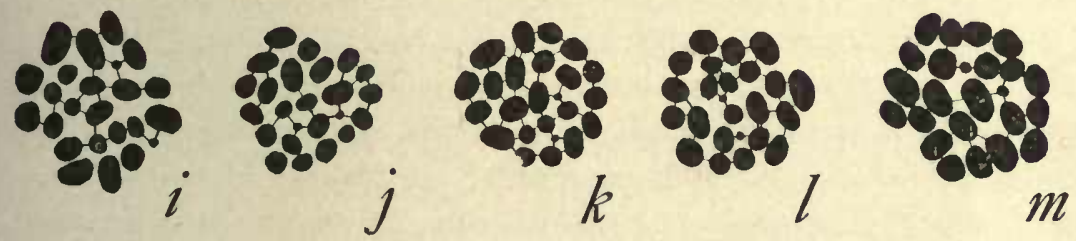
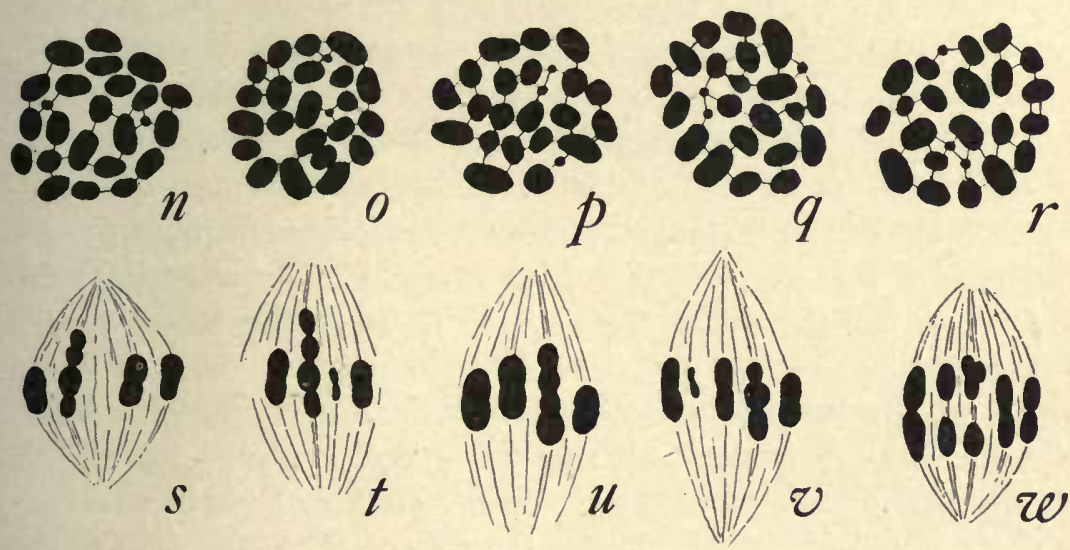

Fig. 11 
are shown in Fig. 9, $p, q$. Fig. 9, $m$, shows a spermatogonial group from fem., No. 42 , that is abnormal in showing with perfect clearness 27 instead of 26 chromosomes (cf. Fig. 2, $h$ ).

\section{Individuals with twenty-four Chromosomes; two Supernumeraries}

The material for these individuals and those of the 25-chromosome class, is less satisfactory than in the preceding case, but the relations are undoubtedly quite analogous to those just described. The 24-chromosome class is represented by 9 males and 4 females, and occurs in all three species. In one of the males one of the supernumeraries is large (of the same size as a small idiochromosome) and one small; in all the others both are large. Additional figures of the first division, showing variations in the grouping, are given in Fig. II, $a-g$; of spermatogonial groups in Figs. II, $i-r$. Of particular interest is the male, term., No. 22, shown in Photo 30 and in Fig. I I, $p-r$. This individual was, unfortunately, immature showing only spermatogonia and cells in the growth-period; but many perfectly clear spermatogonial groups are shown. These groups uniformly show 24 chromosomes, of which three are very small, while in many cases two others are slightly but distinctly smaller than the others. The latter are evidently the small idiochromosome and the larger supernumerary, while the three small ones represent the $m$-chromosomes and the small supernumerary.

In the second division the two idiochromosomes and the supernumeraries are frequently united to form a tetrad element, various forms of which are shown in Fig. I I, $s-w$. The distribution of these four components is not so well shown in this material as in that of the 26-chromosome class, described above. It is, however, clear that this distribution is inconstant. In cases like those shown in Fig. $\mathrm{I}, s, t$, it is probable that the tetrad divides in the middle, so that each idiochromosome is accompanied by a supernumerary, and each pole receives 12 chromosomes. The cases shown in Fig. I I, v, $w$, prove however that this is not always the case; for in $w$ the large idiochromosome is seen passing to one pole while both supernumeraries, attached to the small idiochromosome, 
are passing to the other. In this case one pole receives I I chromosomes, the other 13. It is evident that in this form there is the possibility of forming six classes of spermatozoa, as follows:
(1) $10+I=11$
(2) $10+i+25=13$
(3) $10+I+s=12$
(4) $10+i+s=12$
(5) $10+I+2 s=13$
(6) $10+i=11$

In none of these individuals is the material very favorable for the study of the chromosome-nucleoli. They are always evidently compound, but only in a few cases can the components be clearly recognized (as in Fig. 2, $c$ ).

\section{Individuals with twenty-five Chromosomes; three Supernumeraries}

No individuals of this type were found in M. femoratus. The other two species are represented by three males and three females but here again the material does not admit of exhaustive study. In one of the females, two of the supernumeraries are large and one small, the ovarian cells showing 25 chromosomes, of which three are very small (Fig. I2, $i-k$ ), a condition seen in every group of which a clear view can be had. The two larger supernumeraries cannot, however, be certainly identified in any of these. In all the other individuals the supernumeraries are of the larger form. Fig. 12, $a, b$, show the first division in one of these cases (term., No. 34); $c-g$ are spermatogonial groups from the same individual; $h$, an ovarian group of the same type. Fig. 12, $m-p$, are from a doubtful case in which nearly all the first division figures show three supernumeraries $(n, o)$, but a single case $(m)$ shows distinctly four.

\section{Individuals with twenty-seven Chromosomes; five Supernumeraries.}

This class is represented by a single very interesting male of granulosus (No. 57), in which only the first division can be satisfactorily examined. Many polar views of this division show I7 chromosomes (Fig. 13, $a-i$, Photo Io), of which two are always 
smaller than the others. One of these, always central in position, is evidently the $m$-chromosome bivalent. Of the remaining six, one is in most cases decidedly smaller than the others-a relation

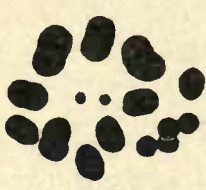

a

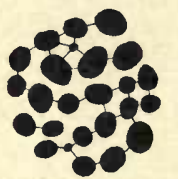

e
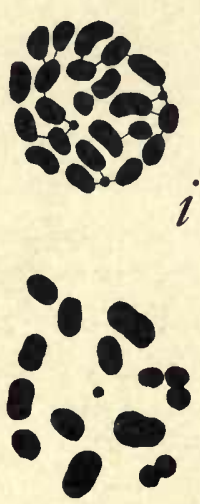

$m$

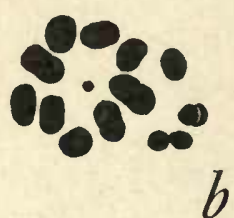

$b$
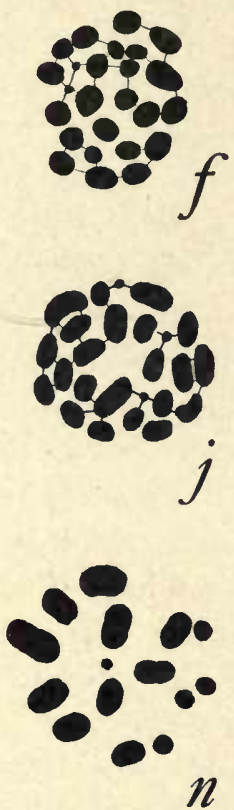

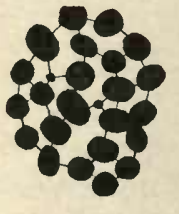

c
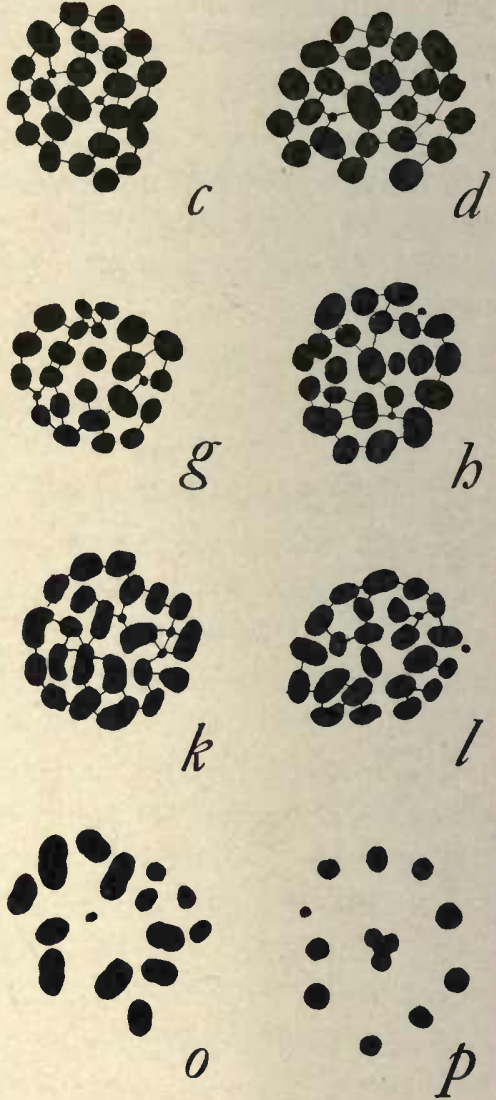

F1G. 12

25-chromosome forms, three supernumeraries

$a, b$, first polar, term., No. $34 ; c-g$, spermatogonial groups from same individual; $h$, term., No. $3^{8}$. ovarian group; $i-k$, ovarian groups from term., No. 27, with two large supernumeraries and one small; $l$, gran., No. $5^{8}$, ovarian groups, three large supernumeraries.

$m, n, 0$, first division, $p$, second division from gran., No. 54 , with three or four supernumeraries.

of which the constancy is attested by the nine figures given of this division. It is evident that in this individual there are four large supernumeraries and one small; and although nospermatogonia 
are clearly shown it may be inferred that the somatic number is 27 . The chromosome-nucleoli in this individual are evidently compound, but in no case can all the components be clearly recognized. The second division shows, as a rule, I I elements in polar view, the central one being compound (Fig. I3, $j-k$ ), but the distribution of the compound element could not be determined.

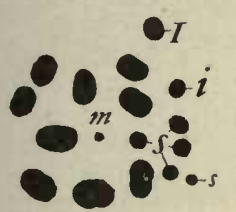

$a$

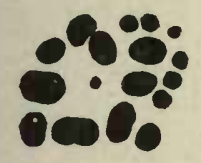

e
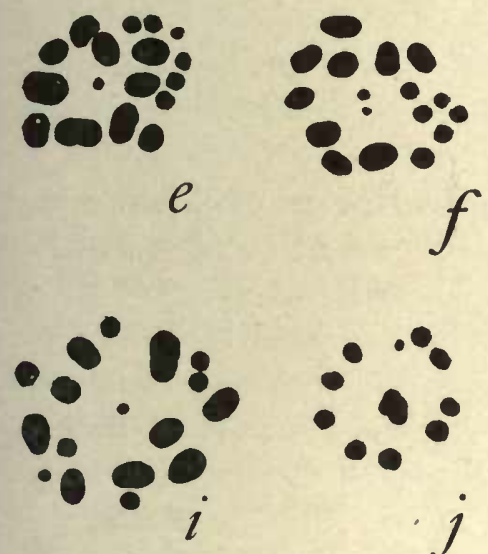

$f$

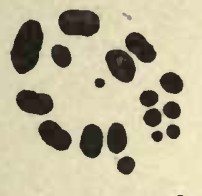

b

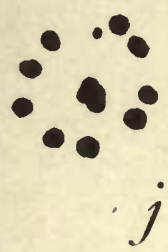

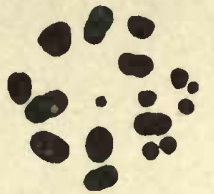

c

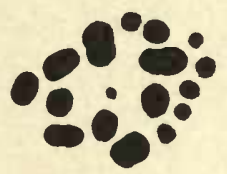

g

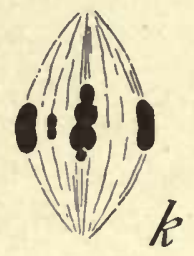

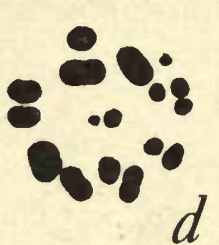
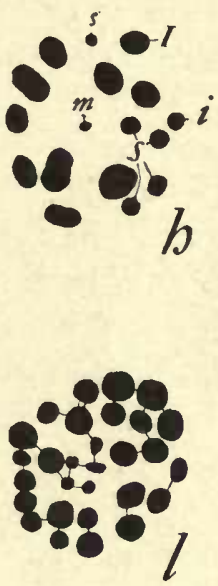

Fig. 13

27 and (?) 28-chromosome forms

$a-i$, first division, from gran., No. 57 , having four large supernumeraries and one small $j$ (polar) and $k$ (side-view), second division, same individual (Photo 10).

l, ovarian group from fem., No. 33 , having three large and two or three small supernumeraries; in this group appear 28 chromosomes.

\section{Individuals with twenty-eight (?) Chromosomes; six} Supernumeraries.

The last case to be considered is that of a single female of femoratus (No. 33), in which the number is either 27 or 28 . A single perfectly clear ovarian group, shown in Fig. $13, l$, shows beyond 
doubt 28 chromosomes, including five smallest ones and three or four next smallest. A few other less clear groups were seen in which appear but 27 chromosomes, the missing one being one of the smallest. In these cases one of the small ones may be hidden among the larger ones; but it is also possible that the 28 -group is an abnormality. In this individual there are probably three larger supernumeraries and either two or three small ones.

\section{C . SUMMARY AND CRITIQUE}

I In the genus Metapodius the number of chromosomes is constant in the individual but varies in different individuals from 21 to 27 or 28 . The number 21 appears only in the males of $\mathrm{M}$. terminalis (Montgomery's material).

2 The number is independent of sex and locality, and is not correlated with constant differences of size or visible structure in the adults.

3 The variation affects only a particular class of chromosomes.

4 The 22-chromosome forms represent the type from which all the others may readily be derived. These forms possess a pair of unequal idiochromosomes which show the same behavior as in Lygæus or Euschistus, all the spermatozoa receiving I I chromosomes, and half containing the large idiochromosome, half the small.

5 In the 2I-chromosome forms the small idiochromosome has disappeared, leaving the large one as an "odd" or "accessory" chromosome. Half the spermatozoa accordingly receive I I chromosomes and half io.

6 Numbers above 22 are due to the presence of from one to five or six additional small chromosomes which show in every respect the same behavior as the idiochromosomes, and are probably to be regarded as additional small idiochromosomes. In the growth period they have a condensed form and are typically associated with the idiochromosomes to form a compound chromosomenucleolus. In the first division they divide as separate univalents. In the second, they are typically (though not invariably) again associated with the idiochromosomes to form a compound element. The components of this element undergo a variable distribution 
to the spermatid nuclei. All the spermatid nuclei receive the haploid type-group of I I chromosomes, half including the small idiochromosomes and half the large; but in addition each may receive one or more supernumeraries. The total number of chromosomes in the sperm nuclei is therefore variable in the same individual.

7 Both the number of the supernumeraries and their size, individually considered, are constant in the individual.

The first question that the foregoing report of results will raise is whether the number and size relations of the chromosomes in each individual are really as constant as I have described them. I have for the most part selected for illustration and description the more typical conditions; but, granting the accuracy of the figures, does such a selection really give a fair presentation of the actual conditions? It is almost needless to say that very many cases might have been shown that would seem to give conflicting results. By far the greater number of these discrepancies are, I believe, only apparent. Numerical discrepancies of this kind are very often evidently due to mere accidents of sectioning or to the superposition or close contact of two or more chromosomes. Again, apparent discrepancies in the size relations of the chromosomes, as seen in polar views, very often arise through different degrees of elongation (particularly in the maturation divisions): But apart from such apparent variations, real deviations undoubtedly occur in almost all of the relations described. Now and then, for example, a spermatogonial or ovarian group is found that clearly shows one chromosome too many (as in Fig. $9, m$ ), ${ }^{9}$ and the same is true of the first spermatocyte-division, but such cases are very rare. The former case is probably a result of an abnormality in the formation of the chromosomes from the resting nucleus, the latter not improbably to a failure of synapsis. Again, both spermatogonial and spermatocyte-cysts are occasionally found in which the number of chromosomes is doubled or quite irregular. These are

-A perfectly clear case of this has been found in the pyrrochorid species Largus cinctus (a particularly fine form for study). In this form the normal male number is iI, the female 12; but in one female three cells were found each of which shows with all possible clearness 13 chromosomes, very many other cells showing the normal number. 
probably due to an antecedent nuclear division without cell division, or to multipolar mitoses such as now and then occur in both spermatogonia and spermatocytes.

As regards the chromosome-nucleoli of the growth-period, the contrast between those of the $2 \mathrm{I}$ and 22-chromosome forms, or between either of these forms and those with higher numbers is usually at once apparent; but in very many cases where more than one supernumerary is present the number of components can only here and there be clearly seen. Contrary to what might be expected from their compact form, the compound chromosome nucleoli seem to be rather difficult of proper fixation, their components often clumping together or breaking up more or less when they coagulate. I infer this from the fact that different slides differ materially in the clearness with which these bodies are shown.

Two discrepancies, apparent or real, should be especially mentioned. One is the difficulty of recognizing the larger supernumeraries in the somatic groups. As already explained, these chromosomes, like the idiochromosomes, appear relatively much larger in the somatic groups than in the first maturation division (owing to their univalence in the latter case); but we should expect to recognize them more clearly, at least in the female groups, than is actually the case. This is perhaps due to their undergoing a greater degree of condensation than the others during the growthperiod; but I am not sure that this explanation will suffice. A second discrepancy, which may involve an important conclusion, is that in perfectly clear views of the first division, the number of supernumeraries is often less than would be expected from the spermatogonial groups. This is notably the case with femoratus, No. 40 (Fig. 9, $h-j$ ), which has clearly 26 spermatogonial chromosomes, but very rarely shows 16 in the first division, the usual number being I5. A similar discrepancy has been noted in other individuals, and in several of the types. Since the typical number in all these cases appears in some or many of the first spermatocytes, I long supposed the occasional deficiency to result from an accident of sectioning. I now incline to believe, however, that in some cases one (or possibly more) of the supernumeraries may really disappear (by degeneration?) during the growth-period, 
and that this may be one way in which their progressive accumulation in number in successive generations is held in check.

For the foregoing reasons it cannot be said that any of the relations described appear with absolute uniformity or fixity. The condition typical of each individual must be discovered by the study and comparison of large numbers of cells. I will only say that prolonged and repeated study has thoroughly convinced me that the relations, as described, may be regarded as being on the whole individual constants. This judgment is based primarily on the exhaustive study of a few of the best series of preparations of individuals of the $21,22,23$, and 26-chromosome types, in which the facts are quite unmistakable and have given the point of view from which the less favorable material of other cases may fairly be examined.

\section{DISCUSSION OF RESULTS}

The principal significance of these phenomena seems to me to lie in their bearing on the general hypothesis of the "individuality" or genetic continuity of the chromosomes; but they are also of interest for a number of more special problems which I will first briefly consider.

The Relation of the Chromosomes to Sex-production in Metapodius

The conditions seen in this genus seem to be irreconcilable with any view that ascribes the sexual differentiation to a general quantitative difference of chromatin, whether expressed in the number or the relative size of the chromosomes. In all known cases of constant sexual differences in the chromosomes it is invariably the female that possesses the larger number of chromosomes or the greater quantity of chromatin, ${ }^{10}$ and this has naturally suggested the view that this difference per se may be the sex-determining factor. As I have pointed out before ('og), such a view is inapplicable to cases like Nezara or Oncopeltus, where the idiochromosomes are of equal size and no quantitative sexual differences are visible; yet the phenomena in these genera are otherwise so closely similar

${ }^{10}$ See review in Wilson'og. 
to those seen in other insects that I cannot doubt their essential similarity also in respect to sex-production.

In Metapodius the facts are still more evidently opposed to the quantitative interpretation. The number of chromosomes has here no relation to sex-production; and, as will be seen from the table at p. 149 , in the forms with supernumeraries the relative frequency of high numbers and of low is nearly equal in the two sexes. If my general interpretation of the chromosomes in this genus be correct, a like conclusion applies to the total relative mass of chromatin in the two sexes; for all individuals alike possess the type-group of 22 chromosomes (Montgomery's form excepted) while the supernumeraries represent the excess above this amount. I have endeavored to determine whether this appears in direct measurements, independently of my general interpretation; but have found this impracticable for several reasons. Very considerable differences in the apparent size of the chromosomes are produced by different degrees of extraction; but this will not account for the considerable differences seen in the same slide when the extraction is uniform. It is evident that the actual size of the chromosomes varies with the size of the cells; for example, both in Metapodius and in many other genera, the chromosomes in the larger spermatogonia near the tip of the testis are larger (in many cases much larger) than those of the smaller spermatogonia of other regions. How great the differences are may be appreciated by a comparison of the figures. For example, in the spermatogonial groups of No. 2 (23 chromosomes, Fig. 7, $v-x)$, the chromatin mass is obviously much greater than in those of No. 2 I (24 chromosomes, Fig. I1,$i-l$ ). In the 25-chromosome female groups shown in Fig. I2, $i-k$ (No. 27), the chromatin mass is evidently much less than in the $2 \mathrm{I}$-chromosome male group shown in Fig. I, $b$, or in the 23-chromosome male groups of Fig. 7 , $v-x$. Conversely, the 22-chromosome female group of No. 44 (Fig. $4, s$ ) shows a much greater chromatin mass than in the corresponding male group of No. 46 (Fig. 4, o), or the male 24-chromosome group shown in Fig. II, $j$.

Evidently, therefore, the relative mass of chromatin can only be determined by means of accurate measurements of both the 
chromosomes and the mass of protoplasm, but I have found the errors of measurement of the cell size to be too great to give any trustworthy result regarding the relative chromatin mass.

Despite the difficulties in the way of an accurate direct determination, I believe the facts on the whole warrant the conclusion that the relative chromatin mass shows no constant correlation with sex. The most probable conclusion is that the male-producing spermatozoa in Metapodius are distinguished by the same characters as in other forms having unequal idiochromosomes, the former class being those that receive the large idiochromosome, the latter those that receive the small one, irrespective of the supernumeraries that may be present in either class. For reasons that I have elsewhere stated, I believe that if the idiochromosomes be the sex-determinants their difference is probably a qualitative one, and since the small idiochromosome may be lacking it would seem that the large one must in every case play the active rôleperhaps as the bearer of a specific substance (enzyme?) that calls forth a definite reaction on the part of the developing individual. If this be so, we can comprehend the fact that the presence of additional small idiochromosomes (supernumeraries) in either sex does not affect the development of the sexual characters in that sex.

$b$ The possible Origin of the unpaired Idiochromosome ("odd" or "accessory" Chromosome) and of the Supernumeraries

The explanation of the unpaired idiochromosome offered in the second and third of my "Studies on Chromosomes" ('05, 'o6) was suggested by the fact that various degrees of inequality exist in the paired idiochromosomes, there being an almost continuous series of forms connecting those in which the idiochromosomes are equal (Nezara, Oncopeltus) with those in which they are so very unequal that the small one appears almost vestigial (Lygxus, Tenebrio). It is evident that by the further reduction and final disappearance of the small member of this pair the large one would be left without a mate, and its history in the maturation process would become identical with that of an "odd" or "accessory" 
chrumosome. I still believe that this explanation may be applicable to many cases; but a different one seems more probable in the case of Metapodius and perhaps may be more widely applicable. This was suggested by the observation (p. 166) that in a very few cases, in 22-chromosome individuals both idiochromosomes were seen passing to the same pole in the second division. The rareness of this occurrence shows that it is doubtless to be regarded in one sense as abnormal. But even a single such event in an original 22-chromosome male, if the resulting spermatozoa were functional, might give the starting point for the whole series of relations observed in the genus, including the establishment of an unpaired idiochromosome. The result of such a division should be a pair of spermatozoa containing respectively Io and 12 chromosomes. The former might give rise at once to a race having an unpaired idiochromosome and the somatic number $2 \mathrm{I}$ in the male (as in Montgomery's material). The latter might similarly produce an individual having in the first generation a single supernumerary chromosome and in succeeding generations an additional number. This appears from the following considerations:

I If a , Io-chromosome spermatozoön, arising in the manner indicated, should fertilize an egg of the 22-chromosome class (having I I chromosomes after reduction) the result should be a male containing 2 I chromosomes, the odd one being the large idiochromosome derived from the egg. Such an individual would be in no respect distinguishable from those of Montgomery's material, and would similarly form male-producing spermatozoa containing Io chromosomes and female-producing ones containing I I (including the unpaired idiochromosome). A single such male, paired with an ordinary 22-chromosome female, would suffice to establish a stable race identical with the form found by Montgomery at West Chester, Pa., the males having 2I chromosomes, the females having 22, precisely as in Anasa or Leptoglossus. This seems to me the most probable explanation of the conditions found in Montgomery's material; and possibly it may explain the origin of the unpaired idiochromosome in other cases as well.

2 The result of fertilizing the same type of egg by a spermatozoön from the r2-chromosome pole would be an individual having 
23 chromosomes (egg II + spermatozoön I2) including two large idiochromosomes - hence presumably a female - and one small. The eggs produced by such a female should after maturation be of two classes, having respectively i I and I 2 chromosomes. The 12-chromosome class would contain both a large and a small idiochromosome, and if fertilized by ordinary II-chromosome spermatozoa would produce individuals with 23 chromosomes, male or female according to the class of spermatozoön concerned. Such females would, as before, contain two large idiochromosomes and one small. The males would contain one large and two small. and would accordingly produce spermatozoa having either II or I2 chromosomes.

Now, such an additional small idiochromosome in the male would be indistinguishable from a single "supernumerary chromosome" as it appears in the 23-chromosome individuals in my material. The resemblance is shown not only in size but also in behavior; for, as I have shown, the supernumerary, like the idiochromosome, forms a chromosome-nucleolus during the growth period, it divides as a univalent in the first division, and in the second is usually associated with the idiochromosome bivalent. A single such supernumerary chromosome, once introduced into the race would lead to the presence of additional ones in succeeding generations. Thus, 12-chromosome eggs fertilized by 12-chromosome spermatozoa would give individuals (male or female) with 24 chromosomes, including two supernumeraries; and from these might arise, through irregularities of distribution such as I have described, gametes with I I, I2, or 13 chromosomes, giving in the next generation $22,23,24,25$ or 26 chromosomes according to the particular combination established in fertilization. ${ }^{11}$ If this

11 Since the presence of -an unpaired idiochromosome in some individuals and of supernumeraries in others is assumed to be traceable to the same initial cause, we should naturally expect to find the two conditions coexisting side by side, and in approximately equal numbers; but in point of fact the former is very rare and was only found in one locality, while the latter is very common. This may constitute a valid objection to my interpretation. It should be borne in mind, however, that abnormal divisions of the kind assumed to form the starting point are very rare, and that an extremely minute proportion of the total number of spermatozoa produced ever actually enter the eggs. The chances against fertilization by either class of the original modified spermatozoa are therefore very great. Since only sixty individuals have been examined it need not surprise us that one of the two conditions in question 
interpretation be correct, the origin of an unpaired chromosome in certain individuals of this genus has been owing to the same cause that has produced the supernumeraries. Since both conditions coexist in the same species, along with that which may be regarded as the original type ( 22 chromosomes) it may be concluded that Metapodius is now in a period of transition from the second to the third of the types distinguished in my last study. It seems quite possible that other species of coreids that now have constantly an unpaired idiochromosome may have passed through a similar condition, though in all of them thus far examined both the small idiochromosome and the supernumeraries have disappeared. In Metapodius, accordingly, the supernumeraries may be regarded as on the road to disappearance. That such is the case is rendered probable by the fact that their number does not pass a certain limit, and is rarely more than four. The very small chromosomes of this kind, so often observed, are perhaps degenerating, or even vestigial in character. But aside from this, attention has already been called to the probability that one or more of the supernumeraries may be lost during the growth-period (p.I86); and while this is not certain, it may well be that both methods are operative in their disappearance.

The foregoing interpretation of the supernumeraries enables us to understand why variations in their number are not accompanied by corresponding morphological differences in the somatic characters; for they are but duplicates of a chromosome already present and hence introduce no new qualitative factor. It can hardly be doubted that some kind of quantitative difference must exist between individuals that show different numbers, but none

has been more frequently met with. Another objection might be based on the different relations that occur in Syromastes. In this form (see Wilson 'og) the passage of both idiochromosomes to one pole without separation is a normal and constant feature of the second division, yet no supernumeraries appear in any of the individuals, and it is probable that the female groups contain two pairs of idiochromosomes like the single pair that appears in the male. We have no data for a conjecture as to how such a condition can have arisen; but evidently the small idiochromosome does not in this case become an erratic supernumerary but retains a definite adjustment to the other chromosomes. Still, I do not consider this an obstacle to my interpretation of Metapodius, for it is now evident that the history of the idiochromosomes in general has differed widely in different species and families, even among the Hemiptera. We have thus far only made a beginning in their comparative study. [See Addendum, p. 200.] 
has thus far been discerned. Such a difference does not appear in the size of the animals, for there are large individuals with no supernumeraries and small individuals that possess them. An interesting field for experiment seems here to be offered.

\section{c The "Individuality" or Genetic Continuity of the Chromosomes}

It is in respect to this much debated hypothesis that the facts observed in Metapodius seem to me most significant and important. It is evident that the whole series of relations are readily intelligible if the fundamental assumption of this hypothesis be accepted. Without such explanation they seem to me to present an insoluble puzzle. The disposition to reject this hypothesis that appears in a considerable number of recent papers on the subject will doubtless lead to more critical and exhaustive observation of the facts; but when it goes so far as to deny every principle of genetic continuity in respect to the chromosomes, it is, I believe, a backward step. This reaction perhaps reaches a climax in the elaborate and apparently destructive criticism of Fick ('o 7 ) who considers the hypothesis to be thoroughly discredited, and believes his analysis to justify the conclusion: "Dass weder theoretisch noch sachliche Beweise für die Erhaltungslehre vorliegen, sondern dass im Gegentheil unwiderlegliche Beweise gegen sie vorhanden sind, so dass es im Interesse der Wissenschaft dringend $\mathrm{zu}$ wünschen ist, dass die Hypothese von allen Autoren verlassen wird" ('o7, p. II2, italics in original). I incline to think that this sweeping judgment would have carried greater weight had Professor Fick, in certain parts of his able and valuable discussion, taken somewhat greater pains in his presentation of facts and shown a more judicial temper in their analysis. ${ }^{12}$ To some of the objections and difficulties

${ }^{12}$ I will give two specific examples of this. The experimental results of Moenkhaus ('04), on hybrid fishes, which evidently form a strong support to the continuity hypothesis, are unintentionally but completely misrepresented in the statement at p. 75: "So berichtet Moenkhaus bei Fundulus-Moniciakreuzung (sic), dass sich die beiderlei (zuerst sehr verschiedenartigen) Chromosomen in der Regel schon nach der zweiten Teilung nicht mehr unterscheiden lassen." But Moenkhaus'sexplicit statement, based on the examination of "many thousand cells," is that even in the late cleavage "Nuclei showing the two kinds of chromosomes mingled together upon the spindle are everywhere to be found" (op. cit., p. 48). Fick evidently had in mind the fact that the paternal and maternal chromosomes do not as a rule retain their original grouping after the first two or three cleavages. His actual statement, however, 
brought forward in this critique reply has already been made by Boveri ('07), Strasburger ('08), Schreiner ('08) Bonnevie ('08) and others. Some of the difficulties are real, but an attentive study of the matter will show that a large part of Fick's critique is directed against the strict hypothesis of individuality and offers no adequate interpretation of the essential phenomenon that requires explanation. It may be admitted that many of the facts seem at present difficult to reconcile with the view that the identity of the chromosomes as actual individuals is maintained in the "resting" nucleus; and I have myself indicated (The Cell, I900, p. 300) that the name "individuality" was perhaps not the best that could have been chosen. Certainly we have as yet comparatively little evidence that the chromosomes retain their boundaries in the "resting" nucleus. It is evident that the chromosomes are greatly diffused in the nuclear network, and it may be that the substances of different chromosomes are more or less intermingled at this time. Fick's "manœuvre-hypothesis," which treats the chromosomes of the dividing cell as temporary "tactic formations," may therefore be in some respects a more correct formulation of the facts than that given by the hypothesis of "individuality" in the strict sense of the term. But the last word on this question has by no means yet been spoken. A new light is thrown on it by the recent important work of Bonnevie ('o8) which brings forward strong evidence to show that in rapidly dividing cells (cleavage stages of Ascaris, root-tips of Allium), although the identity of the orig-

(both here and in the later passage at p. 98) will wholly mislead a reader not familiar with Moenkhaus' work, in regard to one of the most significant and important discoveries in this whole field of inquiry.

Hardly less misleading is Professor Fick's report of my own observations on the sex-chromosomes of insects, which are stated as follows: "Wilson's Unterschungen beweisen eben sicher nur soviel, dass bei einigen Insektengattungen constante Beziehungen zwischen dem Geschlecht und dem Vorhandensein eines besonderen Chromosomenpaares bestehen, bei anderen Gattungen nicht" (p. 90). I am confident that those who are familiar with the researches referred to will not accept this as a fair statement of the results. The fact is that in one form or other the sex-chromosomes are present in all of the forms that I have examined (now upwards of seventy species) and that with various modifications all conform to the same fundamental type. It is true that in two genera (Nezara and Oncopeltus) the ser-chromosomes are equal in size, and hence afford no visible differential between the somatic groups of the two sexes; but I especially emphasized the fact (cf. 'o6, pp. 17, 34) that these chromosomes are in every other respect identical with those of other forms in which the size-difference clearly appears, and are connected with the latter by a series of intermediate gradations that leaves no doubt of the essential uniformity of the phenomena. 
inal chromosomes is lost in the "resting" nucleus after each mitosis, each new chromosome nevertheless arises by a kind of endogenous formation within and from the substance of its predecessor. In this way an individual genetic continuity of the chromosomes can be directly followed through the "resting period" of the nucleus. "Eine genetische Kontinuität der Chromosomen nacheinander folgender Mitosen konnte in der von mir untersuchten Objekten teils sicher (Allium, Amphiuma) teils mit überwiegender Wahrsheinlichkeit (Ascaris) verfolgt werden. Es ging aber auch hervor, dass eine Identität der Chromosomen verschiedener Mitosen nicht existiert, sondern dass jedes Chromosom in einem fruher existierenden endogen entstanden ist, um wieder am Ende seines Lebens für die endogene Entstehung eines neuen Chromosoms die Grundlage zu bilden" (op. cit, p. 54). Whether this particular conclusion will also apply to more slowly dividing cells remains to be seen. But apart from this direct evidence it seems to me that a denial of every form of genetic continuity between the chromosomes of successive cell-generations-which, despite certain qualifications, seems to be the position of Fick and a number of other recent writers-is only possible to those who are ready to ignore some of the most obvious and important of the known facts, especially those that recent research has brought to light among the insects. The most significant of these are:

I In Metapodius the specific number varies, while in the individual both the number and the size-relations of the chromosomes are constant.

2 In all species where the somatic chromosome-groups show sexual differences in regard to the number and size-relations of the chromosomes, exactly corresponding differences exist between the male-producing and the female-producing spermatozoa.

Both these series of facts demonstrate that the "tactic formation" of a fixed number of chromosomes of particular size is not a specific property of a single chromatin-substance as such, of the species. It has been assumed by some writers that departures from the normal specific number, such as appear in merogonic, parthenogenetic, double-fertilized or giant (double) eggs, are the result merely of departures from the normal quantity of chroma- 
tin." 13 If attentively considered the facts summarized above will, I think, clearly show the inadequacy of such an explanation. Why should a given quantity and quality of chromatin always reappear in the same morphological form as that in which it entered the nucleus? Why, for example, in Metapodius should the minute fraction of chromatin represented by a single small supernumerary always reappear in the form of such a chromosome, showing specific peculiarities of behavior, rather than as a corresponding enlargement of one of the other chromosomes? Why should a larger excess always appear as a group of two, three, or more supernumeraries that differ definitely in behavior from the others and show constant size relations among themselves? Specifically, in individual No. 40, why should two small supernumeraries and two large ones always appear, rather than three large ones? In species where a constant quantitative chromatin-difference exists between the sexes, why should the excess in the female always appear in the same form as that which appears in the femaleproducing spermatozoa-in one case as a large idiochromosome instead of a small (Lygæus), in another as an additional chromosome of a particular size (very large in Protenor, small in Alydus, of intermediate size in Anasa), in a third case as three additional chromosomes (Galgulus)?

To these and many similar questions which the facts compel us to consider, I am unable to find any answer on the merely quantitative hypothesis. Each of them receives a simple and intelligible reply under the view that it is the number, size, and quality of the chromosomes that enter the nucleus that determine the number, size, and mode of behavior of those that issue from

${ }^{13}$ Fick's treatment of these cases is worth citing. "Es muss von vornherein als wabrscheinlich bezeichnet werden, dass unter den abnormen Umständen, da einmal die Zahl der 'Chromatin-Manövereinheiten' (im Sinne meiner Manöverhypothese gesprochen) in der Zelle erhöht ist, diese Zahl sich erbält" (p. 96). Why should the number be maintained? Because, we are told, "Die Erbaltung der erhöhten Zahl und ihre regelmässige Wiederkehr bei den folgenden Teilungungen muss bei dem nun einmal uber die Norm erhöhten Chromosomenbestand der Zelle als der einfachere, leichter verständliche Vorgang erscheinen, als es ein besonderer, ein "Regulation" auf die Norm hervorbringender Akt wäre." To most readers this will seem like an argument for, rather than against, the hypothesis of genetic continusty. But since it is obviously not thus intended I can discover no other meaning in the passage than that with a given "bestimmte Chromatinmanöverart" characteristic of the species (p.I I 5) the number of chromosomes formed is proportional to the quantity of chromatin-substance. 
it. But such an answer implies the existence of a definite individual genetic relation between the chromosomes of successive cellgenerations; and it is this relation, I take it, that forms the essence of the hypothesis of genetic continuity, whether or not we include in the hypothesis the assumption that the chromosomes persist as "individuals" in the resting nucleus where their boundaries seem to disappear. We might, for instance, assume that the chromosomes are magazines of different substances (e. g., enzymes or the like) that differ more or less in different chromosomes, that are more or less diffused through the nucleus in its vegetative phase, but are again segregated out in the original manner when the chromosomes reform. ${ }^{14}$ We have, admittedly, but an imperfect notion of how such a re-segregation may be effected, though the conclusions of Bonnevie already referred to, constitute an important addition to the earlier ones of Boveri (see '07, p. 232) in this direction. However this may be, in my view the most practicable, indeed the almost necessary, working attitude is to treat the chromosomes as if they were actually persistent individuals. The facts in Metapodius, which at first sight seem to present so chaotic an aspect, fall at once into order and become intelligible if regarded as due to the presence in the species of a certain number of erratic chromosomes, one or more of which may be introduced into the zygote at the time of fertilization and which in some sense retain their identity throughout the development. The particular combination established at the time of fertilization is the result of the chance union of two particular gamete combinations. Since the distribution of the supernumeraries to the spermatid nuclei is variable, different gamete combinations occur in the spermatozoa of the same individual; and the same is probably true of the eggs. Moreover, adults of the same species live side by side on the same foodplants and presumably may breed together. Different combinations may thus be produced in the offspring of a single pair, whether the parents possess the same or different numbers. Metapodius thus fulfills the prediction of Boveri, written nearly twenty years ago. "Wenn bei einer Spezies einmal sehr viele und verschieden-

${ }^{14}$ A view similar to this is suggested by Fick himself in bis earlier discussion ('05, p. 204), but it does not reappear in his later one. 
artige Irregularitäten vorkämen, diese sich wohl auf lange hinaus erhalten müssten, so dass unter Umständen Fälle mit ausserordentlich grosser Variabilität der Chromosomenzahl zur Beobachtung kommen könnten, ohne dass selbst diese das Grundgesetz umstossen vermöchten, welches lautet: Es gehen aus jedem Kerngerüst so viele Chromosomen hervor als in die Bildung derselben eingegangen sind" ('9o, p. 6I). To the earlier expression of this "Grundgesetz" Boveri has recently added the statement that the chromosomes that emerge from the nucleus are not merely of the same number but also show the same size-relations as those that entered it. "Was durch den kurzen Ausdruck "Individualität der Chromosomen" bezeichnet werden soll, ist die Annahme dass für jedes Chromosoma, das in einen Kern eingegangen ist, irgend eine Art von Einheit im ruhenden Kern erhält, welche der Grund ist, dass aus diesem ruhenden Kern wieder genau ebenso viele Chromosomen hervorgehen und dass dieses Chromosomen überdies da, wo vorher verschiedene Grössen unterschieden waren, wieder in den gleichen Grössenverhältnissen auftreten" ('o7, p.229).

The facts seen in Metapodius and other insects are thoroughly in accord with the foregoing statement, and justify the additional one that the chromosomes conform to the same principle in respect to their characteristic modes of behavior. In the Hemiptera heteroptera generally the idiochromosomes and supernumeraries, the $m$-chromosomes, and the "ordinary chromosomes" or "autosomes" show each certain constant peculiarities in respect to the time of synapsis and behavior during the growth-period, and assume a characteristic (though not entirely constant) mode of grouping in the first spermatocyte. Perhaps the most obvious of these facts is the very early condensation of the idiochromosomes and supernumeraries in the growth-period as contrasted with the other chromosomes; and in the case of Pyrrochoris I have shown ('og) that the idiochromosome never assumes a diffuse condition after the last spermatogonial division. But even more significant are the definite differences shown in the couplings of the various forms of chromosomes that take place in the course of the spermatogenesis. Nothing in these phenomena is more striking than the accuracy with which these couplings take place. 
As Montgomery and Sutton have shown, the ordinary paired chromosomes of the spermatogonia give rise to bivalents of corresponding size at the time of general synapsis. The actual coupling of the ordinary chromosomes at this time is still a matter of dispute ${ }^{15}$ but no doubt can exist in regard to the couplings that occur at a later period in case of the $m$-chromosomes, the idiochromosomes, and the supernumeraries. These characteristic couplings are not determined merely by the size of the chromosomes. The union of the unequal idiochromosomes after the second division takes place with the same regularity as that of the equal $m$-chromosomes in the prophases of the first. A small supernumerary that is indistinguishable from the $m$-chromosomes in the spermatogonia never couples with the latter in either division, but with the much larger idiochromosomes. The couplings are equally independent of the original positions of these chromosomes, either in the spermatogonia or in the growth-period, as is seen with especial clearness in case of the $m$-chromosomes. These phenomena naturally suggest the conclusion that the couplings result from definite affinities among the chromosomes. The possibility no doubt exists that the couplings are produced by extrinsic causes (such as the achromatic structures) but the evidence seems on the whole opposed to such a conclusion. I consider it more probable that they are due to intrinsic qualities of the chromosomes and that the differences of behavior shown by different forms may probably be regarded as due to corresponding physico-chemical differences. This conclusion is in harmony with Boveri's experimental results, though based on wholly different data. While it does not seem worth while to attempt its wider development here, I may express the opinion that all the chromosomes may consist in the main of the same material basis, differing only in respect to certain constituents; and further that the degree of qualitative difference may vary widely in different species.

Zoölogical Laboratory

Columbia University

August 10, 1908

${ }^{15}$ See for example, Meves ('o7, pp. 453-468) who, like O. Hertwig, Fick and others, rejects the theory of "individuality." 


\section{ADDENDUM}

The probability in regard to the female groups of Syromastes, expressed in the footnote at p. 192 was first stated in my preceding paper ('o9, p. 73 ) after a study of the male only. Since the present paper was sent to press I have had opportunity to examine females of this form. The facts are exactly in accordance with my prediction, the female groups containing 24 chromosomes, while the male number is 22. It now seems clear, however, that the two idiochromosomes of Syromastes do not correspond respectively to the large and the small idiochromosome of Metapodius or Lygæus but are equivalent, taken together, to the large idiochromosome or to the odd chromosome of Anasa, etc.

October 25, 1908.

WORKS REFERRED TO

Bonnevie, K. 'o8-Chromosomenstudien. I. Arch. f. Zellforschung, i, 23. BOVERI, Th. 'go-Zellenstudien. III. Ueber das Verhalten der chromatischen Kernsubstanz bei der Bildung der Richtungskörper und bei der Befruchtung. Jena, 1890 .

'o7-Zellenstudien. VI. Die Entwicklung dispermer Seeigel-Eier, etc. Jena, 1907.

FIck, R. '05-Betrachtungen über die Chromosomen, ihre Individualität, Reduction, und Vererbung. Arch. Anat. u. Phys., Anat. Abth., Suppl. 1905.

'o7-Vererbungsfragen, Reduktions-und Chromosomenhypothesen, Bastard-Regeln. Merkel und Bonnet's Ergebnisse, xvi, r9o6.

Foot, K. And Strobell, E. C. 'o7a-The "Accessory Chromosome" of Anasa tristis. Biol. Bull., xii.

'o7b-A Study of Chromosomęs in the Spermatogensis of Anasa tristis. Am. Journ. Anat., vii, 2.

GRoss, J. '04-Die Spermatogenese von Syromastes marginatus. Zoöl. Jahrb., Anat. Ontog., xii.

Lefevre, G. and McGill, C. 'o8-The Chromosomes of Anasa tristis and Anax junius. Am. Journ. Anat., vii. 4.

Moenkhaus, W. S. '04-The Development of the Hybrids between Fundulus heteroclitus and Menidia notata, etc. Am. Journ. Anat., iii. 
Meves, Fr.-Die Spermatocytenteilungen bei der Honigbiene, etc. Arch. Mikr. Anat., lxx.

Montgomery, T. H. 'or-A Study of the Germ-cells of Metazoa. Trans. Am. Phil. Soc., $x x$.

'o6-Chromosomes in the Spermatogenesis of the Hemiptera Heteroptera. Ibid., xxi, 3 .

Paulmier, F. C. ' $99-$ The Spermatogenesis of Anasa tristis. Journ. Morph., $x v$, Suppl.

Schreiner, K. E. And A. '08-Gibt es eine parallele Konjugation der Chromosomen? Videnskebs-Selskabets Skrifter. i. Math-Naturw. Klasse, 1908 , no. 4 .

Stevens, N. M. 'o6-Studies in Spermatogensis. II. A Comparative Study of the Heterochromosomes in Certain Species of Coleoptera, Hemiptera and Lepidoptera, etc. Carnegie Institution. Pub. 36, ii.

'08a-A Study of the Germ-cells of Certain Diptera, etc. Journ. Exp. Zoöl., v. iii.

'08b-The Chromosomes in Diabrotica vittata, etc. Ibid., v, iv.

Strasburger, E. '08-Chromosomenzahlen, Plasmastrukturen, Vererbungsträger und Reduktionsteilung. Jahr. wiss. Bot., xlv, iv.

Wirson, E. B. '05a-Studies on Chromosomes, I. Journ. Exp. Zoöl., ii.

'o5b-Studies, etc. II. Ibid., ii, iv.

'o6-Studies, etc., III. Ibid., iii, $\mathrm{x}$.

'o9-Studies, etc., IV Ibid., vi, I.

'o7a-Note on the Chromosome-groups of Metapodius and Banasa. Biol. Bull., xii, 5 .

'o7b-The Supernumerary Chromosomes of Hemiptera. Report of May Meeting. N. Y. Acad. Sci. Science, n. s., xxvi, 677.

'o7c-The Case of Anasa tristis. Science, n. s., xxv, 631 . 


\section{APPENDIX}

List of individuals examined, arranged according to locality

\begin{tabular}{|c|c|c|c|c|c|c|}
\hline No. & Species & Sex & Locality & Supernumeraries & $\begin{array}{c}\text { Somatic } \\
\text { No. }\end{array}$ & $\begin{array}{l}\text { No. in } \\
\text { first } \\
\text { div. }\end{array}$ \\
\hline $\mathbf{I}$ & terminalis & $0^{\pi}$ & Madison, N. J. (Paulmier) & I small & 23 & 13 \\
\hline 2 & terminalis & $0^{\pi}$ & Madison, N. J. (Paulmier) & I small & 23 & 13 \\
\hline $3^{-11}$ & terminalis & $0^{7}$ & West Chester, Pa. (Montgomery) & absent & 21 & II \\
\hline 12 & terminalis & $0^{\pi}$ & West Chester, Pa. (Wilson) & absent & 22 & 12 \\
\hline 13 & terminalis & $\sigma^{\pi}$ & West Chester, Pa. (Wilson) & I large & 23 & 13 \\
\hline 14 & terminalis & o & West Chester, Pa. (Wilson) & I large & 23 & \\
\hline 15 & terminalis & 웅 & West Chester, Pa. (Wilson) & 2 large & 24 & \\
\hline 16 & terminalis & \& & West Chester, $\mathrm{Pa}$. (Wilson) & 2 large & 24 & \\
\hline 17 & terminalis & $0^{\pi}$ & Mansfield, Ohio & absent & 22 & 12 \\
\hline 18 & terminalis & $q$ & Mansfield, Ohio & absent & 22 & \\
\hline 19 & terminalis & $\sigma^{\pi}$ & Raleigh, N. C. & absent & 22 & 12 \\
\hline 20 & terminalis & $0^{\pi}$ & Raleigh, N. C. & 1 large & 23 & 13 \\
\hline 21 & terminalis & $0^{\pi}$ & Raleigh, N.C. & 2 large & 24 & 14 \\
\hline 22 & terminalis & $\sigma^{\pi}$ & Raleigh, N. C. & I large, I small & 24 & 14 \\
\hline 23 & terminalis & q & Raleigh, N. C. & absent & 22 & \\
\hline 24 & terminalis & $q$ & Raleigh, N. C. & absent & 22 & \\
\hline 25 & terminalis & $q$ & Raleigh, N. C. & I large & 23 & \\
\hline 26 & terminalis & $q$ & Raleigh, N.C. & 2 large & 24 & \\
\hline 27 & terminalis & $q$ & Raleigh, N. C. & 2 large, 1 small & 25 & \\
\hline 28 & femoratus & $\sigma^{x}$ & Raleigh, N.C. & absent & 22 & 12 \\
\hline 29 & femoratus & $\sigma^{2}$ & Raleigh, N. C. & absent & 22 & 12 \\
\hline 30 & femoratus & ㅇ & Raleigh, N. C. & I large & 23 & \\
\hline $3^{I}$ & femoratus & ㅇ & Raleigh, N. C. & 2 large & 24 & \\
\hline $3^{2}$ & femoratus & $q$ & Raleigh, N. C. & 4 large & 26 & \\
\hline 33 & femoratus & ㅇ & Raleigh, N. C. & 3 large & & \\
\hline & & & & 2-3 small & $27-8$ & \\
\hline 34 & terminalis & $0^{\pi}$ & Southern Pines, N. C. & 3 large & 25 & 15 \\
\hline 35 & terminalis & $0^{\pi}$ & Southern Pines, N. C. & 3 large & 25 & 15 \\
\hline 36 & terminalis & $0^{\pi}$ & Southern Pines, N. C. & 4 large & 26 & 16 \\
\hline 37 & terminalis & $0^{\pi}$ & Southern Pines, N. C. & 2 large & 24 & 14 \\
\hline $3^{8}$ & terminalis & $\sigma^{\pi}$ & Southern Pines, N. C. & 3 large & 25 & \\
\hline 39 & femoratus & $\sigma^{\pi}$ & Southern Pines, N. C. & 2 large & 24 & 14 \\
\hline 40 & femoratus & $0^{x}$ & Southern Pines, N. C. & 2 large, 2 small & 26 & 16 \\
\hline 41 & femoratus & 우 & Southern Pines, N. C. & I large & 23 & \\
\hline 42 & femoratus & $\sigma^{x}$ & Columbia, S. C. & 4 large & 26 & 16 \\
\hline 43 & terminalis & $\sigma^{\pi}$ & Charleston, S. C. & I small & 23 & 13 \\
\hline 44 & terminalis & $q$ & Charleston, S. C. & absent & 22 & \\
\hline
\end{tabular}


List of individuals examined, arranged according to locality-Continued

\begin{tabular}{|c|c|c|c|c|c|c|}
\hline No. & Species & Sex & Locality & Supernumaries & $\begin{array}{c}\text { Somatic } \\
\text { No. }\end{array}$ & $\begin{array}{l}\text { No. in } \\
\text { first } \\
\text { div. }\end{array}$ \\
\hline 45 & femoratus & 우 & Charleston, S. C. & 2 large & 24 & \\
\hline 46 & femoratus & $0^{\pi}$ & Savannah, Ga. & absent & 22 & I2 \\
\hline 47 & granulosus & $0^{\pi}$ & Tucson, Arizona & absent & 22 & 12 \\
\hline $4^{8}$ & granulosus & $\sigma^{2}$ & Tucson, Arizona & I large & 23 & 13 \\
\hline 49 & granulosus & $0^{7}$ & Tucson, Arizona & $I$ large & 23 & 13 \\
\hline 50 & granulosus & $0^{7}$ & Tucson, Arizona & 2 large & 24 & 14 \\
\hline $5^{1}$ & granulosus & $\sigma^{\pi}$ & Tucson, Arizona & 2 large & 24 & 14 \\
\hline 52 & granulosus & $\sigma^{\pi}$ & Tucson, Arizona & 2 large & 24 & 14 \\
\hline 53 & granulosus & $\sigma^{7}$ & Tucson, Arizona & 2 large & (24) & 14 \\
\hline 54 & granulosus & $\sigma^{7}$ & Tucson, Arizona & 3-4 large & $25-26$ & $15-16$ \\
\hline 55 & granulosus & $\sigma^{7}$ & Tucson, Arizona & 4 large & 26 & 16 \\
\hline $5^{6}$ & granulosus & $\sigma^{7}$ & Tucson, Arizona & 4 large & 26 & 16 \\
\hline 57 & granulosus & $\sigma^{7}$ & Tucson, Arizona & 4 large, I small & (27) & 17 \\
\hline $5^{8}$ & granulosus & \% & Tucson, Arizona & 3 large & 25 & \\
\hline 59 & granulosus & $\sigma^{7}$ & Grand Canyon, Arizona & 4 large & 26 & 16 \\
\hline 60 & granulosus & $\sigma^{\pi}$ & Grand Canyon, Arizona & 4 large & $(26)$ & 16 \\
\hline $6 \mathrm{I}$ & granulosus & \& & Grand Canyon, Arizona & 4 large & 26 & \\
\hline 62 & granulosus & ㅇ & Grand Canyon, Arizona & \pm 4 large & \pm 26 & \\
\hline
\end{tabular}




\section{EXPLANATION OF PLATE I.}

The figures are reproduced directly from the original photographs, without retouching, at an enlargement of 1500 diameters. It should be borne in mind that in the photographs considerable apparent sizevariations are produced by differences of focus, and that unless the chromosomes lie exactly in one plane the photograph often gives a less accurate impression than a drawing. Drawings of most of these photographs with designations, will be found among the text figures, as indicated.

I M. terminalis (No. 3, Montogmery's material), 21-chromosome form, first spermatocyte-division polar view; unpaired idiochromosome (odd or accessory) outside the ring, to the right (Fig. $3, b$ ).

2 M. terminalis (No. 19), 22-chromosome form, first division, polar view; the two separate idiochromosomes at the right. (The small idiochromosome, being slightly out of focus, appears too small. Its size is correctly shown in the drawing, Fig. 4, $b$ ).

3 M. terminalis (No. 12), 22-chromosome form similar view; idiochromosomes in contact (Fig.4, $f$ ).

4 M. terminalis (No. 20), 23-chromosome form, one large supernumerary, view similar to the preceding; idiochromosomes and supernumerary to the right (Fig. I, g).

5 M. granulous (No. 49), 23-chromosome form, one large supernumerary, which lies inside the ring with the small idiochromosome and $m$-chromosome (Fig. $7, \mathrm{~g}$ ).

6 M. terminalis (No. 1), 23-chromosome form, one small supernumerary lying inside the ring with the $m$-chromosome and one of the large bivalents (Fig. $7, i$ ).

7 M. granulosus (No. 52), 24-chromosome form, two large supernumeraries (Fig. II, $g$ ).

8 M. femoratus (No. 42), 26-chromosome form, four large supernumeraries (Fig. 2, g).

9 M. terminalis (No. 36), 26-chromosome form, similar to preceding (Fig. 9, e).

Io M. femoratus (No. 57), 27-chromosome form, four large supernumeraries and one small (Fig. $\left.\mathbf{I}_{3}, h\right)$.

II M. femoratus (No. 46), 22-chromosome form, first division in side view, both idiochromosomes dividing (Fig. 4, i).

12 M. granulosus (No. 47) 22-chromosome form, second division, polar view (Fig. 5, c).

13 M. femoratus (No. 42), 26-chromosome form; second division, polar view, showing hexad element near center (Fig. 10, $a$ ).

14 M. terminalis (No. 3, Montgomery's material) 2I-chromosome form, second division side view, showing lagging idiochromosome ("accessory chromosome") (Fig. 3, $f$ ).

15 From the same cyst as the last, later stage of second division; idiochromosome entering one pole (Fig. 3, g).

16 M. femoratus (No. 29), 22-chromosome form, second division metaphase in side view, showing idiochromosome bivalent (like Fig. 5, d).

17 M. granulosus (No. 47), 22-chromosome form, late anaphase of second division, one idiochromosome entering each pole (Fig. $5, l$ ).

18 M. femoratus (No. 46), abnormal late anaphase of second division, showing both idiochromosomes passing to the same pole (Fig. 5, 0).

19 M. femoratus (No. 29), 22-chromosome form, second division showing initial separation of the idiochromosomes (like Fig. 5, $f$ ).

20 M. granulosus (No. 49), 23-chromosome form, one large supernumerary, second division metaphase, showing triad element formed by the union of the supernumerary with the idiochromosomebivalent (like Fig. $8, i$ ).

21 M. granulosus (No. 52), 24-chromosome form, two large supernumeraries, second division, show. ing tetrad element consisting of the idiochromosomes and supernumeraries united in a linear series (Fig. II, $u$ ). 
22 M. femoratus (No. 42), 26-chromosome form, four large supernumeraries; second division showing hexad element formed by the idiochromosomes and supernumeraries (Fig. 10, $h$ ).

23 From the same cyst, similar view (Fig. 10, $k$ ).

24 M. terminalis (No. 3 , Montgomery's material), 2I-chromosome form, nucleus from the growthperiod, showing single spheroidal chromosome nucleolus (like Fig. $3, l$ ).

25 M. femoratus (No. 29), 22-chromosome form, growth-period, showing double chromosomenucleolus (idiochromosome-bivalent) and plasmosome (Fig. 6, b).

26 From the same slide, showing different ordinary chromosomes, separate chromosome-nucleoli and plasmosome (Fig. 6, c).

27 M. terminalis (No. 20), $\bumpeq \hat{\jmath}$-chromosome form, growth-period, showing tripartite chromosomenucleolus formed by the idiochromosomes and supernumerary (like Fig. $1, i$ ).

28 M. granulosus (No. 60), 26-chromosome form, growth-period, showing hexad chromosomenucleoli from three different cells (like Fig. 10, $s-u$ ).

29 M. terminalis (No. 2), 23-chromosome form, one small supernumary; spermatogonial group, showing three small chromosomes (the supernumerary and two $m$-chromosomes); the small idiochromosome distinguishable above towards the left (Fig. 7, y).

30 M. terminalis (No. 22), 24-chromosome form, one small supernumerary and one large (Fig. II, $p$ ) 

(E. B Wilson)
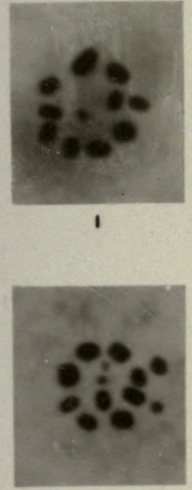

6

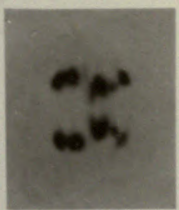

11

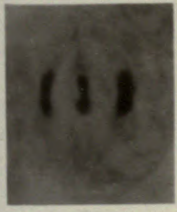

16

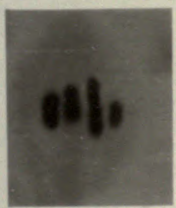

21

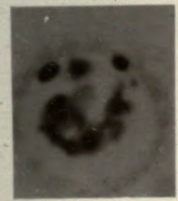

26
2

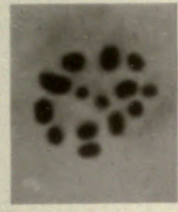

7

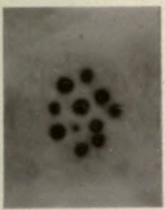

12

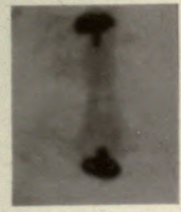

17

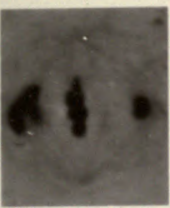

22

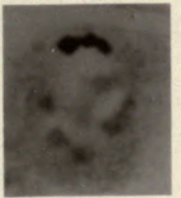

27

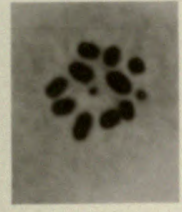

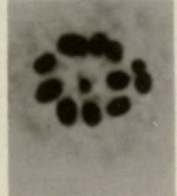

3

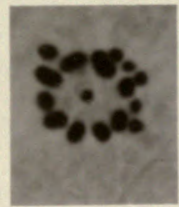

8

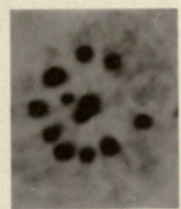

13

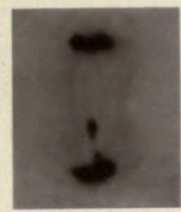

18

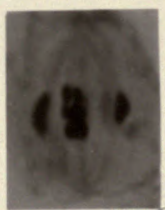

23

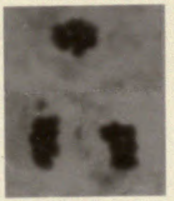

28

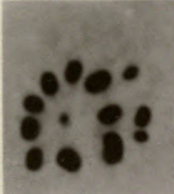

4

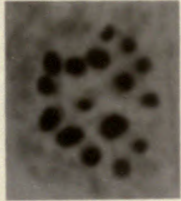

9

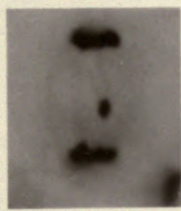

14

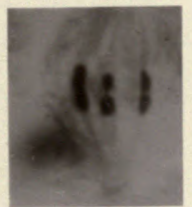

19

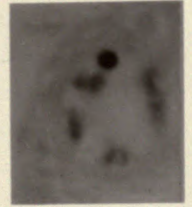

24

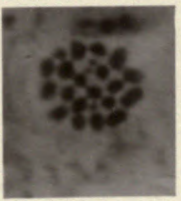

$:{ }^{29} \cdots$

5

10

15

20
${ }^{29}$
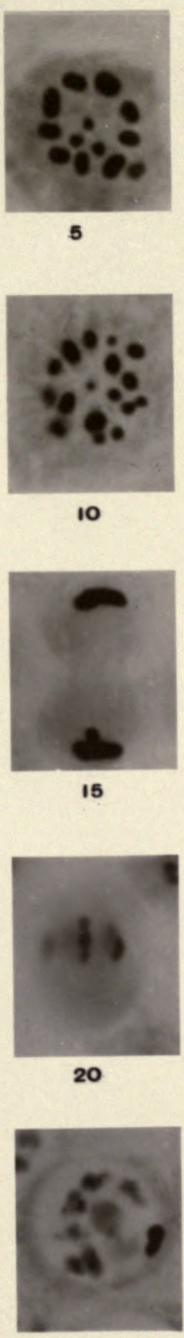

25.

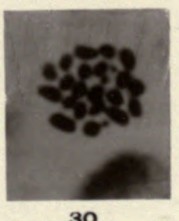

The Journal of Experimental Zoology, Vol. VI. 


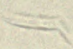

$\because \vdots \vdots \quad \because \vdots \vdots \vdots \vdots \vdots$

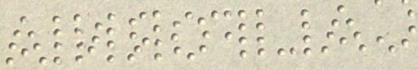




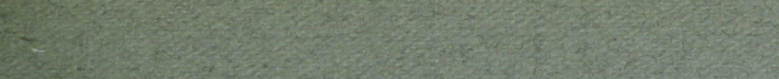

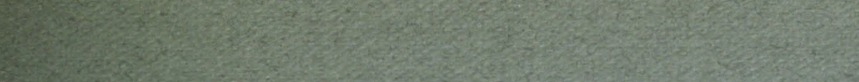
W. 3.1.2.

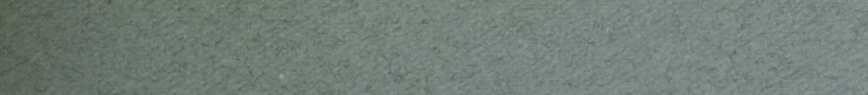

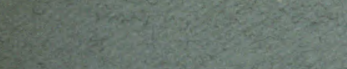

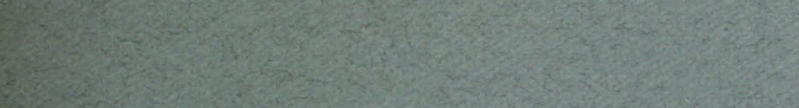

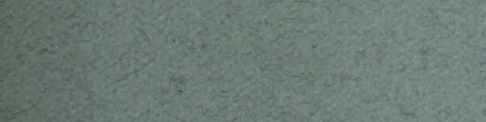
P.

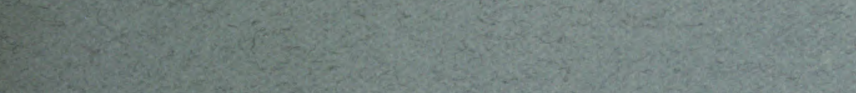

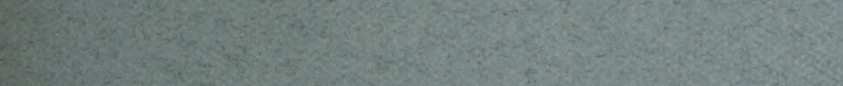

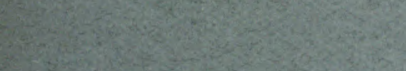

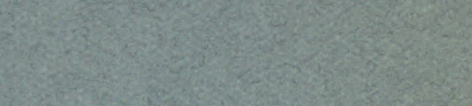

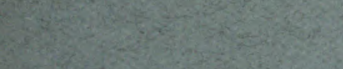

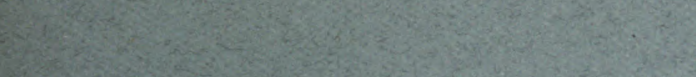

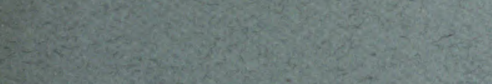

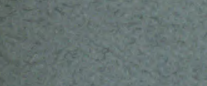

thes

in (10.2)

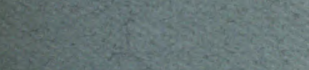

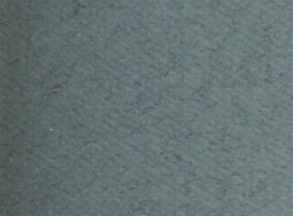

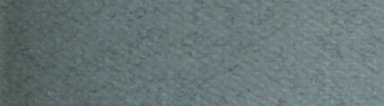

1.75: 2.

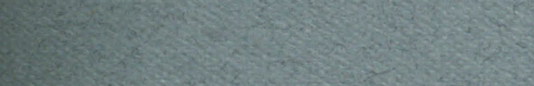
8.: St: 



\section{STUDIES ON CHROMOSOMES}

VI. A NEW TYPE OF CHROMOSOME COMBINATION IN METAPODIUS

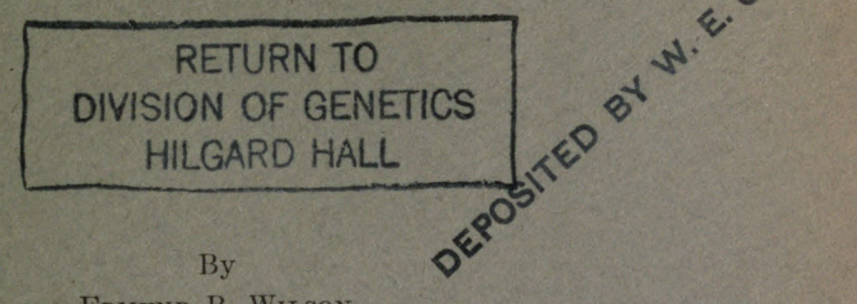

EDMUND B. WILSON

WITH FIVE FIGURES

REPRINTED FROM

THE JOURNAL OF EXPERIMENTAL ZOÖLOGY

Volume IX No.1

SEPTEMBER, 1910

WILLIAMS \& WILKINS COMPANY BALTIMORE 



\title{
STUDIES ON CHROMOSOMES
}

VI. A NEW TYPE OF CHROMOSOME COMBINATION IN METAPODIUS

\author{
EDMUND B. WILSON \\ Professor of Zoölogy, Columbia University. \\ WITH FIVE FIGURES
}

Although the peculiar combination of chromosomes here to be described has been seen in only a single individual, it affords new and I think significant evidence regarding some of the most interesting of the problems connected with the nuclear organization. As was shown in the fifth of my "Studies on Chromosomes," the genus Metapodius is most exceptional and remarkable in that the specific number of chromosomes varies, while that of the individual is on the whole constant. It is true that slight indiscriminate fluctuations in the number of the ordinary chromosomes, or "autosomes," occur, as they do in many other species; but this is only an inconsiderable source of the specific variation. The evidence shows, beyond a doubt in some individuals, and hence with probability for all, that the numerical differences are primarily due to variations in the number of a particular class of chromosomes which I called the "supernumeraries." These may be wholly absent. When present, their number is constant in the individual, but differs in different individuals. They are often recognizable in both sexes by their size, and in the male also by certain very definite peculiarities of behavior in the maturation-process. When they are absent, the diploid groups contain 22 chromosomes; and this condition is almost certainly the fundamental type of the genus, of which all the other conditions are variants. Such a group comprises 18 ordinary chromosomes, or "autosomes" + 2 very small microchromosomes, or $m$-chromsomes +2 unequal idiochromosomes $=22$ (these respective

${ }^{1}$ Wilson: '09c. 
classes having the peculiarities heretofore described). ${ }^{2}$ Numbers above 22 arise through the addition of one or more relatively small "supernumeraries," which agree in behavior with the small idiochromosome, of which they are probably duplicates. None of my own material (53 individuals, of three species) showed less than 22 chromosomes, and at least one small idiochromosome was present in all. In all of Montgomery's material of M. terminalis, however (9 individuals), this chromosome is absent, the spermatogonial number is but 21 , and the large idiochromosome appears without a synaptic mate as a typical odd or accessory chromosome.

The foregoing results were based on the study of 62 individuals in all, representing the three species, terminalis, femoratus and granulosus. In February, 1909, I took at Miami, Fla., two additional male specimens of femoratus, quite typical in structure, and closely similar in external appearance. One of these (No. 63) is an ordinary 23-chromosome form with one large supernumerary (like Nos. 13 or 48 of the general list given in "Study V") and is only of interest for comparison with the other individual. The latter, hereinafter designated as "No. 64," shows a different chromosome-combination from any heretofore seen in this genus or elsewhere. The diploid groups (spermatogonia) contain 22 chromosomes; but both these groups in themselves and their history in maturation proves most clearly that they are not the same as in the typical 22-chromosome forms, differing from the latter in respect to the idiochromosomes and the $m$-chromosomes. In the typical forms there are, as stated above, two of each of these chromosomes. In No. 64 , on the other hand, there are three $m$-chromosomes and but one idiochromosome (the large), the latter appearing as a typical odd or accessory chromosome, as in the material of Montgomery; thus, 18 autosomes $+3 m$-chromosomes +1 odd chromosome $=22$. That this is the true interpretation of the facts is demonstrated by the behavior of these respective chromosomes in the maturation-process. I would emphasize the fortunate fact that both testes of the animal show excellent fixation and staining (strong Flemming, iron haematoxylin) and that they contain multitudes of division-figures which demonstrate

2 Ibid: '05b, 05c, '06, etc. 
all the stages. The agreement of great numbers of division-fig ures from both testes leaves no doubt regarding the constancy of the essential phenomena (with rare minor variations, as indicated beyond). As will be seen, the modification of the diploid groups has led to corresponding modifications of the maturation-process that are most interesting in relation to some of the problems of synapsis and of the qualitative differences of the chromosomes.

\section{DESCRIPTIVE}

\section{a. The spermatogonial groups}

The peculiar anomaly of the chromosome groups, first seen in the spermatocyte-divisions, led me to examine the spermatogonial groups with particular care, and it will be worth while to state both the preliminary and the definitive results. These groups are in the nature of the case more difficult than those of the spermatocytes, owing to the greater number, smaller size, and greater crowding of the chromosomes; hence, only flat metaphase-plates and such as are not very oblique to the plane of section can safely be used. A search through the numerous dividing spermatogonia showed 35 cases that seemed to meet these conditions and also to show no serious obscurity or confusion of the chromosomes. Many of these are of almost schematic clearness, and some are well adapted for photographic reproduction. The first examination showed undoubtedly that 29 of the 35 cases contained 22 chromosomes each, including 19 large and three very small ones. Of the six exceptions, three seemed to lack one of the small ones, two, one of the large ones, and one a large and a small. Closer study of these six cases ultimately showed that in four cases the apparently missing third small chromosome was in reality present, though hidden among the larger chromosomes, while in two cases an apparently missing larger chromosome was found lying immediately below another one, the metaphase-plate not yet having become perfectly flat. This leaves but one exception in 35 cases, and we shall hardly go astray in the conclusion that this exception is probably the result of accident. In any case we may confidently conclude that the chromosome-group shown in 
the 34 cases may be taken as characteristic of the dividing spermatogonia, and that it occurs with a high degree of constancy.

Six of these groups, from the best that could be found, three from each testis, are shown in fig. I, $a-f$. These have been selected particularly to show the different positions of the three small chromosomes. The latter appear to follow no rule whatever, the three lying anywhere in the metaphase-plate; and all may be separate, all together, or two together and one separate. This is an interesting and significant fact, because in the first spermatocyte-division, as described beyond, the three are always associated to form a triad element which invariably occupies the same position in the chromosome-group (see p. 58).

For the sake of comparison, four spermatogonial groups from other individuals are here reproduced (from my fifth Study). Two of these (fig. $1, i, j$ ) are from femoratus, No. 29, which has the typical diploid group of 22 chromosomes, including but two small ( $m$-chromosomes.) The other two (fig. I, $g, h$ ) are from terminalis, No. 2, which has 23 chromosomes, including two $\mathrm{m}$ chromosomes and one small supernumerary ${ }^{3}$. As will appear beyond, this latter chromosome is wholly different in nature from the third small chromosome in individual No. 64, though indistinguishable from it by the eye in the spermatogonial groups.

As the figures show, the larger chromosomes in No. 64 show well marked size-differences, and in most of the groups a largest and second largest pair are usually fairly evident; but it is impossible to pair all of the chromosomes accurately by the eye. It is, however, obvious that not more than 18 of the 19 can be equally paired. One of them must either have no proper mate, or it must form a very unequal pair with the third small chromosome. The following possibilities must, accordingly, be considered:

1. The nineteenth large chromosome and the third small one are respectively a large and an abnormally small idiochromosome which form a pair of synaptic mates, or

2. The nineteenth large chromosome is an odd or accessory chromosome, without a synaptic mate, while the third small one is similar to a small "supernumerary" or

Cf: Photo. 29, Study V. 
All the figures are from camera lucida drawings. With a few exceptions they are a little more enlarged than those of Study V.

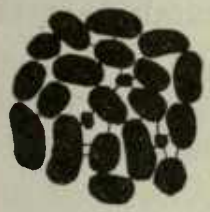

a

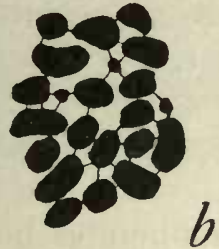

b
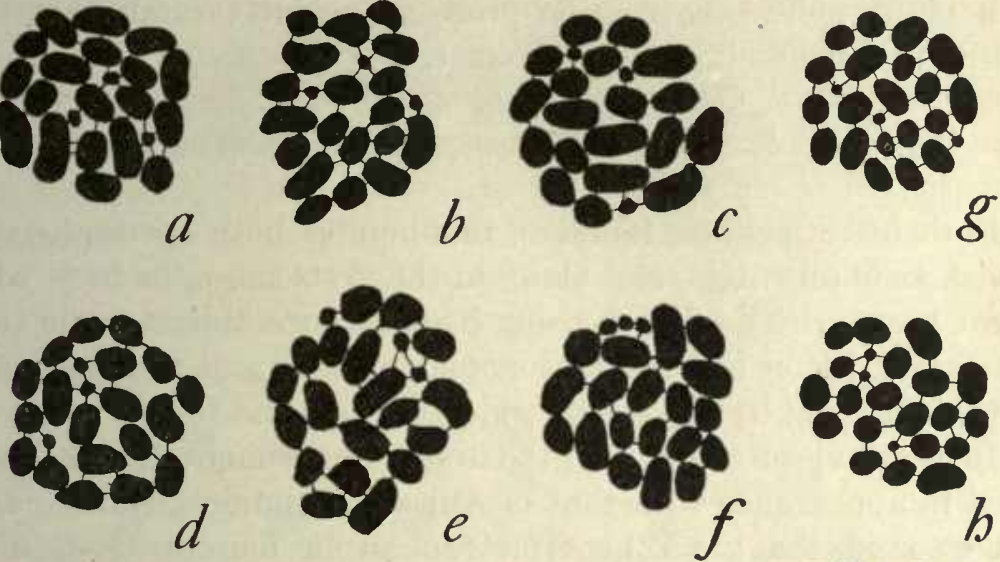
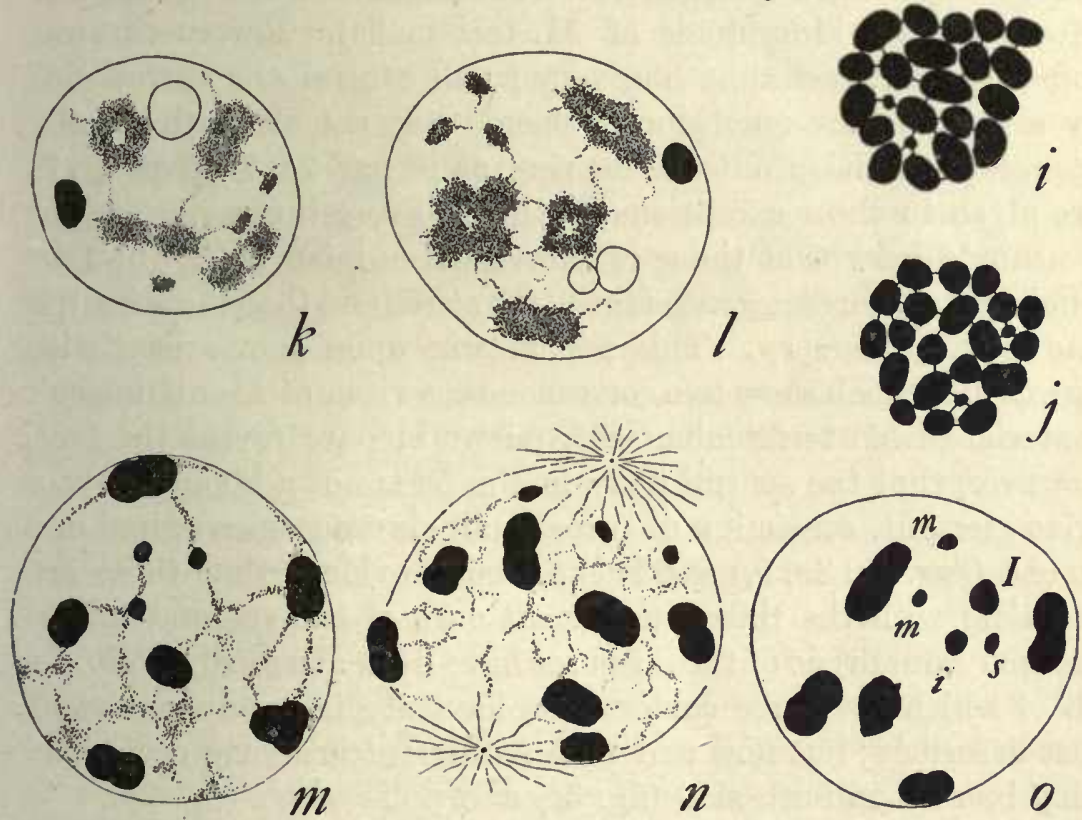

Fig. $1 a-f$, spermatogonial groups, M. femoratus, No. 64 , three from each testis; $g, h$, spermatogonial groups for comparison from M. terminalis, No. 2, with one small supernumerary (23 ehromosomes); $i, j$, spermatogonial groups from"M. fenoratus, No. 29 (22 chromosomes); $k$, $l$, early prophases, No. $64 ; m$, $n$, late prophase of same; $o$, late prophase for comparison, from M. terminalis, No. 43, with one small supernumerary (23 chromosomes). 
3. An odd or accessory chromosome is present, and also a third $m$-chromosome.

A study of the maturation process decisively establishes the third of these possibilities as the fact.

\section{b. The first spermatocyte-division}

Both testes contain immense numbers of both spermatocytedivisions in all stages, and many of the cysts show the facts with great beauty. The first division itself at once indicates the true interpretation of the spermatogonial groups; and this is consisently borne out by the stages which precede and follow.

In polar views (fig. $2, d-g$ ) the first division metaphase is identical in appearance with that of Anasa, Chelinidea, Narnia, and other coreids that have 21 spermatogonial chromosomes (including Montgomery's individuals of M. terminalis). Eleven chromosomes appear, including one very small central one surrounded by a ring of nine much larger ones, while the eleventh usually occupies a position outside the ring, as in figs. $2 d$, $2 f$, (figs. $2 e, 2 g$ are given to show exceptions to this). From these views alone we should infer that the spermatogonial number is 21 , that the small central chromosome is the $m$-bivalent, and that the eccentric one is the accessory. This will appear upon comparison with figs. $2, h, i$, which show two corresponding views of Montgomery's material of M. terminalis. Side views at once reveal the fact, however, that the central body in No. 64 is not a bivalent but a triad element, consisting of three small chromosomes united end to end (figs. $2 b, 3 a, b$ ) and it is perfectly evident that these are identical with the three very small ones of the spermatogonial groups. Hundreds of these figures have been observed, in almost all of which the three components have the linear arrangement just described; but now and then a different grouping occurs, as may be seen in both side (fig. $3 c$ ) and polar views (fig. $2 g$ ).

In the ensuing division the ten larger chromosomes divide equally, showing as they draw apart the curious forms represented in fig. 4, which are closely similar to those described in Anasa by Paulmier ('99). As the figures show, the chromosomes in 

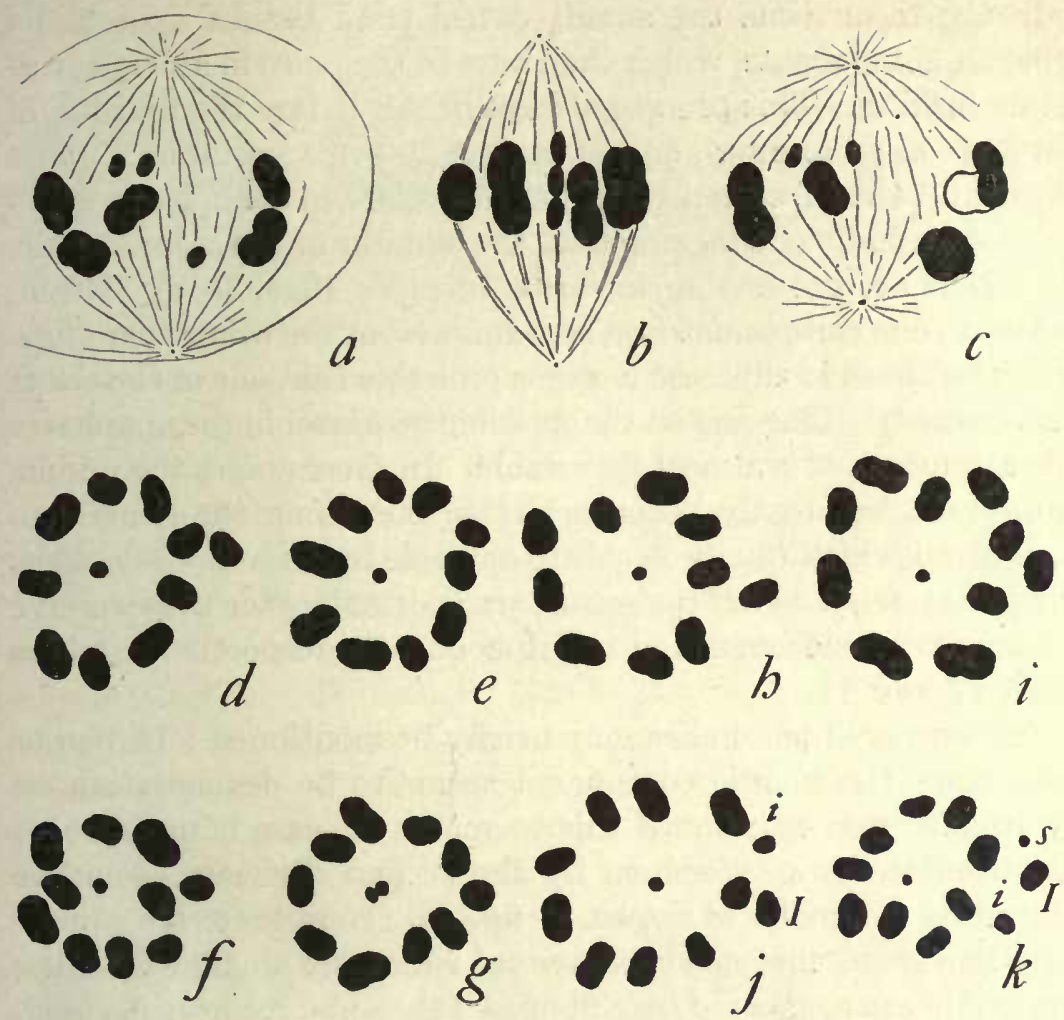

Fig. $2 a$, late prophase No. 64 , spindle forming; $b$, metaphase of same in side view; $c$, late prophase of M. femoratus, No. 29 (22 chromosomes), for comparison with $a ; d-g$, first division metaphase, polar views, No. $64 ; h, i$, similar views of M. terminalis, No. 3 (Montgomery's material), with 21 chromosomes; $j$, similar view of M. femoratus, No. 29 (22 chromosomes); $k$, similar view of M. terminalis, No. 43 (23 chromosomes), with one small supernumerary, for comparison.

the early anaphase are more or less clearly quadripartite, and separate into bipartite daughter chromosomes connected by conspicuous double fibers (figs. $4, b-h$ ) but true tetrads (such for instance, as those observed by Levfere and McGill in Anax, '08) are rarely if ever seen. The quadripartite form, though very characteristic of this division, is by no means invariable in case of the large bivalents, and has not been seen in case of the eccentric odd chromosome. 
In the mean time the small central triad breaks up into its separate components, which then pass to the poles in a very interesting fashion. This process always begins before the division of the large chromosomes, and is subject to some variation. Most frequently the three components draw apart in such a way as to leave the middle one lagging near the equator of the spindle while the others are proceeding towards the poles (flgs. $3 h, i$ ). Often, however, one component first separates from the other two (figs. $3 j, k)$; but even in this case it seems probable that one of the latter is afterwards left lagging on the spindle, since later in the anaphases this arrangement is almost invariable. In these stages the middle component frequently becomes drawn out along the spindle to form a rod which finally passes to one pole to enter the telophase group (figs. $4 e, f$ ). Half the secondary spermatocytes thus receive two small chromosomes and half but one, the respective numbers being 12 and 11.

Two observed anomalies may briefly be mentioned. In two or three cases the middle component seems to be degenerating on the spindle (fig. $4 \mathrm{~g}$ ); but if this be really the case it must be of rare occurrence, as is shown by the second division. Another interesting anomaly is shown in fig. $4 h$. Here there are apparently five small chromosomes, two of which are smaller than the others and are connected by a fiber as if they had recently divided. I am uncertain how to interpret this case, for one of the larger chromosomes (stippled in the figure) is paler than the others and lies at a lower level. This may be a fragment of the original plasmosome. If this be the case we have before us a case in which the central small chromosome has divided precociously. If all the five bodies, on the other hand be chromosomes, one of them would seem to be an extra or adventitious body, comparable to those described and figured by Paulmier in Anasa ('99, fig. 28a).

\section{c. The second spermatocyte-division}

As is to be expected from the asymmetrical distribution of the three small chromosomes in the first division the secondary spermatocytes are of two classes. These divisions are very numerous 

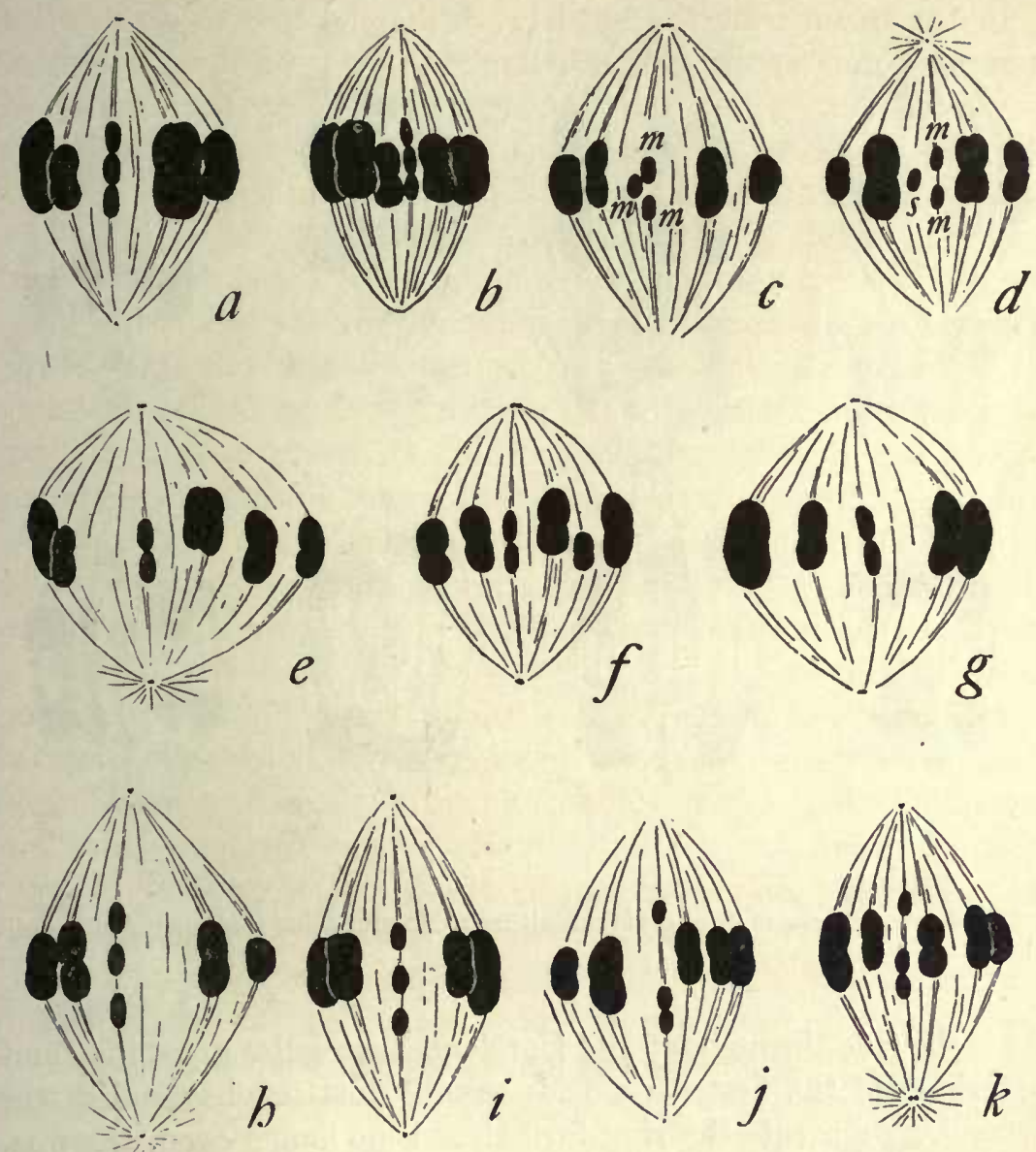

Fig. 3 Metaphases and anaphases of the first division, No. 64, in side view. $a$, $b$, typical side views, with linear central $m$-triad; $c$, unusual grouping; $d$, similar view of M. terminalis, No. 1, for comparison, with one small supernumerary and $m$-bivalent (23 chromosomes); e.g, similar views of M. femoratus, No. 29 (22 chromosomes) for comparison; $f$, the same, M. femoratus, No. 46 (22 chromosomes); $h-k$, No. 64 , initial anaphase, separation of the $m$-triad.

in both testes, and all the stages are shown by hundreds. In polar views of the metaphases about half the cells are seen to contain 11 chromosomes (fig. $5 a, b$ ) and half 12 (fig. $5 c, d$ ), the former containing but one small chromosome and the latter two. 

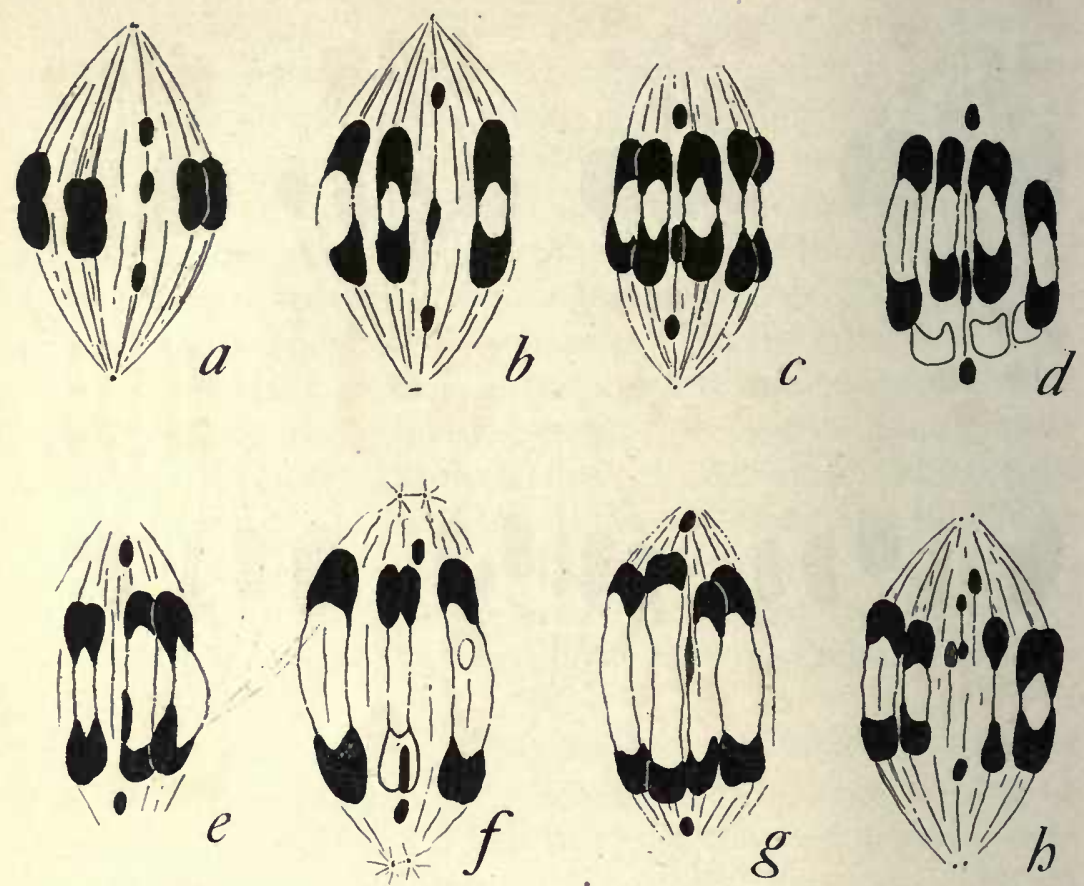

Fig. 4 Anaphases of first division, all from No. $64 ; g$ and $h$ are atypical conditions.

As is the rule throughout the Coreidæ, the regular grouping characteristic of the first division is usually lost or obscured in the second. As a rule the ring formation is no longer seen, there is, no constantly eccentric chromosome, while the $m$-chromosome, invariably central in position in the first division, now occupies any position, though it is more frequently near the center of the group.

In side views of the metaphases all of these chromosomes, with one important exception, are dumb-bellshaped, and in the initial anaphases are seen drawing apart into a pair of daughter-chromosomes (fig. 5e-g). One chromosome, almost invariably central in position, forms an exception in showing no sign of constriction, its form being evenly rounded and often nearly spheroidal. As 

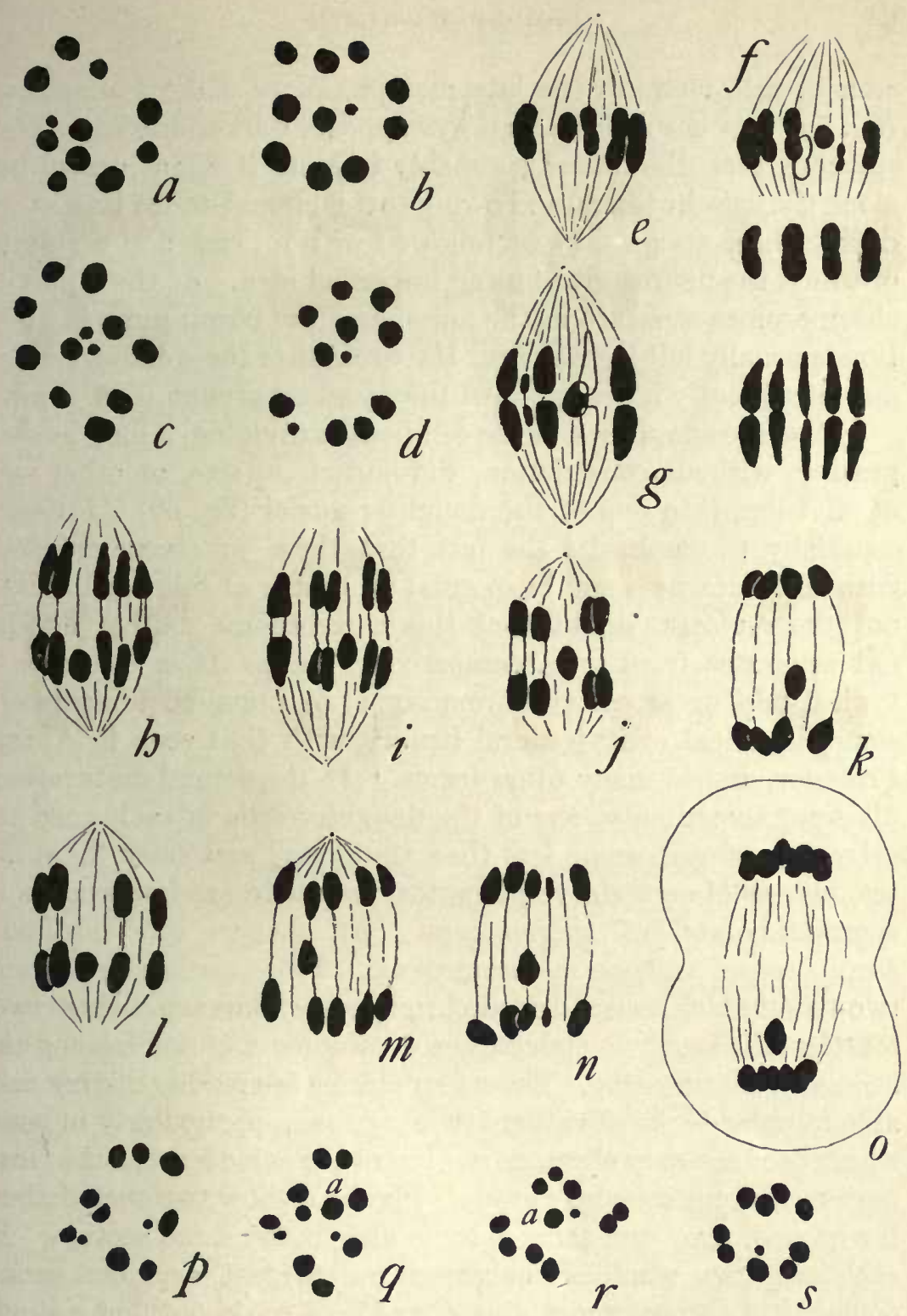

Fig. 5 Second division, No. 64. $a, b$, metaphases, polar views, 11 chromosomes; $c, d$, the same, 12 chromosomes; $e$, side view; $f$, the same showing all the chromosomes (four from lower level shown below); $g$, initial anaphase, all the chromosomes shown (five of them from a lower level at right); $h-0$, later anaphases; $p, q$, sister groups, from the same spindle, late anaphase, $p$, the upper group with 11 chromosomes; $q$, the lower group with $12 ; r, s$, two late anaphase groups (not from the same spindle) to show the third and fourth types of spermatid nuclei. 
seen in side views of the late metaphases or earliest anaphases (fig. $5 \mathrm{~g}$ ) this chromosome always appears darker and more conspicuous than the others (probably because it is not drawn out along the spindle fibers) and owing to this circumstance its history during these stages may be followed with an ease and certainty of which the figures give but an imperfect idea. As the bipartite chromosomes separate in the anaphases the chromsome in question is usually left lagging near the equator of the spindle, though not infrequently it lies in one of the daughter groups (figs. $5, h-n$ ).

In the late anaphases, as the cell-body is dividing, it may be seen passing, without.constriction, diminution in size, or other sign of division, into one of the daughter-nuclei (fig. 50). I desire especially to emphasize the fact that these processes are seen with such clearness and in so great a number of cells, as to leave not the remotest doubt that this chromosome neither divides nor separates from an accompanying mate. It is therefore a typical odd or accessory chromosome, or unpaired idiochromosome, identical in its general history with that seen in Anasa, Protenor, and so many other forms. In the second maturationdivision, accordingly, one of the daughter-cells in each case receives one chromosome less than the other; and since there are two classes of secondary spermatocytes, there are four classes of spermatids and of spermatozoa. All receive nine ordinary chromosomes and one $m$-chromosome. Two-fourths contain and two-fourths lack a second small chromosome; and each of these twofourths falls into two classes, one containing and one lacking the accessory chromosome. These four classes are readily distinguishable in polar views of rather late anaphases, particularly in cases where the accessory chromosome lies at the same level as the chromosomes of one daughter group. Fig. $5 p, q$ show two such daughter groups, from the same spindle and in the same section. In each case two small chromosomes are present, and one group contains 11 chromosomes, the other 12 . I could not find a single case of the other type (with 10 and 11 chromosomes) in which both daughter-groups appear in the same spindle: but two anaphase groups from different spindles are shown in fig. $5 r, s$, the former containing 11 chromosomes, the latter 10 . 


\section{d. The Growth-period and Maturation-prophases}

The foregoing facts demonstrate in the clearest manner that this individual of M. femoratus differs from all other individuals of the genus heretofore examined, with the exception of Montgomery's material of M. terminalis, in having an odd or unpaired idiochromosome (accessory chromosome) which corresponds to the larger member of the pair of unequal idiochromosomes found in other individuals. They show also that the third small chromosome is not a small supernumerary of the type found in other individuals, and is nothing other than a third $m$-chromosome. This is fully borne out by the growth-period and prophases. As I have indicated in earlier papers, the $m$-chromosomes are in general characterized during the growth-period by the fact that they remain univalent (there are some exceptions to this) and in most cases (of which Metapodius is one) are in a diffuse and lightstaining condition. Further, as was first shown by Gross ('04) in Syromastes, as a rule they only conjugate to form a bivalent in the final prophases of the first division-very often not until the spindle is formed and the chromosomes are entering the equatorial plate. Such a late prophase, from a 22-chromosome individual of the same species (No. 29) is shown for the sake of comparison in fig. $2 c$, the two separate $m$-chromosomes appearing above and towards the left. Their final conjugation always takes place at the center of the group (fig. $2 j, 3, e-g$ ).

In individual No. 64, prophases of every stage are shown in hundreds of nuclei. In the latest stages, after the nuclear wall has broken down, three separate small chromosomes are shown (fig. 2a) which may be seen coming together in the final prophases to form the small central triad. Figs. $1 m$, $n$ show two earlier stages from the same cyst with the last, one of them showing the beginning of the spindle-formation, the other an earlier stage when the asters are very small and often invisible. Each of these shows the three separate small chromosomes, as before. At this time all the chromosomes are compact and deeply stained. In still earlier stages, at a time when the bivalents are all diffuse and appear in the form of lightly staining double crosses, rods, etc., 
the three small chromosomes are still easily seen in many of the nuclei; but they are now pale and diffuse like the bivalents. In this respect the third small chromosome differs from a "supernumerary" of the type described in my former paper, and agrees exactly with the $m$-chromosomes.

Each nucleus contains at this period a single compact, rounded and intensely staining chromatic nucleolus, which is no doubt the odd or accessory chromosome (monosome), as in so many other forms, ${ }^{4}$ and in addition there is present a conspicuous, rounded

4 This identification is in agreement with that of most observers in recent years. A few writers have however disputed the view that the chromatic nucleolus of the growth period of the spermatocytes is a chromosome-e. g., Moore and Robinson in case of the cockroach ('05), Foot and Strobell in the case of Anasa('07) and Euschistus ('09), and Arnold ('08), in case of Hydrophilus. The results of Moore and Robinson on this point are opposed by those of Stevens ('05),Wassilieff ('07), and more particularly by the detailed observations of Morse ('09). Those of Foot and Strobell on Anasa are not sustained by the later ones of Lefevre and McGill ('08). Among others who have in the past two years adhered to the view here adopted may be mentioned Otte ('07), Davis ('08), Boring ('07), Jordan ('08), Stevens ('08, '09), McClung ('08), Robertson ('08), Randolph ('08), Nowlin ('08), Payne ('09,) Wilson ('09b, '09c), Gutherz ('09), Wallace ('09), Gerard ('09), and Buchner ('09a). Since I intend to return to the subject hereafter I will take this occasion for only brief comment on some of these results, without attempting a full review of the literature.

Moore and Robinson, who have been followed by Arnold (Strasburger, '07, '09. expresses the same opinion) also regard the body that is seen passing to one pole in one of the maturation divisions ("accessory chromosome") as not a chromosome but a "nucleolus." I find it incredible that anyone can hold to such a view who reckons squarely with the large existing body of direct and detailed observation upon the accessory chromosome itself; and this view seems to be quite ruled out of court by comparative studies on the sex-chromosomes, such for instance as those of Payne on Gelastocoris and the reduvioids. I will not enter here upon the maze of difficulties regarding the numerical relations of the chromosomes which the same view involves, since they have already been indicated by Gutherz ('09), in a recent reply to Strasburger. My own preparations, including an extensive series of sections and smears especially of Protenor, Lygæus and Pyrrhocoris leave in my mind not the least doubt of the identity of the chromatin-(chromosome)nucleolus of the growth period with the odd chromosome (monosome) of the spermatogonia, and with the heterotropic or accessory chromosome of the maturation-divisions.

Certain writers have seemed to take it for granted that the accessory chromosome or "monosome" is always characterized by its nucleolus-like condition in the resting nuclei, not only in the spermatocytes but also in the spermatogonial and other 
pale plasmosome which is considerably larger than the chromosome nucleolus. This body, particularly well shown in these slides, is at once recognizable by its smooth contour, spheroidal form (sometimes double, as in fig. $1 l$ ) and pale yellowish color after the hæmatoxylin, and it forms a striking contrast to the intense blue-black of the chromosome-nucleolus. Nuclei in which all five bodies-the three small chromosomes and both

diploid nuclei. This assumption-which is doing much to confuse the whole subject-may accord with the facts in certain species, but certainly is not generally true. Much of the recent work in this field, as well as some of the earlier (e.g., that of McClung '00, and Sutton '00) goes to show that in many species it is only in the growth-period of the spermatocytes that this chromosome forms a chromosome nucleolus, not in the diploid nuclei of either sex. Such seems to be the case in all the Hemiptera that I have studied. In these animals the accessory chromosome, or its homologue the large idiochromosome, first assumes the nucleolus-like condition in the post-spermatogonial stages, when its origin from an elongate chromosome may in some species readily be followed step by step, as I have shown in Lygæus ('05b) and Pyrrhocoris ('09b). In the spermatogonia of these animals this chromosome does not differ visibly in behavior from the others and cannot be scen in the resting nuclei.

Several years ago, in two successive papers ('05a, '06) I described and commented on the interesting fact that in the female this chromosome (and its fellow, when present) seems in some species not to assume a nucleolus-like condition in the synaptic stage and early growth-period of the oöcytes. Since some doubts on this point were raised in my own mind by the later work of Stevens ('06) and Gutherz ('07)I am now glad to have the very positive confirmation of my results given by the work of Foot and Strobell ('09) on Euschistus (one of several forms I had examined). This confirmation must have been made without knowledge of my previous work, since the latter is referred to in neither text nor literature list, and the supposedly new facts are made the main basis for renewed attack upon my general conclusions. On the other hand Buchner ('09a) has recently found in the synaptic or "bouquet" stage of the oöcytes in Gryllus a nucleolus-like "accessory body" which he believes to be of the same nature as the accessory chromosome of the male, though its history.in maturation was not followed out, nor is other proof of the conclusion given.

It is of some psychological interest to find Buchner on the one hand and Foot and Strobell on the other disputing my conclusions regarding sex-production on diametrically opposing grounds, the first-named author because (as he believes) a chromosome-nucleolus is present in the oöcyte-nucleus, the last named because it is absent(!). In what way either of the mutally contradictory arguments invalidates or weakens my conclusions I am not yet able to perceive, nor need we here consider the contradiction in the data; but it is interesting to observe how each of the arguments goes awry by reason of the confusion regarding the chromosomenucleolus, referred to above. Foot and Strobell, for example, argue that because 


\section{nucleoli-are visible in the same section are not very common.} Two such cases are shown in figs. $1 k, l$., each of which shows also four of the nine larger bivalents.

I have not endeavored to make an exhaustive study of the growth-period as a whole, but the facts reported above taken in

such a body is not present in the oöcyte-nucleus, therefore the odd or accessory chromosome of the male cannot be derived in fertilization from the egg-nucleus -an obvious non sequitur. Buchner's argument, based upon precisely opposite data, shows a somewhat similar, though less obvious entanglement. The essence of his objection is given in the following passage, which at the outset accepts all the essential facts on which the conclusions of Stevens and myself were based. "Auf alle Fälle haben wir nur eine Sorte von Eiern, denn dass er (the accessory chromosome) in einem $\mathrm{Ei}$ ausgestossen und im andern innenbehalten wird, erscheint undenkbar. Die Spermatozoen haben das accessorische Chromosom zur Hälfte. Nehmen wir an, die Eier besässen das accessorische Chromosom schon, so gäbe es Tiere mit zwei Monosomen und solche mit einem-ein Fall der nicht existiert" (op. cit., p. 409, italics mine). This is, indeed, an astounding statement; for it was the very fact that there are individuals that have but one monosome or accessory chromosome (the males), and other individuals of the same species (the females) that have two corresponding chromosomes, upon which the conclusions of Stevens and myself were mainly based(!). This is true, as Gutherz ('08) has shown, of the very form (Gryllus) of which Buchner is writing, the single odd chromosome (monosome) of the male, recognizable by its peculiar form and other characters, being represented in the female by two such chromosomes. This is also in agreement with the results of other recent workers on the Orthoptera including Wassilieff, Davis, Jordan and Morse. I can therefore find no meaning in Buchner's statement unless the word "Monosom" be used to denote simply a chromosome-nucleolus, when the passage becomes at least intelligible. But such a restriction in the meaning of this word is not justified by its etynology, by the original definition of its author (Montgomery, '06a, '06b) nor by the facts; and it does not seem to accord even with Buchner's own usage elsewhere in the paper. That Buchner's statement is totally at variance with the facts when correctly stated is shown by the following summary of my results, quoted from one of the papers in which Montgomery first defined the word "monosome." "When there is a single monosome in the spermatogenesis (as in Protenor, Harmostes, Anasa and Alydus) there are two in the ovogenesis so that the ovogonia possess always an even number of chromosomes"' ('06b, p. 145, italics mine).

But even if we admit that the "accessory body" of the female is a chromosomeand not only is there no proof of this but many reasons for doubting it - what adverse bearing would the fact have upon the "theory"? None as far as I can see, unless this chromosome were proved to be univalent and without a synaptic mate Were all this true,new and unintelligible complications would arise in regard to the numerical relations of the diploid and haploid chromosome-groups in both sexes; but it is not worth while to consider these puzzles since they lie in a region not of observed fact but of pure phantasy. 
connection with the spermatocyte-divisions, are thoroughly decisive in showing the third small chromosome to be an extra $m$-chromosome not distinguishable in any respect from the other two.

\section{DISCUSSION}

It seems to me that in the individual of Metapodius that has here been described nature has performed an experiment which, as far as it goes, is precisely such as we should like to carry out artificially in order to test the hypothesis of the genetic continuity of the chromosomes and the question of their qualitative relations in the maturation-process. . The experiment (if we may call it such) consisted in the omission from the typical 22-chromosome diploid groups of the small idiochromosome, and its replacement by one of different type, a third $m$-chromosome. In what way this was effected can only be conjectured; but it seems altogether probable that the anomaly was present in the original fertilized egg, as a result of one preëxisting in one or both the gametenuclei. ${ }^{5}$ In any case we may be sure that it arose very early in the ontogeny, at a period prior to the separation of the right and left gonads, since both testes show precisely the same characters.

It is certain that the initial anomaly has persisted unchanged through many generations of cells, and that the alteration in the diploid groups has involved corresponding modifications in the maturation-process. The significant fact is that throughout this process the chromosome that has been added does not take the place of the one that has been omitted, but behaves according to its own kind. This is a truly remarkable result when we consider that the number of chromosomes in the diploid groups (22) remains unaltered. These groups still consist of 11 pairs of chromosomes; but one is

${ }^{5}$ We must assume, in this case, that the sperm-nucleus contained no small idiochromosome and that either this nuclcus or the egg-nucleus contained two $m$-chromosomes. The former condition may have resulted from a failure of the idiochromosomes to separate in the second spermatocyte-division (which, as I have shown, may actually occur). The presence of a sccond $m$-chroinosome may be due to a similar cause. 
an unnatural or hybrid pair, which consists of non-homologous members - the large idiochromosome and the third $m$-chromosome. The facts show most decisively that these two chromosomes do not play the part of synaptic mates towards each other, but retain each its own characteristic behavior. In synapsis the third $m$-chromosome invariably couples with its own kind to form a triad element while the large idiochromosome remains unpaired. Thus the substitution of one chromosome for another of a different kind has been followed by no regulative process, and a permanently new combination has been produced. The full force of this conclusion first becomes evident when we compare the present case with those in which there is present a single small supernumerary of the type described in my fifth Study. In the diploid groups such a supernumerary is quite indistinguishable from a third $m$-chromosome - as we may see, for instance, upon comparison with figs. $1 k, 7, t-y$, photograph 29, of Study V. ${ }^{6}$ In the first spermatocytedivision also, in cases where a small supernumerary lies within the ring of large bivalents (as in photograph 6, fig. $7 i$ of my fifth Study) side-views give a picture almost indistinguishable from such a condition as that shown in fig. $3 c$. Such a side view of terminalis No. 1 , is given for comparison in fig. $3 d$, the two $m$-chromosomes just separating at the center of the group, and the supernumerary $(s)$ just to the left. The resemblance between this figure and fig. $3 c$ is so close as to amount almost to identity. It seems incredible that the behavior of the third small chromosome in the ensuing division should not be identical in the two cases; and it should be identical were the history of the individual chromosomes in maturation determined merely by their size or their mechanical relation to the achromatic figure. In point of fact, however, the small supernumerary and the third $m$-chromosome show characteristic differences throughout the whole process. In the growth-period the former appears as a condensed chromosome-nucleolus, usually coupled with the idiochromosome-nucleolus, while the $m$-chromosome remains diffuse and usually free. In the first division the former divides as a univalent (i. e., it is typically uncoupled, though it may be in contact with the idiochromosomes) and is

${ }^{\circ}$ Loc. cit.: '09c. 
usually outside thering (as in fig. $2 h$ ); while the third $m$-chromosome is always coupled with the two others at the center of the ring, and moves to one pole without division. In the second division these relations are almost exactly reversed, the $m$-chromosome dividing equationally as a univalent, while the supernumerary does not divide and is typically coupled with the idiochromosome bivalent near the center of the group. I desire to emphasize the fact that these differences are in no way obscure or difficult to see, but are conspicuously shown in so great a number of cells as to remove all doubt. ${ }^{7}$

${ }^{7}$ This point demands emphasis because of the scepticism expressed by certain writers in regard to the constancy of the chromosomes in respect to number, size and behavior. Conspicuous among these writers is Della Valle ('09) who has brought together a valuable if somewhat uncritical review of the literature, and contributes careful observations of his own upon variations in the chromosomenumber in the somatic cells of Salamandra. Such scepticism is perhaps not surprising in view of the unlucky contradictions that still exist in the literature even of so favorable and well known a group as the insects. But to ascribe this confusion of the literature to a confusion of the facts-i.e., to an inconstancy so great as to preclude the possibility of attaining exact results-would be, I think, a fatal blunder. The confusion in the literature cannot, of course, be attributed altogether to mistakes of observation or to accidents of technique - though both these must be held to a strict reckoning. I am not aware that anyone has maintained that the relations of the chromosomes form an exception to all other biological phenomena in being absolutely fixed and immutable; and due weight should be given to the numerical variations that have been recorded by Della Valle and many others myself included. The fact remains that it is possible to determine accurately what are the normal or typical relations of the chromosomes, as of other structures, and to establish in many cases their high degree of constancy. The same common sense must be used in the treatment of these relations as in the case of other phenomena that are subject to variation. For example, insects have been seen with seven legs, but it is not for this reason to be doubted that insects have six legs. In like manner, in the ovaries of Largus cinctus I have seen as many as three dividing cells that show 13 chromosomes; but I nevertheless do not doubt; after the study of a large number of cases, that the typical number is 12 .

The case of Metapodius is disposed of by Della Valle in the following easy fashion. "Not constancy but variability in the number of chromosomes is the general rule in all organisms; of which the observations published by him (Wilson) are but a special confirmatory case" (op. cit., p. 161, translation). Better acquaintance with the facts in Metapodius would probably render Prof. Della Valle less certain of this; for I am confident that no observer of ordinary competence could confuse such a series of relations as that here displayed with the occasional fluctuations with which we are familiar in many forms, including this very genus. 
It seems to me that such facts have the value of actual experimental evidence in support of the hypothesis of the genetic continuity of the chromosomes and that of their qualitative difference. All will admit that the peculiarities of the later generations of cells in this individual of Metapodius are inherited from earlier ones. It is the obvious, natural and, I think, inevitable conclusion that the third $m$-chromosome, introduced at an early period, has not lost its identity in the later stages. If its presence is merely owing to a corresponding excess of chromatin, how shall we account for the characteristic peculiarities of behavior that differentiate it so sharply from an ordinary "supernumerary" of corresponding size? To reply that the excess represents a particular kind of chromatin that is re-segregated at each division in the form of a particular chromosome is to grant the most vital assumption in the hypothesis of genetic continuity.

I think that sufficient emphasis has not yet been laid upon the support given to this hypothesis by the variable position of the chromosomes in the diploid groups. I have several times pointed out in this paper and preceding ones, that there is no constancy in the relative position of the spermatogonial chromosomes-as may be seen with particular clearness in case of the $m$-chromosomes of the Coreidæ or the small idiochromosome of the Pentatomidæ or Lygæidæ, or of chromosomes distinguishable by their large size, such as are seen in Protenor, Largus or Anasa. This is certainly not what we should expect were the chromosomes merely "tactic" formations that appear in characteristic array, as a crystal form in a solution, merely because of the specific properties of a single chromatin-substance as such. Two answers might be made to this. It might be said that the chromosomes merely represent the segregation of so many different kinds of chromatin that are mixed together in the resting nucleus. ${ }^{8}$ I am disposed to regard this as a tenable hypothesis; but obviously it grants the most essential part of the continuity hypothesis. Again it might be said that the chromosomes are originally formed always in the same ! osition but lose it by subsequent shiftings in the prophases. It

${ }^{8}$ Cf. Fick: '05; Wilson: '09c. 
would be difficult to disprove this in ordinary cases; but fortunately Boveri's studies on Ascaris ('09) have shown beyond all doubt that in this form there is no constancy in the original position of the prophase chromosomes, the only definite order being shown in the close agreement of each pair of daughter-cells. The position of the prophase chromosomes as Boveri shows with great cogency is here a consequence of the position in which they entered the nucleus in the preceding telophases; as the latter position is itself due to causes (which may be quite fortuitous) that determine the position of the preceding metaphase chromosomes.

The facts support no less directly and strongly the conclusion that the chromosomes differ among themselves in a definite way in respect to their behavior, and hence in respect to their functional significance. The differences seen in the maturation-process have thus far taught us nothing whatever in regard to the individual physiological meaning of the chromosomes, in heredity or otherwise, and they are not to be compared in value with the results of direct experiment, such as those carried out by Boveri ('07) in dispermic sea-urchin eggs. It is nevertheless of great interest that the results from these different sources should be in harmony. In my preceding paper I have called attention especially to the significance of the couplings of the chromosomes, pointing out that these certainly do not depend upon the size of the chromosomes (though those which couple in synapsis are in fact equal members of a pair save in certain special cases) nor can they, apparently, depend upon the achromatic mechanism. The various combinations in Metapodius seem to arise simply by the addition or subtraction of certain chromosomes without alteration of the achromatic elements; yet in the resulting new combinations the chromosomes still behave each according to its kind, and (as previously indicated) irrespective of their size. We seem thus driven to accept the view that the chromosomes are physico-chemically different, with all the consequences which such a view may involve.

The cogency of the evidence in favor of the qualitative differences of the chromosomes brought forward in Boveri's masterly work must be generally recognized, as has recently been admitted even by Driesch ('09) who formerly endeavored to find a different 
explanation of the facts. It will be evident to readers of my former papers that I am fully prepared to accept Boveri's conclusions; but there is one very important fact, finally established by the present paper, that must be clearly recognized. If we assume that different factors of heredity are in some sense unequally distributed among the chromosomes, we need feel no surprise that the duplication of one or more of the ordinary chromosomes should produce no perceptible qualitative effect upon development. But it is very surprising that no visible effect should be produced by the removal of a particular chromosome that has no duplicate to take its place. In preceding papers I have called attention to the singular fact that Montgomery's material of M. terminalis differs consistently from my own in the lack of the small idiochromosome or "Y-element" (see Wilson '09a, for this term); but the possibility of two distinct species or races having been confused could not be absolutely excluded. In the present case, however, no doubt can exist, since the two original specimens of M. femoratus from Miami, Florida, are in my cabinet, and in perfect condition for identification. One of these, as already stated, contains both the small idiochromosome and an additional supernumerary, while both are lacking in the other individual; yet the two individuals seem to be otherwise in every essential respect identical. All doubt is thus removed that the small idiochromosome or Y-element, which forms the synaptic mate of the accessory chromosome or $\mathrm{X}$-element, may be present in some individuals and absent in others of the same species, without the appearance of any corresponding differences in the sexual or other characters as far as shown in the external morphology of the animal. ${ }^{2}$ This chromosome, as shown

'It will be seen from this how readily discrepancies regarding the number of chromosomes might arise between different observers working on the same species. It might seem that we have here a simple and plausible explanation of the contradictions that have arisen in the case of Anasa tristis; for we might assume that the diploid number is 21 in some individuals of this species and in others 22 ; and a similar explanation has in fact already been adopted by more than one recent writer (cf. Della Valle: '09, Buchner: '09b).

I am not myself able to take this view of this particular case for several reasons. In the first place, if there be individuals of this species that have 22 spermatogonial chromosomes, as maintained by Foot and Strobell ('07) we should expect to see 
by the work of Stevens and myself, is widely distributed among the insects (Hemiptera, Coleoptera, Diptera) and is strictly confined to the male line (except when supernumeraries are present). In species having an odd or accessory chromosome the Y-element (small idiochromosome) is wanting, and I have urged this fact as showing that this latter chromosome cannot play any essential rôle in sex-production ${ }^{10}$ or in the transmission of the secondary sexual characters, as Castle ('09) ingeniously suggests. What I desire here to point out is that by parity of reasoning we should also conclude that this chromosome is devoid of any special significance in heredity of any kind, at least as far as the visible external charac-

12 instead of 11 separate chromosomes in the first spermatocyte division, as we do in the 22-chromosome individuals of Metapodius(Wilson: '09c). Throughout the Hemiptera, indeed, when the accessory chromosome (or its homologue, the large idiochromosome) is accompanied by a synaptic mate or Y-element, the two are separate in the first division, which accordingly showsone more than the reduced or haploid number-i.e., ${ }_{2}^{n}+1$. The photographs of Foot and Strobell show, however, 11 chromosomes in this division (the two $m$-chromosomes being of course counted as one, like the other bivalents), as they should if the spermatogonial number be 21 .

Still, this might be a case like that of Syromastes, where no Y-element is present, but the accessory is itself double-though such a parallel would hardly help the case, since in no form is the failure of the accessory to divide in one division more indubitably shown than in Syromastes, while Foot and Strobell are persuaded that it does divide in Anasa. But, secondly, my own extended additional observation, like the studies of Lefevre and McGill ('08), still continues to give but one result as before. The living animals (from the same locality as the material of Foot and Strobell) have been kept by hundreds in the greenhouse for months at a time in successive years, and have been regularly employed for class work in cytology and for experimental purposes, in the course of which large numbers of additional sections and smears have been prepared and examined. Others as well as myself have carefully searched among these preparations for cases showing more than 21 spermatogonical chromosomes, without success apart from the double or multiple groups that occasionally appear. The same relation continually recurs, namely 21 spermatogonial chromosomes of which three are larger than the others, while in the dividing ovarian cells the number 22 appears with equal constancy. That not even one case of 22 spermatogonial chromosomes has thus far been found is indeed surprising; for plus variations in the diploid groups are known to occur in some species of Hemiptera, and I have myself described such cases (e.g., Wilson: '09c).

These and other reasons lead me to believe that the conclusions of Foot and Strobell were based on the observation either of very rare fluctuations in the normal diploid number or of accidental products of the technique.

${ }^{10}$ Loc. cit.: '06, '09a, '09d. 
ters and the more obvious internal features are concerned. Taken by itself this case may seem to form a legitimate piece of evidence against Boveri's theory. It cannot, however, be taken alone, but must be viewed from the more general standpoint given by the evidence as a whole. We are still too ignorant of the significance of the "sex-chromosomes" to form an adequate opinion as to the meaning of the Y-element. It may be in this case (as I earlier suggested) a degenerating element, or may represent an excess of a chromatin that is duplicated elsewhere in the chromosomegroup; but these and other speculative possibilites that suggest themselves may well await the outcome of further study.

Department of Zoölogy, Columbia University, December 23, 1909.

\section{BIBLIOGRAPHY}

Arnold, G. 1908 The Nucleolus and Microchromosomes in the Spermatogenesis of Hydrophilus piceus. Arch. Zellforsch., 2, 1.

Boning, Alice N. 1907 A Study of the Spermatogenesis of Twenty-two Species of Jassidæ, Cercopidæ and Fulgoridæ. Journ. Exp. Zoöl., 4, 4.

Boveri, Th. 1907 Die Entwickelung dispermer Seeigel-Eier. Ein Beitrag zur Befruchtungslehre und zur Theorie des Kerns. Zellenstudien, VI, Jena, 1907; also Jena Zeitscher., 42, 1.

1909 Die Blastomerenkerne von Ascaris megalocephala und die Theorie der Chromosomenindividualität. Arch. Zellforsch., 3, 1, 2.

Buchner, P. $1909 a$ Das accessorische Chromosom in Spermatogenese und Ovogenese der Orthopteren, etc. Arch. Zellforsch., 3, 3.

1909 Review of Lefevre and McGill on The Chromosomes of Anasa tristis. Arch. Zellforsch., 2, 4.

Castre, E. 1909 A Mendelian View of Sex-heredity. Science, n. s. March 5.

Davis, Herbert S. 1908 Spermatogenesis in Acrididæ and Locustidæ. Bull. Mus. Comp. Zoöl., 53, 2.

Delda Valle, P. 1909 L'organizzazione della cromatina studiata mediante il numero dei cromosomi. Archivio Zoologico, 4, 1.

Driesch, H. 1909 Die Entwickelungsphysiologie, 1905-1908. Ergebn. der. Anat. u. Entwickelungsgesch., 17.

FIcK, R. 1905 Betrachtungen über die Chromosomen, ihre Individualität, Reduktion and Vererbung. Arch. Anat. u. Phys., Anat. Abt., Suppl. 19. 
Foot and Strobell. 1907 A Study of Chromosomes in the Spermatogenesis of Anasa tristis. Am. Jour. Anat., 4, 2.

1909 The Nucleoli in the Spermatocytes and Germinal Vesicles of Euschistus variolarius. Biol. Bull., 16, 5.

Gerard, P. 1909 Recherches sur la spermatogénèse chez Stenobothrus biguttatus. Arch. Biol., 24, 4.

Gross, J. 1904 Die Spermatogenese von Syromastes marginatus. Zool. Jahrb., Anat. u. Ontog., 20.

Gutherz, S. 1907 Zur Kenntnis der Heterochromosomen. Arch. Mik. Anat. 69. 1908 Ueber Beziehungen zwischen Chromosomenzahl und Geschlecht Zentralbl. f. Phys., 22, 2.

1909 Weiteres zur Geschichte des Heterochromosomes von Gryllus domesticus. Sitzber. Ges. Naturfr., no. 7.

Jornan, H. E. 1908 The Spermatogenesis of Aplopus Mayeri. Carnegie Inst., Wash., Pub. no. 102.

Lefevre ANd McGirl. 1908 The Chromosomes of Anasa tristis. Am. Journ. Anat., 7,5 .

McCuung, C. E. 1900 The Spermatocyte Divisions of the Acrididæ. Kansas Univ. Bull., 1, 2.

1908 Spermatogenesis of Xiphidium fasciatum. Sci. Bull., Kansas Univ., 4, 4.

Montgomery, T. H. $1906 a$ The Terminology of the Aberrant Chromosomes, ete. Science, n. s., 23, 575 .

$1906 \mathrm{~b}$ Chromosomes in the Spermatogenesis of the Hemiptera heteroptera. Trans. Am. Phil. Soc., n. s., 21, 3.

Morse, M. 1909 The Nuclear Components of the Sex Cells in Four Species of Cockroaches. Arch. Zellforsch., 3, 3.

Moore And Robinson. 1905 On the Behavior of the Nucleolus in the Spermatogenesis of Periplaneta Americana. Q. J. M. S., 48, 4.

Nowlin, N. 1908 The Chromosome Complex of Melanoplus bivittatus. Sci. Bull., Univ. Kansas, 4, 12.

Отте, H. 1907 Samenreifung und Samenbildung bei Locusta viridissima. Zool. Jahrb., Anat. u. Ontog., 24.

Paulmier, F. C. 1899 The Spermatogenesis of Anasa tristis. Journ. Morph., 15, Suppl.

Payne, E. 1909 Some New Types of Chromosome Distribution and Their Relation to Sex. Biol. Bull. 16, 4.

Pinney, E. 1908 Organization of the Chromosomes in Phrynotettix magnus. Sci. Bull., Univ. Kansas, 4, 14.

RANDOLPH, H. 1908 On the Spermatogenesis of the Earwig, Anisolaba maritima. Biol. Bull., 15, 2. 
Robertson, W. R. B. 1908 The Chromosome Complex of Syrbula admirabilis. Sci. Bull., Univ. Kansas, 4, 13.

Stevens, N. M. 1905 Studies in Spermatogenesis with Especial Reference to the "Accessory Chromosome." Carnegie Inst., Wash., Pub. no. 36.

1906 A Comparative Study of the Heterochromosomes in Certain Species of Coleoptera, Hemiptera and Lepidoptera, with Especial Reference to Sex Determination. Ibid., No. 36, Part 2.

1908 A Study of the Germ-cells of Certain Diptera, with Reference to the Heterochromosomes and the Phenomena of Synapsis. Journ. Exp. Zoöl., 5, 3.

1909 Further Studies on the Chromosomes of Coleoptera. Ibid., 6, 1.

Strasburger, E. 1907 Ueber die Individualität der Chromosomen und die Pfropfhybridenfrage. Jahrb. Wiss. Bot., 44, 3.

1909 Histologische Beiträge, 7, Zeiptunkt der Bestimmung des Geschlechts, Apogamie, Parthenogenesis und Reduktionsteilung. Jena, 1909.

Sutron, W. S. 1900 The Spermatogonial Divisions in Brachystola magna. Kansas Univ. Bull., 1, 3.

Wallace, L. B. 1909 The Spermatogenesis of Aglaena nævia. Biol. Bull., 17, 2 .

Wassilieff, A. 1907 Die Spermatogenese von Blatta germanica. Arch. Mik. Anat., 70.

WrLson, E. B. $1905 a$ The Chromosomes in Relation to the Determination of Sex. Science, n. s., 27, 564 .

$1905 b$ The Behavior of the Idiochromosomes in Hemiptera. Studies on Chromosomes, I. Journ. Exp. Zoöl., 2, 3.

$1905 c$ The Paired Microchromosomes, Idiochromosomes and Heterotropic Chromosomes in Hemiptera. Studies on Chromosomes, II. Journ. Exp. Zoöl., 2, 4.

1906 The Sexual Differences of the Chromosomes in Hemiptera, etc. Studies on Chromosomes, III. Ibid., 3, 1.

1907 The Case of Anasa tristis. Science, n. s., 25, 631.

1908 The Accessory Chromosome of Anasa tristis. Report of Am. Soc. Zoöl., Science, n. s., 27, 690.

$1909 a$ Recent Researches on the Determination and Heredity of Sex. Vice-presidential Address, A. A. A. S., Science, n. s., 732.

$1909 b$ The Accessory Chromosome in Syromastes and Pyrrhocoris. Studies on Chromosomes, IV. Journ. Exp. Zoöl., 6, 1.

$1909 c$ The Chromosomes of Metapodius. A Contribution to the Hypothesis of the Genetic Continuity of Chromosomes. Studies on Chromosomes, V. Ibid., 6, 2.

$1909 d$ Secondary Chromosome-couplings and the Sexual Relations in Abraxas. Science, n. s., 29, 748. 



\section{STUDIES ON CHROMOSOMES}

VII. A Review of the Chromosomes of Nezara; with Some More General Considerations

By EDMUND B. WILSON

Zoölogical Department, Columbia University

Reprinted from ThE JouRNal OF MORPHOLOGX Vol. 22, No. 1, March 20, 1911 



\section{STUDIES ON CHROMOSOMES}

\section{A REVIEW OF THE CHROMOSOMES OF NEZARA; WITH SOME MORE GENERAL CONSIDERATIONS}

EDMUND B. WILSON

From the Zoölogical Department, Columbia University

NINE FIGURES AND ONE PLATE

\section{CONTENTS}

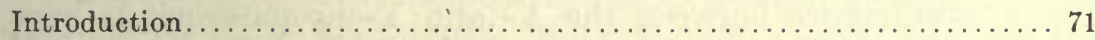

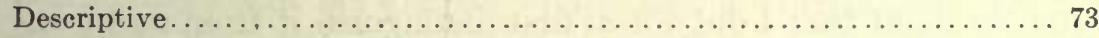

1 The second spermatocyte-division in Nezara................. 73

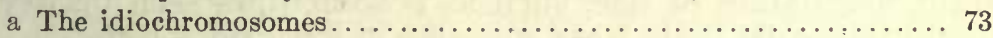

b The double chromosome.......................... 77

2 The first spermatocyte-division......................... 78

3 The growth-period and spermatocyte-prophases............. 80

4 The diploid chromosome-groups ........................ 83

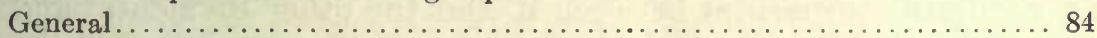

5 The idiochromosomes............................... 84

a Composition and origin of the XY-pair ............... 85

b Modifications of the X-element...................... 88

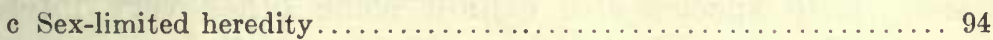

d Secondary sexual characters............................. 99

6 Modes in which the chromosome-number may change............. 99

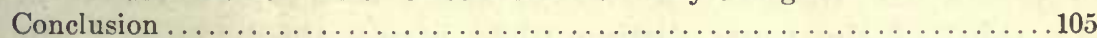

\section{INTRODUCTION}

In the first of these 'Studies' ('05a) I described the idiochromosomes ( $\mathrm{X}$ and $\mathrm{Y}$-chromosomes) of Nezara hilaris as being of equal size in the male, and reached the conclusion that in this species no visible dimorphism appears in the spermatid-nuclei. In my third 'Study' ('06), after examination of the female diploid groups, this species was assigned a unique position as the single then 
known representative of a type in which a pair of idiochromosomes can be identified in both sexes, but are of equal size in both, and in which, accordingly, no visible sexual differences appear in the diploid nuclei. These conclusions, as is now apparent, were based upon a wrong identification of the idiochromosome-pair, which is not the smallest pair, as I then believed, ${ }^{1}$ but one of the largest. When this fact was recognized, the true conditions soon became evident.

I was led to re-examine Nezara hilaris by the fact (very surprising to me) that in Nezara viridula, a southern species closely similar to $\mathrm{N}$. hilaris, the idiochromosomes of the male are extremely unequal in size, and the dimorphism of the spermatidnuclei is correspondingly marked. Upon returning to the study of $\mathrm{N}$. hilaris it soon became manifest that the dimorphism is present in this species also, though in far less conspicuous form. The size-difference between the $\mathrm{X}$ - and $\mathrm{Y}$-chromosomes is here often so slight that I did not at first distinguish it from an inconstant fluctuation of size, such as is sometimes seen between the members of the other chromosome-pairs. When, however, the identity of the XY-pair was correctly recognized, its constancy of position and of size in the second division enabled me to make an accurate comparison between it and the other bivalents; and this fully established the constant inequality of its members, which is constantly greater than that now and then seen in other pairs. Both species also exhibit some other very interesting features that I overlooked in my former studies.

Nezara can therefore no longer stand as a representative of the third of the types distinguished in my third 'Study,' but belongs with Euschistus, Lygaeus, etc., in the second type

${ }^{1}$ This was in part because in most of the other forms known at the time the idiochromosomes are in fact the smallest, or one of the smallest, pairs. In part, also, I followed Montgomery ('01) who described in this species two small "chromatin nucleoli" in the spermatogonial groups, and believed them to be identical with the chromatic nucleolus of the growth-period. In a later paper ('06) Montgomery states these "chromatin nucleoli" to be "apparently not quite equal in volume," and asserts that I was in error in describing them as equal. In my material they are certainly equal in the great majority of cases. However, this is not the idiochromosome-pair. 


\section{DESCRIPTIVE}

Since the two species agree very closely save in respect to the idiochromosomes they may conveniently be considered together. Before describing the divisions, attention may be called to a striking difference between the two species in respect to the size of the cells and karyokinetic figures. As a comparison of the figures will show, the spermatocytes and maturation divisionfigures of $\mathrm{N}$. hilaris are much larger than those of $\mathrm{N}$. viridula. In the spermatogonia this difference is also apparent, though less marked. In the ovaries, strange to say, it cannot certainly be detected, either in the dividing cells or in the nuclei of the folliclecells or of the tip-cells at the upper end of the ovary. It would be interesting to make a more accurate study of these relations; but I will here only state that the differences between the two species seem to arise mainly through greater growth of the spermatocytes in N. hilaris. With this is correlated a greater size of the testis as a whole; but the size of the entire body in this species is but little larger, as far as I have observed, than in $\mathrm{N}$. viridula.

As regards the general features of the divisions, the diploid groups of both sexes uniformly contain fourteen chromosomes, the first spermatocyte-division eight and the second seven, the idiochromosomes being, as is the rule in Hemiptera, separate and univalent in the first division.

\section{The second spermatocyte-division}

a. The idiochromosomes. Polar views of the second division always show 7 chromosomes which are usually grouped in an irregular ring of -six with the seventh near its center (fig. $3 j-m$, figs. 14, 15). In both species one chromosome of the outer ring (s) can usually be distinguished as the smallest, though this is not always evident owing to the apparent variations produced by different degrees of elongation. This is the chromosome that I formerly supposed to be the idiochromosome-bivalent, despite its peripheral position, and despite the fact, which I had myself described, that a similar small chromosome, also peripheral in posi- 


\section{EXPLANATION OF TEXT FIGURES}

Figures 1 to 9 are from camera drawings, and are not schematized except that in a few instances the chromosomes have been artificially spread out in a series in order to facilitate comparison. Figs. $2 k-l$ are somewhat more enlarged than the others. In all the figures $d$ denotes the double chromosome or ' $d$-chromosome,' $s$ the small chromosome, $X$ the large idiochromosome and $Y$ the small.
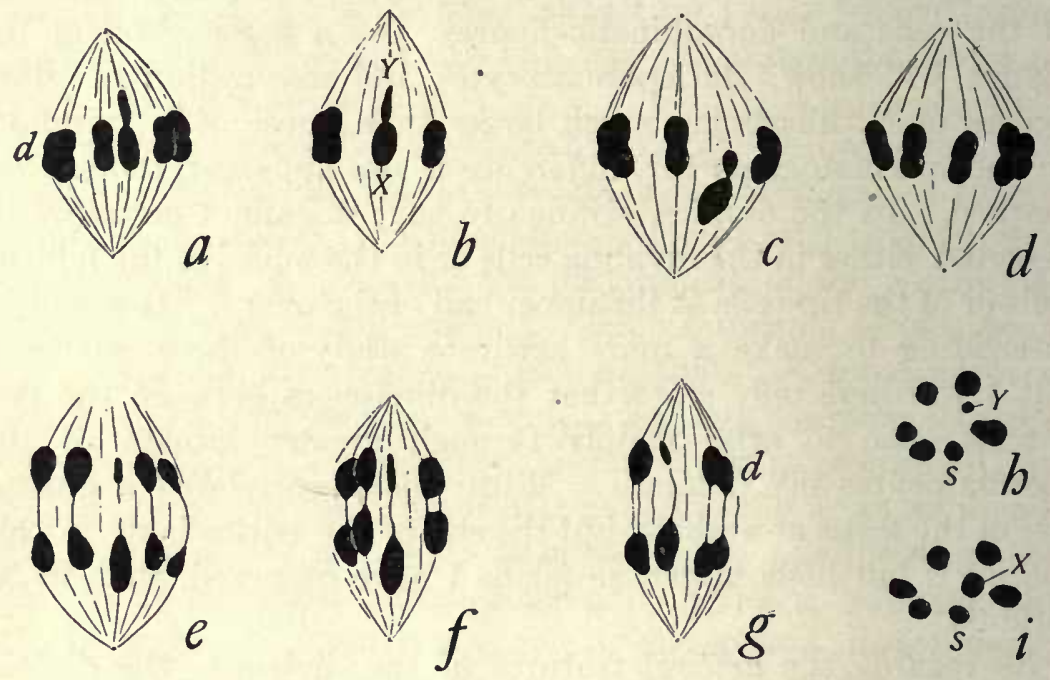

Fig. 1 The second spermatocyte-division in Nezara viridula. $a-d$, metaphases in side view; $e-g$, anaphases; $h, i$, polar views of two sister-groups, middle anaphase, from the same spindle and in the same section.

tion, appears in several other pentatomids (e.g., in Euschistus, Coenus and Mineus). But Nezara forms no exception to the rule that the central chromosome is the idiochromosome-bivalent. In N. viridula this is immediately apparent in side views (often also in polar views) where the central chromosome is seen to consist of two very unequal components, the smaller being not more than one fourth or one fifth the size of the larger (fig. $1 a-c$ ). In the anaphases these separate and pass to opposite poles; while all the others divide equally (fig. $1 e-g$ ). Polar views of middle or rather late anaphases, when both daughter-groups can be seen superposed in the same section, clearly show the marked difference of the two groups in respect to the idiochromosomes (fig. $1 h-i$ ). All the facts are here so nearly similar to those seen in Euschistus or Lygaeus as to require no further description. 
In $\mathrm{N}$. hilaris the conditions differ only in that the two components of the central chromosome are but slightly unequal; but in the examination of at least two hundred of these divisions I have never failed to detect the inequality. A series of side views are shown in fig. $2 a-i$, figs. $16-21$, two of which show all the chromosomes. These figures illustrate practically all the variations that have been seen in the idiochromosomes. The most characteristic condition is that seen in $2 a, b, d$, in which both idiochromosomes ( $\mathrm{X}$ and $\mathrm{Y}$ ) are more or less elongated and united end to end. Less often one of them assumes a more spheroidal form (fig. $2 e, h, i$, fig. 17). The size-difference, though always evident, seems to vary slightly (perhaps because one or the other component may be more or less compressed laterally), but is always distinctly greater than that now and then seen in other bivalents.

Fig. $2 j$ shows a mid-anaphase ${ }^{2}$ (cf. figs. 21-23) in which the inequality would hardly be noticed without close study and the comparison of other cases. Figs. $2 k$ and $l$ are similar stages showing all the chromosomes spread out in a series for the sake of comparison. In both, the two idiochromosomes are easily distinguishable, ${ }^{3}$ and the larger is seen to be one of the three largest chromosomes. Figs. $2 m-n, o-p, q-r$ and $s-t$ are pairs of sistergroups, in each case from the same spindle in anaphase. All of these are selected from cases in which a distinct size-difference appears between $\mathrm{X}$ and $\mathrm{Y}$, but there are also many cases in which this cannot be seen. Such a case was figured in fig. $4 e-f$ of my first 'Study' the correctness of which is confirmed by re-examination of the original section. This condition is due simply to the fact that the large idiochromosome is more elongated than the small, so that the size-difference cannot be seen in polar view; and for the same reason it is often not evident in polar views of the metaphase.

2 This and the two following figures are a little more enlarged than the others.

${ }^{3}$ Fig. $2 l$ is the same group figured in fig. $4 d$ of my first 'Study,' carefully redrawn and corrected. A comparison of the two drawings will show that in the latter a distinct size-difference between $\mathrm{X}$ and $\mathrm{Y}$ is actually shown but is minimized by the fact that the former is represented a trifle too small, the latter a little too large. It is now also evident that they are connected by two connecting fibres instead of by one. 

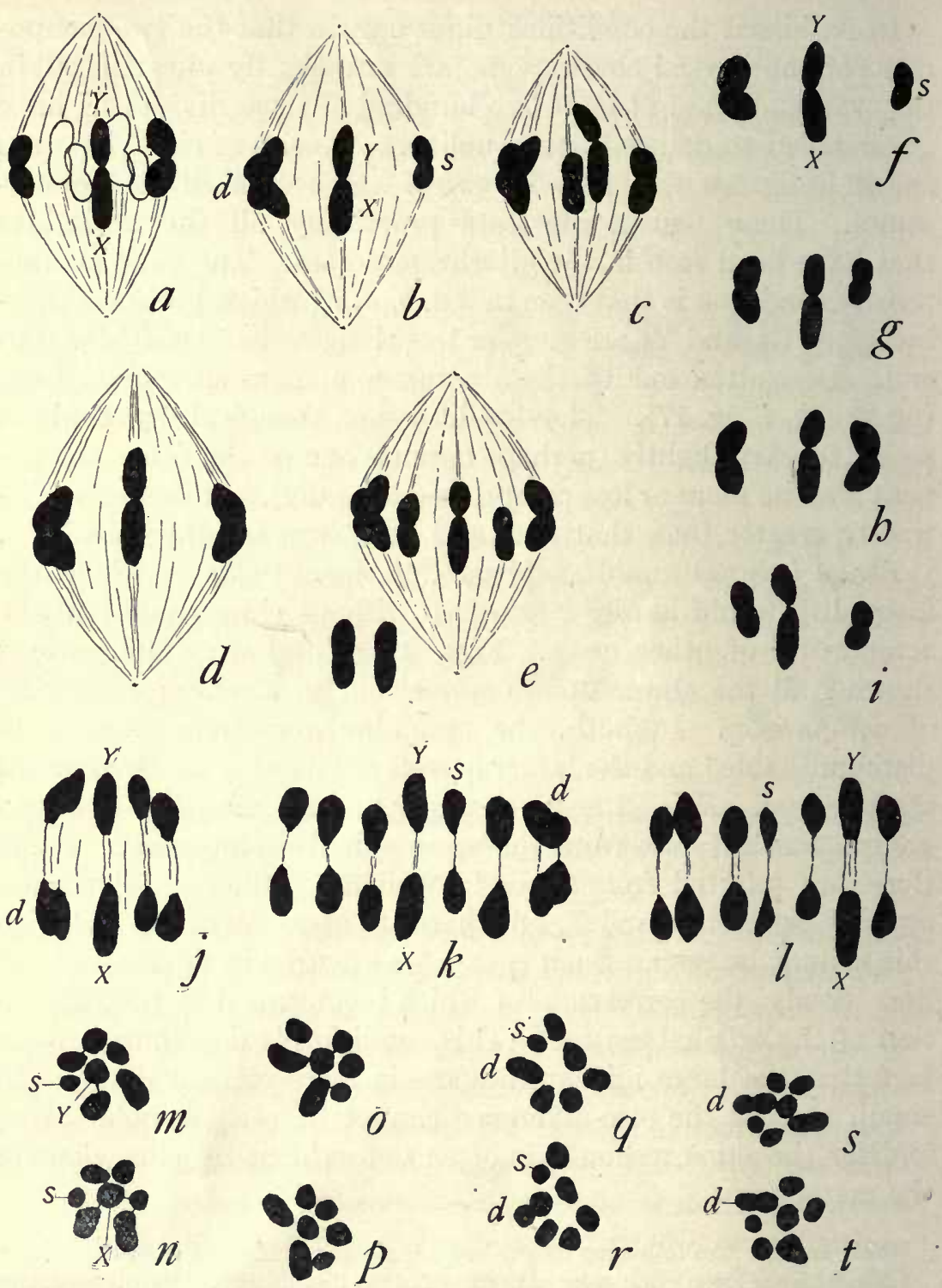

Fig. 2 The second spermatocyte-division in Nezara hilaris. $a-i$, metaphase figures in side view, $a$ and $e$ showing all the chromosomes; $j-l$, mid-anaphases; in $k$ and $l$ all the chromosomes are shown artificially spread out in series; $m-n, o-p$, $q-r, s-t$, four pairs of sister-groups from late anaphases, in polar view, in each case from the same spindle. 
b. The double chromosome. A second interesting feature of the second division that I formerly overlooked is the presence of a remarkable double chromosome which in the metaphase has exactly the appearance of a butterfly with widespread wings. This chromosome (which may be called the $d$-chromosome) is shown in profile view in $2 b-e$ and $1 a-d, 16,17,20,24,25$. This is the only chromosome in the second division that shows any approach to a quadripartite form, andits characters are so marked as to constitute the most striking single feature of the division. As the figures show, it is one of the largest of all the chromosomes. It always has an asymmetrical tetrad shape, giving exactly the appearance of a smaller and a larger dyad in close union; and it always lies in the outer ring, so placed as to undergo an equal division, and with the larger wings of the butterfly turned towards the axis of the spindle. In polar view $(3 j-m)$ the duality is far less apparent and sometimes invisible, even upon careful focussing. In $\mathrm{N}$. viridula the duality is always apparent in side view, but the butterfly shape is usually less evident than in N. hilaris.

In the initial anaphases the $d$-chromosome divides symmetrically, drawing apart into two bipartite chromosomes $(2 j, k, 1 \mathrm{~g})$; but this is seldom evident save in profile view. Viewed from the pole the duality does not now ordinarily appear, though it may still sometimes be seen upon careful focussing. In the later anaphases the two components tend to fuse, and often can no longer be distinguished. Not seldom, however, the duality is visible even in the final anaphases; and sometimes this is so conspicuous that the spermatid-group seems at first sight to comprise eight instead of seven separate chromosomes $(n, r, s, t)$.

Since the duality of this chromosome does not certainly appear in the spermatogonial groups or in the first spermatocyte-division, its peculiar form in the second division might be supposed to result from some special mechanical relation to the spindle-fibers in that division. This is, however, excluded by examination of the interkinesis, in which the chromosomes are irregularly scattered. In these stages, even when the spindle is still very small and the chromosomes lie in a quite irregular group, the butterfly shape is already perfectly evident; and it shows no constancy of 
relation to the spindle-axis, often lying at right angles to the latter. Apparently therefore its duality arises quite independently of the spindle or astral rays, and its constant position in the fully formed spindle is the result of a later adjustment. In this species, as in many others, each chromosome is connected with the pole by a bundle of delicate fibers. In case of the $d$-chromosome this bundle is very broad, but I cannot be sure that it is double.

At first sight any observer would, I think, take the $d$-chromosome to be merely a result of the accidental superposition or close adhesion of two separate dyads of unequal size; but such an interpretation is inadmissible. When all the chromosomes can be unmistakably seen, the $d$-chromosome is found to constitute one of the seven separate elements invariably present in this division; and since the diploid number is 14 in both sexes this chromosome must represent one chromosome, not two, of the original spermatogonial groups. It is certain, therefore, that the double appearance does not result from close apposition of two separate chromosomes; it is therefore not a "tetrad" in the ordinary sense of the word-i.e., not one that results from the synapsis of two chromosomes that are originally separate in the diploid groups.

\section{The first spermatocyte-division}

This division requires only brief mention. As stated, it shows eight separate chromosomes, of which the only one that can be positively identified is the $\mathrm{Y}$-chromosome of $\mathrm{N}$. viridula. This chromosome, always immediately recognizable in this species by its small size $(3 c, d, f, g, i)$, figs. 12,13$)$, is usually central in position, like the $m$-chromosome of the Coreidae, but this is not invariable. Since it divides equally, and without association with any other chromosome $(3 \mathrm{~g})$ it is evident that the two idiochromosomes must be separate and univalent in this division. In $\mathrm{N}$. hilaris $(3 a, b$, figs. 10,11$)$ the eight chromosomes usually form an irregular ring, there is no central chromosome, and neither idiochromosome can be certainly recognized. It nevertheless seems a safe inference from what is seen in N. viridula that the two idiochromosomes are here also separate and univalent. 

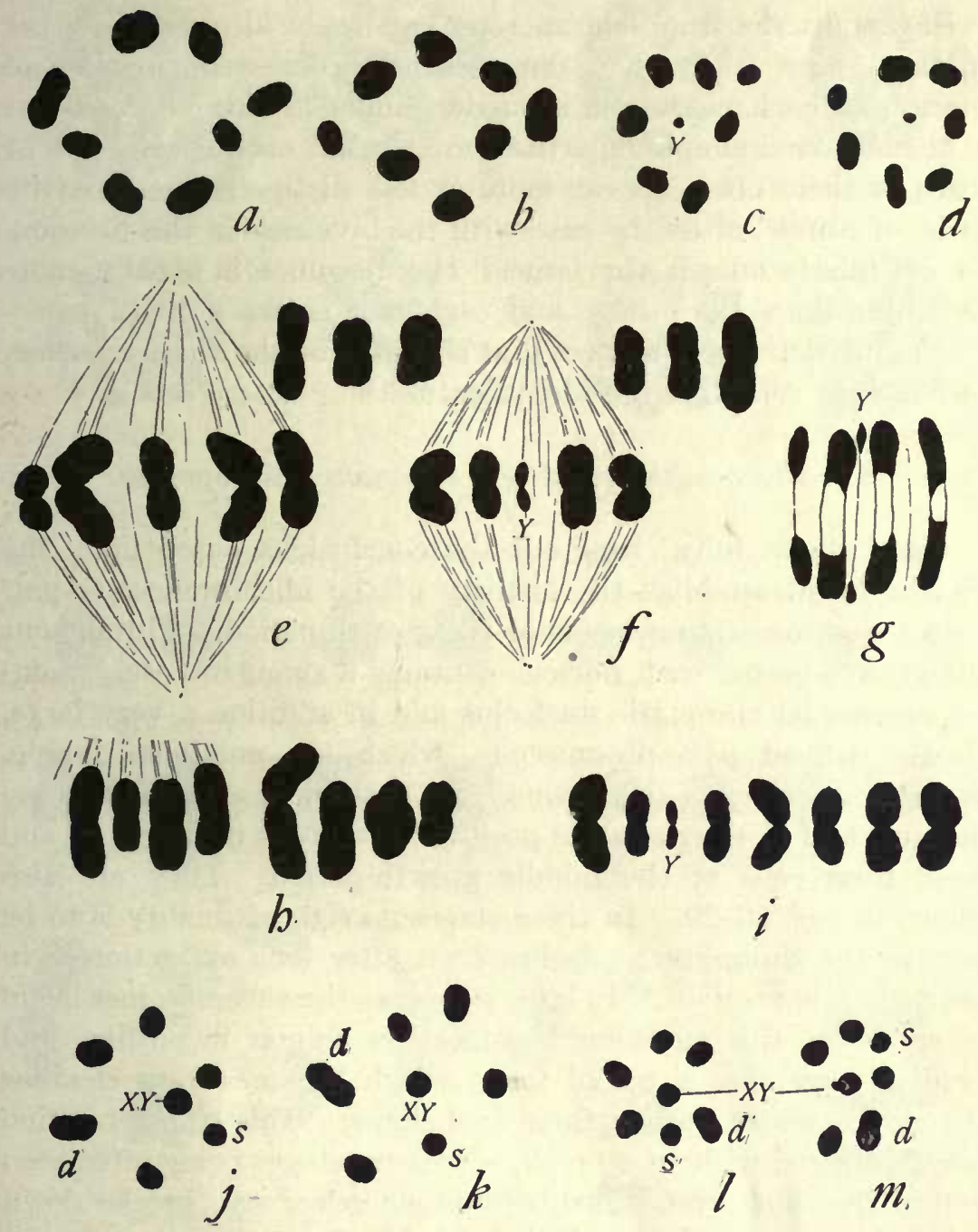

Fig. 3 First and second spermatocyte-divisions in the two species of Nezara. $a, b$, first division, hilaris, polar views: $c, d$, corresponding views of viridula; first division, hilaris, side view showing five of the chromosomes in position and the other three to the right above; $f$, corresponding view of viridula; $g$, middle anaphase, viridula, showing division of $Y$; $h$, first division metaphase, hilaris, all the chromosomes artificially spread out in series; $i$, corresponding view of viridula; $j, k$, second division metaphase, hilaris, polar views; $l, m$, corresponding views of viridula. 
In this division the $d$-chromosome can not be identified in either species. Figs. $3 e, f, h, i$, show all the chromosomes of the two species, in each case from a single spindle in side view. Most of them have a simple bipartite form, but in each species two or three of them often appear more or less distinctly quadripartite as is, of course, often the case with the bivalents in this division. In $\mathrm{N}$. hilaris one of the largest chromosomes is usually more elongated than the others, and each half shows a slight transverse constriction. I suspect that this may be the $d$-chromosome, but cannot establish the identification.

\section{The growth-period and spermatocyte-prophases}

These stages fully bear out the conclusions based upon the divisions and establish the identity of the idiochromosome-pair with the chromatic nucleolus of the growth-period. Throughout the growth-period each nucleus contains a single intensely staining spheroidal chromatic nucleolus and in addition a very large, clearly defined pale plasmosome, which is sometimes double. Series of drawings of these two bodies (in each case from the same nucleus, and in their relative position) are given in figs. $4 i-l$ and $m-p$, from cells of the middle growth-period. They are also shown in figs. 26-29. In these stages no sign of duality is to be seen in the chromatic nucleolus, even after long extraction or in saffranin preparations. In later stages, as the chromosomes begin to condense, this nucleolus becomes less regular in outline, and gradually assumes a tetrad form, which becomes very clear as the chromosomes assume their final shape. This transformation may be traced without a break, successive stages being often seen within the same cyst. Just before the nuclear wall breaks down this tetrad is still clearly distinguishable from the others by its asymmetrical quadripartite form, as seen in $4 y, z$, which show all the chromosomes (in each case from two successive sections). Figs. $4 q-t$ show four views of this tetrad at this period in $\mathrm{N}$. hilaris, while $u-x$ are corresponding views of $\mathrm{N}$. viridula. These figures (which might be indefinitely multiplied) show the marked differences between the two species in respect to this tetrad, obviously corresponding to that seen between the idiochromosome 

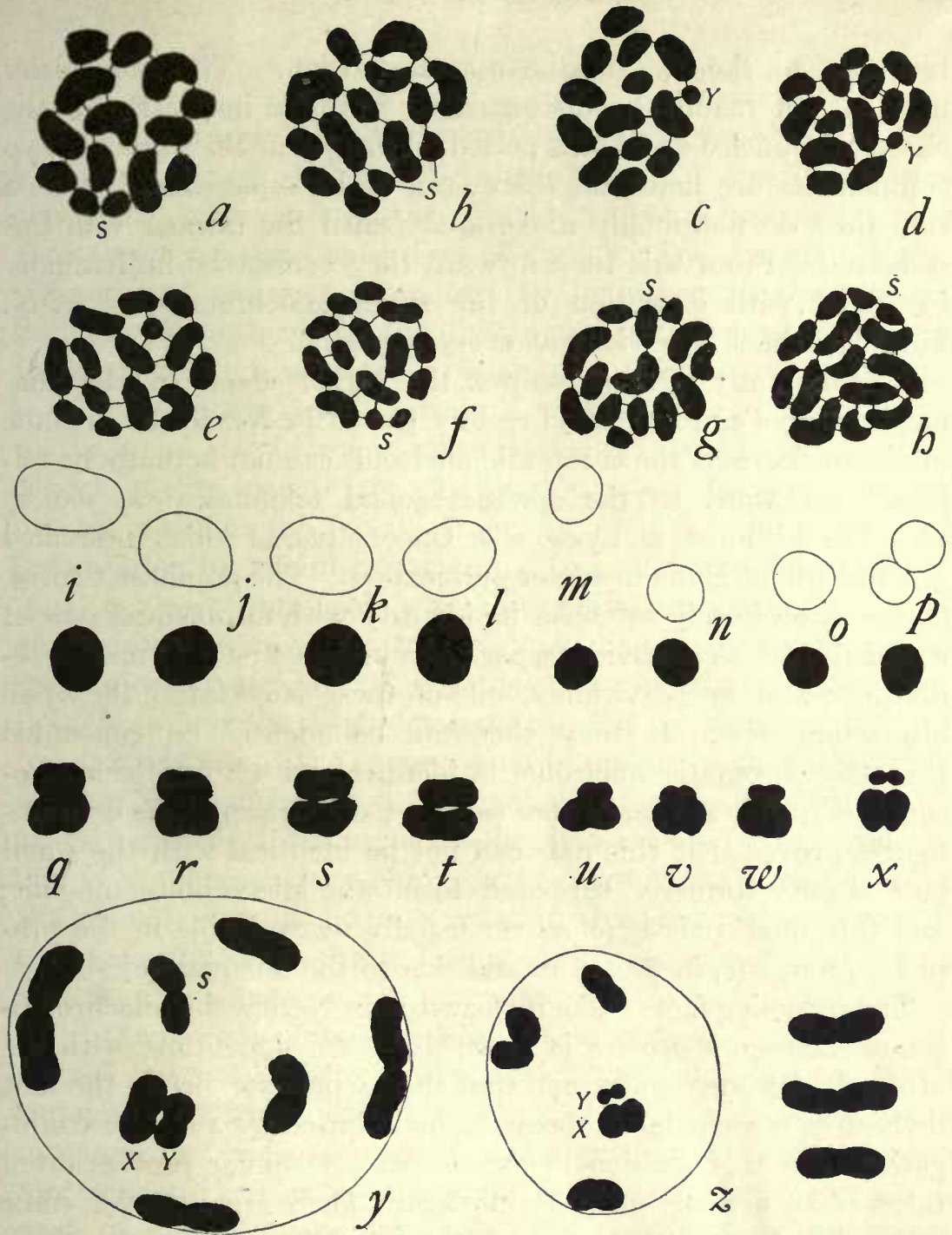

ข

$w$

$x$

Fig. 4 The diploid groups, nucleoli of the growth-period, and late prophasefigures of the two species of Nezara. $a, b$, spermatogonial groups, hilaris; $c, d$, the same, viridula; $e, f$, ovarian groups, hilaris; $g, h$, the same, viridula; $i-l$, chromatic nucleolus and plasmosome from the growth-period, in each case from the same nucleolus in their relative position; $m-p$, corresponding views, viridula; $q-t$, the idiochromosome-tetrad (chromatic nucleolus) from prophase nucleoli, hilaris; $u-x$, corresponding views, viridula; $y$, late prophase nucleus, showing all the chromosomes, hilaris (combination figure from two sections); $z$, corresponding stage, viridula, three of the chromosomes from adjoining section at the right. 
bivalents of the two in the second division. ${ }^{4}$ The two species may in fact readily be distinguished by mere inspection of the chromatic nucleolus at this period. Already at this time the two components are here and there seen to be separating, but as a rule they do not finally move apart until the nuclear wall has dissolved. From this time forward they cannot be individually identified with exception of the small idiochromosome of $\mathrm{N}$. viridula, which is obvious at every period.

As far as my material shows, the earlier stages of the idiochromosomes can not be so readily traced in Nezara as in some other species, and the chromatic nucleolus can not actually be followed backward to the spermatogonial telophases-as can be done in such forms as Lygaeus or Oncopeltus, of which a detailed account will be given in a later publication. The prophase-figures, however, decisively establish its identity with an unequal pair of chromosomes that divide separately in the first spermatocytedivision; and in $\mathrm{N}$. viridula, one of these is certainly the small idiochromosome. It may therefore confidently be concluded that the chromatic nucleolus is identical with the idiochromosome-pair, as in so many other cases. Comparison of the divisionfigures proves that this pair can not be identical with the small pair that I formerly supposed to be the idiochromosome-pair; and this small pair is moreover usually recognizable in the prophase groups $(s$, in $5 y, z)$ in addition to the unequal pair.

The foregoing facts make it clear that in Nezara the idiochromosomes undergo a process of synapsis at the same time with the other chromosome-pairs, and that their separation before the first division is a secondary process, to be followed by a second conjugation after this division is completed. A similar process often takes place in many other Hemiptera. There are, however, some forms, like Oncopeltus, in which the idiochromosomes are always separate, from the last spermatogonial division through all the succeeding stages up to the end of the first division. In this case, which I shall describe more fully hereafter, there can be no doubt that the conjugation which follows the first division is a primary synapsis, to be immediately followed by a disjunction.

${ }^{4} C f$. the earlier figures of the corresponding tetrad in Brochymena in my first 'Study,' fig. 7. 


\section{The diploid chromosome-groups}

In these groups the interest centers again in the identity of the idiochromosomes and the $d$-chromosome. Of the 14 separate chrosomomes present in the diploid nuclei of both sexes, none shows any constant indication of duality (figs. $4 a-h$ ). The $d$ chromosome can not, therefore, be identified in these stages. Secondly, in both species the diploid groups of the two sexes show the same relation as in other Hemiptera of this type, though this is, of course, more readily seen in $\mathrm{N}$. viridula than in hilaris, owing to the small size of the Y-chromosome. In the spermatogonial groups of this species $(4 c, d)$ this chromosome is at once recognizable while in the female groups $(g, h)$ it is lacking, its place being taken by one of larger size. In both sexes the small pair $(s, s)$ is also recognizable. In this species, accordingly, the $\mathrm{Y}$ chromosome is confined to the male line, and the Y-class of spermatozoa must be male-producing, as in other forms.

In $\mathrm{N}$. hilaris the $\mathrm{Y}$-chromosome can not be identified $(4 a, b)$, but the relation of the spermatozoa to sex-production is shown in another way, though less unmistakably than in $\mathrm{N}$. viridula. As already described, the large idiochromosome or X-chromosome is one of the largest three chromosomes seen in the second division. We should therefore expect to see five largest chromosomes in the male diploid groups. This is clearly apparent in figs. $4 a, b$, and is also shown in the corresponding figures of $\mathrm{N}$. viridula $(c, d)$ though not quite so clearly. One of these five in the male should be the $\mathrm{X}$-chromosome; and if the usual relation of the spermatozoa to sex hold true, there should be six largest chromosomes in the diploid groups of the female. This relation actually appears in nearly all cases, and is shown in figs. $4 e, f, g, h$, in each of which, again, the small pair $(s, s)$ may be recognized. Though this evidence is in itself less convincing than that afforded by $N$. viridula (since the relation can not always be made out with certainty) it is fully in harmony with the latter, and sustains the same conclusion. ${ }^{5}$

5 This relation is shown in my original figures of $\mathrm{N}$. hilaris, though not quite as clearly as in the groups here figured. In my first 'Study' ('05) the five largest chromosomes are very clearly shown in fig. $4 h$, and are also evident in $4 q$. In the third 'Study' the relation is hardly evident in the male but fairly so in the female (figs. $5 l, m$ ). 


\section{GENERAL}

\section{The idiochromosomes}

The case of Nezara shows how readily a morphological dimorphism of the spermatid-nuclei may be overlooked when the $\mathrm{X}$ - and Y-chromosomes do not differ markedly in size; and it emphasizes the necessity for the closest scrutiny of these chromosomes in the study of this general question. In my fourth 'Study' I placed with Nezara hilaris, as a second example of my original 'third type,' the lygaeid species Oncopeltus fasciatus (Dall.), on the strength of Montgomery's account of the conditions in the male ('01, '06) and my own unpublished observations on both sexes. While I have carefully re-examined this case also, I am not yet prepared to express an unqualified opinion in regard to it. Certainly, in very many of the cells of this species, at every period of the spermatogenesis, the idiochromosomes (which are always separate up to the second division) seem to be perfectly equal. A slight inequality may indeed be seen in some cases; but as far as I can yet determine this seems to fall within the range of the sizevariation in other chromosomes and gives no positive ground for the recognition of a morphological dimorphism in the spermatozoa. A similar condition has been described in several other insects, notably in some of the Lepidoptera (Stevens, '06; Dederer, '08; Cook, '10), in the earwig Anisolaba (Randolph, '08) and apparently also in the beetle Hydrophilus according to Arnold ('08). I see no reason to question these observations; but the interpretation to be placed on them is by no means clear at present. The experimental evidence on the Lepidoptera seems to demonstrate that in at least one case - that of Abraxas according to Doncaster and Raynor,it is the eggs and not the spermatozoa that are sexually dimorphic; that is, in the terms that I have recently suggested ('10a), in this case it is the female that is sexually 'digametic' while the male is 'homogametic.' Baltzer's careful work on the seaurchins ('09) clearly demonstrates a cytological sexual dimorphism in the mature eggs of these animals, and shows that the spermnuclei are all alike. In cases, therefore, where no visible dimorphism of the spermatid-nuclei is demonstrable, two possibilities 
are to be considered, namely, (1) that it may be the female which (as in sea-urchins) is the digametic sex, and (2) that one sex or the other may still be physiologically digametic even though this condition is not visibly expressed in the chromosomes. The first of these possibilities may readily be tested by cytological examination of the female groups. The second can only be examined by means of experiment, and especially by experiments on sex-limited heredity. It is interesting that the work of Doncaster and Raynor, cited above, and the more recent one of Morgan on Drosophila ('10) have given exactly converse results, the former demonstrating a sexual dimorphism of the eggs, the latter of the spermatozoa. This agrees with the cytological data, as far as they have been worked out. The researches of Stevens ('08, 10), on the Diptera establish the cytological dimorphism of the spermatozoa in these animals, while all observers of the Lepidoptera have thus far failed to find such dimorphism in this group. It thus becomes a very interesting question whether a cytological dimorphism of the mature eggs may be demonstrable in the Lepidoptera; though a failure to find it would in no wise lessen the force of the experimental data. Physiological differences between the chromosomes are of course not necessarily accompanied by corresponding morphological ones-indeed such a correlation is probably exceptional.

(1) (a) Composition and origin of the XY-pair. The facts seen in Nezara again force upon our attention the puzzle of the Y-chromosome or 'small idiochromosome.' It is remarkable that two species so nearly akin as N. hilaris and N. viridula should differ so widely in respect to this chromosome; though this is hardly so surprising as the fact that in Metapodius this chromosome, as I have shown ('09, '10) may actually either be present or absent in different individuals of the same species. These facts show, as I have urged, that although the Y-chromosome shows a constant relation to sex when it is present, it can not be an essential factor in sex-production. As the case now stands this might be taken as a direct piece of evidence against the view that the idiochromosomes are concerned with sex-heredity. Further, as I have pointed out ('10) in Metapodius the introduction of super- 
numerary $\mathrm{Y}$-chromosomes into the female has no visible effect upon any of the characters of the animal, sexual or otherwise; and this might be urged against the whole conception of qualitative differences among the chromosomes and of their determinative action in development. It is especially in view of these and certain other general questions that I wish to indicate some of the many possibilities that must be taken into account in the consideration of this problem. My discussion is throughout based upon the assumption that the chromosomes do in fact play some definite role in determination, and that they exhibit qualitative differences in this respect. I do not hold that they are the exclusive factors of determination; though it is often convenient, for the sake of brevity, to speak of them as if they were such.

(2) Cytologically considered, the morphological dimorphism of the spermatozoa seems to have arisen by the transformation of what was originally a single pair of chromosomes comparable to the other synaptic pairs. We have at present no information as to whether the members of this pair were equal or unequal in size; but in either case there are grounds for the assumption that its two members differed in some definite way in respect to the quality of the chromatin of which they were composed. This pair, which may be called the primitive XY-pair, has undergone many modifications in different species, but without altering its essential relation to sex. In the insects (Hemiptera, Coleoptera, Diptera) its most frequent condition is that of an unequal pair, consisting of a 'large idiochromosome' or 'X-chromosome,' and a "small idiochromosome" or 'Y-chromosome,' the latter being confined to the male line, while the former appears in both sexessingle in the male and paired in the female. That all gradations exist between cases where $\mathrm{X}$ and $\mathrm{Y}$ are very unequal (as in many Coleoptera and Diptera and in some Hemiptera) and those in which they are nearly or quite equal (Mineus, Nezara, Oncopeltus) gives some ground for the conclusion that in the original type the $\mathrm{XY}$-pair was but slightly if at all unequal.

By disappearance of the free $\mathrm{Y}$-member of this pair has arisen the unpaired odd or 'accessory' chromosome, which accordingly 
has no synaptic mate. This condition seems to have arisen in more than one way. It is almost certain that in many cases the Y-chromosome has disappeared by a process of gradual and progressive reduction (as indicated by the graded series observed in the Hemiptera (Wilson, '05b, '06). In some cases (of which Metapodius is an example) the same result may have been produced suddenly by a failure of the idiochromosomes to separate in the second spermatocyte-division (Wilson, '09b). A third possibility, first suggested by. Stevens ('06), is that the X-element may have separated from a YY-pair with which it was originally united. This possibility seems to be supported by recent observations on Ascaris megalocephala, where the $\mathrm{X}$-chromosome is sometimes fused with one of the other pairs, sometimes free (Edwards, '10).

(3) We have as yet no positive knowledge as to how the Xmember of the XY-pair originally differed, or now differs, from the $Y$, or as to how this difference arose-a definite answer to these questions would probably give the solution of the essential problem of sex. There are, however, pretty definite grounds for the hypothesis that the $\mathrm{X}$-member contains a specific ' $\mathrm{X}$-chromatin' that is not present in the $\mathrm{Y}$-member, and that the XY-pair is heterozygous in this respect. If this be so, the primary sexual differentiation is therefore traceable to a condition of plus or minus in this pair, accompanied by a corresponding difference between the nuclear constitution of the two sexes. (Cf. Wilson, '10a.) Further, there is also reason for regarding the heterozygous condition of this pair as due to the presence of the X-chromatin in one member of a pair which is (or originally was) homozygous in respect to its other constituents. The latter may be called collectively the 'Y-chromatin'; and we may, accordingly, think of the XY-pair as being essentially a YY-pair with one member of which the $\mathrm{X}$-chromatin is associated. ${ }^{6}$ Both the $\mathrm{X}$ -

${ }^{6}$ This suggestion is in principle the same as one earlier made by Stevens ('06, p. 54) that the Y-chromosome represents "some character or "characters which are correlated with the sex-character in some species but not in others," with one member of which the X-chromosome is fused; and that "a pair of small chromosomes might be subtracted from the unequal pair, leaving an odd chromosome." 
chromatin and the $\mathrm{Y}$ may themselves be composite, thus giving the possibility of many secondary modifications. The point of view thus afforded opens many possibilities for an understanding of sex-limited heredity, as indicated beyond.

(b) Modifications of the X-element. This view of the XY-pair is based upon two series of facts, of which the first includes the various modifications of the $\mathrm{X}$-member of the pair seen in different species. It is, perhaps, most directly suggested by a study of the pentatomid species Thyanta custator. In this common and widely distributed species I have found two races, which thus far can not be distinguished by competent systematists, ${ }^{7}$ but which differ in a remarkable way in respect to both the total number of chromosomes and the XY-pair. In one of these races (which I will call the 'A form'), widely distributed throughout the south and west, the total number in both sexes is 16, and the XY-pair of the male is a typical unequal pair of idiochromosomes, exactly like that seen in many other pentatomids (e.g., Euschistus, Coenus or Banasa). These are shown in fig. $5 a, b$, their mode of distribution being the usual one. The second race (the ' $B$ form') is thus far known from only a single locality in northern New Jersey. It differs so remarkably from the A form that I could not believe the observations to be trustworthy until repeated study of material, collected in four successive years, established the perfect constancy of the cytological conditions and the apparent external identity of the two forms. In this race the XY-pair is represented by three small chromosomes of equal size, which are always separate in the diploid groups and in the first spermatocyte-division (fig. $5 i$ ), but in the second division are united to form a linear triad series $(5 c, d)$. This group so divides that one component passes to one pole and two to the other $(5 e, h)$, the

${ }^{7}$ I am indebted to Mr. E. P. Van Duzee for a careful study of my whole series of specimens of both races. He could find no constant differential between them. Additional studies of this material are now being made by $\mathrm{Mr}$. $\mathrm{H}$. G. Barber.

Addendum. Since this paper was sent to press Mr. Barber, after prolonged study, has reported his conclusion that the 'A form' is Thyanta custator of Fabricius, while the 'B form' is probably Thyanta calceata of Say, which has long been regarded as a synonym of former species. 

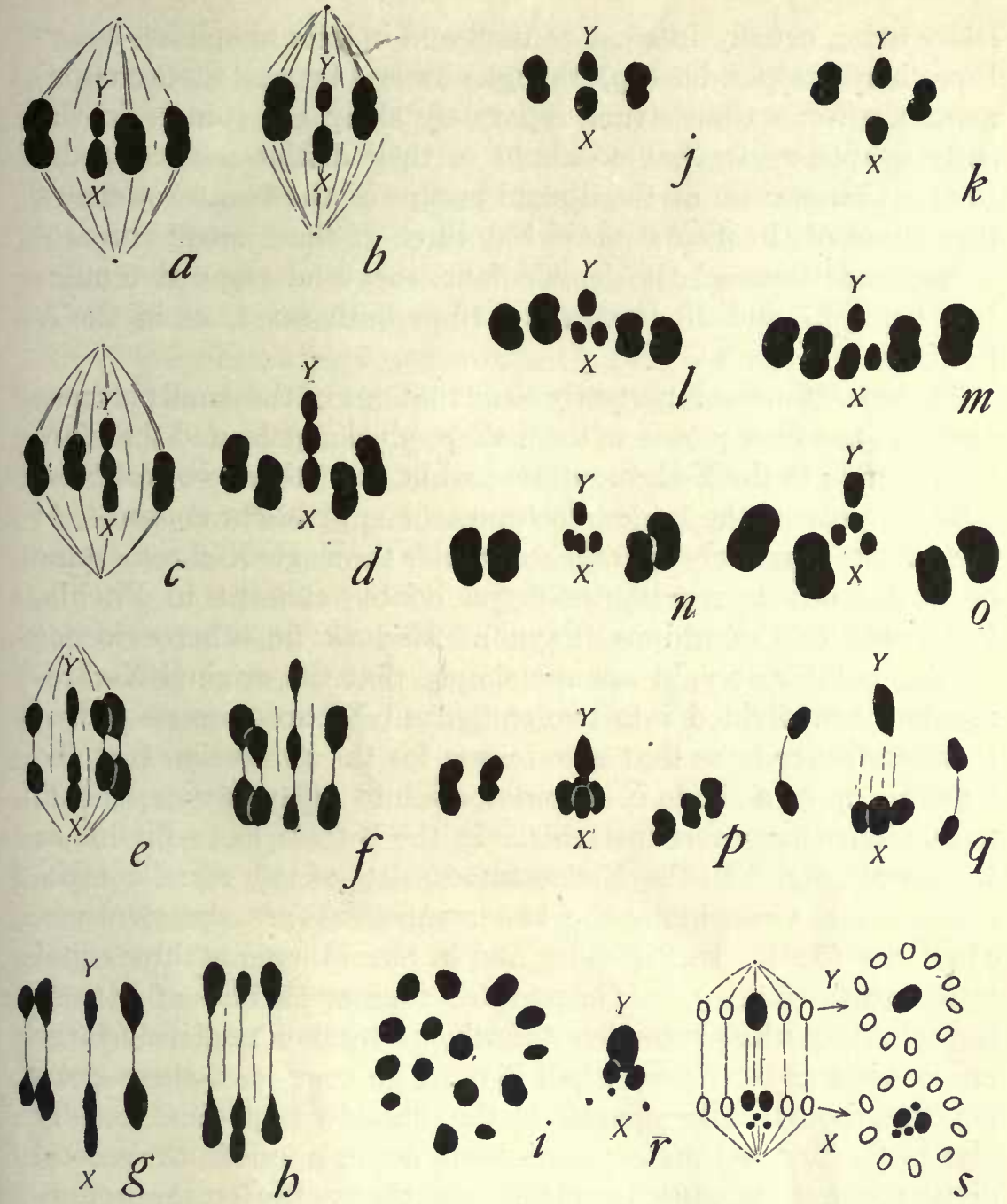

Fig. 5 Comparison of the XY-group in various Hemiptera. ( $a-i$ are original; the others from Payne.) $a, b$, Thyanta custator, 'A form,' second division in side view; $c, d$, corresponding views of the 'B form'; $e-h$, anaphases of same; $i$, polar view of first division of same; $j, k$, metaphase chromosomes, second division, Diplocodus exsanguis; $i$, similar view of Rocconota annulicornis; $m$, similar view of Conorhinus sanguisugus; $n$, Sinea diadema; $o$, Prionidus cristatus; $p$, Gelastocoris oculatus; $q$, anaphase chromosomes of the same species; $r$, the XYgroup, from the second division, Acholla multispinosa; $s$, diagram, slightly modified from Payne, to show the distribution of the XY-components in the second division of the same species. 
latter being usually in close contact and in later anaphases sometimes hardly separable $(5 g)$, though now and then all three components are for a time strung separately along the spindle in the early anaphases, so that no doubt of their distinctness can exist $(5 \mathrm{f})$. Comparison of the diploid groups of the two sexes shows that those of the male contain but three of these small chromosomes and those of the female four, the total respective numbers being 27 and 28 (instead of 16 in both sexes, as in the A form).

These facts make it perfectly clear that one of the small chromosomes in the male passes to the male-producing pole, and therefore corresponds to the Y-chromosome; while the other two, taken together, represent the large idiochromosome, or X-chromosome, of the A form - precisely as in the reduvioids the single $\mathrm{X}$-chromosome of Diplocodus is represented by a double element in Fitchia, Rocconota or Conorhinus (Payne). Had we no other evidence on this point we might assume simply that the original $\mathrm{X}$-chromosome has divided into two equivalent $\mathrm{X}$-chromosomes. But there are other facts that give reason for the conclusion that the breaking up of a single $\mathrm{X}$-chromosome into separate components means something more than this. In the B form, as in Fitchia or Rocconota (fig. 5 l), the X-element consists of two equal components, but in Conorhinus the two components are always of unequal size $(5 \mathrm{~m})$. In Prionidus and in Sinea there are three equal components $(5 n, o)$, in Gelastocoris four equal ones ( $5 p$, and in Acholla multispinosa five, of which two are relatively large and equal and three very small $(5 r, s)$. In every case these components, though quite separate in the diploid groups (and usually also in the first spermatocyte-division) act as a unit in the second division, though not fused, and pass together to the female-producing pole (Payne, '09, '10).

In the foregoing examples the $\mathrm{X}$-element is accompanied by a synaptic mate or Y-chromosome. The following are examples of a similar breaking up of the $\mathrm{X}$-element into separate components when such a synaptic mate is missing. In Phylloxera (Morgan) the X-element consists of two unequal components, sometimes separate, sometimes fused together. In Syromastes (Gross, 
Wilson) it consists of two unequal components, always separate, in the diploid groups but closely in contact (not fused) in both spermatocyte-divisions. The recent work of Guyer ('10) indicates a similar condition in the X-element of man. In Agalena (Wallace) there are two equal components, always separate. Finally, in Ascaris lumbricoides (Edwards, '10) there are five components, separate, and scattered in the diploid groups but closely associated in the spermatocyte-divisions.

In all these cases the significant fact is that not only the number but also the size-relations of these components are constant; and in many of these forms this fact may be seen in such multitudes of cells, and with such schematic clearness, as to leave no manner of doubt. : It seems impossible to understand this series of phenomena unless we assume that the single $\mathrm{X}$-chromosome is essentially a compound body-i.e., one that consists of different constituents that tend to segregate out into separate chromosomes. We are led to suspect, further, that the composition of the $\mathrm{X}$ element, even when it is a single chromosome, may differ widely in different species because of its great variations of size as between different species. For instance, in the family of Coreidae it is in some cases very large (Protenor), in others of middle size (Chelinidea, Narnia, Anasa), in others one of the smallest of the chromosomes (Alydus). Similar examples might be given from other groups.

In the case of Thyanta, therefore, it seems a fair assumption that the double $\mathrm{X}$-element of the $\mathrm{B}$ form likewise represents at least a partial segregation of the $\mathrm{X}$-chromatin from other constituents; and the latter may plausibly be regarded as representing the 'Y-chromatin' of the original X-member of the pair. In other words, we may think of the triad element as a YY-pair, one member of which is accompanied by a separate $\mathrm{X}$-chromosome. In accordance with this its formula should be X.Y.Y, while that of the A form is XY.Y; and this may also be extended to other forms of similar type. If this be admissible, the male formula, as regards essential chromatin-content, becomes in general XY.Y and the female XY.XY, both sexes being homozygous for the $\mathrm{Y}$-constituents, while in respect to $\mathrm{X}$ the male is heterozygous, 
the female homozygous. The puzzle of the Y-chromosome would thus be solved; for although a separate Y-chromosome, when present, is confined to the male line, its disappearance only reduces the male from a homozygote to a heterozygote in respect to the Y-chromatin, and the introduction of supernumerary $\mathrm{Y}$-chromosomes into the female (as in Metapodius) brings in no new element.
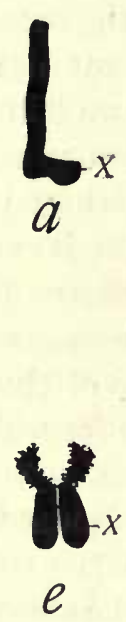
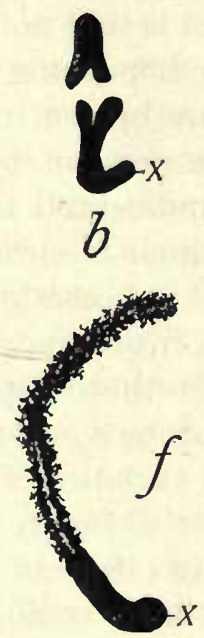
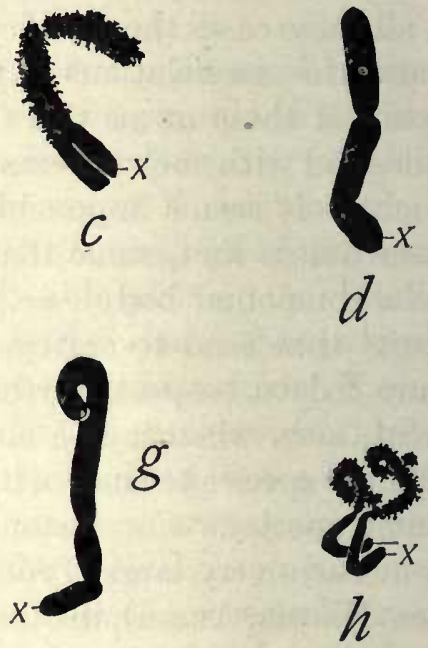

Fig. 6 Compound groups formed by union of the X-chromosome with other chromosomes in the Orthoptera. ( $a$ and $b$, from Sinety, the others from McClung.) $a$, triad group, first division of Leptynia, metaphase; $b$, division of similar triad in Dixippus; $c$, triad group formed by union of the X-chromosome with one of the bivalents, first spermatocyte-prophase, Hesperotettix; $d$, the same element from a metaphase group; $e$, the same element in the ensuing interkinesis; $f$, the compound element of Mermiria, from a first spermatocyte prophase; $g$, the same element in the metaphase (now, according to McClung, united to a second bivalent to form a pentad); $h$, the same element after its division, in the ensuing telophase.

The same general view as that outlined above is suggested by the constant relation known to exist in some cases between the $\mathrm{X}$ chromosome and a particular pair of the 'ordinary chromosomes.' The first observed case of this was recorded by Sinéty ('01) in the phasmid genera Leptynia and Dixippus (fig. $6 a, b$ ), where the $\mathrm{X}$-chromosome is always attached to one of the bivalents in the 
first spermatocyte-division, and passes with one half of the bivalent to one pole. Since the spermatogonial number in Leptynia (36) is an even one and twice that of the separate chromosomes present in the first spermatocyte-division, it may be inferred that the $\mathrm{X}$-element is already united with one of the ordinary chromosomes in the spermatogonia, though Sinéty does not state this. Somewhat later McClung ('05) discovered essentially similar relations in the grasshoppers Hesperotettix and Anabrus (fig. 6, $c-e$ ), and in case of the first named form was able to establish the important fact that it is always the same particular bivalent with which the $\mathrm{X}$-chromosome is thus associated. In respect to the intimacy of this association, a progressive series seems to exist, since in Leptynia it seems to take place in the spermatogonia, in Hesperotettix only in the prophases of the first spermatocytedivision, while in Thyanta the union is only effected after the first division is completed.

Finally, the recent observations of Boring ('09), Boveri ('09) and Edwards ('10) seem to establish the fact that in Ascaris megalocephala the X-element, whether in the diploid groups or in the maturation-divisions, may either appear as a separate chromosome (which has the usual behavior of an accessory chromosome) or may be indistinguishably fused with one of the ordinary chromosomes.

These relations may, of course, be the result of a secondary coupling; and I myself formerly so interpreted them ('09c). But in view of what is seen in Thyanta or the reduvioids we may well keep in mind the possibility that they are expressions or remnants of a more primitive association, like that which I have assumed for an original XY-pair. Whatever be their origin, the effect is the same - a definite linking of the $\mathrm{X}$-chromatin with that of one of the other pairs.

Fig. 7 shows, in purely schematic form, the general conception of these relations that has been suggested above, the $\mathrm{X}$-chromatin being everywhere represented in black. $A$ is the primitive XYpair from which all the other types may have been derived. By simple reduction of such a pair arises the ordinary or typical idiochromosome-pair (B); and from either A or B may be derived 
the other types $(\mathrm{C}-\mathrm{G}),{ }^{8}$ or the more complicated ones shown in fig. 5. I represents the possible mode of separation of the $\mathrm{X}$-element from a YY-pair, as suggested by Stevens; and this may be realized in Ascaris megalocephala $(\mathrm{H}) . \mathrm{J}$ and $\mathrm{K}$ are schemes of the relations seen in Hesperotettix, Anabrus and Mermiria (cf. fig. 6). These may be direct derivatives of a primitive XY-pair, as the diagram suggests, or may be a result

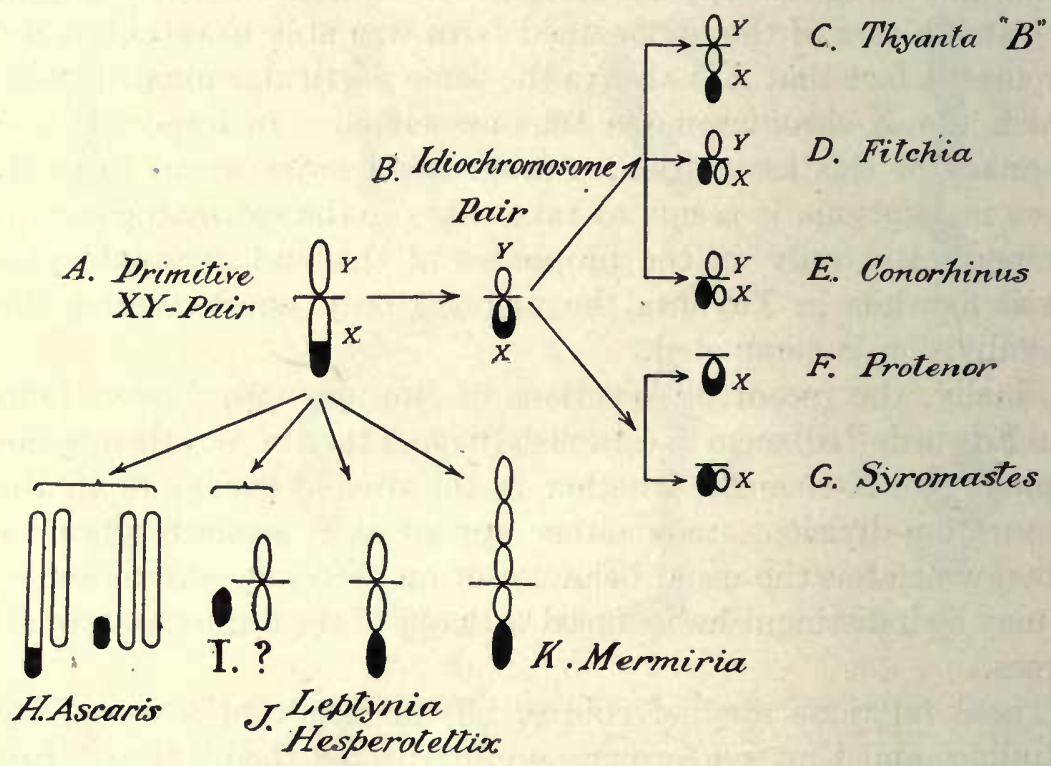

Fig. 7 Diagram illustrating the possible relation of the various types of idiochromosomes to a primitive XY-pair. Explanation in text.

of secondary coupling of $\mathrm{X}$ with other elements. In either case $\mathrm{X}$ may itself have such a composition as is indicated in $\mathrm{F}$ (Protenor).

(c) Sex-limited heredity. (1) The foregoing considerations have an important bearing on the problem of sex-limited heredity, for they give us a very definite view of how such heredity may be effected. It is not my intention to consider this subject in ex-

8 These figures are not intended to indicate the precise mode of segregation of the $\mathrm{X}$ - and $\mathrm{Y}$-chromatins of the $\mathrm{X}$-element, but only illustrate possible modes. 
tenso; but I wish to indicate some of the possibilities that have been opened by the cytological results, even at the risk of offering what may be regarded as too speculative a treatment of the matter. It is obvious that any recessive mutation should exhibit sex-limited heredity when crossed with the normal or dominant form, if it be due to a factor contained in (or omitted from) the X-element. For instance, in the remarkable Drosophila mutants discovered by Morgan ('10) the experimental data establish the fact that white eye-color (which seems to follow the same type of heredity as color-blindness in man) is linked with a sex-determining factor in such a way that when the white-eyed male is crossed with the normal red-eyed female, the former character is neyer transmitted from father to son, but through the daughters to some of the grandsons (theoretically to 50 per cent), though the daughters are not themselves white-eyed; that is, after such an initial cross, white eyes fail to appear in the $F_{1}$ generation in either sex and in the $\mathrm{F}_{2}$ generation appear only in some of the males. As Morgan points out, this follows as a matter of course if the factor for white eye be identical with, or linked with, a sex-determining factor in respect to which the male is heterozygous or simplex, the female homozygous or duplex. The $\dot{X}$-element exactly corresponds in mode of distribution to such a sex-determining factor; for this chromosome, too, is simplex in the male, duplex in the female and its introduction into the egg by the spermatozoon produces the female condition, its absence the male. This chromosome therefore, as I have shown ('06), is never transmitted from father to son, but always from father to daughter. Conversely, the male zygote always receives this chromosome from the mother. So precise is the correspondence of all this with the course of sexlimited heredity of this type that it is difficult to resist the conclusion that we have before us the actual mechanism of such heredity -in other words, that some factor essential for sex is associated in the $\mathrm{X}$-element with one that is responsible for the sex-limited character.

This will be made clearer by the accompanying diagram (fig. 8) where the $X$-element assumed to be responsible for a recessive sex-limited character is underscored $(\underline{\mathrm{X}})$. This character may 
be regarded as due to the absence of some particular constituent that is present in the normal $\mathrm{X}$-element.

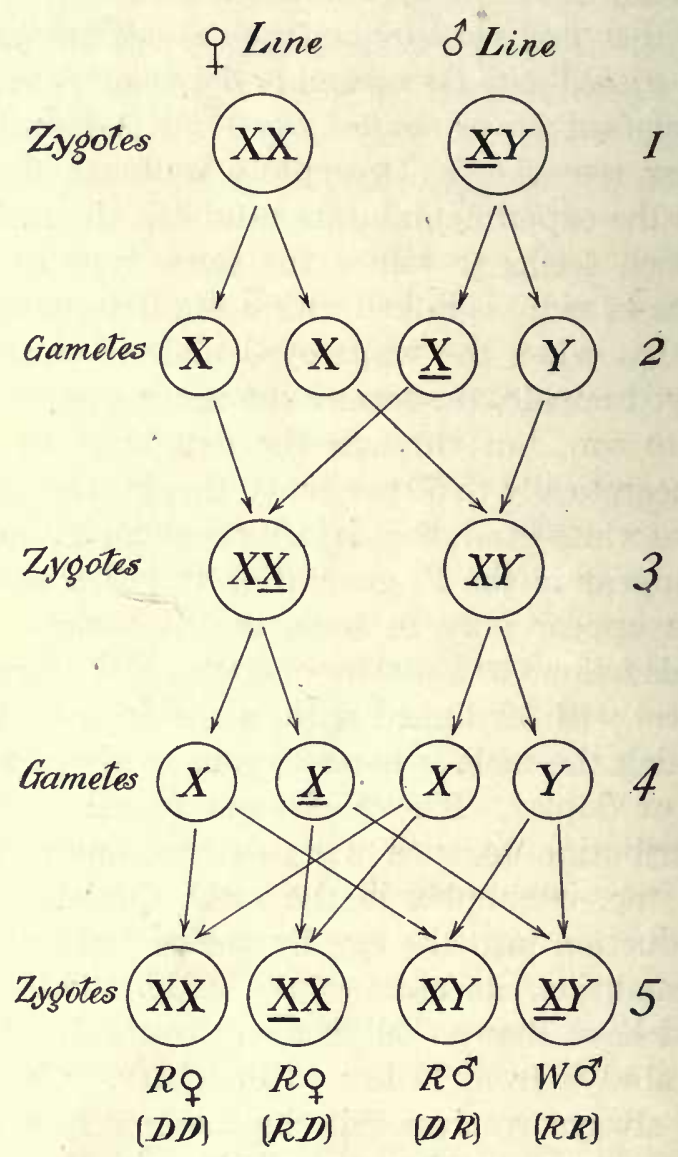

Fig. 8 Diagram of the distribution of the $\mathrm{X}$ - and $\mathrm{Y}$-elements in successive generations, illustrating sex-limited heredity. The underscored $\mathrm{X}$-element $(\underline{X})$ is assumed to bear a factor for a recessive character (e.g., white eye-color), while $\mathrm{X}$ represents the normal or dominant character (e.g., red eye-color). Y (being the absence of $\mathrm{X}$ ) likewise represents the recessive character.

Upon pairing the affected male (XY) with the normal female (XX) there are in the $F_{1}$ generation but two possible combinations, $\mathrm{XX}$ and $\mathrm{XY}$. The affected $\underline{\mathrm{X}}$-chromosome here passes 
into the female, and the male is normal; but the female of course likewise shows only the normal (dominant) character. In the following $\mathrm{F}_{2}$ generation (5) there are four possible combinations $\mathrm{XX}, \underline{\mathrm{XX}}, \mathrm{XY}$ and $\underline{X} Y$, two of each sex. Though $\underline{\mathrm{X}}$ is present in half of each sex, the character appears only in the males, $X Y$, again because of its recessive nature. By crossing together males of the composition XY and females of composition XX, some of the resulting females will have the composition $\mathrm{XX}$, and the sexlimited character is thus made to appear in the female.

When the female is the heterozygous or digametic sex-as in sea-urchins, in Abraxas, the Plymouth Rock fowls, etc.- exactly the converse assumption has to be made. Here, as Spillman ('08) and Castle ('09) have pointed out, the observed results follow if the sex-limited character (e.g., lacticolor color-pattern in Abraxas) be allelomorphic to, or the synaptic mate of, a sexdetermining factor, $X$, that is present as a single element in the female but absent in the male. The formulas now become ${ }^{9}$ (as Spillman has indicated) XG (o grossulariata), GG (o gross.) $\mathrm{XG}$ ( $\%$ lacticolor) and $\mathrm{GG}$ (o lact). $\mathrm{XG} \times \mathrm{GG}$ then gives in $\bar{F}_{1} X G$ and $G G$ (gross. of and $\sigma^{7}$ ), $\underline{G}$ having passed from the female to the male. The following cross, $\mathrm{XG} \times \mathrm{GG}$ gives in $\mathrm{F}_{2}$ the four types $\mathrm{XG}, \mathrm{X} \underline{\mathrm{G}}, \mathrm{GG}$ and $\mathrm{GG}$,-i.e., grossulariata appearing in both sexes but lacticolor only in the female. By crossing $\mathrm{XG}$ with $\underline{\mathrm{GG}}$ some of the progeny will have the composition GG (o lacticolor). The other combinations follow as a matter of course.

This interpretation is in all respects the exact converse of that made in the case of Drosophila, which is also the case with

- These formulas are in substance the same as those stated by Mr. Spillman in a private letter to the writer, and are a simplified form of those suggested by Castle ('09). The interpretation thus given seems both the simplest and the most satisfactory from the cytological point of view of all those that have been offered. It obviates the cytological difficulties that I urged ('09) against Castle's formulas, and renders unnecessary the secondary couplings that I suggested. All these ways of formulating the matter conform, of course, to the same principle and differ only in details of statement. Whether the synaptic mate of $\mathrm{X}$ is directly comparable to the $\mathrm{Y}$-chromosome of other insects (in which case the female formula becomes $\mathrm{XY}$ and the male $\mathrm{YY}$ ) is an open question. 
the experimental results, as Morgan has pointed out. It seems probable that all the observed phenomena may be reduced in principle to one or the other of these schemes, though many modifications or complexities of detail probably exist. A possible basis for many such modifications seems to be provided by the cytological facts already known.

(2) We might assume that in cases of the first type (e.g., Drosophila) both sex and the characters associated with it are determined by the same chromatin; and such a possibility should certainly be borne in mind. But in view of the widely different nature of the characters already known to exhibit sex-limited heredity it seems improbable that we can regard them as all alike due to the same chromatin. In the light of the conclusions that have been indicated in regard to the composition of the X-element, it seems more probable that such characters may be determined by the various other forms of chromatin ('Y-chromatin') associated with the $\mathrm{X}$-chromatin. If these constituents be identical with those contained in the free $\mathrm{Y}$-chromosome (the synaptic mate of $\mathrm{X})$ sex-limited heredity would of course not appear, since the two members of the pair would be homozygous in this respect. It should make its appearance as a result of the dropping out, or other modification, of certain $\mathrm{Y}$-constituents of the $\mathrm{X}$-element, and such a mutation might arise in either sex.

We may perceive here the possibility of understanding many different kinds of sex-limited heredity, perhaps of complex types that have not yet been made known. Such a possibility is suggested, for example, by the remarkable relation discovered by McClung ('05) in Mermiria (fig. $6 f-h$, fig. 7 in diagram), where the X-chromosome is in the first spermatocyte-division attached at one end to a linear chain of four other elements to form a pentad complex, to which may be given the formula XA.ABB. This so divides as to separate $\mathrm{XA}$ from $\mathrm{ABB}$. The interpretation to be placed upon this is a puzzling question under any view, and apparently must await more extended studies on both sexes, perhaps also on other forms, before it can be fully cleared up. Even here the possibility exists, I think, that the entire complex may have arisen by the differentiation of a single original XY-pair; 
but this question is clearly not yet ready for discussion. However such associations have arisen, the result is equally applicable to the explanation of sex-limited heredity.

(d) Secondary sexual characters. Castle ('09) has offered the interesting suggestion that the free Y-chromosome may be responsible for the determination of secondary sexual characters in the male. Though I have criticized this view ('09c) I now believe it may be true for certain cases. It is obviously excluded when the Y-chromosome is missing; and since nearly related species-in Metapodius even different individuals of the same species - show the same or similar secondary male characters whether this chromosome be present or absent, it seems probable that these characters are in general determined in some other way. But if, as I have suggested, sex-limited heredity may arise through a modification of the $\mathrm{Y}$-constituents of the $\mathrm{X}$-element, it follows that the YY-pair thereby becomes heterozygous. In such case, the free $\mathrm{Y}$-chromosome, being confined to the male line, should continue to represent characters that are no longer present in the female, and hence would be indistinguishable from secondary male characters otherwise determined. It has further become evident (as is indicated below) that the chromosome-groups are so plastic that their specific composition may vary widely from species to species. It may very well be, therefore, that Castle's suggestion may apply to some forms.

\section{Modes in which the chromosome-number may change}

The constant and characteristic duality of the ' $d$-chromosome' in the second division suggests a series of questions regarding the mode in which the chromosome-number may change that have an important bearing on those already considered. The appearance of this chromosome must suggest to any observer that it is a compound body, consisting of two closely united components that are invariably associated in a definite way; but it is especially noteworthy that its duality does not certainly appear before the last division. This case must be added to the steadily increasing evidence that chromosomes which appear single and homoge- 
neous to the eye may nevertheless be compound bodies. An important part of it is derived from the modifications of the $\mathrm{X}$ element reviewed above; but the evidence is now being extended to the 'autosomes' or ordinary chromosomes as well. The double chromosome of Nezara suggests either the initial stages of a separation of one chromosome into two or the reverse process-in either case that we have before us one way in which the number, and the composition, of the chromosomes may change from species to species. This is supported by the recent observations of Miss E. N. Browne ('10) on Notonecta. In N. undulata there are always, in addition to a typical unequal XY pair, two small chromosomes that appear in all the divisions as separate elements. In $\mathrm{N}$. irrorata there is always but one such chromosome, the total number in each division being accordingly one less than in $\mathrm{N}$. irrorata. N. insulata presents a condition exactly intermediate, there being sometimes one and sometimes two such small chromosomes. When, however, but one seems to be present, the second may often be seen closely adherent to one of the larger chromosomes; and the latter may positively be identified, by its size, as always the same one. It can hardly be doubted, as the author points out, that we here see three stages in a process by which the chromosome-number is changing, either by the fusion of two originally separate chromosomes, or by the separation of one into two. It is of the utmost importance that this process affects a chromosome that can be positively identified as the same in each case; for this proves that the change is not an indefinite fluctation but the expression of a perfectly orderly process. While there is here (as in the case of the $d$-chromosome of Nezara) no way of knowing in which direction the change is taking place, either alternative involves the conception that the individual chromosomes may be compound bodies, whether as a result of previous fusion or as possible starting points for a subsequent segregation.

The facts now known indicate at least four possible ways in which the chromosome-number (and in three of these also the composition of the individual chromosomes, may change from species to species. 
One is that suggested by the foregoing phenomena, i.e., the gradual fusion of separate chromosomes into one or the reverse process.

A second mode may be the gradual reduction and final disappearance of particular chromosome-pairs, as was suggested by Paulmier ('99), and afterwards by Montgomery and myself, in case of the microchromosomes, or ' $m$-chromosomes' of the coreid Hemiptera. In respect to the size of these chromosomes, a graded series may be traced from forms in which they are very large (as in Protenor) through those where they are of intermediate size down to cases where they are very small (as in Archimerus) and finally to such a condition as that seen in Pachylis (fig. $9 j-l$ ) where they are almost as minute as centrioles and may almost be regarded as vestigial. Four of these stages are shown in fig. 9. In Protenor $(a-c)$ the $m$-chromosomes are so nearly of the same size as the next smallest pair that they often can not be positively identified in the spermatogonial groups. In Leptoglossus phyllopus $(d-f)$ they are always recognizable, though not much smaller than the next pair. In L. oppositus or L. corculus they are a little smaller. In Anasa (the form in which they were first discovered by Paulmier) they are of middle size $(g-i)$, representing perhaps a fair average of the group. Several other genera (e.g., Metapodius) show intermediate stages between this condition and that seen in Archimerus (figured in my second 'Study,' and more recently by Morrill) where the $m$-chromosomes are almost as small as in Pachylis. It is most remarkable that throughout this whole series the $m$-chromosomes exhibit essentially the same behavior (Wilson, '05b, '06), usually remaining separate throughout the entire growth-period and only conjugating in the final prophases of the first spermatocyte-division, to form a bivalent which with rare exceptions occupies the center of the metaphase group; in some forms, also (e.g., Protenor, Alydus) they show a marked tendency to condense at a much earlier period than the other chromosomes. The $m$-chromosomes of Pachylis, excessively minute though they are, exhibit a behavior in all respects as constant and characteristic as even the largest of the series. In the Lygaeidae they seem to be present in some 

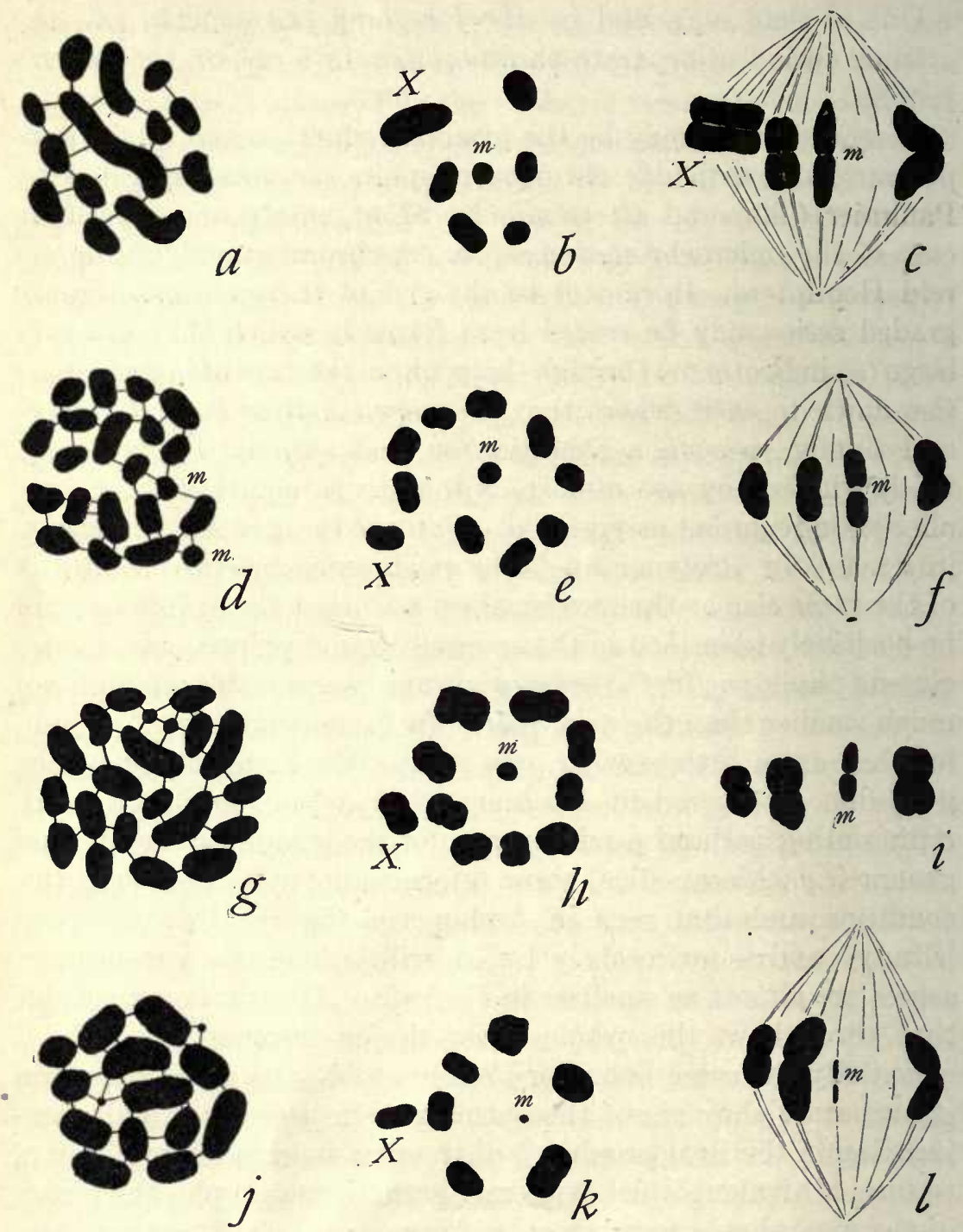

Fig. 9 Comparison of the $m$-chromosomes in Hemiptera. (In each horizontal row are shown at the left a spermatogonial group, in the middle a polar view of the first spermatocyte-division, at the right a side-view of the same division.) $a-c$, Protenor belfragei; $d-f$, Leptoglossus phyllopus; $g-i$, Anasa tristis; $j-l$, Pachylis gigas. 
species (Oedancala, $t$. Montgomery), in others absent (Lygaeus). In the Pyrrhocoridae (Pyrrhocoris, Largus) they are absent as far as known. So characteristic is the behavior of these chromosomes as to leave not the least doubt of their essential identity throughout the whole series; and this series may be regarded as a progressive one, in one direction or the other, with the same reason as in case of any other graded series of morphological characters. The series thus shown in case of the $m$-chromosomes is as gradual and complete as in case of the $\mathrm{Y}$-chromosome, and may with the same degree of probability be regarded as a descending one.

Thirdly, it is probable that the chromosome-number may change by sudden mutations that produce extensive redistributions of the chromatin-substance without involving any commensurate change in its essential content. Were gradual changes, chromosome by chromosome, the usual mode of modification, we should certainly expect to find such conditions as are seen in Nezara, in Notonecta, or in the Coreidae, more frequently. In some groups, however, we find wide differences of chromosomenumber between species even of the same genus, and even between those that are very nearly related, without any accompanying evidence of a gradual process of transition-for instance, among the aphids and phylloxerans (Stevens, Morgan) or in the heteropterous genera Banasa and Thyanta. (Wilson, '09d.) In Banasa dimidiata the diploid number is 16 in both sexes, in the nearly related B. calva 26 . Of the two races of Thyanta custator described above, apparently identical in other visible characters, one has in both sexes the diploid number 16 , with a simple X-chromosome, while in the other the diploid number of the male is 27 and that of the female 28 , and the $\mathrm{X}$-chromosome consists of two components. It is improbable that the differentiation of these two forms has been accomplished by gradual modifications, chromosome by chromosome. It seems far more likely that the change took place by sudden mutation, involving a redistribution of the nuclear material which changed its form but not in an appreciable degree its substance. In the well known case of Oenothera gigas, derived by sudden mutation from Oe. Lamarckiana, a change by sudden mutation is known to be 
PLATE 1

\section{EXPLANATION OF FIGURES}

All the figures from photographs of sections. Enlargement 1500 diameters.

10, 11 First spermatocyte-division (N. hilaris)

12,13 The same ( $\mathrm{N}$. viridula)

14, 15 Second spermatocyte-division (hilaris)

16-25 Side views of second division (hilaris). The XY-pair shown in 16-23, the $d$-chromosome in $16,17,20,24,25$; the small chromosome is evident in $10,12,13,14,15,17,18$.

22 Initial separation of $\mathrm{X}$ and $\mathrm{Y}$

23 Early anaphase, $\mathrm{X}$ and $\mathrm{Y}$ separating near the center (hilaris)

26-28 Nuclei from the growth-period, showing chromosome-nucleolus and plasmosome (hilaris)

29 Corresponding stage (viridula) 


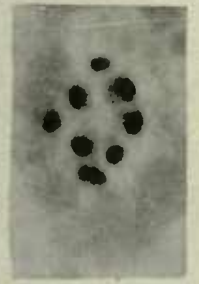

I0

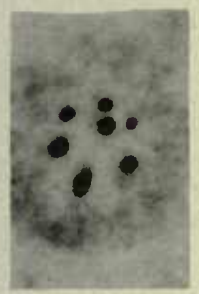

14

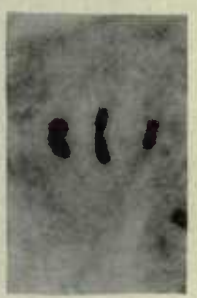

I8

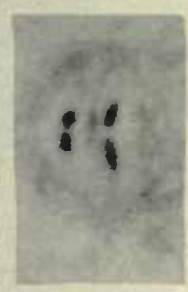

22

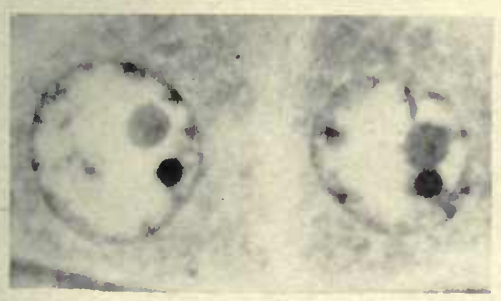

26

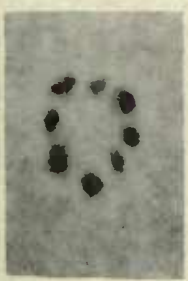

I I

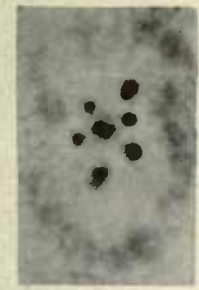

15

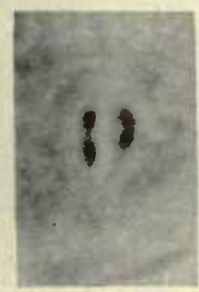

19

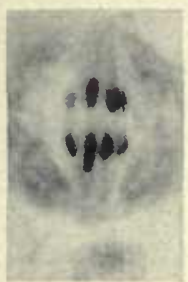

23

27

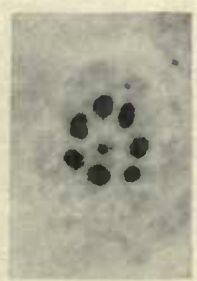

I2

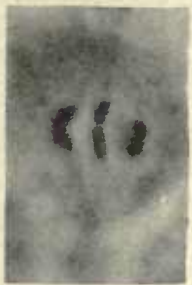

I6

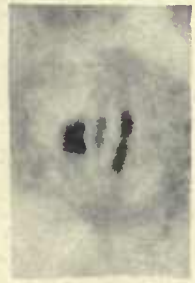

20

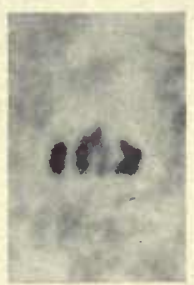

24

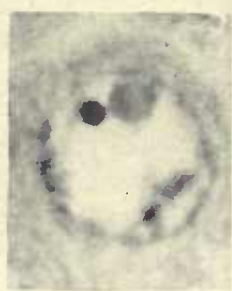

28

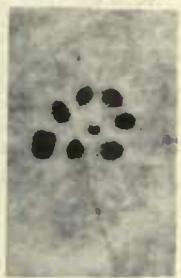

I3

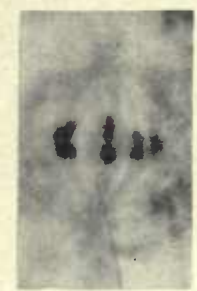

17

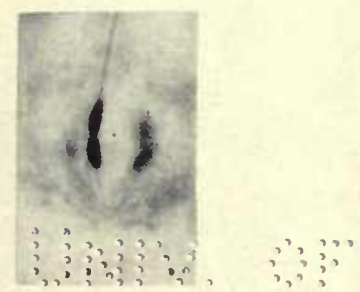

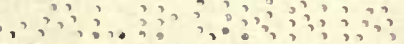

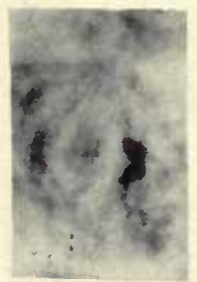

25

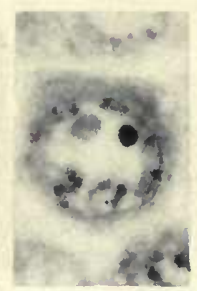

29

JOURNAL OF MORPHOLOGY, VOL. 22 , No. 1 

a fact (Lutz, '07; Gates, '08), though it may be due in this instance to a simple doubling of the whole group. Such cases led me several years ago to the conclusion "that the nucleus consists of many different materials that segregate in a particular pattern and that the particular form of segregation may readily change from species to species"' (Wilson, '09d, p. 2).

Such changes must involve corresponding ones in the morphological and physiological value of the individual chromosomes; and we must accordingly recognize the probability that these individual values, though constant for the species, may change from species to species as readily as does the number. Despite the conformity to a general type often exhibited by particular genera or even by higher groups, the individual chromosomes are therefore per se of subordinate significance; and it may often be practically impossible to establish exact homologies between those of different species. How closely this bears on the origin of the diverse conditions seen in the composition of the XY-pair is obvious.

Lastly, it is almost certain that changes of number may sometimes arise as a result of abnormalities in the process of karyokinesis, such as the passage of both daughter-chromosomes, or of both members of a bivalent, to one pole. In Metapodius I found ('09b) direct evidence of this in case of the XY-pair itself, and endeavored to trace to this initial cause the remarkable variations of number that occur in this genus. Many other observers have recorded anomalies of this kind, in both plants and animals. It seems entirely possible, as has been suggested by McClung ('05) and by Gates('08)that definite mutations may be traceable to this cause; though probably such abnormalities may in general be expected to lead to pathological conditions.

\section{CONCLUSION}

Some of the suggestions offered in the foregoing discussion are admittedly of a somewhat speculative character; but they are not, as I venture to think, mere $a$ priori constructions, but are forced upon our attention by the observed facts. The time has come 
when we must take into account more fully than has yet been done the new complexities and possibilities that have continually been unfolded as we have made better acquaintance with the chromosomes. In this respect the advance of cytology has quite kept pace with that of the experimental study of heredity; and it has established so close and detailed a parallelism between the two orders of phenomena with which these studies are respectively engaged as to compel our closest attention.

Studies on the chromosomes have steadily accumulated evidence that in the distribution of these bodies we see a mechanism that may be competent to explain some of the mostcomplicated of the phenomena that are being brought to light by the study of heredity. New and direct evidence that the chromosomes are in fact concerned with determination has been produced by recent experimental studies, notably by those of Herbst ('09) and Baltzer ('10) on hybrid sea-urchin eggs. But the interest of the chromosomes for the study of heredity is not lessened, as some writers have seemed to imply, if we take the view-it is in one sense almost self-evident-that they are not the exclusive factors of determination. Through their study we may gain an insight into the operation of heredity, that is none the less real if the chromosomes be no more than one necessary link in a complicated chain of factors. From any point of view it is indeed remarkable that so complex a series of phenomena as is displayed, for example, in sex-limited heredity can be shown to run parallel to the distribution of definite structural elements, whose combinations and recombinations can in some measure actually be followed with the microscope. Until a better explanation of this parallelism is forthcoming we may be allowed to hold fast to the hypothesis, directly supported by so many other data, that it is due to a direct causal relation between these structural elements and the process of development.

A second point that may be emphasized is the remarkable constancy of the chromosome-relations in the species, and their no less remarkable plasticity in the higher groups. The scepticism that has been expressed in regard to constancy in the species finds, I think, no real justification in the facts. It is perfectly true that 
individual fluctuations occasionally are seen in the number of the chromosomes, in the process of synapsis, in the distribution of the daughter-chromosomes, and in all other cytological phenomena. It is, however, also true that most observers who have made prolonged, detailed and comparative studies of any particular group, have sooner or later reached the conviction that in each species all the essential relations in the distribution of the chromosomes conform with wonderful fidelity to the specific type. So true is this that the species may often at once be identified by an experienced observer from a single chromosome-group at any stage of the maturation-process. No one, I believe, who has engaged for a series of years in the detailed study of such a group, for instance, as the Hemiptera or the Orthoptera, returning again and again to the scrutiny of the same material, can be shaken in the conviction that the distribution of the chromosomes follows a perfectly definite order, even though disturbances of that order now and then occur. But it is equally important to recognize the fact that this order has undergone many definite modifications of detail from species to species, and that while all cases exhibit certain fundamental common features, we cannot without actual observation predict the particular conditions in any given case. It is now evident that the larger groups vary materially in respect to specific conditions. For instance, in the orthopteran family of Acrididae (McClung) the relations seem to be far more uniform than such a group as the Hemiptera, where great specific diversity is exhibited, the details often changing from species to species in a surprising manner-witness the species of Aphis or Phylloxera (Stevens, Morgan), those of Acholla (Payne) or of Thyanta (Wilson). In these respects, too, the cytologist finds his experience running parallel to that of the experimenter on heredity; and here, once more, we find it difficult not to believe that both are studying, from different sides, essentially the same problem.

December 13, 1910. 


\section{LITERATURE CITED}

ARnold, G. 1908 The nucleolus and microchromosomes in the spermatogenesis of Hydrophilus piceus. Arch. Zellforsch., vol. 2,

BAltzer 1909 Die Chromosomen von Strongylocentrotus lividus und Echinus microtuberculatus. Arch. f. Zellforsch., Bd. 2.

1910 Ueber die Beziehung zwischen dem Chromatin und der Entwicklung und Vererbungsrichtung bei Echinodermenbastarden. Habilitationsschrift, Würzburg. EngeImann, Leipzig.

Boring 1909 A small chromosome in Ascaris megalocephala. Arch., f. Zellforsch., vol. 4.

Boveri, Тн. 1909 "Geschlechtschromosomen" bei Nematoden. Arch. f. Zellforsch., Bd. 4.

Browne, E. N. 1910 The relation between chromosome-number and species in Notonecta. Biol. Bull., vol. 20,1.

Castle, W. E. 1909 A Mendelian view of sex-heredity. Science, n. s., March 5.

Cook, M. H. 1910 Spermatogenesis in Lepidoptera. Proc. Acad. Nat. Sci., Philadelphia, April.

Dederer, P. 1908 Spermatogenesis in Phyllosamia. Biol. Bull., vol. 13.

Edwards, C. L. 1910 The idiochromosomes in Ascaris megalocephala and Ascaris lumbricoides. Arch. f. Zellforsch., vol. 5.

Gates, R. R. 1908a The chromosomes of Oenothera. Science, n. s:, vol. 27, Aug. 2.

1908b A study of reduction in Oenothera rubrinervis. Bot. Gazette, vol. 46 ,

1909 The behavior of the chromosomes in Oenothera lata $\times$ O. gigas. Ibid., vol. 48 .

Gross, J. 1904 Die Spermatogenese von Syromastes marginatus. Zool. Jahrb. Anat. u. Ontog., vol. 20.

GOYER, M. 1910 Accessory chromosomes in man. Biol. Bull., vol. 19.

Herbst, C. 1909 Vererbungsstudien, VI. Die cytologischen Grundlagen der Verschiebung der Vererbungsrichtung nach der mütterlichen Seite. Arch. Entwicklungsm., Bd., 27.

Lotz, A. M. 1907 A preliminary note on the chromosomes of Oenothera La. marckiana and one of its mutants. Sci., n. s. 26.

MCClung, C. E. 1905 The chromosome complex of orthopteran spermatocytes. Biol. Bull., vol. 9. 
Montgomeri, T. H. 1901 A study of the chromosomes of Metazoa. Trans. Am. Phil. Soc., vol. 20.

1906 Chromosomes in the spermatogenesis of the Hemiptera Heteroptera. Trans. Am. Phil. Soc., vol. 21.

Morgan, T. H. 1909a A biological and eytological study of sex-determination in phylloxerans and aphids. Jour. Exp. Zoöl., vol. 7,

1910 Sex-limited inheritance in Drosophila. Science, n. s. 32, July 22.

Morrill, C. V. 1910 The chromosomes in the oogenesis, fertilization and cleavage of coreid Hemipters. Biol. Bull., vol. 19.

Padlmier, F. C. 1899 The spermatogenesis of Anasa tristis. Jour. Morph., vol. 15, Suppl.

PAYne, F. 1909 Some new types of chromosome distribution and their relation to sex. Biol. Bull., vol. 16.

1910 The chromosomes of Acholla multispinosa. Biol. Bull., vol. 18.

Randolph, Harriet. 1908 On the spermatogenesis of the earwig, Anisolaba maritima. Biol. Bull., vol. 15.

SinÉtY, R. DE 1901 Recherches sur la biologie et l'anatomie des phasmes. La Cellule, t. 19.

Spillman, W. J. 1908 Spurious allemorphism. Results of some recent investigations. Am. Naturalist, vol. 42.

Strevens, N. M. 1906 Studies in spermatogenesis, II. A comparative study of the heterochromosomes in certain species of Coleoptera, Hemiptera and Lepidoptera, etc. Carnegie Inst. Pub. 36.

1908 A study of the germ-cells of certain Diptera, etc. Jour. Exp. Zoöl., 5, 3.

1910 The chromosomes in the germ-cells of Culex. Jour. Exp. Zoöl., vol 8.

Wallace, L. B. 1909 The spermatogenesis of Agalena nævia. Biol. Bull., vol. 17 .

WrLson, E. B. 1905a Studies on chromosomes, I. The behavior of the idiochromosomes in Hemiptera. Jour. Exp. Zoöl., vol. 2.

$1905 \mathrm{~b}$ Studies on chromosomes, II. The paired microchromosomes, idiochromosomes, and heterotropic chromosomes in Hemiptera. Jour. Exp. Zoöl., vol. 2.

1906 Studies on chromosomes, III. The sexual differences of the chromosomes in Hemiptera. Jour. Exp. Zoöl., vol. 3.

1909a Studies on chromosomes, IV. The accessory chromosome in Syromastes and Pyrrhocoris. Jour. Exp. Zoöl., vol. 6. 
1909b Studies on chromosomes, V. The chromosomes of Metapodius, etc. Jour. Exp. Zoöl., vol. 6.

1909c Secondary chromosome-couplings and the sexual relations in Abraxas. Science, n. s. 29, p. 748.

1909d Differences in the chromosome-groups of closely related species and varieties, etc. Proc. Seventh Internat. Zoöl. Congress, Aug. 1907.

1910a The chromosomes in relation to the determination of sex. Science Progress, no. 16, April.

1910b Studies on chromosomes, VI. A new type of chromosome-combination in Metapodius. Jour. Exp. Zoöl., vol. 9.

1910c Note on the chromosomes of Nezara. Science,n. s. 803, May 20. 



\section{Studies on Chromosomes}

VIII. Observations on the Maturation-Phenomena in Certain Hemiptera and Other Forms, with Considerations on Synapsis and Reduction

EDMUND B. WILSON

From the Department of Zoölogy, Columbia University

Reprinted from The Journal of Experimental ZoöLoàx, Vol. 13, No. 3, October, 1912

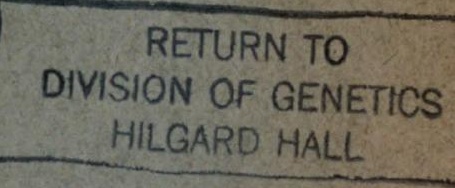




\section{9}

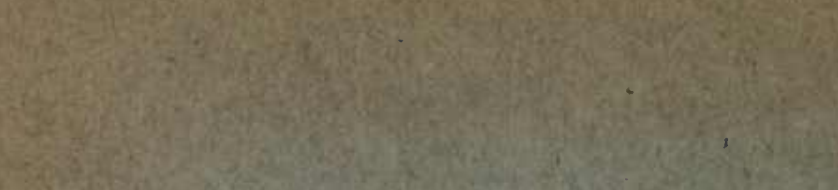

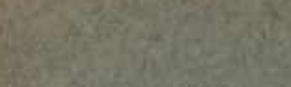

(2)

ansing

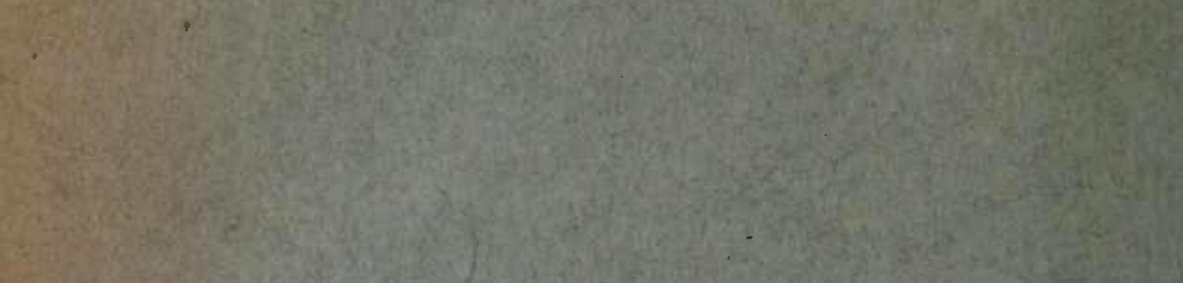

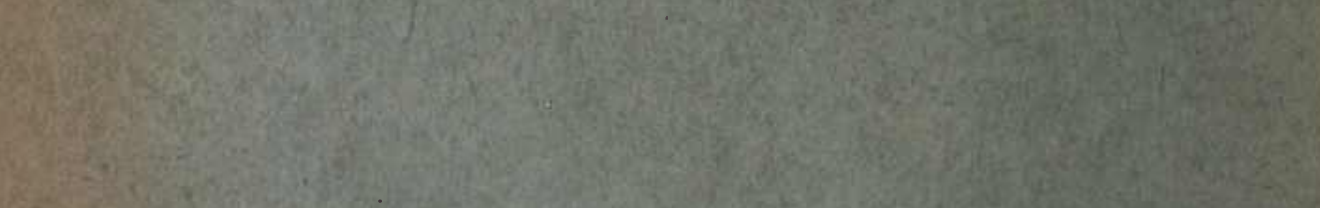

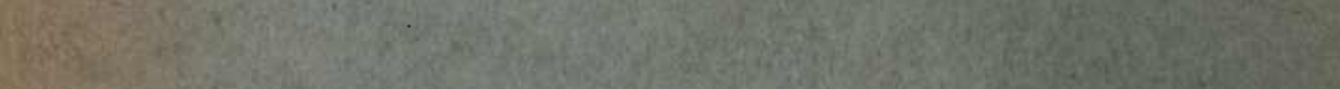

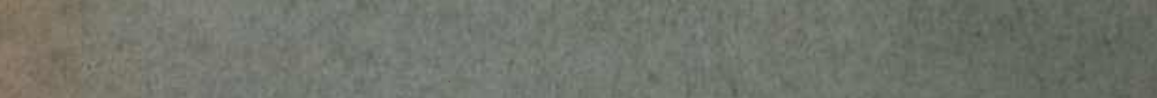

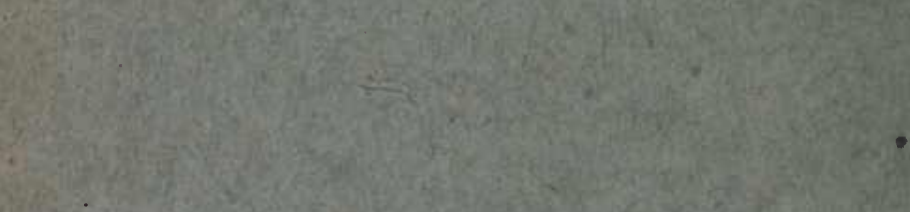

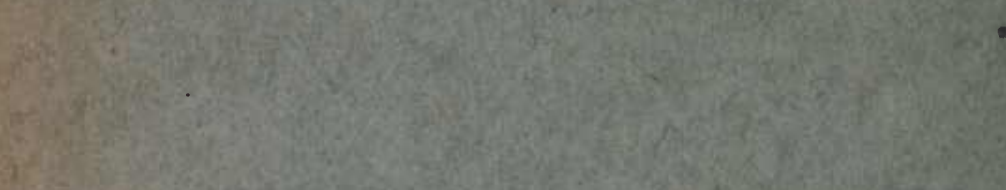

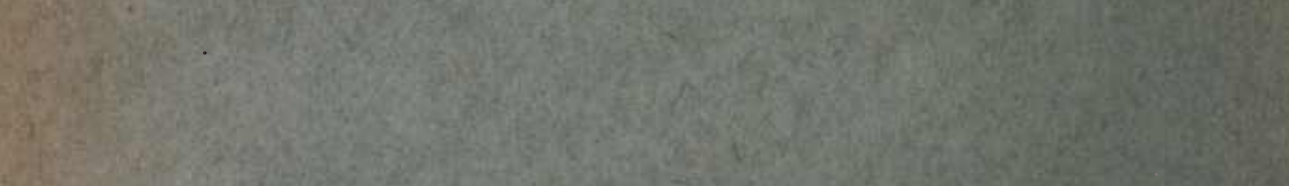

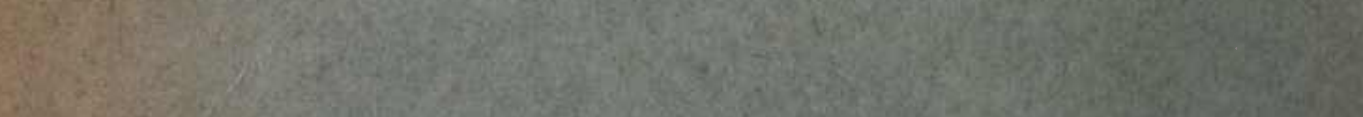

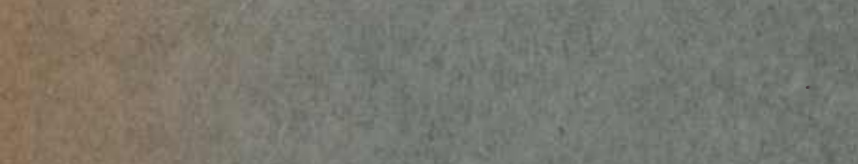
(2)

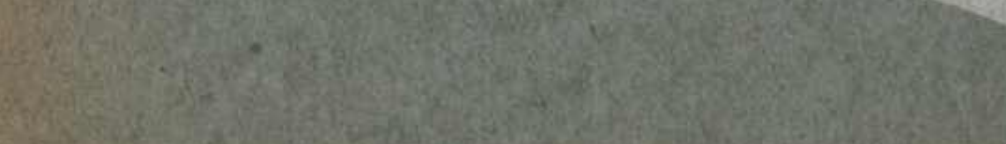

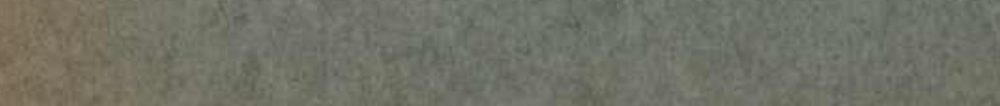

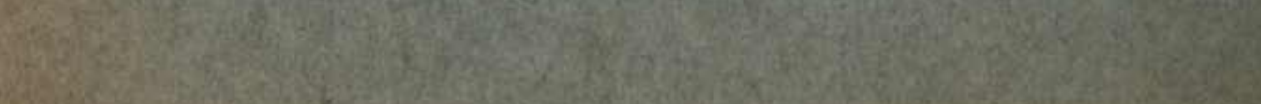

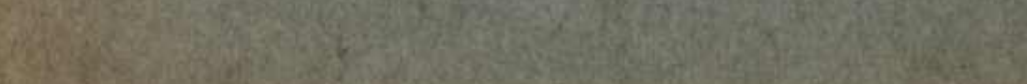

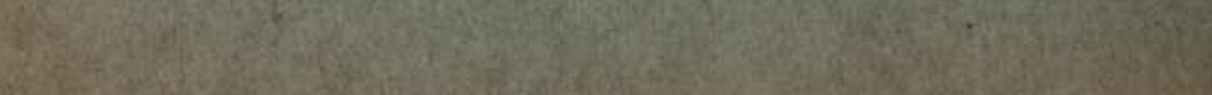




\title{
STUDIES ON CHROMOSOMES
}

\section{OBSERVATIONS ON THE MATURATION-PHENOMENA IN CER- TAIN HEMIPTERA AND OTHER FORMS, WITH CONSIDERATIONS ON SYNAPSIS AND REDUCTION}

\author{
EDMUND B. WILSON
}

From the Department of Zoölogy, Columbia University

\author{
NINE PLATES
}

\section{CONTENTS}

Introduction.

I. The maturation-divisions in Oncopeltus and Lygaeus with reference to the sex-chromosomes

1. The diploid chromosome-groups..................... 350

2. The first spermatocyte-division........................ 351

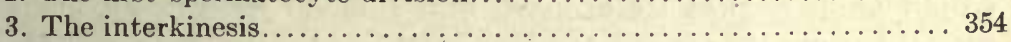

4. The second spermatocyte-division..................... 355

5. Size-relations of the sex-chromosomes in Oncopeltus............ 357

II. The growth-period

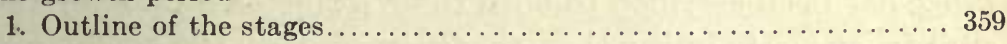

2. Stages $a$ to $d$. The pre-synaptic stages. Comparison with other

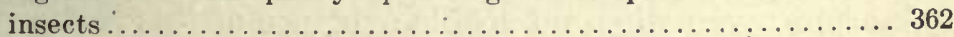

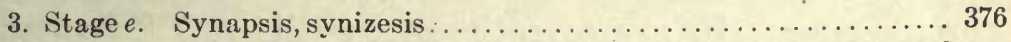

4. Stages $f$ and $g$. Post-synaptic spireme (pachytene, diplotene), and the diffuse or confused stage....................... 377

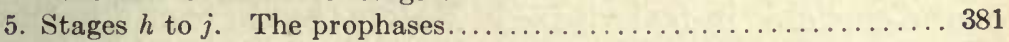

6. Comparative considerations regarding the growth-period........ 387

7. Comment on the sex-chromosomes in Oncopeltus............. 390

III. Critical considerations on the maturation-phenomena based on a comparison of the Hemiptera with Tomopteris, Batracoseps and other forms

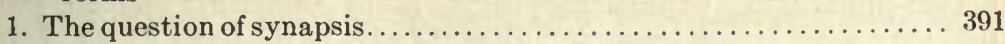

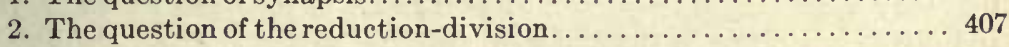

3. The chromosomes and heredity...................... 419 


\section{INTRODUCTION}

In this paper are described observations on certain phases of the maturation-process in Oncopeltus fasciatus (Dall.), Lygaeus bicrucis (Say), and some other Hemiptera, together with the results of a comparison of these species with some other insects, with Tomopteris and with Batracoseps. It was my original object to clear up the relations of the sex-chromosomes in Oncopeltus and to trace as completely as possible their history in the maturation-process; but in doing this it was found necessary to take into consideration many other features of the spermatogenesis, and I will take this opportunity to present some conclusions based on a broader study of these problems on which I have long been engaged.

In respect to the sex-chromosomes, Oncopeltus is of especial interest because it stands on the border line between species in which the $X$ - and $Y$-chromosomes are visibly unequal in size, and in which a corresponding visible difference appears between the diploid chromosome-groups of the two sexes, and those in which such sexual differences can not be seen. ${ }^{1}$ In my fourth 'Study' ('09 a) Oncopeltus was classed with Nezara hilaris as an example of the latter class of cases; and much theoretic importance has been ascribed to both these forms as indicating the possibility or probability that the spermatozoa are really sexually dimorphic even when no visible evidence of this is shown by the chromosomes. In my seventh 'Study' ('11 a) ) I showed, contrary to my original account, that a dimorphism of the spermatid-nuclei is in fact visible in Nezara; but in regard to Oncopeltus judgment was reserved as I was still baffled by apparently contradictory data. I am now in a position to clear up these

1 The first account of Oncopeltus was given by Montgomery ('01), who described the sex-chromosomes ('chromatin-nucleoli') as of equal size in the male, and found that they remain always separate, without fusing at the time of general synapsis, and divide separately in the first spermatocyte-division. Subsequently ('06) he added that these chromosomes (now called 'diplosomes') conjugate to form a bivalent after the first division, and undergo disjunction in the second division. In my fourth 'study' ('09 a) I briefly confirmed these accounts, and stated that the female diploid chromosome-groups are not to be distinguished by the eye from the male. 
contradictions and to announce a definite result. Oncopeltus is indeed a case in which the $X$ - and $Y$-chromosomes are very often sensibly equal in size, and in which the sexual differences of the diploid groups are too elusive to be certainly distinguished by the eye. These differences, nevertheless, almost certainly exist. In certain individuals a distinct size-difference between $X$ and $Y$ is clearly evident in a large percentage of the cells at every stage of the spermatogenesis; and even in individuals where they usually appear equal, inequality is unmistakably seen in a small percentage of the cells. Aside from this, the close similarity-almost identity - between Oncopeltus and Lygaeus bicrucis in all other features of the spermatogenesis makes it extremely probable that the same essential relations of the chromosomes to sex exist in both, though they are only clearly obvious in Lygaeus.

Like many other insects of this order, Lygaeus and Oncopeltus are distinctly unfavorable objects for the direct study of synapsis and the reduction-division -indeed the problem of synapsis seems to be practically insoluble in these particular forms. They nevertheless present some very interesting features for comparison with other forms. In the first place, the history of the sex-chromosomes may here be traced with almost unique clearness. They. may be identified at a very early pre-synaptic period, and followed thence as individual bodies through every later stage up to the time of their final delivery to the spermatid-nuclei. Every step may be followed in their conjugation and subsequent disjunction without any intervening process of fusion. In case of these particular chromosomes, therefore, I consider synapsis and disjunction to be indisputable facts. It is far otherwise with the ordinary chromosomes or 'autosomes.' It is extremely difficult to gain any clear idea of their behavior in the synaptic period, and I fear quite impossible to trace them individually through the growth-period. On the other hand, their behavior in the pre-synaptic period and in the maturation-prophases exhibits some very interesting features when compared with other forms in which the process of synapsis and its sequel are more accessible to observation. In making such a comparison I have been fortunate in the opportunity to make use of some remarkably fine 
preparations of other investigators, to whom I am under great obligations. To Professor McClung I owe the loan of a beautiful series of orthopteran preparations, especially of Phrynotettix, Mermiria, Chortophaga and Achurum, which display on a larger scale some of the same phenomena seen in the pre-synaptic stages of the Hemiptera, and leave no doubt of the close parallel between the two groups in this regard. Even more, however, I am indebted to Dr. and Madame Schreiner, and to Professor Janssens, for some of their admirable original preparations of Tomopteris and Batracoseps, which have enabled me to make a prolonged study of the phenomena of synapsis in these classical objects. In particular, two magnificent slides of Batracoseps by Janssens demonstrate both the complete seriation of the stages and the finest details of the nuclear structures with incomparable clearness. Though I have also made many preparations of this form, as well as of Plethodon and other Amphibia, I must admit my failure to equal in all respects the standard set by the slides of Janssens. A close comparison of these various preparations has more than ever impressed me with the futility of attempting the study of these problems with material that is unfavorable for the purpose, or with preparations that in any respect fall short of the highest standard of technical excellence. Nothing is more certain than that different objects differ enormously in the clearness with which the relations are displayed, and in their reaction to fixing and staining reagents. Had this been more generally recognized, many erroneous conclusions and some ill-considered criticism might have been avoided. .

Through the study of Batracoseps and Tomopteris I have finally been convinced - for the first time, I must confess, as far as the autosomes are concerned-(1) that synapsis, or the conjugation of chromosomes two by two ${ }^{2}$ is a fact, and (2) that in these ani-

${ }^{2}$ A number of writers have suggested that the term synapsis, as here employed, should be abandoned in favor of some less ambiguous word (such as Haecker's term 'syndesis') because it has so frequently been applied to the contraction-figure ('synizesis' of McClung). I am, however, in favor of the retention of the word, for the ambiguity has arisen simply through a misunderstanding of Moore's meaning. He applied the term 'synaptic phase,' or 'synapsis,' to the series of changes following the last diploid division (during the 'rest of transformation') 
mals (perhaps also in the Orthoptera) the conjugation is a side by side union, or parasynapsis. On the other hand, the evidence of a 'reduction-division' in the ordinary use of the term-i.e., the disjunction of the same chromosomes that unite in synapsisseems to me to be far short of a demonstration. In these forms synapsis is followed by a union so intimate that no adequate evidence of duality can for a time be seen in the resulting bivalents. I do not for this reason argue against the conception of the reduction-division. On the contrary, I shall offer new considerations in favor of this conception in a somewhat modified form; but in case of the autosomes it must for the present rest mainly upon indirect evidence. In this respect the autosomes differ notably from the sex-chromosomes, at least in the male sex; and this difference may be of significance for some of the most interesting phenomena of sex-heredity.

in the course of which the apparent number of chromosomes is redueed to one-half. "There are thus, after the rest of transformation, only one half as many chromosomes, i. e., separate chromatin-masses, as there were before, and the halving of their number, being brought about while the nuclei are still at rest, is to that extent comparable to what is now known to go forward during the maturation of the reproductive elements of plants. I therefore propose the term Synaptie Phase (from $\sigma v \nu a \pi \tau \omega$, to fuse together) to denote the period at which this most important change appears in the morphological character of reproductive cells" ' ' 95 , p. 287. In subsequent pages the phrase 'synaptic phase' is of ten shortened to 'synapsis' in the same sense). This 'most important change' is obviously the halving of the number of chromosomes; and nowhere in his paper is the word applied to the contraction-figure, though the latter is stated to be "characteristic of this particular phase in the spermatogo- and ovogenesis of a great variety of animal forms" (p. 305). Though there was, perhaps, some obscurity in his original use of the word, all doubt as to Moore's meaning is removed in a later paper, published jointly with Farmer ('05), where synapsis is precisely defined as "that series of events which are concerned in causing the temporary union in pairs of pre-maiotic chromosomes" (p. 490). The fact that so many later writers have misapplied it should not debar us from the continued use of so convenient and appropriate a term-one that seems particularly fitting if it be a fact, as a number of excellent observers have concluded, that synapsis is followed by actual fusion. I can discover no reason why McClung's term 'synizesis' should not be generally employed for the contraction-figure, as it already is by most American writers. 
I. THE MATURATION-DIVISIONS IN ONCOPELTUS AND LYGAEUS WITH REFERENCE TO THE SEX-CHROMOSOMES

In Oncopeltus the diploid number of chromosomes is sixteen in both sexes (figs. 1 to 5, photos, 1, 2) in Lygaeus fourteen (fig. 6), and the second spermatocyte-division shows half these numbers that is, eight in the former case, seven in the latter. The first division shows in each case one more than the haploid number, owing to the fact, repeatedly described heretofore in other Hemiptera, that in the first division the $X$ - and $Y$-chromosomes divide as separate univalents, while in the second they are united to form a bivalent. In both Oncopeltus and Lygaeus these chromosomes conjugate in the final anaphase of the first division, just as the cell is about to divide.

\section{The diploid chromosome-groups}

The spermatogonial and oogonial divisions require but brief description since they present no striking features and the sizedifferences are but slightly marked. In Lygaeus bicrucis (fig. 6) the fourteen chromosomes are in the main similar to those of $\mathrm{L}$. turcicus as described in my first and third 'Studies' ('05, '06) though the $Y$-chromosome is relatively smaller in the latter species. The $X$-chromosome can not be identified by the eye, but must be at least twice the size of the $Y$-chromosome, as indicated by the maturation-divisions and by the spermatogonial groups themselves. Unfortunately my material of this species does not show a single good equatorial plate in the female; but the relations are here no doubt the same as in $\mathrm{L}$. turcicus.

In Oncopeltus, of which I have abundant material of both sexes, the size-differences of the chromosomes are even less marked than in Lygaeus, and it is impossible to identify pairs of different sizes. Careful study fails to reveal any differences between the diploid groups of the two sexes that are sufficiently marked or constant to give any certain result (figs. 1 to 5). In the male one chromosome not infrequently is somewhat smaller than the others (fig. 2), and this may be the $Y$-chromosome; but very often this is not evident, even in other spermatogonia from the same cyst (figs. 1, 3). As will be shown beyond, the $X$ - and $Y$-chromosomes 
are often somewhat unequal in later stages; but this, too, is inconstant. Oncopeltus is in fact, therefore, a form in which the sexual differences of the chromosome-groups are too slight or too elusive to be distinguished by the eye. It is, however, perfectly certain that an $X Y$-pair is present, the members of which show all the characteristic peculiarities of behavior that characterize these chromosomes in other forms.

\section{The first spermatocyte-division}

The maturation-divisions are shown with remarkable clearness in Oncopeltus and fygaeus-indeed, either of them might be taken as a model of those Hemiptera in which a simple $X Y$-pair is present. The following account applies primarily to Oncopeltus, Lygaeus being described only by way of comparison.

In the first division appear nine separate chromosomes in a grouping of remarkable constancy. Seven of the nine bivalents are grouped in an irregular ring, near the center of which lie the univalent $X$ - and $Y$-chromosomes, side by side but not in contact (figs. 8,9,11, photo. 3). The constancy of this grouping appears from the following data. Two hundred clear polar views, taken at random, did not show a single case of more than nine chromosomes; plus variations of number in this division (such as are occasionally seen in many species) must therefore be very rareindeed, I have never seen such a case. ${ }^{3}$ Of the two hundred cases, one hundred and seventy-two showed the grouping just described. In the remaining twenty-eight the deviations were unimportant; the ring may show a gap at one side (figs. 10, 12), one of the bivalents may lie inside it (fig. 10), or (rarely) one or both the sexchromosomes may lie in the ring (fig. 13). In only two cases did the sex-chromosomes not lie side by side; in these, they were separated by one bivalent (fig. 13).

The size-relations alone sufficiently indicate that the two small central chromosomes are the univalent sex-chromosomes (a fact

${ }^{3}$ Apparent minus deviations are of course common, but are disregarded because evidently due in most (all?) cases to the fact that one or more chromosomes lie outside the plane of section. 
fully established by study of the growth-period and the prophases); for, were one or both bivalent, the spermatogonial groups should show one or two corresponding pairs, which is not the case. In Lygaeus (fig. 7) the grouping is the same, but only six bivalents are present, and the sex-chromosomes are conspicuously unequal in size.

The composition of these chromosomes is better seen in smears than in sections, and better in Protenor (described beyond) than in either of these forms. In sections, side views of the full metaphases (figs. 14 to 17, photo.4) usually show all of the chromosomes as simple dumb-bell figures, though indications of a quadripartite form sometimes appear. In smears the bivalents are often seen to be quadripartite, owing to the presence of a longitudinal split in addition to the transverse constriction; but this never appears in the univalents. All the chromosomes alike divide 'transversely' - that is, across the constriction of the dumb-bell. In case of the bivalents, therefore, the early anaphase-chromosomes are double bodies, while the sex-chromosomes are single, but this contrast only appears clearly in smears, owing to the close union of the two halves. In this respect the relations are less clearly seen in these forms than in some others, such as Anax, where the anaphase-chromosomes are clearly double ( $c f$. Lefevre and McGill, '08), or Aprophora, where the same condition is conspicuously shown (Stevens, '06).

The anaphases are of particular interest because, as has been mentioned, a conjugation between the $X$ - and $Y$-chromosomes takes place in the later stages. As, the division begins and the daughter-chromosomes are separating, a marked contrast in form often appears between the sex-chromosomes and the autosomes (figs. 19 to 21). The latter are more or less extended transversely and often show a slight constriction, thus giving evidence of their double nature, which is accentuated by the very conspicuous double fibres by which they are connected. The latter are so thick, and stain so deeply, as to appear as if. spun out from the chromosomes themselves (as has been noted by other observers). On the other hand, the sex-chromosomes do not show such a constriction, remaining nearly circular in outline, while the connect- 
ing fibers are much less conspicuous, and often appear single. Up to the middle anaphases the sex-chromosomes remain always separate (figs. 18, 19). In the later anaphases all the chromosomes draw more closely together, and often come more or less into contact, though without losing their original grouping; but in case of the autosomes the contact is but casual and temporary, while the sex-chromosomes become definitely attached to each other to form a dumb-bell.shaped body at the center of the group (figs. 19,21). By this process the total number of separate chromatin-elements is reduced from nine to eight (the haploid number). In Lygaeus the process takes place in exactly the same way and may be seen with equal clearness, both in polar views and in side-views. In both species polar views of the final anaphases show that the chromosomes, save for their more crowded condition, have retained the same grouping as in the metaphase (figs. 24 to 29 ), the $X Y$-bivalent being at the center, surrounded by the other chromosomes in the form of a ring. These facts are seen so clearly and in so great a number of cases as to remove every doubt that in these species the conjugation between $X$ and $Y$ regularly takes place at the poles of the spindle before the first maturation-division has been completed. ${ }^{4}$

In Lygaeus the $X Y$-bivalent thus formed is readily distinguishable by the inequality of its two components (figs. 28, 29, 46). In Oncopeltus it is often not thus marked but its identity is no less certainly revealed in another way. As the figures show, the autosomes still show but slight indication of a transverse constriction, and can hardly be described as dumb-bell shaped until a later period. The $X Y$-bivalent, on the other hand, is invariably deeply constricted, so as to have a conspicuously dumb-bell shape, and it often still appears like two chromosomes that are merely in contact. This characteristic difference persists throughout the entire interkinesis, and is still perfectly obvious in the ensuing metaphase of the second divicion.

'I described and figured this process in the case of Coenus in my first 'Study' ('05 b) but did not recognize its constancy. I now incline to think that it will be found to occur in the same way in many other forms. 


\section{The interkinesis}

The interkinesis has hitherto been very briefly treated by myself and other observers of the insects, because in most species the chromosomes are so closely crowded at this time as to preclude accurate study. Lygaeus (at least in my material) is no exception to this, but Oncopeltus fortunately shows every stage of the interkinesis with remarkable clearness. There is no 'resting stage' between the two divisions, no nuclear vacuole is formed, and both the chromosomes and the centrioles retain their individuality throughout.

At the moment when the equatorial furrow has appeared and the conjugation of $X$ and $Y$ has taken place, the centrioles are already rather far apart, and still lie at some distance from the chromosome-group (fig. 22). All the achromatic elements are now so delicate that it is difficult to make sure of the exact structure; but it is certain that each centriole is surrounded by a small, but very distinct aster, and the two seem to be connected by a delicate central spindle. As the cell divides several other changes take place. The chromosomes; without otherwise changing their grouping become still more crowded together, and thus become massed in a nearly flat plate, while the centrioles move still farther apart (fig. 23). Shortly after the division these relations are unchanged save that the centrioles are still farther separated and lie nearly on opposite sides of the chromosome-group. The asters are still present, and between them lies a rather large, irregularly spindle-shaped area. It is difficult to say whether this should be-regarded as an actual spindle; but delicate fibrillae often may be seen extending into it from the poles.

The chromosome-group lies somewhat excentrically within this area in the form of an irregular flattened plate. In side-view (fig. 30) it is usually impossible to distinguish more than a few of the chromosomes. In face view also, the crowding is often so great that the grouping can not be exactly made out. Here and there, however, it is evident that the original grouping has not been lost, and occasionally plates are to be found in which every chromosome may be clearly seen (figs. 31, 32). Study of such 
cases makes it certain that no fusion or process of disintegration has taken place, nor is any evidence of a nuclear vacuole to be seen. The chromosomes still retain the same form as in the preceding anaphases, the $X Y$-bivalent lying near the center, and still very clearly distinguished by its markedly bipartite form. A very characteristic feature of this stage is the massing of mitochondrial granules on one side of the spindle-area as seen in sideview (figs. 30,31 ). This results from the fact that during the division the chondriosomes are mainly massed around the spindle and do not extend to any great extent into the polar areas (fig. 22). After completion of the division therefore the chondrisomes still lie mainly on the side of the chromosome-plate. In. a general way this relation persists throughout the interkinesis. In the condition just described the cells remain until the prophases of the second division. It is probable that the interkinesis is of rather brief duration, because in some cysts all stages may be found between the closing anaphases of the first division and full metaphases of the second. Cysts may, however, be found in which practically all of the cells are in the condition described; from which it may be inferred that a brief pause follows thecompletion of the first division.

\section{The second spermatocyte-division}

The prophases of the second division, which follow directly upon the stage just described, are marked by a resumption of activity on the part of the astral systems, which rapidly increase in development while a definite spindle is formed between them. As this takes place, the chromosomes spread further apart and take up a position at the equator of the spindle in the same grouping as before. It is rather difficult to follow this change completely, as these stages are not very abundant in my preparations, and are almost always seen in oblique view. It is, however, certain that a double movement of the chromosomes takes place, involving (1) a rotation of each chromosome through about 90 degrees, so as to assume a position with its long axis parallel to the axis of the spindle, and (2) a virtual rotation of the entire group, so as 
to lie at right angles to the spindle. I am uncertain whether the latter movement is a simple rotation of the group as a whole, or whether the relative position of the individual chromosomes changes more or less as they spread apart. It is certain, however, that when the metaphase has been attained (figs. 34-37, photo. 4) the chromosomes have the same general grouping as in the final anaphases of the first division or in the interkinesis, save that they are less crowded. As before, the $X Y$-bivalent lies near the center, surrounded by the seven other chromosomes, very often arranged in an irregular ring, though this is somewhat variable.

It seems probable from the facts just described that in these animals the general grouping of the chromosomes is determined in the prophases of the first spermatocyte-division. Already at this time the $X$ - and $Y$-chromosomes are brought into position for their ensuing conjugation; and their topographical relation to the autosomes remains thenceforward unchanged until their final delivery to the spermatid-nuclei. In this respect these species agree with such forms as Fitchia or Rocconota among the reduvioids (Payne, '09) and differ from the coreids and other forms in which a marked change of grouping occurs after the first division. I conclude, further, that neither the chromosomes nor the centrioles lose their identity in the period between the first and second divisions, and that a complete relation of continuity exists between the two generations of spermatocytes in this respect.

In side-views of the second metaphase the $X Y$-bivalent is still almost always distinguishable from the other chromosomes by its deeply constricted dumb-bell shape (figs. 36, 37, 42, 43); and in correlation with this, this element is apparently always the first to divide, its two components having often completely separated before the others have even become deeply constricted (figs. 38 to $41,44,46$, photo. 6 ). This precocious division of the $X Y$-bivalent is a very common phenomenon among the Hemiptera (as I have heretofore described). It is obviously due to the comparatively loose union of $X$ and $Y$ after their conjugation, so that they yield more readily to the poleward force (whatever it may be) that operates during the division. 
The later stages of this division have been so often described as to call for no further account. The final result is that the spermatid-nuclei receive the haploid number of chromosomes-seven in Lygaeus, eight in Oncopeltus - half the nuclei in each case receiving $X$ and half $Y$. The facts seen so clearly in both these species remove every possible doubt that the $X$ - and $Y$-chromosomes which thus enter the spermatid-nuclei are the same individual chromosomes that conjugate at the end of the first division and persist throughout the interkinesis to disjoin in the course of the second division. It is of course possible that some exchange of material may take place between them during the brief period of their association. Of this, however, there is no evidence; and it is certain that their individual boundaries are not lost to view, and that not even an apparent fusion takes place at this period or any earlier one.

\section{The size-relations of the sex-chromosomes in Oncopeltus}

In my first examination of this species my attention was given mainly to some excellent preparations from two individual males (designated by the numbers 711 and 712 ) in which the $X$ - and $Y$ chromosomes appear equal in a large majority of the nuclei. The facts in Nezara hilaris (Wilson, '11 a) led me to extend the examination to other individuals of Oncopeltus, when to my surprise one individual was found (later two others) in which a slight but evident inequality was obvious in a large percentage of the cells at all stages. Upon reëxamination of the entire series the interesting discovery was made that in every individual cases could be found of both equality and inequality, the ratio between them varying widely in different individuals. In the extreme cases this is perfectly apparent to the eye, so that individuals of predominantly equal or unequal type may readily be distinguished even by casual inspection. In other cases one is often in doubt until large numbers of the nuclei have been tabulated. As examples of the extreme types I give below the results of a study of two individuals (nos. 712 and 760) representing the best material as to fixation and staining. In these a comparison of the $X$ - and 
$Y$-chromosomes as to apparent size was made in one hundred nuclei, taken at random, from each of the following five stages of the spermatogenesis: (1) the pre-synaptic leptotene, (2) the synaptic period (synizesis), (3) the post-synaptic spireme, (4) the first spermatocyte-metaphase, (5) the second spermatocyte-metaphase. The best of these stages for the purpose are the maturation-divisions as seen in side-view (because of the elimination of foreshortening) and the pre-synaptic leptotene (because of the clearness with which both sex-chromosomes may be seen at this time), but in neither individual was the requisite number of side-views of the first division available. In the latter case, therefore, both sideviews and polar views have been included. The cases are classed as equal (eq.), unequal (uneq.) and doubtful (dbf.), the latter including those in which there was reason to suspect error due to foreshortening or the like.

\begin{tabular}{|c|c|c|c|c|c|c|c|c|c|c|c|c|c|c|c|}
\hline & \multicolumn{3}{|c|}{ PRE-SYNAPTIC } & \multicolumn{3}{|c|}{ SYNAPTIC } & \multicolumn{3}{|c|}{ POST-SYNAPTIC } & \multicolumn{3}{|c|}{ FIRST DIVISION } & \multicolumn{3}{|c|}{ SECOND DIVISION } \\
\hline & eq. & uneq. & dbf. & eq. & uneq. & dbf. & eq. & uneq. & dbf. & eq. & uneq. & dbf. & eq. & uneq. & dbf. \\
\hline 712 & $\begin{array}{r}83 \\
5\end{array}$ & $\begin{array}{l}10 \\
93\end{array}$ & $\begin{array}{l}7 \\
2\end{array}$ & $\begin{array}{r}80 \\
7\end{array}$ & $\begin{array}{l}13 \\
89\end{array}$ & $\begin{array}{l}7 \\
4\end{array}$ & $\begin{array}{r}65 \\
5\end{array}$ & $\begin{array}{l}23 \\
86\end{array}$ & $\begin{array}{r}12 \\
9\end{array}$ & $\begin{array}{r}55 \\
2\end{array}$ & $\begin{array}{l}37 \\
95\end{array}$ & $\begin{array}{l}8 \\
3\end{array}$ & $\begin{array}{r}83 \\
6\end{array}$ & $\begin{array}{r}9 \\
87\end{array}$ & $\begin{array}{l}8 \\
7\end{array}$ \\
\hline
\end{tabular}

There is no doubt a considerable error in these figures due to foreshortening, since these chromosomes, though often spheroidal, are often slightly elongated (ellipsoidal), and are of course seen in all positions. But.after making a large allowance for this, the contrast between the two individuals is manifest at every stage of the spermatogenesis, and nowhere more so than in side-views. of the second division. I have made tabulations of several other individuals which give percentages of equality ranging from ninety down to ten; but in most cases the data from intermediate types are less consistent and the probable error is much larger, for obvious reasons. ${ }^{5}$

From these observations I draw the conclusion that in Oncopeltus the $X$ - and $Y$-chromosomes show a certain tendency

${ }^{5}$ Compare figs. 9, 10, 14, 15, 24, 25 (equal type, no. 711), 18, 19,3 6-40 (equal type, no. 712 ) with $11,12,16,17,20,31,32,42$ to 44 (unequal type, no. 760 ). See also photos. 7 to 11 . 
towards inequality in all individuals, so marked in some cases as to characterize a large percentage of the cells, so slight in others that it can not be distinguished by the eye in more than a small percentage. A noteworthy fact remains to be mentioned. Among my few smear-preparations of Oncopeltus is one slide, showing great numbers of nuclei at nearly all stages, in which the $X$-chromosomes are almost always equal in the growth-period and earlier stages but invariably unequal in the prophases. I distrust this evidence somewhat, for it is notorious that variations of size are very readily produced in smears owing to different degrees of flattening. Were this the only explanation, however, we should expect to see the size-differences as great in the earlier as in the later stages. If the result be trustworthy, it is interesting as indicating the existence of some kind of material difference between $X$ and $Y$ that is expressed in a greater enlargement of one of them at the period when both expand somewhat and undergo longitudinal splitting.

\section{THE-GROWTH-PERIOD}

For the direct study of the actual process of synapsis and its relation to the reduction-division, Oncopeltus and Lygaeus present practical difficulties that I have thus far found insuperable; hence no attempt will be made to describe synapsis in detail. The transformations of the chromatin during the growth-period will nevertheless be considered at some length, partly in order to trace the complete history of the sex-chromosomes, partly because of the interest of many features presented by the autosomes, and I will also describe certain facts observed in other animals that may help to elucidate some of the problems here encountered.

\section{Outline of the stages}

In Oncopeltus it is necessary to distinguish not less than twelve well marked stages following the last spermatogonial division, as follows:

a. (Figs. 47 to 49 .) The final spermatogonial telophases, in which the anaphase-chromosomes break up into a confused net- 
like structure, and for a short time their boundaries can not certainly be distinguished. In the latter part of this stage the $X$ and $Y$-chromosomes become clearly recognizable as compact, deeply staining bodies; but in the earlier stages they too seem to be in a diffused condition. This stage, of short duration, corresponds to Davis's 'Stage $a$ ' in the Orthoptera ('08), and probably may be compared to the 'resting stage' that has been described as following the last diploid division in many other forms.

b. (Figs. 50 to 51.) Post-spermatogonial nuclei of somewhat larger size, in which the chromatin appears in the form of separate, massive bodies, approximately equal in number to the chromosomes of the diploid groups. Two of these, of more even contour and staining more deeply, are now recognizable as the sex-chromosomes. The other masses are more or less irregular in form, often ragged in texture, and stain more lightly.

c. (Figs. 52 to 55.) The lightly staining masses are in this stage transformed into delicate, closely coiled or convoluted threads, while the sex-chromosomes retain their massive form, and are thus rendered very conspicuous. In the latter part of this stage the fine threads are seen uncoiling or unravelling from the massive bodies to form the leptotene-threads of the following stage.

d. (Figs. 56 to 59.) The pre-synaptic leptotene. The autosomes now have the form of long delicate threads, while the sexchromosomes retain their massive form as 'chromosome-nucleoli.'

e. The synaptic stage or synizesis (figs. 60 to 61 ). The threads are now much thicker, stain more deeply, and are closely convoluted in a contraction-figure or synizesis. A plasmasome can sometimes be distinguished at this time, but is usually first seen in the ensuing stage.

f. Post-synaptic spireme (pachytene, diplotene, figs. 62 to 65). Separate thick threads are now again spread through the nucleus, approximately of the haploid number, and in the latter part of the period longitudinally divided. The plasmasome is now nearly always present, though rather small.

g. The diffuse or confused stage (figs. 66 to 67 ). The double segmented spireme disappears from view, giving rise to a rather 
coarse, vague, lightly staining net-like structure, in which are suspended the chromosome-nucleoli and the plasmasome, the latter at its maximum size. In this stage the nuclei remain throughout the greater part of the growth-period.

h: Early prophases (figs. 105, 107). The staining capacity of . the chromatin increases, while more definite and apparently single threads are evident. The sex-chromosomes are more elongate and longitudinally split. The plasmasome now diminishes in size and disappears.

i. Middle prophases (figs. $108^{\circ}$ to 114 ). The threads rapidly condense, stain more deeply, and draw together to form tetradrods, double crosses, double $V$ 's, or (rarely) double rings. The sex-chromosomes are short rods, longitudinally split.

j. Late prophases. In these all the chromosomes are converted . into compact, deeply staining dumb-bell shaped bodies, rarely quadripartite in outline, which are ready to enter the spindle. This stage is often found in the same cysts with the preceding, all intermediate gradations being readily seen.

k. The division-period, including the two spermatocyte-divisions.

1. Differentiation of the spermatids. Spermiogenesis in the narrower sense.

With various modifications the foregoing stages are found in many Hemiptera, among the best of which for study of the early stages are the pyrrhocorid species Largus cinctus and L. succinctus. Some doubt exists in regard to Stage $\alpha$; and it is possible that in some forms (of which Largus may be an example) the spermatogonial chromosomes do not lose their identity at this time but give rise directly to the massive bodies of Stage $b$. In some cases (Largus, Pyrrhocoris, Alydus) the latter part of Stage $g$ is characterized by a second synizesis or contraction-figure, in which the autosomes are again closely massed together. In such cases the early prophases are much more difficult to analyze.

I feel confident that the seriation of the stages is correctly determined -indeed the only possible doubt concerns the earliest presynaptic stages. The seriation is indicated by the general topography of the testis, which consists of very definite lobes in which 
the cysts develop progressively in a nearly continuous series from one end to the other. All the cells in each cyst are nearly, but often not quite, in the same stage. While the order of succession is not demonstrated so accurately as in some objects (e.g., in Batracoseps) it is placed practically beyond doubt by the study of transitional conditions in cysts where slightly earlier and later stages occur side by side.

Throughout the whole complicated series of changes in the autosomes, the sex-chromosomes are at once recognizable at every stage (save the very first) by their condensed and deep-staining character. Lygaeus differs from Oncopeltus in the fact that the $X$-chromosome always retains a rod-like form and is longitudinally split at least as early as Stage $f$. In Oncopeltus both sexchromosomes remain in the form of rounded and apparently undivided chromosome-nucleoli up to Stage $h$, when they too assume the form of short, longitudinally split rods. At the period of synizesis the $X$-chromosome in Lygaeus shortens somewhat, but at no time does it assume the rounded form characteristic of Oncopeltus and many other forms. In this respect Lygaeus bicrucis differs from $\mathrm{L}$. turcicus, where the $X$-chromosome has the form of a much elongated and longitudinally split rod in the early post-synaptic stages, but later contracts to a spheroidal form (Wilson, '05 b). These species of Lygaeus remove every doubt, could such longer exist, of the identity of the chromatic 'nucleoli' of the growth-period with a pair of chromosomes.

\section{The pre-synaptic period. Stages a to $d$}

The study of this period in these animals is of much interest in relation to a series of questions, frequently raised in late years, that are of the utmost importance for the theory of synapsis. These are: (1) Are the leptotene-threads of this period chromosomes? (2) Is their number equal to that of the spermatogonial chromosome-groups? (3) Can they be traced directly as individuals to the anaphase-chromosomes of the last spermatogonial division? As will be seen, the facts in the Hemiptera, in the dragon-fly Anax, and in certain Orthoptera give good reason to 
answer the first two of these questions in the affirmative, while the third remains unanswered.

Stage b. It will be advantageous to consider this important stage before that which precedes it, as there are doubts concerning the latter: This stage and the following one are characteristic of many Hemiptera and Orthoptera, and is seen also in the dragon-fly; and some of these forms are much better adapted for its critical study than are Oncopeltus and Lygaeus. ${ }^{6}$

In the latter forms numerous cysts in Stage $b$ are seen in the region between the spermatogonial cysts and the synaptic zone, often abutting directly upon the former. For this reason I long supposed this stage to follow immediately upon the last spermatogonial division, i.e., to be the last spermatogonial telophase. Such indeed is possibly the case in Largus, as already stated; but in some other forms it is certainly separated from the telophase by an intervening net-like stage. In Oncopeltus and Lygaeus Stage $b$ is characterized by rather small spheroidal nuclei in which may be very distinctly seen a group of separate, more or less irregular, massive chromatic bodies, the number of which is approximately, in some cases exactly, equal to the diploid number of chromosomes (figs. 50, 51, 71, 72). In preparations but slightly extracted (after haematoxylin or saffranin) all these masses stain alikedeep blue or red. Upon further extraction a very striking contrast appears between two of these bodies and the others, the former retaining their deep color and having a fairly even contour, while the latter become pale and are more or less irregular in shape. As will be shown, the two dark bodies are the $X$ - and $Y$-chromosomes, which may be traced individually through all the succeeding stages up to the spermatocyte-divisions. In Oncopeltus they are spheroidal or ovoidal in shape and nearly equal in size (figs. 50, 51). In Lygaeus the $X$-chromosome is much larger than the $Y$, and always has the form of a more or less elongate rod, which shows a good deal of variation, being sometimes quite straight, sometimes curved in various ways (figs. 71, 72). In

'In my fourth 'Study' ('09 a) I gave a brief account of this stage in Pyrrhocoris, illustrated by photographs, describing it as a 'spermatogonial post-phase,' but did not endeavor to work out the history of the autosomes. 
Largus and Pyrrhocoris but one dark body is seen; and this, as I earlier showed in the latter case, is the unpaired $X$-chromosome.

These massive bodies strongly suggest those to which Overton ('05, '09) has given the name of 'prochromosomes' in the case of plant cells. Since however they differ from the latter in some important respects I will not here employ this term; and for a similar reason will not designate them by Strasburger's term 'gamosomes' ('05), though they undoubtedly give rise to the chromosomes that enter synapsis.

Even a casual inspection of these nuclei is enough to show that the number of chromatic masses is not far from the spermatogonial number of chromosomes, while here and there a nucleus may be found in which this number may be exactly counted. The enumeration is most readily made in the case of Largus cinctus where the spermatogonial number is eleven. In this species, which has eleven spermatogonial chromosomes (photo. 33), nuclei may readily be found in which ten of the paler chromatic masses may be definitely counted. In L. succinctus their number is often seen to be about twelve (the spermatogonial number being thirteen). In like manner, the number of the pale masses in Lygaeus is sometimes seen undoubtedly to be twelve, in Oncopeltus about fourteen, the spermatogonial numbers being respectively fourteen and sixteen, though in neither of these species can the number be exactly determined in many cases. I do not hesitate however to draw the conclusion definitely that in these animals the full diploid number of separate chromatic masses is present in a stage that shortly follows the last spermatogonial division and precedes the formation of the leptotene-threads. In the dragon-fly, Anax junius, there is a closely corresponding stage, but in this case all of the chromatic masses stain nearly alike, and the $X$-chromosome can often not be certainly distinguished until a little later.

The stage described above evidently corresponds to one in the Orthoptera (Davis's 'Stage $b$ ' in Dissosteira, Chortophaga and other grasshoppers) and is clearly shown in some of McClung's slides. In all these forms, however, the chromatic masses stain more deeply than in the Hemiptera, are of elongate form, and are 
more or less definitely polarized. In Anax and the Hemiptera, on the other hand, they are of more less or rounded or irregular form, and show no definite polarization. This is corelated with a corresponding difference in the form and position of the spermatogonial anaphase-chromosomes. In the Orthoptera the latter are in general rod-shaped, with their long axes parallel to the spindleaxis; in Anax and the Hemiptera they are much shorter, often rounded in form, and with their long axes (when distinguishable) lying at right angles to the spindle-axis. The conditions described above are occasionally varied by the appearance of one or two deep-staining bodies in addition to the sex-chromosomes, usually of smaller size (ef. the photographs of Pyrrhocoris in my fourth 'Study'). Owing to their inconstancy I am uncertain as to their nature.

Whether all of these chromatic masses are chromosomes is a question that probably can not be directly or certainly determined in the case of Oncopeltus and Lygaeus. We must rely here upon indirect evidence. But there can be no doubt that two of them are chromosomes, for the two deeply staining bodies of Lygaeus and Oncopeltus may be traced step by step, with no break of continuity, into the two chromatic 'nucleoli' of the synizesis and all succeeding stages, and thence throughout the growth-period into the $X$ - and $Y$-chromosomes of the maturation-divisions. Since the paler bodies correspond in number to the spermatogonial number of autosomes, and since they undoubtedly give rise to the leptotene-threads that enter the synaptic stage, it is at least a fair inference that they too are chromosomes, or are destined to become such.

Stage a. As stated above, I long supposed the stage just described to follow immediately after the last spermatogonial division; but it now seems certain that in Oncopeltus and Lygaeus, as in the Orthoptera (Davis, op. cit.) it is preceded by one which more nearly approaches the condition of a 'resting' nucleus. In this stage only the sex-chromosomes can be clearly identified, and there is reason to conclude that in a still earlier telophase not even these can be distinguished. 
In certain cysts that obviously precede those of Stage $b$ the nuclei are still smaller, the sex-chromosomes more elongated, while the autosomes form a lightly staining, vague net-like structure in which individual chromosomes can not be distinguished. This stage evidently corresponds to Davis's 'Stage $a$ ' in the Orthoptera, and is well shown in McClung's preparations. A similar stage has been described by several other students of the Orthoptera, especially by McClung.

It is difficult to represent these nuclei accurately in drawings; but a fairly good idea of them may be obtained from figs. 68 to 70 , which are from careful studies. They seem to contain a rather coarse and close network, with thickened and irregular nodes of varying size and number. In both species the sex-chromosomes are more elongated than in Stage $b$, and in Lygaeus the $X$-chromosome often assumes an almost vermiform shape, as is shown in the figures. That these nuclei follow almost immediately upon the last spermatogonial telophase is proved both by their small size and by the transitional stages seen in the same nuclei. This is most clearly seen in Lygaeus, where the elongate $X$-chromosome enables us to identify the early spermatocytes with certainty (these chromosomes do not appear as condensed bodies in the spermatogonial nuclei). In the cyst from which figs. 68 to 70 were drawn both sex-chromosomes are perfectly clear in many of the nuclei, but in many the $Y$-chromosome can not be found, and in a considerable number of nuclei, which seem to lie entirely within the section, not a trace of either sex-chromosome can be seen (fig. 68). In this particular cyst no spermatogonial divisions are seen; but in other cysts in the same region of the testis, nuclei of exactly the same type as those last mentioned (with neither sexchromosome in evidence) are seen together with the spermatogonial anaphases. That the latter are the final spermatogonial divisions can not be proved; but in Lygaeus the evidence seems nearly decisive that there is a short period following the last division in which the identity of all the chromosomes is lost to view. I believe this to be true also in Oncopeltus, though the evidence is less satisfactory. On the other hand, it is possible that in Largus the final anaphase-chromosomes give rise directly to the massive bodies of 
Stage $b$. It is at any rate certain that the telophase-chromosomes in this form retain their identity much longer than in Lygaeus, as is shown by figs. 74 and 75 , which are connected by all intermediate stages with anaphase-figures in the same cyst. In a recent paper on Euschistus, Montgomery ('11) describes a stage that seems to correspond to my Stage $b$, and identifies the massive bodies with the telophase-chromosomes." I must confess, however, that neither this account nor my own observations on Euschistus convinces me that this is correct. It seems to me that we have as yet no safe demonstration in any animal that the presynaptic chromosomes are actually the same individual chromosomes as those of the last diploid division.

I am unable to state in exactly what way the massive bodies of Stage $b$ arise, for there is no way of demonstrating the seriation at this time, and the change is probably effected rapidly. Different cysts of Stage $b$ vary considerably, the massive bodies being more irregular and less sharply defined in some; but I have not gained any clear idea of the succession.

Stage c. We may now consider the most interesting changes that take place during the transition to the leptotene stage, the earlier of which may in some cases be seen in the same cysts with the preceding stage. In Oncopeltus and Lygaeus the minuteness and delicacy of the structures are such that I was long in doubt as to how the process takes place; but Largus, Anax, and some of the grasshoppers constitute a series in which the same essential phenomenon is seen on a successively larger scale, and which leaves no doubt as to its nature. In all these forms the process involves the resolution of the paler massive bodies into closely convoluted or coiled threads, which then uncoil or unravel to form the leptotenethreads of the succeeding stage. The sex-chromosomes, on the other hand, fail to undergo such a transformation, and retain their massive form, though in some cases (Largus) there is some evidence that they too may have an internal thread-like structure.

${ }^{7}$ Arnold ('08) gives a similar account of a corresponding stage in Hydrophilus, and describes the massive bodies as conjugating directly two by two, before giving rise to spireme-threads. 
A process of this type was long since described by Janssens ('01) in both the spermatogonial prophases and the pre-synaptic nuclei of Triton (figs. 27, 67), where it somewhat resembles the resolution into threads of the 'nucleoli' of the germinal vesicle of the same animal, as earlier described by Carnoy and Lebrun ('98). A process more or less similar was described by the Schreiners ('06, '08) in the post-spermatogonial (pre-synaptic) stages of Tomopteris, by Pinney ('08) in the spermatogonial prophases of Phrynotettix, and especially by Davis ('08) and more recently by Brunelli ('11) in the pre-synaptic stages of Chortophaga, Tryxalis and other grasshoppers; Grégoire describes a similar process in plant-cells, first in the somatic cells of the root-tip in Allium ('06, p. 330), later in the pre-synaptic sporocyte-nuclei ('07, p. 391). The analogous relations discovered by Bonnevie and other recent observers are referred to beyond.

In Oncopeltus as the process begins, the pale chromatic masses become looser in texture and more ragged in contour, and each of them gradually assumes the appearance, though somewhat vaguely, of a closely convoluted thread (figs. 52, 53). In the stages that follow (figs. 54,55 ) the coiling becomes looser, so that contorted or spiral threads are clearly evident, and at the same time the massive bodies progressively disappear from view. These stages unmistakably show the nature of the process that is taking place. It is now clear that each of the original compact masses (excepting the sex-chromosomes) has resolved itself into a tightly convoluted thread, which is uncoiling to form a leptotene-thread. The spiral or contorted course of the threads is still very evident when the massive bodies as such have disappeared from view (fig. 55), but is finally lost in the completed leptotene-stage (figs. 56 to 59). In Lygaeus the process is closely similar and requires no separate description. Figs. 71 and 72 show two nuclei in Stage $b$, in each of which twelve of the paler masses can be counted (not all shown in the drawing), while the $X$-and $Y$-chromosomes are conspicuously seen. Whole cysts full of these nuclei are seen in nearly all of my sections. Figs. $73 a$ and $73 b$ show two early leptotene-nuclei of this species after the unravelling is completed. 
These stages have been described in Oncopeltus mainly. because of the importance of following the sex-chromosomes at this period; but, as already mentioned, they are shown more clearly in Largus, spermatogonial telophases of which are shown in figs. 74 and 75, and Stage $c$ in figs. 76 to 78. Photos. 26 and 27 show nuclei of this form in Stages $b$ and early $c$, the character of which I hope will appear in the reproductions. The threads are here coarser and show a more definitely spiral disposition, especially evident as the uncoiling progresses. This is clearly evident in many nuclei in the negative from which photo. 27 is reproduced. Though these nuclei are still rather small, they afford demonstrative evidence in regard to the main fact. I am further confident that the threads are separate and undivided, and that but one thread is formed from each mass; but the latter conclusion is less certain than the former. In the dragon-fly, Anax, the facts are similar, and in some respects still more clearly shown. Stage $b$ is shown in fig. 85 (the massive bodies all deeply stained); and in fig. 86 (closely similar to Janssen's fig. 67 of the spermatogonial prophases of Triton) are shown three nuclei lying side by side, in which appear three successive stages of the unravelling. The spiral disposition of the threads in this form is sometimes conspicuous, and may be clearly seen because of the tendency of the chromatic masses to assume a peripheral position in the nucleus. Not infrequently are seen nuclei like fig. 87 in which a striking effect is given by the uncoiling threads. In such cases it is very evident that the spirals are single, and the evidence is strong that one thread is forming from each massive body.

These stages may be studied to still greater advantage in the grasshoppers, where they have been accurately described and figured by Davis ('08). This observer describes the post-spermatogonial nuclei (Stage $b$ ) as containing a series of elongated massive bodies, very definitely polarized, approximately equal in number to the spermatogonial autosomes, and having "approximately the same orientation that the autosomes had during the preceding telophases of the last spermatogonial division" (op. cit., p. 38). In figs. 43 and 44 he represents the unravelling of a single thread 
from each of these masses, and concludes that each of the latter thus "becomes converted into a single chromatin-thread." A study of McClung's preparations, particularly of Achurum, leads me to a confirmation of this conclusion. Stage $b$ is better seen in the slides of Phrynotettix than in Achurum; but as the latter shows the unravelling stages more clearly a figure of Stage $b$ from this form is here given (fig. 88). In Achurum the unravelling process is quite unmistakable (figs. 89 to 92 , less highly magnified than the other figures of the plate; see also photo. 28). The threads here form closely convoluted knots (much like those figured by Janssens in the spermatogonial prophases of Triton), and a spiral arrangement is seldom seen. In Phrynotettix and Mermiria the process is less evident, but appears to be of the same general nature.

Especially in Largus, Anax and Achurum the definiteness of the pictures and the succession of the stages seen side by side in the same cysts or in adjacent ones, entirely excludes, I think, the possibility that they are a merely accidental appearance due to vacuolization of the massive bodies, corrosion-products or fixation-artifacts. The only question is whether the thread that unravels from each massive body is single, double or longitudinally divided. I am nearly certain that the threads are single and undivided. In this respect my conclusions agree with those of Davis, and differ from those recently announced by Brunelli ('11) in the case of Tryxalis. This author describes a similar unravelling process but believes the threads to consist of two separate longitudinal halves which result from a longitudinal split that is evident already in the preceding telophases, and which separate still more widely as they uncoil. The evidence for both these conclusions seems to me very incomplete, as none of the unravelling stages are shown, and the massive bodies of Stage $b$ are assumed to arise directly from the telophase chromosomes without proof of this important point. This assumption may be correct, but it seems more probable that Stage $a$ has been overlooked by this observer.

The question here involved is so important that I have endeavored to reach a more certain result by study of the analogous processes seen in the spermatogonial prophases. The first of these 
cases, as already mentioned, was described by Janssens in Triton. The chromatin-masses ('blocs') from which the spireme-threads unravel are here of irregular shape, and show no polarization, but are nevertheless believed to be directly traceable to the preceding telophase-chromosomes. The threads are already in evidence in the latter, and sometimes show an irregularly spiral course (Janssens's fig. 80) but in the later stages are irregularly convoluted in a manner very similar, as far as can be judged from the figures, to that seen in the pre-synaptic stages of the grasshoppers. Janssens emphasizes his belief that in general a single thread is formed from each 'block,' though in certain cases the latter are double and give rise to two threads. An essentially similar process is described for the pre-synaptic stages. "La première transformation qu'on observe dans les auxocytes est analogue à celle qui annonce le commencement de la division dans les spermatogonies . . . et consiste en un résolution des blocs de nucléine" ('01, p. 68). An essentially similar phenomenon in the spermatogonial prophases is brilliantly demonstrated in two admirable slides of Phyrnotettix by McClung, one stained with iron haematoxylin, the other by Flemming's triple method. In this form the 'chromatin blocks' are elongate and polarized (fig. 93, photos. 29 to 31 ), and the thread later forms a beautiful and very definite spiral, as was first described by Pinney (08). This observer describes the spiral threads as formed separately within the vesicles or sacculations to which the preceding anaphasechromosomes give rise (as first made known by Suttolı '00, in Brachystola and confirmed by several others subsequently).

As far as can be judged from the figures and brief description of Pinney, the threads are formed as rather loose and open spirals directly out of a fine reticulum within each chromosome-vesicle. The rather limited material at my disposition shows somewhat different conditions, though confirming the main point. The conditions in McClung's slides differ from those described by Pinney in that none of the resting nuclei or early prophases show the nuclear sacculations so distinctly, nor is the chromatin so diffusedifferences which may well be due to the fact that none of the earlier generations of spermatogonia are shown. In these nuclei 
the thread-formation is preceded by a stage in which the chromosomes appear in the form of deeply staining, elongated, and more or less definitely polarized bodies (fig. 93, photo. 29), ragged in contour and loose in texture, but showing as yet no definite coiled thread. This condition must shortly precede the threadformation because the latter may clearly be seen in other nuclei in the same cysts. Whether these bodies are individually derived from the anaphase-chromosomes of the preceding anaphase can not here be determined, but Miss Pinney's observations make it highly probable that such is the case. In any case, already in the early prophases each of these masses is seen to be resolving itself into a closely coiled or convoluted thread, similar to that seen in the pre-synaptic stages but disposed in more definitely spiral fashion (figs. 94 to 96 , photos. 30 to 32 ). In some cases there are indications that each of these spirals is still enclosed in a more or less separate nuclear sacculation, in other cases this can not be seen. In some cysts the threads are seen tightly coiled within the massive bodies at one side of the cyst, while stages of uncoiling are seen progressively towards the opposite side. In slightly later stages all gradations are seen in the uncoiling of the threads to form separate threads, which still show a distinct spiral course even after they have begun to shorten and thicken (fig. 96, photo. 32). From this stage it is easy to trace every step up to the time' when the prophase-chromosomes are about to enter the metaphase. The longitudinal division is not evident until the uncoiling is well advanced, and the two halves remain in close apposition until the metaphase.

The points that I would here emphasize are:

1. The extreme clearness with which the spiral threads are seen, which removes every possible doubt as to what is taking place.

2. The fact that the threads are-separate from the time of their first formation. Apparently there can be no question here of a continuous spireme.

3. The transitional conditions seen in the same cysts, which prove these stages to be prophases, not telophases. In this re- 
spect the phenomenon is different from that discovered by Bonnevie ('08, '11) in Ascaris and Allium, where the spiral thread is formed in the telophase-chromosomes and uncoils to form the thread-work of the resting nucleus.

4. The certainty that the spirals are single, not double, i.e., do not consist of two interlacing spirals, as has recently been described in the telophase-chromosomes of Tryxalis by Brunelli ('10), and in the final anaphase-chromosomes of Amphibia by Schneider ('11) and Dehorne ('11).

5. The strong evidence thus afforded that only one thread arises from each chromatin-mass.

There can be no doubt that the process here so clearly demonstrated is of the same general nature as that seen in the pre-synaptic nuclei of these animals and of the. Hemiptera. I therefore consider it at least probable that in the latter case also a single thread is formed from each chromatin-mass and hence that the number of pre-synaptic leptotene-threads is equal to the diploid number of chromosomes.

Résumé of Stages a to c. The close parallel that exists between the pre-synaptic stages of the Hemiptera, Odonatata and Orthoptera is obvious. In all these forms the pre-synaptic chromosomes first appear in the form of massive 'prochromosome'-like bodies, approximately of the diploid number, of which one $(X)$ or two $(X$ and $Y$ ) are already recognizable as the sex-chromosomes by their more compact structure, regular contour, and deep-staining quality. Each autosome is converted into a tightly coiled or convoluted thread which ultimately unravels to form a leptotene-thread of the stage which immediately precedes synapsis. This process is clearly analogous to that seen in the spermatogonial prophases, and in each case the evidence is that a single thread arises from each massive body. The pre-synaptic leptotene-threads are thus seen to be of the same nature, and probably of the same number, as the spermatogonial prophase-threads, and are therefore to be regarded as forming a diploid group of chromosomes. The sex-chromosomes, on the other hand, persist in the massive form to constitute 'chromosome-nucleoli,' 
which may be traced into and through the growth-periods. ${ }^{8}$ It is however very doubtful whether the massive bodies of Stage $b$ can actually be traced individually back to the anaphase-chromosomes of the preceding division, though this may be possible in some forms.

Stage d. The leptotene-nuclei. It is impossible to draw any definite line of demarkation between this stage and the preceding one, since they are connected by insensible gradations. An excellent idea of these stages is given by Miss Hedge's careful drawings (figs. 56 to 59 ; cf. photos. 7,8 ). In the earlier nuclei the threads still have a more or less spiral or wavy course, and still show distinct evidence of clumping together in masses. A little later both these appearances are lost, and the threads form an evenly diffused, delicate spireme, always separated from the nuclear wall by a considerable clear space. Still later the threads become somewhat thicker, more open in arrangement, and stain a little more deeply (figs. 58, 59, $73 a, 73 b, 78$ to 80 ).

These nuclei are now ready to enter the synaptic or synizesis stage, which immediately follows. They show essentially the same characters in Oncopeltus, Lygaeus, Largus, and many other Hemiptera; but their composition is difficult to analyze precisely. It is certain that the spireme is not continuous at this stage, for free ends of the threads are readily seen; but the number of threads can not be determined. In general they show no trace of polarization, though in Lygaeus traces of such an arrangement are sometimes visible. Whether the threads branch or not is a very difficult question. At first sight they give the impression that they do branch; and in my fourth 'Study' (on Pyrrhocoris) I described them in fact as forming a "net-like structure in which traces of a spireme-like arrangement may sometimes be seen." The more carefully one studies these nuclei, however, the more doubtful this becomes. Certainly the threads may often be followed

\footnotetext{
8 The mitotic transformation of the massive bodies is not however diagnostic of the autosomes, for in some of the Orthoptera, as McClung and his successors have shown, the $X$-chromosome is also converted into a closely convoluted thread at a later period. I have some reason to suspect that the $X$-chromosome of Largus may also consist of a very tightly convoluted thread in the earlier stages; but there is never any sign of its uncoiling, and in the later stages it appears homogeneous.
} 
individually for a considerable distance without branching; and it it my belief, after prolonged study, that the threads do not really branch or form a network, though such an appearance is often given by fine strands of 'linin' (perhaps coagulated nuclear sap) connected with the threads.

A point to be emphasized is that these threads do not show the least sign of longitudinal division, and in this respect offer a marked contrast to the longitudinally double threads seen in the post-synaptic stages.

As Stage $c$ passes into Stage $d$, the contrast between the sexchromosomes and the others becomes still more pronounced. In Oncopeltus the former often become nearly spheroidal in shape, and stain so intensely as to appear exactly like chromatic nucleoli. In Lygaeus they stain with equal intensity, but still retain more or less of a rod-like form, particularly in case of the $X$-chromosome. In Largus (as in Pyrrhocoris) the unpaired $X$-chromosome becomes as a rule spheroidal. In all these forms the sex-chromosomes always occupy a peripheral position with respect to the mass of chromatin-threads, sometimes in the clear space outside the latter, more often embedded in its peripheral zone. A very striking fact (to which I formerly called attention in case of Pyrrhocoris) is that the sex-chromosomes in these stages are always separated from the threads by a vacuole-like space. This is most conspicuous in Largus (figs. 78 to 80 ) where the vacuole is unusually large and clearly defined; but it also appears in the other forms when seen in the right position. No definite wall to the vacuole can be seen, but the chromatin-threads are often seen encircling its outer limit, as if lying upon a definite substratum. This fact is interesting as indicating that the sex-chromosomes really lie in separate compartments or chambers of the nucleus, even though their walls can not be seen. Is this, conceivably, true of other chromosomes, and may this possibly be the basis of the genetic continuity of chromosomes in general? 


\section{Stage e. The synaptic period. Synizesis}

We now approach a problem that I have thus far found insoluble in these animals, and which will therefore be considered very briefly. This involves the changes by which the leptotene-nuclei pass into the pachytene stage, which here begins with the contraction-figure, or synizesis. This stage is initiated by a rapid thickening of the threads, accompanied by an increase in staining capacity and a further contraction of the mass which they form. A very good idea of this stage may be obtained from fig. 60 , which is carefully studied in every detail. As this figure shows, the synaptic knot distinctly shows two kinds of threads, thick and thin, closely convoluted, but showing no definite polarization or other visible arrangement in loops. The results shows that the process of synapsis must be in progress at this time, but the closest study has thus far failed to reveal the true relation of the thick threads to the thin, and I doubt the practicability of determining precisely what is taking place. In these Hemiptera, as Digby has recently remarked of Galtonia, "synapsis faces one as an impenetrable wall" ('10, p. 739). A little later the synaptic knot undergoes still futher contraction (fig. 61) and is till more difficult to analyze; but in favorable cases it may be seen to consist of thick threads, closely convoluted, and still showing no trace of polarization. This stage evidently corresponds to the early pachytene of other forms; but the 'bouquet' figure, so characteristic of many animals, seems to be entirely wanting here, and $I$ have found no indication of it in any of the Hemiptera.

Of one hundred nuclei of this stage in Oncopeltus, taken at random, seventy-five showed $X$ and $Y$ entirely separate, sometimes on opposite sides of the synaptic knot, while in twenty-five cases they lay side by side, just in contact. Not one of these nuclei has been found after a search of many hundreds, in which these chromosomes were fused, or even flattened together. 'In Lygaeus, on the other hand, there is a stronger tendency for these chromosomes to come together at this time, one hundred nuclei showing them separate in forty-five cases and in contact in fiftyfive. In the latter case they are often pressed together to form 
an unsymmetrical dumb-bell shaped body (photo. 12) but are never fused to form a single body. In thirty-six of the foregoing fifty-five cases in Lygaeus, $X$ and $Y$ were attached end to end (photos. 12, 13), in seventeen side by side (photo. 13)

There is a good deal of variation in the degree of contraction, even in cells of the same cyst; and this may be due in part to differences of response to the fixing agent. That the contractio figure can not be regarded as an artifact, however, is proved by the fact (which I briefly described in my fourth 'Study') that in some Hemiptera it may be readily seen in the living cells, as has also been shown by other observers. Gates ('08) has suggested, in case of certain plants, that the synizesis is not produced by a contraction of the chromatin-mass but by enlargement of the nucleus due to rapid accumulation of liquid about the chromatin. Such a view can hardly apply to these insects, I think, though studies of the living material would give a more trustworthy result than those upon sections.

The synaptic knot often lies excentrically in the clear space. Just outside it, or embedded in its periphery lie the sex-chromosomes, still surrounded in many cases by the vacuole, though this is now less evident. They retain the same appearance as in the preceding stage, except that in Lygaeus they are somewhat shorter than before. In none of these Hemiptera does either sex-chromosome elongate, or show any definite relation to the nuclear pole at this stage. In this respect these animals differ markedly from some of the Orthoptera, where the $X$-chromosome becomes elongated and takes part in the general polarization of the chromatin in the 'bouquet' stage. There is no evidence of a giving off of material from this chromosome or from the nucleus at this time. ${ }^{9}$

\section{Stages $f$ and $g$}

Stage f. The post-synaptic spireme. Pachytene and diplotene. In the stage immediately following synizesis the chromatinthreads quickly spread apart through the nuclear cavity, and are

' Cf. Moore and Robinson ('04) and Morse ('09) on the cockroach, Buchner ('09) on Gryllus. 
now very clearly seen to be separate, constituting a segmented spireme. All the threads still stain deeply and are very much thicker than in the leptotene-stage; hence these nuclei may be called the pachytene-nuclei. In the earlier part of this stage it is uncertain whether the threads are longitudinally split or not; in many cases the closest study fails to reveal such a split in sections (figs. 62, 63), though in smears (fig. 65, photo. 10) the split is very clearly seen in nuclei that seem to belong to this period. In the later part of this period the threads become still thicker and shorter and very often show a conspicuous longitudinal cleft. This is less readily seen in Oncopeltus and Lygaeus (figs. 64, 83, 84) in which, indeed, the threads sometimes do not show a trace of such a cleft at this time (which I attribute to defective fixation). In Largus, on the other hand, the cleft appears in the most conspicuous way, especially in sections fixed with Hermann's fluid (figs. 81, 82), where the threads are often seen to consist of double rows of granules often showing a distinctly paired arrangement.

In Oncopeltus and Largus the sex-chromosomes are at this time hardly changed, still having the form of undivided, rounded chromosome-nucleoli. In Lygaeus, the $Y$-chromosome is still of this type, but the $X$-chromosome (usually near the nuclear membrane) is now very clearly split lengthwise (fig. 84), in which condition it persists from this time throughout the whole growth-period. The plasmasome is considerably larger than before although not yet at its maximum size.

The number of chromosomes (separate chromatin-masses) is now obviously approximately half that of the diploid groups. In Oncopeltus and Lygaeus this can be determined only approximately; but it is certain that the number is not far from the reduced or haploid number - that is to say, there are in Oncopeltus, in addition to the two chromosome-nucleoli, about seven separate diplotene-threads, in Lygaeus about six. In Largus cinctus (where the spermatogonial number is eleven) nuclei may readily be found in which the number of double threads may be exactly counted. Such nuclei show, in addition to the single chromosome-nucleolus, five double threads (figs. 81, 82) of which 
one is much longer than any of the others and evidently. corresponds to the large pair of chromosomes that are a constant feature of the diploid groups in this species (photos. 33, 34). From these facts it is clear that each of the double threads is a bivalent, which corresponds to a pair of spermatogonial chromosomes, and that synapsis must have taken place during the period of contraction or synizesis, as many other observers have concluded in both animals and plants. ${ }^{10}$ In what manner synapsis takes place, and whether the longitudinal halves of the diplotene-threads represent the original conjugants in side by side union are questions that here present very great practical difficulties to direct observation.

Stage g. The diffuse or confused period. The diplotene-nuclei now undergo a remarkable transformation, characteristic of many Hemiptera, in the course of which the double threads as such completely disappear from view, giving rise to a diffuse, lightly staining net-like stage in which the boundaries of the individual bivalents are indistinguishable (figs. 66, 67, 97, photos. 11, 14, 16). In Oncopeltus and Lygaeus I have found it impossible to arrive at any clear notion as to the exact nature of this transformation. In Hermann preparations of Largus all the transitional stages are shown with great apparent clearness, yet even here it is difficult to reach a certain result. This question-one of the most important involved in the maturation-process-will, I believe, repay careful study in smear-preparations, which I hope to undertake hereafter with more adequate material than I have at present.

As the process begins, the threads become less regular and at the same time longer and thinner, while the longitudinal cleft is still more evident. A little later the two halves of the double threads become more or less contorted, more granular and irregular in structure, and at the same time are often seen to be separating in an irregular way (figs. 101-103). By the continuation of this change the double threads as such disappear from view, and the whole nucleus is traversed by rather thin, irregular, con-

${ }^{10}$ In Syromastes Gross ('04) believed that the somatic or diploid number of double threads could be counted in the post-synizesis stages, and that synapsis took place at a later period. 
torted, more or less interrupted granular threads, which often seem to branch more or less. These nuclei show a certain resemblance to the pre-synaptic leptotene-nuclei of Stage $d$; but both their position in the testis and their structure render a confusion between these stages impossible. When the process is completed the threads are greatly diminished in staining-capacity, seem to branch more freely, and in Oncopeltus and Lygaeus often give almost the appearance of a network with thickened nodes (figs. $66,67,97)$. In Largus, however, the threads remain more in evidence, and the nuclei do not so nearly approach the 'resting' condition (fig. 104).

At the height of this stage it is, I believe, quite impossible to distinguish the individual chromosomes (bivalents) or to analyze exactly the composition of the nuclei. I nevertheless incline to the conclusion that the autosomes do not actually lose their identity at this time. The phenomena which follow in Stage $h$, especially as shown in Protenor, give considerable reason to conclude that the prophase-figures are already formed in the diffuse stage but are lost to view by their intricate extension, contortion and interlacing. In Euschistus, as recently described by Montgomery ('11), the confused period is much less marked; and this observer believes that the bivalents may be individually recognized at every period. In Tomopteris and Batracoseps the confused period is entirely omitted.

In the condition described the nuclei remain throughout the greater part of the growth-period. In Oncopeltus the sex-chromosomes remain always spheroidal or ovoidal (photos. 11, 16) and apparently undivided. In Lygaeus both sex-chromosomes (of which a more detailed account is given at p. 384) are rod-like and longitudinally split (photos. 13 to 15). In Largus the $X$-chromosome is spheroidal but often shows a small but very distinct central cavity. In all these forms the plasmasome is conspicuous throughout, and attains its greatest size in this stage. In Oncopeltus and Lygaeus the chromatin undergoes no contraction during this period. In Largus, on the other hand (as in Pyrrhocoris, Alydus and some others) the latter part of this period is characterized by a very marked second contraction-figure or synizesis, 
forming a spheroidal and rather dense mass separated from the nuclear wall by a considerable clear space (cf. Gross's figures of this stage in Pyrrhocoris, '07).

\section{Stages $h$ to $j$. The prophases}

a. The bivalents. At the end of the diffuse period the nuclei undergo a rapid change which marks the appearance of the definitive prophase-chromosomes. This is accompanied by a progressive condensation and increase of staining capacity, which reaches a climax in the final prophases, and by the disappearance of the plasmasome. These changes may be studied to better advantage in smears than in sections, and is better shown in my material of Protenor than in the other forms. As seen in sections, the initial stage (figs. 105, 106) shows the nuclear threads more distinct, less crowded and straighter, often giving an appearance somewhat similar to the beginning of Stage $g$, but the bivalents are not yet defined. In smears of Protenor (figs. 115 to 117) it is clearly apparent that the threads are separate, single (i.e., not longitudinally split) and much contorted. Alittle later the threads are seen to be forming themselves into the characteristic bivalent figures, still in a very diffuse and irregular form, but plainly showing their individual boundaries, and in some cases also their characteristic forms (figs. 107, 108, 118, 119, photo. 17). In Protenor the $m$-chromosomes are first clearly seen at this time but are much less definite in contour than in the following stage. As the condensation proceeds the bivalents become more definite in shape and can be more readily analyzed. In Stage $i$ (figs. 109-14, photos. 18 to 23) they have the forms which have been familiar to us since the early work of Paulmier ('98, '99) on the Hemiptera. The most characteristic of these is (1) the double cross, consisting of four arms, at right angles to each other, and longitudinally split. The four arms may be equal in length. More commonly one pair is shorter than the other. In the later stages the four arms typically lie in the same plane. In earlier ones they are often curved; and the two longer arms may be curved towards each other until they nearly meet to form a ring. 
In some cases the two arms actually meet, uniting to form a closed ring, of the type first made known by Paulmier ('98) and often observed since, both in insects and in other animals, such as Tomopteris, or the grasshoppers; but this type is much rarer in Oncopeltus and Lygaeus than in some other species. (3) The third type is that of the tetrad-rod, which consists of a straight rod, which shows both a longitudinal split and a transverse median suture. These forms are readily deducible from the double cross by reduction and final disappearance of the lateral arms, the position of which is now indicated by the transverse suture. As will be shown hereafter (especially in the case of Protenor) the double crosses undergo in their later stages precisely this change; but the evidence indicates that some of the tetrad-rods never pass through the double cross stage. (4) The fourth type is the double- $V$, best described as a $V$-shaped figure that is longitudinally split in the plane of the two branches, from the apex of the $V$ towards the free ends, accompanied by a greater or less degree of separation of the two halves thus produced. Figures of this type are especially common in the earlier stages (fig. 108, photos. 17, 18) and may be recognized soon after the beginning of Stage $h$ in a much more elongate form, as shown in fig. 107, photos. 17 (from a smearpreparation). In the final prophases (Stage $j$ ) all the bivalents finally condense to form dumb-bell figures, though the double crosses (now much condensed, and often more or less opened out in a ring form) may sometimes still be distinguished in the early metaphases. In the course of this process the lateral arms of the crosses sooner or later disappear, and a cross constriction appears at the points where they have been. These conditions will be more fully considered later in the case of Protenor.

Owing to the uncertainty regarding synapsis and the impossibility of tracing the bivalents individually through the confused period, it is not possible to offer more than a somewhat conjectural interpretation of the origin and relationships one to another of these various forms. Paulmier, McClung and other earlier students of the insects assumed the primary type to be a tetrad-rod, representing two univalent chromosomes, united end to end, and longitudinally split. From this type the doublecross was assumed 
to arise by a drawing out of the central region of each longitudinal half from the synaptic point to form the 'lateral arms' of the cross. The ring-form was supposed to arise by a secondary bending around of the two principal arms until the free ends united; the $V$-forms by a sharp flexure of the bivalent at the synaptic point (cf. Paulmier, '98, '99). On the whole, however, it seems to me that the evidence points more strongly to the opposite interpretation, first clearly worked out by the Schreiners ('06) for the closely similar figures seen in Tomopteris. According to this, the original condition is that of two parallel threads or rods, in parasynaptic association, each of which sooner ar later undergoes longitudinal fission. The rings are described as arising by an opening apart of the two rods along their middle portions while remaining attached by both ends; the $V$ 's by an opening apart from one end, while remaining attached at the other; and from the latter, by complete opening out of the two limbs until they are in a straight line, arise the tetrad-rods. From the latter the crosses are readily derived by drawing out of the lateral arms in the manner assumed by Paulmier.

Though all this is somewhat hypothetical as applied to these insects, I consider it the more probable view for several reasons. The first of these is the evidence that the lateral halves of the diplotene-threads begin to separate already at the end of Stage $f$ as the nuclei are passing into the confused stage, and the correlated fact that in the initial prophases the bivalents are seen drawing together out of more or less widely separated single threads. A second is the prevalence of the double $V$-figures in the early prophases, and their gradual disappearance as the prophases advance. It is evident that these $V$-figures are opening apart in these stages, not closing up. Elongated $V$ 's with their limbs often nearly parallel (figs. 107, 108, photo. 17) are commonly seen in smearpreparations of these stages, and in slightly later ones all intermediate stages connect them with the tetrad-rods or double crosses (figs. 109 to 114 , photo. 18). These facts are quite independent of any particular conception of synapsis, but they seem to fit best with the view that the original type is a longitudinally double rod following parasynapsis, as maintained by the Schrei- 
ners. This view receives strong support from Montgomery's recent paper on Euschistus, in which all stages of such opening out are shown, and in which the process of parasynaptic union is described in detail. It may be pointed out that the accurate figures of Sutton ('02) from smear-preparations of Brachystola, are entirely in accordance with such an interpretation, as has been also indicated by Grégoire ('10). I nevertheless adopt this conclusion only in a provisional way, as it is still based to large extent upon indirect evidence much of which is not inconsistent with the earlier and opposite conception of Paulmier.

The facts that have been described, especially as seen in Protenor, point very definitely to the conclusion that the initial stages of the formation of the bivalents are passed through with as the nuclei pass into the confused stage, and that they do not really lose their identity in this stage but are only lost to view by their looseness of structure, great extension, and intricate entanglement. This interesting question will repay more adequate study; for if my conclusion be correct it may help us to solve the difficult problem of the disentanglement of the leptotene-loops in the synaptic process of such forms as Tomopteris and Batracoseps (cf. p. 407).

b. The sex-chromosomes. The history of the sex-chromosomes during these stages is very easily followed throughout, particularly in smear-preparations, and affords a complete demonstration of their identity with the chromatic 'nucleoli' of the growthperiod. In Stage $h$ these chromosomes almost always lie close against the nuclear wall, and in Lygaeus still show but littlechange, both retaining the form of short longitudinally split rods. In Oncopeltus they show a marked change, being now more or less elongated into a rod-like form, often a little irregular in shape, and now for the first time plainly longitudinally split (figs. 105, 106). In Stage $i$ they are regular, short, compact longitudinally divided rods, essentially like those of Lygaeus save for their nearly equal size. This may be studied to best advantage in smear-preparations, where the composition of the chromosome-groups may be completely analyzed. In such preparations the total number of chromosomes in Oncopeltus is nine, including seven bivalents 
and the two univalent sex-chromosomes. The later may at once be recognized by (1) their smaller size, (2) more compact texture, and (3) simple, rod-like form and longitudinal split (figs. 108 to 110 , photos. 20 to 23 ). In both sections and smears all gradations are seen between these nuclei and those of the growth-period which remove all doubt as to the identity of these chromosomes with the chromatic 'nucleoli' of the latter. On the other hand, it is easy to trace these chromosomes step by step through the later prophases into the two small chromosomes of the first division. In these stages the double rods are seen progressively shortening until they assume the dumb-bell shape in which they enter the spindle (figs. 112-114). It is clear from the transitional stages that the transverse constriction of the dumb-bell corresponds to the original longitudinal split of the rod before its shortening, while the long axis of the dumb-bell represents the original transverse axis of the rod. The apparent 'transverse' division of the dumb-bell is therefore in reality a longitudinal division.

We may with advantage consider at this point some very interesting features presented by the $X$-chromosome in Lygaeus bicrucis $^{11}$ especially during the stages preceding the prophases. In the earliest stage $(a)$ this chromosome is an elongate, almost vermiform body, which appears homogeneous in structure (figs. 69, 70). In Stages $b$ to $d$ (figs. 71 to 73 ) it is shorter and thicker, and still usually appears homogeneous, though in much extracted preparations of Stage $c$ it may appear longitudinally divided. In Stage $e$ (synizesis) it is considerably shorter and shows no sign of division (photo. 12). In Stage $f$ it is again more elongated and unmistakably split lengthwise (photos. 13 to 15), and in this condition persists throughout the whole growth-period, gradually shortening in the prophases until it assumes a dumb-bell shape, quite as in Oncopeltus (photo. 25).

At every period from the post-synaptic spireme onwards many cases may be found in which the double rod appears nearly or quite homogeneous (figs: 84, 98, 99, $100 a, b$ : photos. 14, 15); but

${ }_{11}$ This account applies only to this species. The facts in L. turcicus are very different, as already mentioned (see Wilson, '05 b, '06). 
in many other cases it very clearly shows a double series of varicosities that are accurately paired in the two longitudinal halves, as if the rod originally consisted of a series of large granules or segments that afterwards underwent longitudinal fission. Some of the various forms that appear are represented in fig. 100. Of these forms one is far more frequent than any of the othersthat which shows three pairs of segments $(100 d)$, which in the best cases are very sharply marked, in others less distinct though evident, in others barely perceptible. In some cases (usually more elongate forms), four segments are apparent (fig. 100, c), but no case has been seen with more than four. In a few cases, where the rod seems to be shorter, less than three segments appear, and an almost quadripartite form results (fig. $100 \mathrm{e}$ ). Some of these cases are obviously due to a sharp curvature of the rod, so that in foreshortened view only the end segments are seen; but I have seen a few cases in which the rod seems to have simply shortened and two pairs of the segments seem to have fused together. In fig. $100 f$ the rod seems to show six segments (the only such case seen); but it is nearly certain that this represents the $X$ - and $Y$-chromosomes lying end to end, as a separate $Y$-chromosome can not be found elsewhere in the nucleus. This case, as well as others where $Y$ is separate, indicates that the $Y$-chromosome also may consist of segments, but not more than two such have been seen in any case. Figs. $100, g$ and $h$, show two isolated $Y$-chromosomes of the homogeneous type.

It seems to me hardly possible that this striking appearance is an accidental artifact, first because of the frequency of the trisegmental type, and second because of the correspondence of the segments of the two halves in each double rod, which is often rendered more striking by. a decided inequality of the segments (well shown in fig. $100 \mathrm{f}$ ) in each half. All such cases that I have seen show the segments accurately paired. For these reasons I believe the segmented structure to be comparable to the linear arrangement of 'chromatin-granules' so often described in the spireme-threads of the ordinary chromosomes, and to be an expression of some kind of internal structure in the $X$-chromosomes. These facts may be added to the evidence reviewed in my preced- 
ing 'Study' ('11 a) that the $X$-chromosome (like other chromosomes) is a compound body. They help us to understand how an $X$-chromosome that is originally single may break up into two or more components that behave as separate chromosomes in the diploid groups but become associated in a coherent group (' $X$ element') at the maturation-period (Payne, '09, Wilson, '11, Edwards, '10), and provide a still more definite basis for the conclusion that this chromosome may be the bearer of many other factors than the one for sex (Wilson, '11, Morgan, '11, Gulick, '11). The bearing of this.on sex-limited heredity is obvious.

It is a very important fact that at no time in their history do the individual sex-chromosomes in these Hemiptera exhibit a crossform or tetrad structure comparable to that which is so characteristic of the bivalents. Such a tetrad structure only appears when the two sex-chromosomes are united to form a bivalent-as is seen for instance in Brochymena or Nezara (Wilson, '05 b, '11 a). The only apparent exception to this is the $X$-chromosome in Lygaeus, as already mentioned; but this exception is evidently only apparent. The essentially bipartite structure of these chromosomes is a significant fact that is obviously correlated with their univalent nature, and with their approaching single division in the course of the two spermatocyte-divisions. The wider implications of this will be considered in Part III, in connection . with the facts seen in Protenor.

\section{Comparative considerations regarding the maturation-period}

A comparison of the growth-period in these Hemiptera with the conditions seen in such forms as Tomopteris or Batracoseps shows some striking, though I think secondary points of difference.

In the first place, the formation of compact, massive bodies from which the leptotene-threads unravel in the pre-synaptic period, which is so characteristic of these insects, seems not to take place in Batracoseps and some other forms; though, as will be indicated beyond, the Schreiners have found indications of an analogous process in Tomopteris.

Secondly, the polarized amphitene, or 'bouquet-stage,' that is characteristic of Tomopteris, Batracoseps and other forms, seems 
to be entirely wanting in these insects, where in its place we find the closely convoluted and apparently non-polarized synaptic knot or synizesis. The controversy as to whether the latter is an artifact, due to the coagulating effect of the reagents, seems to be terminated by the fact, determined by Sargant ('97), Overton ('05), Berghs ('04), Oettinger ('09), and myself ('09 a, '09 b) that the synizesis may be clearly seen in the fresh (living?) material immediately after gentle teasing apart of the cells in a normal fluid (Ringer's solution) in which the spermatozoa continue actively to swim. Neither at this stage nor. in those that immediately precede or follow is there the least sign in these animals of an elongation of the sex-chromosomes or of a giving off of nuclear material to the protoplasm.

Third, in Tomopteris and Batracoseps the pachytene-loops formed in synapsis persist as such throughout a large part of the growth-period, without undergoing at any period an apparent loss of identity in a 'diffuse' stage such as is so characteristic of the Hemiptera. In Batracoseps the pachytene-loops become longitudinally divided ('diplotene') near the end of the growth-period, when they give rise directly to the prophase-figures. In Tomopteris the diplotene-threads are apparent at a much earlier period (Schreiner), but here too give rise directly to the prophase-figures. In the Hemiptera here considered the diplotene is likewise formed very early, but the diffuse stage is interpolated between it and the definitive formation of the prophase-figures, and the greater part of the growth-period is passed in this condition (in some cases accompanied by a second contraction-figure in the later period). There is, however, an analogy in this respect between these Hemiptera and Tomopteris, where the Schreiners describe and figure ('06, p. 19, figs. 31, 32) a stage following the early diplotene in which the parallel halves of the double threads become longer, thinner, less regular, and spread more or less widely apart, though still retaining their connection at certain points. It is very probable that this process corresponds to that which marks the beginning of the diffuse stage in the Hemiptera, but does not proceed so far; and that in this respect Tomopteris is intermediate between these animals and the Amphibia. Perhaps 
we may here find a clue to the more extreme forms of diffusion observed in the oogenesis of many animals and in the ordinary somatic nuclei. ${ }^{12}$

As regards the problem of synapsis and reduction, the existence of the synizesis and diffuse stages renders the Hemiptera very unfavorable objects as compared with Tomopteris or Batracoseps, and we are here thrown back upon analogies. Emphasis may however be laid upon the essential similarity of the prophasefigures in Tomopteris and these insects; and if my interpretation of the diffuse stage be correct, it is probable that these figures have essentially the same mode of origin from the diplotenethreads. Following this analogy, I provisionally assume the latter to follow an original side by side union, or parasynapsisnot an end to end union or telosynapsis, as was assumed by Paulmier, Montgomery and (in Orthoptera) McClung, Sutton, and more recently by Davis. In his latest paper ('11) Montgomery rejects his former interpretation in favor of the one here adopted. If the double cross-figures (or the tetrad-rods) arise in the manner assumed, it is clear that their 'transverse' division is the last remnant of the original longitudinal cleft of the diplotene-thread; and it is certain, as Paulmier first showed, that this 'transverse' division corresponds to the planeof the first spermatocyte-division. If we accept this, and if the original longitudinal cleft of the diplotene corresponds to the plane of synapsis, it follows that the first spermatocyte-division is the 'reductiondivision,' as Paulmier and Montgomery concluded. I repeat, however, that this conclusion is here adopted only in a tentative way; since the case is by no means proved; and, as will appear, my conception of the reduction-division differs materially from the one more commonly held.

As regards the sex-chromosomes, on the other hand, all is clear. The observations here recorded remove every doubt, I think, in

${ }^{12} \mathrm{~A}$ more or less wide divergence of the longitudinal halves of the diplotenethreads appears to be the rule among many animals and plants. It has been especially emphasized by Grégoire ('04, '10) who has called attention to the striking contrast in this respect between the bivalent chromosomes of the maturationperiod and the longitudinally split spireme-threads of the somatic divisions. Sec also Strasburger, '09, pp. 98 to 100. 
regard to the following points. First, it is certain that each of these chromosomes divides but once in the course of the maturation-process, namely, in the first division; and this division is clearly longitudinal and equational. The second 'division' of the XY-pair is obviously not a division at all but only the disjunction of two separate chromosomes that have for a short time been in contact without loss of their identity. This process is an evident and typical reduction-division in the original sense. In these animals, therefore, it is quite certain that the $X Y$-pair undergoes a process of 'post-reduction' (cf. Wilson, '05 c). It is a remarkable fact, proved by the studies of Stevens, that in the Coleoptera and Diptera the $X Y$-pair follows the reverse order, as is also the case with the $m$-chromosomes of the coreid Hemiptera. ${ }^{13}$

\section{Comment on the sex-chromosomes in Oncopeltus}

The extremely close correspondence between Oncopeltus and Lygaeus at every stage of the spermatogenesis leaves not the least doubt of the identity of the sex-chromosomes of the two forms. Apart from the size-differences, of these chromosomes, Lygaeus differs from Oncopeltus only in (1) the retention throughout of a rod-like form by the $X$-chromosome, (2) the earlier appearance of the longitudinal split in both sex-chromosomes, (3) a slightly more marked tendency for the sex-chromosomes to conjugate at the time of general synapsis. On the other hand, the sex-chromosomes of the two forms agree in all the characteristic peculiarities of these chromosomes shown in the Hemiptera generally, namely, (1) the retention of a compact and deeply staining character from an early pre-synaptic period down to the spermatocyte-prophases, (2) their division as separate univalents in the first spermatocyte-division, (3) their subsequent conjugation to form a bivalent, which occupies a nearly central position in the second spermatocyte metaphase-group, and (usually) divides in advance of the other chromosomes. These facts.fully establish

\footnotetext{
${ }^{13}$ I may point out that it is inadmissible to designate as ' $m$-chromosomes' any pair of especially small chromosomes without respect to their other characteristics,

- as has been done by several writers. The $m$-chromosomes of the Coreidae are not always distinctly smaller than the other chromosomes, and they are characterized by certain very definite peculiarities of behavior. Cf. Wilson, '05 c, '11 a.
} 
the identity of the sex-chromosomes in Oncopeltus. It may therefore be taken as an established fact that a pair of sex-chromosomes may be recognizable as such even in cases where they show no perceptible difference of size, and where no constant differences between the diploid chromosome-groups of the two sexes can be seen.

Such cases are of course fatal to the view that the nuclear differences between the sexes are reducible to one of general chromatinmass; and, as I have elsewhere urged, these chromosomes can be regarded as factors in sex-production only by assuming some kind of difference between the substance of $X$ and $Y$. I will not here enter upon the discussion of a point that has been fully considered in several earlier papers (see especially Wilson,' 11 a, '11 b). I will only again express the view that the differential factor between $X$ and $Y$ may plausibly be regarded a specific chemical substance (the ' $X$-chromatin') that is either confined to the $X$-chromosome or is there present in relative excess, and in respect to which the two sexes differ correspondingly. If this is correct, the sexual differences may be at bottom dependent upon a fundamental quantitative difference of metabolism, as stated in my first paper on this subject ('05 a). Such nuclear differences between the sexes may of course exist not only in forms where no difference of total chromatin mass is visible, but even where no special 'sex-chromosomes' are differentiated. The surprising thing, indeed, is that they should in some instances be expressed in, or accompanied by, visible sexual differences of the chromosome-groups.

III. CRITICAL CONSIDERATIONS ON THE MATURATION-PHENOMENA BASED ON A COMPARISON OF THE HEMIPTERA, TOMOPTERIS, BATRACOSEPS AND SOME OTHER FORMS

As has been indicated, my conclusions concerning synapsis and reduction in Hemiptera are largely tentative in character. If I nevertheless venture to make some critical comment on the general problem it is mainly because of the opportunity I have had to reëxamine these phenomena in Tomopteris and Batracoseps. 


\section{The question of synapsis}

The cytological problem of synapsis and reduction involves four principal questions, as follows: (1) Is synapsis a fact? Do the chromatin-elements actually conjugate or otherwise become associated two by two? (2) Admitting the fact of synapsis, are the conjugating elements chromosomes, and are they individually identical with those of the last diploid or pre-meiotic division? (3) Do they conjugate side by side (parasynapsis, parasyndesis), end to end (telosynapsis, metasyndesis) or in both ways? (4) Does synapsis lead to partial or complete fusion of the conjugating elements to form 'zygosomes' or 'mixochromosomes,' or are they subsequently disjoined by a 'reduction-division?' Upon these questions depends our answer to a fifth and still more important question, namely, (5) Can the Mendelian segregation of unitfactors be explained by the phenomena of synapsis and reduction?

Despite the prodigious accumulation of data regarding these questions the unprejudiced student of the literature finds himself compelled to admit that not one of them has yet received a really demonstrative answer - at least not one that has brought conviction to the minds of all competent cytologists. I do not propose to consider them exhaustively, or to give any approach to a complete review of the literature. This has been done by other writers, notably by Grégoire ('05, '10) in two extended and masterly memoirs, by Strasburger in a most valuable series of critical essays ('07, '08, '09, '10), and by Haecker ('07, '10). (See also Davis, '08, Gérard, '09, Gates, '11, Montgomery, '11, and the series of papers by the Schreiners and by Janssens.). I will however indicate some of the conclusions to which I have been led in an effort to form an independent judgment concerning the facts, especially in Tomopteris and Batracoseps, which are probably unsurpassed as objects of observation, have become classical through the well known studies of the Schreiners ('06, '08) and of Janssens ('03, '05), and have formed a main center of controversy in recent years.

The conclusions of these observers (more especially those of the Schreiners) have been the object of repeated criticism on the part of Goldschmidt ('06, '08), Fick ('07, '08), Meves ('07, '08, '11), Haecker ('07, '10) and many others. These criticisms, too well 
known to call for extended review, were substantially at one in the contention that what had been described as a parallel or sidewise conjugation of spireme-threads during the 'bouquet,' 'synaptene' or 'amphitene' (synaptic) stage is nothing other than a modified form of longitudinal splitting, in which double threads, longitudinally divided from the beginning, are progressively differentiated out of the nuclear substance from one pole of the nucleus towards the opposite pole. In the course of his able critique Meves ('07) endeavors to break down the distinction between such a process and that which is seen in the prophases of somatic cells, contending that in both cases the longitudinal duality is brought about by a biserial grouping of the chromatin-granules of the resting nucleus, and urging that the process seen in the amphitene-nuclei is of essentially the same nature as the early division of spireme-threads in the diploid nuclei long ago described by Flemming. Unquestionably, this objection is worthy of the most attentive consideration, especially in view of the conclusion of several recent observers (considered more in detail beyond) that the longitudinal division of the spireme-threads is in some cases already in evidence in the chromosomes of the preceding anaphases or telophases, and that the two halves thus arising may separate more or less widely before the nuclei have entered the 'resting' state. For Meves there is no problem of synapsis. The Gordian knot is cut with the statement, "Die Geschlechtszellen bezw. ihre Kerne haben nach meiner Vorstellung (1907) die besondere Eigenschaft ererbt, beim Eintritt in die Wachstumsperiode nur die halbe Zahl von Chromosomen auszubilden" ('11, p. 296). Certainly the adoption of this simple solution would save a great deal of trouble; but I fear that the facts compel us to take a more roundabout way out of our difficulties. Goldschmidt and Haecker, on the other hand, do not doubt the fact of synapsis, and take issue only with the parasynaptic mode of conjugation. Concerning the latter Haecker's latest expression of opinion is as follows:

Vielmehr hat sich in mir die Ueberzeugung befestigt, dass der Eindruck einer Parallelkonjugation im wesentlichen durch die teilweise Koinzidenz zweier voneinander unabhängiger Erscheinungen 
hervorgerufen kann, nämlich erstens eines mehr zufälligen oder, besser gesagt, selbsverständlichen teilweisen Parallelismus der Fäden, wie er durch die in der Synapsisphase bestehende polare Anordnung der Kernsubstanzen bedingt wird, und zweitens einer verfrühten, bei den einzelnen Objekten und Individuen je nach dem physiologischen und Konservierungszustand bald früher, bald später, bald regelmässiger auftretenden primären Längsspaltung ('10, p. 185).

Without citing other zoölogical critics at this point, attention may be called to the increasing tendency now apparent among botanical cytologists to reject, or at least to restrict, the theory of parasynapsis held by Strasburger, Allen, Berghs, Grégoire and a large number of other botanical ' zygotenists,' in favor of a telosynaptic conception like that of Farmer and Moore('05), itself essentially like that many years earlier maintained by Haecker and Rückert among zoölogists. Among these may be mentioned Mottier ('07, '08), Gates ('08, '11), Davis ('09, '11) and Digby ('10). These observers and others, though differing more or less as to the details, are in agreement on the essential point that in some species at least the synaptic connection of the chromosomes is end to end, not side by side; and that a longitudinal duality of the spireme-threads at the synaptic period (synizesis, or earlier) is either absent, or if present is due either to an accidental parallelism or to a longitudinal splitting comparable to that seen in the diploid prophases. These observers are in substantial agreement that the chromosomes (if persistent entities) are originally arranged in linear series, and united end to end, in a spireme-thread which ultimately breaks apart into bivalent segments, each consisting of two chromosomes in parasynaptic union. The sidewise pairing, which undoubtedly occurs in some plants, is believed by Farmer and Moore, Mottier, and others to result from a secondary looping of these segments, which takes place long after the synizesis stage. Gates, however, expressly adopts the view that synapsis may take place by either method in different species, possibly even in the same species.

I must admit that my own faith in parasynapsis (such as it was) and even in synapsis itself, was materially shaken by some of the criticisms and observations that have just been indicated, and that $I$ took up the study of the question in a distinctly scepti- 
cal spirit. It was only after prolonged and repeated study of the same objects, in part of the same preparations, as those of the Schreiners and of Janssens, that this scepticism gave way to the belief that the conclusions of these observers (not to mention others) are probably well founded. I will not at this time publish new figures or photographs of these forms (of which I have a large number, particularly of Batracoseps) but will here confine myself to a brief statement of the main reasons why I do not find it possible to accept the adverse criticisms that have been indicated.

There are two points that demand especial emphasis. One is the complete demonstration of the seriation of the stages that is afforded in the testis of Batracoseps. The regular and panoramic progression of stages from the spermatogonial end of the testis to that of the spermatids renders error on this point out of the question; and in particular, there is no possibility of confusing the post-synaptic with the pre-synaptic stages, or the synaptic nuclei with those of the early prophases (pro-strepsinema) of the spermatocyte-divisions. The second point of importance is the essential accuracy of the figures of the Schreiners and of Janssensindeed, my only criticism of those of Janssens might be that the relations are often shown even more clearly in the preparations than in his figures, perhaps because the latter were in some cases made from material not quite as perfectly fixed as the best that has come under my observation. In the case of Tomopteris I have been able in a considerable number of cases to compare the figures of the Schreiners with the identical nuclei from which they were drawn. Here and there, perhaps, certain details might be somewhat differently represented by different observers; but a study of very numerous cells at every stage of the spermatogenesis has thoroughly convinced me that as a-whole the figures of these authors present a faithful picture of what any observer may see in the preparations. The only question that can be raised seems to me therefore to be a matter of interpretation.

I think that any observer, whatever be his individual prepossession, who will take the trouble to study these preparations thoroughly, will find himself compelled to admit the following facts: 
1. That the 'amphitene' stage (to employ Janssens's appropriate term for the synaptic nuclei) is preceded by one in which the nucleus is traversed by fine, undivided, leptotene threads the free ends of which are from an early period polarized towards the pole of the nucleus near which lie the centrioles.

2. That from this pole, during the amphitene stage, thick and often plainly double threads are formed progressively towards the opposite pole near which (in Batracoseps) lies the chromoplast.

3. That pari passu with the growth of the thick threads the thin threads disappear until all have vanished.

The conclusion is irresistible, and will hardly be disputed, that the thick (pachytene) threads grow at the expense of material supplied by the thin ones (leptotene).

It is further indisputable that in many cases the thick (and often double) threads terminate anti-polewards in two undivided diverging thin threads like the branches of a $Y$, which often separate at a wide angle and may be traced for a long distance, sometimes to opposite sides of the nucleus, as continuous threads. This fact may be seen in both Tomopteris and Batracoseps with a clearness that admits of no doubt. These $Y$-figures are so numerous, so clear, and in their more striking forms so different from anything seen at other stages as to constitute a highly characteristic feature of the nuclei at this particular stage.

Janssens, the Schreiners, Grégoire and others have with good reason insisted on the fact, seen with especial clearness in Batracoseps, that not more than two thin threads are thus seen diverging from the anti-poleward ends of the thick threads. Fick ('07) after examination of the Schreiners' preparations of Tomopteris, stated that he could sometimes observe more than two such diverging threads. Even Janssens in his earlier work (with Dumez) on Plethodon, believed that he had seen a similar appearance "Un chromosome naissant est parfois en relation avec plusieurs filaments, de tel manière qu-il devient très difficile à l'observateur de faire un choix" ('03, p. 423); but in his later work on Batracoseps he insists that such is not the case.

I have studied this point with the greatest care of which I am capable in both Tomopteris and Batracoseps. In the former 
case one may indeed often be in doubt, particularly in the earlier stages, though many perfectly clear $Y$-figures are evident. Such doubtful cases may however very well be due either to a confusion produced by threads of linin, to defects of fixation, or to coagulation-products of the nuclear enchylema. Batracoseps seems to me, however, decidedly more favorable for study of this point than Tomopteris, partly because of the much greater size of the nuclei, partly because of the greater brilliancy of the pictures in detail, especially evident in material fixed with Carnoy's fluid. At its best this method, in my experience, is much superior to Flemming's or Hermann's fluids for study of this point. Prolonged search among the huge amphitene-nuclei of Batracoseps has failed to show even a single clear case in which more than two leptotene-threads can be traced into connection with a single pachytene. When the latter terminate anti-polewards in more than one leptotene-thread two are always seen, very often diverging like the branches of a $Y$; and these bifid figures appear with the utmost clearness in every view-sidewise, endwise and obliquely. It is of course true that such bifid figures are often not in evidence. Not infrequently pachytene-threads seem to end abruptly without connection with the leptotene; sometimes they seem to end in single leptotene-threads. But in the nature of the case the true relation of the latter to the former must often fail to appear in the sections. This may result from many causes -accidents of sectioning, entanglement of the threads, unfavorable position, and the like-and it is very probable that in the coagulation-process of fixation the delicate thin threads may often break away from their normal connections. When allowance is made for these sources of error it is in fact surprising that so many demonstrative $Y$-figures are seen; and it is a significant fact that these figures, though often bent or distorted, always show the same orientation in the nucleus with respect to the centrosome pole.

That the $Y$-figures represent the normal and typical relation of the pachytene-threads to the leptotene seems to me indisputable; and I consider it utterly impossible to interpret these figures as an expression of a progressive longitudinal splitting of previously 
undivided threads. The $Y$-figures are not opening apart, they are closing up, as is placed beyond doubt by the magnificent demonstration of the seriation given in the testis of Batracoseps. $Y$-figures with a short stem and long arms precede, they do not follow, those with long stem and short arms. What is taking place is evidently a coming together of the thin threads side by side in pairs to form the thick ones - a process exactly. opposite to longitudinal division. I do not hesitate, therefore, to confirm positively the description of the facts given by Janssens and the Schreiners.

I desire to emphasize the striking contrast that exists between the amphitene-nuclei and the spermatogonial or other diploid prophase-nuclei. . It seems to me that Meves goes much too far when he directly compares the process of 'parallel conjugation' to the early fission of spireme-threads in the diploid nuclei as described by Flemming and his successors; for one is led from this to suppose that figures may be seen in the two cases that are essentially similar. But no one can study the early spermatogonial prophases in Tomopteris or Batracoseps without being struck by the very great contrast which they present to the amphitenenuclei. ${ }^{14}$ Never in the former case, as far as I have been able to find, are the two halves of the double spireme-threads seen diverging like the branches of a $Y$; nor have I been able to discover such pictures in the early prophases of other diploid nuclei, such as the epithelial and connective tissue cells of larval salamanders. Even though such pictures could be found, the amphitene nuclei undeniably offer peculiarities that differentiate them in the most

\footnotetext{
14 In considering this question it is necessary to point out that the single figure of the amphitene stage that Meves offers in favor of his interpretation ('07, textfigure, p. 460) conveys no real idea of the characteristic relation of the leptotenethreads to the pachytene. Many pictures similar to this are seen in my own sections of Batracoseps and Plethodon, especially after fixation by Flemming's fluid or Hermann's, often also in inferior preparations from Carnoy's fluid; but as a rule it is only in the best Carnoy preparations that the exact relations can be clearly and generally seen. It is evident that the least defect due to fixation or to the shrinkage of embedding process tends to obscure the leptotene-threads and cause them to assume a more netlike appearance.
} 
conspicuous way from the earlier generations of cells in the testis; and these are not to be ignored in the study of this problem.

Another very striking fact in the case of Batracoseps is that the two branches of the $Y$ often give exactly the appearance of twisting together to form the stem-a condition very clearly shown in many of Janssens's figures; though I do not find it mentioned in the text. The pictures seen in Tomopteris also sometimes suggest a similar condition, though less clearly; but in neither case am I entirely sure of the case, since the torsion often can not be seen. A twisting together of the longitudinal halves of the diplotenethreads at a later stage ('strepsinema') is of course a very familiar fact; but I can find only a few indications here and there in the literature of such a twisting at the synaptic stage. Two very definite accounts of such a process have recently been given by Agar ('11) in the case of Lepidosiren, and by Bolles Lee ('11) in Helix. The latter author believes the double spiral to persist as such in the succeeding pachytene stage. "Jamais, à aucune moment, même dans les enroulements les plus étroits du bouquet tassé, on ne voit rien qui puisse faire conclure à une fusion des "deux éléments" (p. 70). It is quite possible that a close torsion of the threads may explain the fact that it is in many cases diffcult or impossible to distinguish a longitudinal duality for a certain time after the synaptic process is completed.

Concerning synapsis in the Orthoptera I can only speak with considerable reserve. Most observers of this group have concluded that the longitudinal duality of the diplotene-threads is due to a process of longitudinal splitting (McClung and all of his pupils, Sinéty, Montgomery, Davis, Buchner, Jordan, Granata, Brunelli) and only a few have attributed it to parasynapsis (Otte, Gérard, Morse). The few observations I have been able to make on McClung's preparations of Achurum, Phrynotettix and Mermiria nevertheless lead me to the impression that a side by side union of leptotene-threads takes place here also. The case is however much less clear than in the other forms since the polarization is less marked, and the amphitene stage, though clearly apparent in some cases, is correspondingly less conspicuous. 
Nevertheless, in these nuclei also the leptotene-threads may often be seen to lie parallel and in pairs on one side of the nucleus, while on the opposite side they are quite irregular. ${ }^{15}$.Here too may be seen $Y$-shaped figures, in some cases almost exactly like those of Batracoseps, except that the stem is more clearly double and shows no indication of torsion. It may again be pointed out that Sutton's observations on Brachystola are entirely consistent with a parasynaptic mode of conjugation. I think therefore that the case for telosynapsis in these animals is not yet established, and that in spite of the careful work of Davis, Brunelli and others, the question must still be considered open.

As to the Hemiptera, sufficient emphasis has already been laid upon the practical difficulties which they present. In Euschistus however, as described in Montgomery's recent valuable paper ('11) the difficulties are less baffling than in many other forms; and he has had the advantage of working with a species in which the total number of threads can be determined in at least some of the nuclei at every stage. In this work the author, reversing his earlier conclusions concerning these insects, definitely accepts the theory of parasynapsis. As I have pointed out, the prophasefigures in Oncopeltus and Protenor are certainly not out of harmony with this conclusion. I therefore accept the probability of a side by side union in these animals, though I think the possibility of an end to end conjugation is not yet excluded.

But if, now, the fact of a side by side union of parallel leptotene-threads be granted, we have still not arrived at a demonstration of parasynapsis; for there are some very important possibilities yet to be reckoned with. Before such a demonstration can be admitted, we must first make sure of the number of separate pre-synaptic chromosomes (cf. Fick, Meves) and secondly must exclude the possibility, which has been suggested by several writers, that the parallel union is no more than a reunion of sister-threads that have been derived by an earlier longitudinal fission of a single thread (or chromosome) and have subsequently

${ }^{15}$ This was also noted by Davis but attributed by him to "an accidental arrangement, which is more common near the pole since in this region the threads are crowded more closely together"' ('08, p. 127). 
undergone wide separation. Such, for instance, is the view of Brunelli ('11) in an interesting recent work on Tryxalis; and a similar view is suggested on the botanical side by the recent work of Digby ('10) on Galtonia, and of Fraser and Snell ('11) on Vicia. All these observers believe the longitudinal duality of the spireme threads at the synaptic period to be quite comparable to that of the diploid prophases, and to be traceable to a longitudinal split that is already present in the preceding telophase-chromosomes (cf. also the work of Dehorne and of Schneider, already cited), and these writers emphasize the fact that the products of this fission do in fact separate more or less widely as the nuclei enter the 'resting' period. As to the subsequent changes Brunelli concludes, "Successivamente, le due metà longitudinale degli individuî cromosomici si parallelizzerebbero: donde gli aspetti intermedî che sono stati descritti come scissione di un filo unico, o come l'acollimento di due fili cromatici avendi il valore di due cromosomi (ipotesi della zigotenia)" ('11, p. 9). It is evident from this how essential it is to determine the number of pre-synaptic threads; for if they have such an origin as has just been indicated, their number should be tetraploid (double the diploid), whereas if they represent whole chromosomes the number should be diploid.

In the insects that I have studied the pre-synaptic stages are of especial interest as affording almost a demonstration that the presynaptic number of threads is the diploid number. I attach great weight to the history of the sex-chromosomes in these stages; for, owing to the fortunate circumstance that they are individually recognizable at this time, we can be perfectly sure that at least one pair of chromosomes of the diploid groups is here represented by two separate chromosomes that afterwards undergo synapsis. When we consider that these chromosomes are hardly distinguishable from the other chromatic masses of Stage $b$ until after considerable extraction, that the latter are of the same or nearly the same number as that of the spermatogonial autosomes, and that they give rise to the separate leptotene-threads that enter synapsis, we must admit that strong ground is given for the conclusion that the latter are individually representatives of 
the spermatogonial autosomes. In some at least of the objects I have examined these threads are single, not double; and I can find no evidence that they consist or have consisted of two interlacing spirals or closely associated halves. In this respect they seem to be quite like the spirals that uncoil from massive bodies in the spermatogonial prophases in Phrynotettix. In this case I can speak with complete assurance; for the evidence afforded by McClung's brilliant preparations of this form is absolutely demonstrative that the spirals are single, and that the longitudinal duality is produced by a subsequent longitudinal split of the spiral thread (which is essentially in agreement with Janssens's earlier conclusions in the case of Triton).

For the foregoing reasons I accept the probability that the parallel union of leptotene-threads does not form part of a peculiarly modified process of longitudinal division, but should be regarded as a true conjugation or parasynapsis of entire chromosomes. Apart from the convincing evidence afforded by the sex-chromosomes, my observations are essentially in agreement with those of the Schreiners in regard to the origin of the leptotene-threads. These observers describe the latter in Tomopteris as arising from much thicker, loose, polarized loops of the diploid number (18) which transform themselves into convoluted threads in a manner somewhat similar to that seen in the insects: "Nicht selten haben wir Bilder gesehen, die uns den Eindruck gegeben haben, dass das Chromatin der lockeren Schlingen sich zuerst zu einem unregelmässig aufgebauten, stark gewundenen und gefalteten Bande sammelt, aus dem wieder die deutlich begrenzten dünnen Fäden hervorgehen" ('06, p. 18). Later, "Die Chromatinfädchen, die auf Stadien wie Fig. 18 und $20 a$ hervortreten sind, vovon uns fortgesetzte Untersuchungen immer fester ünberzeugt haben, in den breiten aufgelockerten Chromatinbänder der vorgehenden Stadien spiral aufgerollt oder zusammengefaltet sind'' ('08, p. 10, italics mine). My own study of the Tomopteris slides gives me the same impression; and I think it probable that the phenomenon here seen is of the same nature as that which so clearly appears in the insects, though the thick 'Chromatinbänder' are here much less sharply defined. Jans- 
sens's somewhat similar account of the pre-synaptic stages in Triton have already been mentioned (p. 368). In Batracoseps, on the other hand, there is as yet nothing to show that the leptotene-threads arise directly from the irregular and variable chromatin-masses that are seen in the earlier stages ('protobroch' and 'deutobroch' nuclei).

Opinion still differs so widely in respect to the pre-synaptic conditions in plants that its discussion can hardly be undertaken here. Overton ('05, '09) and those who have adopted the 'prochromosome-theory' find the leptotene stage preceded by one in which massive 'prochromosomes' are present, of the diploid number, and already showing an association in pairs which is a forerunner of actual synapsis; but other observers have found no support for this view in the objects they have examined (cf. Mottier, '07, '09). It seems possible that different species may differ in this respect, as is certainly the case in animals.

It is impossible to leave this discussion without mention of two additional series of facts which lend strong indirect support to the theory of synapsis. One is the remarkable discovery that in the diploid groups the chromosomes are often found to correspond two by two in respect to size, as was first pointed out by Montgomery ('01) and Sutton ('02); and that in some cases the chromosomes are actually arranged in pairs according to their size. The latter fact was also first described, I believe, by Montgomery ('04) in Plethodon, later in a number of the Hemiptera ('06); and in the latter work first appears the view that such an actual arrangement in pairs is characteristic of the diploid nuclei (p. 148). A similar arrangement was later described by Janssens and Willems ('08) in Alytes; and a most striking, unmistakable case of the same kind was found by Stevens ('08) in several of the Diptera. On the botanical side similar facts have been described by Strasburger ('05), Geerts ('07), Sykes ('09), Müller ('09), Gates ('09), Stomps ('11) and others. Overton, Rosenberg, Lundegard, and others likewise describe the "prochromosomes" as arranged in pairs in the diploid nuclei, as well as in the presynaptic stages of the auxocytes. So many of these cases have been described, some of them of quite demonstrative character 
(Diptera), that no doubt can exist of the widespread tendency of the chromosomes to assume this arrangement already in the diploid nuclei. It appears to me however that those authors who consider the paired grouping to be a general characteristic of the diploid nuclei go much too far. ${ }^{16}$ Not only are numerous exceptions seen in the case of individual chromosome-pairs, but in many cases no trace of paired grouping appears. Such exceptions may readily be seen in the figures of Montgomery, Stevens, Morrill and myself of the Hemiptera, which are probably unsurpassed among animals for the clearness with which the size-relations are shown. In cases where certain pairs may be unmistakably recognized (as in Protenor and other Coreidea) the two members frequently show no constancy of relative position-compare for instance the accurate figures of the diploid groups of Protenor, Anasa, Alydus and Nezara in my third 'Study' ('06) or those of Morrill ('10) of Archimerus, Chelinidea, Anasa and Protenor.

But this does not in the least lessen the significance of the remarkable cases that have been established. The tendency towards such an association of the chromosomes in pairs is undoubtedly widespread; and the very fact that it does not follow a fixed order may be used as an argument in favor of a conjugation at the synaptic period. When the pairing is already evident in the diploid groups the way for synapsis has been prepared in advance. This process must take place at some time subsequent to the association of the germ-nuclei in fertilization; and that such a process undoubtedly occurs nullifies all the a priori objections that might be urged against the possibility of a corresponding process that is delayed until the maturation-period is reached.

10 Strasburger, for example, says, "Ich zweifle nicht im geringsten daran, dass es sich um eine allgemeine Erscheinung in diploiden Kernen dabei handelt, wenn sie auch nicht immer auffällig ist" ('09, p. 90). Gates is still more specific. "It is evident that the pairing of the chromosomes is not brought about at synapsis or at any other period of meiosis, but that the chromosomes are really paired throughout the life cycle of the sporophyte . . Synapsis plays no special part in the pairing . . Meiosis and reduction consists essentially in the segregation of the members of these pairs that have been in association since soon after fertilization"' ('11, pp. 334, 335). 
Lastly may be mentioned the interesting facts observed in the maturation of hybrids between parental forms having different numbers of chromosomes. Well known as Rosenberg's results on Drosera are ('04, '09), the main facts may be again outlined, especially as exactly analogous results have recently been reached by Geerts ('11) in hybrid Oenotheras. On crossing Drosera longifolia (having forty chromosomes) with D. rotundifolia (having twenty chromosomes) the hybrids have the intermediate number of chromosomes, thirty $(20+10)$. In the firstmaturation-division appear ten double and ten single chromosomes, the former undergoing a regular division and distribution to the poles, while the latter fail to divide, undergo an irregular distribution, and often fail to enter the daughter-nuclei. Rosenberg's interpretation is that the ten rotundifolia chromosomes conjugate with ten of the longifolia ones to form the ten bivalent (double) chromosomes, leaving ten longifolia chromosomes as unpaired univalents which undergo irregular distribution. The results of Geerts are exactly analogous. Oenothera gigas (twenty-eight chromosomes) crossed with Oe. lata (fourteen chromosomes) gives hybrids with twenty-one chromosomes $(14+7)$. The first division shows seven double (bivalent) and seven single (univalent) chromosomes; and, as in Drosera, the bivalents divide equally and symmetrically, while the univalents wander irregularly along the spindle and often fail to enter the daughter-nuclei. His interpretation is the same as that of Rosenberg. ${ }^{17}$ If correct, these results, indirect though they be, constitute almost an experimental demonstration of both synapsis and reduction.

In summing up, it is my opinion that in spite of all the apparent contradictions and conflict of opinion concerning the modus oper-

17 Gates however ('09) in an earlier study of the same hybrid examined by Geerts, was led to quite different results, concluding that half the pollen cells receive $10 \mathrm{chromosomes}$ and half 11 . I can not, however, find evidence in his paper to sustain his conclusion that "there is not here a pairing and separation of homologous chromosomes of maternal and paternal origin" (op. cit., p. 195). Gates gives no account of the exact mode of distribution of the chromosomes in the heterotypic division, but only the end result. This result would however follow if exactly such a pairing and disjunction took place as is described by Geerts, provided the remaining chromosomes also underwent an approximately equal distribution. It remains therefore to be seen whether the apparent contradiction of results is real. 
andi of synapsis, the cumulative force of the evidence in favor of the fundamental fact is irresistible. This question is not to be judged alone by the study of any one of its single phases. The whole extensive series of facts must be reckoned with; and despite their variations in detail the data are too consistent in their fundamental aspects, to be explained away. On the other hand, it is obvious that the problem as to how the parental chromatin-homologues become definitely associated in pairs is still far from a definitive solution. We should certainly expect a phenomenon so fundamental to follow everywhere the same type; but I am in agreement with the opinion that has been expressed by some other writers (cf. Gates, '11) that the particular mode of union may be of subordinate significance. In accepting the main conclusions of the Schreiners and of Janssens in regard to Tomopteris and Batracoseps I do not mean to imply that end to end conjugation, or telosynapsis, may not also take place in other forms. I hold no brief for parasynapsis; and I fully recognize the weight of the evidence in favor of telosynapsis recently brought forward especially by the botanical cytologists that have been cited. The studies of King ('07, '08) on Bufo should also be emphasized in this connection. I repeat that the phenomena seen in the insects by no means exclude the possibility of synapsis of this type, at least in some forms. Nearly all observers are agreed that the side by side union begins at or near the free ends of the leptotene-threads and advances step by step along their course. We can here see how readily the one mode might pass into the other; and the suggestion of Gates that the mode of synapsis may be correlated with the shape of the conjugating elements at the time of their union seems well worthy of consideration.

It is not to be denied that the acceptance of parasynapsis involves some very puzzling difficulties. It is, for instance, hard to comprehend how long loop-shaped chromosomes can become so disposed as to undergo progressive side by side union from their free ends towards the central points, as both the Schreiners and Janssens have concluded. Janssens appears to recognize this when he says: "L'éloignement des filaments (i.e., the wide divergence of the branches of the $Y$-figures) avant leur soudure est un 
fait très étonnant, mais .absolument certain;" but he very justly adds, "De ce que nous ne pouvons pas expliquer par quel méchanisme la soudure se réalise dans de tels cas, il ne s'en suit rien contre son existence indubitable"' ('08, p. 167). However diffcult such a mode of union may seem a priori, the preparations of the Schreiners actually demonstrate double loops that are united at both ends while widely separate along their middle portionsshown for example in figs. 16, 17 and 18 of their paper of 1908 , which accurately represent the facts, as I am able to confirm from examination of these identical nuclei in the original preparations. We must seek to discover by observation how the conjugating loops disentangle themselves from the apparent chaos of the leptotene-spireme. The chaos may however be apparent rather than real. The interesting facts worked out by Janssens in regard to the persistent orientation of the loops in the pre-synaptic stages of Batracoseps indicate that their polarity is not lost at any time between the final spermatogonial anaphases and the amphitene stage, and that their free ends always converge towards the centrosome. It seems quite possible that the way for synapsis may be prepared already in a very early pre-synaptic stage, by a definite regrouping of the chromosomes that may take place before the leptotene loops are formed as such. It is evident that the central portions of the loops are constantly shortening as the peripheral portions come together (possibly as a result of the progressive torsion of the latter). It seems therefore by no means a hopeless task to undertake a definite solution of the puzzle by observation.

\section{The question of the reduction-division}

The history of the sex-chromosomes in Oncopeltus affords, I believe, complete demonstration of the occurrence both of synapsis and of a true reduction-division in the original sense-i.e., of the disjunction of two entire chromosomes that have previously conjugated in synapsis. But, although this creates a certain presumption in favor of the occurrence of a similar process in case of the other chromosome-pairs, this argument must not be pushed too far-indeed, there is reason to believe that in case of the ordi- 
nary chromosomes ('autosomes') the process may be different in some important respects from that seen in the sex-chromosomes; and we must not lose sight of the wide difference of behavior in other respects that differentiates the latter from the former. It is possible that the sharply marked process of conjugation and disjunction characteristic of the sex-chromosomes and $m$-chromosomes may becorrelated with their specific functional relations. The case for the autosomes must therefore rest upon their direct study, and a reduction-division can only be fully established by tracing the bivalents as double bodies or 'gemini' through every stage from the time of their conjugation to that of their disjunction.

Whatever view of synapsis be adopted, this is a difficult task. If we take the view that the chromosomes are arranged in linear series in a spireme-thread which breaks into bivalent segments each consisting of two chromosomes in telosynaptic union there is no guarantee, as far as I can see, that the latter ultimately separate at the synaptic point. If we accept parasynaptic conjugation the difficulty is of a different kind, namely, the extremely close union of the conjugants side by side, which as nearly all observers are agreed, follows upon synapsis. The most that has been asserted by these observers has been that evidence of longitudinal duality can always be seen in some of the bivalents at every stage. Without reviewing all these cases, I will only recall that in the case of Batracoseps, for example, Janssens says, "Pendant le long stade du bouquet, les anses sont simples et par aucune méthode cytologique nous ne parvenons à y reconnaître la moindre trace de dualité" ('05, p. 401). In case of Tomopteris the Schreiners admit that at a period shortly following synapsis no longitudinal division can be seen in the pachytene-threads; and these authors are compelled to fall back upon indirect evidence in support of their conclusion that the duality is not really lost. Again, in Montgomery's recent work on Euschistus ('11) he expresses the conviction that there is no valid evidence of any actual fusion between the conjugants; yet in point of fact states that after the completion of synapsis "The autosomal loops (bivalents), are in one-half the normal number and, for the most 
part, each of them appears solid and undivided." The most that can be said appears to be that "in the greater number of cases there is to be seen in each geminus at least traces of a clear space which marks the original point of meeting of two univalents" ('11, p. 738). It seems to me that this is hardly a sufficient basis for so important a conclusion. Many similar statements might be cited from other authors. On the other hand some very competent observers not only find no evidence of duality in the early post-synaptic bivalents but definitely conclude that the conjugants completely fuse to form 'zygosomes' or 'mixochromosomes' (e.g., Vejdovsky, '07, for the Enchytraidae, Bonnevie, '08, '11, for Allium and other forms, Winiwarter and Saintmont, '09, for the cat).

In the case of Batracoseps I can fully confirm Janssens's statement that no evidence of longitudinal duality can be seen in the pachytene-loops throughout the greater part of the growthperiod, even in the most perfect preparations, and after various modes of fixation, staining and extraction. It is only in the earlier period that the duality appears, and then of ten only here and there and in a small portion of the thread. It is the same in Tomopteris. The longitudinal cleft often so clearly seen at the time of synapsis seems soon to disappear, so that for a time nothing can be seen in the pachytene-threads to indicate their bivalent nature. Theoretically it is of course quite possible that this appearance is deceptive, and that the two elements are in reality always distinct; but if we resort to theory an equally strong case can be made out, I think, in favor of partial or complete fusion. It seems at any rate certain that in some of the most favorable material thus far found among animals, synapsis is followed by a union so intimate that no adequate evidence of duality is afterwards seen until the diplotene stage is reached in the prophases of the first division. It is very possible that this may be due in some cases to a close twisting together of the threads (cf. p. 399) but it would hardly be safe to accept such an explanation at present.

I am myself inclined to accept the evidence at its face value, and to conclude that parasynapsis is followed by at least a partial 
fusion of the two conjugants; and that the synaptic processinvolves not merely an association of the chromosomes to form 'gemini' but a process of reconstruction which may profoundly change their composition (cf. Boveri, '04). I am, however, by no means in agreement with those writers who for a similar reason would reject in toto the conception of the reduction-division in the case of these chromosomes. Very important evidence upon this point is afforded by the contrast in structure and behavior between bivalents and univalents in the maturation-divisions; and this has not yet received sufficient attention on the part of writers on this general subject. In the first place, it is a rule, without exception so far as I am aware, that univalent chromosomes divide but once (of course equationally) in the course of the two maturation-divisions, while bivalents divide twice. The additional division in case of the bivalents must, therefore, be in some manner a consequence of synapsis. In the second place, the difference between univalents and bivalents is often clearly displayed in a characteristic difference of structure in the prophases. In the insects that I have studied the former are always bipartite bodies, the latter often quadripartite obviously in preparation for a single division in the former case, for two divisions in the latter. The best examples of these facts are offered by the sex-chromosomes; but they are also exhibited by the $m$-chromosomes of Hemiptera, and by certain anomalies sometimes seen in the autosomes.

Perhaps the most striking of these cases is that of the $X$-chromosome because of the different conditions seen in different species. In some forms this chromosome is accompanied by a synaptic mate (the $Y$-chromosome) with which it unites to form a bivalent. before the spermatocyte-divisions (Coleoptera, Diptera); in other species $X$ and $Y$ divide as separate univalents in the first division and afterwards conjugate (many Hemiptera); while in still others $Y$ is missing and $X$ is always univalent. In the first case the $X Y$ bivalent 'divides' in both spermatocyte-divisions - reductionally in the first, equationally in the second, as may be clearly seen because of the inequality of $X$ and $Y$ (Stevens). In the second case (e.g., Oncopeltus Lygaeus) this order is reversed, the first division being of course equational, the second reductional. In 
the third case $\mathrm{X}$ divides in but one spermatocyte-division (either the first or the second according to the species) and in the other passes undivided to one pole. It is here perfectly clear, as has been urged by McClung ('01, '02) and myself ('05 c), that the failure to divide in one division is due to the absence of a synaptic mate; and it is thus rendered doubly certain that in case of the $X Y$-pair but one true division (an equational) takes place, the other 'division' (reductional) being merely the separation of the synaptic mates. This is, I think, a conclusive demonstration in the case of these chromosomes of the reality (1) of the conception of bivalence, (2) of the reduction-division in its original and unmodified sense. That these conclusions are not limited to the sex-chromosomes is shown by the $m$-chromosomes of the Hemiptera, which have no relation to sex as far as known. In this case conjugation (synapsis) is usually delayed until the last possible moment before the first division. Their separation, which immediately ensues, is again a true reduction-division of the $m$ bivalent; but what we here call a 'division' is obviously not properly such but only the disjunction of two distinct bodies that have but just come into momentary contact. In this case, as in that of the $X Y$-pair, the term reduction 'division' is a misnomer. But one actual division takes place, the equation-division. This is fully borne out by the interesting anomaly that I described in a single individual of Metapodius in my sixth 'Study,' consisting in the presence of three $m$-chromosomes instead of two. Here all three uniformly couple to form a triad element in the first division, which immediately breaks up into its components, of which one passes to one pole and two to the other. In the second division all three divide equationally, so that half the spermatids receive but one $m$-chromosome half two. This is, of course, exactly in accordance with expectation; and it is a remarkable fact that the two $m$-chromosomes that are present in half the secondary spermatocytes do not disjoin but divide equationally; as they should.

The case of the autosomes is different, owing to the intimate union and possible fusion that follows synapsis; and it seems probable that the reduction-division must here be regarded in a differ- 
ent light. The history of these chromosomes as contrasted with that of those just considered, nevertheless affords some important evidence bearing on this question. As has been stated, the prophase-bivalent is of quadripartite composition (though this may fail to become visible until the later prophases) while the prophase-univalent is bipartite. This has already been emphasized in the case of Oncopeltus, but may studied to still greater advantage in Protenor, because of the greater size of the chromosomes and of their very marked individual size-differences. In the male of this form, as heretofore described by Montgomery, Morrill and myself, the spermatogonial groups contain thirteen chromosomes, and the unpaired $X$-chromosome is very nearly twice the size of the largest pair of autosomes (photos. 35, 36). In the female two such $X$-chromosomes are present (photos. 37,38 ). In the male the $X$-chromosomes of course remains univalent throughout the entire maturation-process, while the large pair of autosomes produces a bivalent that is of nearly the same size as the univalent $X$. The latter is at every period distinguishable. In the earlier stages of the growth-period, when the autosomes are in a diffuse and lightly-staining condition, it remains compact, in the form of a somewhat elongate vermiform body, that is closely coiled about or within a plasmasome to form a rounded "chromosome-nucleolus the true nature of which only appears after considerable extraction (cf. Montgomery, '01, fig. 127). In smears (as is the rule among the Hemiptera) the plasmosome usually collapses, setting free the $X$-chromosome, when its rod-like form becomes clearly apparent (photo. 39). In later stages it shortens, thickens and splits lengthwise, so as to appear in the prophases as a rather short; thick rod, very plainly split (figs. 120-131, photos. 40 to 51). The large bivalent, on the other hand, first becomes recognizable in the prophases, as the process of condensation occurs, when it may be studied to the best advantage in smear-preparations, in which all the chromosomes are spread out in one plane.

In such preparations, of which I have a large number, the large bivalent is invariably distinguishable by its large size-nearly twice that of any of the others; and we thus have opportunity to compare it accurately, side by side in the same nucleus, with a 
univalent chromosome $(X)$ of the same size. It is most interesting to observe in Protenor the gradual emergence of the bivalents from the confused nuclear threadwork of Stage $g$. Early stages of the process are seen in figs. 115 to 117 , which clearly demonstrate (1) that the threads do not constitute a continuous spireme, but are separate, (2) that they do not lie side by side in pairs to form a diplotene, but are single and undivided. Figs. 118 and 119 show two nuclei, only a little later than the preceding, in which all the bivalents are clearly seen and the large one is perfectly evident. It is of course only now and then that a nucleus in this stage can be found in which all the chromosomes are thus clearly distinguishable; the bivalents are still so extended and irregular as to present a hopelessly confused picture in sections, and very frequently also in smears. It is however my firm belief that the bivalent-figures, intricately entangled though they are, are already quite distinct at least as early as fig. 115, and I do not hesitate to accept this as probable for the still earlier and more confused nuclei that precede.

From the latter part of Stage $h$ (i.e., figs. 118, 119) every step may readily be followed as the chromosomes continue to condense, contract and increase in staining capacity. Of the innumerable nuclei showing these stages in my smear-preparations a few are shown in figs. 120 to 131, and in photos. 40 to 51. As these figures show, the typical number of separate chromatinbodies is eight, which may however be reduced to seven by the coupling of the two smallest ( $m$-chromosomes, figs. 124, 129, 130 , photos. $45,46,48$ ), or in certain rare abnormalities may be increased to more than eight (fig. 128, photo. 43). Of these eight, three are univalent, namely, the two smallest ( $m$-chromosomes) and the large $X$-chromosomes, the latter always distinguishable by its more compact consistency, greater staining capacity, rod-like form, and simple longitudinal split.

The remaining five are the bivalent autosomes, which from the beginning have the same forms as in Oncopeltus-i.e., double crosses, double $V$ 's, or longitudinally split rods which sooner or later develop a transverse suture at their middle points and thus are plainly seen to be of quadripartite nature. 
A particular interest attaches to the striking and constant contrast between the $X$-chromosome and the large bivalent; I do not here refer to their conspicuous difference in texture and staining capacity in the earlier stages but to a characteristic difference in morphological composition that persists up to the very metaphase of the first division. The $X$-chromosome is always bipartite - a simple rod, longitudinally split. Sometimes it is curved, sometimes slightly constricted at the middle point, sometimes irregular in form; but many of these variations are evidently quite accidental. It never shows the least approach to the double cross form nor does a transverse suture at the middle point make its appearance. In the final prophase it enters the spindle at right angles to. the latter, and undergoes a longitudinal division (figs. 132 to 134, cf. Montgomery, '01, '06, Wilson, '11 a). The large bivalent, on the other hand, is always, sooner or later, a quadripartite body. In most cases it forms a fine double cross, of the same type as that already described in case of Oncopeltus, and showing the same variations. All gradations are found, in different nuclei, on the same slide, between forms in which the arms of the cross are equal (figs. 120,122, 123, photos. $40,41,49$ ) and those in which one pair (the 'lateral' arms) are but just perceptible (figs. 121, 124, 125). In some cases, comparatively rare, the lateral arms are quite wanting, and the bivalent appears as a longitudinally split rod (figs, 126, 129, 130) but in these forms a distinct transverse cleft or suture is often seen at the middle point; and in a few cases the rod is sharply bent at this point to form a $V$-shaped figure (fig. 127). Sometimes the transverse cleft is not seen in the earlier stages: but always in the later prophases it becomes evident (figs. 129, 130, 131, photos. 48, 51). In these stages, as in Oncopeltus, the lateral arms of the double crosses sooner or later disappear, being apparently progressively drawn in to the axial arms, and in their place appears first a transverse suture and later a constriction across which the first division takes place. In the early metaphases the large bivalent shows two extreme types, connected by all intermediate transitions. At one extreme are ring-like tetrads (fig. 133) which are evidently derived from crosses by shortening of the arms and per- 
sistence of the central opening. At the other extreme are short tetrad-rods (fig. 132) which clearly show two division-planes at right angles to each other. These forms may be derived either from the more elongate rod-like forms of earlier stages or from crosses by disappearance of the lateral arms. In both types the quadripartite structure is unmistakable. The large bivalent ultimately assumes a dumb-bell form, with its longer axis parallel to that of the spindle-axis, and undergoes a 'transverse' division; while, as already stated, the $X$-chromosome always enters the spindle with its long axis transverse to that of the spindle, and undergoes longitudinal division (figs. 132, 134; see also Wilson, '11, fig. 9, Montgomery, '01, figs. 136, 138).

No observer who studies these nuclei attentively can fail to be struck by the remarkable difference between the large bivalent and the large $X$-univalent. Its explanation is obvious; the former is preparing to divide twice, the latter once, in the course of the two maturation-divisions: But this does not yet touch the root of the matter. We have still to ask why two chromosomes of equal size in the same nucleus differ so widely in respect to their mode of division. The reply is again obvious. It is because one of them has double the chromosomic value (or valence) of the other, the bivalent representing two chromosomes of the original diploid groups, while the univalent represents but one. This conclusion, which is hardly more than a statement of fact, is confirmed by a very interesting anomaly shown in fig. 128, and in photo. 43. This nucleus contains nine chromosomes, and the large bivalent is absent as such, while in its place appear two separate and equal chromosomes of half its size $(B, B$ in the figure), while the four smaller bivalents are plainly recognizable $(b-b)$. The explanation obviously is that through an abnormality of synapsis the two members of this particular pair have failed to unite and have therefore remained univalent. Both of these chromosomes have the form of simple, longitudinally divided rods, without trace of a quadripartite structure, while the four smaller bivalents all show the cross-form (though the lateral arms are but slightly developed in one of them). It may be surmised, I think, that if the later history these separate univalents could be followed out, they would be 
found to divide but once (equationally) in the course of the two spermatocyte-divisions, as in case of the $X$-chromosomes, or the $m$-chromosomes.

When these facts are taken together the conclusion seems to me unavoidable that one of the divisions of the bivalent chromosome (and hence, one of the division-planes seen in the bivalent prophase-figures) is a consequence of its bivalence-i.e., of its origin from two chromosomes instead of one, of the original diploid groups. An almost conclusive demonstration of this is given by the fact that when the $X$-and $Y$-chromosomes are united to form a bivalent in the prophases, this body, like the others, often shows a tetrad structure (as in Brochymena, Wilson, '05 b, or Nezara, Wilson, '11 a); and in the case of Ascaris felis, recently described by Edwards ('11) this bivalent has a double cross-form, closely similar to that of the other bivalents save for the inequality of two of the components (op. cit., fig. 2). I do not mean to imply that either division-plane of the tetrad represents the actual plane of separation of the same two chromosomes that have united in synapsis; on the contrary, I think it probable, as already indicated, that the original chromosomes may have undergone reconstruction. What may be said is that one division is independent of bivalence, the other a consequence of it; and it is further clear that the former effects no reduction of valence, while the latter does. Whether we regard the autosome-bivalent as to its origin or its fate, it has, irrespective of its relative size, double the chromosomic value of a univalent in the maturation-process; and in this respect it is exactly comparable to an $X Y$-bivalent or an $m$ bivalent, in which one of the divisions is demonstrably a reduction-division in the original sense. This value, or 'valence' is reduced to one-half in one of the maturation-divisions. May we not here find a definition of the reduction-division that may be accepted even by those who deny the individuality of the chromosomes, or who believe that synapsis is followed by actual fusion? We may define an equation-division as one that effects no reduction of valence, a reduction-division as one that reduces the valence to one-half. This conforms exactly to the observed facts; and such a definition is, I think, equally consistent with complete disjunc- 
tion or with a process of reconstruction subsequent to synapsis. I do not see how the analysis can be carried further without entering upon theoretical ground. Nevertheless, I do not hesitate to accept the probability that the reduction-division, as thus defined, involves a disjunction of chromatin-elements of some kind that are involved in the production of the unit-factors of heredity and that.the Mendelian disjunction may here find its explanation (cf. De Vries, '03, Boveri, '04). It seems to me that the conclusions indicated by Boveri several years ago ('04) still remain the most probable; that is to say, that the degree of union may vary in different cases, involving sometimes no fusion (as is suggested by the history of the $X Y$-pair), sometimes complete fusion, in other cases no more than a partial exchange of material. This point will be again touched upon in connection with Janssens's theory of the "chiasmatype."

The point that I wish here to emphasize is the validity of the conceptions of bivalence and the reduction-division, which have been more or less explicitly denied by a number of writers. I accept, of course, the conclusion of Haecker ('07), Bonnevie ('08, '11), Della Valle ('07), Popoff ('08) and others, that neither the heterotypical form of division nor an apparent tetrad-structure is necessarily diagnostic of bivalence or a reduction-division. Tetrad-like chromosomes have been repeatedly described in somatic divisions (see especially Della Valle, Popoff, cited above), and also in the univalent chromosomes of the second maturation-division a striking example of which is the ' $d$-chromosome' of Nezara, described in my seventh 'Study.' Bonnevie, especially, has demonstrated in Nereis and other forms the very close similarity of the somatic chromosomes (in the cleavage of the egg) to the heterotype-rings of the maturation-divisions. Her conclusion is:

Ich habe in meinen Objekten nicht nur für die Annahme einer Reduktionsteilung keinen einzigen Beweis finden können; meine Untersuchung hat auch ergeben, dass die früher in der heterotypischen Natur der ersten Reifungsteilung gesehene Stütze einer solchen Annahme sämmtlich hinfällig sind . . Die beiden Reifungsteilungen müssen daher, bis anderes bewiesen worden ist, als Aequationsteilungen aufgefasst werden ('08, p. 271, '11, p. 241). 
Again, from the existence of tetrad-shaped chromosomes in certain somatic divisions of Amphibia, Della Valle ('07) concludes, "Tutte le precedente formazioni e molto probabilmente, quando esistono, anche quelle della profase del primo fuso di maturazione, non hanno alcun rapporto con la reduzione cromatica, ma sono indice di una costituzione patologica dei cromosomi"(!) Both the foregoing passages, I think, like others of like import that might be cited, overshoot the mark. The significance of ringshaped or tetrad-like chromosomes must be judged from a more critical standpoint than this. We must endeavor to discover their real meaning in each case by the study of their origin, their behavior in successive divisions, their morphological composition (whether simple or compound bodies), their relation to the conditions seen in other species, and any other facts that may throw light upon them. By way of illustration certain specific cases may be mentioned. A very interesting one is afforded by the $X$-chromosome of Syromastes (Gross, '04, Wilson, '09 a,'09 b), which is unaccompanied by a synaptic mate ( $Y$-chromosome) yet forms a conspicuous 'tetrad' in the first spermatocyte-division. The reason here is that this 'chromosome' consists of two components, which appear as separate chromosomes in the spermatogonial groups but in the maturation-divisions are associated to form a double element which behaves precisely like the single $X$-chromosome of other species, and obviously corresponds to the latter because four such components are present in the diploid groups of the female (Wilson, '09 b). The ' $d$-chromosome' of Nezara is a different but not less interesting case, always forming a conspicuous 'tetrad' in the second spermatocyte-division, but appearing as a single chromosome in the diploid groups. These two apparently contradictory cases are brought under the same point of view-especially when compared with the analogous relations described by Morgan ('09) in Phylloxera, and by Browne ('10) in Notonecta - if we assume that in each case the chromosome in question was originally a single body which in Nezara shows a slight tendency to separate into two components, in Syromastes has already done so (cf. Wilson, '11 a). Neither case offers 
a real exception to the rule; for such 'tetrads' obviously differ entirely from the true tetrads of the maturation-processes, which represent synaptic pairs of the diploid groups. Perhaps an analogous case is that of Cyclops and Diaptomus, where Haecker ('95, '02) long since demonstrated a cross-bar or suture in each component of each bivalent; and since each of these component, is sooner or later longitudinally divided, double tetrads or 'ditetrads' are thus produced. The work of Braun ('09) and Matschek ('09, '10) confirms this but, like that of Haecker himself, proves that the cross suture is without significance for the divisions, since both the latter are longitudinal (cf. also Lerat, '05); while Krimmel ('10) has shown that in Diaptomus the transverse constriction or suture is also present in the univalent chromosomes of the diploid groups in somatic cells. Haecker's conclusion that the cross-suture represents the point of end to end synapsis is a quite unproved, and I think very improbable, assumption. In view of what is seen in Syromastes, Notonecta, Phylloxera and Nezara, it seems more likely that each univalent chromosome in these forms consists of two closely united components, of which the cross-suture is an expression. It seems quite possible that the number of chromosomes might change in these animals by complete separation of the two components in case of one or more of the chromosomes.

Keeping in view all such apparent exceptions, the fact remains that the heterotypic (or tetrad) form is a highly characteristic feature of the maturation prophase-bivalents, and that in this respect they show in general a marked contrast to the univalents, here and elsewhere. The meaning of this in case of the maturation-divisions is unmistakable; and the burden of proof may be left with those whose theoretical prepossession will not allow them to accept the natural explanation that here is manifest.

\section{The chromosomes and heredity}

That the chromatin-substance (more specifically that of the chromosomes) plays some definite rôle in determination has 
received fresh support in recent years from several sources. Direct experimental evidence that is nearly if not quite demonstrative has been produced through the work of Boveri ('07) on multipolar mitosis, of Baltzer ('09, '10) on reciprocal crosses in sea-urchins, and of Herbst ('09) and Godlewski ('11) on the combined effects of artificial parthenogenesis and fertilization in hybridization. Indirect but very strong evidence has been given by the demonstration of a constant relation between particular chromosomes and sex and especially sex-limited characters (cf. Wilson, '11 a, b, Morgan, '11, Gulick, '11). This evidence in no manner precludes the view that the protoplasmic substances are also concerned in determination-indeed experimental embryology and cytology have produced very clear evidence that such is the case. The study of the nucleus, and especially of the chromosomes, offers however one of the most available paths of approach to a study of the activity of the germ-cells in determination, and for a detailed analysis of genetic problems in their cytological aspects.

It has been widely assumed that the Mendelian segregation depends upon a disjunction of chromatin-elements in the reductiondivision, as was originally suggested by Guyer, Sutton, Boveri and Cannon. It has, however, becomes obvious from the experimental data that if this be so, these elements can not be individual chromosomes of fixed composition. This was first seen to follow from the fact, now apparently well determined in certain cases, that the number of independent allelomorph-pairs may be greater than the number of chromosome-pairs. More recently the same result is demonstrated by the work of Bateson and Punnett ('11) on 'coupling' and 'repulsion' in certain plants, and by that of Morgan ('11 a) on sex-limited characters in Drosophila, which proves that an interchange of unit-factors must to some extent take place between homologous bearers of these factors in the germ-cells. The results of Morgan in particular nevertheless bring strong support to the view that the chromosomes are such bearers of unit-factors; for the whole series of phenomena determined in Drosophila, complicated as they seem, become at once intelligible under the assumption that certain factors necessary 
for the production of the sex-limited characters are born by the $X$-chromosome; and without this assumption they are wholly mysterious.

Adopting this explanation, the history of certain of these sexlimited characters, as Morgan points out, demands the further assumption that in the female the factors for these characters may to some extent undergo an interchange between the two sex-chromosomes (here two $X$-chromosomes) while in the male such interchange does not take place. Such a difference between the sexes finds a perfectly simple explanation in cases where the $X$-chromosome of the male has no synaptic mate ( $Y$-chromosome). When a $Y$-chromosome is present-as may be the case in Drosophila according to the cytological observations of Stevens ('08)the problem becomes more complicated, but there are some facts that may be significant in this direction. It is well known that in the male the sex-chromosomes commonly retain a compact and rounded form (as 'chromosome-nucleoli') throughout the entire spermatogenesis, and in some cases (Oncopeltus) they conjugate while in this condition, and subsequently disjoin without ever having undergone fusion or even intimate union, such as is so characteristic of the autosomes during the maturation-process. Unfortunately the oögenesis is as yet but imperfectly known; but there is considerable evidence that in some forms the sexchromosomes exhibit a quite different behavior from that characteristic of the spermatogenesis. My own observations ('06) seemed to show that in some of the Hemiptera the sex-chromosomes do not in the oöcyte retain a compact form at the period of synapsis, or in the early growth-period, and the later observations of Foot and Strobell ('07) show that the same is true for later stages of the germinal vesicle. The work of Morrill ('10) further shows that in the female the sex-chromosomes have already conjugated before the first oöcyte-division. These fact make it probable that in these forms the sex-chromosomes of the female show the same behavior in synapsis and reduction as the autosomes, and enter into the same intimate union that characterizes the latter. It is quite possible that in these facts we may find an 
explanation of the difference of the sexes in respect to the interchange of sex-limited factors that is proved to take place by the experimental results. ${ }^{18}$

There is no a priori reason why such a process of free interchange between homologous chromosomes may not take place in the somatic or diploid. nuclei. It is, however, far simpler to assume that it occurs during or subsequent to synapsis; for it is only at this period that the homologous chromosomes are intimately and regularly associated (cf. Boveri, Strasburger, '08, '09). It is these facts, taken together with the cytological evidence, that lead me to the conclusion already indicated that the synaptic union results in a reorganization of the chromosomes, and that the two moieties of the final prophase-chromosomes are probably not identical with the original conjugants. Janssens's theory of the chiasmatype ('09) gives the only simple mechanical explanation thus far offered as to how such an orderly exchange of materials may be effected; and his conception has recently been applied in an ingenious manner by Morgan (' $11 \mathrm{~b}$ ) to an explanation of both 'repulsion' and 'coupling' in heredity. The chiasmatypetheory has been criticized by Grégoire and by Bonnevie on the ground that a strepsinema stage occurs in the division of somatic nuclei as well as in the maturation-prophases, and that the original twisting together of the threads is in some cases followed by untwisting, without such a process of partial fusion and subsequent secondary splitting as is postulated by Janssens. There are two replies to this. One is that Janssens's theory is not an a priori construction but a conclusion based on a most accurate and detailed study of the actual conditions seen in the prophases of Amphibia which prove that such a process as he postulates must here take place. The other is that there is considerable evidence that a twisting together of the conjugating threads takes place in the process of synapsis, leading to a most intimate union of the

${ }^{18}$ It would, however, be rash to generalize this statement at present, for the observations of Buchner on the female Gryllus ('09) and those of Winiwarter and Saintmont ('09) on the cat, have demonstrated a nucleolus-like body in the synaptic and later stages of the oocytes which may be the $X X$-bivalent, though this is unproved, and doubt is thrown upon the second of these cases by the recent work of Gutherz ('12). 
two members of each pair, and followed by a longitudinal split. It would, no doubt, be premature definitely to accept this conclusion at present; but it seems worthy of more attentive consideration than it has yet received.

Turning to the more general aspects of these problems, in what sense may we be justified in speaking of the chromosomes as bearers of the 'determiners' or factors of determination? I have recently outlined in another place (Wilson, '12) my position in regard to this question, and will here only indicate its most essential features. The 'determiners' or 'factors' on which unit-characters depend need not be regarded as independent, self-propagating germs (pangens, biophores, or the like); it is sufficient for our purpose to think of them in a more vague way as only specific chemicals entities of some kind. However they are conceived in this regard, it would be a fundamental error to regard them as 'bearers' of the characters that depend upon their presence or absence; for every character is produced as a reaction of the germ considered as a whole or unit-system. Characters are 'borne' (if the expression is permissible at all) by this system as a whole; and the 'unit-factors' or 'determiners' postulated by students of genetics need be considered only as specific, differential factors of ontogenetic reaction in a complex organic system. Many 'unit-characters' are known to depend upon a number of such unit-factors, in some cases probably upon a large number; and they may be definitely altered this way or that by varying the particular combinations of these factors. But any unit-factor produces its characteristic effect only in so far as it forms a part of a more general apparatus of ontogenetic reaction constituted directly or indirectly by the organism as a whole. In all this, a striking parallel exists between the physical basis of heredity and the complex molecular groups of organic substances such as the proteins. The relation of the 'determiners' to the qualities of the organism considered as a whole may be compared to that which exists between the protein 'Bausteine' and the qualities of the protein molecular group as a whole.

"Just as the qualities of a particular protein may be definitely altered by the addition, subtraction or the substitution one for another of parti- 
cular side-chains or molecular 'Bausteine,' so the addition, subtraction or substitution of particular 'determiners' or 'factors' in the zygote. calls forth specific responses that lead to the production of corresponding characters. The reasoning that applies to the first of these cases seems equally applicable to the second. No one, I suppose, would hold in the first case that the particular molecular groups or 'Bausteine' concerned in the change are 'bearers of' (i.e., are alone responsible for) the resulting new qualities. The qualities of any protein, as Kossel has recently urged, belong to the molecule as a whole, and are not to be regarded as the sum of the qualities of its constituent 'Bausteine.' Why should we regard in a different light the 'determiners' (chemical substances?) concerned in the second case? They are, clearly, not to be regarded as 'bearers' or 'physical bases' of the characters which depend upon their presence or absence. They are, I repeat, only differential factors of ontogenetic reactions that belong to the germ considered as a whole or unit-system"' (Wilson, '12).

- Kossel ('12) makes the pregnant remark that every peculiarity of the species and every occurrence affecting the individual may be indicated by special combinations of protein 'Bausteine.' The facts lead us to seek for such compounds (substances) in the chromatin or the chromosomes. It can hardly be said that even a beginning has been made.in the chemical investigation of the distribution of the chromatin-substances within the nucleus. Cytologically, however, a long series of the most significant facts have been made known in respect to their groupings and modes of distribution. Evidence steadily accumulates that these processes are perfectly ordered; and the fact is now more than ever evident that they run parallel to the factors of determination and heredity. There has been a disposition on the part of certain writers of late to minimize the definite order of the morphological transformations of the nucleus (cf. Fick, '07, Della Valle, '09); and these authors, among others, have undoubtedly helped to create an impression that these phenomena, particularly as regards the chromosomes, are too vague and fluctuating to afford trustworthy results on the side of cytological research. I believe this to be a backward step, though I am very ready to admit the service to accuracy of observation that may be rendered by so critical and sceptical a spirit. Plastic, and in some respects variable like other biological phenomena, these processes undoubtedly are; but the more one studies them in detail the stronger grows the con- 
viction of an exact order that underlies their superficial fluctuations, and one of which the main outlines are steadily becoming clearer.

It appears to me that many of the recent cytological advances support the view that the true key to this order was found by Flemming when he chose the word 'mitosis' (' 82 ), and by Roux in his attempt to find the essential meaning of the mitotic process ('83). This is well illustrated by the pre-synaptic phenomena that have here been considered, and by the increasing body of observations that emphasize the importance of the mitotic transformation of the chromatin-substance. It is difficult to see what meaning such processes can have if they do not involve a linear alignment of different elements (substances) which are thus brought into a particular disposition for ensuing processes of division (Roux) or of paired association (Strasburger). ${ }^{19}$ The practical utility of such a conception for the analysis of genetic problems has already become apparent. It is still only an hypothesis, but one which we may hope sooner or later to see subjected to definite experimental test.

March 1, 1812.

\section{LITERATURE CITED}

AgAR, W. E. 1911 The spermatogenesis of Lepidosiren paradoxa. Quart. Journ. Mic. Sci., n. s., no. 225, vol. 87, part I.

Arnold, G. A. 1908 The nucleolus and microchromosomes in the spermatogenesis of Hydrophilus piceus. Arch. f. Zellforsch., Bd. 2, no. 1.

BALtzer, F. 1909 Ueber die Entwicklung der Echinidenbastarde mit besonderer Berücksichtigung der Chromatinverhältnisse. Zool. Anz., Bd. 5.

1910 Ueber die Beziehung zwischen dem Chromatin und der Entwicklung und Vererbungsrichtung bei Echinodermenbastarden. Arch. f. Zellforsch., Bd. 5.

Bateson and Punnett 1911 On the inter-relation of genetic factors. Proc. Roy. Soc. Eng., B., vol. 84.

Berghs, J. B. 1904 La formation des chromosomes hétérotypiques. II. Depuis la sporogonie jusqu'au spirème definitif. La Cellule, tom. 21, no. 2.

19 This point has been forcibly urged by Strasburger (see '08, pp. 563-568; '09, pp. 95-97). 
Bonnevie, K. 1907 'Heterotypical' mitosis in Nereis limbata. Biol. Bull., vol. 13 , no. 2 .

1908 a Chromosomenstudien, I. Chromosomen von Ascaris, Allium und Amphiuma. Arch. f. Zellforsch., Bd. 1.

1908 b Chromosomenstudien, II. Heterotypische Mitosen als Reifungscharakter. Ibid., Bd. 2.

1911 Chromosomenstudien, III. Chromatinreifung in Allium cepa. Ibid., Bd. 6.

Boveri, Th. 1904 Ergebnisse über die Konstitution der chromatischen Substanz des Zellkerns. Jena.

1907 Zellen-Studien, VI. Die Entwicklung dispermer Seeigel-Eier. Ein Beitrag zur Befruchtungslehre und zur Theorie des Kerns. Jena.

BraUn, H. 1909 Die specifische Chromosomenzahlen der einheimischen Arten der Gattung Cyclops. Arch. f. Zellforsch., Bd. 3, no. 3.

Browne, E. N. 1910 The relation between chromosome-number and species in Notonecta. Biol. Bull., vol. 20, no. 1.

Brunelui, G. 1910 La Spermatogenese della Tryxalis. I. Divisioni spermatogoniale. Mem. Soc. Ital. Sci., Ser. 3, tom. 16.

1911 La Spermatogenese della Tryxalis. II. Divisioni maturative. Reale Acc. d. Lincei, Ser. 5a, tom. 8.

Buchner, P. 1909 Das akzessorische Chromosom in Spermatogenese und Ovogenese der Orthopteren, zugleich ein Beitrag zur Kenntnis der Reduktion. Arch. f. Zellforsch., Bd. 3.

1910 Uber die Beziehungen zwischen Centriol und Bukettstadium. Ibid., Bd. 5 .

Carnoy and Lebrun. 1897, 1898, 1899 La vesicule germinative et les globules polaires chez les batraciens. I, II, III, La Cellule, tom. 12, 13, 14 .

Davis, B. M. 1909 Pollen development of Oenothera grandiflora. Ann. Bot., vol. 23.

1910 The reduction divisions of Oenothera biennis. Ibid., vol. 24.

1911 Cytological studies on Oenothera, III. A comparison of the reduction divisions of Oenothera lamarckiana and $O$. gigas. Ibid., vol. 25 .

DAvis, H. S. 1908 Spermatogenesis in Acrididae and Locustidae. Bull: Mus. Comp. Zool. Harvard, vol. 52, no. 2.

Dehorne, A 1911 Recherches sur la division de la cellule. I. Le duplicisme constant du chromosome somatique chez Salamandre maculosa et chez Allium cepa. Arch. f. Zellforsch., tom. 6.

Della Value, P. 1907 Osservazioni di tetradi in cellule somatiche. Contributo alla conoscenza delle tetradi. Atti Acc. Napoli, tom. 13.

1909 L'organizzione della chromatina studiata mediante il numero dei cromosomi. Arch. Zoologico, tom. 4.

1911 La continuità della forme in divisione nucleare ed il valore mor. fologico dei cromosomi. Ibid., tom. 5.

DigBy, L. 1910 The somatic, premeiotic and meiotic nuclear divisions of Gal tonia candicans. Ann. Bot., vol 24. 
EDwards 1910 The idiochromosomes in Ascaris megalocephala and Ascaris lumbricoides. Arch. f. Zellforsch., Bd. 5.

1911 The sex-chromosomes in Ascaris felis. Ibid., Bd. 7.

Farmer and Moore 1905 On the meiotic phase (reduction divisions) in animals and plants. Quart. Journ. Mic. Sci., vol. 48, no. 4 .

FICK, R. 1907 Vererbungsfragen, Reduktions- und Chromosomenhypothesen, Bastard-Regeln. Merkel u. Bonnet's Ergebnisse, Bd. 16.

1908 Zur Konjugation der Chromosomen. Arch. f. Zellforsch., Bd. 1.

Foot AND STRoBell 1907 The nucleoli in the spermatocytes and germinal vesicles of Euschistus variolarius. Biol. Bull., vol. 16.

Fraser and Snell 1911 The vegetative divisions in Vicia faba. Ann. Bot., vol. $25, \mathrm{c}$.

Gates, R. R. 1907 Pollen development in hybrids of Oenothera lata x O. lamarckiana and its relation to mutation. Bot. Gaz., vol. 43.

1908 A study or reduction in Oenothera rubrinervis. Ibid., vol. 46.

1909 The behavior of the chromosomes in Oenothera lata x O. gigas.

Ibid., vol. 48.

1911 The mode of chromosome reduction. Ibid., vol. 51.

Geerts, J. M. 1909 Beiträge zur Kenntniss der Cytologie und der partiellen. Sterilität von Oenothera lamarckiana. Rec. Trav. Bot. Néerland., Bd. 5.

1911 Cytologische Untersuchungen einiger Bastarde von Oenothera gigas. Ber. d. deutsch. Bot. Ges., Bd. 29, no. 3.

Gerard, P. 1909 Recherches sur las spermatogénèse chez Stenonothrus biguttulus. Arch. Biol., Bd. 24.

Gregoire, V. 1904 Le réduction numérique des chromosomes et les cinèses de maturation. La Cellule, tom. 21.

1905 Les résultats acquis sur les cinèses de maturation dans les deux règnes. I. Ibid., Tom. 22.

1906 La structure de l'élément chromosomique au repos et en division dans less cellules végétales. Ibid., Tom. 23 .

1907 La formation des gemini hétérotypiques dans les végétaux. Ibid. Tom. 24.

1910 Les cinèses de maturation dans les deux règnes. II. Ibid., Tom. 26.

GodLewski, E. 1911 Studien über die Entwicklungserregung, I. Kombination der heterogenen Befruchtung mit der künstlichen Parthenogenese. Arch. f. Entwicklm., Bd. 33, nos. 1, 2.

Goldschmidt, R. 1906 Review of Schreiner ('06) in: Zool. Centrb., Bd. 13, nos. $19,20$.

1908 a Ueber das Verhalten des Chromatins bei der Eireifung und Befruchtung des Dicrocoelium lanceatum (Distomum lanceolatum). Arch. f. Zellforsch., Bd. 1, no. 1.

1908 b Ist eine parallele Chromosomenkonjugation bewiesen? Ibid., Bd. 1 , no. 4 .

Gross,.J. 1904 Die Spermatogenese von Syromastes marginatus. Zool. Jahrb., Anat. u. Ontog., Bd. 20.

1907 Die Spermatogenese von Pyrrhocoris apterus. Ibid., Bd. 2\%. 
Golıck, A. 1911 Ueber die Geschlechtschromosomen bei einigen Nematoden, etc. Arch. f. Zellforsch., Bd. 6.

Gotherz, S. 1912 Ueber ein bemerkenswertes Strukturelement (Heterochromosom?) in der Spermiogenese des Menschen. Arch. Mik. Anat., Bd. 79, no. 2.

HAECKER, V. 1895 Ueber the Selbständigkeit der väterlichen und mütterlichen Kerbestandteile während der Embryonalentwicklung von Cyclops. Arch. Mik. Anat., Bd. 46.

1902 Ueber das Schicksal der elterlichen und gross-elterlichen Kernanteile. Jen. Zeitschr., Bd. 37.

1907 Die Chromosomen als angenommene Vererbungsträger. Ergebn. Fortschritt. Zool., Bd. 1.

1910 Ergebnisse und Ausblicke in der Keimzellforschung. Zeitschr. f. indukt. Abst. u. Verebungslehre. Bd. 3.

Herbst, C. 1909 Die cytologischen Grundlagen der Verschiebung der Vererbungsrichtung nach der mütterlichen Seite. Arch. f. Entwicklm. Bd. 27. no. 2 .

Janssens, F. A. 1901 La spermatogénèse chez les tritons. La Cellule, tom. 19. 1905 Evolution des Auxocytes males du Batracoseps attenuatus. Ibid., tom. 22.

1909 La théorie-de la chiasmatypie. Nouvelle interprétation des cinèses de maturation. Ibid., tom. 25.

JANSSENS ET DUMEZ 1903 L'élément nucléinien pendant les cinèses de maturation des spermatocytes chez Batracoseps attenuatus et Plethodon cinereus. Ibid., tom. 20.

JANSSENS ET Willems 1908 Spermatogénèse dans les batraciens. IV, Le spermatogénèse dans l'Alytes obstetricus. Ibid., tom. 25.

KING, H. D. 1907 The spermatogenesis of Bufo lentiginosus. Am. Jour. Anat., vol. 7 .

1908 The oögenesis of Bufo lentiginosus. Jour. Morph., vol. 19.

Kossel, A. 1912 The proteins. Herter Lecture, Johns Hopkins, Oct., 1911. Johns Hopkins Hospital Bull., March, 1912.

KrImmeL, O. 1910 Chromosomenverhältnisse in generativen und somatischen

- Mitosen bei Diaptomus coeruleus, etc. Zool. Anz., Bd. 35.

LEE, A. BoLLes 1911 Le réduction numérique et la conjugasion des chromosomes chez l'escargot. La Cellule, tom. 27.

Lefevre AND MCGiul 1908 The chromosomes of Anasa tristis and Anax junius. Am. Jour. Anat., vol. 8.

LERAT, P. 1905 Les phenomènes de maturation dans l'ovogénèse et la spermaotogénèse du Cyclops strenuus. La Cellule, tom 22, no. 1.

Matschek, H. 1910 Ueber Eireifung und Eiablage bei Copepoden. Arch. f. Zellforsch., Bd. 5 .

McClong, C. E. 1899 A peculiar nuclear element in the male reproductive cells of insects. Zool. Bull., vol. 2.

1901 Notes on the Accessory Chromosome. Anat. Anz., vol. 20. 1902 a The Accessory Chromosome-Sex Determinant? Biol. Bull., III.

1902 b The spermatocyte divisions of the Locustidae. Kansas Univ. Sci. Bull., 14, p. 8. 
Meves, F. 1907 Die Spermatocytenteilungen bei der Honigbiene nebst Bemerkungen über Chromatinreduktion. Arch. Mik. Anat., Bd. 70.

1908 Es gibt keine parallele Konjugation der Chromosomen. Arch. f. Zellforsch., Bd. 1.

1911 Chromosomenlänge bei Salamandra, nebst Bemerkungen zur Individualitätstheorie der Chromosomen. Arch. Mik. Anat., Bd. 77.

Montgomery, T. H. 1901 A study of the chromosomes of the germ cells of Metazoa. Trans. Am. Phil. Soc., vol. 20.

1904 Some observations and considerations upon the maturation phenomena of the germ cells. Biol. Bull., vol. 6 , no. 3 .

1906 Chromosomes in the spermatogenesis of the Hemiptera Heteroptera. Ibid., vol. 21.

1911 The spermatogenesis of an Hemipteron, Euschistus. Jour. Morph., vol. 22, no. 3.

Moore, J. E. S. 1895 On the structural changes in the reproductive cells of Elasmobranchs. Quart. Journ. Mic. Sci., vol. 38.

Moore AND Robinson 1904 On the behavior of the nucleolus in the spermatogenesis of Periplaneta americana. Quart. Jouın. Mic. Sci., vol. 48.

Morgan, T. H. 1909 A biological and cytological study of sex determination in Phylloxerans and Aphids. Jour. Exp. Zool., vol. 7, no. 2.

1911 a An attempt to analyze the constitution of the chromosomes on the basis of sex-limited inheritance in Drosophila. Jour. Exp. Zool., vol. 11, no. 4 .

$1911 \mathrm{~b}$ Random segregation versus coupling in Mendelian inheritance. Science, n. s., vol. 34 , no. 873.

Morrill, C. V. 1910 The chromosomes in the oögenesis, fertilization and cleavage of Coreid Hemiptera. Biol. Bull., vol. 19, no. 2.

Morse, M 1909 The nuclear components of the sex-cells in four species of cockroaches. Arch. f. Zellforsch., Bd. 3.

Motrier, D. M. 1907 The development of the heterotype chromosomes in pollen mother-cells. Ann. Bot., vol. 21.

1909 On the prophases of the heterotypic mitosis in the embryo-sac mother-cell of Lilium. Ibid., vol. 23.

MüLLER, C. 1909 Ueber karykinetische Bilder in den Wurzelspitzen von Yucca. Jahrb. f. Wiss. Bot., Bd. 47, no. 1.

Oetringer, R. 1909 Zur Kenntniss der Spermatogenese bei den Myriopoden. Samenreifung und Samenbildung bei Pachyiulus varius. Arch. f. Zellforsch., Bd. 3, no. 4.

Overton, J. B. 1905 Ueber Reduktionsteilung in den Pollenmutterzellen einiger Dikotylen. Histologische Beiträge zur Vererbungsfrage. Jahrb. f. Wiss. Bot., Bd. 42.

1909 On the organization of the nuclei in the pollen mother-cells of certain plants, with especial reference to the permanence of the chromosomes. Ann. Bot., vol. 23.

Padlmier, F. C. 1898 Chromatin reduction in the Hemiptera. Anat. Anz., Bd. 14.

1899 The spermatogenesis of Anasa tristis. Jour. Morph., vol. 15, Supplement. 
Payne, F. 1909 Some new types of chromosome distribution and their relation to sex. Biol. Bull., vol. 16.

Pinney, E. 1908 Organization of the chromosomes in Phrynotettix magnus. Kansas Univ. Sci. Bull., vol. 4, no. 14.

Popoff, M. 1908 Ueber das Vorhandensein von Tetraden-Chromosomen in den Leberzellen von Paludina vivipara. Biol. Centrb., Bd. 28, no. 17.

Robertson, W.R. B. 1908 The chromosome complex of Syrbula. Kansas Univ. Bull., vol. 4, no. 13.

Rosenberg, O. 1904 Ueber die Tetradenteilung eines Drosera-Bastardes. B Ber. d. deutsch. bot. Ges., Bd. 22 .

1909 Cytologische und morphologische Studien an Drosera longifolia $\mathrm{x}$ rotundifolia. Kungl. Svenska Vetenskapsakad. Handl., Bd. 43, no. 15 .

SARgant, E. 1897 The formation of the sexual nuclei in Lilium martagon. Ann. Bot., vol. 9.

Schrller, J. 1909 Ueber künstliche Erzeugung "primitiver" Kernteilungsformen bei Cyclops. Arch. f. Entwicklm., Bd. 27, no. 4.

Schnerder, K. C. 1910 Histologische Mitteilungen. III. Chromosomengenese. Festschrift R. Hertwig. Bd. 1.

Schreiner, A. UND K. E. 1906 a Neue Studien über Chromatinreifung der Geschlechtszellen.- I. Die Reifung, der männlichen Geschlechtszellen von Tomopteris onisciformis. Arch. Biol., Bd. 22.

1906 b II. Reifung der männlichen Geschlechtszellen von Salamandra maculosa, Spinax niger und Myxine glutinosa. Ibid., Bd. 22.

1908 Gibt es eine parallelle Konjugation der Chromosomen? Erwiderung an die Herren Fick, Goldschmidt und Meves. Videnskbs-Selskab. Skrifter. I. Math.-Naturv. Klasse, No. 4.

Stevens, N. M. 1906 Studies on spermatogenesis. II. A comparative study of the heterochromosomes in certain species of Coleoptera, Hemiptera and Lepidoptera, with especial reference to sex determination. Carnegie Institution Pub. no. 36 .

1908 A study of the germ cells of certain Diptera, with reference to the heterochromosomes and the phenomena of Synapsis. Journ. Exp. Zool., vol. 5, no. 3 .

Stomps, T. J. 1911 Kernteilung und Synapsis bei Spinacia oleracea. Biol. Centralb., Bd. 21, nos. 9-10.

Strasburger, E. 1904 Ueber Reduktionstheilung. Sitzungsber. k. k. Preuss. Akad. Wiss., Bd. 18.

1905 Typische und allotypische Kernteilung. Jahrb. f. Wiss. Bot., Bd. 41.

1907 Ueber die Individualität der Chromosomen und die Propf-hybriden-Frage. Jahrb. f. Wiss. Bot., Bd. 44.

1908 Chromosomenzahlen, Plasmastrukturne, Verebungsträger und Reduktionsteilung. Ibid., Bd. 45.

1909 Zeitpunkt der Bestimmung des Geschlechts, Apogamie, Parthenogenese und Reduktionsteilung. Jena, G. Fischer.

1910 Chromosomenzahl. Ibid. 
Sutron, W. S. 1900 The spermatogonial divisions in Brachystola magna. Kansas Univ. Quart., vol. 9.

1902 On the morphology of the chromosome-group in Brachystola magna. Biol. Bull., vol. 4, no. 1 .

Sykes, M. G. 1909 On the nuclei of some unisexual plants. Ann. Bot., vol. 23. Vejdovsky, F 1907 Neue Untersuchungen über die Reifung und Befruchtung. Königl. Böhmische Ges. d. Wiss., Prag.

De Vries, H. 1903 Befruchtung und Bastardierung, Leipzig.

Winiwarter ET SAintMont 1909 Nouvelles recherches sur l'ovogénèse et

- l'organogenèse de l'ovaires des mammifères (chat). IV. Ovogénèse de la zone cortical primitive. Arch. Biol. tom. 25.

Wruson, E. B. 1905 a The chromosomes in relation to the determination of sex in insects. Science, vol. 22, p. 564.

$1905 \mathrm{~b}$ Studies on chromosomes. I. The behavior of the idiochromosomes in Hemiptera. Jour. Exp. Zool., vol. 2, no. 3.

1905 c Studies, II. The paired microchromosomes, idiochromosomes and heterotropic chromosomes in Hemiptera. Ibid., vol. 2, no. 4.

1906 Studies, III. The sexual differences of the chromosome-groups in Hemiptera, with some considerations on the determination and heredity of sex. Ibid., vol. 3, no. 1.

1909 a Studies, IV. The 'accessory' chromosome in Syromastes and Pyrrhocoris, with a comparative review of the types of sexual differences of the chromosome-groups. Ibid., vol. 6, no. 1 .

$1909 \mathrm{~b}$ The female chromosome-groups in Syromastes and Pyrrhocoris. Biol. Bull., vol. 16, no. 4.

1909 c Photographic illustrations of the morphological and physiological individuality of the chromosomes in Hemiptera (Abstract). Proc. Seventh Internat. Zool. Congr., Boston, August, 1907.

1911 a Studies, VII. A review of the chromosomes of Nezara, with some more general considerations. Jour. Morph., vol. 22, no. 1.

$1911 \mathrm{~b}$ The sex chromosomes. Arch. Mik. Anat., Bd. 77.

1912 Some aspects of cytology in relation to the studv of genetics. Am. Naturalist, vol. 46, February. 
PLATE 120

\section{EXPLANATION OF FIGURES}

All of the figures are from Oncopeltus fasciatus excepting 7 and 8, which are from Lygaeus bicrucis. Enlargement 2250 diameters.

1-3 Spermatogonial metaphases. Oncopeltus.

4-5 Metaphases from ovarian cells.

6 Spermatogonial metaphase. Lygaeus.

7 Metaphase of first spermatocyte-division. Lygaeus.

8-13 Metaphases of first spermatocyte-division in polar view. Oncopeltus.

14-17 Metaphases of same in lateral view, showing the sex-chromosomes near the center.

18 Early anaphase of same division, showing all the chromosomes. The two at the right and the one at the left have been drawn displaced so as not to confuse the central group.

19 Later anaphase, showing approach of the sex-chromosomes.

20 . Slightly later stage, showing the sex-chromosomes in contact at each end of the spindle.

21 Slightly earlier anaphase, from a smear-preparation, showing all the chromosomes. The sex-chromosomes already in conjugation at each pole.

${ }^{20}$ All of the figures of plates 1 to 7 have been drawn as far as possible with the camera lucida, though of course at so great an enlargement it has been necessary to finish much of the finer detail freehand. Many of them are the work of Miss Mabel L. Hedge, to whose skill and painstaking accuracy especial acknowledgment is due. In many cases (as indicated in brackets) the same objects are shown by photographs on plates 8 to 9 . It should be added that some of the finer details appear less clearly in the reproductions of these photographs than in the original negatives. 

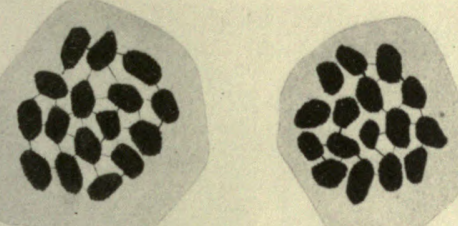

2
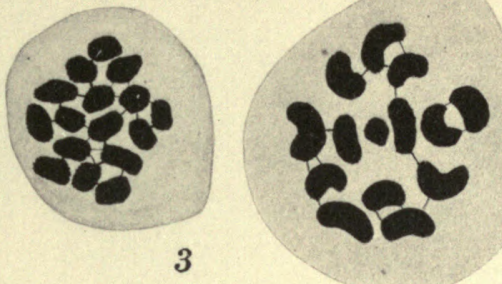

1
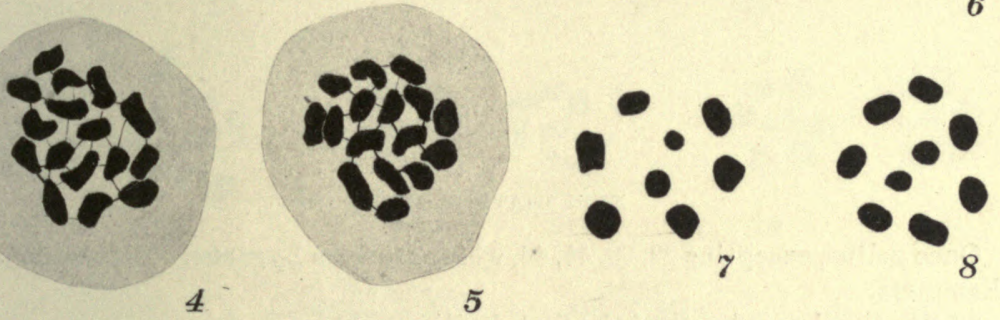

$0_{10}^{1}$

308080000

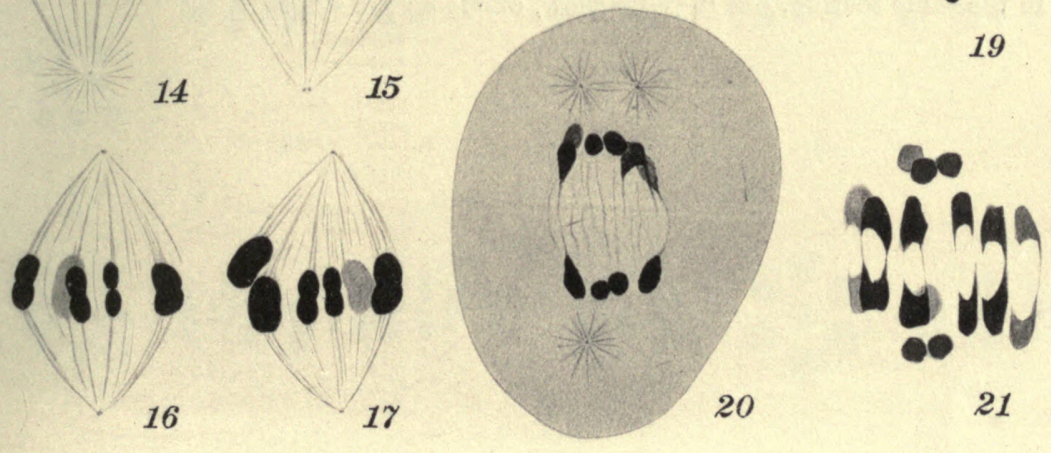


PLATE 2

EXPLANATION OF FIGURES

Onco peltus, excepting $28,29,45,46$, which are from Lygaeus. Enlargement 2250 diameters.

22-23 Final anaphases of the first division. The sex-chromosomes not visible in these views.

24-25 Polar views of two daughter-chromosome plates from the same stage as the foregoing (from different spindles) showing the $X Y$-bivalent near the center (no. 712).

26-27 Similar views of sister-groups from the same spindle (no. 760).

28-29 Sister-groups from the same spindle. Lygaeus.

30-32 Interkineses, showing the chromosome-groups, the first in side-views, the others in face-view showing all the chromosomes (no. 760).

33 Prophase of second division.

34-35 Second division metaphase in polar view.

36-44 The same in side-view, the sex-chromosomes seen separating in several of the figures (36-41, equal type, no. 712; 42-44, unequal type, no. 760).

45-46 (photo. 6) Second division metaphases of Lygaeus, lying side by side in the same section, one in polar view, one from the side. 

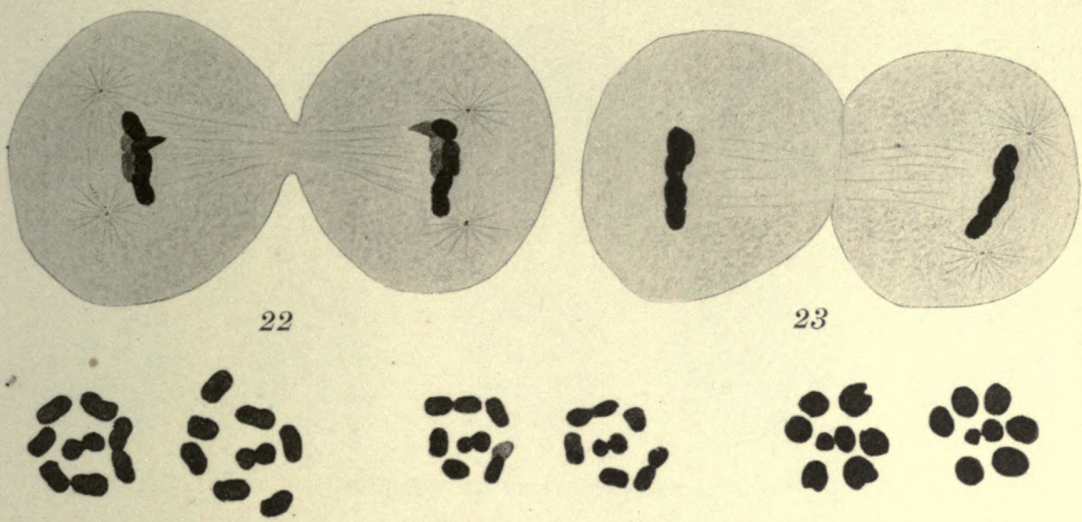

:

24

25

26

27

28

29

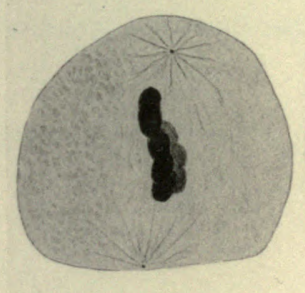

30

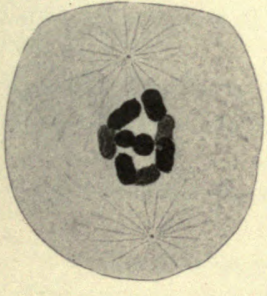

31

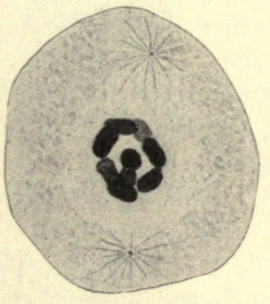

32

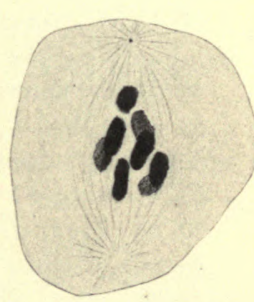

33

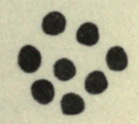

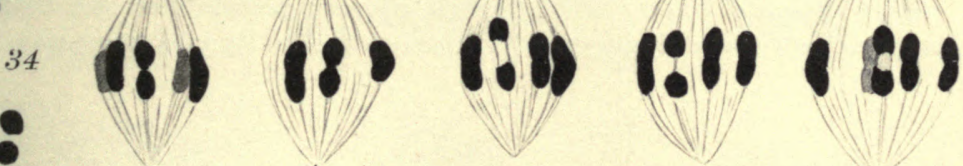

00 - 1

36

37

35

in

41

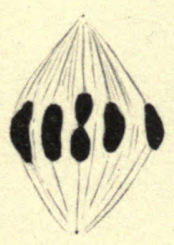

43

38

39

40

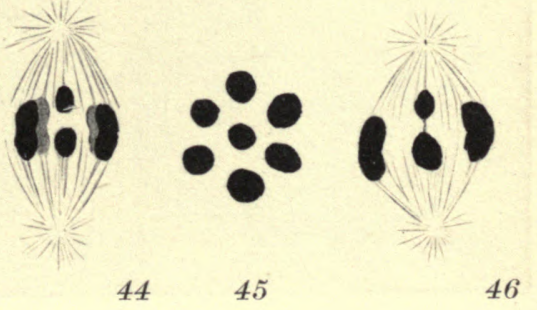

THE JOURNAL OF KXPERIMENTAL ZÖ̈l.ogy, vol. 13, NO. 3.

Wilson, Del. 


\section{PLATE 3}

\section{EXPLANATION OF FIGURES}

Oncopeltus, from sections excepting 65, which is from a smear-preparation. Enlargement 2250 diameters.

47-48 Spermatogonial telophases. It is uncertain whether this is the last division or an earlier one.

49 Later spermatogonial telophase. Probably Stage $a$.

50-51 Stage $b$, showing the massive chromatic bodies, the sex-chromosomes readily distinguishable.

$52-55$ Stages $b-c$, showing the process of unravelling.

56-59 Stage $d$. Leptotene-nuclei.

60 Transition to the synizesis. Synaptic period.

61 Stage $e$. Synizesis, from a very clear specimen.

62 Transition to the following stage.

63 Stage $f$. Pachytene-nucleus. The threads apparently undivided.

64 Stage $f$. Diplotene-nucleus.

65 (photo. 10). Stage $f$. Diplotene-nucleus, from a smear-preparation.

66-67 Stage $g$. The confused stage, showing plasmasome and both chromosome-nucleoli (sex-chromosomes). Plasmasome at its maximum size. 

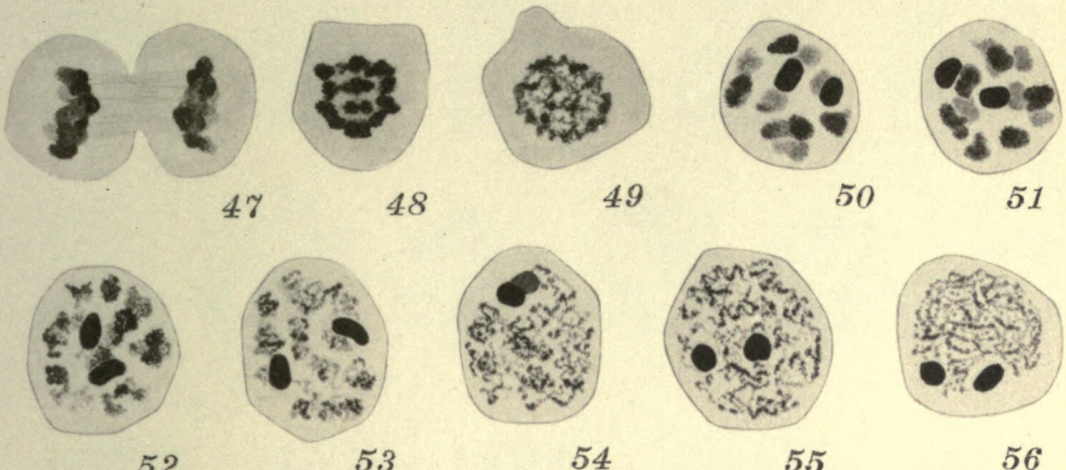

52

53

55

56
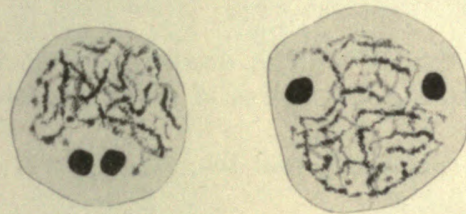

57

58
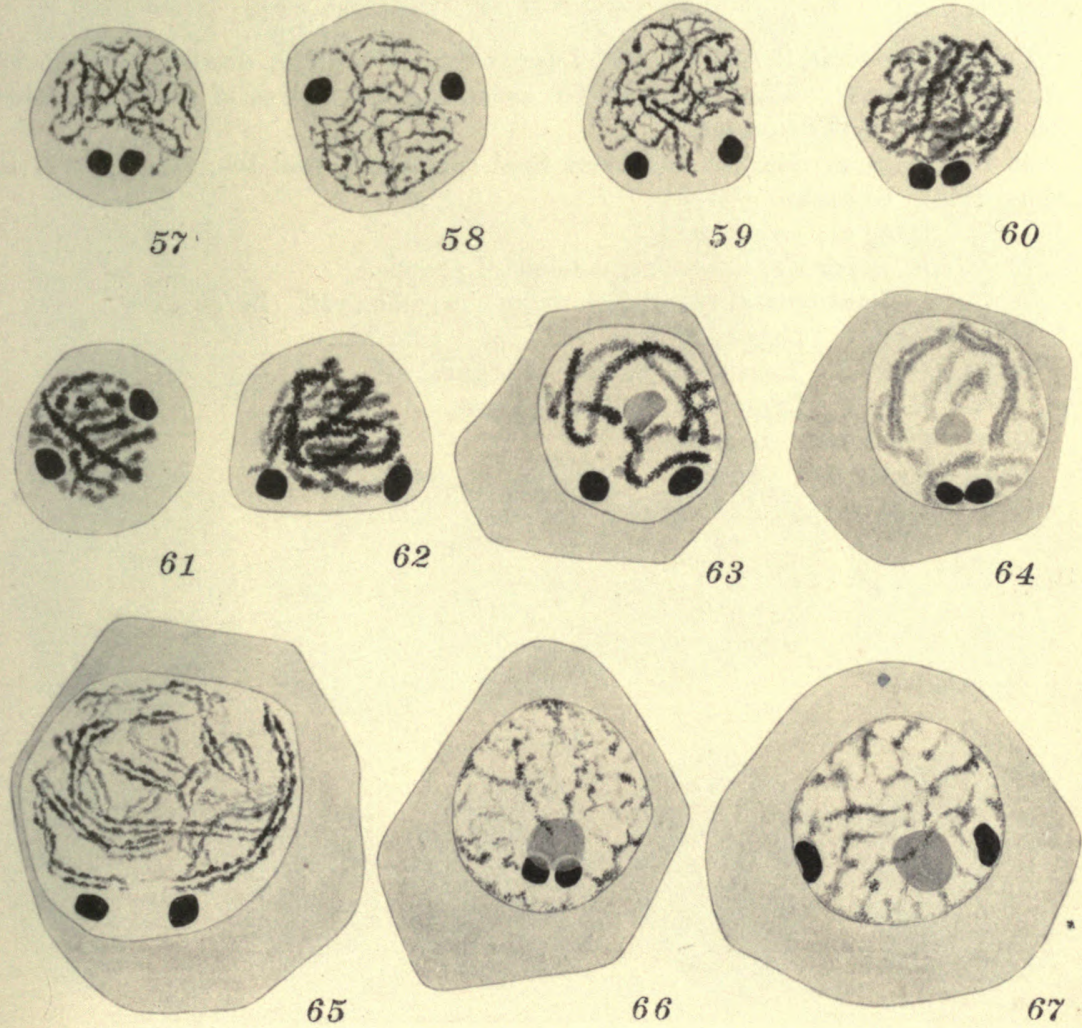


\section{PLATE 4}

\section{EXPLANATION OF FIGURES}

Lygaeus bicrucis (68-73, 83, 84), Largus cinctus (74-82), Anax junius (85-87), Achurum (88-92). Enlargement 2250, excepting the figures of Achurum, which are enlarged 1500 diameters.

68-70 Stage $a$. Earlier and later final spermatogonial telophases, from the same cyst. Lygaeus.

71-72 Stage $b$. Lygaeus.

$73 a-73 b$ Stage $d$. Leptotene-nuclei. Lygaeus.

74-75 Spermatogonial telophases, from the same cyst. Largus.

76-78 Stage $c$. Largus.

79-80 Stage $d$. Leptotene-nuclei. Largus.

81-82 Stage $f$. Diplotene-nuclei. Largus.

83-84 The same. Lygaeus.

85-87 Stages $b-c$. Anax.

88-92 Stages $b-c$. Achurum. 

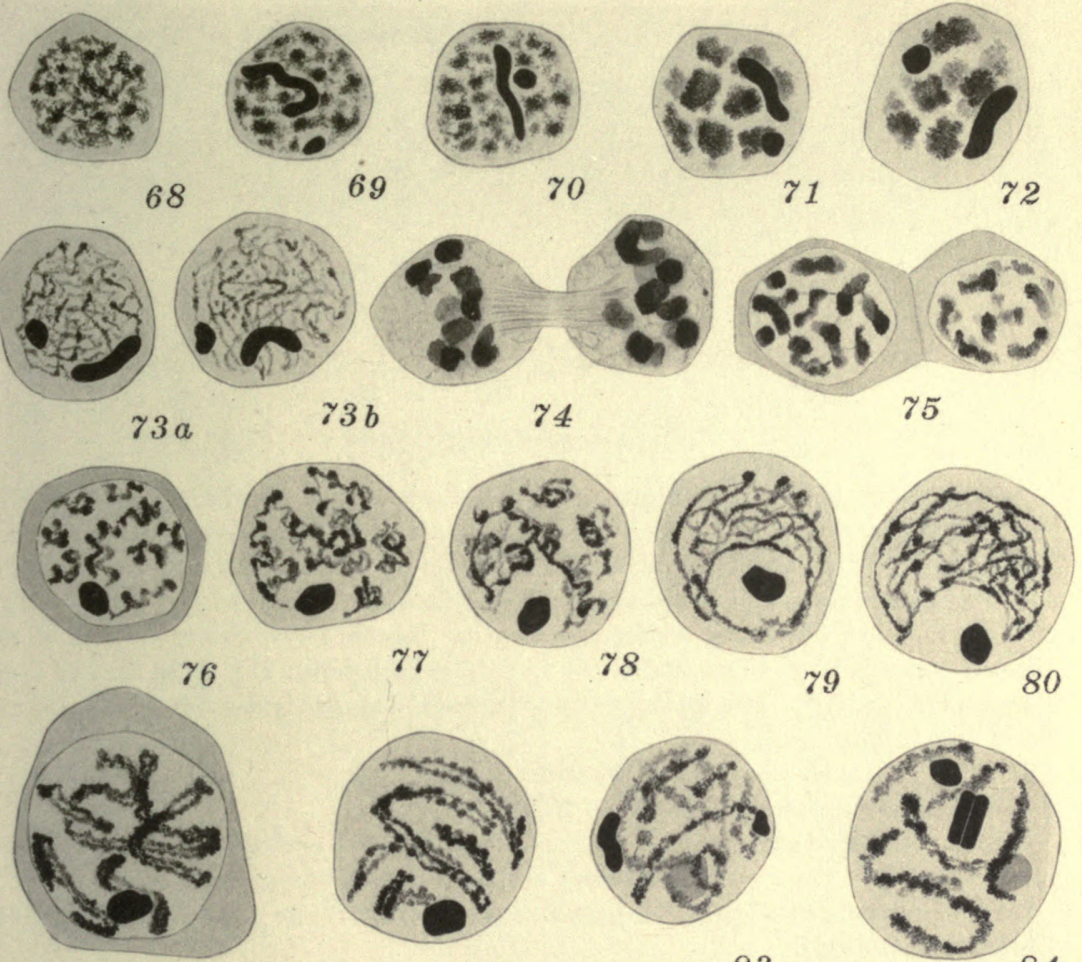

81

82

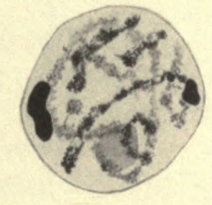

83
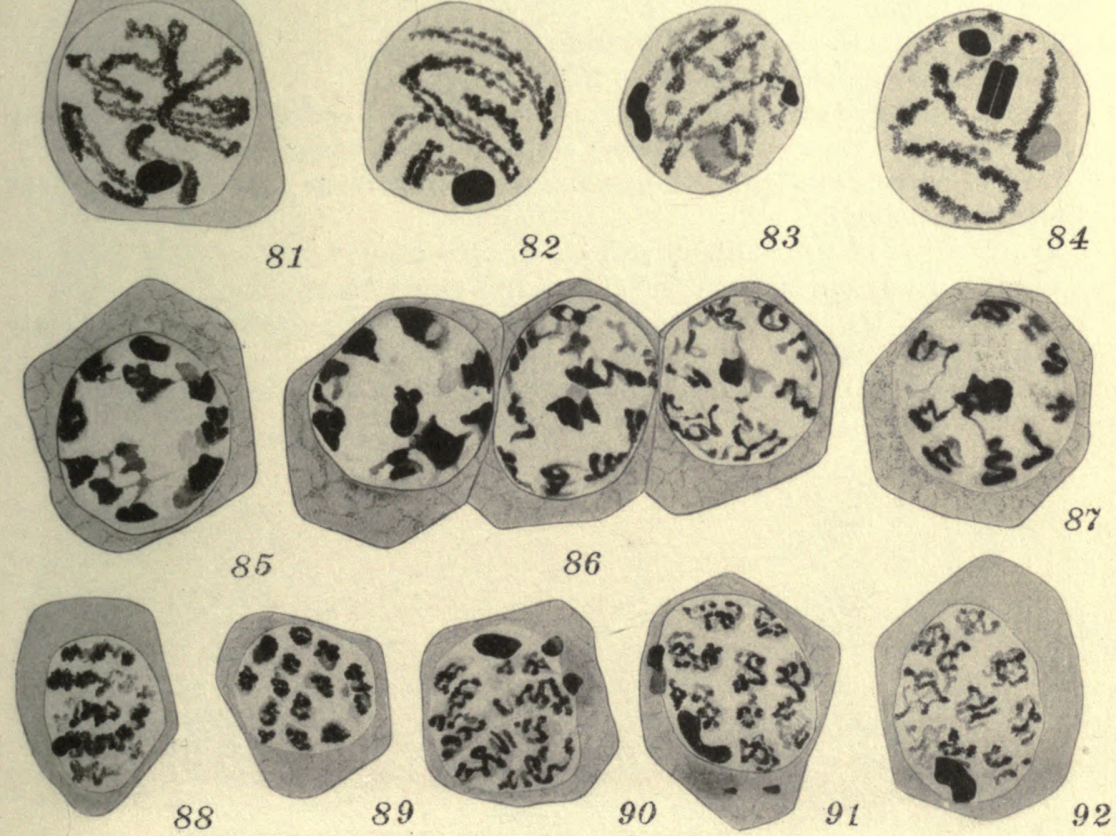


\section{PLATE 5}

\section{EXPLANATION OF FIGURES}

Phrynotettix (93-96), Lygaeus (97-100), Largus (101-104), Oncopeltus (105108). From sections, excepting 107, 108, which are from smear-preparations. The figures of Phrynotettix enlarged 1500 diameters, the others 2250 diameters. 93-95 Spermatogonial prophases of Phrynotettix. Fig. 93 is an early stage, showing massive polarized bodies. The other figures show the uncoiling of the spireme-threads from these bodies, 94 in side view (photo. 31), 95 as viewed from the pole (photo. 30 ). Fig. 96 shows two successive stages lying side by side in the same cyst (photo. 32).

97 The confused stage (Stage $g$ ) in Lygaeus.

98-99 Early prophases (Stage $h$ ) from Lygaeus.

$100 a-h$ Isolated chromosome-nucleoli, from Stages $f$ and $g$ in Lygaeus. showing various forms of the sex-chromosomes assumed during the growth-period.

101-103 Largus cinctus. Nuclei transitional from Stage $f$ (diplotene) to Stage $g$ (confused period).

104 Nucleus of the confused period. Largus cinctus.

105-106 Early prophase-nuclei, Oncopeltus (early Stage $h$ ).

107 (photo. 17) Slightly later prophase-nucleus of Oncopeltus, showing early bivalents.

108 (photo. 18) Later prophase of the same, early Stage $i$. 

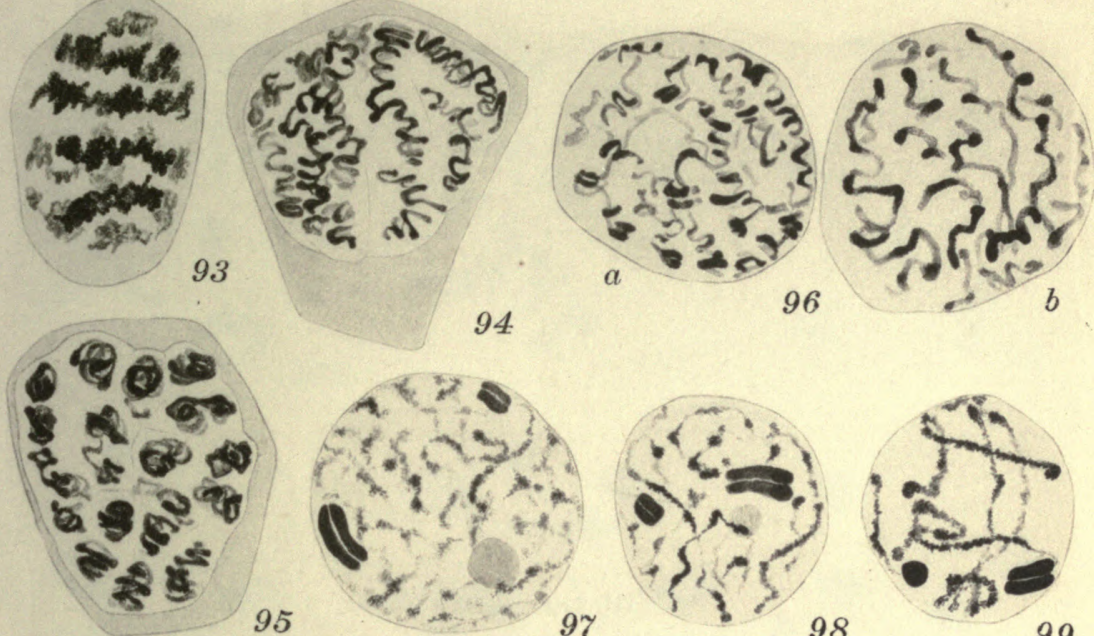

94 b

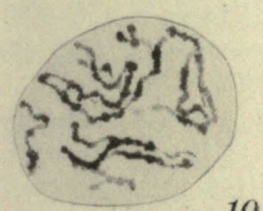

$95+2+29$
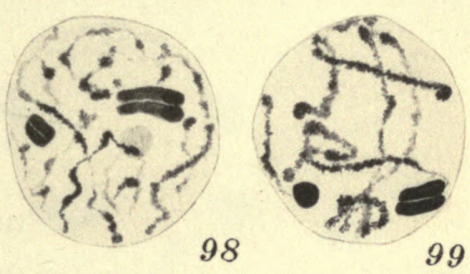

\section{8}

99

101

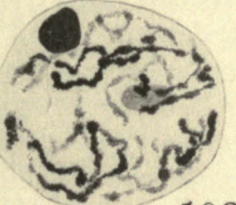

\section{2}
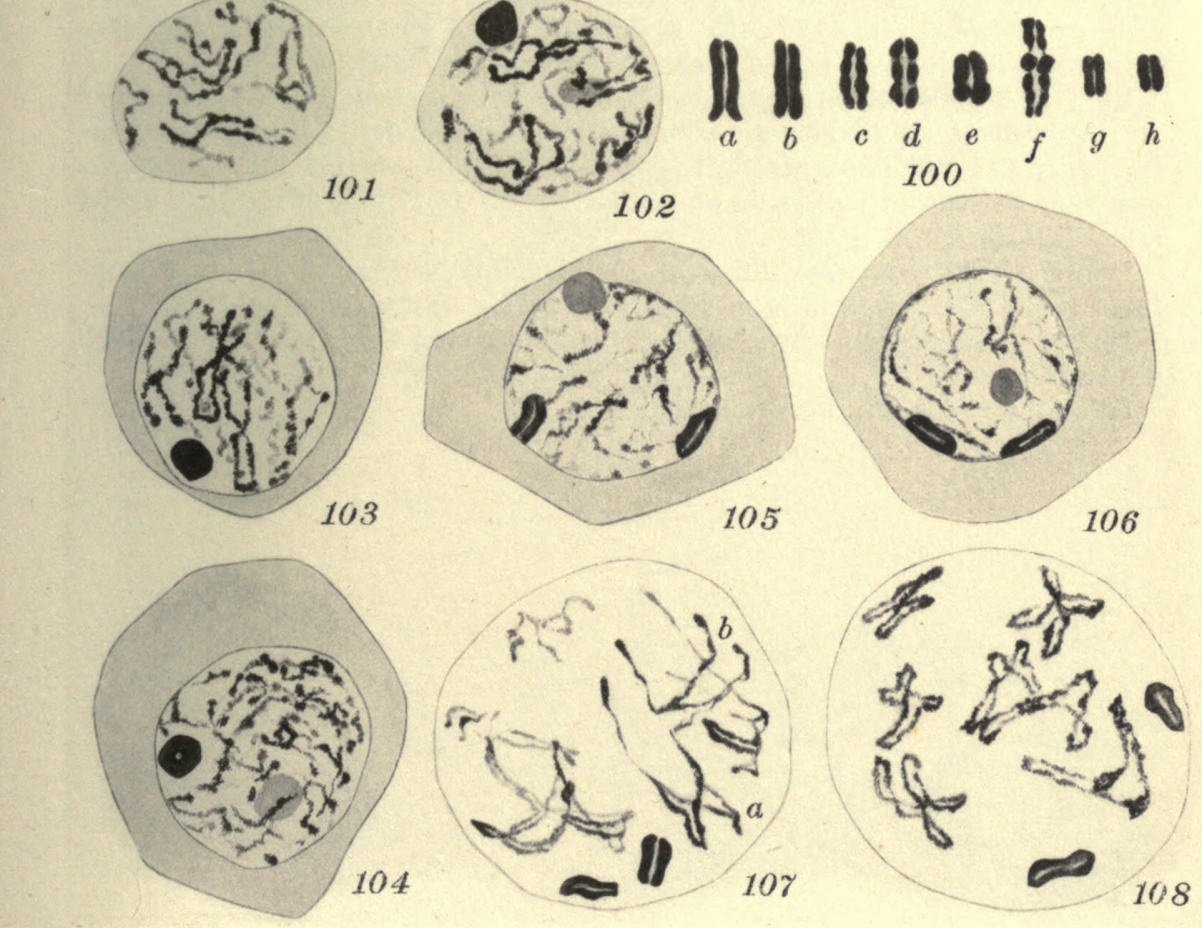


\section{PLATE 6}

\section{EXPLANATION OF FIGURES}

From smear-preparations of Oncopeltus (109-114) and Protenor (115-120); Enlargement 2250 diameters. ( $X$ designates the $X$-chromosome, $B$ the large bivalent in Protenor, $m, m$, the $m$-chromosomes in the latter form.

109-114 Middle and late prophases of the first spermatocyte-division, showing various forms of the bivalents during their condensation. In the earlier figures the $X$-and $Y$-chromosomes are short, longitudinally split rods (109-111); in the later ones they are shortening to a dumb-bell form (112-114). Two of the same nuclei are shown in photos. $20,21$.

115-117 Early prophases (Stage $h$ ), the bivalents just emerging from the confused stage. The $m$-chromosomes are but vaguely distinguishable.

118-119 Late Stage $h$, showing all the chromosomes, the bivalents still much diffused.

120 (photo. 40) Nucleus from Stage $i$. 

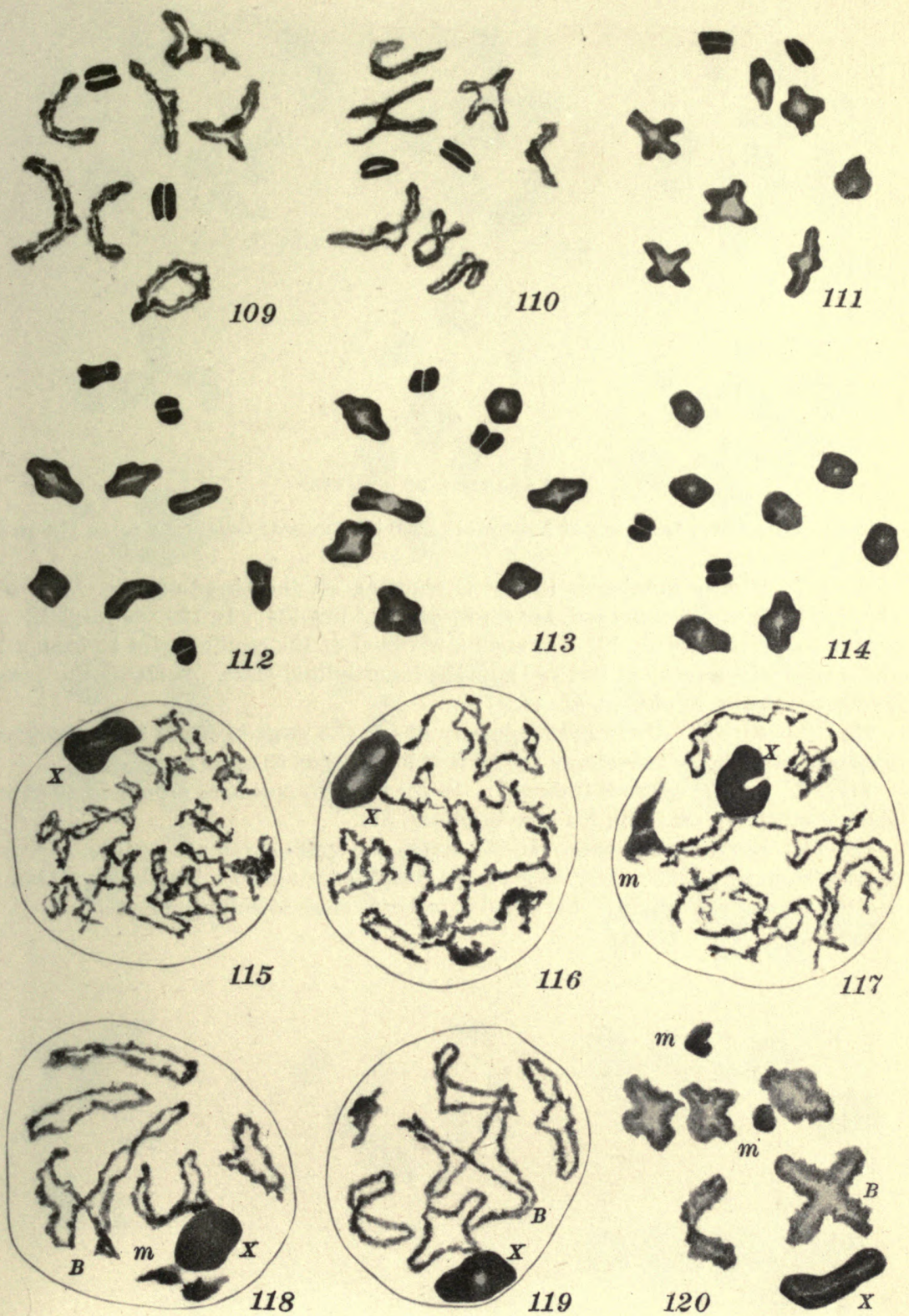

$m$
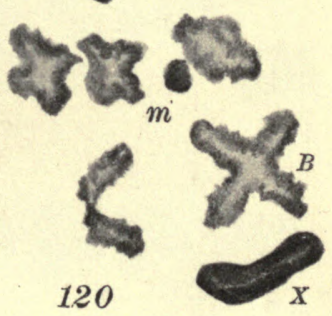


\section{PLATE 7}

\section{EXPLANATION OF FIGURES}

From smear-preparations of Protenor; 2250 diameters; (lettering as in the preceding plate).

12t-127 Middle prophases (Stage $i$ ) showing all the chromosomes. The $m$ chromosomes, now condensed, are separate in all but 124 . In 126 the large bivalent is a straight rod; in 127 it is such a rod bent at the middle point to form a $V$ (here seen edgewise so as not to show the longitudinal cleft. Some of the same nuclei are shown in photos. $41,44,47$.

128 (photo. 43) Abnormal nucleus in which the large bivalent is represented by a pair of separate univalents $(B, B)$ that have failed to unite in synapsis.

129-131 Late prophases (Stage $j$ ). In 131 the chromosomes are ready to enter the metaphase-plate; (fig. 131 also in photo. 51).

132-134 Early metaphases. In 132 one of the small bivalents and the $m$-chromosomes appear abnormally large, owing to flattening. In 134 the ring tetrad to the left has been slightly displaced in order to show it more clearly. 

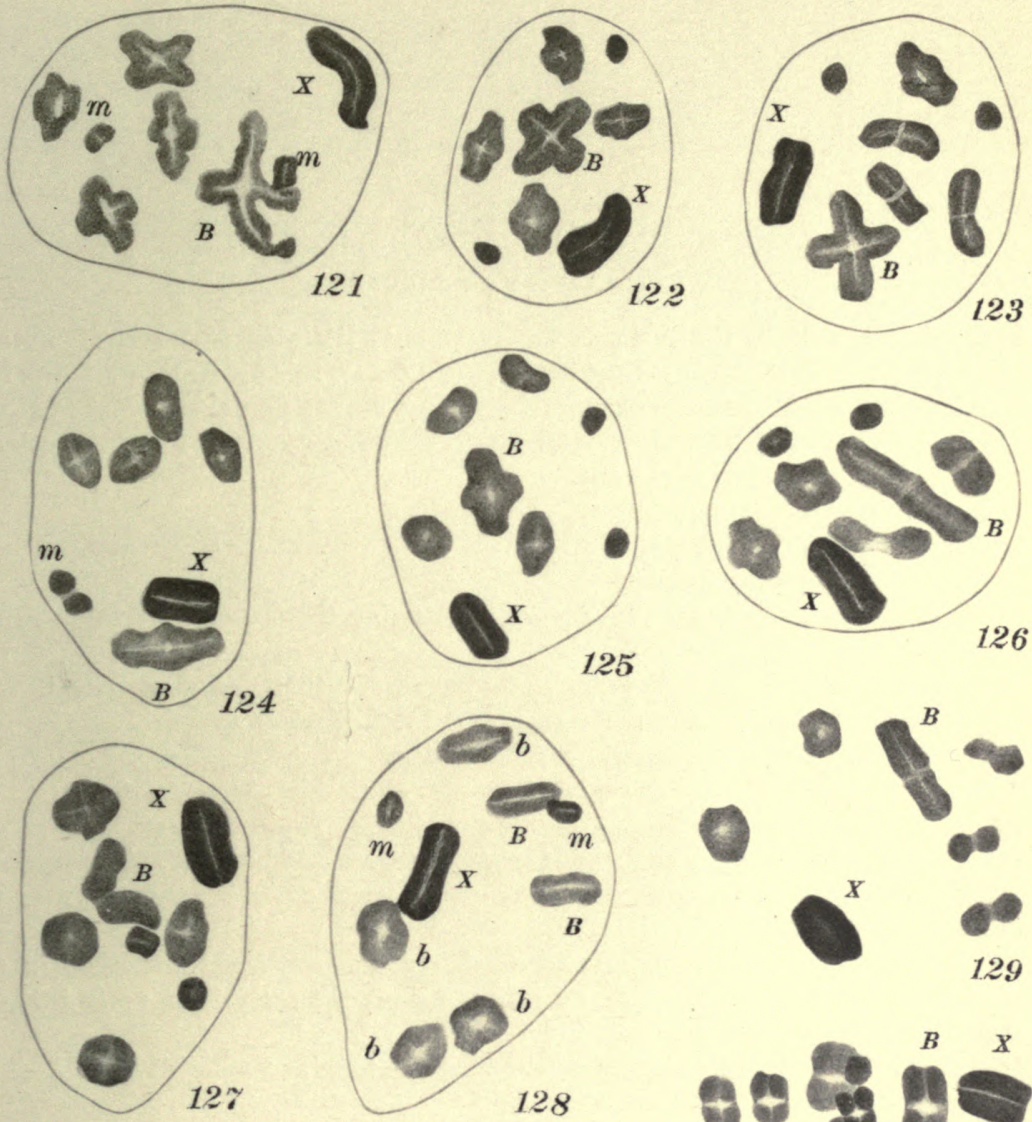

8
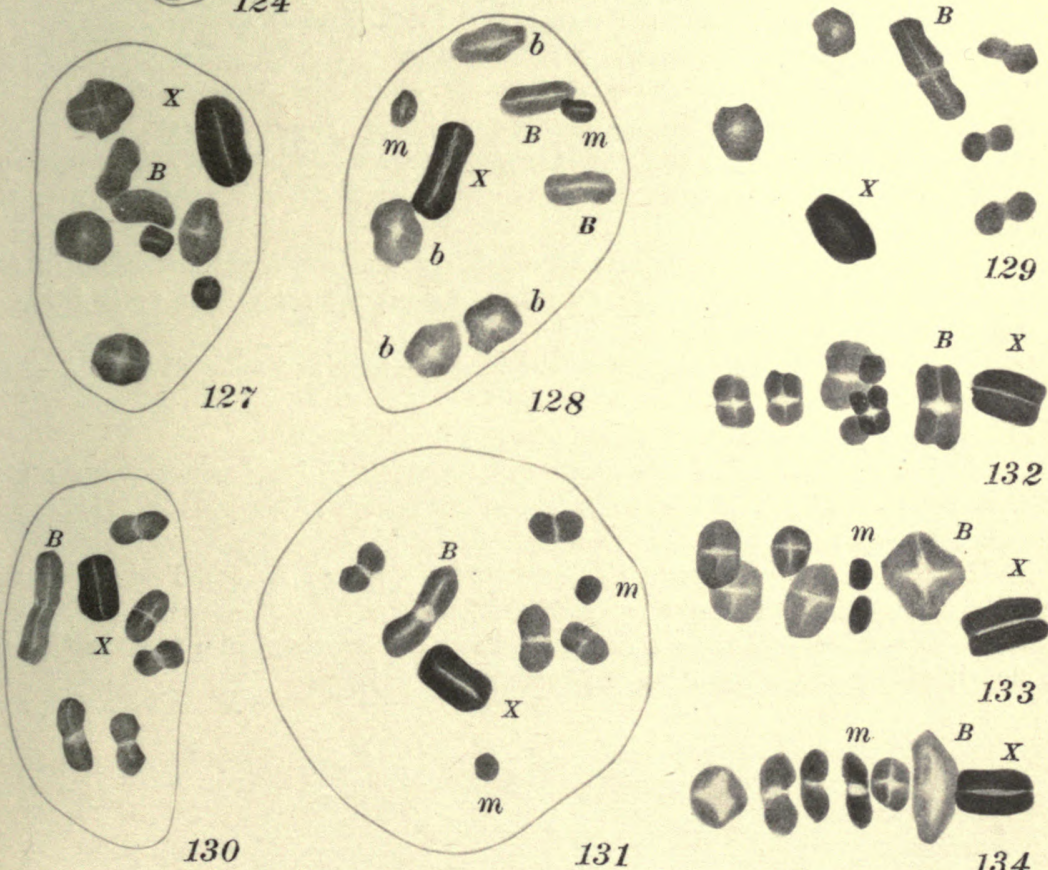

132

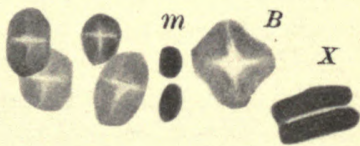

133

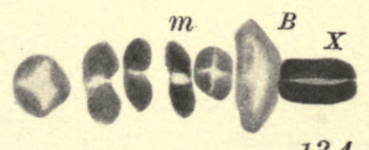

134 


\section{PLATE 8}

\section{EXPLANATION OF FIGURES}

From photographs by the author. Enlargement a little less than 1250 diameters. Oncopeltus (1-5, 7-11, 16-24), Lygaeus bicrucis $(6,12-15,25)$, Largus cinctus (26, 27). Many figures of the same preparations, from drawings, are reproduced in the preceding plates, as indicated in brackets. Photos. 1-15, 26,27, from sections, the others from smear-preparations.

1,2 Spermatogonial metaphases. Oncopeltus.

3 First division metaphase.

4 Second division metaphase.

5 First division metaphase in side view, showing the sex-chromosomes in the center.

6 (Figs. 45, 46) Second division metaphases, one in polar view, one in side view, showing the initial separation of $X$ and $Y$. Lygaeus bicrucis.

7-8 Stage $d$. Leptotene-nuclei of Oncopeltus.

9 Pachytene-nuclei, just emerging from the synizesis. Oncopeltus.

10 (Fig. 65). Stage $f$. Early diplotene-nucleus. From a smear.

11 Stage $g$. Confused stage, showing plasmasome and both sex-chromosomes.

12 Stage $e$. Synizesis, Lygaeus, from much extracted preparation, showing the $X$ - and $Y$-chromosomes united.

13 Above, the $X$-and $Y$-chromosomes of Lygaeus in Stage $f$, attached in one case end to end, in the other side by side. Below, the same from early Stage $g$, showing also the plasmasome.

14-15 Nuclei of Stage $g$, Lygaeus, showing the longitudinally divided $X$-chromosome, and (at the left) the plasmasome.

16 Nucleus of the confused period (Stage $g$ ). Oncopeltus.

17-24 Early, middle and late prophases (Stages $h-j$ ) from smear-preparations of Oncopeltus. Photo. 17 (fig. 107), 18 (fig. 108), 20 (fig. 109), 21 (fig. 111). The sex-chromosomes distinguishable in each case.

25 Late prophase-nucleus of Lygaeus (Stage $i-j$ ), the $X$-and $Y$-chromosomes readily distinguishable above towards the left.

26-27 Stage $b-c$, in Largus cinctus. The uncoiling of spiral leptotene-threads is clearly visible in the negative of photo. 27. 


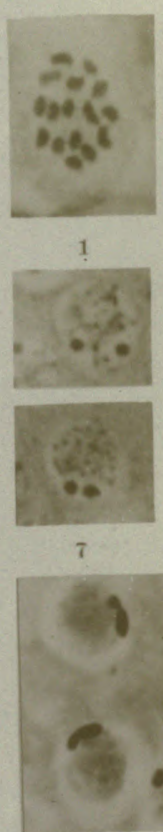

12

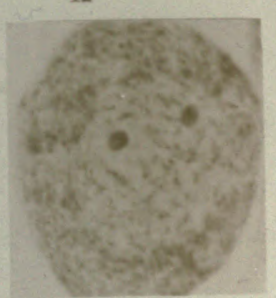

16

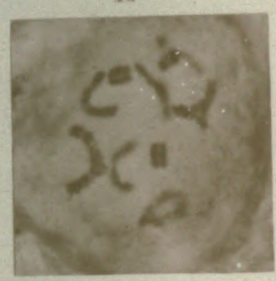

20

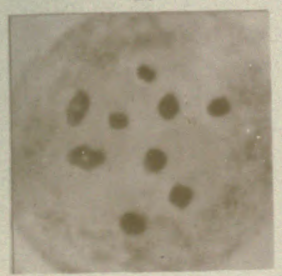

24

2

8

13
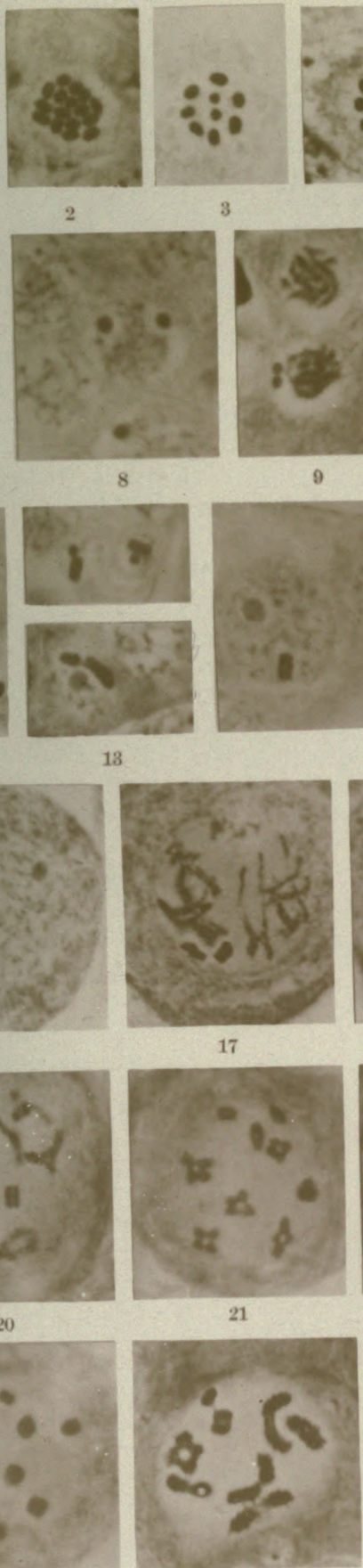

17

21

25

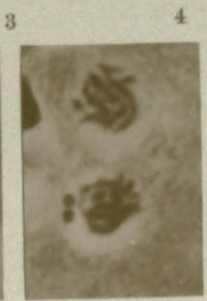

9

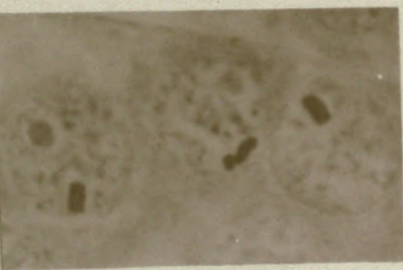

14

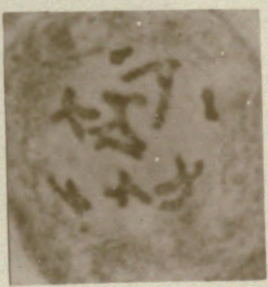

18

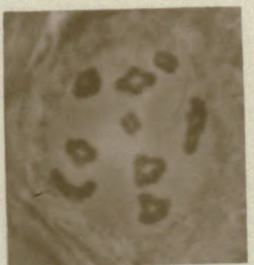

22

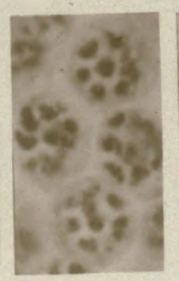

26

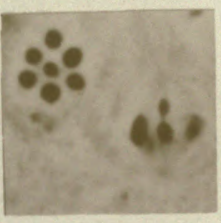

6

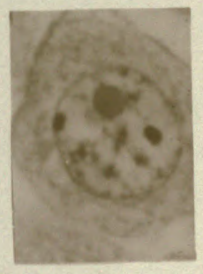

11

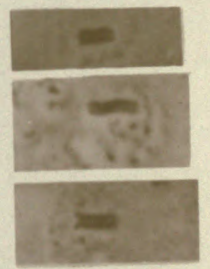

15

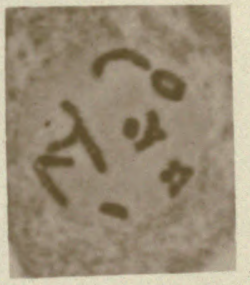

19

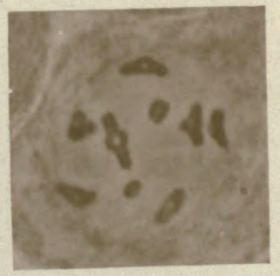

23

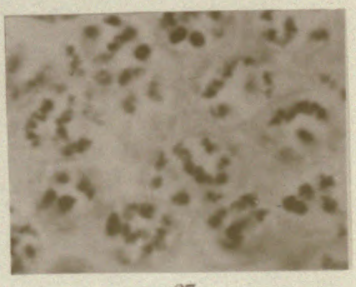

27

THE jOURNAL OF EXPERTMENTAL ZoöLogY, vol. 13, No, 3 


\section{PLATE 9}

\section{EXPLANATION OF FIGURES}

From photographs by the author. Enlargement as in the preceding plate. Photos. 28-38 from sections (28-32 from McClung's preparations), 39-51 from smear-preparations. Photo. 28, Achurum, 29-32 Phrynotettix, 33, 34 Largus cinctus, 35-51 Protenor belfragei.

28 Stage $c$ in Achurum. The unravelling threads clearly shown in the negative.

29 Early spermatogonial prophase of Phrynotettix, showing the massive chromatin-bodies just before the spiral thread is evident.

30 (Fig. 95). At the left, polar view of the coiled threads during the early uncoiling, spermatogonial prophase.

31 (Fig. 94). The same stage (from a nucleus immediately adjoining in the same section) seen in side-view.

32 (Fig. 96). Two adjoining nuclei, showing at the left an earlier, and at the right a later stage of the uncoiling of the spireme-threads. The drawings of these and the preceding photo. show threads at other levels as well.

33 Spermatogonial metaphase of Largus cinctus, 11 chromosomes, including one large pair. The $X$-chromosome is one of the smaller ones, and can not be distinguished by the eye.

34 Metaphase of diploid group of the female of the same species, 12 chromosomes.

35 (Fig. $1 e$, Wilson, '06). Spermatogonial metaphase of Protenor; 13 chromosomes.

36 The same. In both these photos. the large $X$-chromosome and the large pair of autosomes are readily distinguishable.

37-38 Diploid chromosome-groups of the female Protenor, showing the $X$-pair and the large pair of autosomes; 14 chromosomes.

39 Protenor. Above, a nucleus of the confused stage $(g)$ showing the elongate $X$-chromosome. Below are three final anaphases of the second division, showing the passage of the undivided $X$-chromosome to one pole.

40-51 Prophase-nuclei of Protenor. Photo. 40 (fig. 120), 41 (fig. 122) 43 (fig. 128), 44 (fig. 121), 46 (fig. 129), 47 (fig. 127), 51 (fig. 131). 

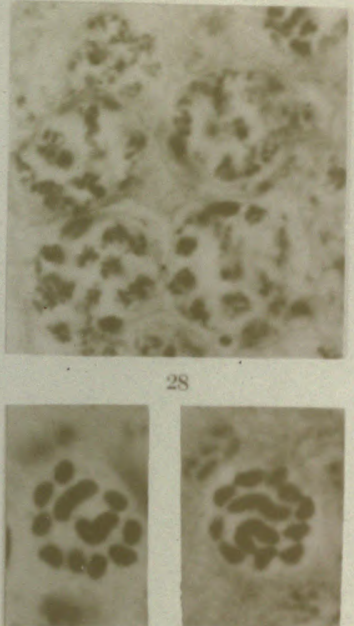

38

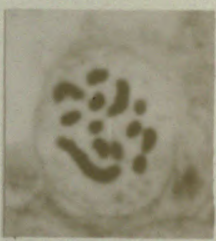

35

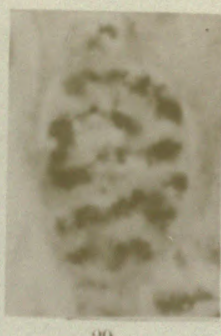

29

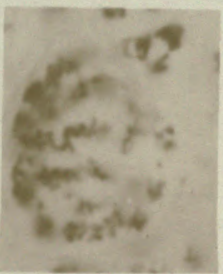

31

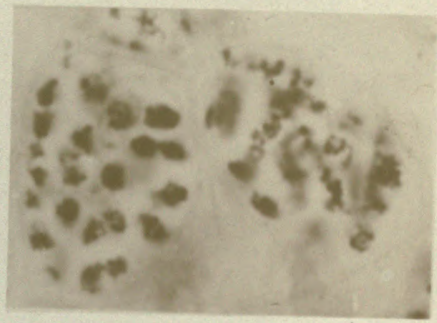

30

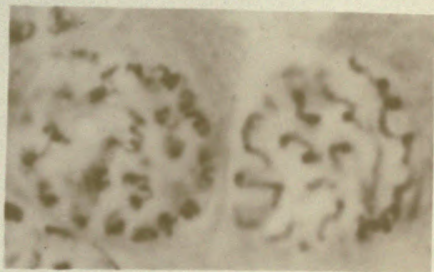

32

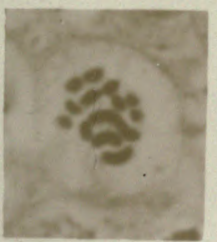

36

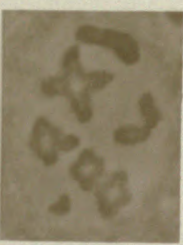

40

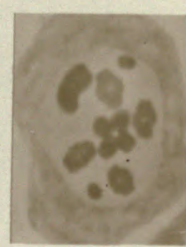

41

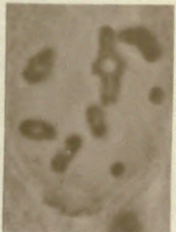

42

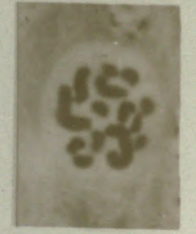

37

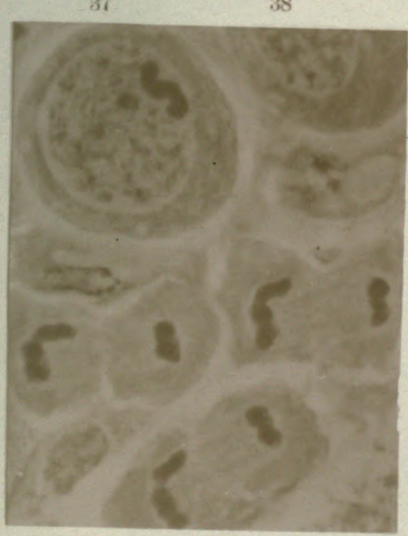

39

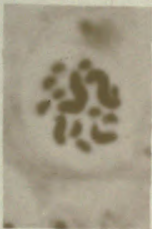

38

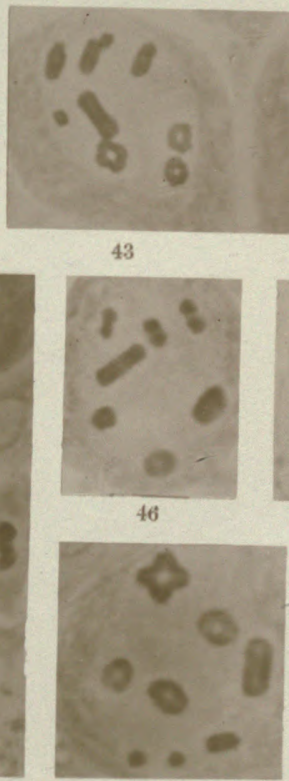

49
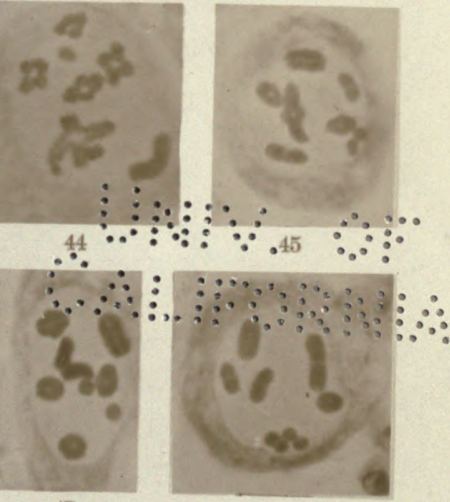

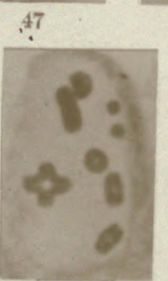

50

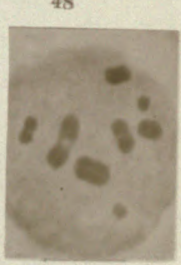

51

THE JOURNAL OF EXPERTEXTAL, zö̈LOGY, voL, 13, No, 3

WHAON PFOTO 



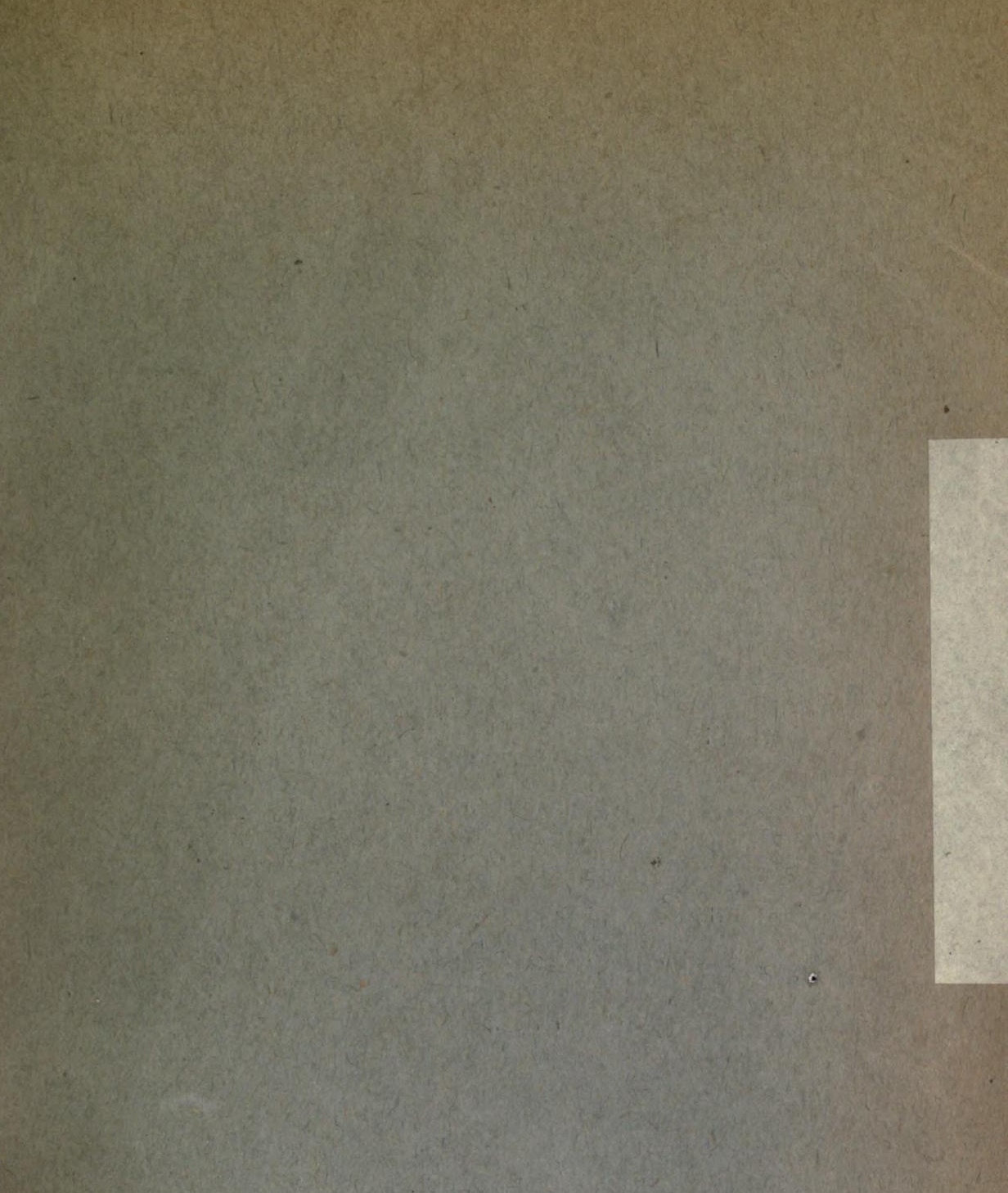

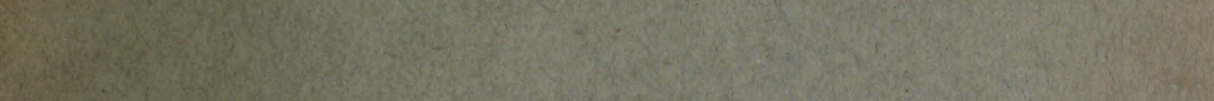

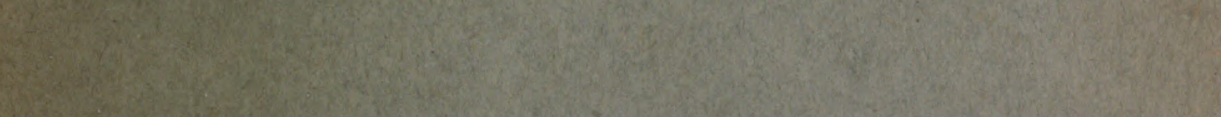

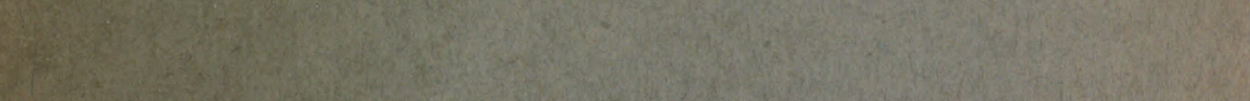

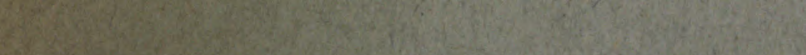

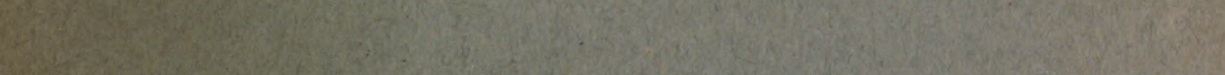

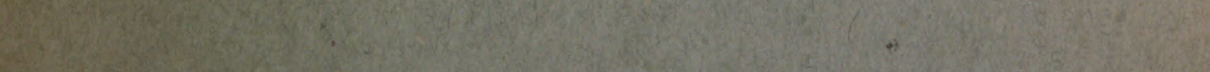

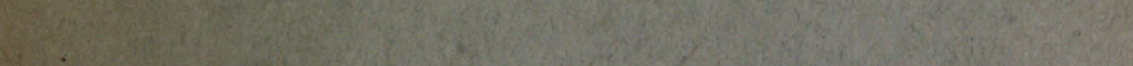

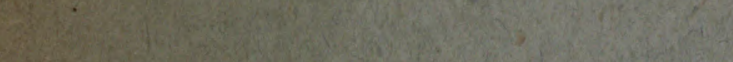

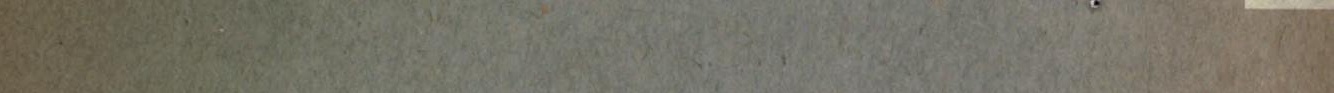
.

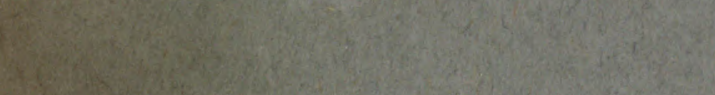

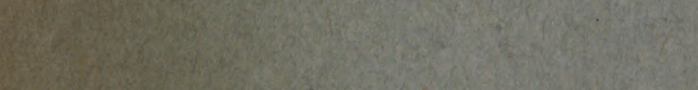
28

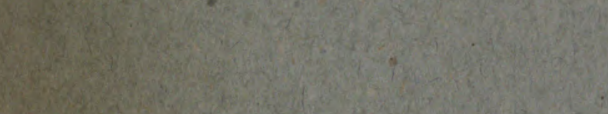
is $x^{2}=$

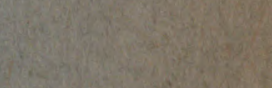

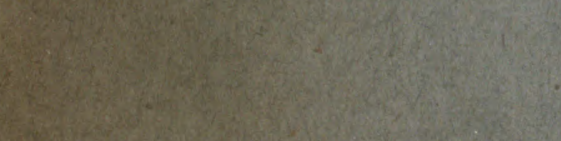

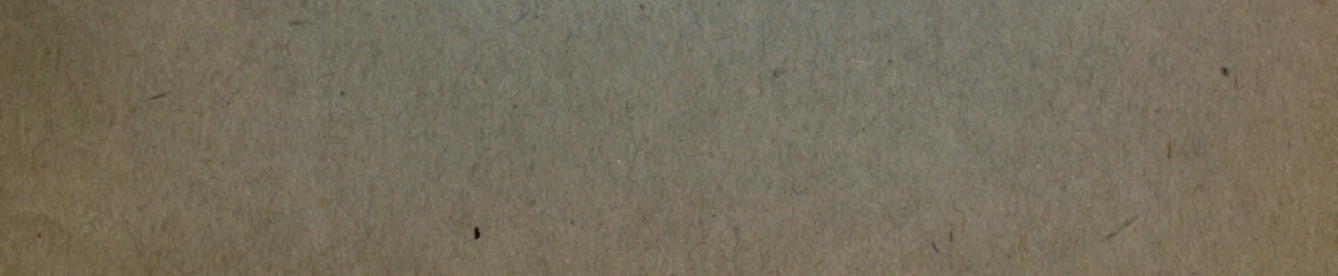
$\sin (-100$

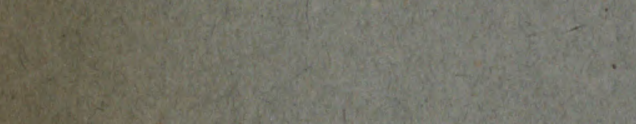

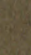
$6-2=2-2=0$ 


\section{THE JOURNAL OF EXPERIMENTAL ZOÖLOGY,}

VOLUME 13, NUMBER 3, OCTOBER, 1912

\section{CONTENTS}

\section{Edmund B. Wilson}

Studies on chromosomes. VIII. Observations on the maturation-phenomena in certain Hemiptera and other forms. with considerations on synapsis and reduction. From the

- Department of Zoölogy, Columbia University. N ne plates 345

\section{Wayland M. Chester}

Wound closure and polarity in the tentacle of IVtridium marginatum. From The Museum of Comparative Zoölogy, Harvard College. Eight figures................. 451

\section{Max Morse}

Artificial parthenogenesis and hybridization in the eggs of certain invertebrates. From Trinity College.......... 471 


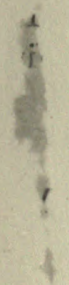

s

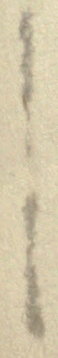





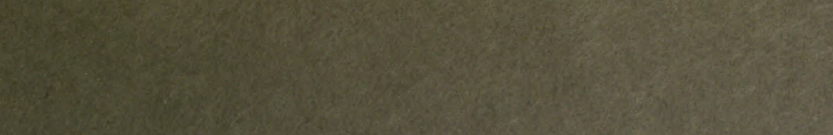

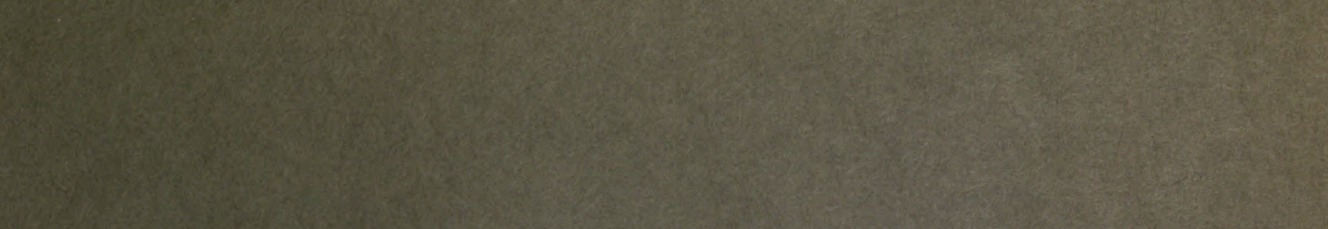

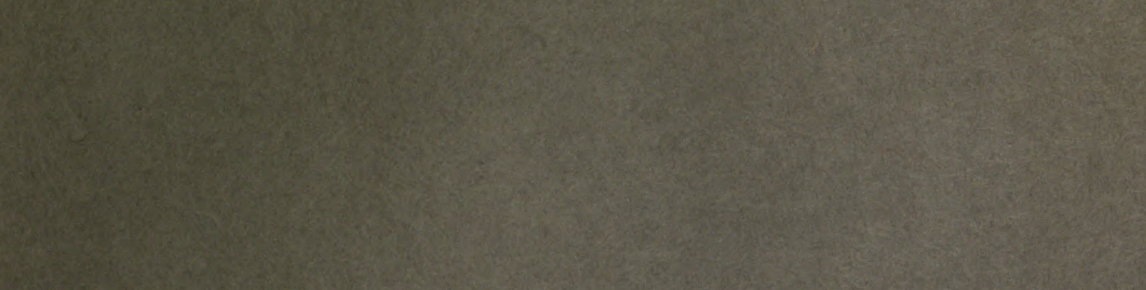

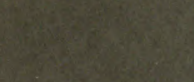

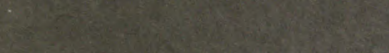

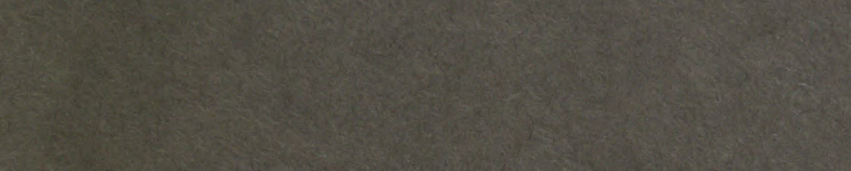




\section{U.C. BERKELEY LIBRARIES |||||||||||||||||||||||||||||||||||||| || Cㄹㄴ 95243}

THE UNIVERSITY OF CALIFORNIA LIBRARY 
